DOE/NASA/0179-1

NASA CR-165143

STOR-80-22

\title{
ANALYTICAL INVESTIGATION OF CRITICAL PHENOMENA IN MHD POWER GENERATORS
}

STD Researci Corpcration

Post Office Box 'C"

Arcadia, California 91006

31 July 1980

Prepared for NATIONAL AERONAUTICS AND SPACE ADMINISTRATION Lewis Research Center Under Contract DEN 3-179

for

U.S. DEPARTMENT OF ENERGY Office of Magnetohydrodynamics Assistant Secretary for Fossil Energy

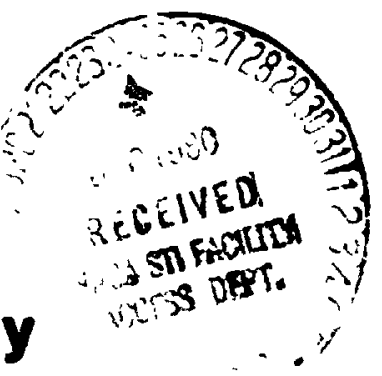


DOE/NASA/0179-1

NASA CR-165143

STDR-80-22

ANALYTICAL INVESTIGATION OF

CRITICAL PHENOMENA IN

MHD POWER GENERATORS

STD Research Corporation

Post CFfice Box "C"

Arcadia, California 91006

31 JuLY 1980

Prepared for

NATIONAL AERONAUTICS AND SPACE ADMINISTRATION

Lewis Research Center

Cleveland, Ohio 44135

Under Contract DEN 3-i79

FOR

U.S. DEPARTMENT OF ENERGY

Office of Magnetohydrodynamics

Assistant Secretary for Fossil Energy

WASHINGTON, D.C, 20545

Under Interagency Agreement EC-77-AA-012674 



\section{ABSTRACT}

Critical phenomena in the Arnold Engineering Development Center (AEDC) High Performance Demonstration Experiment (HPDE) and the U.S. U-25 Experiment, are analyzed. Also analyzed are the performance of a NASA-specified 500 MW(th) flow train and computations carried out by STD Research under Contract AC-01-79ET15501 concerning critical issues for the scale-up of MHD Generators.

The HPDE is characterized by computational simulations of both the nominal conditions and the conditions during the experimental runs. The steady-state performance is discussed along with the Hall voltage overshoots during the start-up and shutdown transients. The results of simulations of the HPDF runs with codes from the Q3D and TRANSIENT code families are compared to the experimental results. The results of the simulations are in grod agreement with the experimental data.

Additional critical phenomena analyzed in the AEDC/HPDE are the optimal load schedules, parametric variations around the simulations of HPDE Run 006-014, the parametric dependence of the electrode voltage drops, the boundary laver behavior, near electrode phenomena with finite electrode segmentation, and current distribution in the end regions.

The U.S. U-25 experiment is characterized by computational simulations of the nominal operating conditions. The steady-state performance for the nominal design of the U.S. U-25 ixperiment is analyzed, as is the dependence of performance on the mass flow rate. 
A NASA-specified $500 \mathrm{MT}(\mathrm{th})$ MHD flot train is characterized for computer simulation and the electrical, transport, and thermodynamic properties at the inlet plane are analyzed.

Issues for the scale-up of MHD power trains are discussed. The AEDC/HPDE performance to date is analyzed to compare these experimental results to scale-up rules. The optimum Mach number distribution is analyzed with emphasis upon its effect on part-load and transient behavior. The effects of alternate cross-sectional shapes on channel performance are also evaluated. 
page

ABSTRACT

TABLE OF CONTENTS

iv

LIST OF TABLES

vii

LIST OF FIGURES

ix

1.0 INTRODUCTION

1

2.0 DESCRIPTION OF THE STD/MHD CODES

3.0 HPDE DATA ANALYSIS AND INTERPRETATION 12

3.1 Channel Characterization $\quad 12$

3.1.1 AEDC/HPDE - Nominal Conditions 12

3.1.1.1 Geometry 12

3.1.1.2 Magnetic Field 14

3.1.1.3 Loading 14

3.1.1.4 Working Fluid 15

3.1.1.5 Wall Conditions 15

3.1.1.6 Channel Inlet Conditions 16

$\begin{array}{ll}3.1 .1 .7 & \begin{array}{l}\text { Other Conditions and } \\ \text { Assumptions }\end{array}\end{array}$

3.1.2 AEDC/HPDE Run - Experimental

Conditions

3.1.2.1 Magnetic Field 17

3.1.2.2 Loading 17

3.1.2.3 Working Fluid 18

3.1.2.4 Wall Conditions 19

3.1.2.5 Channel Inlet Conditions 20

3.2 Acquisition and Processing of HPDE Data 21

3.2.1 Data Acquisition System 21

3.2.2 Meter Panel System 21

3.2.3 STD Interface with HPDE Data Display
Systers

3.3 Steady-State Performance at the Nominal
Operating Condition

3.4 Hall Voltage Overshoots 25 
Table of Contents (cont'd)

3.5 Shock Location 29

3.6 Analysis of Run 006-008 3')

3.7 Steady-State, Q3D Simulation of
Run 006-014

3.8 Steady-State, TRANSIENT Simulation of
Run 006-014

3.9 Comparison of Run 006-014 Measurements
with Q3D Simulations

4.0 CRITICAL PHENOMENA IN THE AEDC/hPDE 172

4.1 Parametric Variation Around the Q3D
Simulation of Run 006-014

4.2 Parametric Dependence of Electrode
Voltage Drops and Plasma Uniformity

$\begin{array}{ll}\text { 4.2.1 Dependence on Electrode } & \begin{array}{l}\text { Nal1 } \\ \text { Temperature }\end{array}\end{array}$

4.2.2 Dependence on Magnetic Field 183

4.2.3 Dependence on Load Fastor 185

4.3 Parametric Variations Around the

TRANSIENT Simulation of Run 006-014 187

$\begin{array}{ll}4.4 \text { Optimal Load Schedules } & 190\end{array}$

4.5 Near Electrode Phenomena with Finite
Electrode Segmentation

4.6 Current Distribution in the End Regions 196

5.0 CRITICAL PHENOMENA POR THE U.S. U-25 EXPERIMENT 225

5.1 Channel Characterization: U-25 Nominal
Operating Conditions

5.1.1 "As-Bul1t" Geometry 225

5.1 .2 Magnetlc Field 226

5.1.3 Loading 226

5.1 .4 Working Fluid 226

5.1.5 Mall Conditions 228

5.1.6 Channel Inlet Conditions 228

5.1.7 Other Conditions and Assumptions 228 
Table of Contents (cont'd)

5.2 Steady-state Performance for Nominal Design

229

5.3 Quasi-Steady Performance as a Function of Mass Flow Rate

231

6.0 PERPORMANCE OF NASA-SPECIFIED 500 MT(TH) MHD GENERATOR

6.1 Charnel Characterization

277

6.2 Generator Inlet Plane Blectrical

Conductivity Calculations

277

7.0 SCALE-UP CONSIDERATIONS FOR MHD POWER TRAINS 283

7.1 AEDC/HPDE Performance To Date 284

7.2 Optimum Mach Number Distribution and Its Bffect on Part-Load and Transient Behavior

286

7.3 Effect of Alternate Cross-Sectional Shapes on Channel Performance

8.0 SUMMARY AND CONCLUSIONS

8.1 HPDE Data Analysis and Interpretation

8.2 Criticai Phenomena in the AEDC/HPDE

8.3 Critical Phenomena in the 0.s. U-25 Experiment

8.4 Blectrical Conductivity Calculations in Coal Flames 


\section{LIST OF TABLES}

Page

Table 3-1 Channel Electrode Spacing

Table 3-2 Nominal Flow Rates of Combustion Gases

Table 3-3 Nominal Magnetic Field for AEDC/HPDE Experiments

Table 3-4

HPDE Load Resistance Settings

Table 3-5 Water Conductivity in Load Buckets for AEDC Experiments

Table 3-6

Flow Rates of Combustion Gases for Runs 006-008 and 006-014

Table 3-7

Load Resistances for AEDC Run 006-014

60

Table 4-1 Results of TRANSIENT Computations Performed for Task I

Table 4-2. Electrical Performance Parameters at $x=2.77 \mathrm{~m}$ in the AEDC/HPDE Run 006-014 200

Table 5-1 Design Data for the U.S. U-2E MHD Generator

Table 5-2 The U.S. U-25 1-D Magnetic Fieli:

Distribution

Table 6-1 Full Load Operating Characteristics

For a NASA-Specified $500 \mathrm{MW}(\mathrm{th})$ MHD

Channel

Table 6-2 Thermodynamic Data to Define the Working fluid for a NASA-Specified 500 MW(th) MHD Channel

Table 6-3

List of Species Used to Define the Working Fluid at the Inlet of the NASA-Specified $500 \mathrm{MW}(\mathrm{th})$ Generator 


\section{List of Tables (cont'd)}

Table 7-1 Interactio. Parameters and Performance Indices for Evaluation of MHD

Generator Performance Scaling

Table 7-2 List of Final TRANSIENT Ccmputations

Performed Under Contract AC-01-79ET 15501

Table 7-3 List of Final Q3DYZ Computations

Performed Under Contract AC-01-79ET-15501

299

Table 7-4 Data from Q3DYZ Computations Performed Under Contract AC-01-79ET 15501 from the Nominal $20 \mathrm{MW}(\mathrm{th})$ Channel

Table 7-5 Data from Q3DYZ Computations Performed Under Contract AC-01-79ET15501 from the Nominal $50 M W(t h)$ Channel

Table 7-6

Data from Q3DYZ Computations Performed Under Contract AC-01-79ET15501 from the Nominal $300 \mathrm{MW}(\mathrm{th})$ Channel

Table 7-7

Data from Q3DYZ Computations Performed Under Contract AC-01-79ET15501 from the Nominal $2000 \mathrm{MT}(\mathrm{th})$ Channel 


\section{LIST OF FIGURES}

Page

Figure 3-1 AEDC/HPDE internal geometry with designated cross sections

61

Figure 3-2 Axial variation of the channel geometry in the AEDC/HPDE at the nominal $2 \mathrm{~T}$ operating conditions

Figure 3-3 Magnetic field strength distribution along magnet bore

Figure 3-4 AEDC/HPDE nominal wall surface temperature distribution

Figure 3-5 Location of the Hall probes on the floor of the magnet bore

Figure 3-6 Time history of electrode wall surface temperature for various heat transfer rates, as calculated by ARO, Inc. $[3-11]$

Figure 3-7 AEDC/HPDE electrode wall surface temperature distribution (revised), calculated by ARO, Inc. [3-12]

Figure 3-8 Computed time history of pressure distribution throughout flow train of typical $300 \mathrm{MWTh}$ MHD generator during start-up

Figure 3-9 Voltage anode to ground, HPDE Run 006-008

Figure 3-10 Electrical power output as a function of mass flow rate ind magnetic field for the nominal operating conditions of the AEVC/HPDE

Figure 3-11 Enthalpy extraction ratio as a function of mass flow rate and magnetic field for the nominal operating conditions of the AEDC/HPDE

Figure 3-12 Axial variation of the channel geometry in the AEDC/HPDE at the nominal $2 \mathrm{~T}$ operating conditions: Q3D calculation 
List of Figures (cont'd)

Figure 3-13 Axial variation of the centerline gasdynamic variables in the AEDC/HPDE at the nominal $2 \mathrm{~T}$ operating conditions: Q3D calculation

Figure 3-14 Axial variation of the plasma parameters in the AEDC/HPDE at the nominal $2 \mathrm{~T}$ operating conditions: Q3D calculation

Figure 3-15 Axial variation of the electric loading in the AEDC/HPDE at the nominal $2 \mathrm{~T}$ operating conditions: Q3D calculation

Figure 3-16 Axial variation of the power extraction parameters in the AEDC/HPDE at the nominal $2 T$ operating conditions: Q3D calculation

Figure 3-17 Axial variation of the electrical field variables in the AEDC/HPDE at the nominal 2 operating conditions: Q3D calculation

Figure 3-18 Axial variation of the short circuit fields and the local internal impedence in the AEDC/HPDE at the nominal $2 \mathrm{~T}$ operating conditions: Q3D calculation

Figure 3-19 Axial variation of the electrical stresses on the electrodes in the AEDC/HPDE at the nominal $2 \mathrm{~T}$ operating conditions: Q3D calculation

Figure 3-20 Axial variation of the electric potential in the AEDC/HPDE at the nominal $2 T$ operating conditions: Q3D calculatiud

Figure 3-21 Axial variation of the elertrode vol age drops in the AEDC/HPDE at the nomina. $2 \mathrm{~T}$ operating conditions: Q3D calculation

Figure 3-22 Axial variation of the electrode boundary layer parameters in the AEDC; : $P D E$ at the nominal 2 T operating conditiuns: Q3D calculation 


\section{List of Figures (cont'd)}

Figure 3-23 Axial variation of the sidewall boundary layer parameters in the AEDC/HPDE at the nominal $2 \mathrm{~T}$ operating conditions:

Q3D calculation

Figure 3-24 Axial variation of the heat transfer parameters in the AEDC/HPDE at the nominal $2 \mathrm{~T}$ operating conditions: Q3D calculation

Figure 3-25 Anode to ground voltage of electrode 336 for AEDC/HPDE Run 006-008

Figure 3-26 Anode Voltage anode to ground, HPDE Run 006-008

Figure 3-27 Cathode to ground voltage distribution for AEDC/HPDE Run 006-008

Figure 3-28 Total Hall voltage for AEDC/HPDE Run 006-008

Figure 3-29 Axial variation of static pressure for the AEDC/HPDE at the $4 \mathrm{~T}$ nominal operating conditions, load resistance

Figure 3-30 Axial variation of Mach number sor the 4 Tominal operating conditions

Figure 3-31 Position of the normal shock as a function of mass flow rate and magnetic field for the nominal operation conditions of the AEDC/HPDE

Figure 3-32 Axial variation of the centerline gasdynamic variables from computation CMSIPUFIKW for the AEDC/HPDE

Figure 3-33 Axial variation of the plasma properties from computation CMSIPUFIKT for the AEDC/HPDE

Figure 3-34 Axial variation of the short circuit variables and internal impedance from computation CMSIPUFIKW for the AEDC/HPDE 


\section{List of Figures (cont'd)}

Figure 3-35 Axial variation of electrical loading from computation CMSIPUFIKW for the AEDC/HPDE

Figure 3-36 Axial variation of th power extraction parameters from computation CMSIPUFIKW for the AEDC/HPDE

Figure 3-37 Axial variation of the electrical field variables from compita:ion CMSIPUFIKW for the AEDC/HPDE

Figure 3-38 Axial variation of the electric potential from computation CMSIPUFIKW for the AEDC/HPDE

Figure 3-39 Axial variation of the electrode voltage drops from computation CMSIPUFIKW for the AEDC/HPDE

Figure $3-40$

Axial variation of the heat transfer parameters from computation CMSIPUFIKW for the AEDC/HPDE

Figure 3-41 Axial variation of the electrode boundary layer parameters from computation CMSIPUFIKW for the AEDC/HPDE

Figure 3-42 Axial variation of the sidewall boundary layer parameters from computation CMSIPUFIKW for the AEDC/HPDE

Figure 3-43 Axial ariation of the channel geometry from :omputation CMSIPUFIKW for the AEDC/HP'JE

Figure 3-44 Axial variation of the centerline gasdynamic variables from computation CMSIQBEIKW for the AEDC/HPDE

Figure 3-45 Axial variation of the plasma properties from computation CMSIZBEIKW for the AEDC/HPDE

Figure 3-46 Axial variation of the short circuit variables and internal impedence from computation CMSIQBEIKW for the AEDC/HPDE 


\section{List of Figures (cont'd)}

Figure 3-47 Axial variation of the eiectrical loading from computation CuSIQBEIKI for the AEDC/HPDE

Figure 3-48 Axial variation of the power extraction parameters from computation CUSIQBEIXI for the $A B D C / H P D E$

Figure 3-49 Axial variation of the electrical field variables from computation CMSIQBEIKI for the ABDC/HPDE

Figure 3-50 Axial variation of the electric potential from computation CuSIQBEIK for the AEDC/HPDE

Figure 3-51 Axial variation of the electrode voltige drops from computation CuSIQBEIK for the AEDC/HPDE

Figure 3-52 Axial variation of the heat transfer parameters from computation CMSIQBEIKT for the AEDC/HPDE

Figure 3-53 Axial variation of the electrode boundary layer parameters from computation CASIQBEIK for the AEDC/HPDB

Figure 3-54 Axial variation of the sidewall boundary layer parameters from computation CUSIQBEIKT for the AEDC/HPDE

Pigure 3-55 Axial variation of the channel geometry from computation CuSIQBEIKT for the AEDC/HPDE

Figure 3-56 Calculated and measured static pressure distribution in channel

Figure 3-57 Cathode potential from Run 006-008 and Q3D Run QMSIQBEIKT

Figure 3-58 Anode and cathode potential: Run 006-008

Figure 3-59 Normai current density - experimental and theoretical 


\section{List of Pigures (cont'd)}

Figure 3-60 Faraday voltage - experimental and theoretical

120

Figure 3-61 Power per electrode - experimental and theoretical

121

Figure 3-62 Faraday voltage, normal current density and power: $A E D C$ Run 006-008

122

$\begin{array}{lll}\text { Figure 3-63 Power, HPDE Run 006-014 } & 123\end{array}$

$\begin{array}{llr}\text { Figure 3-64 Power, HPDE Run 006-014 } 124 & \end{array}$

Pigure 3-65 Paraday voltage, HPDE Run 006-014 125

Figure 3-66 Faraday vol tage, HPDE Run 006-014 126

Figure 3-67 Faraday current, HPDE Run 006-014 127

Figure 3-68 Faraday current, HPDE Run 006-014 128

Pigure 3-69 Voltage anode to ground, HPDE Run 006-014 129

Pigure 3-70 Voltage anode to ground, HPDE Run 006-014 130

Figure 3-71 Voltage cathode to ground, HPDE Run 006-014 131

Figure 3-72 Voltage cathode to ground, HPDE Run 006-014 132

Figure 3-73 Load resistance for HPDE Run 006-014 133

Figure 3-74 Channel geometry: HPDE Run 006-014 134

Figure 3-75 Electrical loading: HPDE Run 006-014 135

$\begin{array}{lll}\text { Figure 3-76 Interaction parameter based on velocity: } & 136 \\ \text { HPJE Run 006-014 } & \end{array}$

Figure 3-77 Interaction parameter based on pressure:
HPDE Run 006-014

$\begin{array}{lll}\text { Figure 3-78 Centerline gasdynamic variables: } & \\ & \text { HPDE Run 006-014 } & 138\end{array}$

$\begin{array}{ll}\text { Figure 3-79 Electrode boundary layer parameters: } & \text { i39 } \\ & \text { HPDE Run 006-014 }\end{array}$ 


\section{Iist of Figures (cont'd)}

Figure 3-80 Sidewall boundary layer parameters:

HPDE Run 006-014

140

Figure 3-81 Heat transfer parameters: HPDE Run 006-014

Figure 3-82 Plasma properties: HPDE Run 006-014 142

Figure 3-83 Nonuniformity factors: HPDE Run 006-014 143

Figure 3-84 Short circuit variables and internal impedance: HPDE Run 006-014

Pigure 3-85 Electrical stresses on the electrodes: HPDE Run 006-014

Figure 3-86 Power extraction parameters: HPDE Run 006-014

Figure 3-87 Electrical field variables: HPDE Run 006-014

Figure 3-88 Electric potential: HPDE Run 006-014

Figure 3-89 Electrode voltage drops: HPDE Run 006-014

Figure 3-90 Flow train geometry for AEDC Run 006-014

Figure 3-91 Axial distribution of average gasdynamic variables for AEDC Run 006-014

Figure 3-92 Axial distribution of electrode boundary

layer parameters for AEDC Run 006-014

Figure 3-93 Axial distribution of boundary layer heat transfer parameters for AEDC Run 006-014

Figure 3-94 Axial distribution of plasma properties for AEDC Run 006-014

Figure 3-95 Axial distribution of electrical loading for AEDC Run 006-014

Figure 3-96 Axial distribution of electrical field variables for AEDC Run 006-014 
List of Figures (cont'd)

Figure 3-97 Axial distribution of power extraction parameters for AEDC Run 006-014

Pigure 3-98 Axial distribution of electric potential for AEDC Run 006-014

Figure 3-99 Comparison of the axial distributions of static pressure from the data acquisition system and STD simulations of HPDE Run 006-014

Figure 3-100 Comparison of axial distributions of Fare ay voltage from the experimental data and STD simulations of HPDE Run 006-014

Figure 3-101 Comparison of the axial distributions of current per electrode from the experimental data and STD simulations of SPPDE Run 006-014

Figure 3-102 Comparison of the axial distributions of normal current density from the experimental data and STD simulations of HPDE Run 006-014

Figure 3-103 Comparison of the axial distributions of load resistance from the experimental data and STD simulations of HPDE Run 006-014

Figure 3-104 Comparison of the axial distribut ns of power per electrode from the experimental data and STD simulations of HPDE Run 006-014

Figure 3-105 Comparison of the axial distributions of integrated pows output from the experimental data and STD simulations of HPDE Run 006-014

Figure 3-106 Comparison of the axial distributions of 2-D electrode vol tage drop VD(V) for HPDE run 006-014 


\section{List of Figures (cont'd)}

Figure 3-107 Cumparison of the axial distributions of centerline potential from the experimental data and STD simulations of HPDE Run 006-014

Figure 3-108 Comparison of the axial distributions of cathode (STD notation) potential from the experimental data and STD simulations of HPDE Run 006-014

Figure 3-109 Comparison of the axial distributions of anode (STD notation) potential from the experimental data and STD simulations of HPDE Run 006-014

Figure 3-110 Comparison of the axial distributions of anode interelectrode voltage from the experimental data and STD simulations of HPDE Run 006-014. Experimental data represent averages over 10 electrodes

Figure 3-111 Comparison of the axial distributions of the anode Hall field from the experimental data and STD simulations of HPDE Run 006-014. Experimental data represents averages over 10 electrodes

Figure 4-1 Generator isentropic efficiency vs. enthalpy extraction ratio for simulation of HPDE Run 006-014 and simulations with variations in the conditions from those of Computation CHPQUUUJBO

Figure 4-2 Coordinate systems and stations at which total voltage drops are measured and computed voltage drops are tabulated

Figure 4-3 Variation of total (anode + cathode) electrode voltage drop with electrode surface temperature under the conditions of the Q3D simulation COBQUUUJBO of the HPDE Run 006-014

Fibure 4-4 Variation of the plasma nonuniformity factor G with electrode wall temperature:

Parametric variations around the $A E D C / H P D E$ Run 006-014 simulation conditions 


\section{List of Figures (cont'd)}

Figure 4-5 Variation of the conductivity nonuniformity factor with electrode wall temperature:

Parametric variations around the AEDC/HPDE

Run 006-0144 simulation conditions

Figure 4-6 Variation of the total electrode voltage drop with peak magnetic field at various stations in the AEDC/BPDC: Parametric variations around the Q3D simulation of the HPDE Run 006-014

Figure 4-7 Variation of the relative electrode voltage drops with peak magnetic field at various stations in the $A B D C / H P D E$ :

Parametric variations around the Q3D simulation of the HPDE Bun 006-014

Figure 4-8 Variation of the plasma nonuniformity iactor with peak magnetic field in the AEDC/HPDE: Parametric variations around the Q3D simulation of the HPDE Run 006-014

Pigure 4-9 Variation of the conductivity nonuniformity factor with peak magnetic field in the AEDC/HPDE: Parametric variations around the Q3D simulation of the HPDE Run 006-014

Figure 4-10 Variation of the conductivity nonuniformity factor with Faraday load factor:

Parametric variations around the Q3D

simulation of the AEDC/HPDE Run 006-014

Figure 4-11 Variation of the plasma nonuniformity

factor with Faraday load factor:

Parametric variations around the Q3D

simulation of the AEDC/HPDE Run 006-C14

Figure 4-12 Variation of total electrode voltage drop at various stations in the AEDC/HPDE with Faraday load factor: Parametric variations around the Q3D simulation of Run 006-014

Figure 4-13 Generator isentropic efficiency vs. enthalpy extraction ratio for simulation of APDE Run 006-014 ana simulations with variations in the magnetic field and wall temperature distribution 


\section{List of Figures (cont'd)}

Figure 4-14 Generator isentropic efficiency vs. enthalpy extraction ratio for simulation of $A E D C / H P D E$ Run 006-014 with variations in load factor and a magnetic field of 4 tesla

Figure 4-15 Generator isentropic efficiency vs. enthalpy extraction ratio for simulation of AEDC/HPDE Run 006-014 with variations in load factor and a magnetic field of 5 tesla

Figure 4-16 Generator isentropic efficiency vs. enthalpy extraction ratio for simulation of AEDC/HPDE Run 006-014 with variations in load factor and a magnetic field of 6 tesla

Figure 4-17 AEDC/HPDE Run 006-014 simulation - Code Family: ARRAY. Current streamlines in the near-cathode region at station 77.4 $(x=2.779 \mu$ from inlet flange)

Figure 4-18 AEDC/HPDE Run 006-014 simulation Code Pamily: ARRAY. Current streamlines in the near-anode region at station 77.4 $(x=2.779$ M from inlet flange).

Figure 4-19 AEDC/HPDE Run 006-014 simulation Code Family: INLET. Current streamlines in midchannel - no shorted loads - contour interval: $50 \mathrm{~A} / \mathrm{m}$

Figure 4-20 AEDC/HPDE Run 006-014 simulation Code Pamily: INLET - Equipotentials in midchannel - no shorted loads - contour interval: $50 \mathrm{~V}$

Pigure 4-21 AEDC/HPDE Run 006-014 simulation Code Family: INLET - Equipotentizls in midchannel - single-pair Faraday short contour interval: $50 \mathrm{~V}$

Figure 4-22 AEDC/HPDE Run 006-014 simulation Code Family: INLET - Current streamlines in midchannel single-pair Faraday short contour interval: $500 \mathrm{~A} / \mathrm{m}$

Figure 4-23 AEDC/HDPE Run 006-014 simulation Code Family: INLET - Inlet region current streamlines contour interval: $50 \mathrm{~A} / \mathrm{m}$ 


\section{List of Figures (cont'd)}

Figure 4-24 AEDC/HPDE Run 006-014 simulation -

Code Family: INLET - Inlet region

equipotentials contour interval: $50 \mathrm{~V}$

Figure 5-1 Axial variation of open and short

circult variables in the $U-2.5$ at

$50 \mathrm{~kg} / \mathrm{s}$ operating conditions. STD

computation BRCYKDXJGJ

figure 5-2 axial variation of the electrical loading in the u-25 at $50 \mathrm{~kg} / \mathrm{s}$ operating conditions. std computation BRCYKDXJGJ

Figure 5-3 Axial variation of the plasma properties in the $0-25$ at $50 \mathrm{~kg} / \mathrm{s}$ operating conditions. STD computation BRCYKDXJGJ

Figure 5-4 Axial variation of electrical field variables in the $U-25$ at $50 \mathrm{~kg} / \mathrm{s}$ operating conditions. STD computation BRCYKDX.GJ

Figure 5-5 Axial variation of the average gasdynamic variables in the U-25 at $50 \mathrm{~kg} / \mathrm{s}$ operating conditions. STD computation BRCYKDXJGJ

Figure 5-6 Axial variation of electric potential in the U-25 at $50 \mathrm{~kg} / \mathrm{s}$ operating conditions. STD computation BRCYKDXJGJ

Pigure 5-7 Axial variation of the channel geometry in the U-25 at $50 \mathrm{~kg} / \mathrm{s}$ operating conditions. STD computation BRCYKDXJGJ

Figure 5-8 Axial variation of the heat transfer parameters $\pm \mathrm{n}$ the $U-25$ at $50 \mathrm{~kg} / \mathrm{s}$ operating conditions. STD computation BRCYKDXJGJ

Figure 5-9 Axial variation of the power extraction parameters in the $0-25$ at $50 \mathrm{~kg} / \mathrm{s}$ operating conditions. STD computation BRCYKDXJGJ

Figure 5-10

Axial variation of the optimization variables in the U-25 at $50 \mathrm{~kg} / \mathrm{s}$ operating conditions. STD computation BRCYKDXJGJ

Figure 5-11 Axial variation of open and short circuit variables in the $U-25$ at $50 \mathrm{~kg} / \mathrm{s}$ operating conditions. STD computation BRCYEHZIGK 


\section{List of Figures (cont'd)}

Figure 5-12 Axial variation of the electrical loading in the $U-25$ at $50 \mathrm{~kg} / \mathrm{s}$ operating conditions. STD computation BRCYEHZJGK

Figure 5-13 Axial variation of the plasma properties in the $0-25$ at $50 \mathrm{~kg} / \mathrm{s}$ operating conditions. STD computation BRCYEHZJGK

Figure 5-14 Axial variation of electrical field variables in the $U-25$ at $50 \mathrm{~kg} / \mathrm{s}$ operating conditions. STD computation BRCYEHZJGK

Figure 5-15 Axial variation of the average gasdynamic variables in the $U-25$ at $50 \mathrm{~kg} / \mathrm{s}$ operating conditions. STL compuation BRCYEHZJGK

Figure 5-16 Axial variation of electrical potential in the U-25 at $50 \mathrm{~kg} / \mathrm{s}$ operating conditions. STD computation BRCYEHZJGK

Figure 5-17 Axial variation of the channel geometry in the $\mathrm{U}-25$ at $50 \mathrm{~kg} / \mathrm{s}$ operating conditions. STD computation BRCYEHZJGK

Figure 5-18 Axial variation of the heat transfer parameters in the $\mathrm{U}-25$ at $50 \mathrm{~kg} / \mathrm{s}$ operating conditions. STD computation BRCYEHZJGK

Figure 5-19 Axial variation of power extraction parameters in the $0-25$ at $50 \mathrm{~kg} / \mathrm{s}$ operating conditions. STD computation BRCYEHZJGK

Figure 5-20 Axial variation of the optimization variables in the U-25 at $50 \mathrm{~kg} / \mathrm{s}$ operating conditions. STD computation BRCYEHZJGK

Figure 5-21 Axial variation of open and short circuit variables in the $U-25$ at $50 \mathrm{~kg} / \mathrm{s}$ operating conditions. STD computation BRCYJCGJGK

Figure 5-22 Axial variation of the electrical loading in the $0-25$ at $50 \mathrm{~kg} / \mathrm{s}$ operating conditions. STD computation BRCYJCGJGK

Figure 5-23 Axial variation of the plasma properties in the $0-25$ at $50 \mathrm{~kg} / \mathrm{s}$ operating conditions. STD computation BRCYJCGJGK 


\section{List of Pigures (cont'd)}

Figure 5-24 Axial variation of electrical field variables in the $U-25$ at $50 \mathrm{~kg} / \mathrm{s}$ operating conditior 3. STD computation BRCYJCGJGK

Figure 5-25 Axial variation of the average gasdynamic variables in the $0-25$ at $50 \mathrm{~kg} / \mathrm{s}$ operating conditions. STD computation BRCYJCGJGK

Figure 5-26 Axial variation of electric potential in the U-25 at $50 \mathrm{~kg} / \mathrm{s}$ operating conditions. STD computation BRCYJCGJGK

Figure 5-27 Axial variation of the channel geometry in the $0-25$ at $50 \mathrm{~kg} / \mathrm{s}$ operating conditions. STD computation BRCYJCGJGK

Figure 5-28 Axial variation of the heat transfer parameters in the $U-25$ at $50 \mathrm{~kg} / \mathrm{s}$ operating conditions. STD computation BRCYJCGJGK

Figure 5-29 Axial variation of power extraction parameters in the $0-25$ at $50 \mathrm{~kg} / \mathrm{s}$ operating conditions. STD computation BRCYJCGJGK

Pigure 5-30 Axial variation of the optimization variables in the U-25 at $50 \mathrm{~kg} / \mathrm{s}$ operating conditions. STD computation BRCYJCGJGK

Figure 5-31 Generator isentropic efficiency vs. gross power for simulation of U-25 with hot and cold walls

Figure 5-32 Shock location and choke points as a function of mass flow rate for the $0-25$ channel

Figure 5-33 Output power as a function of mass flow rate for the $U-25$ channel

Figure 5-34 Entbalpy extraction as a function of mass flow rate for the $U-25$ experiment

Figure 7-1 Variation of enthalpy extraction rate with the interaction parameter based on pressure 304

Figure 7-2 Variation of isentropic efficiency with interaction parameter based on pressure 


\section{List of Figures (cont'd)}

Figure 7-3

Figure 7-4

Figure 7-5

Figure 7-6

Figure 7-7

Figure 7-8

Figure 7-9

Figure $7-10$

Variation of specific energy extraction with the product of interaction parameter bascd on pressure and the Faraday load factor $K$ or diagonal generator electrical conversion efficiency $\eta_{e}$

Variation of conductivity nonuniformity factor $g$, averaged over the channel length, with sensible heat rate or thermal input to the MHD generator.

Development of the axial velocity profiles in the Q3D simulation CHPQUUUJBO of the AEDC/HPDE Run 006-014

Variation of the ratio of sidewall to average electrode wall displacement thickness with the interaction parameter based on velocity

Variation of the MHD generator exit blockage with interaction parameter based on velocity

Average skin friction coefficient on the electrode walls and sidewalls as a function of the interaction parameter based on velocity

Schematic diagram representing the cases which were completed with the TRANSIENT code under contract AC-01-79ET15501

Time and spatial variation of Mach number for case $1.1 .530 \%$ quasi-steady mass flow and power reduction from STD computation A5MYFALIE5

Figure 7-11 Time and spatial variation of Mach number for case $1.1 .930 \%$ quasi-steady mass flow and power reduction from STD computation A5MYSHS IFA

Figure 7-12 Time and spatial variation of Mach number for case $2.1 .530 \%$ quasi-steady mass flow and power reduction from STD computation A5MYCJGIFE 


\section{List of Figures (cont'd)}

Figure 7-13 Time and spatial variation of Mach number for case $2.1 .930 \%$ quasi-steady mass flow and power reduction from STD computation A5MY J IKIEY

Figure 1-14 Time and spatial variation of Mach number for case $3.1 .530 \%$ quasi-steady mass flow and power reduction from STD computation A5MYBGN IEM

Figure 7-15 Time and spatial variation of Mach number for case $3.1 .930 \%$ quesi-steady mass flow and power reduction from STD computation A5MYFVGIFD

Figure 7-15 Time and spatial variation of Mach number for case $4.1 .530 \%$ quasi-steady mass flow and power reduction from STD computation A5MYCXGIEN

Figure 7-17 Time and spatial variation of Mach number for case $4.1 .930 \%$ quasi-steady mass flow and power reductionfrom STD computation BRCYKPS IFS

Figure 7-18 Normalized gross power vs. normalized mass flow rate for channels with their nominal electrical hook-up

Figure 7-19 Normalized gross power vs. normalized mass flow rate for 4 channels with their alternate electrical hook-up

Figure 7-20 Combustor stagnation pressure vs. normalized mass flow rate for different size generators with mass flow varying between $70 \%$ and $100 \%$ at nominal design value

Figure 7-21 Combustor stagnation pressure vs. normalized mass flow rate for different size generators with mass flum varying between $70 \%$ and $100 \%$ of nominal design value

Figure 7-22 Net power vs. normalized mass flow rate for two generators with mass flow varying between $70 \%$ to $100 \%$ of nominal design value 


\section{List of Figures (cont'd)}

Figure 7-23 Net power vs. normalized mass flow rate for two generators with mass flow varying between $70 \%$ to $100 \%$ of nominal design value 326

1. 3 7-24 Normalized net power vs. normalized mass flow rate for two generators with mass $f$ ! ow varying between $70 \%$ and $100 \%$ of nominal design value

Figure 7-25 Normalized net power vs. normalized mass flow rate for two generators with mass flow varying between $70 \%$ and $100 \% r^{\circ}$ nominal design value

Figure 7-26 Gross power/mass flow rate vs. inte lon parameter times electrical efficiencj for four different size channels with mass flow varying from design to $70 \%$ of design

Figure 7-27 Gross power/mass flow rate vs. interaction parameter times electrical efficiency for four different size channels with osass flow varying from design to $70 \%$ of design

Figure 7-28 Normalized gross power and normalized mass flow rate for an inputted $+5 \%$ s:.ne wave in mass flow for a $20 \mathrm{uth}$ channel with Faraday connection

Figure 7-29 Normalized gross power and normalized mass flow rate for an inputted $+5 \%$ sine wave in mass flow for a $300 \mathrm{Mt} t \bar{h}$ channel with Paraday connection

Figure 7-30 Schematic diagram representing the cases which were completed with the Q3DYZ code under contract AC-01-79ET15501

Figure 7-31 Schematic diagrams of the hexagonal, rectangular, and elliptical geometries used for UHD channel cross-sections

Figure 7-32 Case 2.3.1 nominal 50 MM(th) crosssectional variation of electrical potential ( $V$ ) axial distance $=2.00 \mathrm{~m}$ STD computation in BRCYBIIIPQ 


\section{List of Figures (cont'd)}

Figure 7-33 Case 2.3.1 nominal $50 \mathrm{MW}(\mathrm{th}$; crosssectional variation of current stream function $(A / M)$ axial distance $=2.00 \mathrm{~m}$. STD computation BRCYBIIIFQ

Figure 7-34 Case 2.3.9 uominal $50 \mathrm{MH}$ (th) crusssectional variation of electrical potential (V) axial distance $=2.00 \mathrm{~m}$. STD computation BRCYBRNIFQ

Figure 7-35 Case 2.3.9 nominal $50 \mathrm{MH}(\mathrm{th})$ crosssectional variation of current stream function $(A / M)$ axial distance $=2.00 \mathrm{~m}$. STD computation BRCYBRNIFQ

Figur- 7-36 Case 1.3.1 nominal $20 \mathrm{MW}(\mathrm{th})$ crosssectional variation of electrical potential (V) axial distance $=1.00 \mathrm{~m}$.

STD computation BRCYCJAIFQ

Pigure 7-37 Case 1.3.1 nominal $20 \mathrm{Mm}(\mathrm{th})$ crosssectional variation of current stream function $(A / M)$ axial distance $=1.00 \mathrm{~m}$. STD computation BRCYCJAIFQ

Figure 7-38 Case 1.3.5 nominal $20 \mathrm{MW}(\mathrm{th})$ crosssectional variation of electrical potentiai (V) axial distance $=1.00 \mathrm{~m}$. STD computation BRCYCIUIFQ

Pigure 7-39 Case 1.3.5 nominal $20 \mathrm{Mm}(\mathrm{th})$ crosssectional variation of current stream function $(A / M)$ axial distance $=1.00 \mathrm{~m}$. STD :omputation BRCYCIUIFQ 


\section{ANALYTICAL INVESTIGATION OP CRITICAL PHENOMENA IN MHD PONER GENGRATORS}

1.0 INTRODUCTION

U.S. MHD technology for commercial power generation has made a significant transition in 1980. Test data is being obtained for wid power trains of sufficient scale, interaction, and durability to bridge the gap between frior laboratory scale speriments and the requirements of comercial systems. In addition, detailed design studies are being initiated for advanced power train concepts which will ultimately lead to pilot-scale tests of a coal-fired uHD power plant. The significant power train projects in this transitional phase of MHD technology include the AEDC High Performance Demonstration Experimen: (HPDE), the U.S. U-25 generator!diffuser; U.S. participation in the U-25B experiments, the UTSI Coal Fired Flow Facility (CPFF), the Advanced Power Train (APT) for the Componert Development and Integration Facility (CDIF), and the Engineering Test Facility (ETF).

These projects each represent an important advance beyond previous laboratory experience. In contrast to past laboratory experiments, these projects test MHD power trains in which the interaction between the electrical forces and working fluid will be strong enough to cause important departures from classical internal duct gasdynamics. There are at present only limited experimental data to guide the prediction of the performance and behavior of MHD channels in such moderate-tohigh interaction situations. Likewise, there is a lack of experimental data against which the test results of these programs might be compared. Under NASA Contract DEN3-179, STD Research Corporatior bas continued the application of its 
comprehensive analytical capabilities to predict and interpret the behavior of high interaction MHD power trains. This Final Report for Contract DEN3-179 describes the results of analyses of high interaction experiments and designs carried out by STD during this transitional phase of MHD technology.

The planning, detailed analysis, and interpretation of the AEDC/HPDE and the U-25 experiments have been the subject of concern at STD Research Corporation from their initial conception [1-1]. During many years of contributions to the initiation and evaluation of these experiments, STD Research Corporation has analyzed many of the interim designs for the experimental hardware. Under U.S. Department of the Interior, and U.S. Department of Energy contracts since 1971, STD Research has made numerous specific recommendations for improved test parameters and procedures for the HPDE and U.S. 0-25 tests. Many of the STD recommendations have resulted in changes in test hardware and/or procedures.

Under DOE contract AC-01-79ET15501 and NASA contract DEN3-179, STD Research has analyzed the "as-built" specifications of the HPDE and U-25 experiments under nominal operating conditions. These analyses have quantitatively predicted the effects of critical, nonideal processes which will control the performance of the HPDE and U-25 tests. Yoreover, the work undel these contracts has mapped the predicted nominal operating characteristics of those experiments over the likely ranges of mass flow rate, working fluid composition, diffuser recovery coefficient, wall temperature, and other operating conditions.

Under Contract DEN3-179, STD Research has carried out analysis of preliminary data from the first tests of the HPDE. 
These experimental data confirm earlier analytical predictions by STD Research Corporation, such as the presence of large axial voltages during the start-up transient and the presence of much-largor-than design electrode voltage drops. This experimental confirmation of effects predicted by the STD analytical tools further validates the accuracy of these tools.

Studies of the nominal operation of the HPDE have yielded estimates of the relative importance of critical phenomena expected in the flow train tests. Unfortunately, the experiments can never run at precisely the nominal conditions. A significant test of an analytical tool is its capability to utilize inputs from instantaneous measurements taken during a test, and then to predict other quantities measured at the same instant during the same test. Such data are now available from the HPDE, and comparisons between such data and the STD/MHD code results are presented in this report.

Previous test data analyses at STD Research Corporation [1-2] have amply demonstrated the benefits of careful test data analysis for providing data consistency and understanding of the relative importance of the operative physical processes. As data have been received from the APDB, STD Research Corporation has undertaken a detailed analysis of appropriate, self-consistent data sets which have led to (1) better understanding of the significance of each test and (2) reoommendations for improved test procedures.

The survey of the nominal performance of the U-25 generator designed and constructed in the United states was carried to the point of evaluatiun of the "as-built" performance of the channel. The channel has not been operated to date, and therefore the analytical predictions oi the 
studies of nominal performance have not been checked against experimental data. Insights gained by the comparison between the analytical simulation of $A E D C / H P D B$ experimental data were applied to the U-25 nominal operating conditions, however. These analyses focused on the implications of operating the U-25 channel with cold walls without modification of the original design loft. The results indicated that the electrode boundary layer voltage drops would assume a major role in the achievement of the $10 \mathrm{MT}$ design goal with the "as-built" U-25 nominal operating conditions.

Perhaps the most important objective of analytical modeling of any physical process is the development of the ability to extend the results of experiments under one set of conditions to the conditions of other experiments. To do this, one must have the ability to exercise sufficiently rigorous and complete physical models which do not depend upon the application of adjustable, empirical factors in order to obtain agreement with experimental data. The use of such rigorous analytical tools, as are employed at STD Research Corporation, place a substantial burden on the experimentalist to provide precise, complete measurements for all of the physical data required as input to the calculation. Experience at STD Research Corporation [1-4] has shown that the combination of well diagnosed experimental data and rigorous analyses can pay dividends in understanding complex physical mechanisms and the experimental data itself. After the initial "shake down" phases of HPCE testing, the HPDE is beginning to produce such data. The simulations performed under Contract DeN3-179 have been fruitful as a consequence.

It is of interest to extrapolate the results of the AEDC/HPDE experimental data analyses to-date to conditions of 
higher interaction. These conditions include operation of pilot scale and commercial scale MHD generators. Under Contract DEN3-179 and its predecessors, STD hesenrch. Corporation has characterized the behavior of MHD power trains from laboratory scale to commercial scale utilizing a selfconsistent set of analytical tools. It was shown in [1-3] that the performance and fluid behavior of MHD power trains correlates well with the appropriate ateraction pare?.?c The performance and interaction parameters of the AEDC/HPDE relative to generators of other scales are survevad in the present report.

Utilizing the results of calculations carried out under Contract AC-01-79ET15501, the scale dependence of the performance of generators of alternate cross-sections (elliptical and hexagonal) also has been analyzed. The benefits of alternate cross-sectional geometries are strongly dependent on the boundary laye: characteristics present at a particular station in a given channel. The powel generation potential of each of hree cross-sections in each of four channels ranging in scale from $20 \mathrm{MH}$ thermal input to $2000 \mathrm{Mm}$ thermal input is compared. The comparison has been carried out between the power actually generated and (1) the power generation potential predicted by simplified electrical alculations, (2) power generation with rectangular geometry, and (3) ideal power generating zapability based on center-line conditions at each axial station. While the analyses are indicative of the potential benefits of alternate crosssectional configurations in MHD generators at various scales, the results do not represent optimum configurations. A number of additional computational degrees of freedom which were not considered in the previous study may also serve to improve the performance of generators of alternate cross-sections beyond the levels demonstrated. 
Finally, an analytical study of the scale dependence of part load and transient behavior of MHD power generators is summarized in the present report. It is shown that a critical parameter for the successful part load operation of MHD power generation is the velocity or Mach number distribution in the MHD generator. The conclusion of this study has been that the key to successfui off-design or part load operation of MHD generators is the maintenance of the Mach number distribution at or near the optimum value for the stagnation conditions existing at each station in the MHD generator.

This Final Report is organized according to the major topics addressed during the course of Contract DEN3-179. Section 2 presents a brief description of the cudes utilized duriig the analytical investigations, and points out documents and literature in which fuller descriptions of the codes may be found. Section 3 focuses on the analysis of the "as-built" AEDC/HPDE, including the analysis of actual test data. Section 4 is devoted to special itudies based upon the simulations of AEDC/HPDE experimental data described in Section 3 . Section 5 describes the characteristics of the nomina?, "as-built", operating conditions and specifications of the U.S. U-25 flow train. Section 6 describes the results of thermodynamic calculations of coal combustion products, including estimates of the electical conductivity at the inlet of a NASA specified $500 \mathrm{Mm}$ th MHD generator. The purpose of section 7 is to put the results of the present contract and contract AC-01-79ET15501 into the context of the overall development program for MHD power generation. Sections 8 and 9 summarize the findings of the refort and enumerate the major recommendations resulting from the studies presented. 


\subsection{DESCR IPTION OF THE STD/MHD CODES}

The STD/MHD codes are constructed from a large collection of modules or subprograms which address various aspects of the MHD problem. Taken together, these codes define and solve the MHD problem, including, where applicable:

1. Viscosity and wall roughness

2. Volume viscous losses

3. Vall roughness effects on skin friction

4. Other sinin friction effects

5. Compressibility

6. Turbulence, including MHD effects

7. Turbulence structure parameters and transport coefficients

8. Convective heat transfer

9. Radiative heat transfer

10. Anisotropic, nonuniform, fluctuating electrical transport properties

11. Nonuniform, fluctuating plasma/fluid properties

12. Nonequilibrium effects

13. Finite reaction rates, including ionization/recombination

14. Electron energy relaxation

15. Electron radiation losses

16. Electron thermal and concentration diffusion

17. Nonlinearity of the plasma as a conducting medium

18. Electrode surface and sheath phenomena

19. Electrochemical effects at the electrodes and slag layer

20. Finite electrode effects

21. Flow separation

22. Current leakage due to imperfect segmentation or slag layer leakages

23. Axial current leakage in the plasma and interelectrode arcing

24. Trans-boundary laye: arcing

25. Shock waves and shock wave/boundary layer interactions

26. MHD instabilities (including magnetoaerothermal)

27. Ionization instability modes

28. Current streamer formation

29. Start-up transients, including sequencing of fuel, oxidizer, and seed valves, secondary infection of fuel, seed and oxidizer, and load circuit switching

30. Shut-down transients

31. Perturbations from steady state operation, including steam plant upsets, load circuit faults, part-load operatinn and load iollowing transients

32. Generator/power conditioning system interactions

33. Generator end region losses

34. Slag layer dynamic phenomena 
35. Channel geometry effects, including nonrectangular cross-sectional shapes

36. Transverse pressure gradients and nonuniformities

37. Secondary flows induced by MiD or viscous effects

38. Fundamental combustion processes, including particle burnout, particle trajectories, liquid layer formation on combustor walls, seed mixing and uniformity, pressure loss, etc.

39. Alfvén waves

40. Hartmann flow

41. Nucleation effects

42. Electron capture by particles

43. Radiative ionization and recombination

44. Nonequilibrium radiation and coherent radiation

45. Effects of nonideal mixtures in condensed phase chemistry

46. "Faraday Catastrophe" load faults

47. Three-dimensional and time-dependent induced magnetic field effects

48. Ion slip effects

49. Nonideal plasma effects

50. Combustor-generated inlet swirl

The modules or subprograms describing these and other processes can be coupled in a variety of ways, depending upon the desired focus of a given calculation.

Detailed descriptions of the formulations, solutions, and applications of the appropriate STD/MHD codes are contained in Ref. [2-1] through [2-5]. A capsule description of each of the TRANSIENT, Q3D, PROPERTIES, FIN, INLET, BONDLAY, and GRAPHICS code families follows.

\section{Code Family: TRANSIENT}

Time-dependent magnetohydrodynamics, plasmadyaamics, and compressible gasdynamics for internal flows with MHD power extraction and body forces. Comprehensive code family accounts for two-dimensional effects, wall effects (heat transer and friction) and kinetics. Applicable to MHD power systems, gas-dynamic lasers, and other high temperature flow systems in which start-up, shutdown or unsteady operation are important. TRANDEE is one of the specialized codes within this family. The User's Guide to this code is available, Ref. $[2-3]$. 
Code Family: Q3D

Quasi-three-dimensional, steady MHD, electrical, and fluid mechanical effects in equilibrium (combustion-driven) or nonequilibrium (noble gas) MHD flow trains (combustor, nozzle, channel, and diffuser) of arbitrary cross section. This code family includes various cross sectional MHD generator performance calculations which may be coupled to predict three dimensional performance and behavior. While finite segmentation options exist, they were not utilized in the performance of the Q3D calculations described in this report. QUE3DEE is one of the specialized codes within this family. A User's Guide is available for this code, Ref. $[2-4]$.

\section{Code Family: PROPERTIBS}

Generalized transport and thermodynamic properties of equilibrium combustion flames. These codes consider reactions between more than 500 gas, liquid, or solid state species. Plexible inputs include initial fuel, oxidizer, and additive (seed, ash, etc.) compositions and states. Outputs include electrical and fluid transport. coefficients and all thermodynamic state variables.

Code Family: FIN

Two-dimensional MHD electrode solutions with electron energy relaxation, electron ionization/recombination kinetics, effects of slag coatings, and finite electrode/ insulator width. Codes in this family apply to the region of the MHD generator channel which may be considered "periodic"; i.e., regions in which changes from electrode pair to adjacent electrode pair are essentially negligible.

Code Family: BONDLAY

Integral boundary layer models with MHD body forces, Joule dissipation, wall roughness effects. 


\section{Code Family: GRAPHICS}

General and customized graphics packages for post-processing raw data files generated by other code families. Includes codes for contour generation and three dimensional projection of bivariate data.

Code Family: INLET

Codes in this family solve the two- dimensional electrical part of the MHD problem in the part of the MHD generator which cannot be considered periodic. These codes take given gasdynamic variables and compute the two dimensional distributions of electron temperature, Ohm's law coefficients, current density, and electric field in the end regions of an MHD channel. 


\section{REFERENCES}

[2-1] S. T. Demetriades, "Computer Analysis and Evaluation of MHD Systems," STD Research Corporatiou Final Report FE2243-22 prepared for ERDA, Divn. Kagnetohydrodynamics, Fossil Energy Category UC-908, January 1979

[2-2] "STD/MHD Codes - Overview and Catalogue of Some of the STD/MHD Code Families Available to the Public," STD Research Corporation Report No. STDR-104-79, July 1979

[2-3] "STD/MHD Codes - Introductory Users Guide; Code: TRANDEE, Pamily: TRANS! ENT," STD Research Corporation Report No. STDR-101-79, July 1979

[2-4] "STD/MHD Codes - Introductory Users Guide; Code: QUE3DEE, Family: Q3D," STD Research Corporation Report No. STDR-102-79, July 1979

[2-5] "STD/MHD Code Descriptions for Computer Programs Utilized under Contract AC-01-79-ET15501," STD Research Corporation Final Report, Tasi 1 , Contract No. AC-01-79-ET15501; Report No. STDR-79-3 prepared for ERDA, Divn. Magnetohydrodynamics, DOE/ET/15501-1, Fossil Energy Category $\mathrm{UC}-90 \mathrm{~g}$, January 1979 
STD Research Corporation has performed calculations to survey the power producing characteristics of the AEDC/HPDE over the nominal operating range as well as calculations to simulate actual experiments which have occurred to date. The results of these studies are discussed in this section.

\subsection{Channel Characterization}

Subsection 3.1 .1 describes the physical parameters used for simulations of the nominal operation of the AEDC/HPDE. Descriptions of operating parameters which differed from these nominal conditions during actual tests are provided in subsequen: subsections.

\subsubsection{AEDC/HPDE - Nominal Conditions}

The flow train of the Arnold Engineering Development Center High Performance Demonstration Experiment (AEDC/HPDE) consists of a combustor, transition nozzle, electrode test section, generator, generator/diffuser adapter section and diffuser. Pig. 3-1 is a schematic of the final, "as-built" internal dimensions of the device (axial locations are referenced from the combustor bsick plate).

\subsubsection{Geometry}

The combustor for the HPDE is based on renovation and modification of what was formerly the LORHO burner and is described in $[3-1]$ through $[3-4]$. For the purpose of performance analysis, the combustor may be characterized as a 
$0.610 \mathrm{~m}$ diameter cylinder of $1.195 \mathrm{~m}$ length. The transition nozzle is a three-dimensional contraction irom the $0.605 \mathrm{~m}$ diameter burner to $a 0.264 \mathrm{~m}$ by $0.489 \mathrm{~m}$ rectangular electrode test section. The electrode test section is of no consequence during the MHD experiments and may be regarded simply as an unloaded portion of the generator. The MHD generator channel is constructed in 5 sections, the dimensions of which are shown in Fig. 3-1. In the STD computer codes the $x$-axis is located at the center of the channel cross-section. The $y$-axis is in the electrode-to-electrode direction with the positive direction being from cathode toward anude. The z-axis is in the sidewall-to-sidewall direction. The magnetic field vector is in the positive $z$ direction. The channel height, width, area, and aspect ratio are plotted as a function of the axial coordinate in Fig. 3-2.

There are 485 pairs of electrically accessible electrodes, of which only 417 pairs spanning $7.15 \mathrm{~m}$ in the interior of the channel are loaded under the nominal operating load sinedule.

The electrodes are electrically isolated from each otutr with refractory, and each electroae spans the transverse $r^{2}$ itance across the channel. The gap between electrodes is 1.52 m throughout the channei [3-5]. Graphite caps are attached to eacn electrode pair to allow operation at a high surface temperature.

The "pegwall" insulator walls are composed of 0.019 square conducting pegs which are spaced $1.58 \mathrm{~mm}$ apart and are insulated with refsactory naterial [3-4].

The generator is itted (via an adapter section) with a plane-walled diffuser with parallel sidewalls (extensions of 
the electrode walls) and diverging top and bottom walls. The mechanical design [3-1] of the diffuser allows for setting the upper and lower walls at any angle between 0 and $3^{\circ}$. Experiments to date have fixed the divergence angle at $1^{\circ}$ on both walls.

\subsubsection{Magnetic Field}

The magnet for the MHD High Performance Demonstration Experiment is designed to provide (at $27 \mathrm{MW}$ and $16 \mathrm{kA}$ ) a peak field of $6 \mathrm{~T}^{\prime}$ when sulsed from $77 \mathrm{~K}\left(\mathrm{LN}_{2}\right)$ and $3.5 \mathrm{~T}$ when operated continuously water-cooled. The magnet bore is $0.89 \mathrm{~m}$ wide by $0.71 \mathrm{~m}$ high at the entrance, $1.4 \mathrm{~m}$ wide by $1.17 \mathrm{~m} \mathrm{high}$ at the exit, snd the poles are $7.1 \mathrm{~m}$ long. The total length of the magnet is a $9.16 \mathrm{~m} \cdot[3-6]$

Fig. 3-3 shows the agreement between tine predicted and measured magnetic field distributions. STD simulalions of the AEDC/HPDE experiments to date have used, as inp.t for the field distribution, the MEA design values $[3]-i j]$. These values are scaled by the measurement of Hall prote 49 for each particular run under analysis.

\subsubsection{Loading}

The nominal loading scheme for the HPDE generator is in a Faraday configuration. For purposes of performance survey calculations, the load distribution is given as follows [3-5].

$\begin{array}{ll}\frac{x(m)}{2.735} \leq x \leq 8.875 & \frac{R L(O h m-m)}{0.8} \\ 8.875<x \leq 9.885 & 1.6\end{array}$

where $x$ is measured from the burner backplate. The electrode 
spacing is given in [3-7] and is included in Table 3-1 for convenience.

\section{1 .1 .4 Vorking Fluid}

The working fluid for the channel is composed of the products of the combustion of toivene and oxyben with nitrogen alluent. The seed is potassium hydroxide dissolved in methanol. The fuel/seed mixture is injected into the com" stor as a liquid at ambient temperature. The nominal mass flow rates for the facility are $45 \mathrm{~kg} / \mathrm{s}$ for operation between 2 and $3 \mathrm{~T}, 50 \mathrm{~kg} / \mathrm{s}$ for ope ration between 3 and $4 \mathrm{~T}$, and 53 to $54 \mathrm{~kg} / \mathrm{s}$ for operation at $4 T$ and greater $[3-5]$. The nominal value for both the $\mathrm{N}_{2} / \mathrm{O}_{2}$ mole ratio and the stoichiometry is 1.0 . Table 3-2 contains a list of the flow rates which describe the working fiuid under nominal conditions ( $2 \mathrm{~T}, 45 \mathrm{~kg} / \mathrm{s}$ ).

\subsubsection{Iall Conditions}

The nominal values for the surface temperatures on both the insulating and electrode walls are shown in Fig. 3-4. These temperatures were determined by a one-dimensional ransient heating analysis performed by AEDC personnel and described in $(3-7)$. For all calculations the duration of heating was taken to be $15 \mathrm{~s}$, after which there was a cool-down cycle during wich atmospheric air is forced through the channe:. The temperature history for each wall is calculated at the channel inlet and exit, and the distribution of the wall temperatures along the channel were obtained by assuming a linear variation between these locations (for values corresponding to $t=15 \mathrm{~s})$. The combustor operates with a wall temperature of $450 \mathrm{~K}$, and the rolghness of all walls is charactrrized by an equivalent sand roughness of $3 \mathrm{~mm}$ in the 
electrode test section and channel, and as "smooth" in the combustor, nozzle, and diffuser [3-5].

\subsubsection{Channel Inlet Conditions}

The character of the flow as it enters the MHD generator is not completely defined by the experimental data. Ideally, one would prefer to know with confidence the complete distribution of mass, momentum, and energy at the inlet plane. Avallable data are sparse due to the experimental difficulty of obtaining these measurements. The heat loss to the watercooled components of the burner is on the order of $3 \%[3-4]$. The total heat loss to all components upstream of the first loaded electrode was calculated, as described in section 3.1.2.5, by STD Research; the value of 5.7\% was used for the nominal case. The original design calculations based on the nominal operating conditions indicated that the boundary layer thickness is approximateiy $12 \mathrm{~mm}$ at the inlet plane.

\subsubsection{Other Conditions and Assumptions}

The generator exit condition is determined by the fact thic the HPDE ilow train exhausts to the atmosphere. Hence, the static pressure in the exit plane of the diffuser is assumed to have the nominal value of one atmosphere.

\subsubsection{AEDC/HPDE - Experimental Conditions}

The HPDE experimental conditions are chosen according to experience learned from each successive test and a test plan which includes variation of the key test parameters. Consequently, the HPDE runs have not, to date, operated at exactly 
the "nominal" conditions pr, ented in the previous section. Post-experimental data analysis is the only way to determine the operating point of a particular experiment. It is the purpose of this section to set for in as completely as possible, using the data at hand, the experimental conditions under which the AEDC/HPDE has actually operated to this date.

\subsubsection{Magnetic Field}

During power producing runs the magnetic field strength is monitored via Hall probes, the locations of wbich are shown in Fig. 3-5. All probes are located on the floor of the magnet bore. A measure of the variation of the magnetic field in the interelectrode direction is provided by probes H2A and $\mathrm{HA}$ which are displaced above and below the midline $(y=0)$ by $0.014 \mathrm{~m}$. The location of these probes with respect to the design magnetic field distribution is shown in Fig. 3-3. Note that they are near the peak magnetic field region. For the purpose of computer simulation of the experiments, the measured values of probes $\mathrm{H} 2 \mathrm{~A}$ and $\mathrm{H} 3 \mathrm{~A}$ are averaged over the time interval of interest. The resulting value of the magnetic field is then used to scale the design curve to yield the complete magnetic field distribution ror the experiment under consideration. Table 3-3 contains the values of the magnetic field so obtained for the AEDC experiments to date.

\subsubsection{Loading}

The load bank is a series of liquid rheostats consisting of polypropylene buckets, each containing two copper plates. The plate spacing can be varied to achieve a resistance range from 10 to 60 ohms. Table 3-4 contains the 
resistance of each electroae pair as set by AEDC personnel [3-8]. $\mid 3-9]$. These values have been normalized by a water conductivity of $150 \mu \mathrm{S} / \mathrm{cm}$. Note that the reference resistances for electrode pairs numbered greater than 357 have been changed for runs subsequent to 006-007 and also that electrode pairs 401 and above are open circuited. Table 3-5 contains the water conductivity during the runs for all runs to date. To determine the resistance schedule for any particular run, the values from Tables 3-4 and 3-5 must be used in the following equation:

$$
R_{\text {run }}=R_{\text {ref }}\left(150 / K_{\text {run }}\right)
$$

The product of the above load resistance and the electrode pitch (width from Table 3-1 plis the insulator thickness of $1.52 \mathrm{~mm}$ ) then uniquely specifies the loading of the channel in the Faraday configuration. It should be noted here that comparisons of the load resistarces determined in the above manner and those determined from the voltago-current characteristics of the experimental data are not very good for Run 006-008, but were better for subsequent runs. Further discussion on this point is contained in Sectiras 3.6 and 3.7.

\subsubsection{Working Fluid}

Table 3-6 contains a list of the flow rates of the various constituents of the working fluid for two typical experimental runs (006-008 and 006-014). These flow rate data are input to codes from the STD THERMODYNAMICS family to generate a thermodynamic data base and other data. Input flow rates for oxidizer, seed, and fuel were obtained by averaging the experimental data over the time period $4.54 \leq T_{2} \leq 5.21 \mathrm{~s}$ for Run 006-014 and 9.5 $\leq \mathrm{T}_{2} \leq 10.2 \mathrm{~s}$ for Run 006-008. 


\subsubsection{Wall Conditions}

Fig. 3-6 illustrates the results of a transient heating analysis performed by AEDC personnel for the electrode surface including the 1/2" graphite caps [3-11]. Experimental data have indicated that the heat trarsfer rates on the electrode surfaces at the inlet flange and at a station $5.59 \mathrm{~m}$ from the inlet flange are $450 \mathrm{Btu} / \mathrm{ft}^{2}-\mathrm{sec}$ and $100 \mathrm{Btu} / \mathrm{ft}^{2}-\mathrm{sec}$, respeciively. Several of the power producing runs to date have yielded their best data about 5 seconds into che run. Reading the curves of Fig. 3-6 (at $t=5 \mathrm{~s}$ ) for the heat transfer rates mentioned above yields electrode surface temperatures of $1130 \mathrm{~K}$ and $540 \mathrm{~K}$ at the inlet flange and a point $5.59 \mathrm{~m}$ downstream, respectively. For the purpose of performance calculations, the complete temperature distribution is assumed to be linear along the channel between these two points. These data were the best available at the time these simulations were performed.

All simulations beginning with the simulations of Run 006-014 utilized the temperature profile described in the above paragraph. Simulations of runs prior to 006-014, which were carried out before this information became available, utilized the nominal temperature distribution, Fig. 3-4.

As the HPDE runs were analyzed, better wall temperature data became available. A further revision to the electrode surface temperatures has been published in [3-12] and is reproduced in Pig. 3.7. This estimate is based on a twodimensional, fully viscous calculation including the effects of pressure gradient. 


\subsubsection{Channel Inlet Conditions}

To determine the boundary layer thickness at the first loaded electrode, a series of computations have been made with a code from the STD BONDLAY Family. Several runs were made assuming various values for the equivalent sand roughness in the components upstream of the channel win the following results at the first loaded electrode:

\begin{tabular}{ccc}
$\begin{array}{c}\text { Sand Roughness Height } \\
(\mathrm{mm})\end{array}$ & $\begin{array}{c}\delta(\text { electrode wall) } \\
(\mathrm{mm})\end{array}$ & $\begin{array}{c}\delta(\text { sidewa } 11) \\
(\mathrm{mm})\end{array}$ \\
\cline { 1 - 1 } 0.0 (smooth) & 17.6 & 13.3 \\
0.1 & 24.0 & \\
1.0 & 34.9 & 38.4 \\
2.0 & 40.0 & 44.3 \\
3.0 & 43.6 & 48.4
\end{tabular}

These calculations also predict that the total enthalpy loss up to the first loaded electrode ranges between approximately $5.7 \%$ for the smooth wall calculation to approximately $7.2 \%$ for the $3.0 \mathrm{~mm}$ roughness calculation. The latter value compares well with the datum of $7.5 \%$ measured by AEDC [3-12]. 
density, integrated power output, ard interelectrode voltages are determined from tne primary measurements and other geometrical data.

\subsubsection{STD Interface with HPDE Data Systems}

The HPDE data acquisition system data are processed by codes from the STD GRAPHICS family and plotted as a function of both space and time. These codes allow the data and computational results to be displayed with any degree of time and space resolution necessary, contingent of course, on the ultimate density of the data, to examine events of importance. Fig. 3-8 is illustrative of the manner in which critical events can be followed throughout their lifetime using this type of cisplay.

A plot of the data acquisition system data for a typical AEDC run (006-008) is given in Fig. 3-9. On this figure the time coorinate increases from left-to-right and starts at $T_{2}=$ 0 ( $\mathrm{T}_{2}$ is the time in seconds from combustcr ignition). Also, the axial distance along the flow trais is plotted from top-to-bottom. On this figure, the value of the data is plotted vertically above the corresponding point in the time-distance coordinate plane. It is possible to define and plot subsets or "windows" in the data as small as necessary to resolve an event of interest if the data are available. Such options have as yet gone unused for the HPDE data due to the relative coarseness of the experimental data in space and time. Much higher data rates will be required ts resolve the spacetime variation of critical phenomena such as MHD instability modes, electrical transients, etc. 


\subsection{Steady State Performance_at the Nominal Operateing}

Conditions

Prior to receiving the experimental data from the initial powered runs, a study was conducted in order to determine the probable performance range of the AEDC generator during the first phase of testing. The nominal operating conditions were defined by AEDC personnel and are documented in Section 3.1.1. During these computations the peak magnetic field was varied between 2.0 and $4.0 \mathrm{~T}$ and the mean flow rate was varied between 45 and $54 \mathrm{~kg} / \mathrm{s}$. Due to the unavailability of sophisticated FIN or ARRAY calculations in the early stages of te HPDE analysis, a variety of wall condition models were employed in Q3D for the initial calulations of the iPDE performance under the nominal operating conditions.

With the arc mode current transfer option, the current transverse through the boundary layer flows with negligible loss when the local electric field exceedes a critical value. Based on previous work, the critical value used for these computations is $-12 \mathrm{kV} / \mathrm{m}$. For the diffuse current mode option, the current transverse through the boundary layer is corputationally forced to be uniform across the width and length of the electrodes.

Figs. 3-10 and 3-11 present the summary of the predicted performance of the HPDE at the nominal operating conditions for $2 \mathrm{~T}, 3 \mathrm{~T}$, and $4 \mathrm{~T}$ operation. Performance predictions were carried out as a function of mass flow rate with the STD TRANSIENT family of codes in the quasi-steady mode of operation. The predicted values fall within the bounds of uncertainty defined by a series of more sophisticated, quasi-three-dimensional calculations with the Q3D family of 
codes. Depending upon the relative importance of seed condensation and current transport mechanisms, the predicted power output was found to vary between 4.28 to $15.3 \mathrm{MW}$ at $2 \mathrm{~T}$ and between 25.96 to 39.76 at $4 \mathrm{~T}$. Fig. 3-11 indicates that the experiment should not be expected to obtain $15 \%$ enthalpy extraction at $4 \mathrm{~T}$ and the nominal operating conditions.

Figs. 3-12 to 3-24 are results of a calculation, COBQFXRIHU, with a code from the Q3D family for the $2 \mathrm{~T}$ nominal condition (Point $A(2 \mathrm{~T})$ in Fig. 3-10). These are axial profiles of certain key variables which are output from the calculation. The origin for the axial coordinate in these figures is located at the inlet flange (end of electrode test section in Fig. 3-1). Note how the sectioning in the channel construction (see Fig. 3-1) is directly reflected in the Mach number and velocity distribution of Fig. 3-13. The load factor decreases from 0.8 to 0.64 over the active channel (Fig. 3-15). Fig. 3-16 shows that approximately $15.3 \mathrm{MW}$ are produced at the nominal $2 T$ conditions at an enthalpy extraction ratio of 0.059. Maximum normal current densities of approximately 0.5 $\mathrm{A} / \mathrm{cm}^{2}$ and maximum Hall fields $C_{-}$approximately $1.8 \mathrm{kV} / \mathrm{m}$ ar 3 present during operation as shown by Fig. 3-17. Maximum voltage drops* of approximately $750 \mathrm{~V}$ occur in the rear end of the generator (Fig. 3-21).

\footnotetext{
*Except as otherwise noted, voltage drops in this report are defined as the difference between the Faraday voltage and the voltage difference across the channel if the centerine transverse voltage gradient were extrapolated to the electrodes.
} 


\subsection{Hall Voltage Overshoots}

The mechanism for the occurrence of Hall field overshoots during start-up and shutdown was described in Vol. I, pp. 5-12 to 5-16 of the STD Research Corporation report FE2243-17 under contract EX-76-C-01-2243 (May 1978). During periods in which the channel is operating at design mass flow rates, but is unseeded or slightly seeded, high velocities are experienced in the channel. In the presence of the magnetic field, B, these higher-than-design velocities can lead to much higher-than-desion Hall fields:

$$
E_{X}=\beta U B(1-K) / G
$$

Taking note that $\beta-B / p$, we find that (1) $E_{x}-B^{2}$, and (2) every factor in the above expression (except the nonuniformity factor $G$ ) changes in the direction to increase the magnitude of $E_{x}$ as the velocity increases. Such unseeded or slightly seeded conditions are present during the start-up and shutdown sequences because the seed valve is opened last and closed first.

In the calculations described in the forementioned report, Hall fields exceeding $20 \mathrm{kV} / \mathrm{m}$ locally were observed at the nominal $6 \mathrm{~T}$ condition during the start-up and shutdown events, and Hall fields exceeding $4 \mathrm{kV} / \mathrm{m}$ extended over more than half the channel. Scaling these fields by the square of the ratio of magnetic flux density in Run 006-008 to the value used in the previous study $(2.35 \mathrm{~T} / 6 \mathrm{~T})^{2}$, we might expect Hall fields of the order of $3 \mathrm{kV} / \mathrm{m}$ over the entire channel due to the increased extent of the supersonic flow region in AEDC/HPDE Run 006-008 (see Section 3.6 for a complete analysis of this run). Thus, instantaneous total Hall voltages up to $25 \mathrm{kV}$ might be expected in the 006-008 start-up and shutdown events. 
Fig. 3-25 is a plot of the anode-to-o,round voltage for electrode pair number 336 during AEDC/HPDE Run 006-008 in which the diffuser became grounded during the run. Figs. 3-26 and 3-27 show the anode and cathode voltages at all instrumented electrodes (for the Data Acquisition system) over the entire duration of the run. It is clear that the overvoltages occurred throughout the channel. The reduced data acquisition system data, which are presented in all plots of data acquisition systems in this report, are averages over five acquisitions in order to filter the noise. Therefore, while voltages as high as $25 \mathrm{kV}$ are not displayed in these figures, the high standard deviations associated with the peaks in the Pig. 3-25 data imply higher instantaneous voltages than the averaged values depicted in Fig. 3-25.

The sequence of events that took place during Run 006-008 are reasonably well understood and are depicted schematically in Fig. 3-28. This figure presents the actual measured diffuser-to-ground voltage along with a curve whict. represents the ideal generator voltage which would occur. $f$ there had not been an external arc from the diffuser to ground. Due to an approximate $2 \mathrm{~s}$ delay between fuel injection and seed injection into the combustor flow, sufficient time is available to establish steady, unseeded combustion flow in the MHD channel during start-up. If no electrical faults develop, the Hall voltage would have reached $25 \mathrm{kV}$ as discussed above. This is indicated by the solid trace in Fig. 3-28. Instead, an electrical breakdown from diffuser to ground developed as the Hall voltage increased in Run 006-008. The exact breakdown vcltage is not known from the experimental data due to the long averaging times of the data acquisition system and panel meter data. If sufficient informatirn about the geometrical configuration of the region near the electrical brakdown were 
known, the air breakdown voltage could be calculated. This is not a critical parameter because the breakdown problem at this location has since been corrected.

During the period between the electrical breakdown and the onset of seed flow to the ccmbustor, the external arc is sustained by passage of a modest amount of current through the plasma frow the combustor to the diffuser breakdown location. Independent assessments of the electrical conductivity in the plasma during the unseeded period yield an estimate of $0.03 \mathrm{~s} / \mathrm{m}$ $\pm 0.02 \mathrm{~s} / \mathrm{m}$. These estimates were arrived at by (1) examination of the STD THERMGDYNAMICS code family predictions for the electrical conductivity of the unseeded combustion flame, and (2) an analysis of the Faraday current and voltage measurements during the unseeded portion of Run 006-008 using calculated values of the gas velocity.

By assuming that the electrical conductivity is relatively constant in the generator during the unseeded portion of the run, by assuming that the conductivity is relatively uniform over the channel cross-section, and by taking note that the measured voltage to ground of anode 1 and anode 10 differ by 1000 volts (the corresponding difference on the cathode side is 1200 volts), it 3 possible to estimate a total current flow during the unseeded period of approximately $10 \pm 5 \mathrm{~A}$. Such a "trickle" of current would be sufficient to maintain the external arc from the diffuser to ground.

As the seed first enters the combustor, the bulk conductivity of the plasma increases, and the current available to the external arc increases substantially. The steady-state current in the external arc, corresponding to the time when the seed flow has reached its steady-state value, is estimated from 
Q3D computations to be in excess of $850 \mathrm{~A}$, and possibly as high as 1200 A (see section 3.6). The buriling voltage of the external arc decreases as the current increases, according to the standard V-I characteristic of electrical arcs. During the seed-on transient, the voltages to ground of the loaded electrodes in the generator undergo a voltage transient which is indicated in Figs. 3-25 to 3-27. Unlike these floating electrode circuits, the diffuser voltage is fixed by the ar: burning characteristics; and, consequently, the difiuser does not experience this "seed-on" transient.

During the HPDE shutdown sequence, the seed vaive is shut prior to fuel cut-off. As the seed is purged from the channel, the conductivity required to sustain the high current discharge gradually diminishes, and the diffuser voltage follows the V-I characteristics of the external discharge. Were there are no electrical faults present, the Hali voltage would again rise to approximately $25 \mathrm{kV}$. However, the burning voltage of the arc rises to some lower value, and then the external diffuser-to-grouid discharge is extinguished. When the discharge is extinguished, the diffuser-to-ground voltage rises but is intercepted by the voltage trace which would be present without external electrical discharges. Tais trace falls as the fuel supply to the combustor diminishes and combustion ceases.

The practical lesson from all this: over-voltage protection for many tens of thousands of volts rather than hundreds or thousands of volts should be provided between power train and ground. 


\subsection{Shock Location}

The range of possible mass flow rates and magnetic field strengths obtained in whe AEDC/HPDE facility allow for operation in both the supersonic and subsonic flow regimes. The nominal operating conditions for the initial testing phase (described in section 3.1.1) were designed to insure operation in the supersonic flow regime. Ideally, the shick-down to subsonic flow would occur dowastream of the active channel. While surveying the nominal operating conditions with a code from the TRANSIENT family, particular attention was given to following the position of the shock as it aried with mass flow rate and magnetic field strength.

Figs. 3-29 and 3-30 are representative of the results of this study at the $2 \mathrm{I}$ nominal conditiun. The orizin of the axial coordinate for these plots is the burner backplate (channel exit is at $9.8 \varepsilon \mathrm{m}$ ). Fig. 3-31 summarizes the predicted location of the normal shock during operation at $2 \mathrm{~T}$, $3 \mathrm{~T}$, and $4 \mathrm{~T}$ with the nominal operating conditions and various mass flow rates. It is seen from these figures that the shock is predicted to lie outside the active portion of the generator, except during $4 \mathrm{~T}$ operation. The $4 \mathrm{~T}$ shock can be pushed out of the generator by a small increase of the mass flow rate within the facility limits. Also s' un are the shock locations predicted for unseeded operation for the nominal N/O ratio and for a combustion mixture, designater $N / O=1.25$, which simulates the off-design conditions for the first hot flow tesc of the generator. The data from this test show i a pressure disturbance indicative of a shock system at approximately $9.1 \mathrm{~m}$. The STD calculation based on the best available interpretation of experimentil conditions predir is a normal shock to exist $1.6 \mathrm{~m}$ downstream of the pressure anomaly. 
This is ronsistent with the empirically determined observation in supersonic flows that the duct stalls 2-3 diameters upstream of a normal shock computed by one-dimensionel gas dynamics.

\subsection{Analysis of Run 006-008}

During start-up of Run 006-008 an electrical breakdown from diffuser to ground developed as the Hall voltage increased. Calcul. ons were initiated with codes fron the Q3D family to determine the leakage current from the diffuser to ground for this run and the associated power loss due to operating with the grounded diffuser. An input data set, which contains magnetic field and load resiotance distributions and thermodynamic data from the AEDC Data Package [3-9], was constructed for use in these computations. These inputs are discussed more fully in Section 3.1.2.

It should be remarked here that this simulation was performed prior to receiving the revised wall temperature schedules of Pigs. 3-6 or 3-7 and as such the calculation was done using the nominal electrode wall temperature schedule of Fig. 3-4. In addition, the assumption of smooth walls upstream of the gererator was made according to the nominal conditions. Subsequent simulations of the Rur. 005-014 data suggest that the upstream conditions are more accurately characterized by rough walls.

As a first approximation to simulate the effects of the grounded diffuser, a series of Q3D computations was performed in which a constant value of $I_{x}$ is assumed to exist at each crosssection of the gencrator. The actual leakage current would be determined as that current which yields distributions of normal current density, power, Paraday voltage, and Hall potential most closely resembiling those measured during the experiment. 
At the time these calculations were performed, the data for the electrode potentials during the run appeared to contain a number of uncertainties. The data from the meter panel and data acquisition systems were each incomplete. Depending on how the data were combined, an estimate fcr the Hall potential difference across the active channel betveen 1100-1600 $\mathrm{V}$ might be obtained. In addition, calculatious to check the loed resistance at several electrode pairs indicated a few discrepancies between the data from the two systems. Many of the load resistances inferred from the measured currents and voltages differ substantially, particularly at the rear end of the generator, from the values used in the simulations. These input values were provided to STD on the basis of separate measurements of load resistance prior to the run and are documented in Section 3.1.2.

According to AEDC persornel [3-13] there were two causes of data inconsistencies in Rua 006-008:

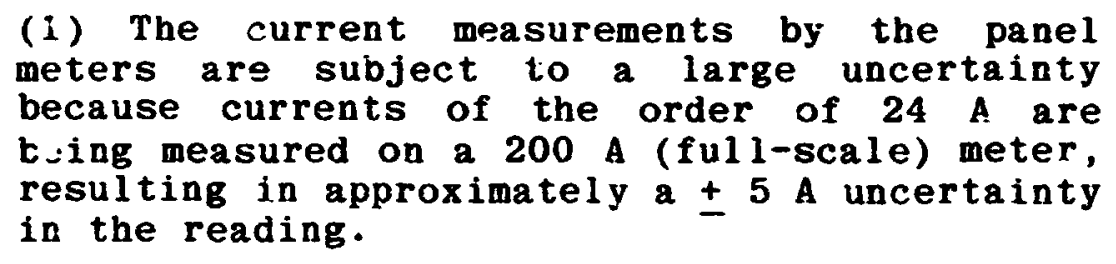

(2) The current transductors used in the data acquisition system were probably measiring such low currents as to be in a nonlinear range of th? probe characteristics.

It is important to note that at the time of the STD Rin 006-008 simulations, the HPDE might be considered to be in a "shake-down" mode in which the data acquisition and reduction procedures were beisg perfected. Some of the data utilized in this simulation have heen confirmed by AEDC to be inconsistent. 
These inconsistencies have been corrected by AEDC since the time of the STD simulations. When the data acquisition system data and the meter panel data for Run 006-008 were refined by AEDC, better agreement with the load resistances was obtaided.

Two Q3D runs were made in an attempt to simulate this experiment: CMSIPUFIKT with $I_{x}=0$ and CMSIQBEIK with $I_{x}=850$ A. The axial variation of the gasdynamic, plasma, and electrical parameters from CMSIPUPIK are given in Figs. 3-32 through 3-43. The axial variation of these same parameters from computation CMSIQBEIKW are given in Figs. $3-44$ through 3-55.

Comparison of the axial variations of the gasdynamic parameters presented in Figs. 3-32 and 3-44 indicates that the values are nearly the same with a slightly higher velocity, Mach number, stagnation temperature, and stagnation pressure from CMSIQBEIKW than for CMSIPUFIKm. Comparison of the axial variation of the plasma properties in Figs. 3-33 and 3-45 indicates that the Hall parameter is larger at the exit for CMSIQBEIKT. As seen by comparison of Figs. 3-35 and 3-47, the Paraday load factor and electrical conversion efficiency are not identical when there is a net $I_{x}$. For CMSIQBEIKT, the Paraday load factor decreases to about 0.4 at the back end of the channel.

Due to the large value of $I_{x}$ and power dissipation in the arc from diffuser to ground, the values of the power extraction parameters are much smaller for cuSIQBEIKT, Fig. 3-48, than for CMSIPUPIKT. Fig. 3-36. The axial variations of the electrical field variables in Pigs. $3-37$ and $3-49$ indicate that the net $I_{x}$ causes the Hall field to be negative at the front and back ends of the channel. In addition, the Faraday 
voltage and normal current density are lower with a net $I_{x}$. Comparison of the axial variation of the electrode potential from Figs. 3-38 and 3-50 shows the effects of $a$ ne: $I_{x}$ : the potentials decrease at the front end of the channel (due to negative $\left.E_{x}\right)$; potentials are smaller throughout the channel; and the differences from cathode to anode (Faraday voltage) are smaller throughout the channel.

Comparison of the electrode voltage drops in Figs. 3-39 and 3-51 indicates that the voltage arops are the same at the entrance, but peak at about a 20\% lower value for CMIQBEIKI. Comparison of the electrode boundary layer parameters on Figs. 3-41 and 3-53 indicate that the net $I_{x}$ causes an increase in the anode shape factor and a decrease in the cathode shape factor. Comparison of the sidewall boundary layer parameters on Figs. 3-42 and 3-54 indicates that the sidewall boundary, displacement, momentum, and enthalpy thicknesses are all greater with a net $I_{x}$.

Computation CASIPUFIKW simulated the conditions which might have been obtained in the HPDE if the external short from diffuser to ground had not occurred. Computation CMSIQBEIKI is a first approximation to simulate the conditions of Run 006-008 at the time corresponding to $T_{2}$ of approximately $9.0 \mathrm{~s}$. This computation is only a first approximation because the actual $I_{x}$ caused by the shorted diffuser is not known. Therefore, precise correspondence between calculated and measured values cannot be expected.

Fig. 3-56 illustrates the static pressure distribution, both measured and predicted, in the active generator. The excellent agreement of theory and experiment indicates that there were no large-scale anomalous fluid mechanical phenomena 
present in the channel during this portion of the run, and suggest that the differences between the experimental data and the computations described below may be mainly electrical in nature.

The measured and predicted potential of the cathode are depicted in Fig. 3-57. The experimental distribution from the AEDC meter panel data system was obtained from an AEDC plot, which is given in Fig. 3-58. It should be remarked here that the meter data for the potential is unreliable over the first 10 instrumented electrodes, since negative potertials could not bc accurately measured at the time of the run. Also plotted in F:g. 3-57 is the cathode potential recorded by the data acquisition system. Both experinental distributions have been plotted with an offset from the recorded values such that the potential at electrode no. 168 is zero.

The Q3D calculations as originally planned would determine the constant value of $I_{x}$ in the generator such that the Hall potential difference computed from entrance to exit compared well with the measured values. The unreliability of the meter data at ihe inlet and the sparsity of the data from the acquisition system at the exit prevents a precise determination of the potential difference. Simultaneous consideration of both sets of data indicate a Hall potential difference of about $i 200 \mathrm{~V}$ as a reasonable target (see Fig. 3-57). The Q3D calculation CMSIQBEIKW was made with $I_{K}=850$ A, nominal wall temperatures, and diffuse current transfer. The predicted Hail potential shows good qualitative agreement with the data. Purther computations at an increased $I_{x}$ would probably lower the potential difference to the arget value. 
Figs. 3-59, 3-60 and 3-61 are plots of the experimental and the calculated distributions of normal current density, Faraday voltage, and power respectively. The meter data distributions were obtained from an AEDC figure, which is reproduced as Fig. 3-62, while those for the data acquisition system were taken from [3-9]. Again, there is good qualitative agreement between theory and experiment for these quantities. clearly, the theory and experiment lack quantitative agreement, especially in the rear-end region of the generator. Possible causes of this lack of agreement are (1) failure to match I (2) unreliable electrical data, or (3) the presence of phenomena not revealed by the present computation.

Certainly, some of the discrepancy is expected since the Hall potential comparison of Fig. 3-57 indicates that a higher $I_{x}$ should be used in the computation. in addition, there are discrepancies in the experimental data recorded by the two measuring systems. Perhaps this is best illustrated in the current and voltage graphs of Figs. 3-59 and 3-60. While the voltage measurements at $T_{2}=8 \mathrm{~s}$ agree fairly well, the current distributions vary differently along the channel. In addition to the fact that the data is in some instances perhaps not yet well understood, the vccurrence of additional phenomena such as internal shorting and current loops (resulting in nonconstant $I_{x}$ ) in the generator cannot be excluded. STD computation CMSIQBEIKN utilized a leakage current of $I_{z}=850 \mathrm{~A}$ to predict a Hall potential difference of approximately $1400 \mathrm{~V}$. An additional estimate for the magnitude of $I_{x}$ can be obta. ed from the equation

$$
I_{\mathbf{x}}=\frac{V_{\sigma}}{A} \mathbf{L}
$$


where $L$ is the length over which the short accurs and $A$ is the cross sectional area occupied by the current. At the back end of the channel a typical cross sectional area is $0.8 \mathrm{~m}^{2}$. The measurements from the data acquisition system at $T_{2}=8 \mathrm{~s}$ indicate an anode to ground potential (V) of approximately 1400 $V$ at $6.22 \mathrm{~m}$ from the first loaded electrode. The axial distance from this station to the diffuser (grounded) is 2.67 m. STD computations yield an electrical conductivity ( ) in this region of approximately $3 \mathrm{~s} / \mathrm{m}$. Assuming the leakage current fills the entire cross-sectional area of the channel, application of $\mathrm{Eq}$. (1) yields $I_{x}=1300 \mathrm{~A}$. Similarly, at the front end of the generator typical values of the crosssectional area and gas conductivity are $0.14 \mathrm{~m}^{2}$ and $11 \mathrm{~s} / \mathrm{m}$, respectively. The experimental data at $T_{2}=8 \mathrm{~s}$ indicates an anode to ground potential of approximately $400 \mathrm{~V}$ at $1.82 \mathrm{~m}$ from the channel inlet. Using these values, application of Eq. (1) predicts a leakage current of $340 \mathrm{~A}$.

To obtain a valid value of the leakage current and its effects, it is necessary to take into account the structure and location of the leakage current with sophisticated models. Although the bulk conductivity -veraging presented in the previous paragraph is crude, the order of magnitude of the leakage current may be estimated.

\subsection{Steady State Q3D Simulation of Run 006-014}

AEDC Run 006-014 was a powered run of 8 seconds duration which arced in the breech at approximately $5.3 \mathrm{~s}$ after the seed entered the channei. The experimental data show two distinct performance regimes (see Figs. 3-63 to 3-72)

(1) A period between $4.5 \leq \mathrm{T}_{2} \leq 5.21 \mathrm{~s}$ in which the channel is ungrounded añd the data is relatively steady in time. 
(2) A period between $5.5 \leq T_{\text {. }} \leq 6.5 \mathrm{~s}$ in which the channel is operating in a grounded condition.

The Q3D simulations performed under this contract have pertained only to the channel performance when in the ungrounded condition.

The input data for the Q3D simulations are fully described in Section 3.1.2. These data were obtained by averaging the experimental data over the time period $4.54 \leq \mathrm{T}_{2}$ $\leq 5.21 \mathrm{~s}$. The effect of the bleed resistor was accounted for in the calculation with a constant axial current of $23.54 \mathrm{~A}$. This value was obtained from the average diffuser-to-ground voltage $(9416 \mathrm{~V})$ and the known resistance of the $\# 12$ wire used as the blead resistor ( 400 ohms).

Heat transfer measurements by AEDC personnel have resulted in a modified electrode surface temperature as shown in Fig. 3-6. The sparseness of the heat transfer data prohibits exact specification of the electrode surface temperature. The Q3D simulations have, at the suggestion of AEDC personnel, used temperatures corresponding to the $\mathbf{4 5 0}$ $\mathrm{Btu} / \mathrm{ft}^{2}-\mathrm{sec}$ and $50 \mathrm{Btu} / \mathrm{ft}^{2}-\mathrm{sec}$ curves (at $\mathrm{T}=5 \mathrm{~s}$ ) at the inlet and exit flanges respectively. A linear variation of temperature with axial location was assumed between these po ats. The nominal temperature distribution was retained for the insulating walls (Fig. $3-4$ ).

A list of the nominal load resistances, supplied by AEDC, is contained in Table 3-7 for runs subsequent to Run 006-006. These values of resistances have been normalized ${ }^{2}$, a 
nominal water conductivity factor of $150 \mu \mathrm{s} / \mathrm{cm}$. Also listed in Table 3-7 ale the average load resistances obtained from the experimental data at these locations. To conform as closely as possible to the experimentally observed values of the load resistance, and yet retain the detailed electrode-by-electrode distribution provided by the nominal schedule, STD adjusted the nominal schedule with a conductivity factor of $159 \mu \mathrm{s} / \mathrm{cm}$ for these initial calculations. This agrees with the conductivity values derived from the data acquisition system values for Faraday voltage divided by Faraday current over the first 168 electrode pairs. The load schedule used in these simulations appears to be at variance with the experimentally indicated values in the downstream half of the generator, and the performance predictions may be expected to be less reliable toward the aft end of the generator. A plot of input load resistance schedule and the resistance as determined by the experimental data at each instrumented electrode pair is given in Fig. 3-86.

Three Q3D calculations have been made during the theoretical simulation of Run 006-014:

(1) STD Computation BTAYUYDJBE: assumes all walls prior to inlet flange can be characterized as smooth walls. Inlet boundary layer thickness is $18 \mathrm{~mm}$. Enthalpy loss upstream of the generator inlet flange is $5.7 \%$.

(2) STD Computation CHPQUUUJBO: assumes roughness of all walls prior to generator inlet flange can be characterized by an equivalent sand roughness of $3 \mathrm{~mm}$. Enthalpy loss upstream of generator inlet flange increases to $7.2 \%$ and inlet boundary layer thickness increases to 44 mm. (See Section 3.1.2.5). 
(3) STD computation CHPQQFLJDV: Assumes roughness of all walls prior to generator inlet flange can be characterized as smooth walls. Inlet boundary layer thickness is $18 \mathrm{~mm}$. Enthalpy loss upstream of the generator is increased to $7.2 \%$ to account for a $3 \%$ combustion inefficiency due to incomplete droplet-burnout, as discussed in $[3-14]$. The chemistry was maintained the same, however.

Figures 3-74 to 3-89 contain the results of a typical Run 006-014 simulation calculation (CHPQUUUJBO) in the form of axial profiles of the key gasdynamic ant electrical variables. Fig. 3-75 shows that the Faraday load factor, $K$, decreases along the channel from approximately 0.7 to 0.6 . Figs. 3-76 and 3-77 show that the interaction parameter based on velocity and the interaction parameter based on pressure achieve values at the end of the channel of approximately 2.3 and 0.95 respectively. This interaction is sufficiently high to cause noticeable electrode boundary layer asymmetries (e.g., the electrode shape factors in Fig. 3-79) and rearly zero blockage at the channel exit (Fig. 3-76). Fig. 3-86 shows that the generator power output under these conditions is approximately $22 \mathrm{MT}$. Maximum normal current densities and Hall fields of approximately $0.7 \mathrm{~A} / \mathrm{cm}^{2}$ and $2.0 \mathrm{kV} / \mathrm{m}$ respectively exist at steady-state conditions (Fig. 3-87). Fig. 3-89 indicates that boundary layer voltage drops in excess of $2000 \mathrm{~V}$ may exist at the end of the generator for this relatively cold wall operation.

The boundary layer voltage drop measurements are perhaps not sufficiently refined at the present time to discriminate between various models of current transport at the walls. The diffuse discharge model selected for the Q3D simulation of Run 006-014, has yielded voltage drops within the range of uncertainty of the data and has been approximately confirmed with the more sophisticated FIN and ARRAY calculations described in Section 4.5 . 
Under conditions of higher interaction than were studied with Q3D in the neighborhood of the Run 006-014 operating conditions, the flow will become transonic in the MHD generator. The Q3D family of codes requires additional computational resources to iterate on the downstream pressure condition in transonic or subsonic flows. To economize, the high interaction cases for the Run 006-014 parametric study were carried out with the TRANSIENT family of codes, with the quasi-steady option. The starting point for the TRANSIENT parametric variations is a simulation of Run 006-014 utilizing data generated by the Q3D simulationn CHPQUYDJBE to account for multidimensional phenomena. Figures 3-90 through 3-98 are plots of the results of a computation, BRCYTRCJEB, with a code from the TRANSIENT family to simulate AEDC Run 006-014. The origin of the axial coordinate in these graphs is the burner backplate. The first loaded electrode is at $x=2.7 \mathrm{~m}$ and the last is at $x=9.4 \mathrm{~m}$ (see Fig. 3-90). These calculations were made for conditions similar to those of CHPQUYDJBE (smooth walls upstream of generator and $100 \%$ efficient combustion), which were discussed in the previous section.

Most of the key parameters resulting from TRANSIENT simulations shown in Pigures 3-90 through 3-98 are also quite comparable to the Q3D simulation CHPQUUUJBO, which was described in the previous section. In particular, Figure 3-96 shows that a maximum current density of $0.685 \mathrm{~A} / \mathrm{cm}^{2}$ and a maximum Hall field of $2.005 \mathrm{kV} / \mathrm{m}$ are predicted with the TRANSIENT code. This compares well with the Jy,max of 0.7 $\mathrm{A} / \mathrm{cm}^{2}$ and the $\mathrm{E}_{\mathrm{x}, \max }$ of $2.007 \mathrm{kV} / \mathrm{m}$ predicted with $\mathrm{Q} 3 \mathrm{D}$ (see Fig. 3-87). Similarly, the exit Mach numbers predicted by TRANSIENT and Q3D are 1.73 and 1.74 , respectively (Figs. 3-78 and 3-91). 
These figures also show the shock is located $10.74 \mathrm{~m}$ from the burner backplate. The total heat loss to the wall predicted by TRANSIENT is $31.4 t \mathrm{MH}$, while that predicted by Q3D is $30.97 \mathrm{MW}$. The TRANSIENT calculation yields $23.6 \mathrm{MW}$ for the integrated power output while the Q3D calculation CHPQUUUJBO predicts 21.3 MW or a difference of approximately 10\%. Most of this discrepancy is due to the pre-generator wall roughness characterization which was for smooth walls in the TRANSIENT calculations and for rough walls in CHPQUUUJBO. The power output from the TRANSIENT calculation is more comparable to the result of the Q3D simulation CHPQUYDJBE, $24.6 \mathrm{MW}$.

The simulation of the generator operation with the TRANSIENT code is useful because it gives a broader view, if with slightly less detail, of the entire flow train. It should be noted that the accuracy of TRANSIENT and the agreement between results from computations with the TRANSIENT and Q3D code families is due to inputs to TRANSIENT from 23D calculations to account for fundamentally multidimensional phenomena such as wall losses and plasma nonuniformity factors. This is necessary to ensure the accuracy of any quasi-onedimensional calculation.

\subsection{Comparisor of Run 006-014 Measurements with Q3D}

\section{Simulations}

The resilts from Q3D computations discussed in Section 3.7 were analyzed and compared to the experimental data from HPUE Run 006-014. In this section the results of this comparison are presented with emphasis placed on two Q3D calculations, CHPQUUUJBO and CHPQQFIJDV. The conditions and underlying assumptions for these separate computations (see Section 3.7 ) are identical except for the following: 
(1) The inlet boundary layer thickness and enthalpy flux are determined by a smooth wall boundary layer calculation upstream of the generator for computation CHPQQFLJDV (rather than rough with $3 \mathrm{~mm}$ equivalent sand roughness assumed in CHPQUUUJBO).

(2) A 3\% combustion inefficiency due to incomplete droplet-burnout is assumed for comnutation CIIPQQFLJDV (rather than a 100\% efficient combustor assumed in (HPQUUUJBO).

The experimental data for the static pressure, a primary measurement of the data acquisition data, during the time after ignition, $4.1 \leq \mathrm{T}_{2} \leq 5.2 \mathrm{~s}$ is given in Fig. 3-99. The static pressure is a primazy measurement of the data acquisition data. The axial coordinate in Fig. $3-99$ is referenced to the generator inlet flange. Also given in Fig. 3-99 are the axial distributions of static pressure from computations CHPQuUUJBO and COBQQFLJDV. There is excellent agreement between the experimental and the computed values throughout the channel.

The experimental HPDE meter panel data from Run 00014 at $T_{2}=4.8 \mathrm{~s}$ are compared to the values obtained from computations CHPQUUUJBO and COBQQFLJDV in Figs. 3-100 through - 111. The axial crordinate in these figures is referenced to the inlet flange. The Faraday vc:tage (Fig. 3-100) and the current per electrode (Fig. 3-101) are primary measurements of the meter panel data. The normal current density (Fig. 3-102) and the integrated power output (Fig. 3-105) are determined from the primary measurements and other geometrical data. The computed values from computations CHPQUUUJBO and COBQQFLJDV fall within the measurement tolerances of the experimental values. Some local discrepancies of up to $5 \%$ are evident, but it is our judgment that the accuracy of the input data, especially for load resistance, as well as the $\pm 2 \%$ fluctuations in current and voltage during the period $4.5 \mathrm{~s}<\mathrm{T}_{2}<$ 5.21 s could explain such differences. As shown in Fig. 3-100, 
the computations tend in underpredict the Faraday voltage in the front half of the generator. This can be explained by consideration of the uncertainty of the channel loading.

The difference between the voltage-curyent characteristics measured by the two datd-gathering systems and the nominal values is discussed in sectior 3.7. Tha calculatec values of the load resistances from the meter panul data yield values that are typically $10 \%$ higher than those computed from the data acquisition system (see Fig. 3-103). This is especially true in the latter third of the generator. In addition, the value of a load resistance computed at a particular electrode pair can vary $3-5 \%$ with time.

A rough calculation, under the assumption that the fluid properties change only slightly at this rather low interaction level, indicates that if the downstream load resistance were matched more accurately, a lower current and a higher Faraday voltage could be expected, the differences being approximately 3.5\%. The integrated power output, shown in Fig. 3-105 would be relatively unaffected (the difference is estimated to be less than 1\%) by this adjustmen: to input load resistance.

The integrated power distribution for the meter panel data measurements can be regaided as approximate, but better than the distribution obtained from the data acquisition system measurements. Inese points were calculated by numerically integrating the power through the loads for the instrumented electrode pairs. To obtain a value for the integrated powir, the power was assumed to vary linearly between each set of instrumented electrodes ( 10 electrode pairs apart). The scatter in the power per electrode measured by the meter panel data, and plotted in Fig. 3-10\%, indica tes that such a regular behavior is probably a weak assumption. 
Computation CHPQUUUJBO simulated a bleed resistor from the diffuser-to-ground with a net asial current $0: 24$ A. The power dissipated is this bleed resistor was calculated to be $0.2 \mathrm{MI}$ by cHpquoujBo. This te less than $1 \%$ of the power produced in the channel.

It should be reemphasized that these preliminary computations have arsumed diffuse-mode current transfer. At t'e relatively cold electrode surface temperatures indicated by the experiwental heat transfer measurements to this point, arc mode surrent transfer is a distinct possibility, particularly on the anocies, and should be considered. This will be discissed further in Section 4.5 This will be discussed further in section 4.5. The res.2!ts described in Section 4.5 further suggest the possibilicy of the existence of very small arcs, particularly on the anodes.

The axial distribution of total electrode voltage drop from computation CHfQuUUJBO is given in Fig. 3-106. Experimental vaiues of trtal voltage drop, obtained from the HPDE program manager, at four sxial locations are also given in this figure. The electrode voltage drops computed by STD Computation CHPQUUUJBO appear to agree with the measured voltage drops within the experimental tolerances.

The axial distributions of centerline, cathode, and anode electrical potential are given in Figs. 3-107, 3-108, and $3-109$, respectively. The potentiais have been adjusted to set grount $(0.0 \mathrm{~V})$ at the centerline of the first loaded electrode pair. Thcre is excellent agreement between the meter panel and the results of cumputation CORQGFLJDV. Also given on Fig. 3-109 are the data obtained by the data acquisition system for the time period $4.1 \leq \mathrm{T}_{2} \leq 5.2 \mathrm{~s}$. 


\section{REFERENCES}

[3-1] G. T. Garrison, et al, "Annual Report on the MHD Hi Report on the MHD High Performance Demonstration Experiment," ERDA Report FE-1542-1, July 1975

[3-2] H. J. Schmidt, et al, "Report on the MHD Performance Experiment of the Period May 1, 1975, to September 30, 1976," ERDA Report FE-1542-2, July 1977

[3-3] H. J. Schmidt, et al, "Report on the MHD Performance Experiment for the Period October 1, 1976, to September 30, 1977," DOE Report FE-1542-35, March 1978

[3-4] H J. schmidt, et al, "Report on the MHD Performance Experiment for the Period October 1, 1977 to September 30, 1978," DOE Report FE-2895-3, Apr11, 1979

[3-5] Meeting with R. Starr, J. Lineberry, H. Schmidt, and Milam of ARO, Inc., B. Phillips of NASA/Lewis Research Center, and T. Swean and A. Vetter of STD Research Corporation at AEDC, 9 August 1979

[3-6] R. J. Averill, et al, "Final Engineering Report on the Dual Mode Magnet for the Righ Performance Demonstration Experiment," ERDA Report FE-1542-3, July 1977

[3-7] H. J. Schmidt, "Final Report on the Design of the MhD Generator Channel for the High Perfo.mance Demonstration Experiment," ERDA Report FE-1542. August 1977

[3-8 j R. L. Lowry, "High Performance Demonstration Experiment S:personic Faraday Performance Evaluation," Data Package: Propulsion hind Tunnel Pacility, Arnold Enginetring Development Center; Preliminary Data: Run :-006-006, Sept. 1979

[3-9] R. L. Lowry, "High Performance Demonstration Experiment Supersonic Faraday Performance Evaluation," Data Package: Propulsion Wind Tunnel Facility, Arnold Engineering Development Center; Preliminary Data: Run MI-006-008, Oct. 1979 
[3-10] R. L. Lowry, "High Performance Demonstration Experiment Supersonic Faraday Performance Evaluation," Data Package: Propulsion Wind Tunnel Pacility, Arnold Engineering Development Center; Preliminary Data: Run MI-006-010, 31, Oct. 1979

[3-11] Preliminary Data received during meeting between $R$. Starr, R. Lowry, H. Schmidt, and Milan of AEDC and T. Swean and A. Vetter of STD Research Corporation at AEDC, January 1980

[3-12] R. L. Starr, et al., "Description, Performance, and Preliminary Faraday Power Production Results of the HPDE Facility," Paper presented at the $7 \mathrm{th}$ Int'l. Conference on MHD electrical Power Generation, MIT, June 1980

[3-13] Private Communication: telephone conversation with R.L. Starr 6 Dec. 1979

[3-14] S. T. Demetriades, "Analyses of Critical Transient Phenomena in the AEDC/HPDE, Vol. III, Initial Pre-experiment Combustor Analysis," STD Research Corporation Report FE-2243-17 prepared for ERDA, Division Magnetohydrod, samics, May 1978

[3-15] R. L. Lowry, "High Performance Demonstration Experiment Supersonic Paraday Performance Evaluation," Data Package: Propulsion Wind Tunrel Facility, Arnold Engineering Developwent Center; Preliminary Data: Run MI-006-013, 14 January 1980

[3-16] R. L. Lowry, "High Performance Demonstration Experiment Supersonic Faraday Performance Evaluation," Data Package: Propulsion Mind Tunnel Facility, Arncid Engineering Development Center; Preliminary Data: Run MI-006-014, 22 January 1980 
TABLE 3-1

Channel Electrode Width

Electrode Reference Electrode Midth**
(m) No. of Electrodes No. *

$\begin{array}{rll}-30 & \text { to } & 26 \\ 27 & \text { to } & 58 \\ 59 & \text { to } & 162 \\ 163 & \text { to } & 394 \\ 395 & \text { to } & 444 \\ 445 & \text { to } & 454\end{array}$

0.0254

0.01905

57

0.01588

0.0127

0.0254

0.02223

50

10

*Electrodes are numbered consecutively upstream and downstream from electrode 0 which is the first electrode connected to a load.

** The insulator thickness is $1.52 \mathrm{~mm}$ between adjacent electrodes 


\section{TABLE 3-2}

Nominal Flow Rates of Combustion Gases

Component

Fuel

oxidizer

seed

$\mathrm{N}_{2} / \mathrm{O}_{2}$

Pilot $\left(\mathrm{CH}_{4}\right)$

Seed Concentration
Flow Rate

$5.64 .3 \mathrm{~kg} / \mathrm{s}$

$37.247 \mathrm{~kg} / \mathrm{s}$

$2.069 \mathrm{~kg} / \mathrm{s}$

1.0

$0.034 \mathrm{~kg}$ is

$0.454 \mathrm{~kg} \mathrm{KOH} / \mathrm{kg} \mathrm{CH} \mathrm{CH}_{3} \mathrm{OH}$

Total enthalpy of combustion gases (no losses) $271.7 \mathrm{MH}$

Specific enthaipy of combustion gases $6.04 \mathrm{MJ} / \mathrm{kg}$ (adiabatic conditions)

$T_{\text {flame }}(6 \mathrm{~atm})=3021 \mathrm{~K}$

$0-4356$ 


\section{TABLE $3-3$}

Nominal Magnetic Pield Used for AEDC Simulations

Run no.

$$
\frac{\langle B(H 2 A)+B(H 3 A)\rangle *}{2}
$$

1.537

2.327

2.697

2.810

3.231
Time Interval Ref.

$4.5 \leq \mathrm{T}_{2} \leq 7.5 \quad[3-8 \mid$

$9.5 \leq T_{2} \leq 10.2 \quad[3-9]$

$4.0 \leq T_{2} \leq 4.5 \quad[3-10]$

$4.5 \leq \mathrm{T}_{2} \leq 5.5 \quad[3-11]$

$\left.4.5 \leq T_{2} \leq 5.3 \quad \mid 3-11\right]$

*Due to nonlinear behavior of the instrumentation, the numbers presented in this table are slightly in error. Refined data. [3-12], were not available at the time these simulations were performed. 
TABLE 3-4.

A. HPDE Load Resistance Settings for Runs Prior to 006-008

\begin{tabular}{|c|c|c|c|c|c|}
\hline $\begin{array}{l}\text { Electrode } \\
\text { No. }\end{array}$ & $\begin{array}{l}\text { Reference } \\
\text { Resis. } \Omega \\
(\mathbb{K}=150)\end{array}$ & $\begin{array}{l}\text { Date } \\
1979\end{array}$ & $\begin{array}{l}\text { Electrode } \\
\text { No. }\end{array}$ & $\begin{array}{l}\text { Reference } \\
\text { Resis. } \Omega \\
(K=150)\end{array}$ & $\begin{array}{l}\text { Date } \\
1979\end{array}$ \\
\hline $\begin{array}{r}-24 \\
-23 \\
-22 \\
-21 \\
-20 \\
-19 \\
-18 \\
-17 \\
-16 \\
-15 \\
-14 \\
-13 \\
-12 \\
-11 \\
-10 \\
-9 \\
-8 \\
-7 \\
-6 \\
-5 \\
-4 \\
-3 \\
-2 \\
-1 \\
0 \\
1 \\
2 \\
3 \\
4 \\
5 \\
6 \\
7 \\
8 \\
9 \\
10 \\
11 \\
12 \\
13 \\
11 \\
10 \\
16\end{array}$ & 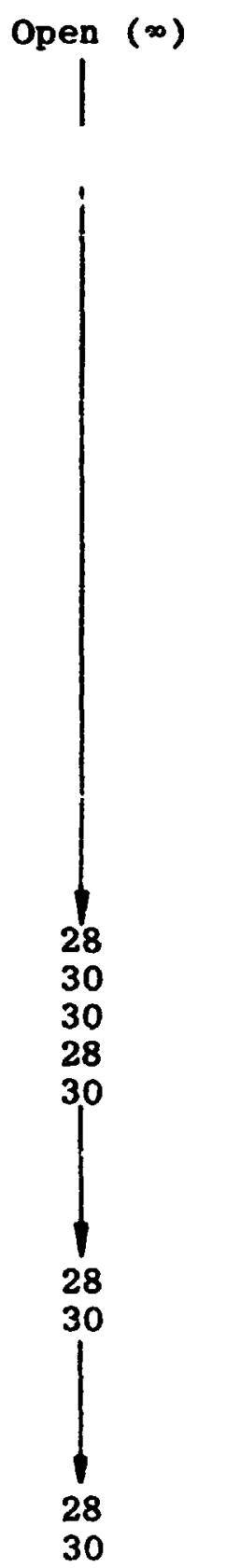 & $i^{\text {Oct. } 10}$ & $\begin{array}{l}17 \\
18 \\
19 \\
20 \\
21 \\
22 \\
23 \\
24 \\
25 \\
26 \\
27 \\
28 \\
29 \\
30 \\
31 \\
32 \\
33 \\
34 \\
35 \\
36 \\
37 \\
38 \\
39 \\
40 \\
41 \\
42 \\
45 \\
44 \\
45 \\
46 \\
47 \\
48 \\
49 \\
50 \\
51 \\
52 \\
53 \\
54 \\
55 \\
56 \\
57\end{array}$ & $\begin{array}{c}28 \\
30 \\
28 \\
28 \\
30 \\
32 \\
30 \\
39 \\
37 \\
39 \\
41 \\
39 \\
37 \\
39 \\
19 \\
38 \\
4 \\
4\end{array}$ & $\left.\right|_{\text {Oct. }} ^{10}$ \\
\hline
\end{tabular}


TABLE 3-4. (cont.)

\begin{tabular}{|c|c|c|c|c|c|}
\hline $\begin{array}{l}\text { Electrode } \\
\text { No. }\end{array}$ & $\begin{array}{l}\text { Reference } \\
\text { Resis. } \Omega \\
(\mathrm{K}=150)\end{array}$ & $\begin{array}{l}\text { Date } \\
1979\end{array}$ & $\begin{array}{c}\text { Electrode } \\
\text { No. }\end{array}$ & $\begin{array}{l}\text { Reference } \\
\text { Resis. } \Omega \\
(\mathbb{K}=150)\end{array}$ & $\begin{array}{l}\text { Date } \\
1979\end{array}$ \\
\hline $\begin{array}{c}58 \\
59 \\
60 \\
61 \\
62 \\
63 \\
64 \\
65 \\
66 \\
67 \\
68 \\
69 \\
70 \\
71 \\
72 \\
73 \\
74 \\
75 \\
76 \\
77 \\
78 \\
79 \\
80 \\
81 \\
82 \\
83 \\
84 \\
85 \\
86 \\
87 \\
88 \\
89 \\
90 \\
91 \\
92 \\
93 \\
94 \\
95 \\
96 \\
97 \\
98 \\
99 \\
100 \\
101\end{array}$ & \begin{tabular}{|}
47 \\
40 \\
47 \\
50 \\
47 \\
1 \\
50 \\
47 \\
1 \\
50 \\
51 \\
51 \\
51 \\
5 \\
5 \\
5 \\
5 \\
5
\end{tabular} & $\left.\right|^{\text {Oct }}$ & $\begin{array}{l}102 \\
103 \\
104 \\
105 \\
106 \\
107 \\
108 \\
109 \\
110 \\
111 \\
112 \\
113 \\
114 \\
115 \\
116 \\
117 \\
118 \\
119 \\
120 \\
121 \\
122 \\
123 \\
124 \\
125 \\
126 \\
127 \\
128 \\
129 \\
130 \\
131 \\
132 \\
133 \\
134 \\
135 \\
136 \\
137 \\
138 \\
139 \\
140 \\
141 \\
142 \\
143 \\
144 \\
145\end{array}$ & 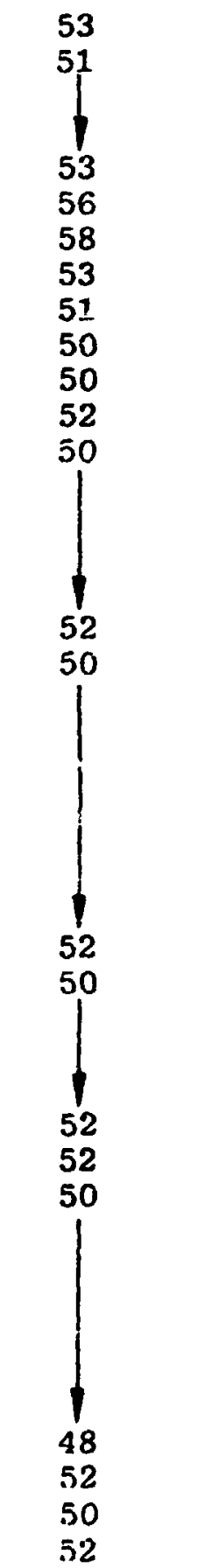 & $\left.\right|_{\text {Oct.16 }} ^{\text {Oct.15 }}$ \\
\hline
\end{tabular}


TABLE 3-4. (cont.)

\begin{tabular}{|c|c|c|c|c|c|}
\hline $\begin{array}{l}\text { Electrode } \\
\text { No. }\end{array}$ & $\begin{array}{l}\text { Reference } \\
\text { Resis. } \Omega \\
(\mathrm{K}=150)\end{array}$ & $\begin{array}{l}\text { Date } \\
1979\end{array}$ & $\begin{array}{l}\text { Electrode } \\
\text { No. }\end{array}$ & $\begin{array}{l}\text { Reference } \\
\text { Resis. } \Omega \\
(K=150)\end{array}$ & $\begin{array}{l}\text { Date } \\
1979\end{array}$ \\
\hline $\begin{array}{l}146 \\
147 \\
148 \\
149 \\
150 \\
151 \\
152 \\
153 \\
154 \\
155 \\
156 \\
157 \\
158 \\
159 \\
160 \\
16: \\
162 \\
163 \\
164 \\
165 \\
166 \\
167 \\
168 \\
169 \\
170 \\
171 \\
172 \\
173 \\
174 \\
175 \\
176 \\
177 \\
178 \\
179 \\
180 \\
181 \\
182 \\
183 \\
184 \\
185 \\
186 \\
187 \\
188 \\
189\end{array}$ & 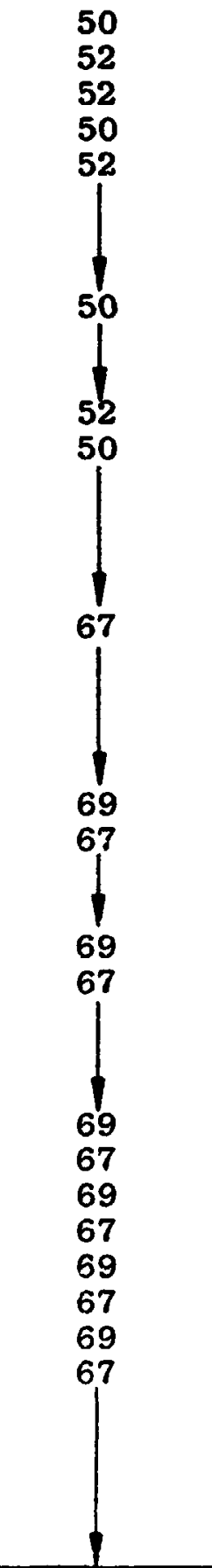 & $\left.\right|_{\text {oct.17 }}$ & $\begin{array}{l}190 \\
191 \\
192 \\
193 \\
154 \\
195 \\
196 \\
197 \\
198 \\
199 \\
200 \\
201 \\
202 \\
203 \\
204 \\
205 \\
206 \\
207 \\
208 \\
209 \\
210 \\
211 \\
212 \\
213 \\
214 \\
215 \\
216 \\
217 \\
218 \\
219 \\
220 \\
221 \\
222 \\
223 \\
224 \\
225 \\
226 \\
227 \\
228 \\
229 \\
230 \\
231 \\
232 \\
233\end{array}$ & 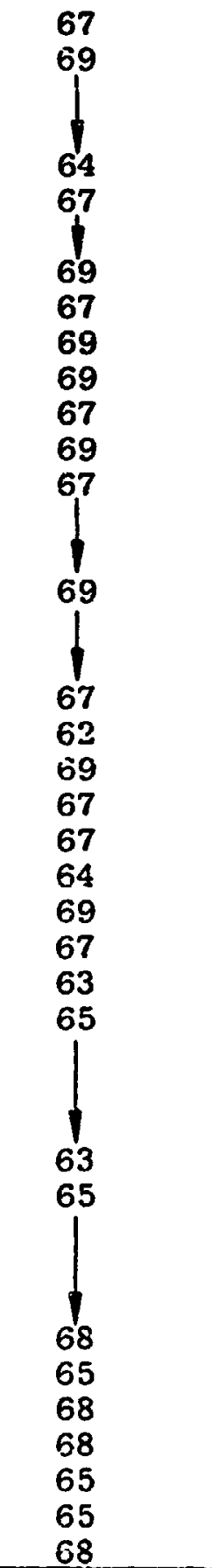 & $\left.\right|_{\mid} ^{\text {Oct. } 17}$ \\
\hline
\end{tabular}


TABLE 3-4. (cont.)

\begin{tabular}{|c|c|c|c|c|c|}
\hline $\begin{array}{l}\text { Electrode } \\
\text { No. }\end{array}$ & $\begin{array}{l}\text { Reference } \\
\text { Resis. } \Omega \\
(\mathrm{K}=150)\end{array}$ & $\begin{array}{l}\text { Date } \\
1979\end{array}$ & $\begin{array}{l}\text { Electrode } \\
\text { No. }\end{array}$ & $\begin{array}{l}\text { Reference } \\
\text { Resis, } \Omega \\
(B=150)\end{array}$ & $\begin{array}{l}\text { Date } \\
1979\end{array}$ \\
\hline $\begin{array}{l}234 \\
235 \\
236 \\
237 \\
238 \\
239 \\
240 \\
241 \\
242 \\
243 \\
244 \\
245 \\
246 \\
247 \\
248 \\
249 \\
250 \\
251 \\
252 \\
253 \\
254 \\
255 \\
256 \\
257 \\
258 \\
259 \\
260 \\
261 \\
262 \\
263 \\
264 \\
265 \\
266 \\
267 \\
268 \\
269 \\
270 \\
271 \\
272 \\
273 \\
274 \\
275 \\
276 \\
277\end{array}$ & 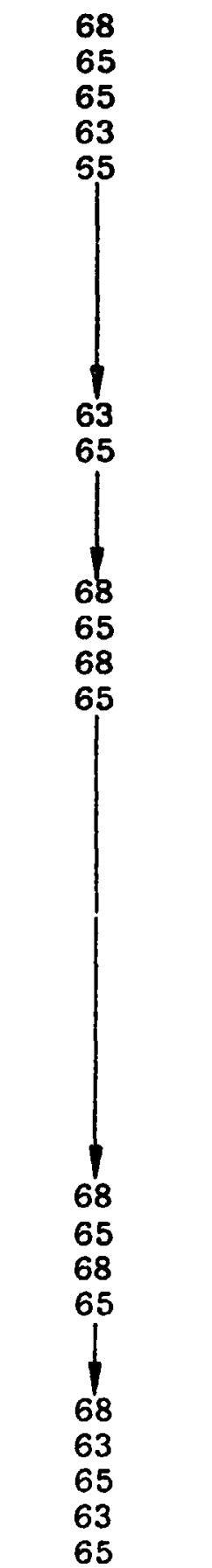 & Oct.18 & $\begin{array}{l}278 \\
279 \\
280 \\
281 \\
282 \\
283 \\
284 \\
285 \\
286 \\
287 \\
288 \\
289 \\
290 \\
291 \\
292 \\
293 \\
294 \\
295 \\
296 \\
297 \\
298 \\
299 \\
300 \\
301 \\
302 \\
303 \\
304 \\
305 \\
306 \\
307 \\
308 \\
309 \\
310 \\
311 \\
312 \\
313 \\
314 \\
315 \\
316 \\
317 \\
318 \\
319 \\
320 \\
321\end{array}$ & 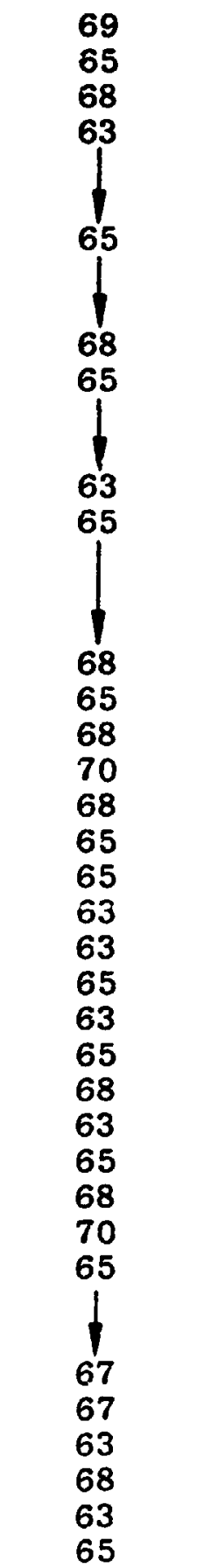 & $\operatorname{Oct}_{i} 18$ \\
\hline
\end{tabular}


TABLE 3-4. (cont.)

\begin{tabular}{|c|c|c|c|c|c|}
\hline $\begin{array}{l}\text { Electrode } \\
\text { No. }\end{array}$ & $\begin{array}{l}\text { Reference } \\
\text { Resis. } \Omega \\
(K=150)\end{array}$ & $\begin{array}{l}\text { Date } \\
1979\end{array}$ & $\begin{array}{l}\text { Electrode } \\
\text { No. }\end{array}$ & $\begin{array}{l}\text { Reference } \\
\text { Resis } \Omega \\
(\mathrm{K}=150)\end{array}$ & $\begin{array}{l}\text { Date } \\
1979\end{array}$ \\
\hline $\begin{array}{l}322 \\
323 \\
324 \\
325 \\
326 \\
327 \\
328 \\
329 \\
330 \\
331 \\
332 \\
333 \\
334 \\
335 \\
336 \\
337 \\
338 \\
339 \\
340 \\
\mathbf{3 4 1} \\
342 \\
343 \\
344 \\
345 \\
346 \\
347 \\
348 \\
349 \\
350 \\
351 \\
352 \\
353 \\
354 \\
355 \\
356 \\
357 \\
358 \\
359 \\
360 \\
361 \\
362 \\
363 \\
364 \\
365 \\
366\end{array}$ & $\begin{array}{r}70 \\
65 \\
68 \\
68 \\
65 \\
63 \\
68 \\
65 \\
65 \\
70 \\
68 \\
65 \\
63 \\
65 \\
65 \\
1 \\
\\
\end{array}$ & $\left.\right|^{\text {oct.18 }}$ & 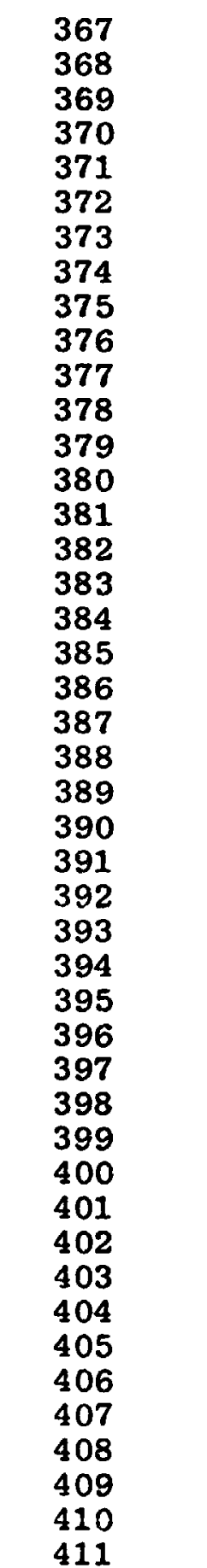 & $\begin{array}{l}70 \\
68 \\
66 \\
70 \\
63 \\
89 \\
91 \\
89 \\
89 \\
105 \\
103 \\
108 \\
127 \\
152 \\
164 \\
176 \\
\text { Open (o) } \\
\mid\end{array}$ & oct.19 \\
\hline
\end{tabular}


TABLE 3-4. (cont.)

\begin{tabular}{|c|c|c|}
\hline $\begin{array}{l}\text { Elect rode } \\
\text { No. }\end{array}$ & $\begin{array}{l}\text { Reference } \\
\text { Resis. } \Omega \\
(\mathrm{K}=150)\end{array}$ & $\begin{array}{l}\text { Date } \\
1979\end{array}$ \\
\hline $\begin{array}{l}412 \\
413 \\
414 \\
415 \\
416 \\
417 \\
418 \\
419 \\
420 \\
421 \\
422 \\
423 \\
424 \\
425 \\
426 \\
427 \\
428 \\
429 \\
430 \\
431 \\
432 \\
433 \\
434 \\
435 \\
436 \\
437 \\
438 \\
439 \\
450 \\
451 \\
452 \\
453 \\
454 \\
455\end{array}$ & Open $(\infty)$ & Oct. 19 \\
\hline
\end{tabular}


TABLE 3-4. (cont.)

B. HPDE Load Resistance Settings for Runs Subsequent to 006-007

\begin{tabular}{|c|c|c|c|c|c|}
\hline $\begin{array}{l}\text { Electrode } \\
\text { No. }\end{array}$ & $\begin{array}{l}\text { Reference } \\
\text { Resis. } \Omega \\
(K=150)\end{array}$ & $\begin{array}{l}\text { Date } \\
1979\end{array}$ & $\begin{array}{l}\text { Electrode } \\
\text { No. }\end{array}$ & $\begin{array}{l}\text { Reference } \\
\text { Resis. } \Omega \\
(K=150)\end{array}$ & $\begin{array}{l}\text { Date } \\
1975\end{array}$ \\
\hline $\begin{array}{l}336 \\
337 \\
338 \\
339 \\
340 \\
341 \\
342 \\
343 \\
344 \\
345 \\
346 \\
347 \\
348 \\
349 \\
350 \\
351 \\
352 \\
353 \\
354 \\
355 \\
356 \\
357 \\
358 \\
359 \\
360 \\
361 \\
362 \\
363 \\
364 \\
365 \\
366 \\
367 \\
368 \\
369 \\
370 \\
371 \\
372 \\
373 \\
374 \\
375 \\
376\end{array}$ & 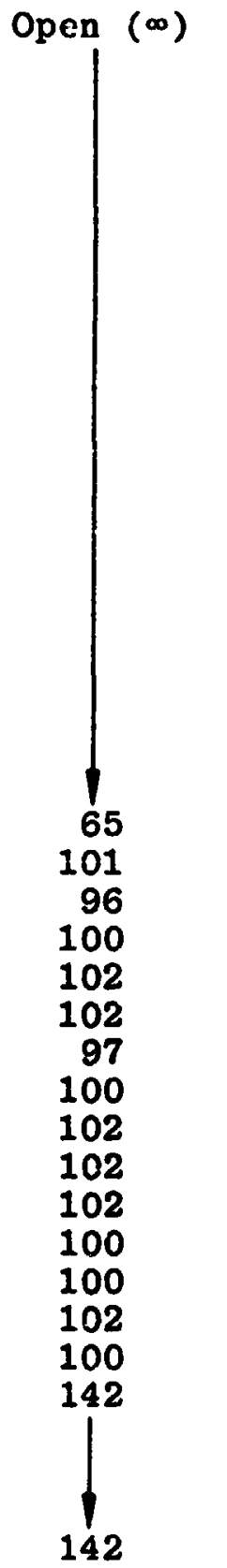 & $\operatorname{Oct}_{\mid} 18$ & $\begin{array}{l}377 \\
378 \\
379 \\
380 \\
381 \\
382 \\
383 \\
384 \\
385 \\
386 \\
387 \\
388 \\
389 \\
390 \\
391 \\
392 \\
393 \\
394 \\
395 \\
396 \\
397 \\
398 \\
399 \\
400 \\
401 \\
402 \\
403 \\
404 \\
405 \\
406 \\
407 \\
408 \\
409 \\
410 \\
411 \\
412 \\
413 \\
414 \\
415 \\
416 \\
417\end{array}$ & $\mid$ & $\begin{array}{c}\text { Oct. } 18 \\
\mid\end{array}$ \\
\hline
\end{tabular}




$$
\pi
$$




\section{TABLE 3-5}

Water Conductivi:; in Load 3uckets for AEDC Experiments

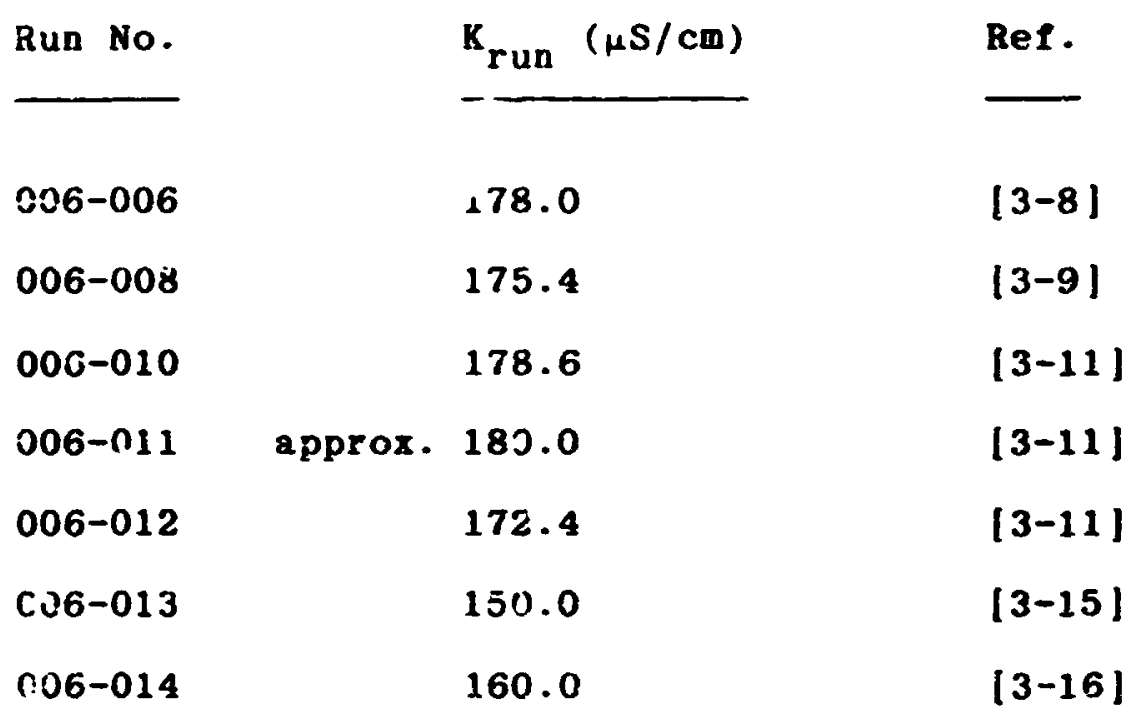

$0-4358$ 
TABLE 3-6

\section{Flow Rates of Combustion Gases and Runs 006-008 and 006-014}

\section{Component}

Fuel $(\mathrm{kg} / \mathrm{s})$

Oxidizer $(\mathrm{kg} / \mathrm{s})$

Seed, $\mathrm{kg} / \mathrm{s})$

Pilot $\left(\mathrm{CH}_{4}\right)(\mathrm{kg} / \mathrm{s})$

$\mathrm{N}_{2} / \mathrm{O}_{2}$ ratio

Seed Concentration

( $\mathrm{kg} \mathrm{KCH} / \mathrm{kg} \mathrm{CH} \mathrm{CH}_{3} \mathrm{OH}$ )

Equivalence Ratio

(fuel lean)

Total enthalpy of combustion

gases (no losses) (MT)

Specific enthalpy of

combustion gases ( $\mathrm{UJ} / \mathrm{kg}$ )

$T_{\text {flame }}(6 \mathrm{~atm})(\mathrm{K})$
Run 006-008

6.60

40.99

1.84

0.034

1.0

0.3

1.5

299.3

6.05

3048
006-018

6.84

39.82

2.54

0.034

0.929

0.3

1.11

303.8

6.16

3063 


\section{TABLE 3-7}

Load Resistances for AEDC Run 006-014

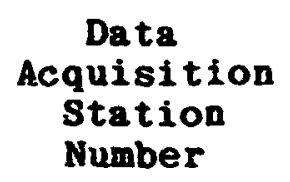

1

2

3

4

5

6

7

8

9
42

84

126

168

210

252

294

336

376

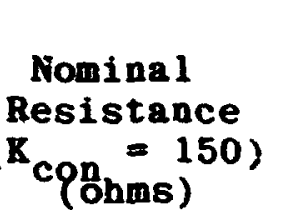

39.1

50.9

50.0

69.1

61.9

67.8

65.5

65.5

141.6
Averaged

Experimental

Resistance

$4.54 \leq \mathrm{T} 2 \leq 5.21$

(ôhms)

36.64

48.19

47.97

65.63

61.25

66.02

65.82

65.70

153.71
Indicated Conductivity Factor $(\mu S / \mathrm{cm})$

160.0

158.4

156.3

157.8

151.6

154.0

149.3

149.5

138.2 


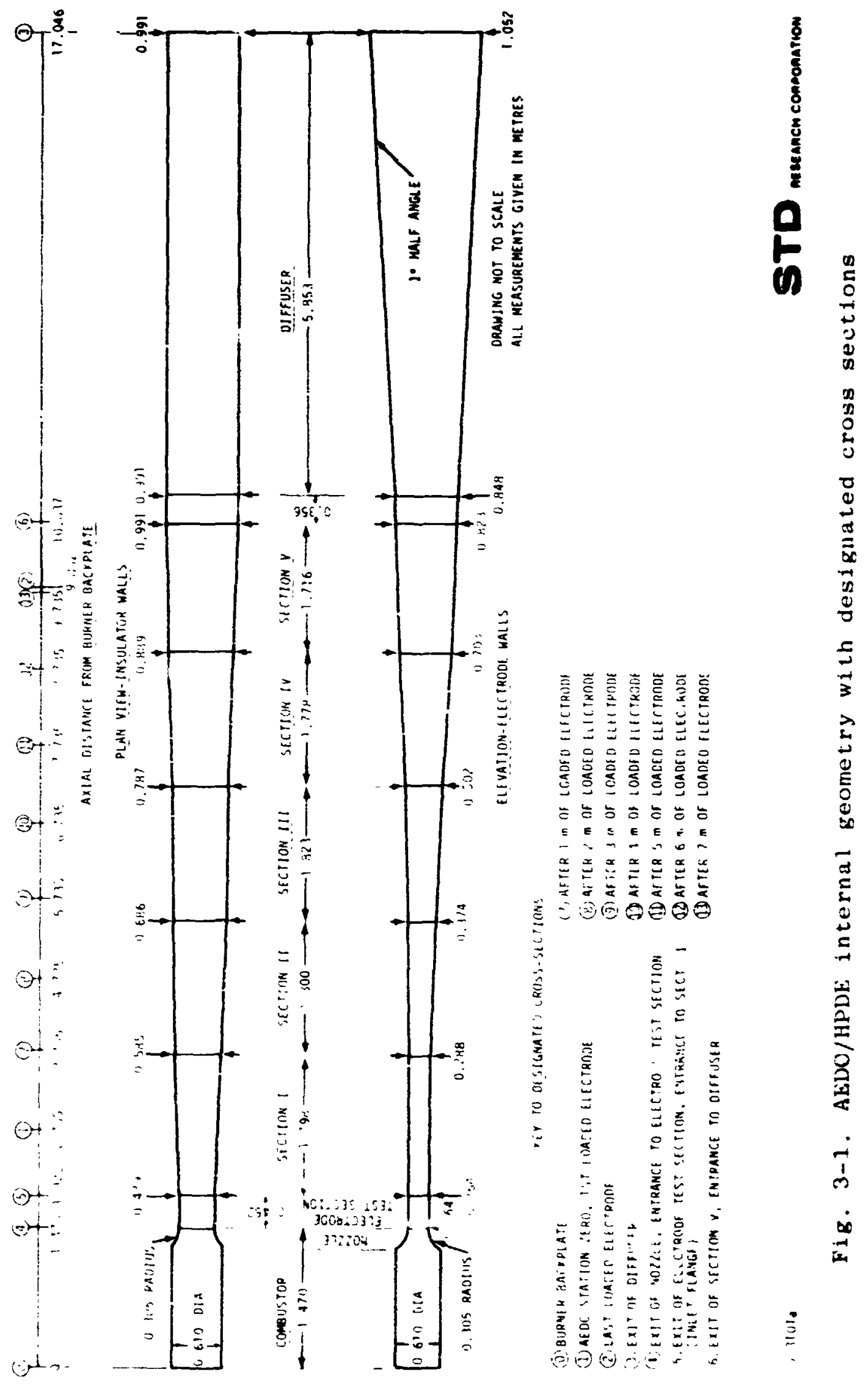


STD arseancm corrcanion

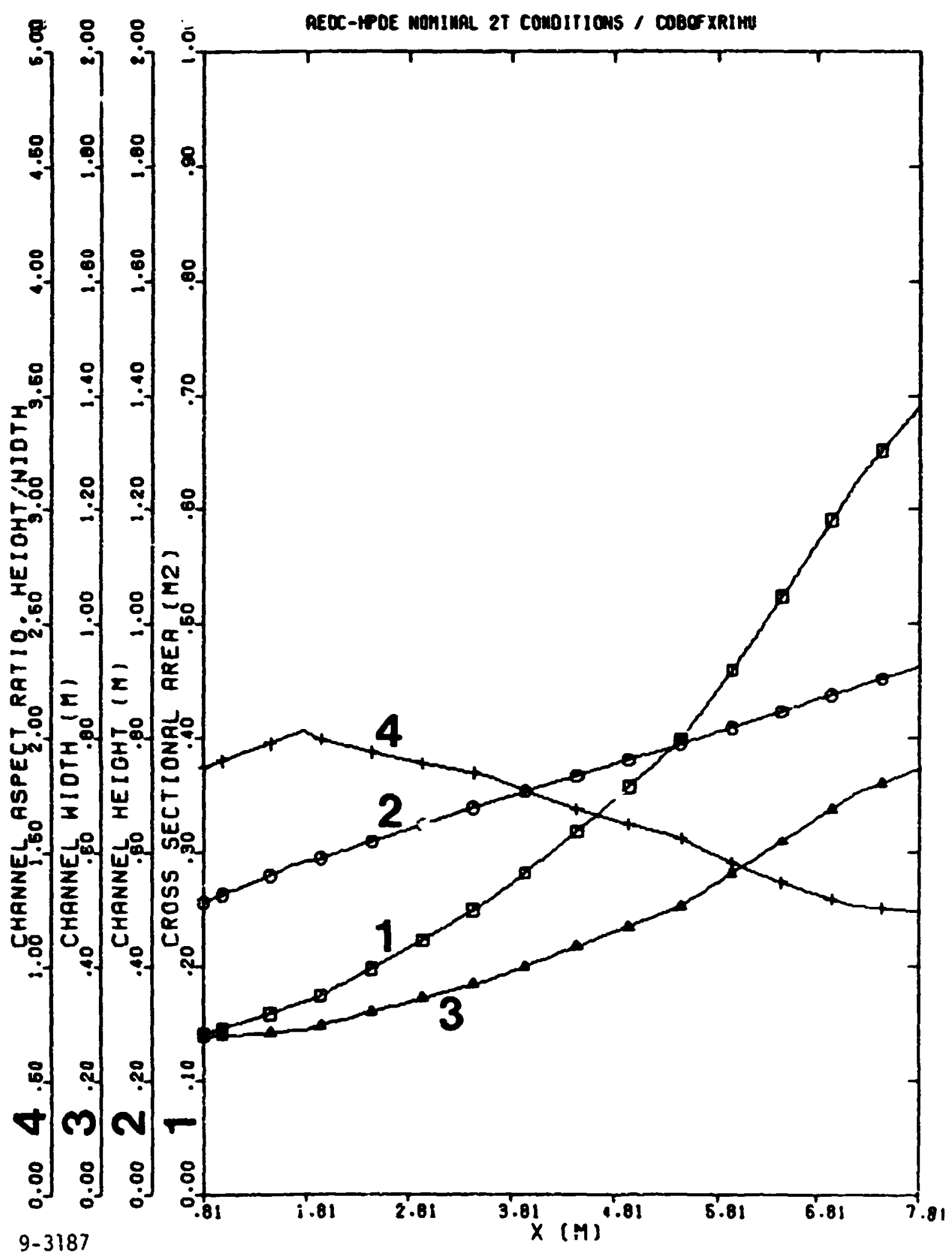

Fig. 3-2. Axial variation of the channel geometry in the $A E D C / H P D E$ at the nominal $2 T$ operating conditions. 


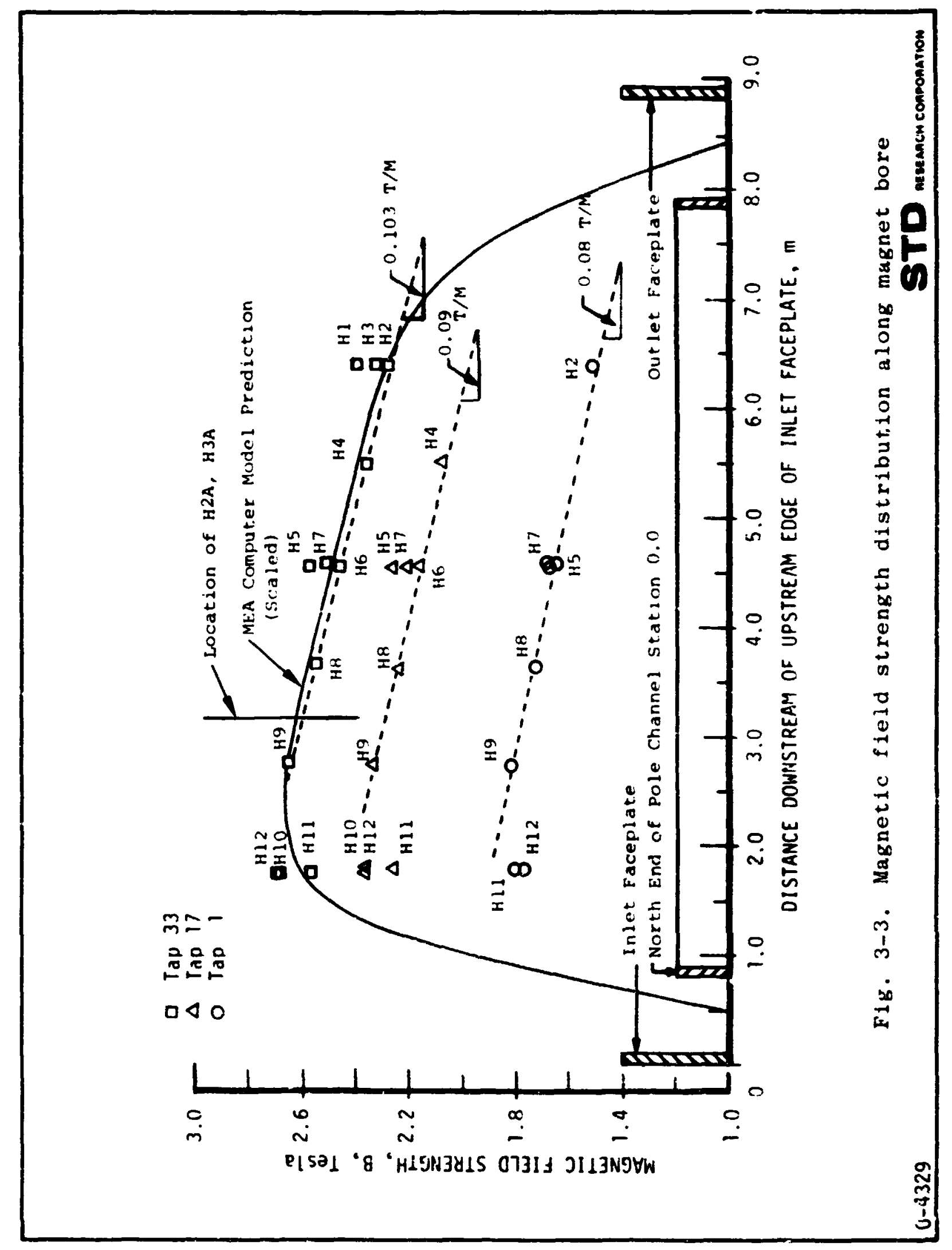




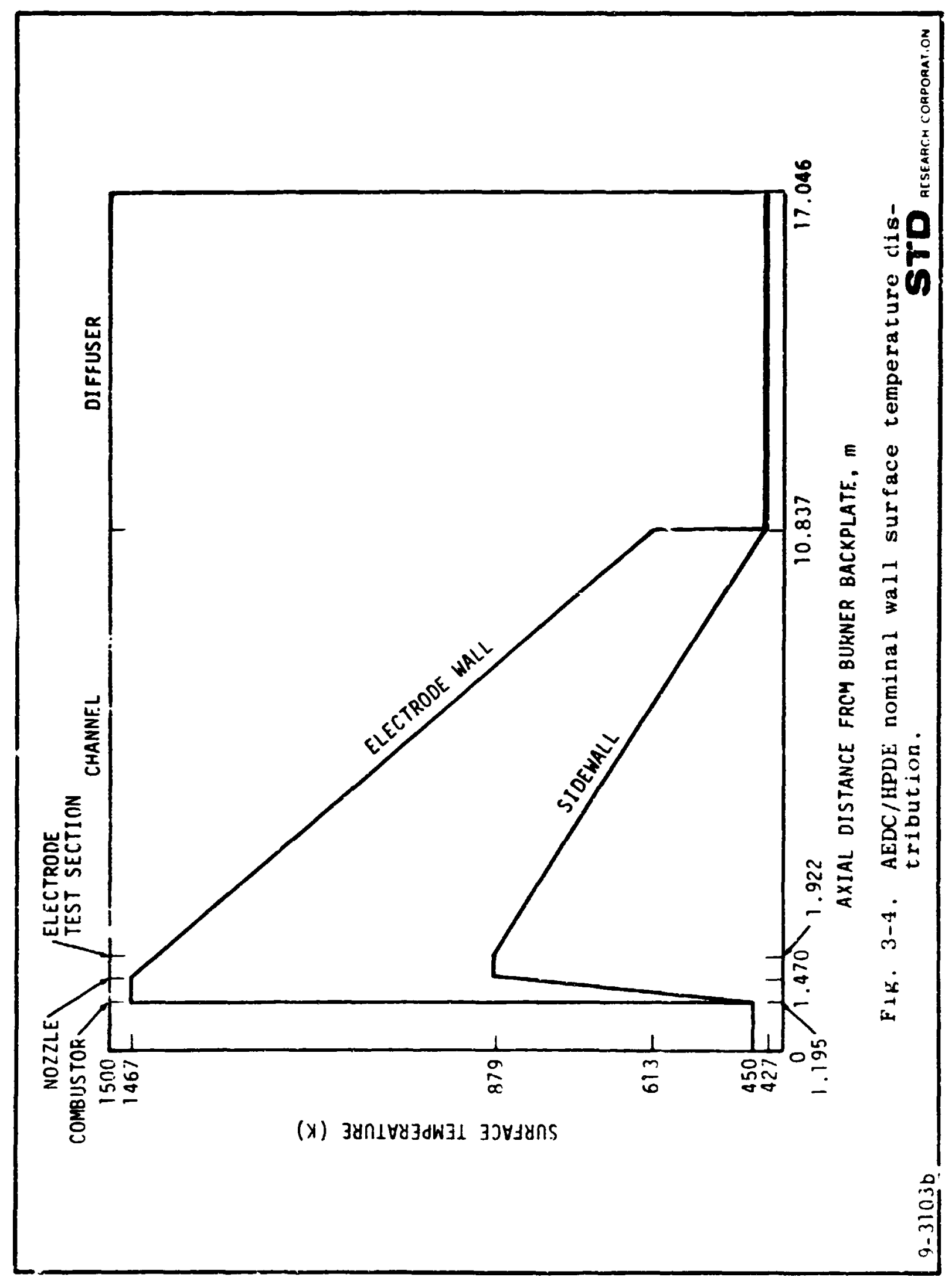




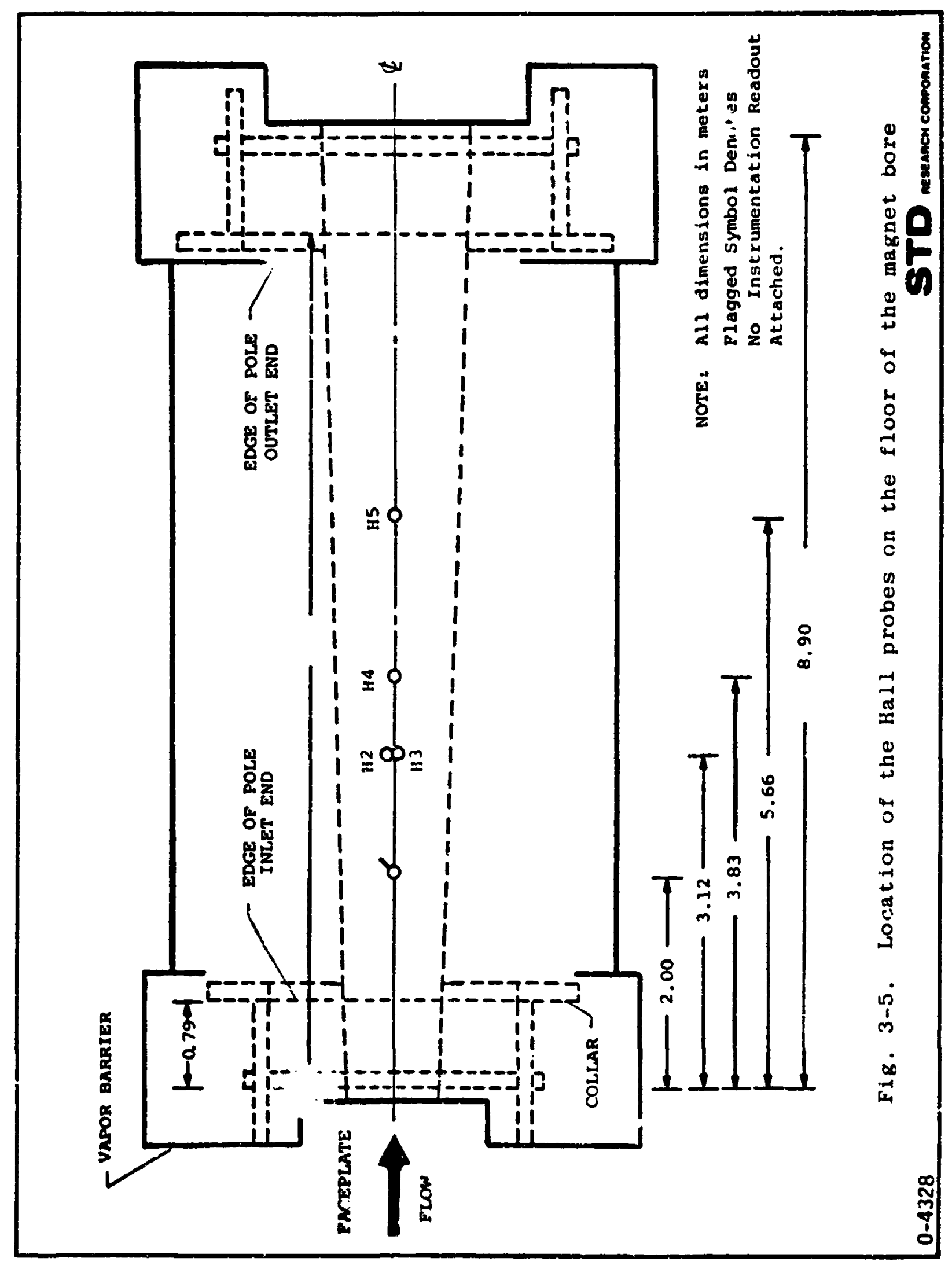




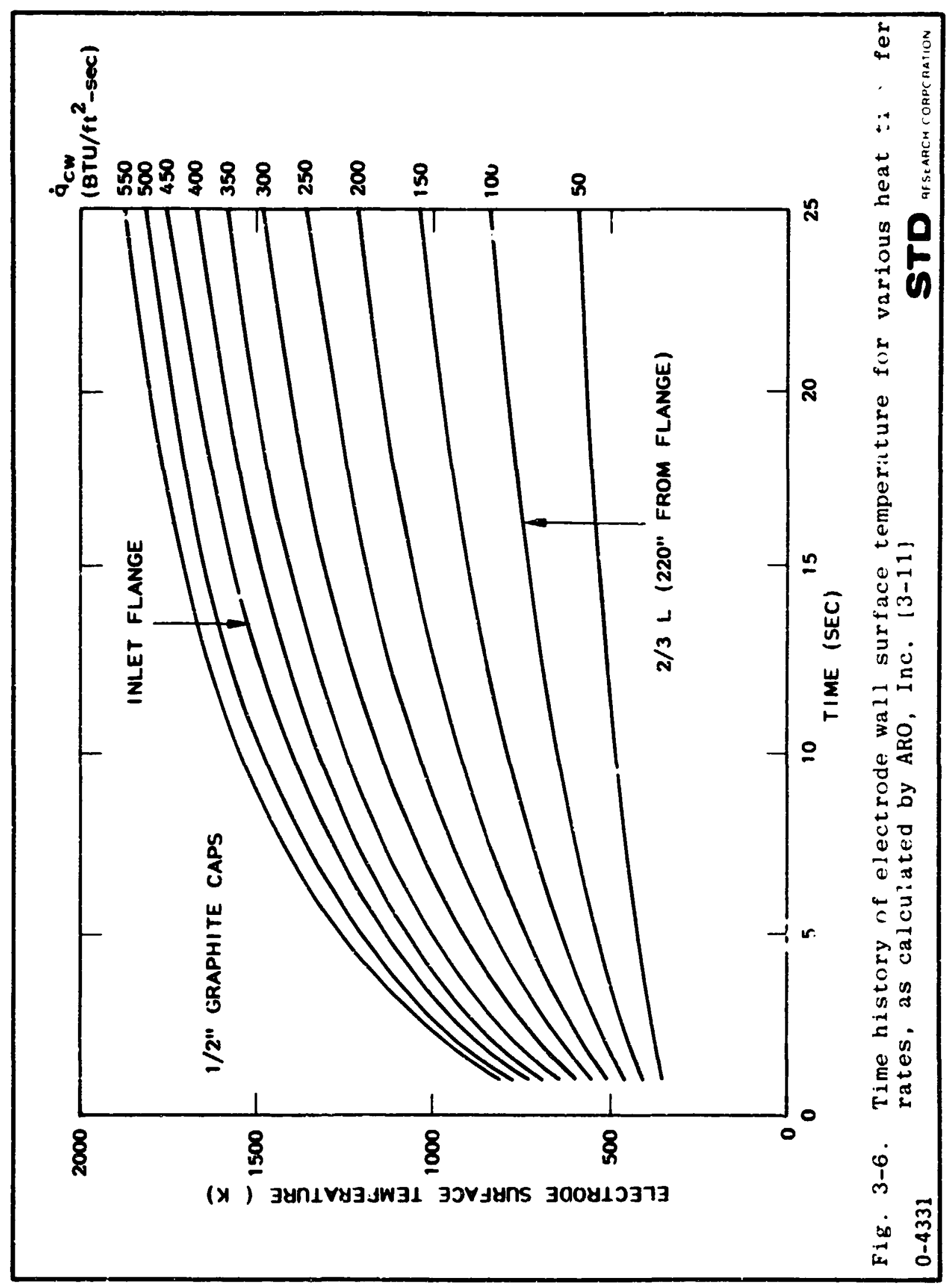




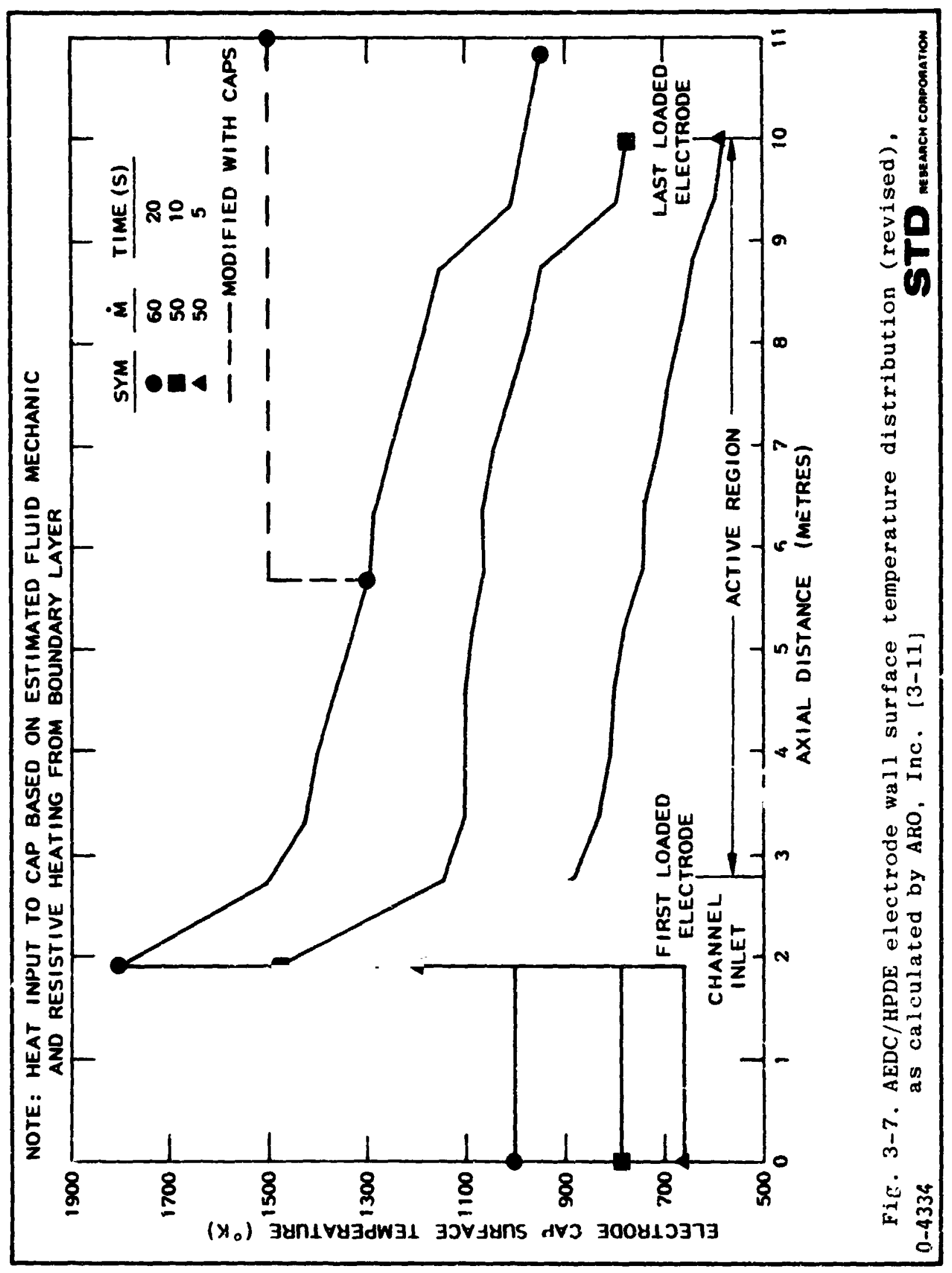




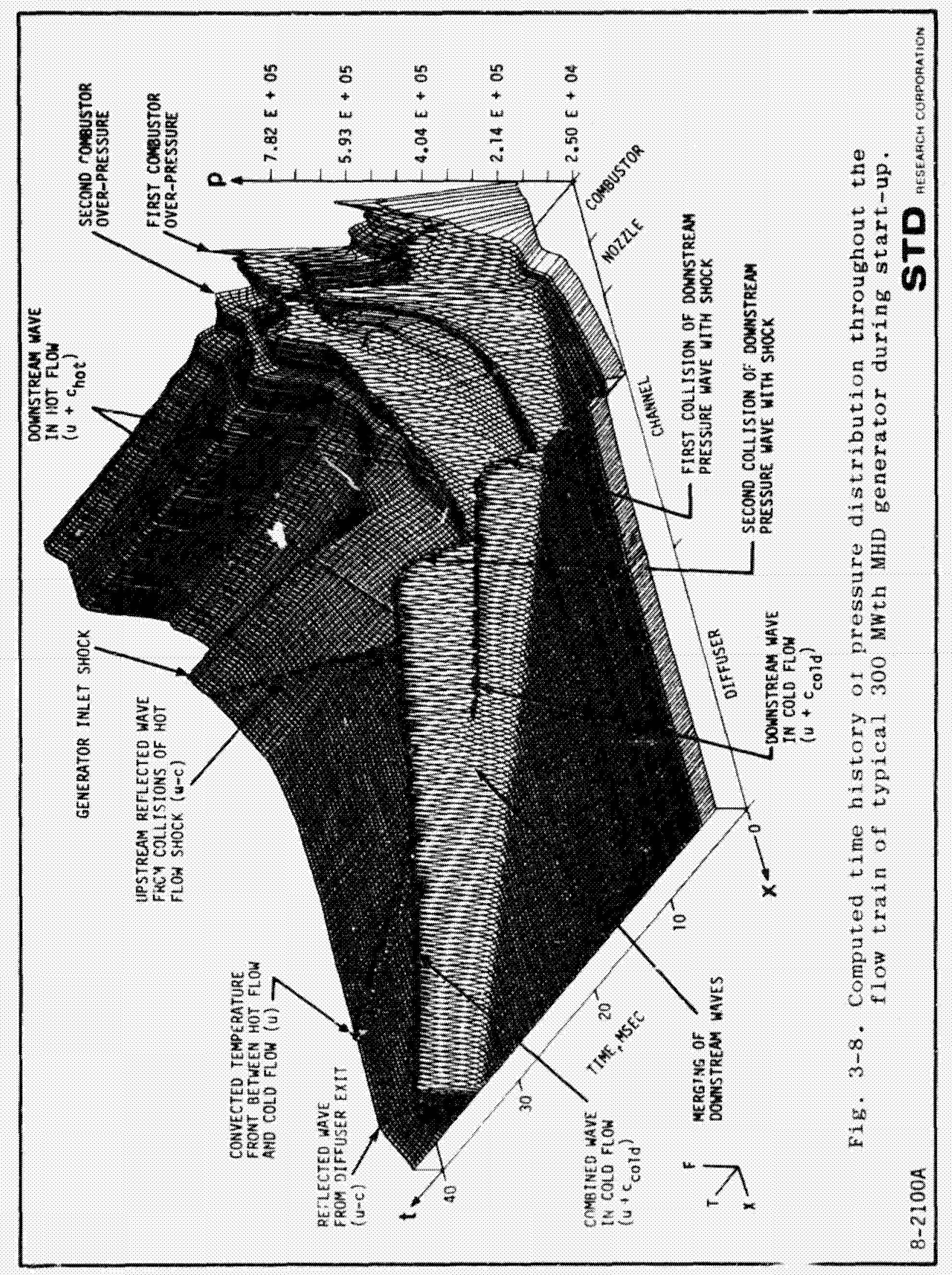




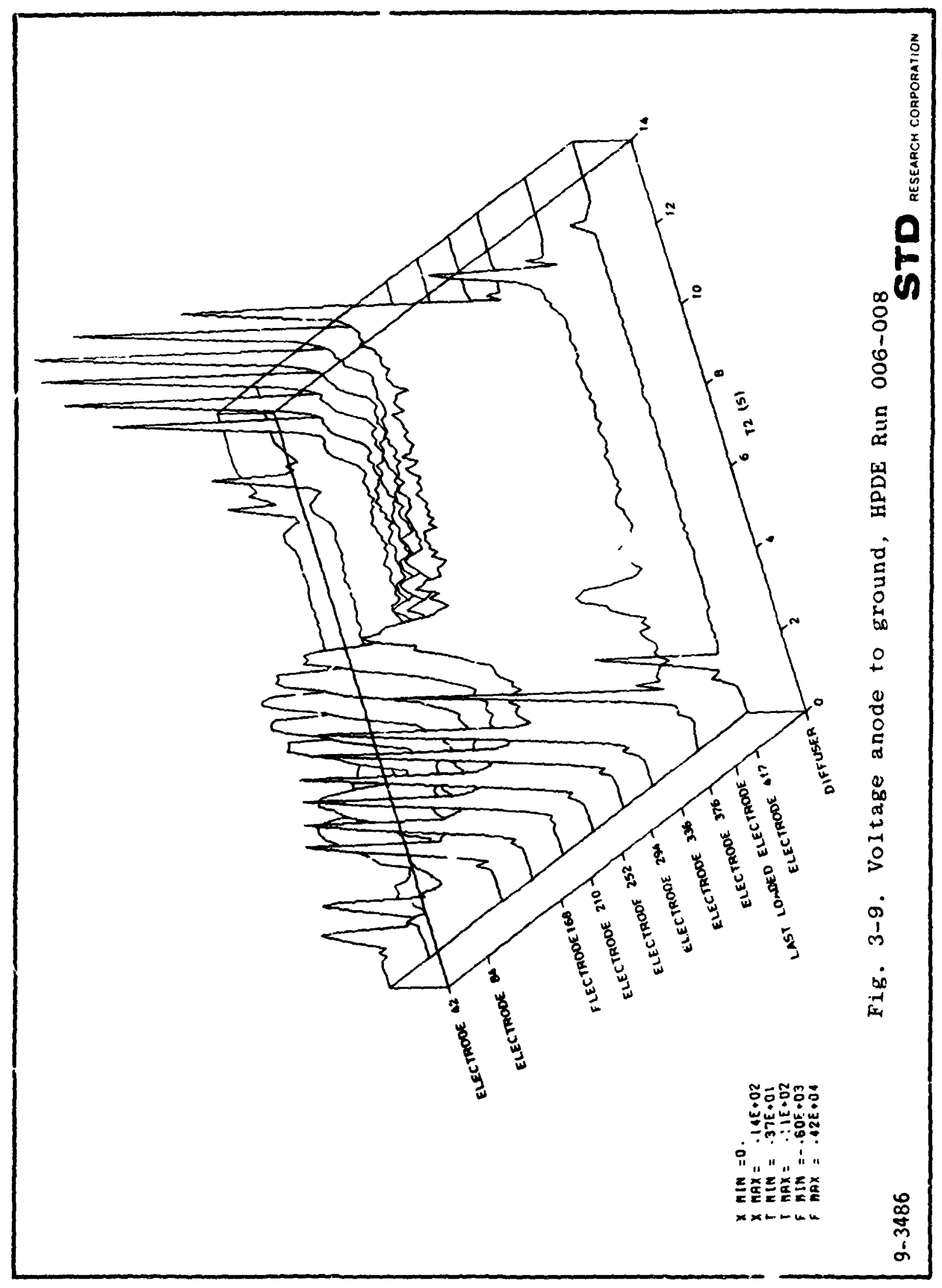




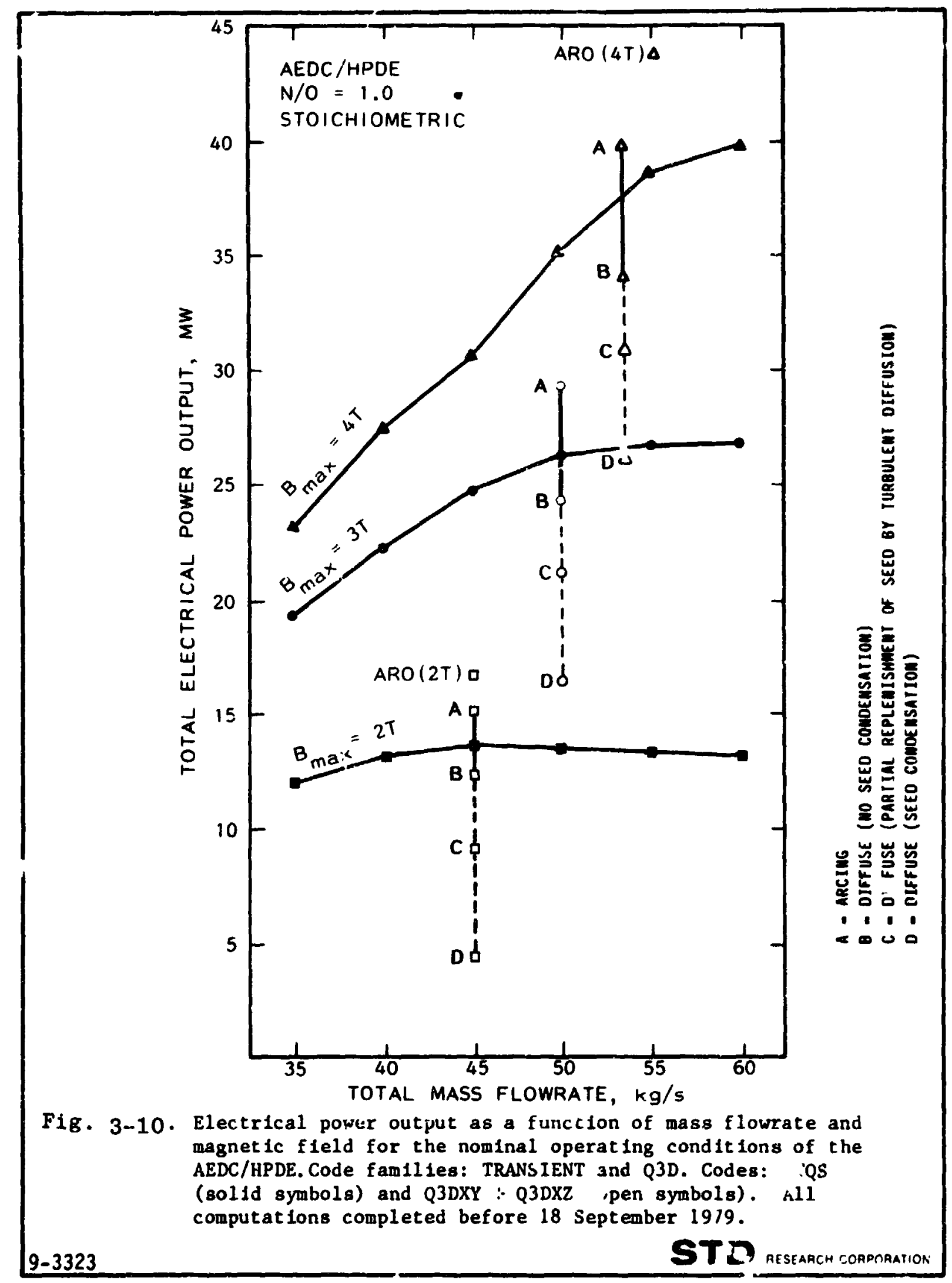




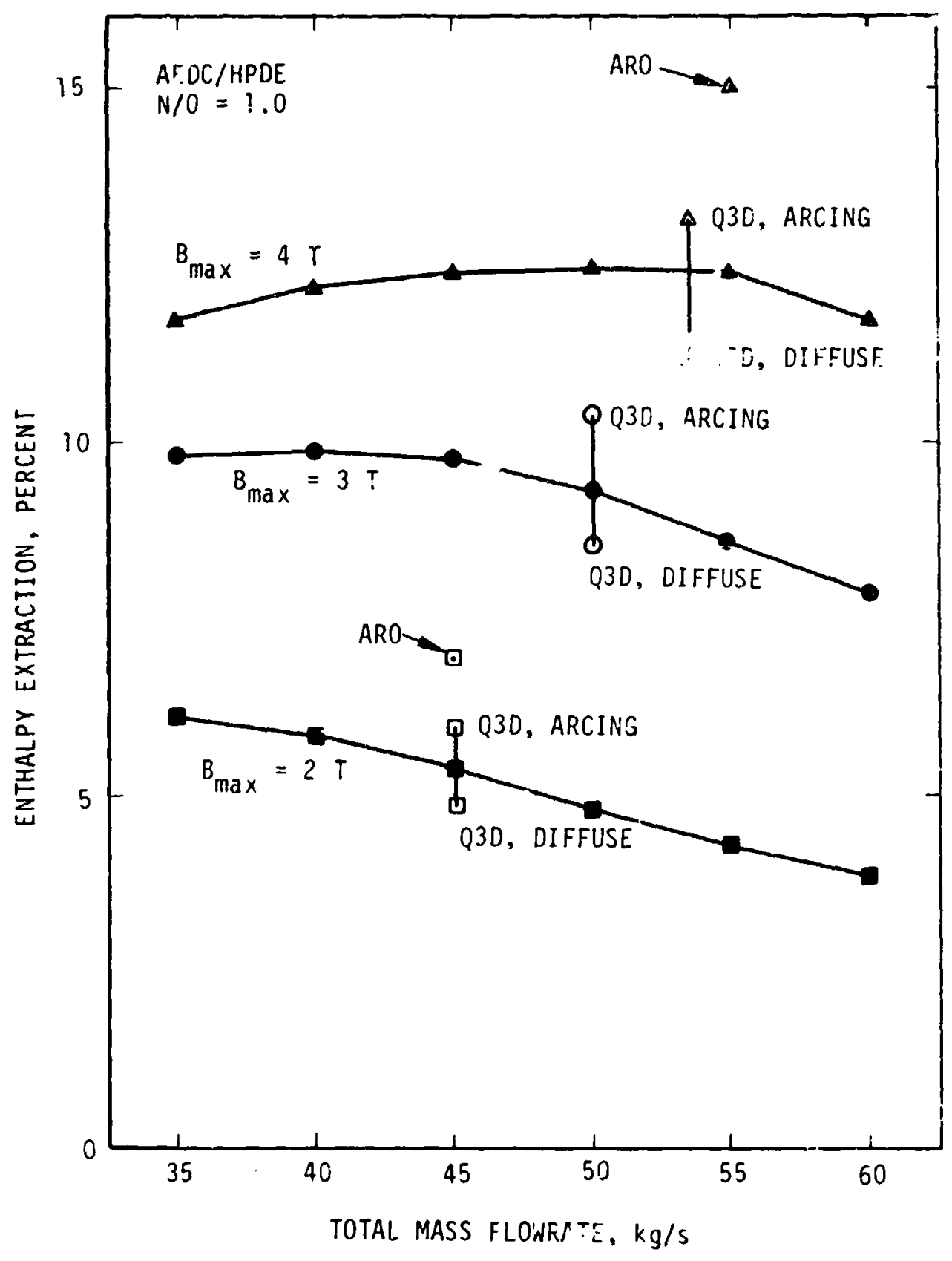

Fig. 3-11. Enthalpy extraction ratic as ifunction of mass fiow rate and magnet 1 : fleid for the nominal c perating conditions of the ALLC/HP n:. Co familles: TRANSIENI and Q3D. Codes: T\&5 *.: Q Q condensation zonsidered.: 
STD meseancen coargasinom

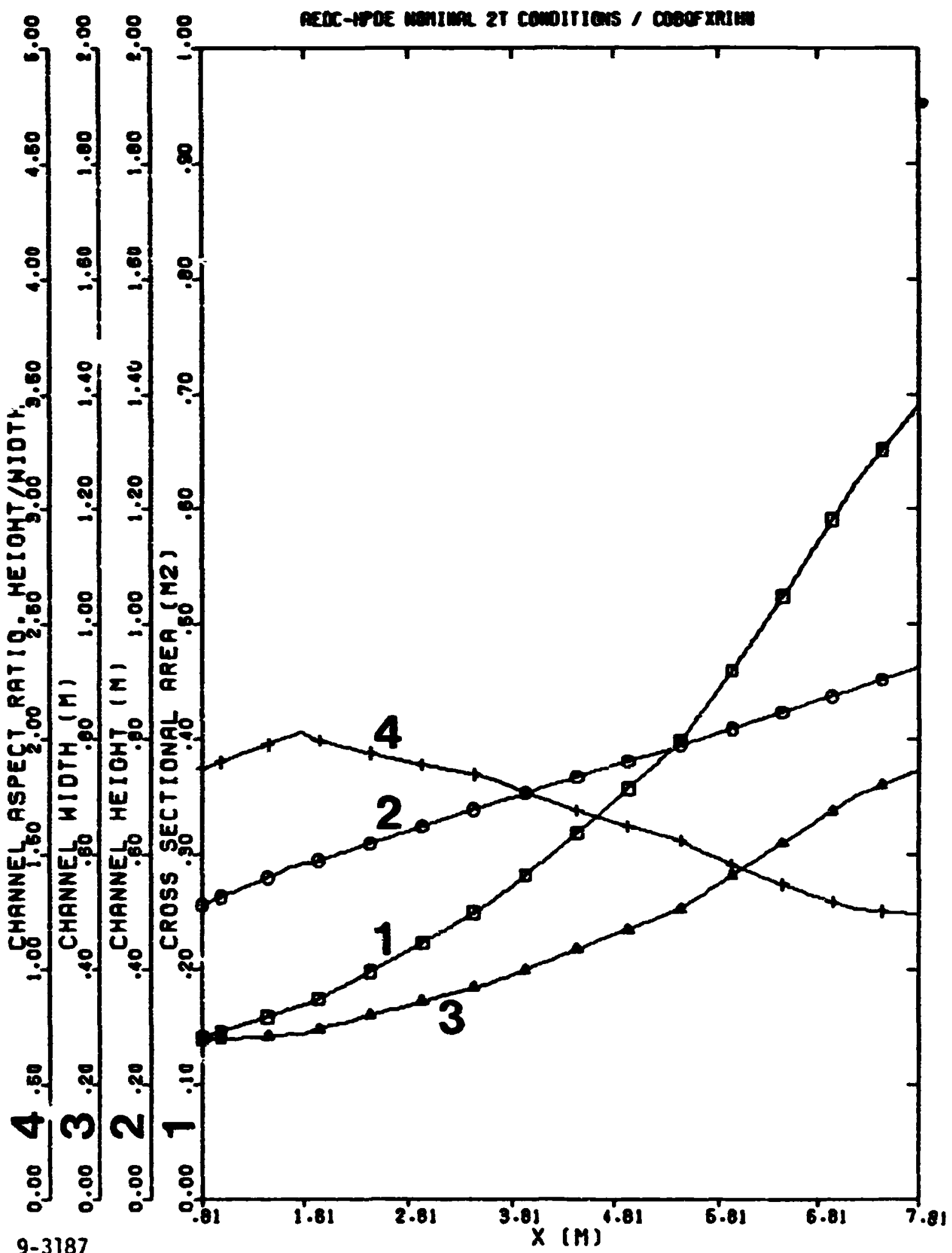

Fig. 3-12. Axial variation of the channel geometry in the AEDC/ HPDE at the nominal $2 \mathrm{~T}$ operating conditions: Q3D calculation.

C -2 
STD RESEARCM COAPORATION

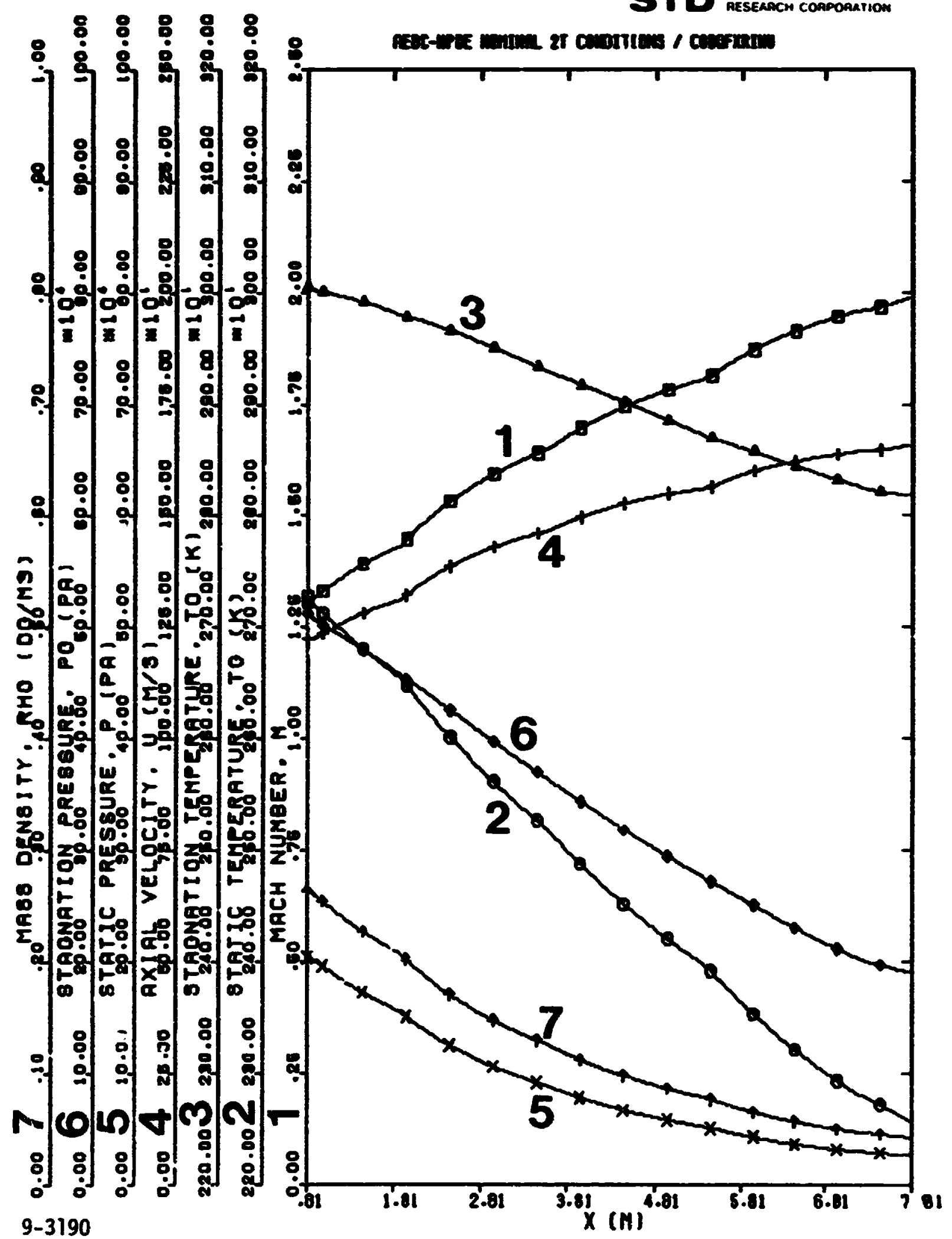

Fig. 3-13. Axial variation of the centerline gasdynamic va-iables in the AEDC/HPDE at the nominal $2 \mathrm{~T}$ operating conditions: Q3D calculation. 


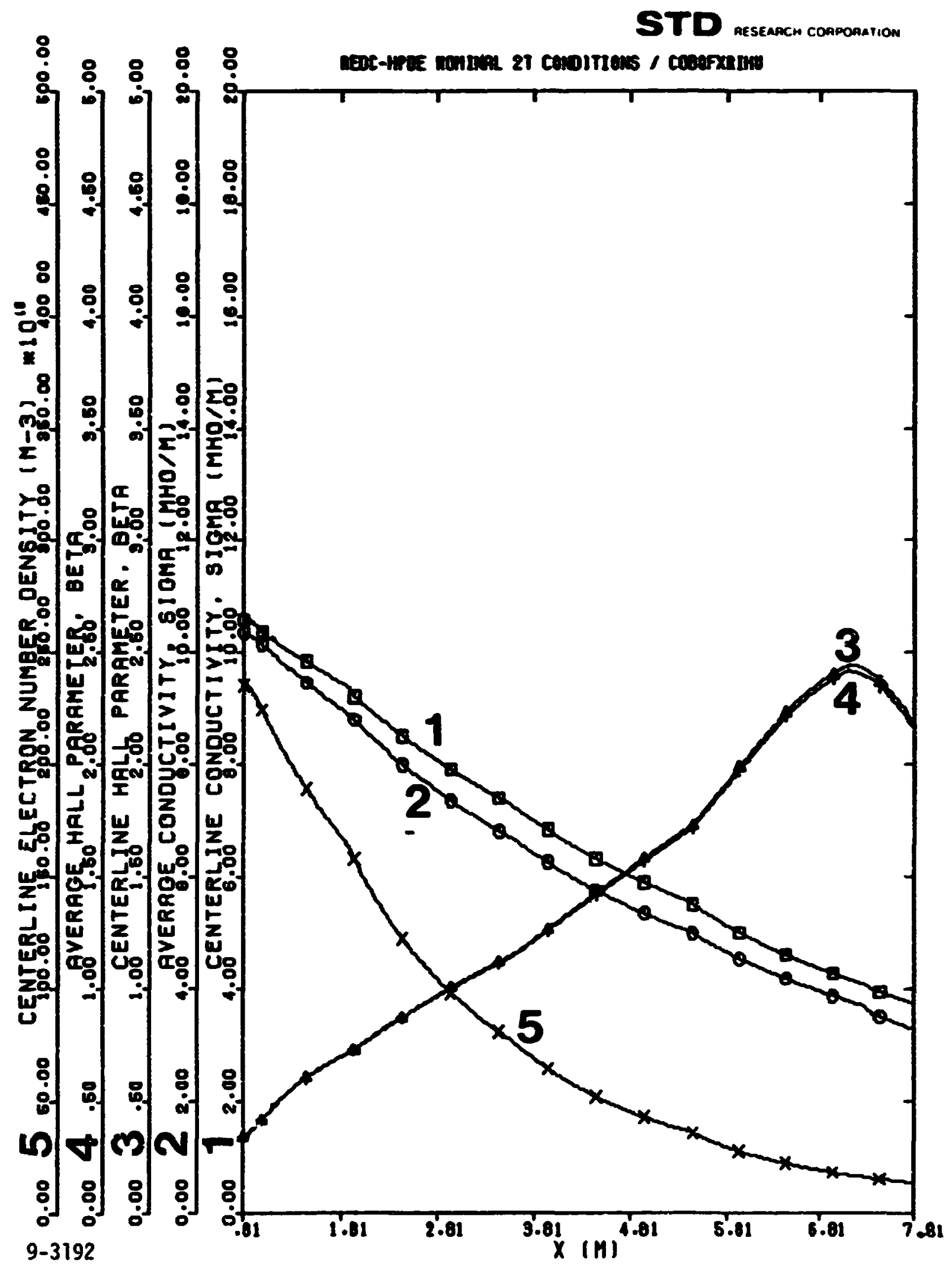

Fig. ?-14. Axial varlation of the plasma parameters in the AEDC/ HPDE at the nominal $2 \mathrm{~T}$ operating conditions: Q3D calculation. 


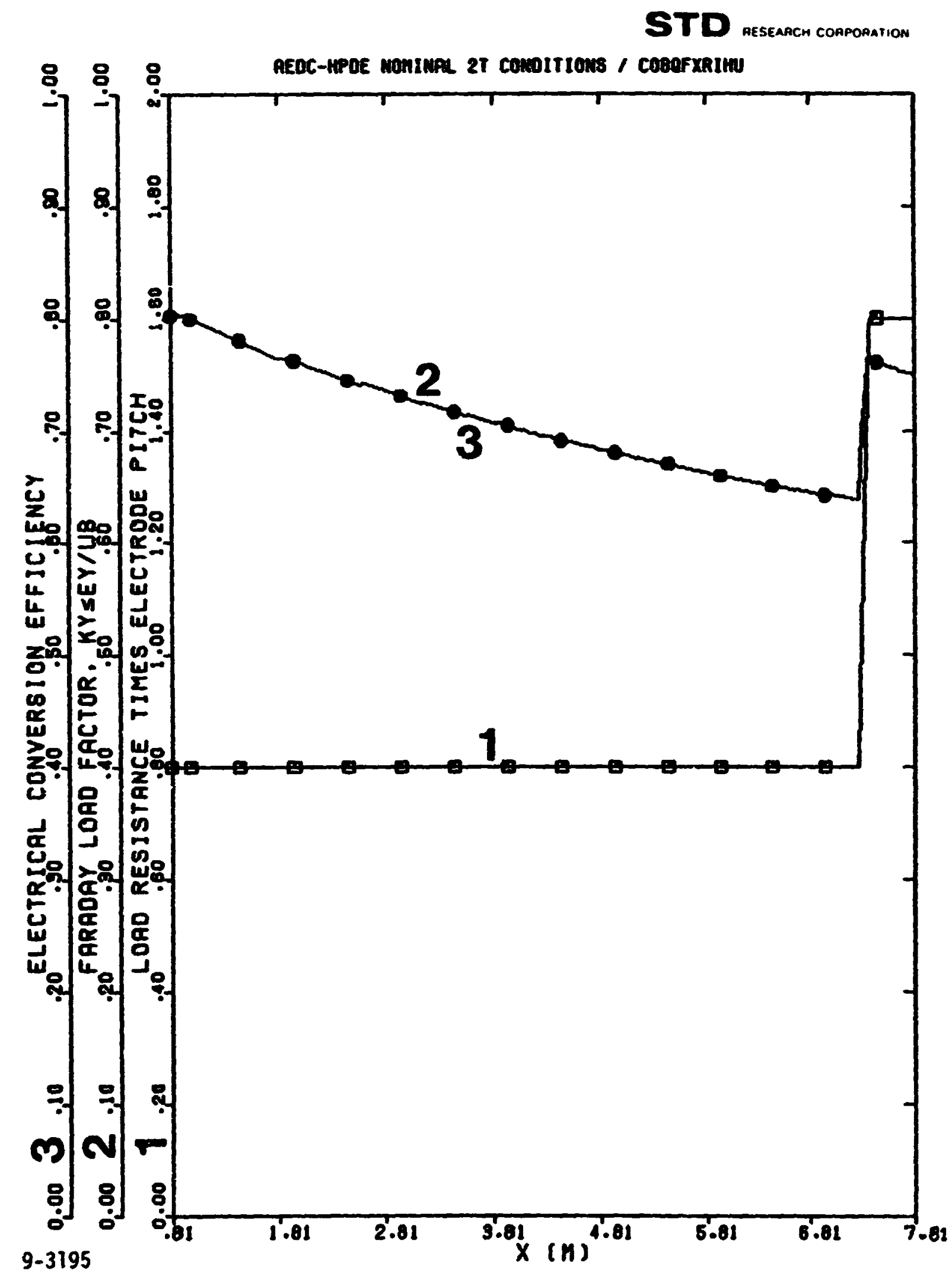

Fig. 3-15. Axial variation of the electrical loading in the AEDC/ HPDE at the nominal $2 \mathrm{~T}$ operating conditions: Q3D calculation. 
STD aEseahch CoRporation

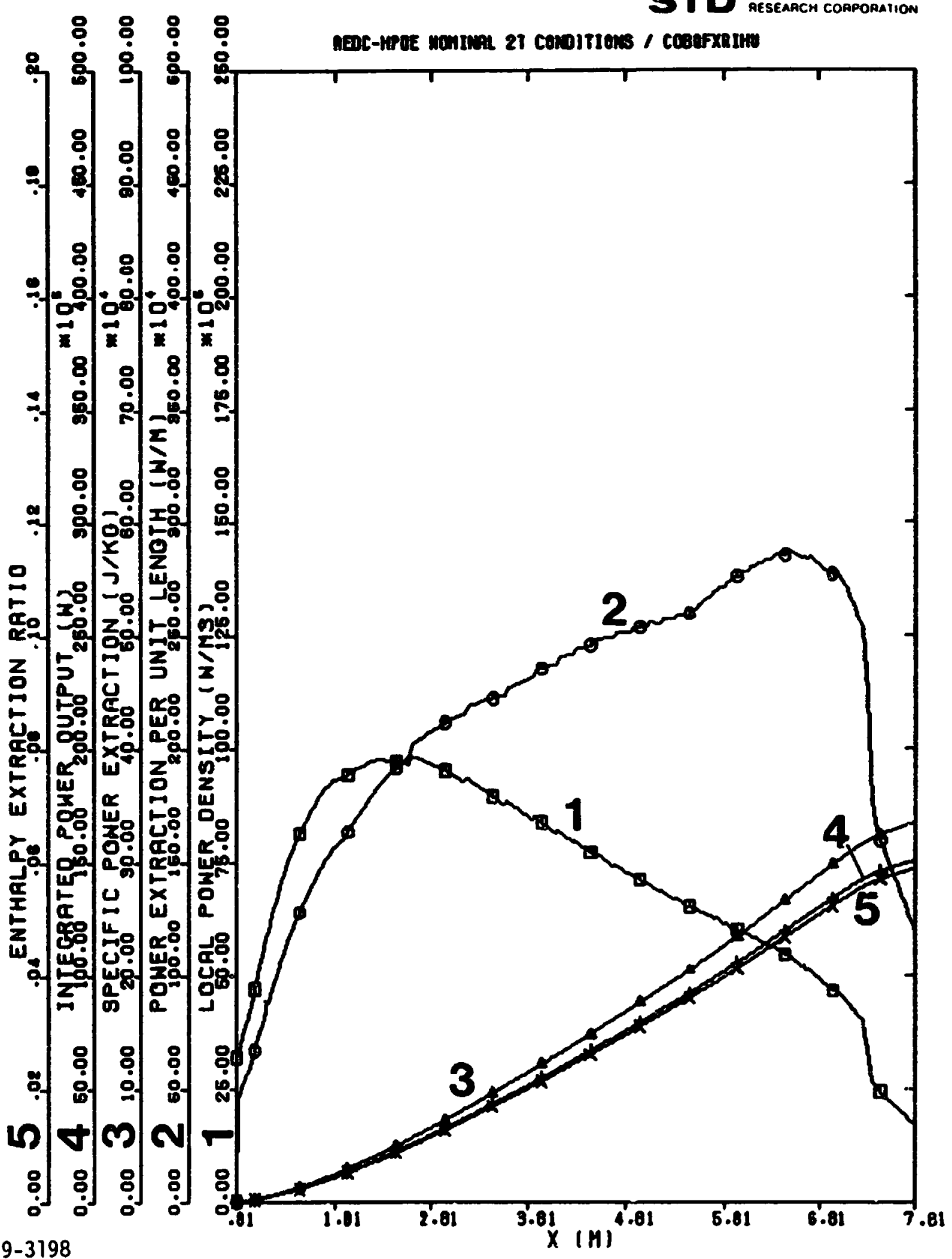

Fig. 3-16. Axial variation of the power extraction parameters in the AEDC/HPDE at the nominal $2 \mathrm{~T}$ operating conditions: Q3D calculation 
STD aeseanch corporation

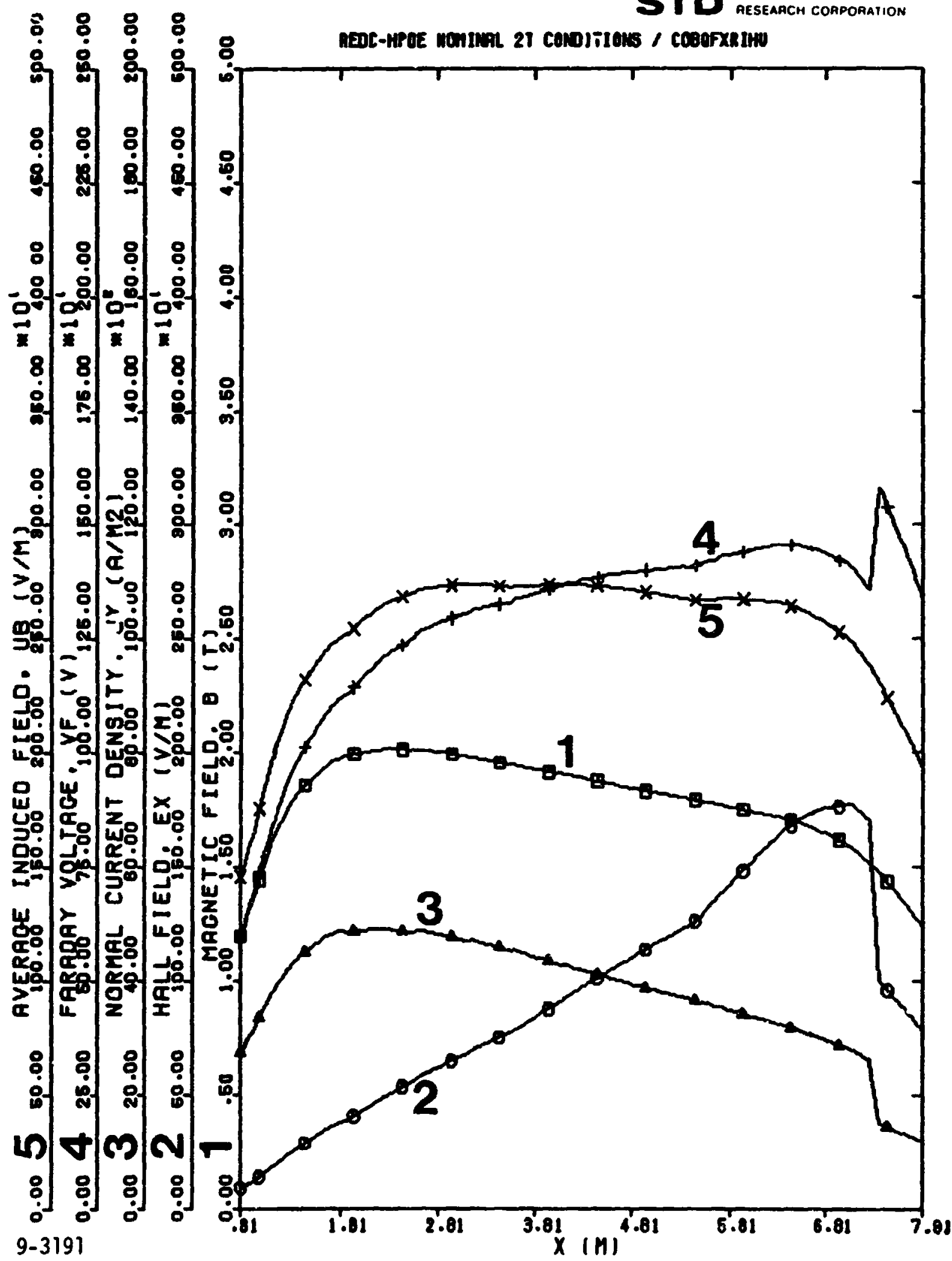

Fig. 3-17. Axial variation of the electrical field variables in the AEDC/HPDE at the nominal 2 T operating conditions: Q3D calculations. 
STD heseanch copporanion

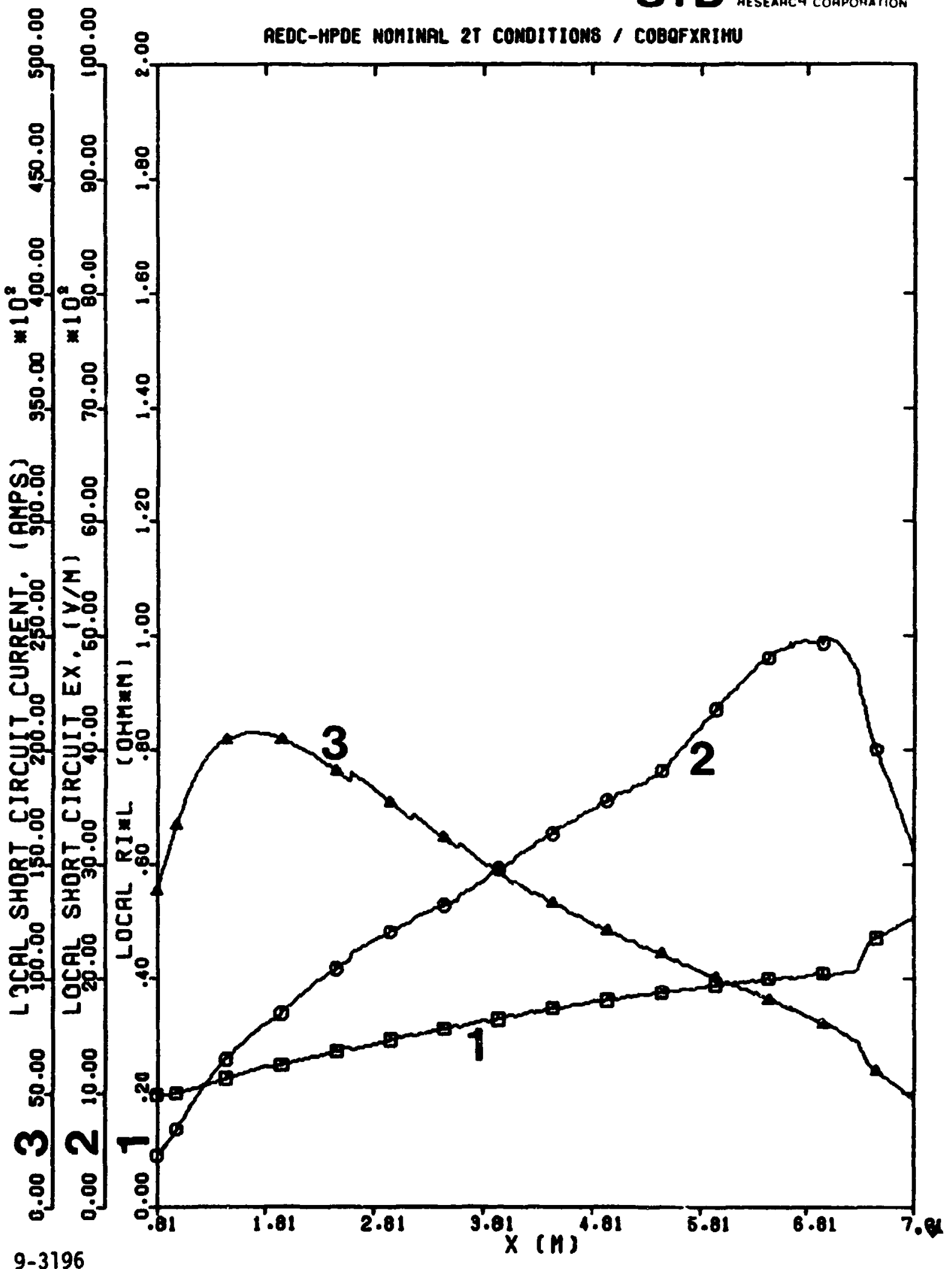

Fig. 3-18. Axial variation of the short circut fields and the local internal impedance in the AEDC/HPDE at the nominal $2 \mathrm{~T}$ operating conditions: Q3D calculation. 


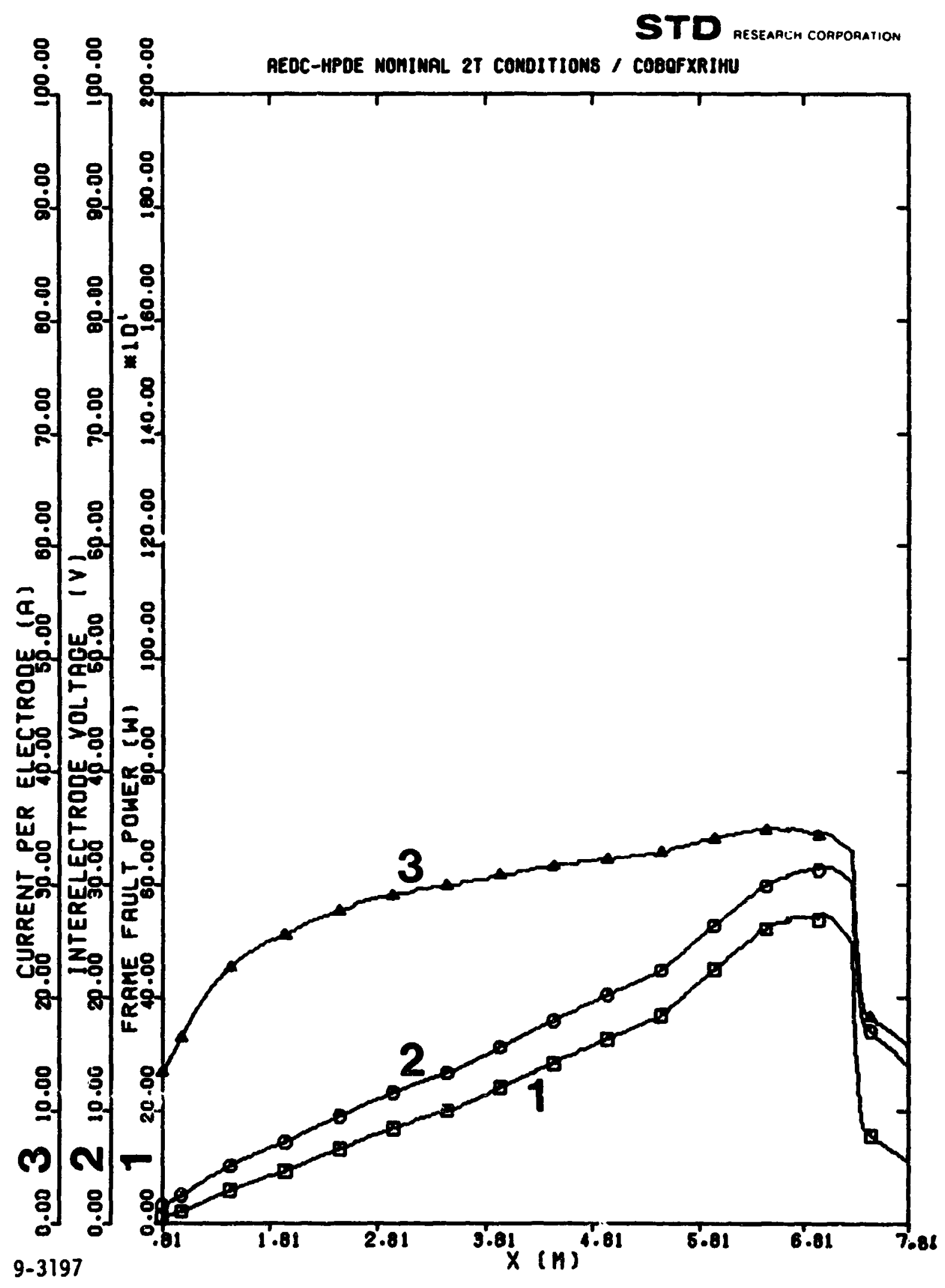

Fig. 3-19. Axial variation of the electrical stresses on the electrodes in the AEDC/HPDE at the nominal $2 \mathrm{~T}$ operating conditions: Q3D calculation. 


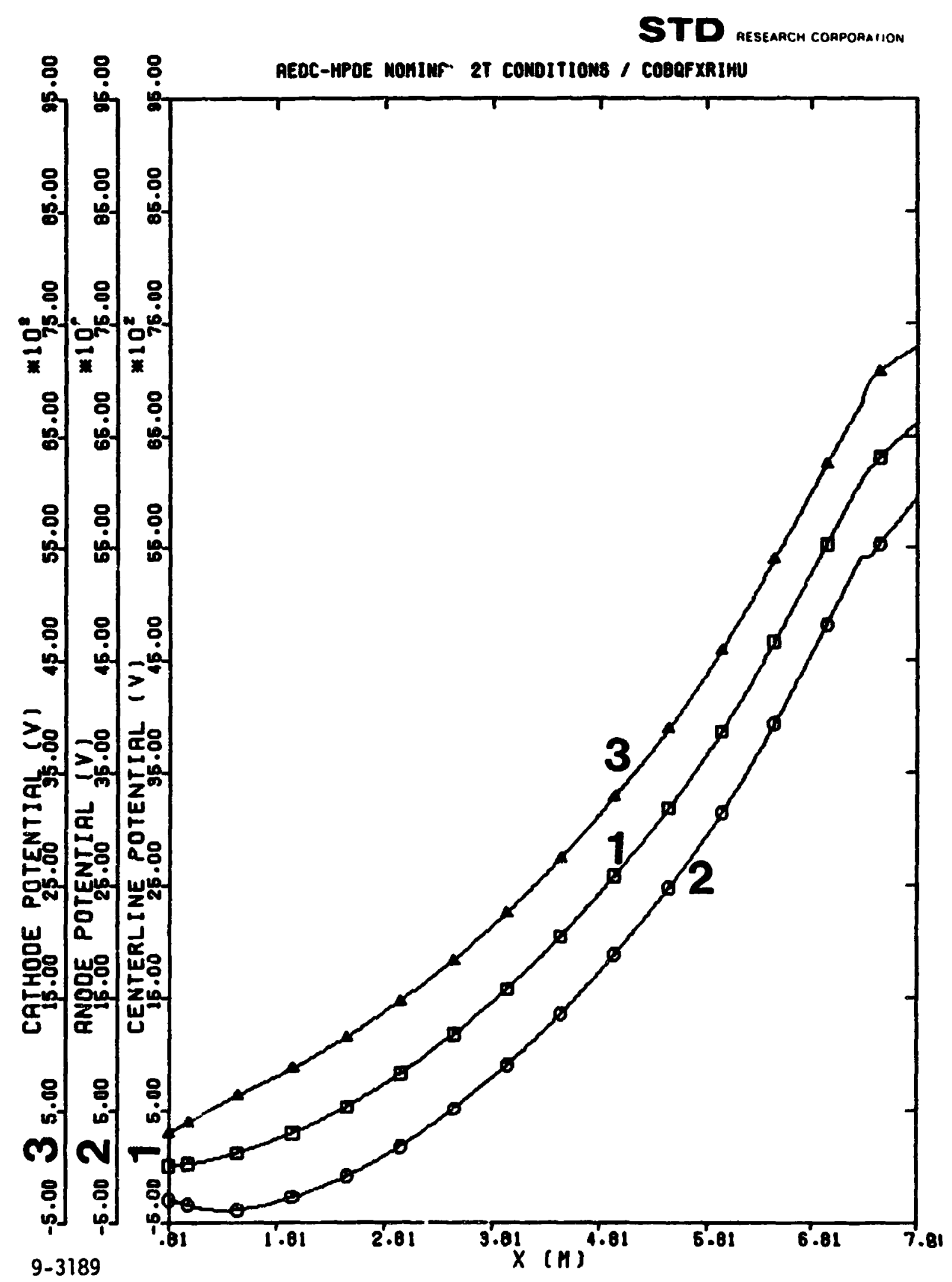

Fig. 3-20. Axial variation of the electric potential in the AEDC/ HPDE at the nominal $2 \mathrm{~T}$ operating conditions: Q3D calculation. 
STD heseabch copporation

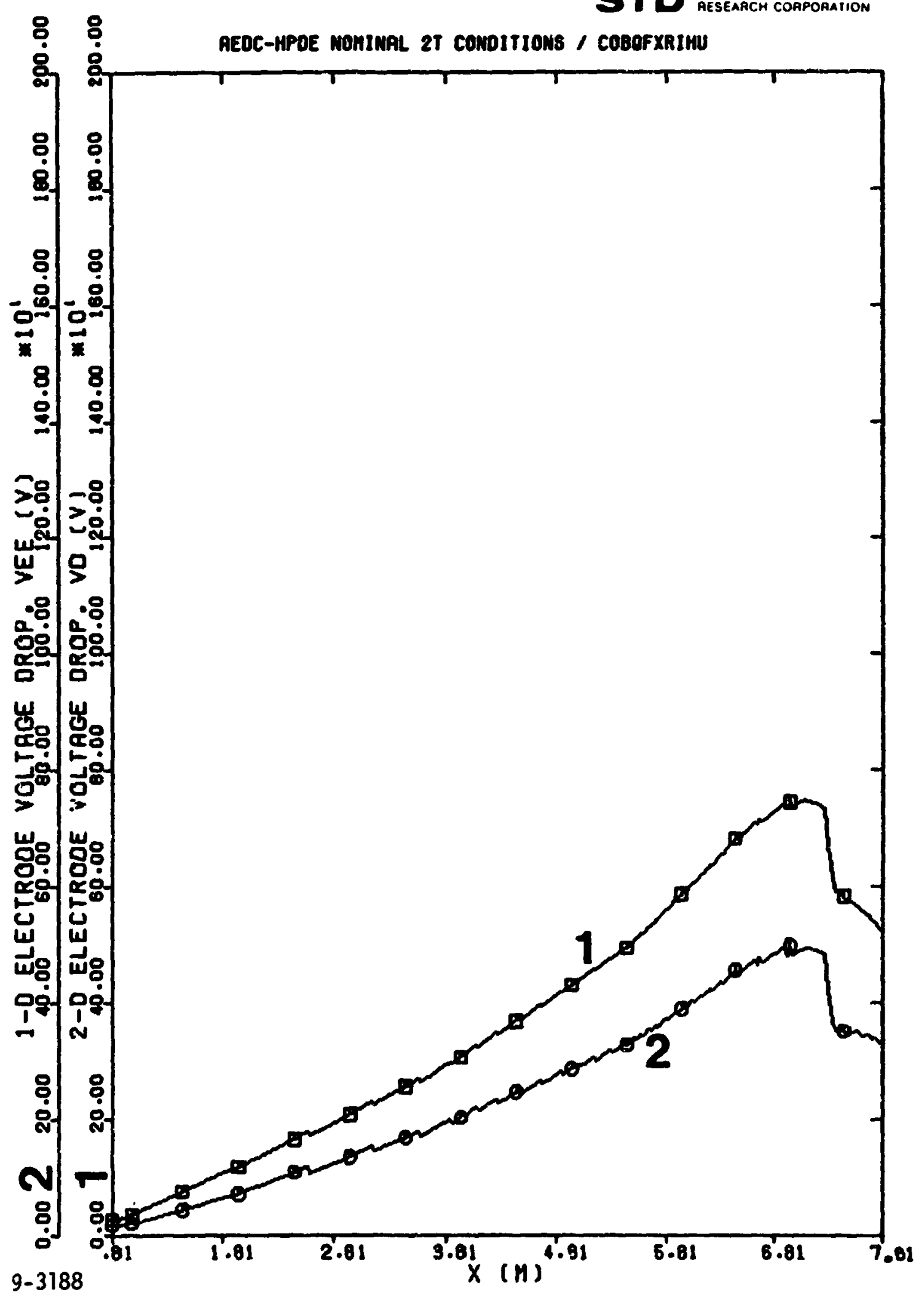

Fig. 3-21. Axial variation of the electrode voltage drops in the AEDC/HPDE at the nominal $2 \mathrm{~T}$ operating conditions: Q3D calculation. 
STD heseapch comporation

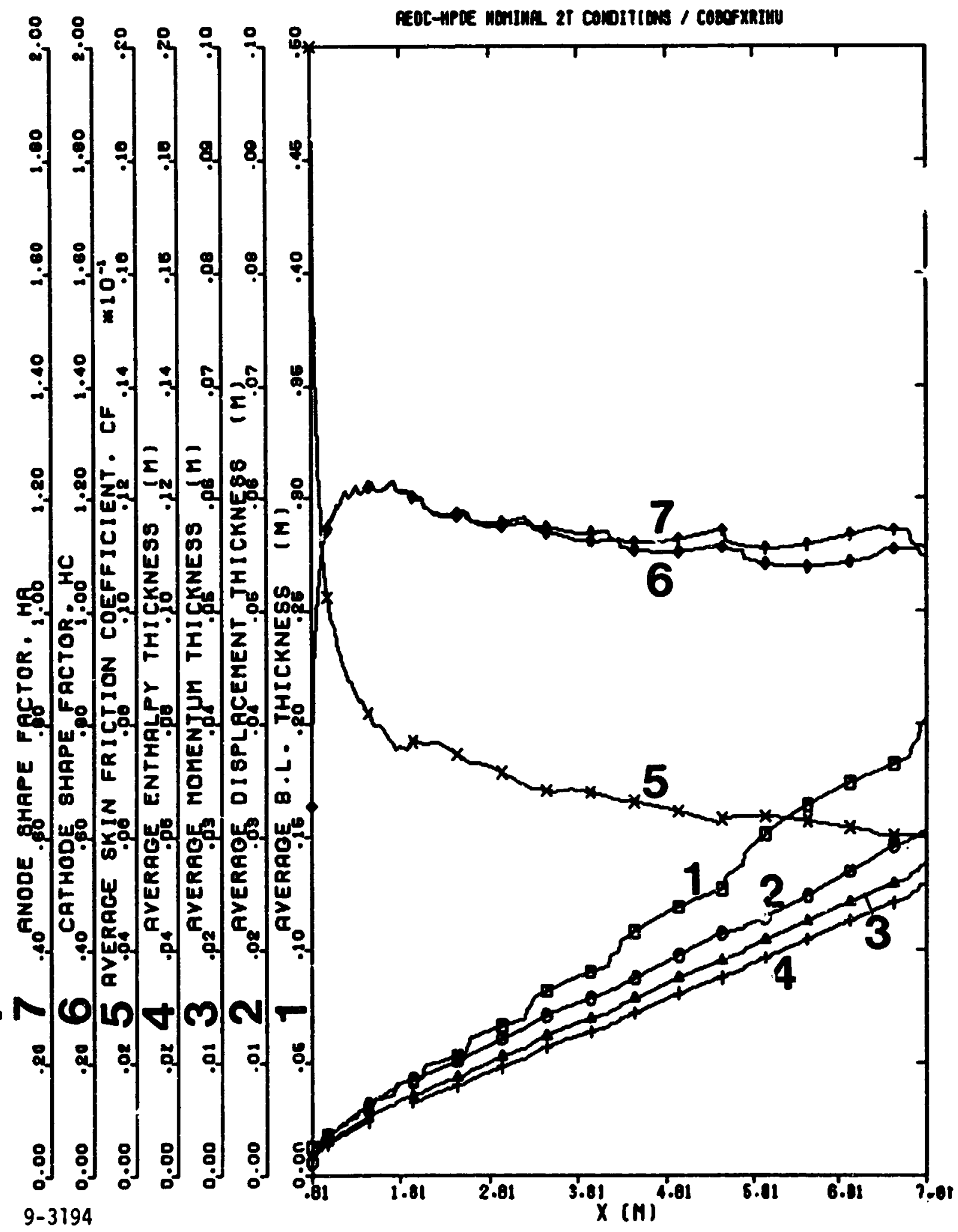

Fig. 3-22. Axial variation of the electrode boundary layer parameters in the AEDC/HPDE at the nominal $2 \mathrm{~T}$ operating conditions: Q3D calculation. 
STD peseabch corporation

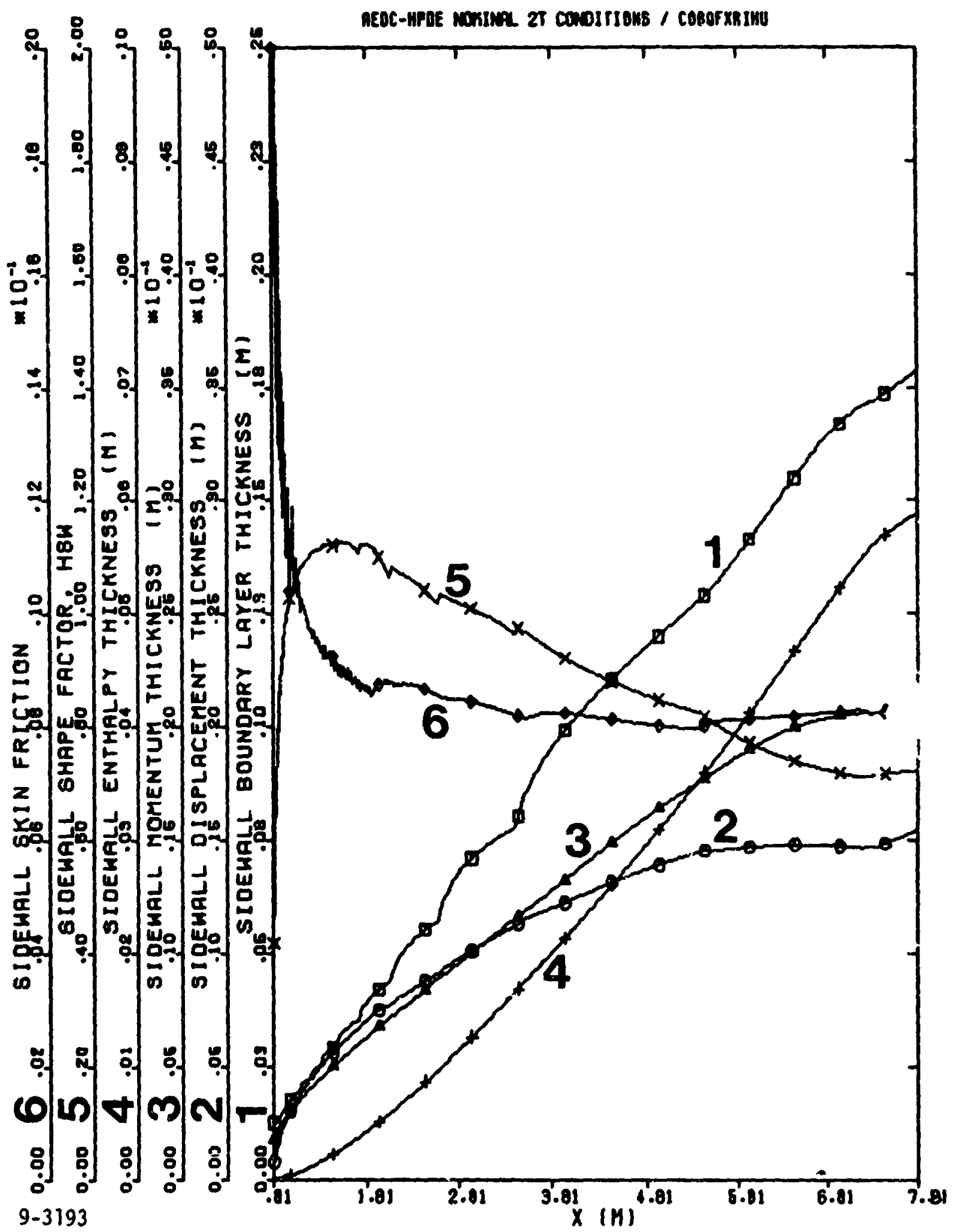

Fig. 3-23. Axial variation of the sidewall boundary layer parameters in the AEDC/HPDF. at the nominal $2 \mathrm{~T}$ operating conditions: Q3D calculation. 


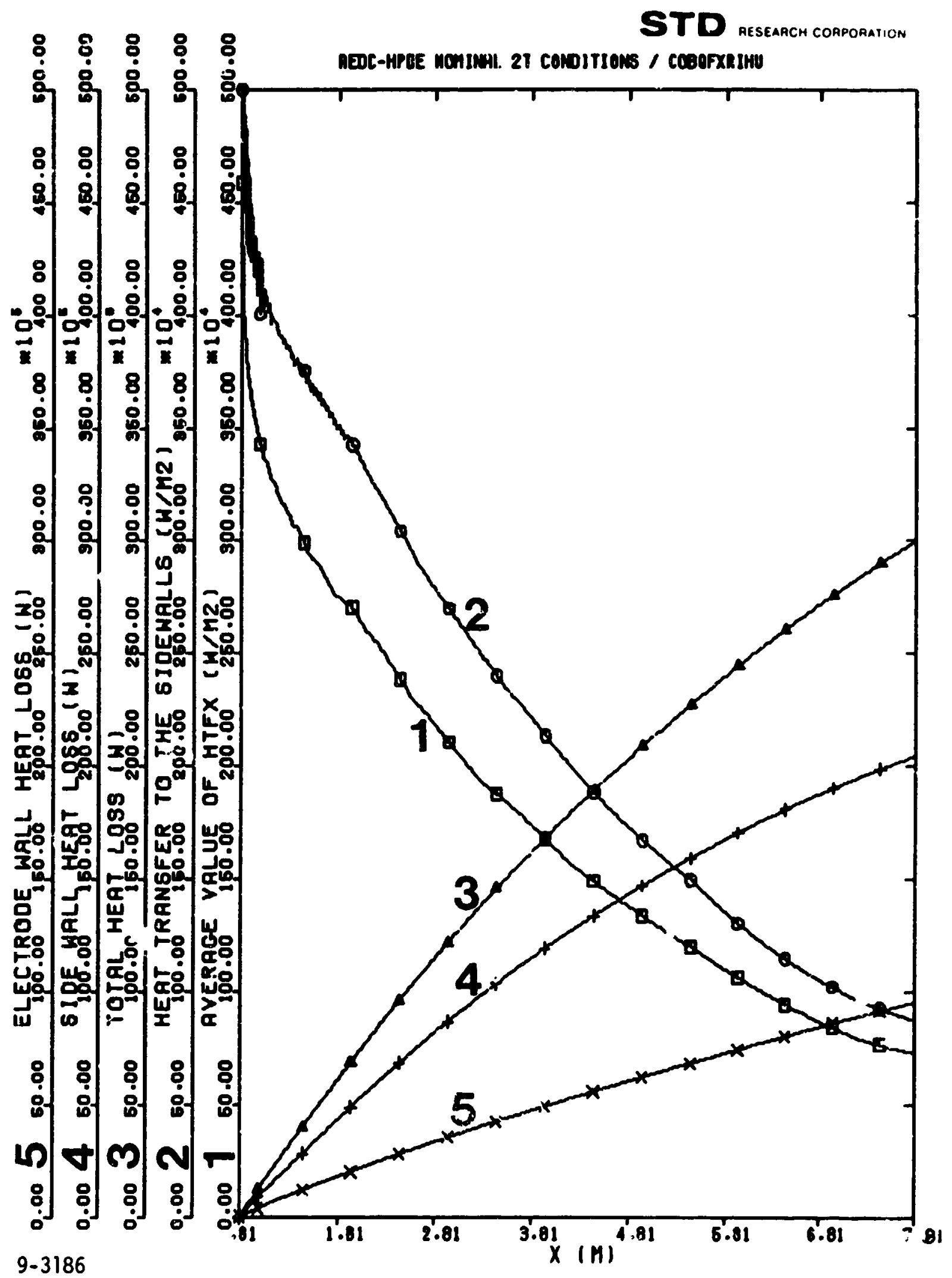

Fig. 3-24. Axial variation of the heat transfer parameiers in the AEDC/HPDE at the nominal 2 T operatine conjit;ons: Q3D calculation. 
STD hesearch conpoamion

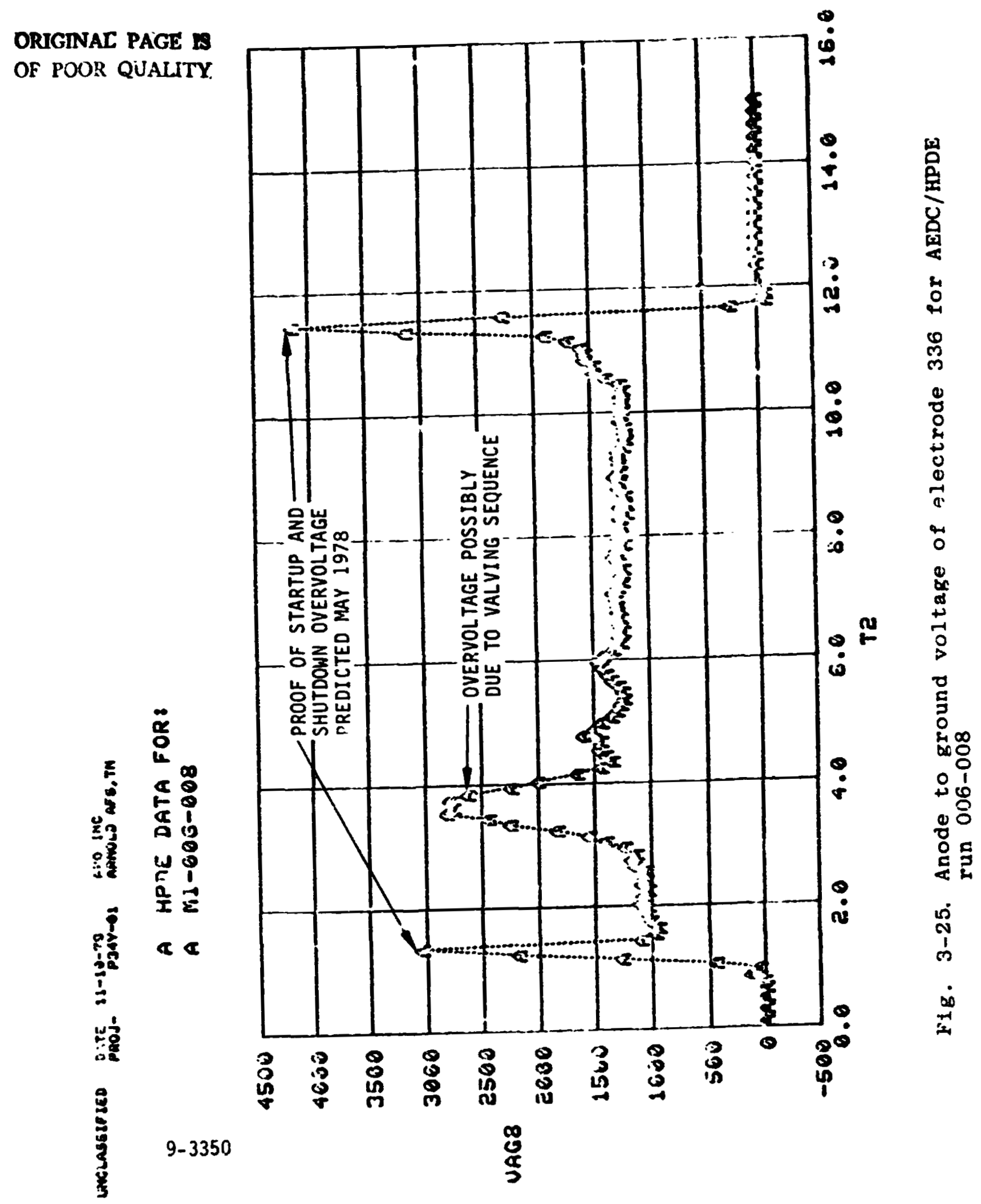




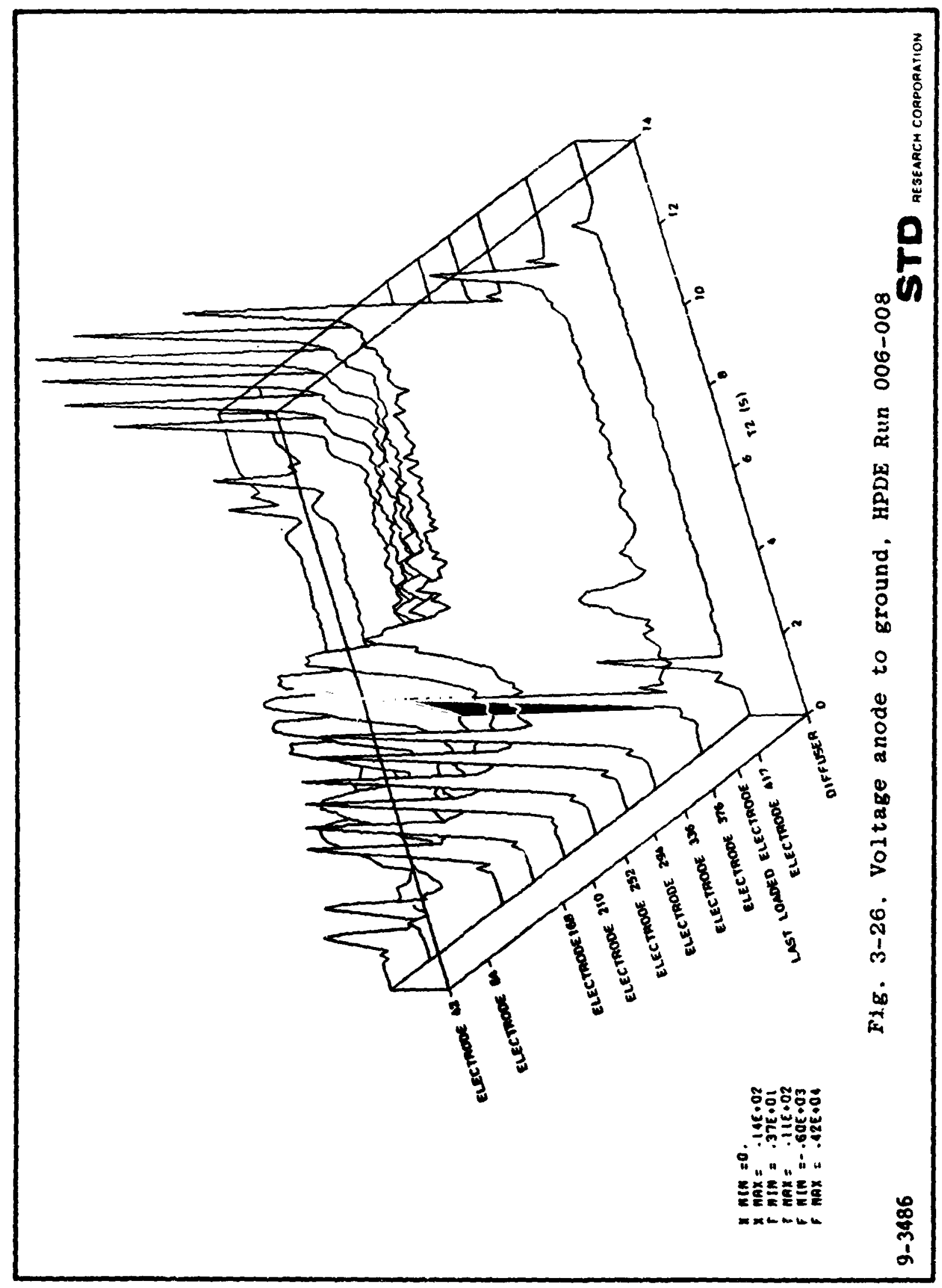




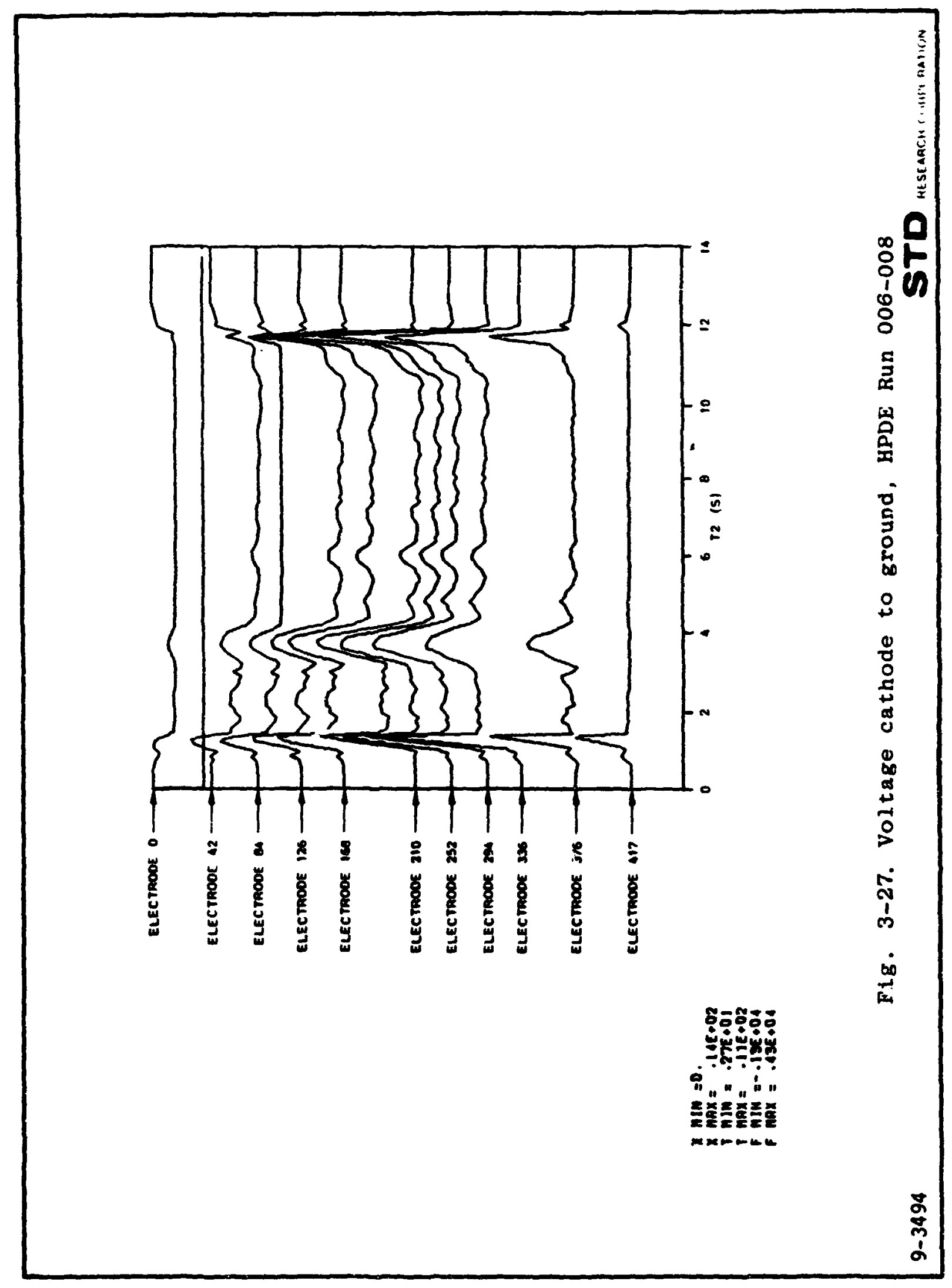


STD aesearch coapoanion

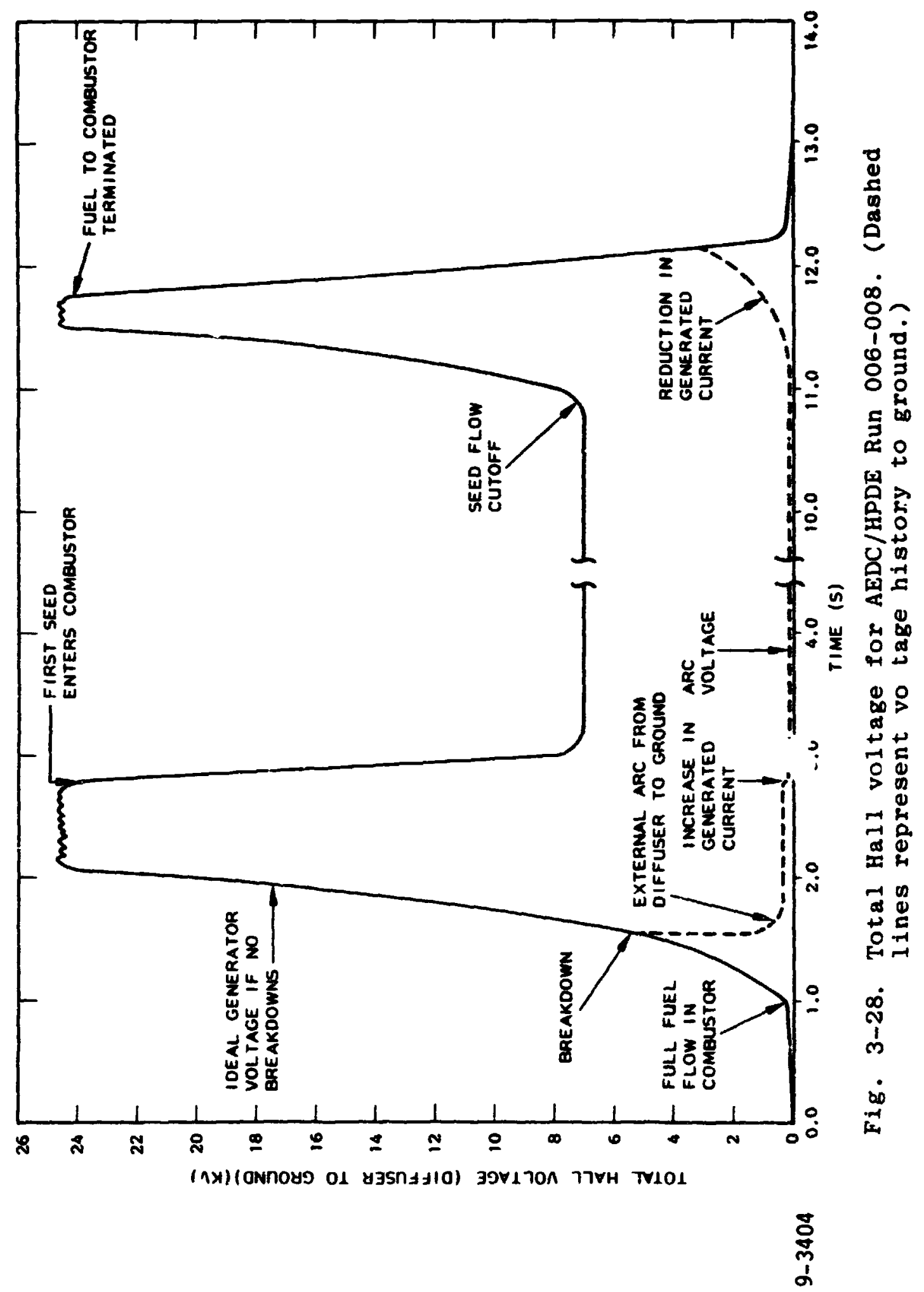




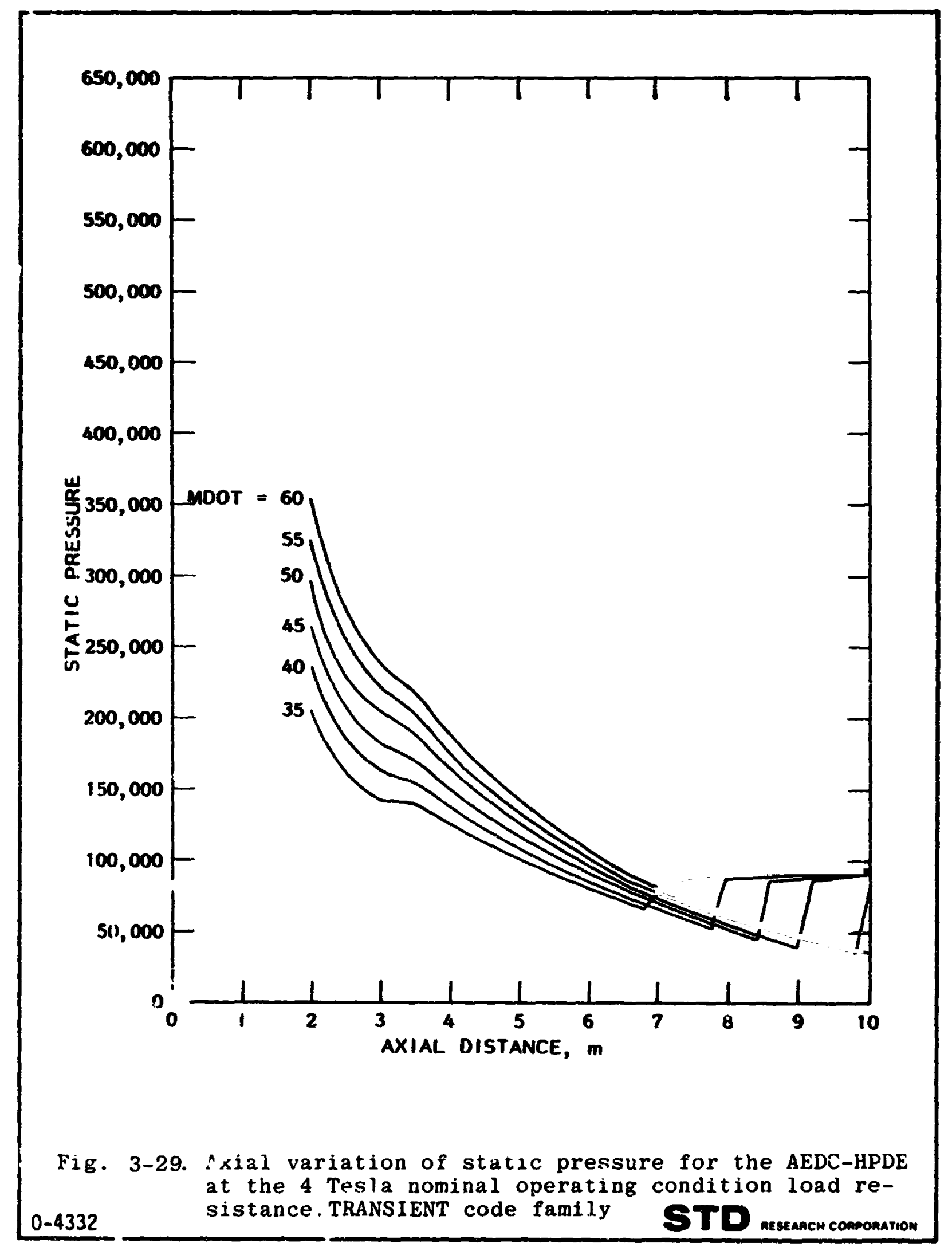




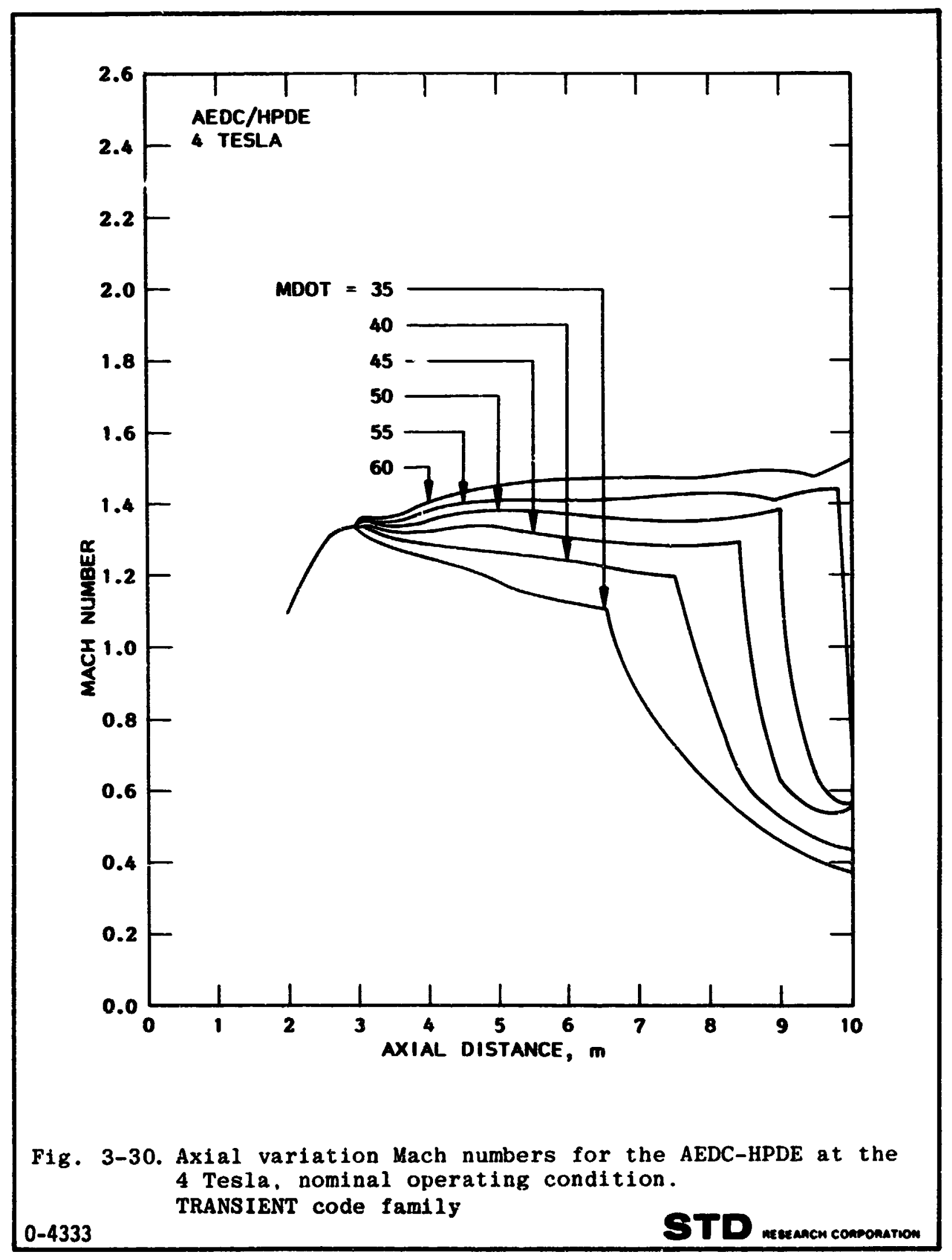


More: A rule of thuab in supersonic flous is that a duct stalls 2-3 diaceters upstreas of a noreal shock coeputed by one-dieensional gas dvnanics. (n $\sim 1.5-5$ )

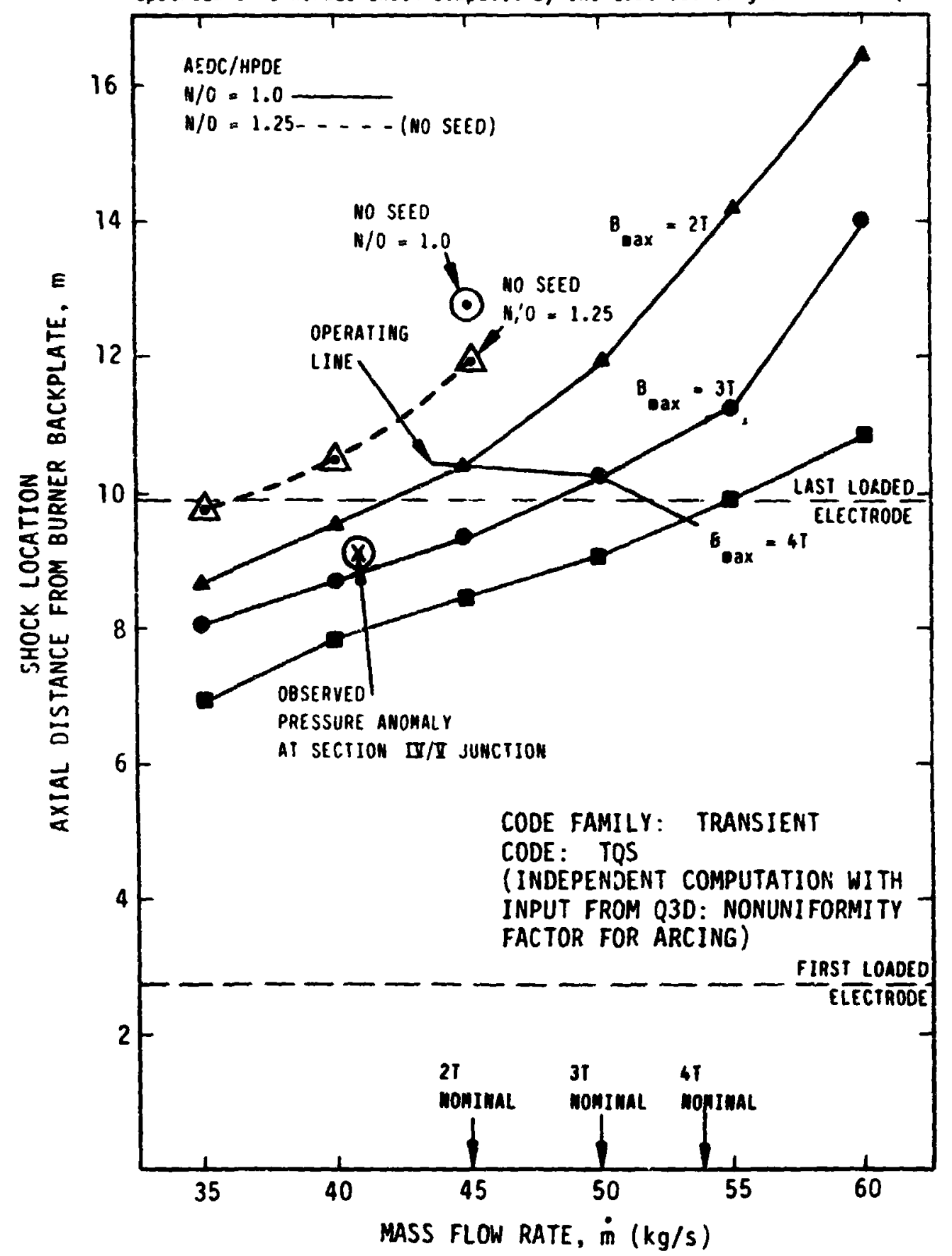

Fig. 3-31. Position of the normal shock as a function of mass flow rate and magnetic field for the nominal operating conditions of the AEDC/HPDE (except for the "NO SEED" point.) Code family: TRANSIENT. Code: TQS. 


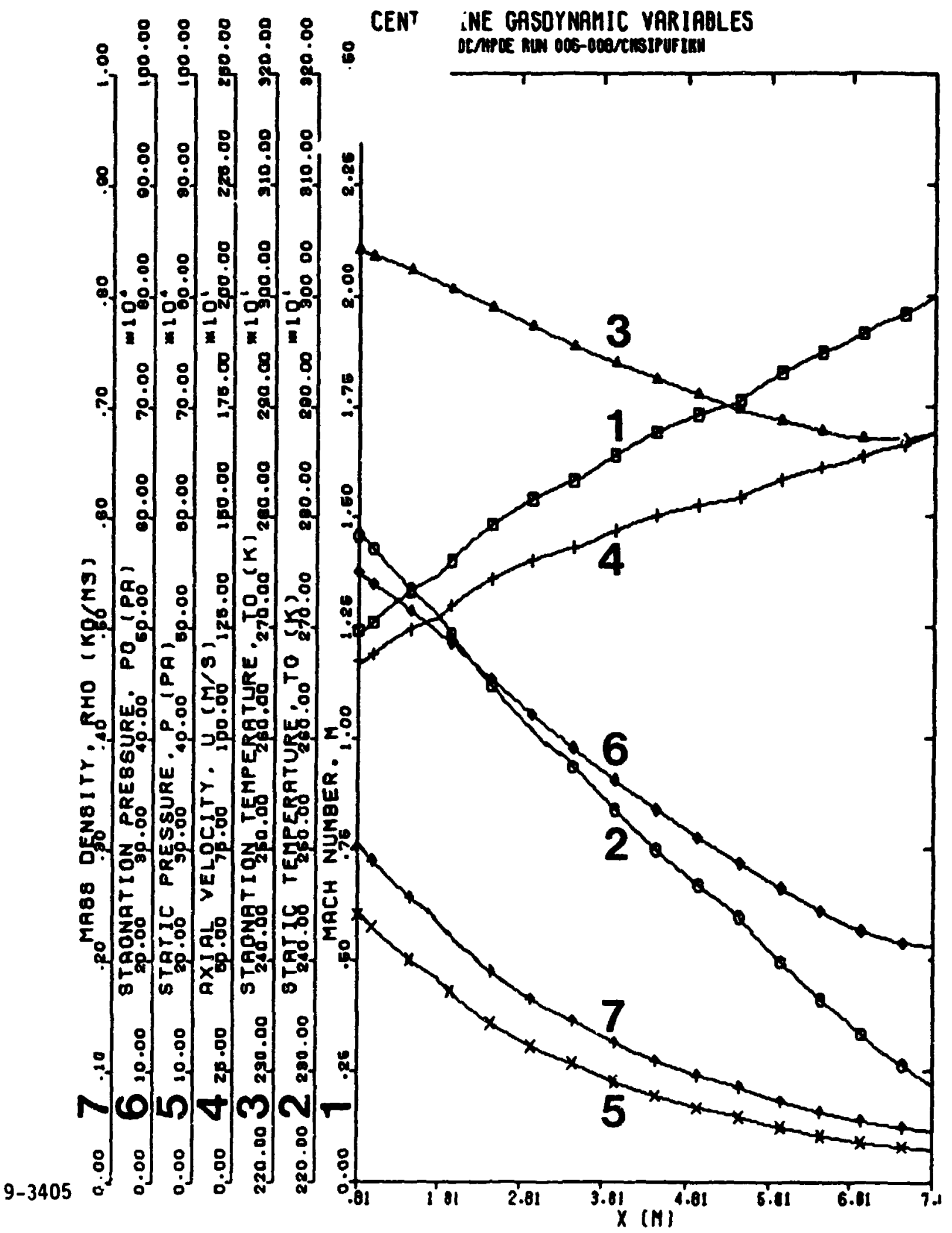

Fig. 3-32. Axial variation of the centerline gasdynamic variables from computation CMSIPUFIKW for the AEDC/HPDr. 
STD REsEAch CORPOAarion

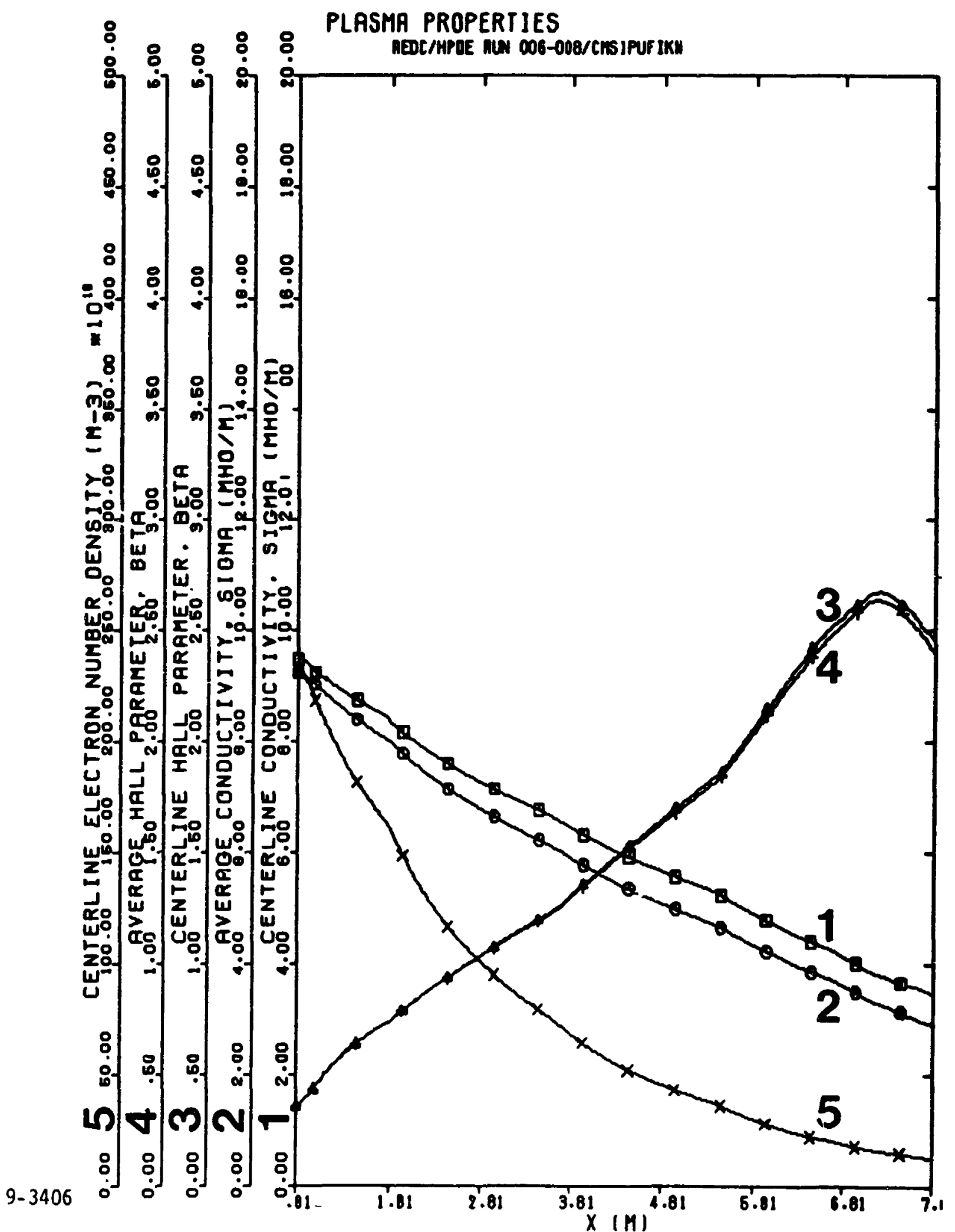

Fig. 3-33. Axial variation of the plasma properties from computation CMSIPUFIKW for the AEDC/HPDE. 
STD RESEAACM COAPDARA ION

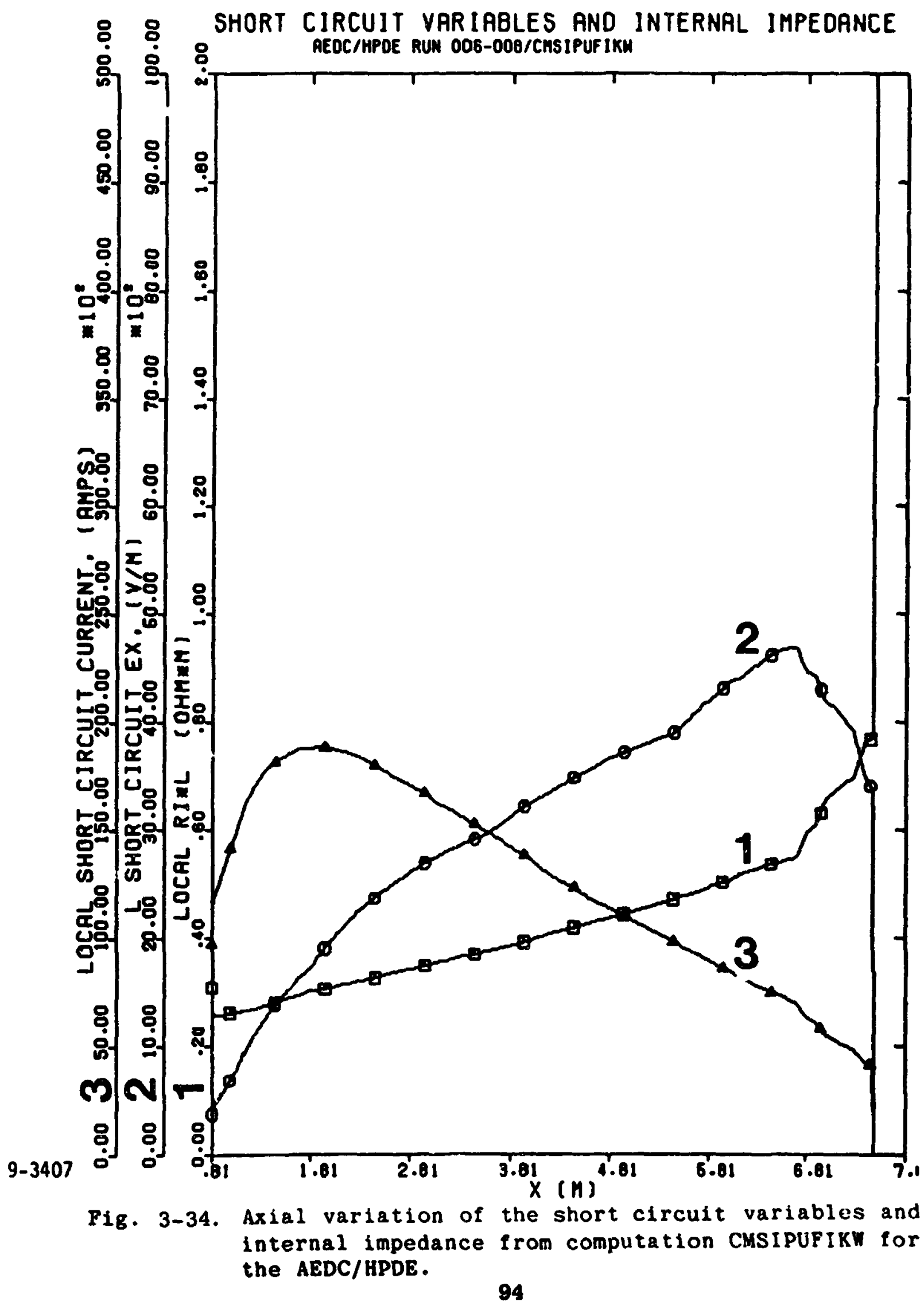


STD reseafch Corporation

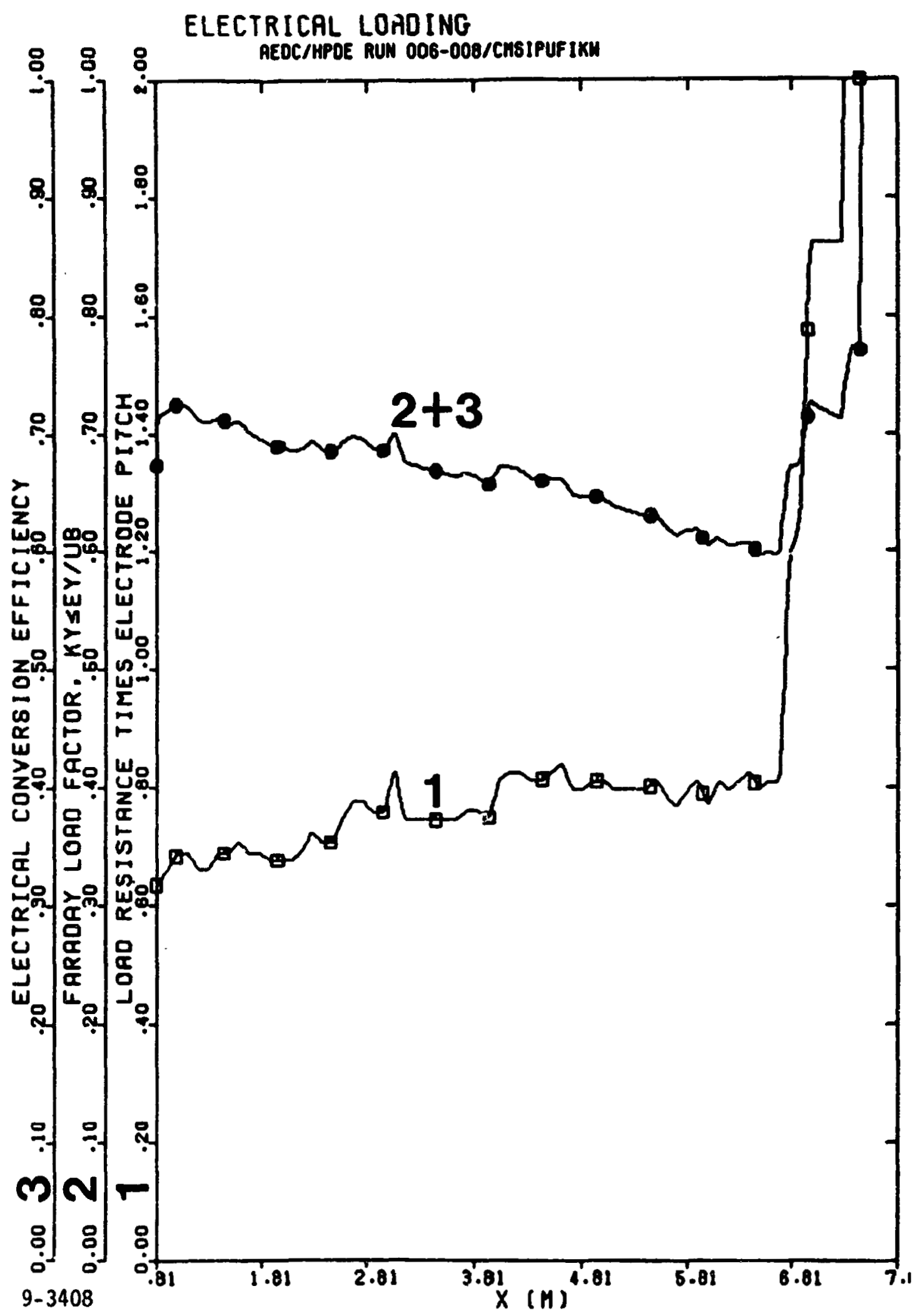

Fig. 3-35. Axial variation of the electrical loading from computation CuSIPUFIKW for the AEDC/HPDE, 
STD aeseanch CoRpoaArion

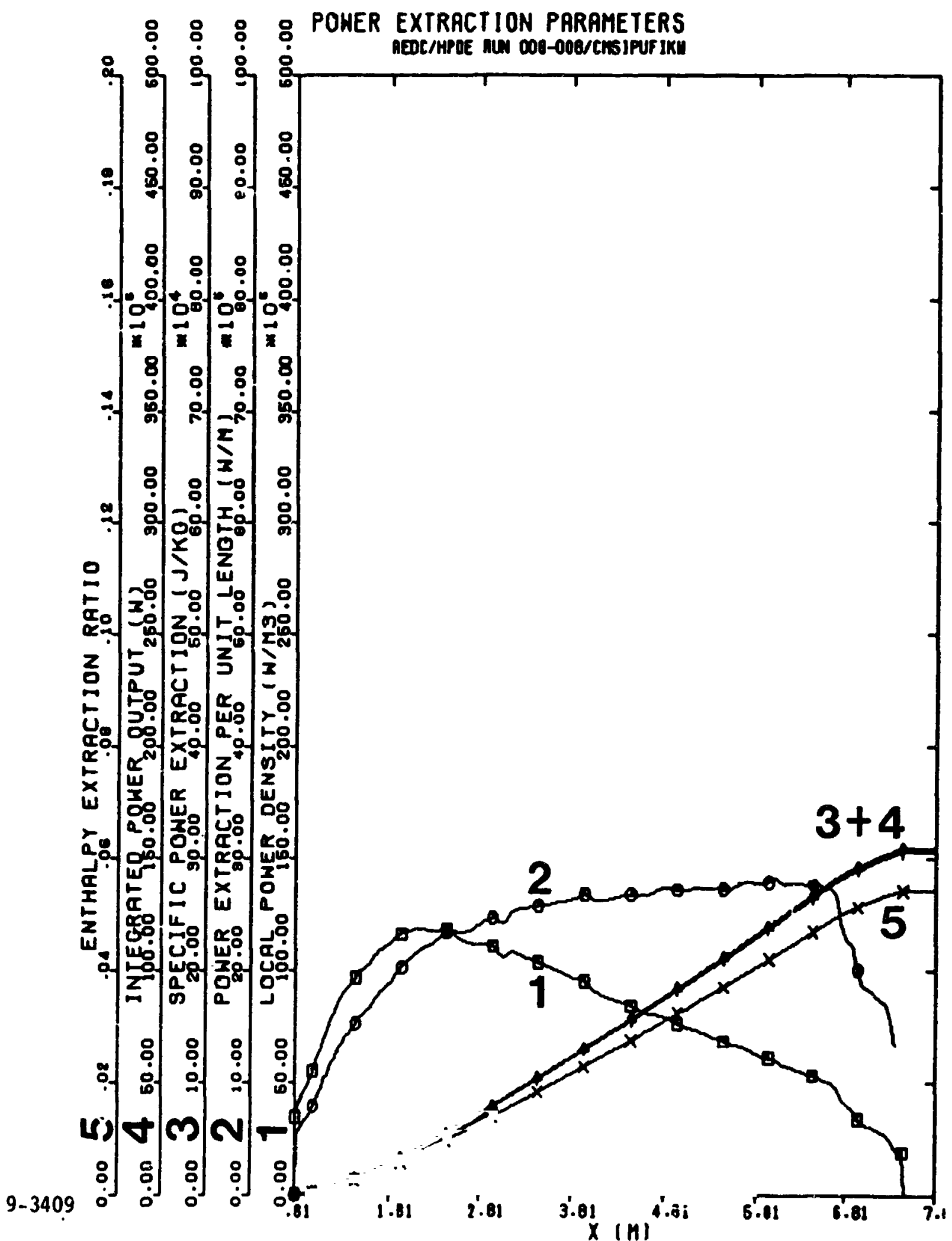

Fig. 3-36. Axial variation of the power extraction parameters from computation CMSIPUFIKW for the AEDC/HPDE. 
STD aEseafch CoRporation

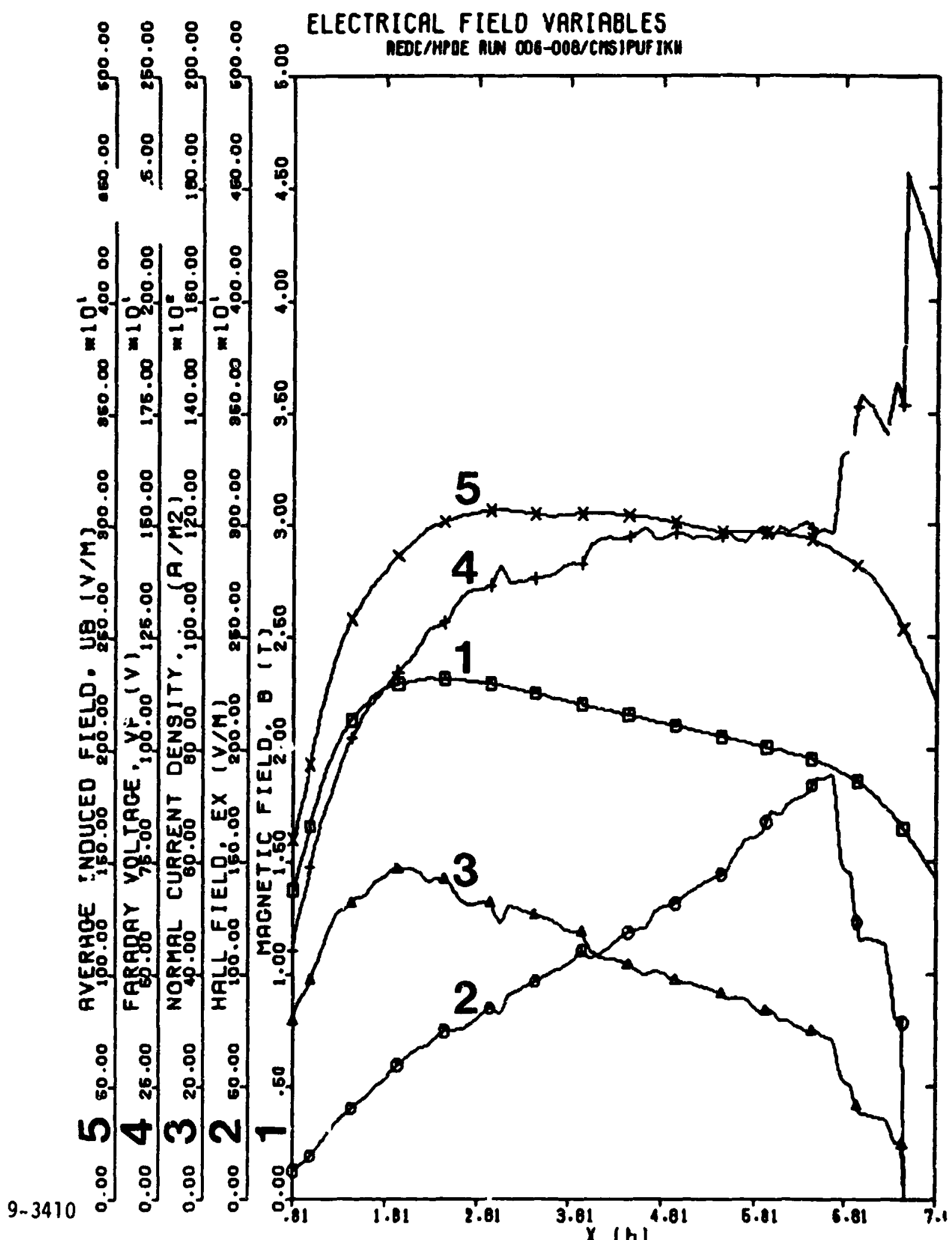

Fig. 3-37. Axial variation of the electrical field variables from computation CMSIPUFIKW for the AEDC/HPDE. 
STD esearch cuppuanion

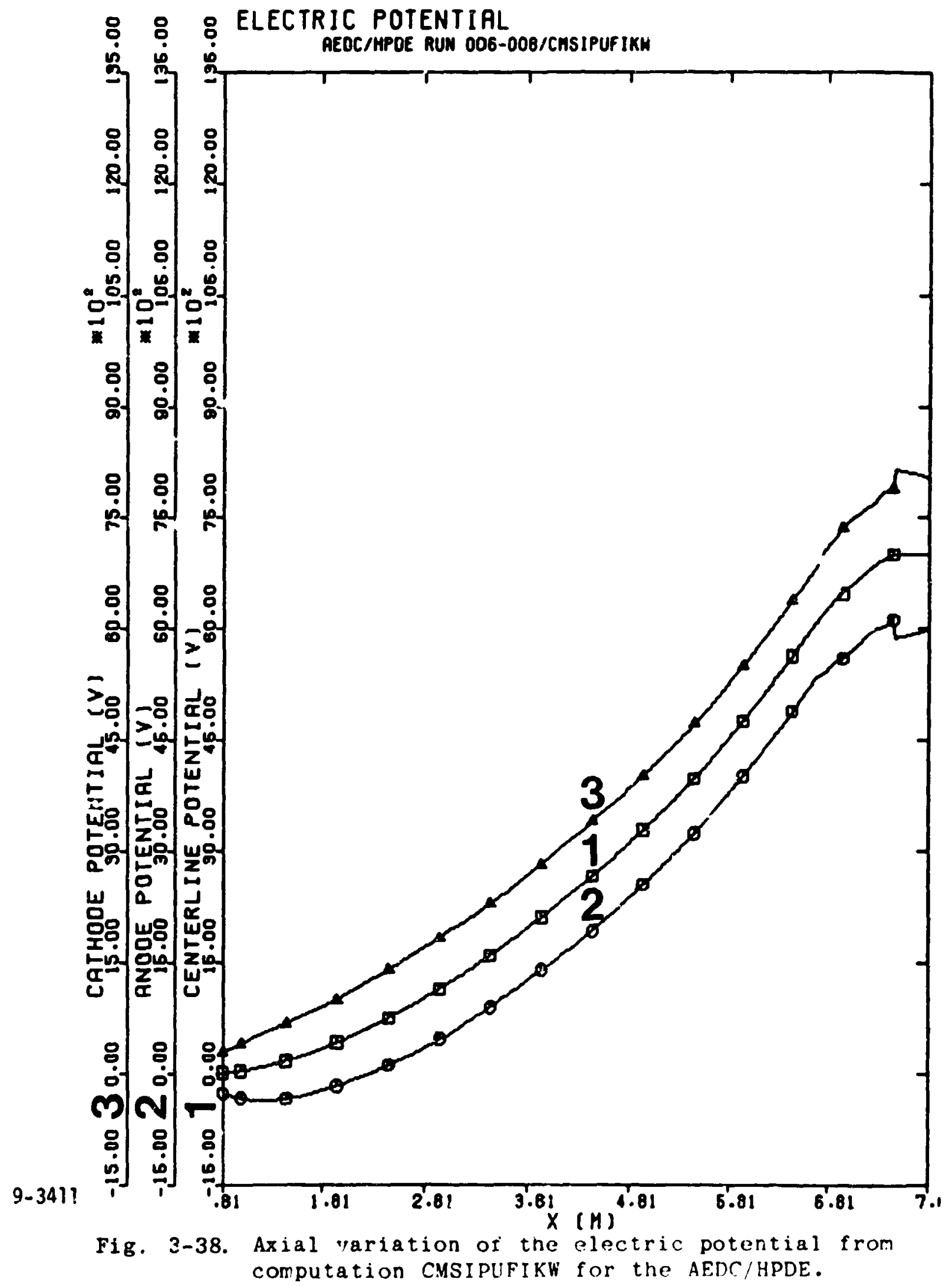


STD reseapcm coppchation

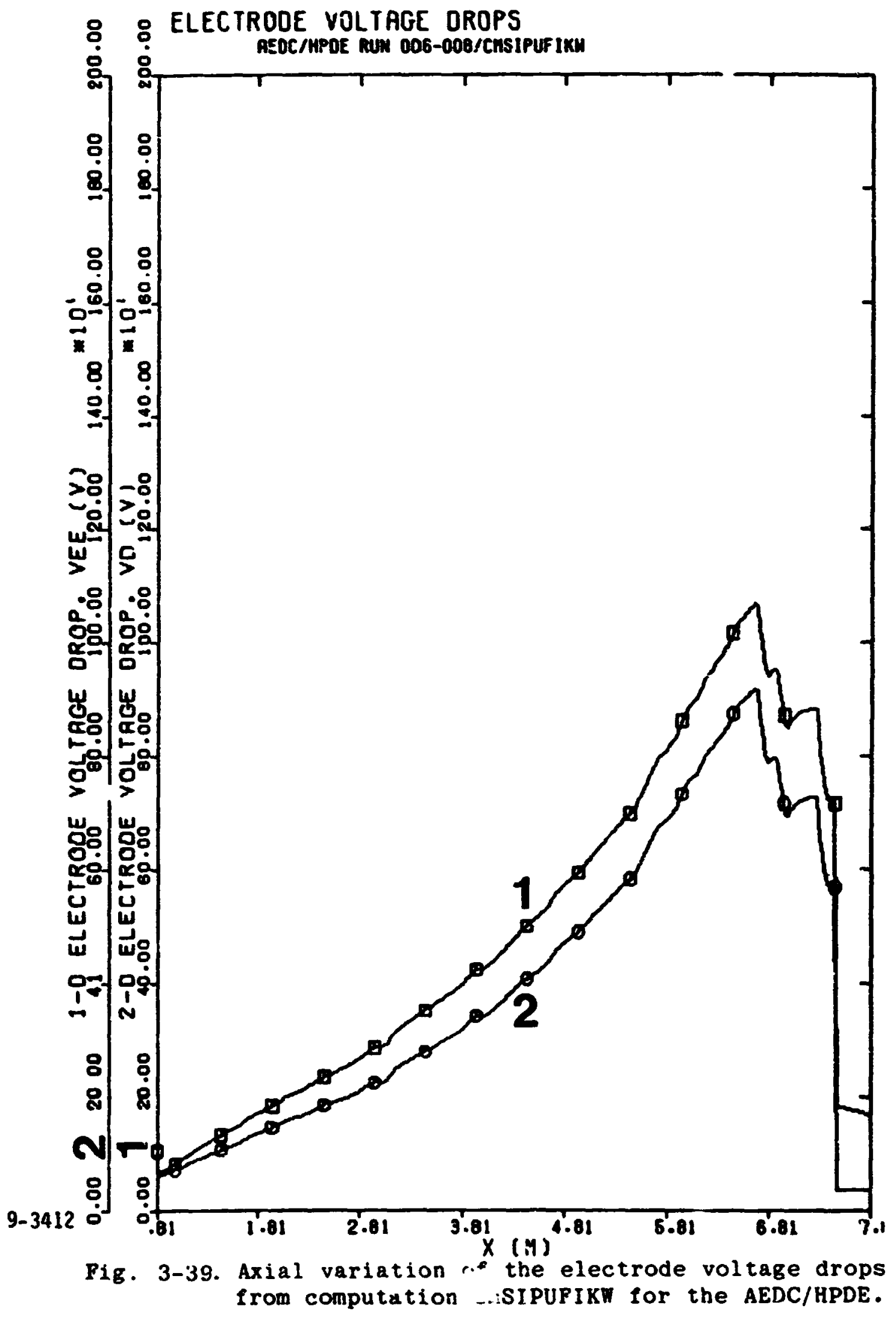


STD REsGAACH CORPOAAIION

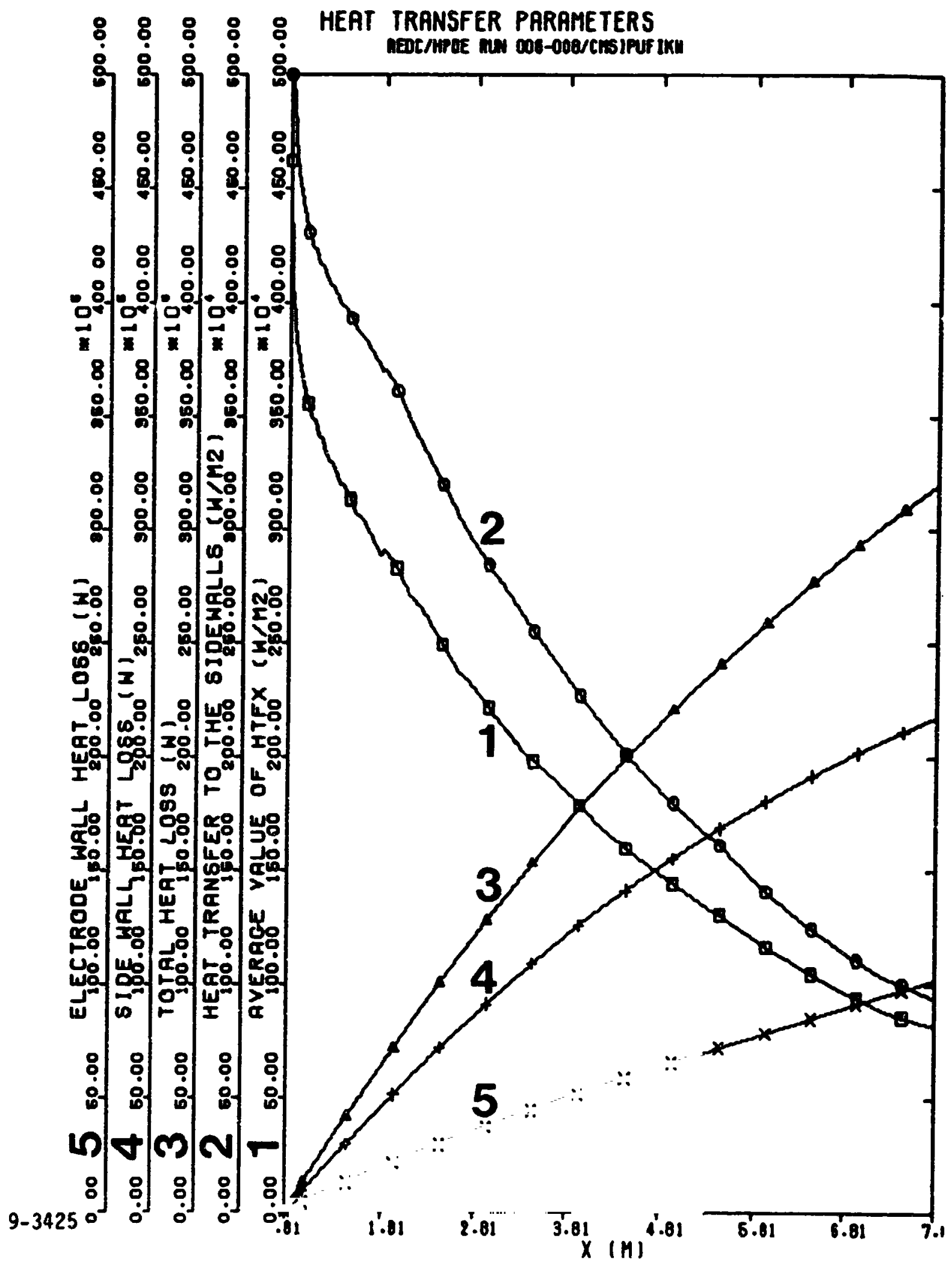

Fig. 3-40. Axial variation of the heat transfer parameters from computation CMSIPUFIKW for the AEDC/HPDE. 
ORIGINal Page is

OE POOR QUALITY

STD aEsearch COaporation

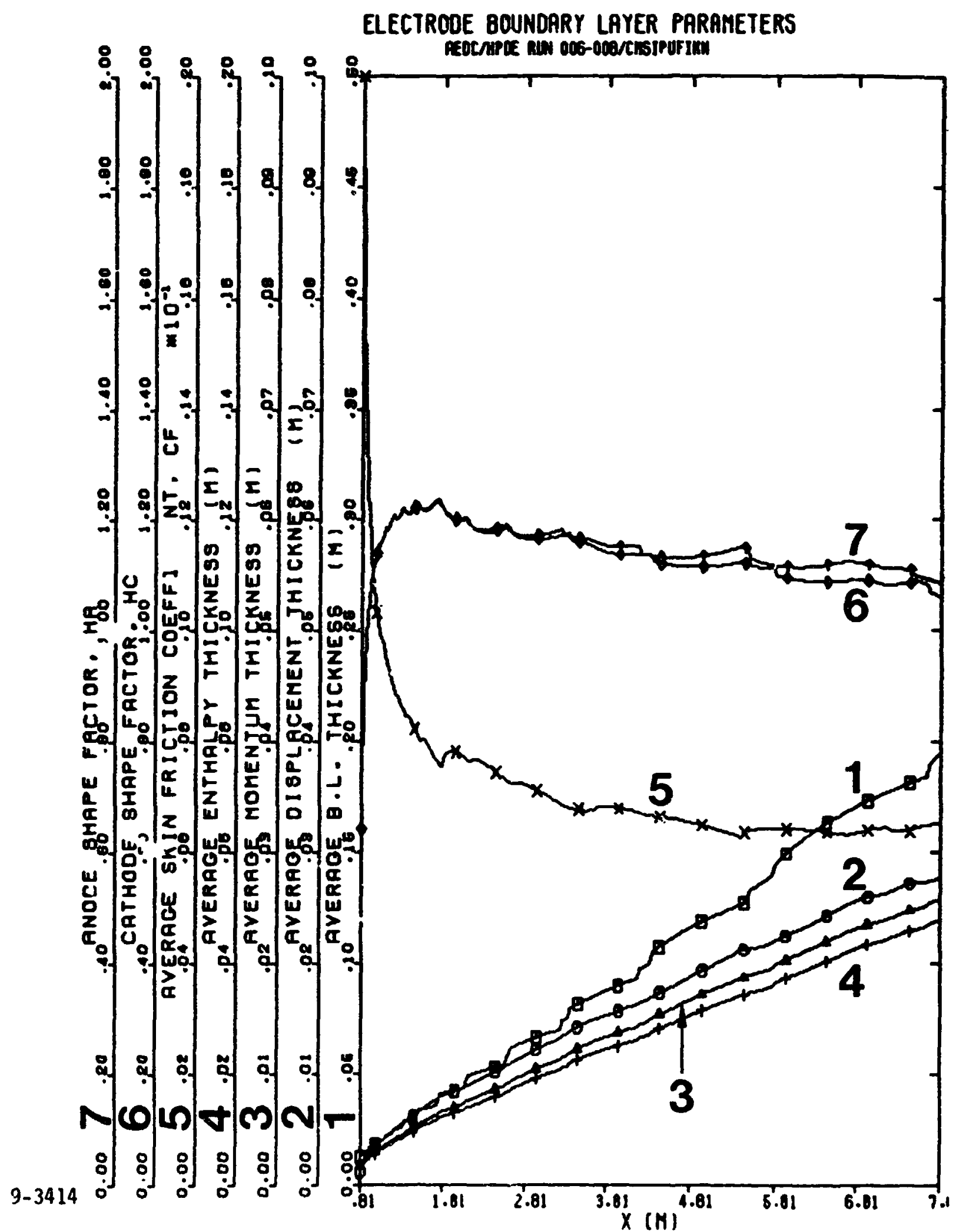

Fig. 3-41. Axial variation of the eiectrode buindary layer parameters from computation CMSIPUFIKW for the AEDC/HPDE. 
STD RESEAACH CORPORATION

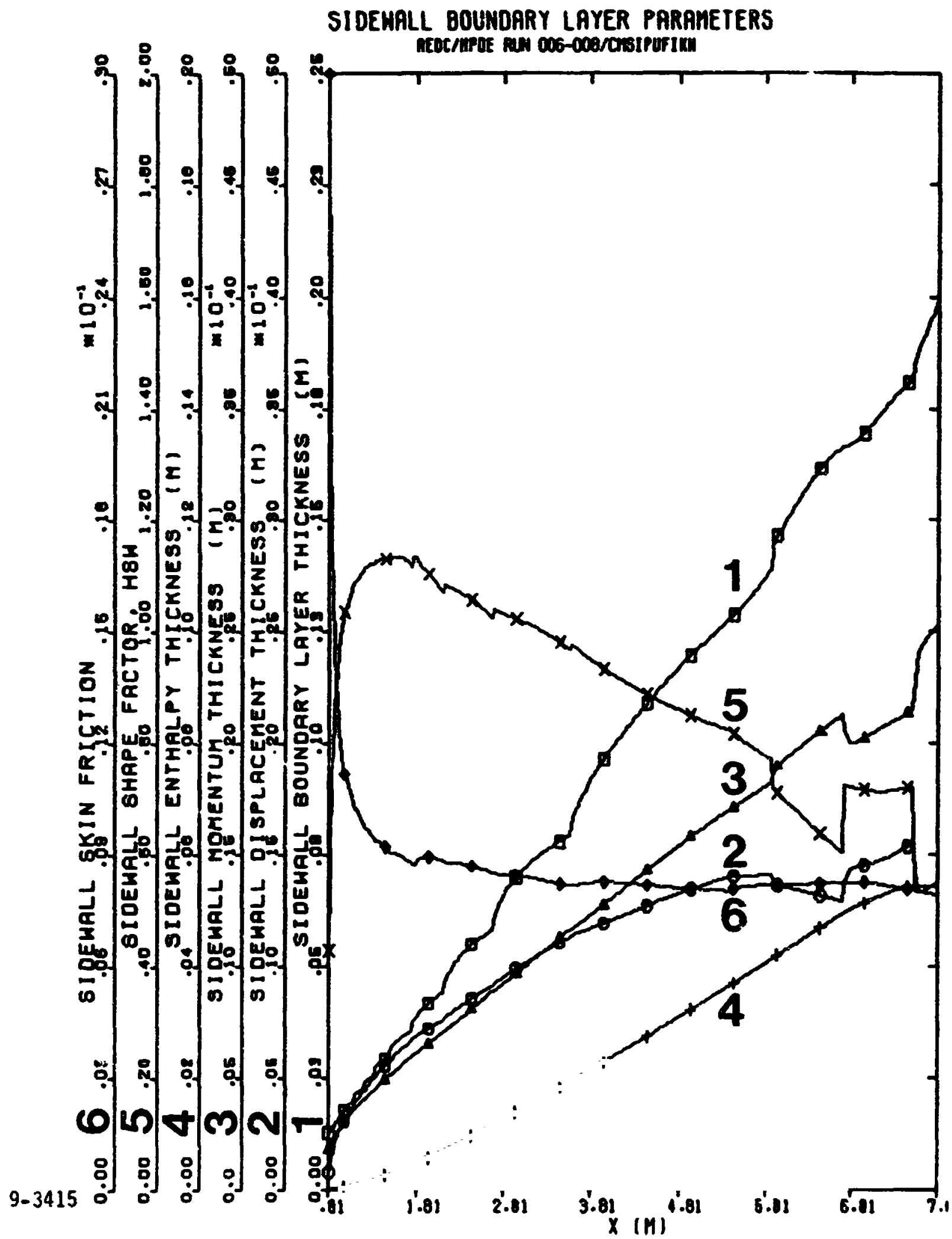

rig. 3-42. Axiai varialiun of tise sidewail boundary laycr parameters frum computation CMSIPUFIKW for the AEDC/HPDE. 
STD ResearCh CORPoRarion

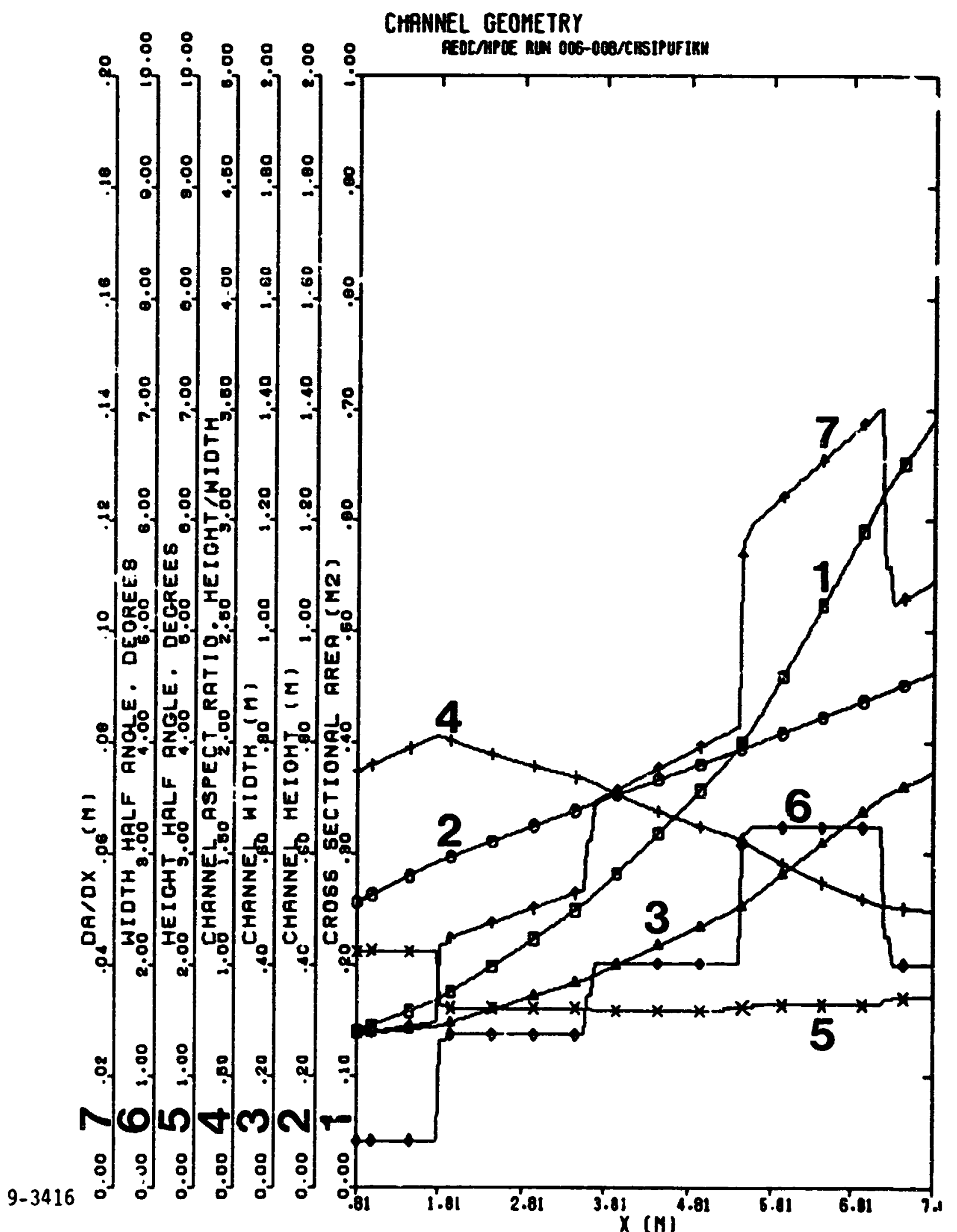

Fig. 3-43. Axial variation of the channel geometry from corputation CMSIPUFIKW for the AEDC/HPDE. 
STD PESEAACH CORPOAaIION

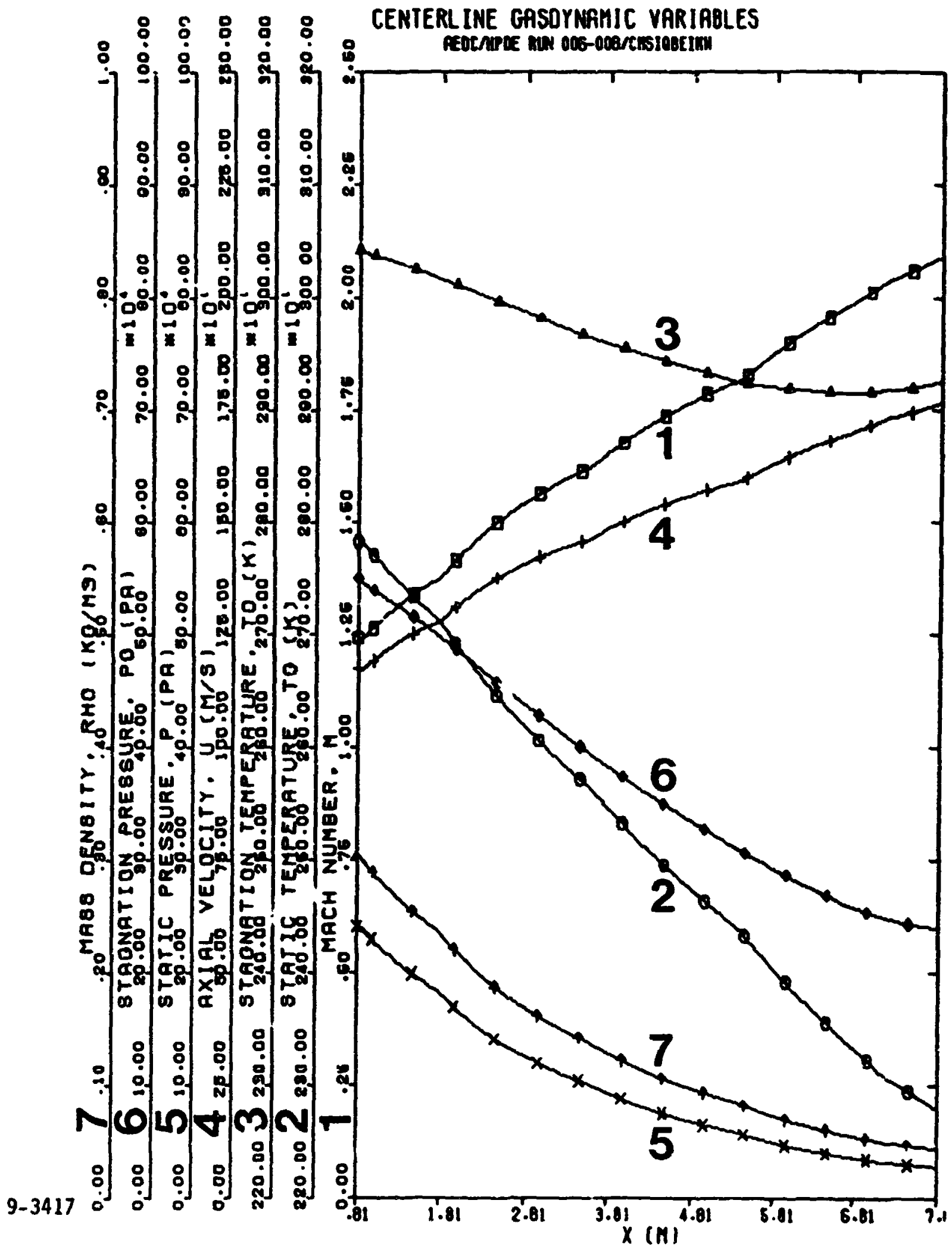

Fig. 3-44. Axial variation of thz centerline gasdynamic variabies from computation CMSIQBEIKW for the AEDC/HPDE. 
STD REsEAach CoRpoaarion

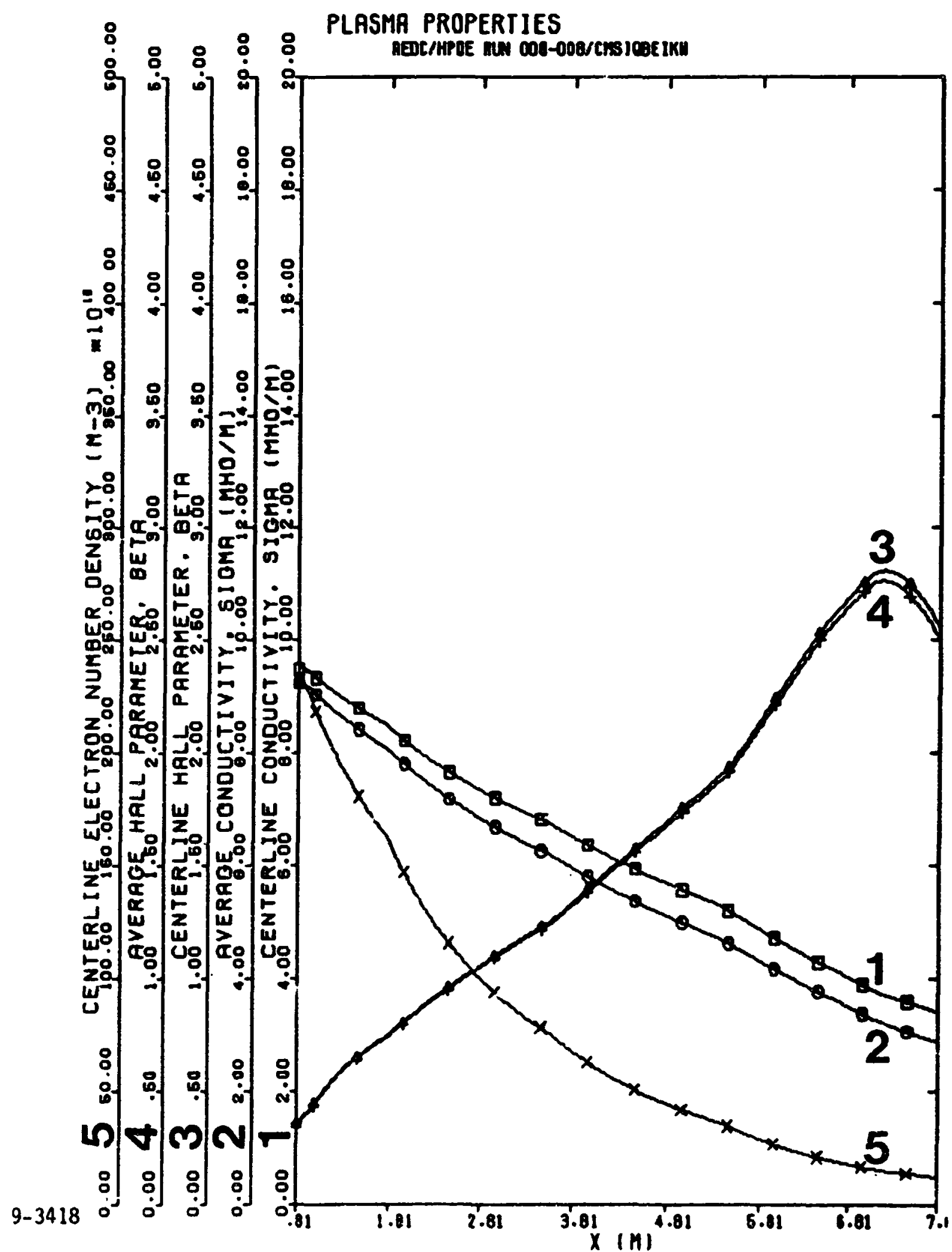

Fig. 3-45. Axial variation of the plasma properties from computation CMSIQBEIKW for the AEDC/HPDE. 
STD aesearch CoAporation

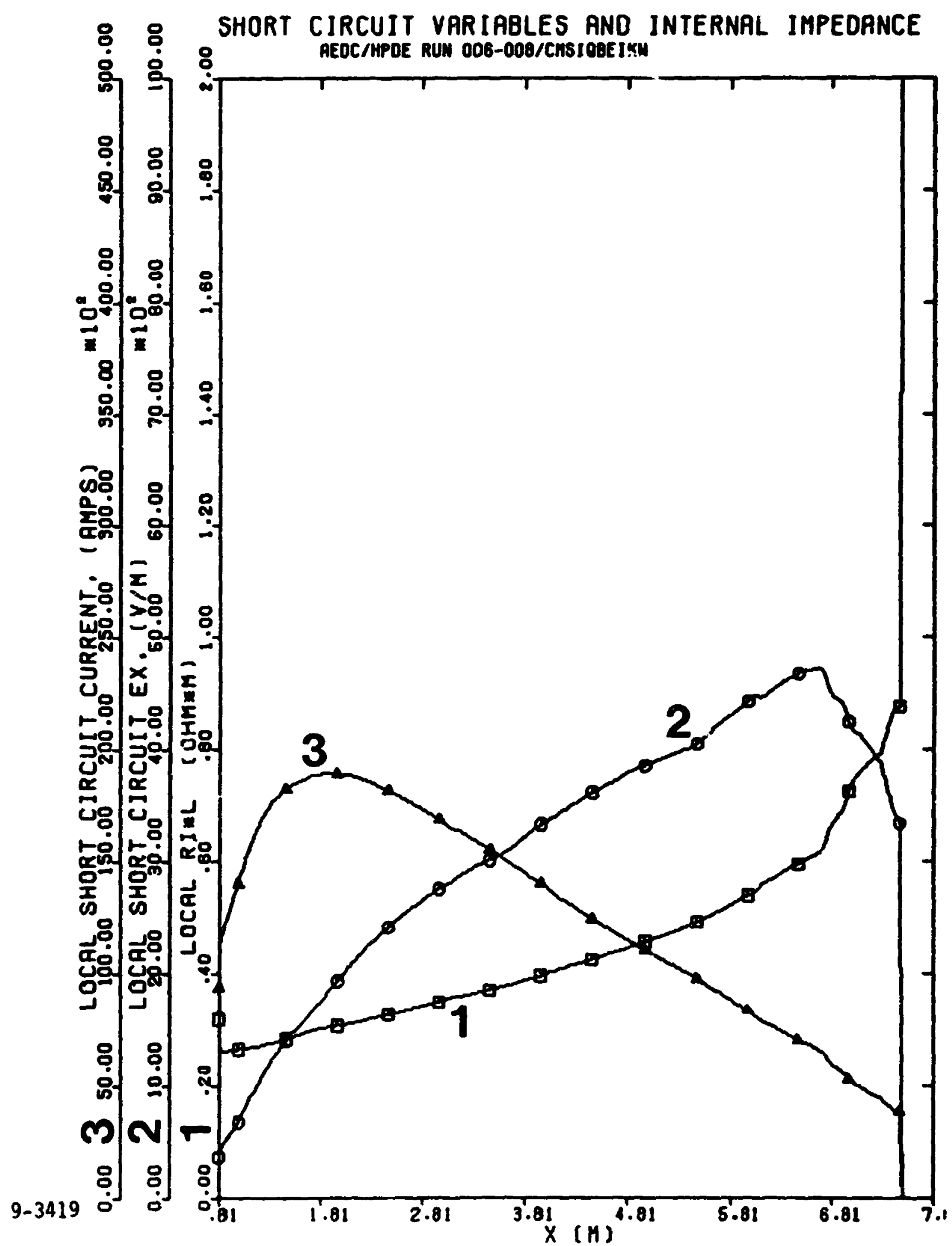

Fig. 3-46. Axial variation of the short circuit variables and internal impedance from computation CMSIQBEIKW for the AEDC/HPDE . 
STD atseabch comporation

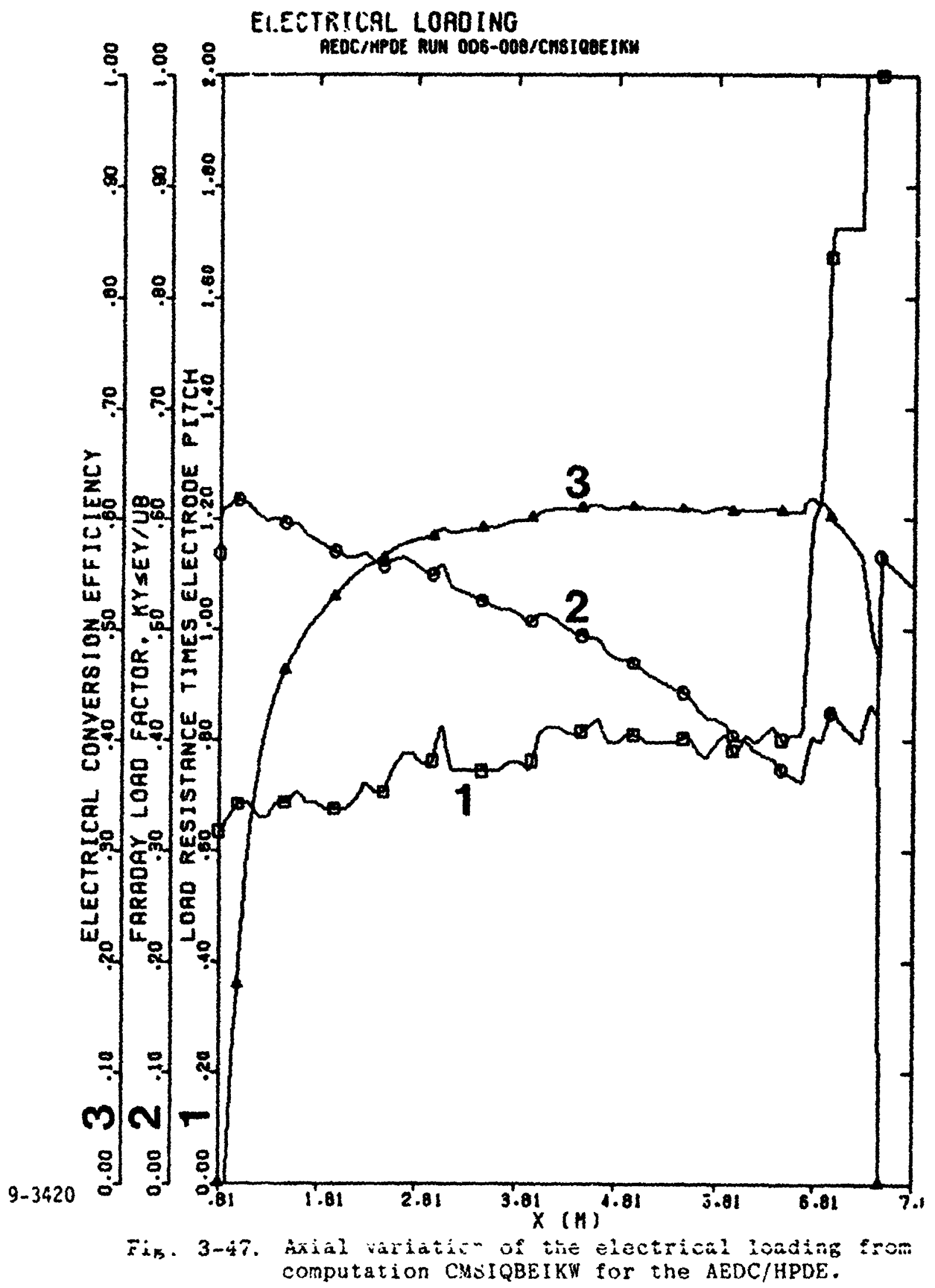


STD heseanch coaporanion

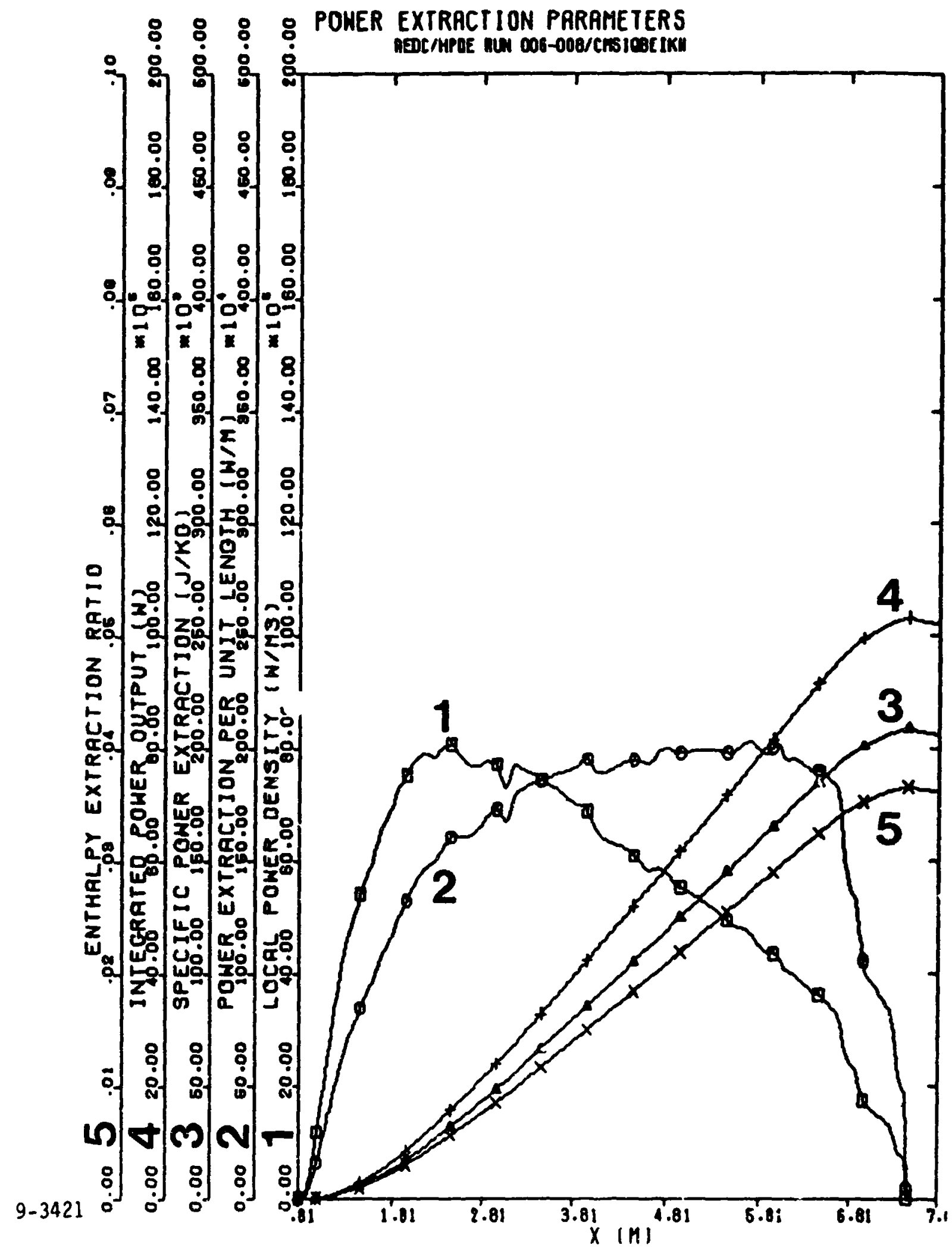

Fig. 3-48. Axial variation of the power extraction parameters from computation CMSIQEEIKW for the AEDC/HPDE. 
STD aEsEABCH COPPOQATION

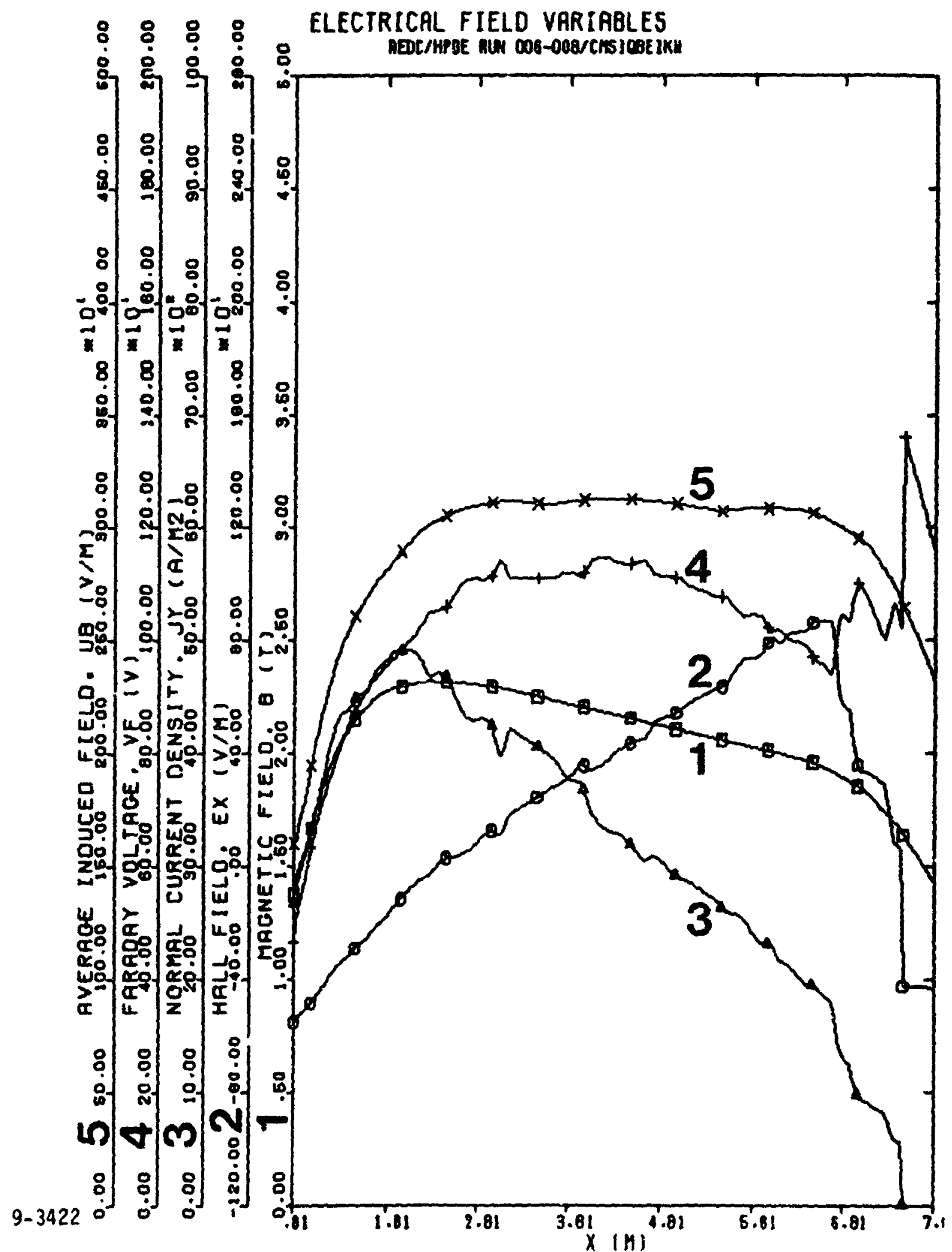

Fig. 3-49. Axial variation of the electrical field variables from computation CMSIQBEIKW for the AEDC/HiPDE. 
STD aeseapch comporation

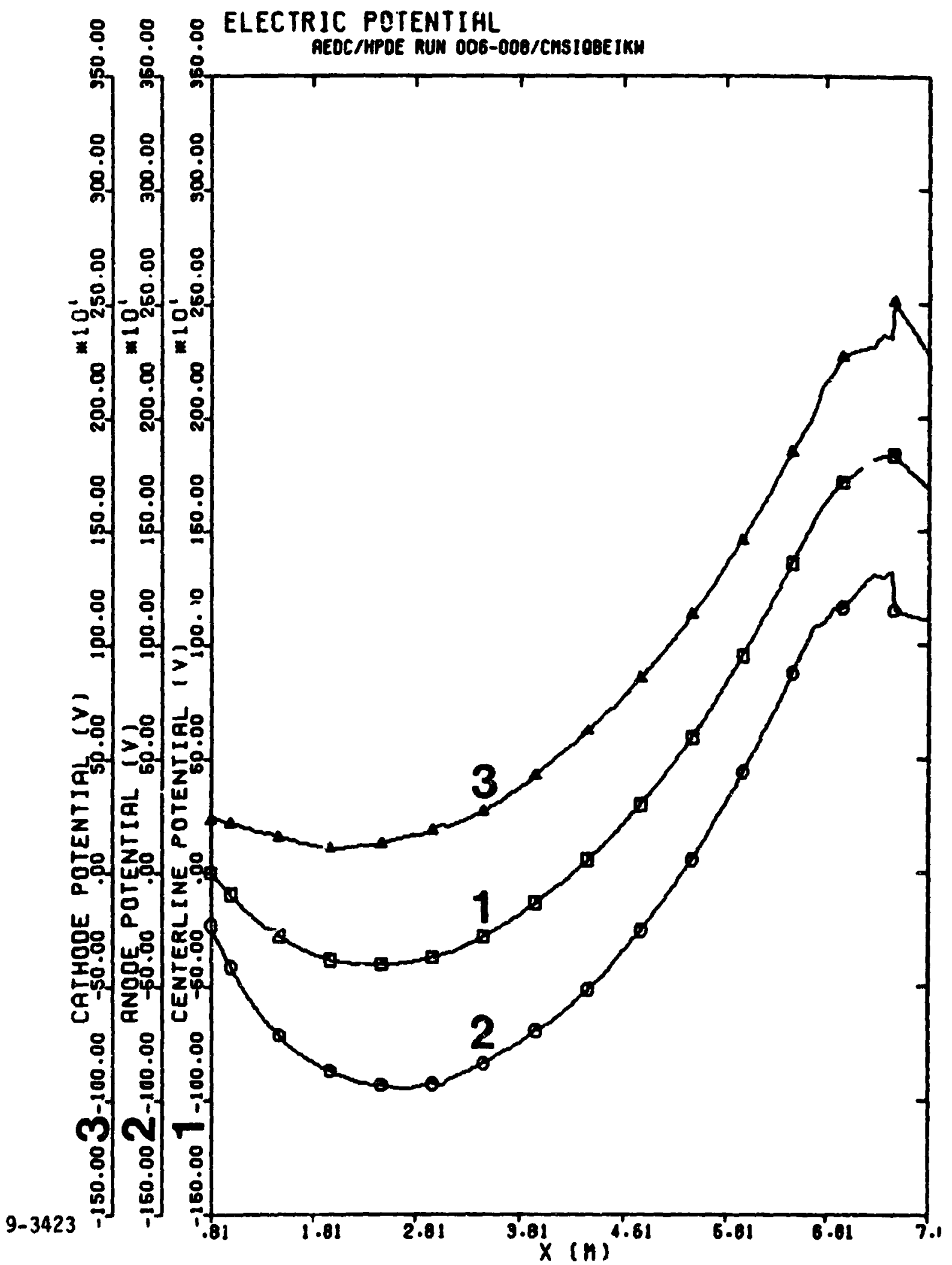

Fig. 3-50. Axial variation of the electric potential from computation CMSIQBEIKW for the AEDC/HPDE. 
STD hesearcm Corporation

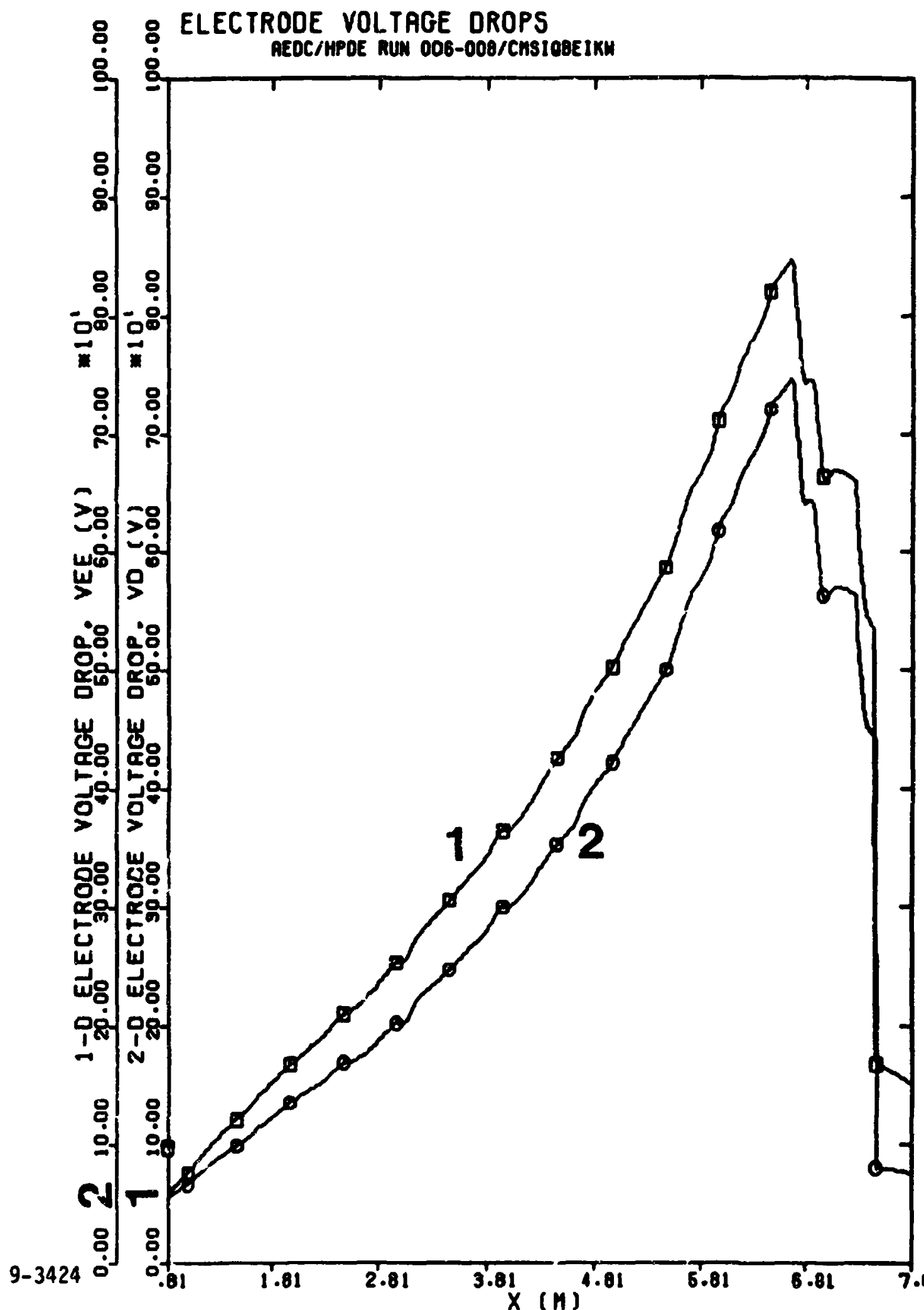

Fig. 3-51. Axial variation of the electrode voltage drops from computation CMSIQBEIKW for the AEDC/HPDE. 
STD aEsearCm CORPDPatic

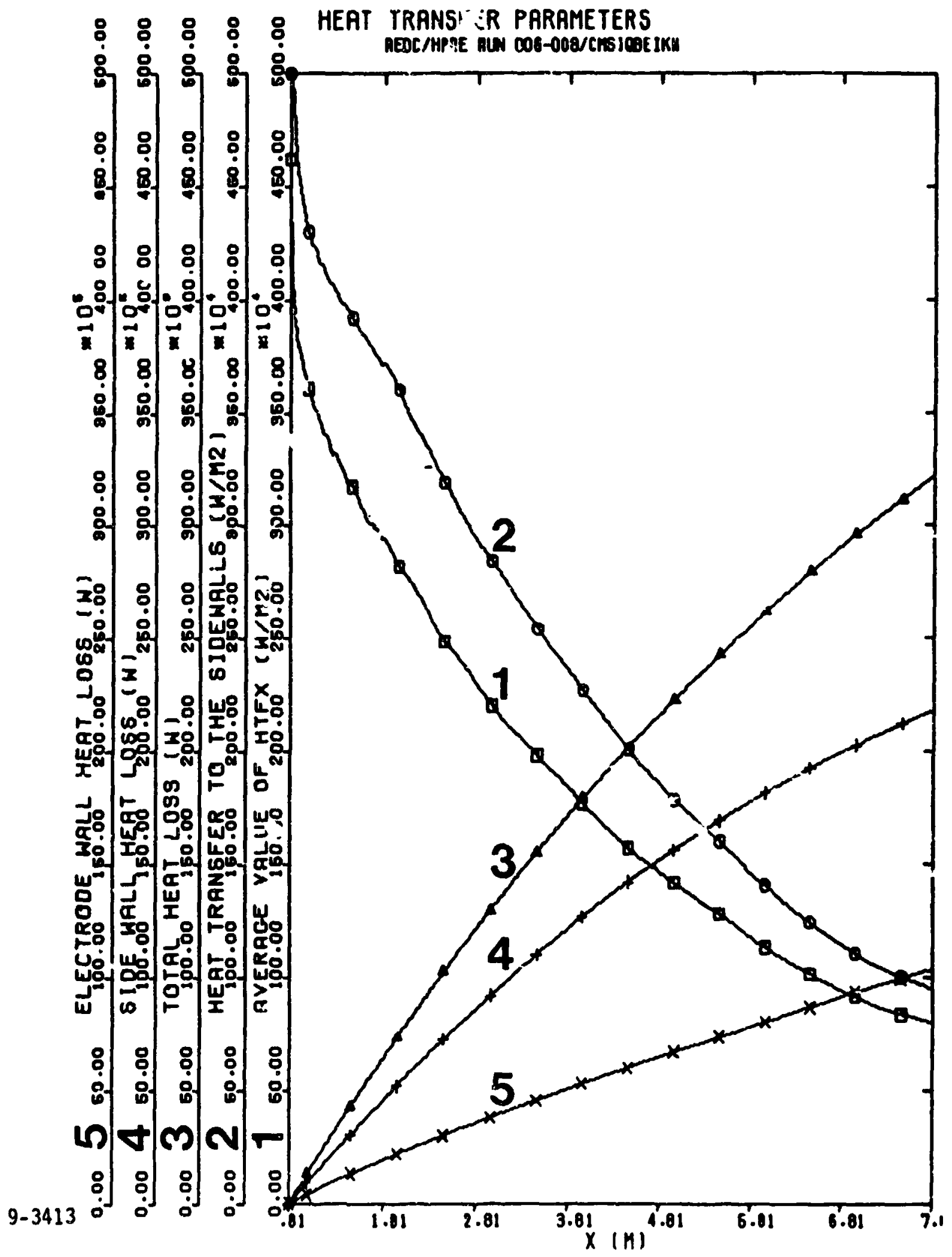

Fig. 3-52. Axial variation of the heat transfer parameters from computation CMSIQBEIKW for the AEDC/HPDE. 
STD aesearcm conpopation

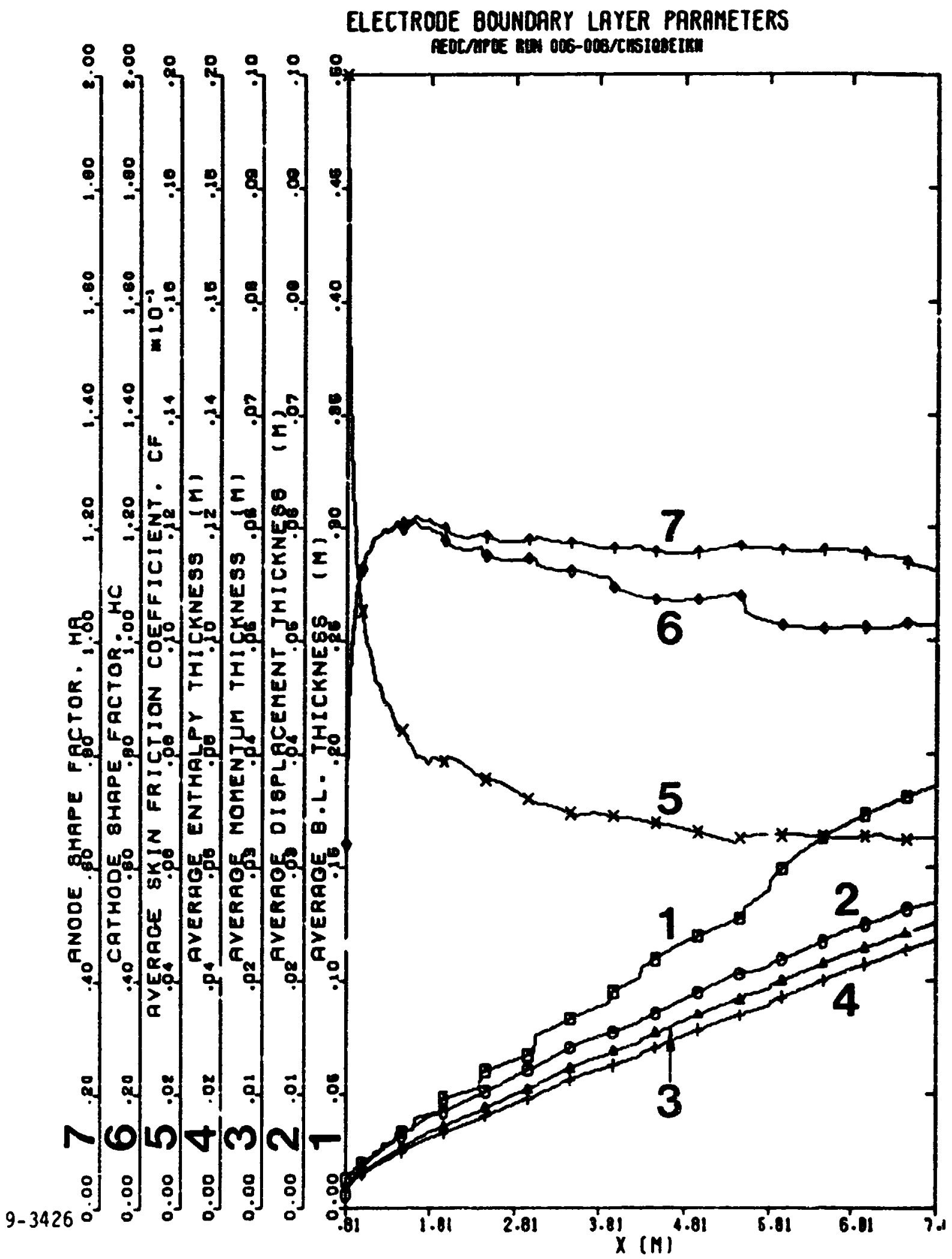

Fig. 3-53. Axial variation of the electrode boundary layer parameters from computation CMSIQBEIKW for the AEDC/HPDE . 
STD aesearcm coppopation

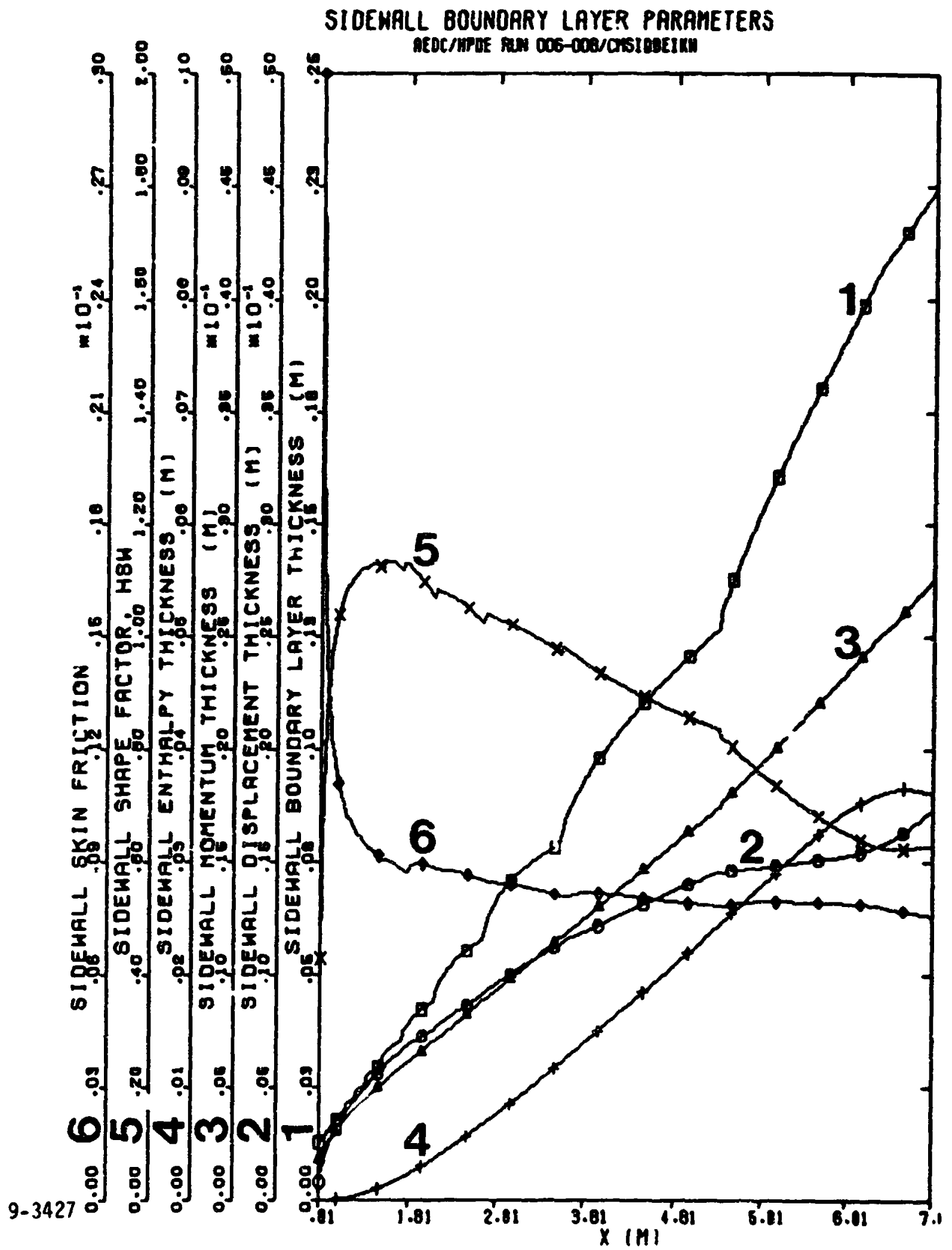

Fig. 3-54. Axial variation of the sidewall boundary layer parameters from computation CMSIQBEIKW for the AEDC/HPDE . 
STD aEseaAch CORDoRarion

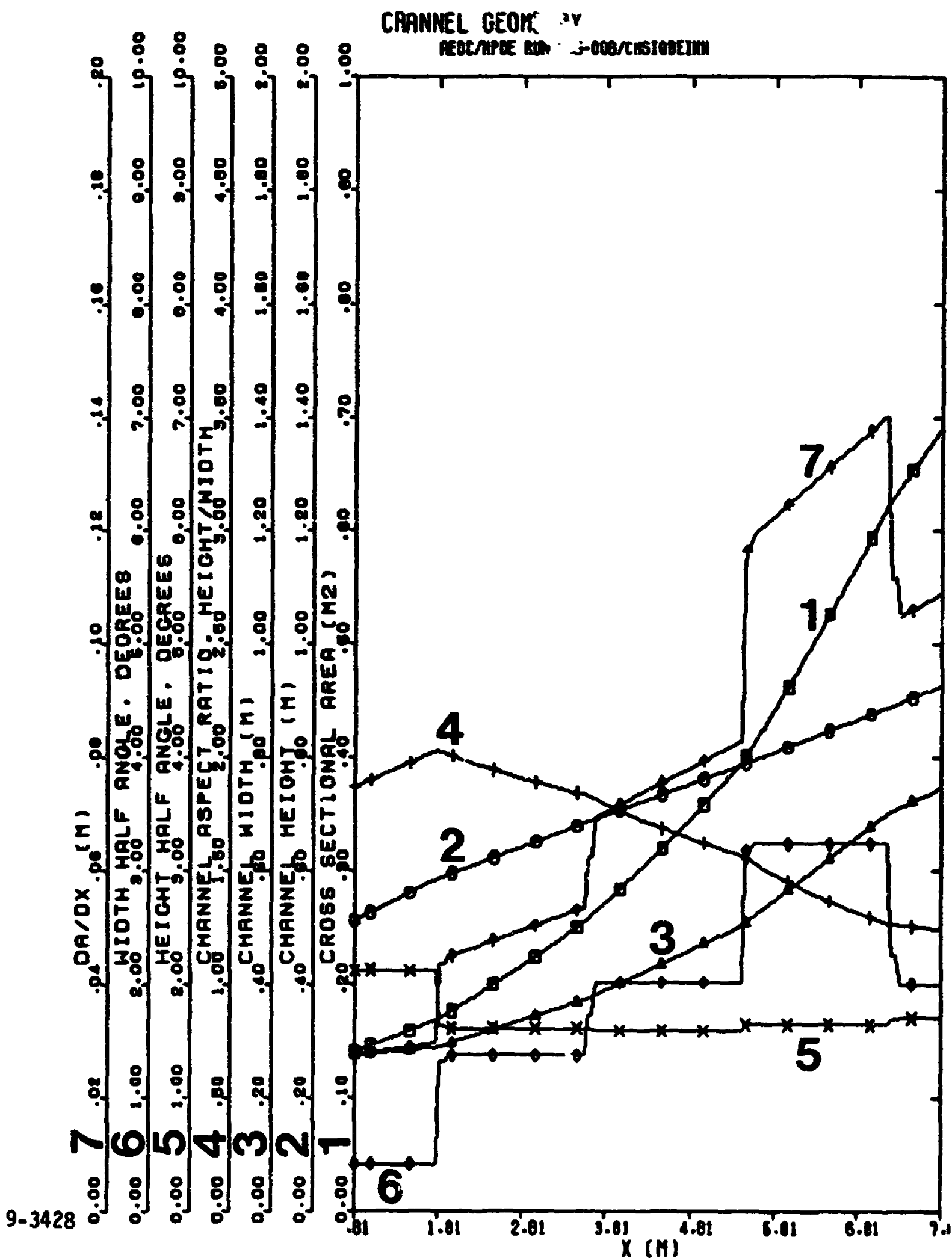

Fig. 3-55. Axial variation of the channel geometry from computation CMSIQBEIKW for the AEDC/HPDE. 


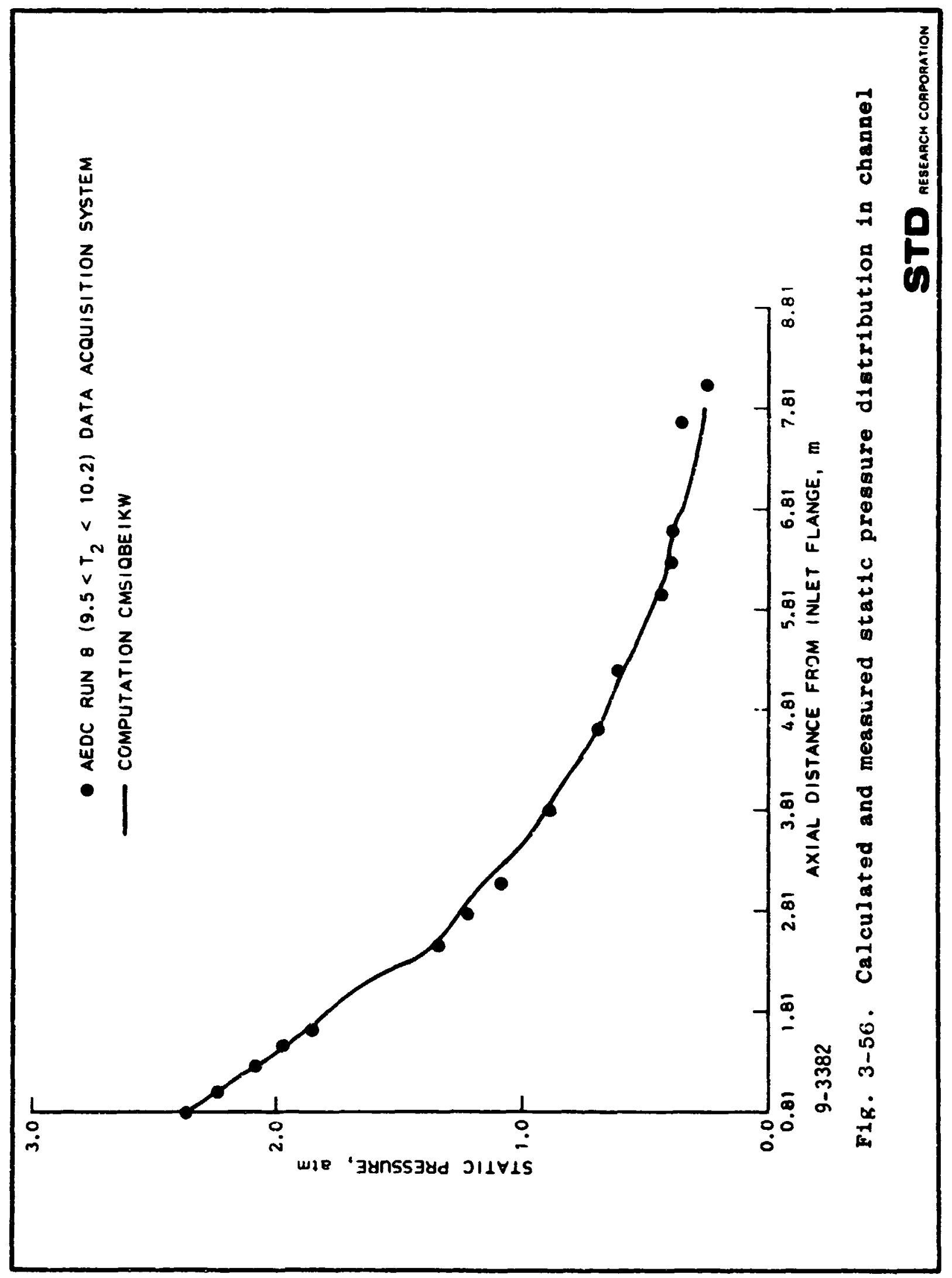




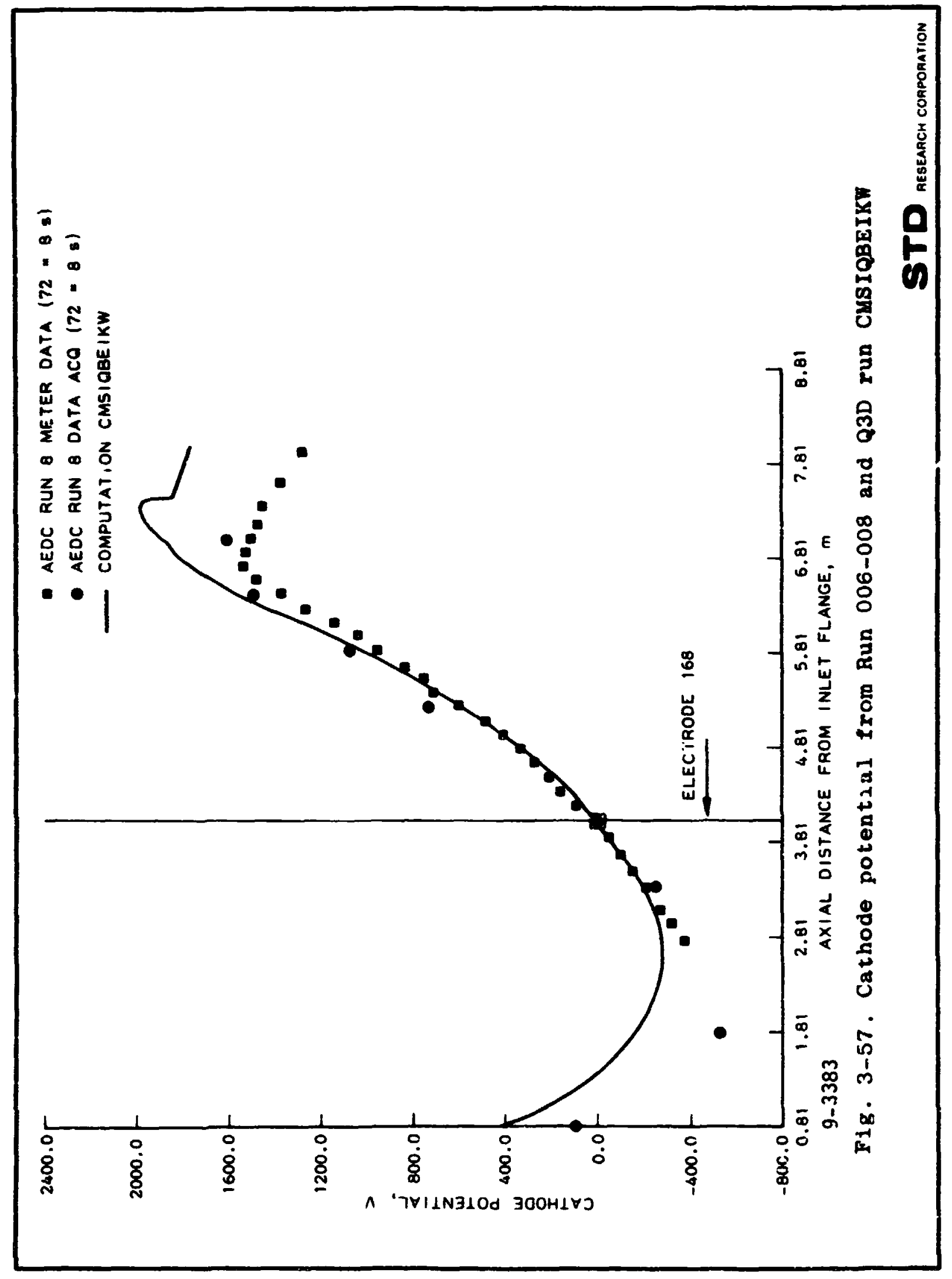



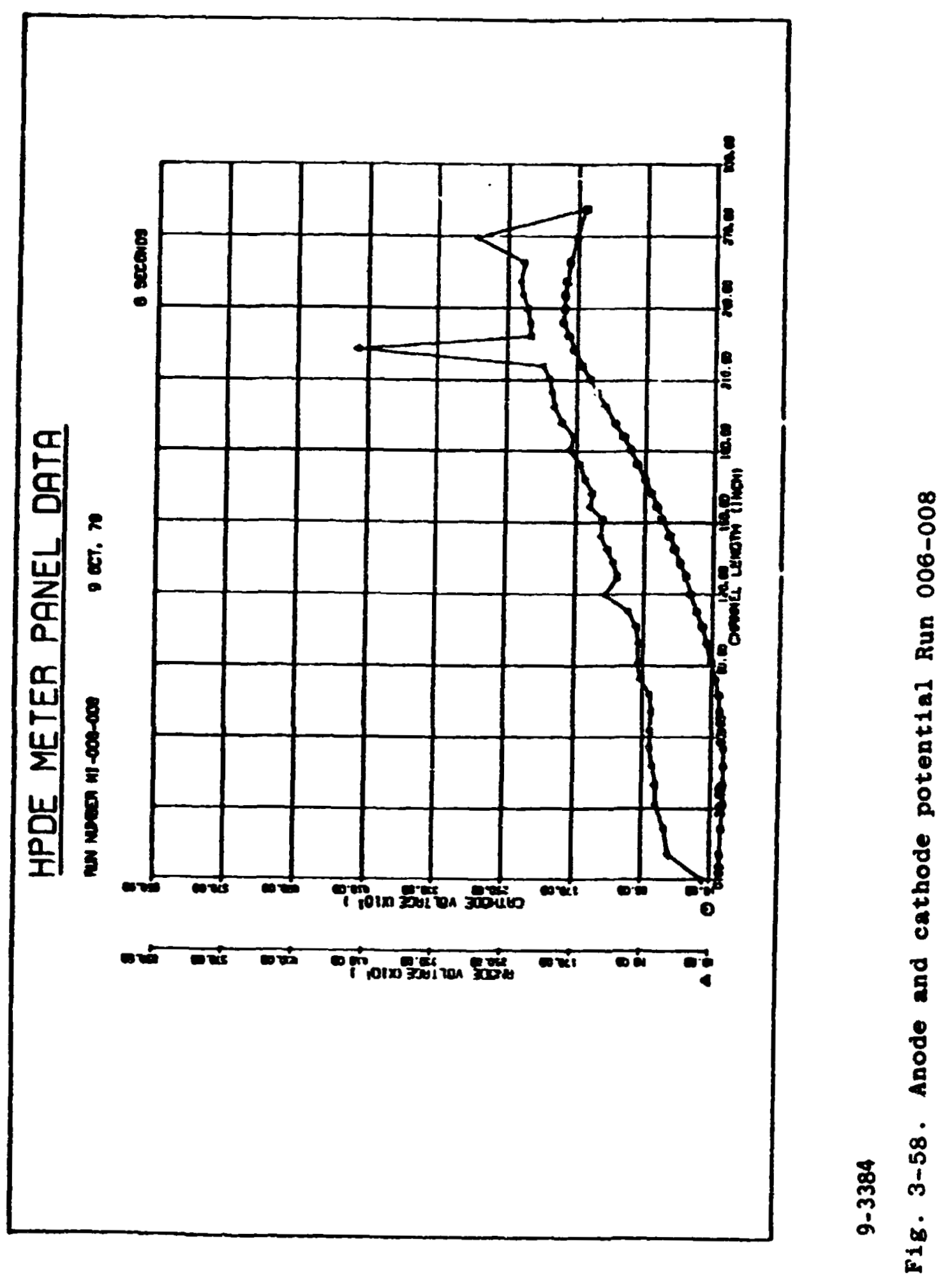


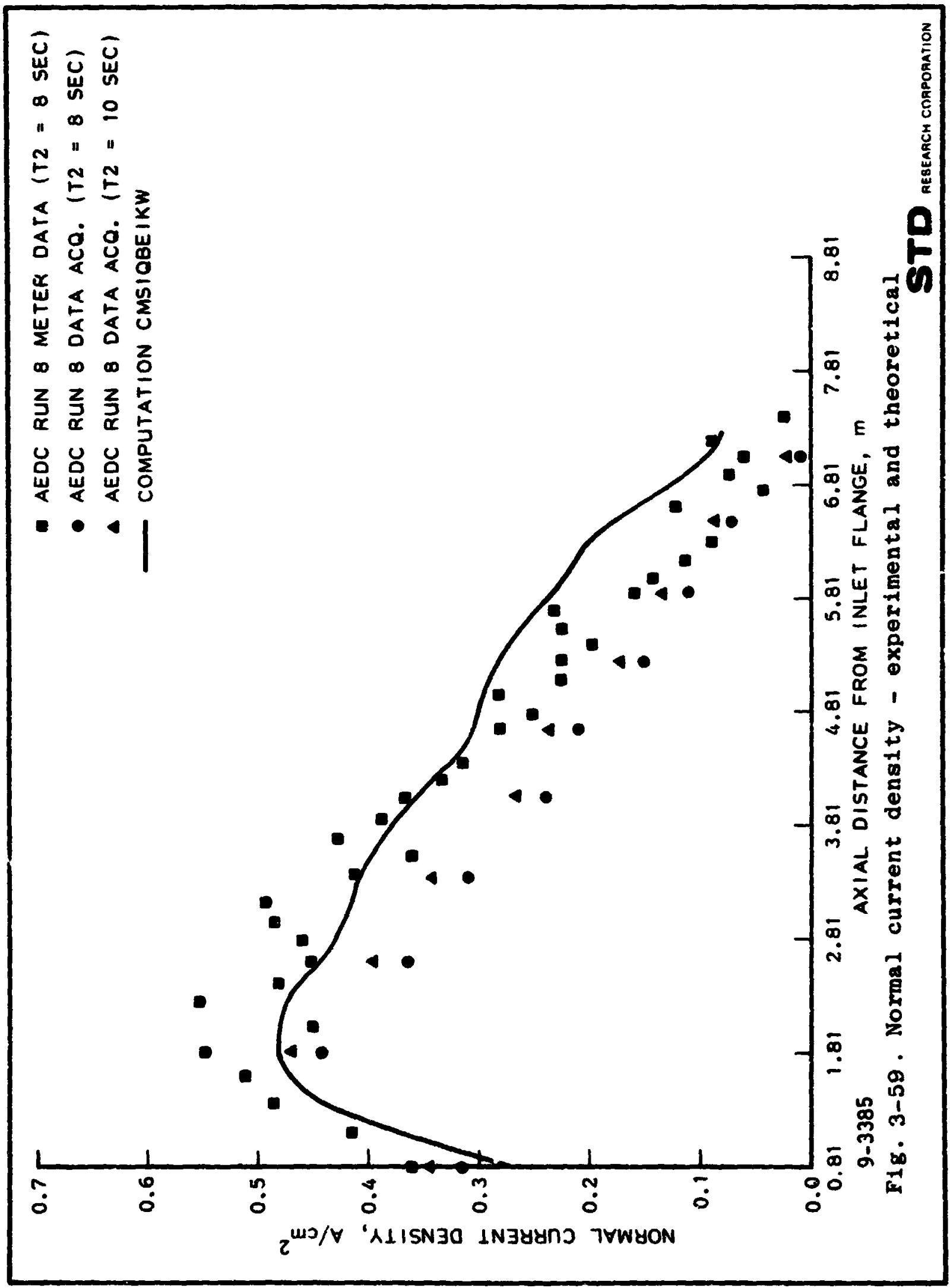




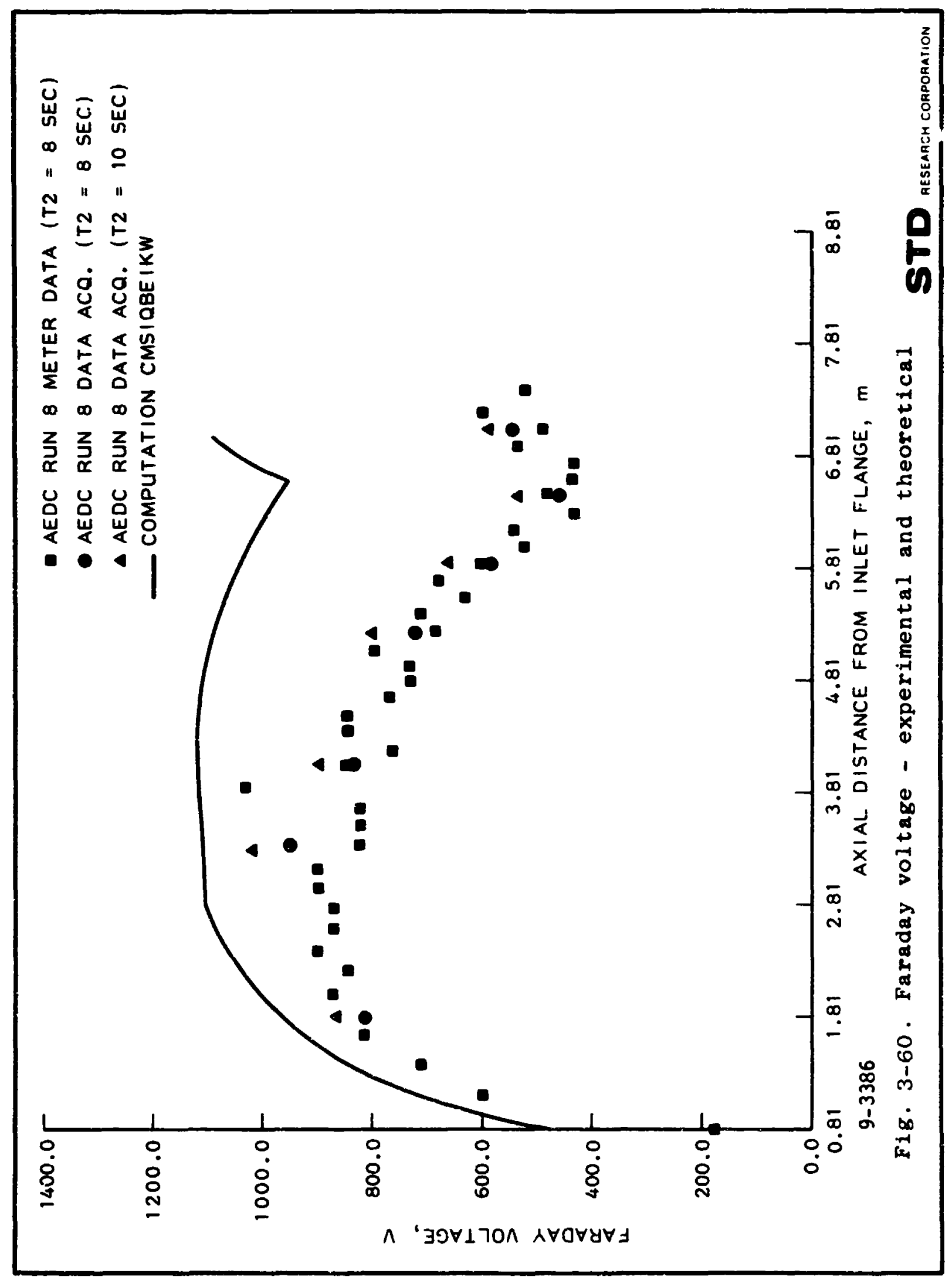




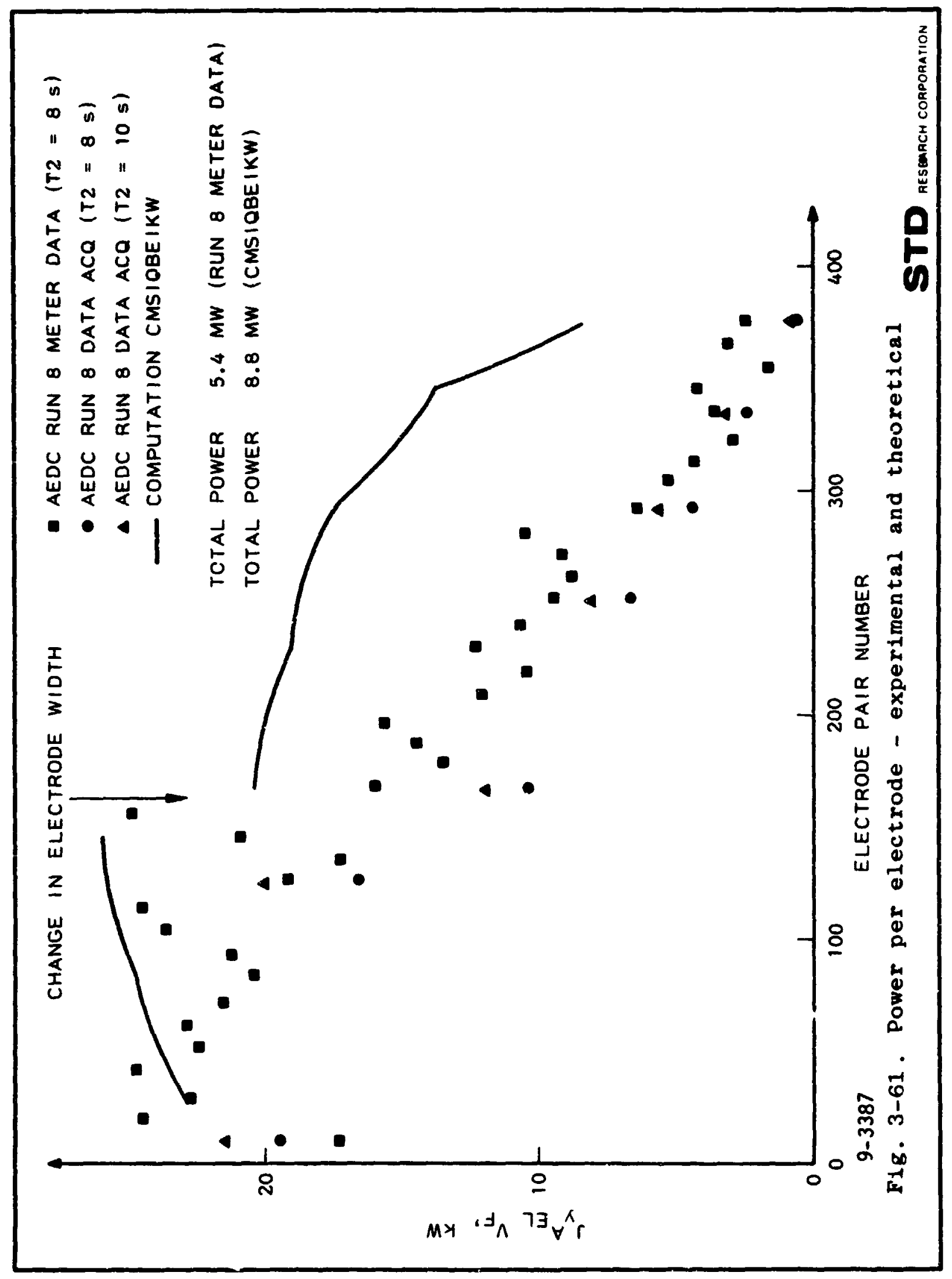



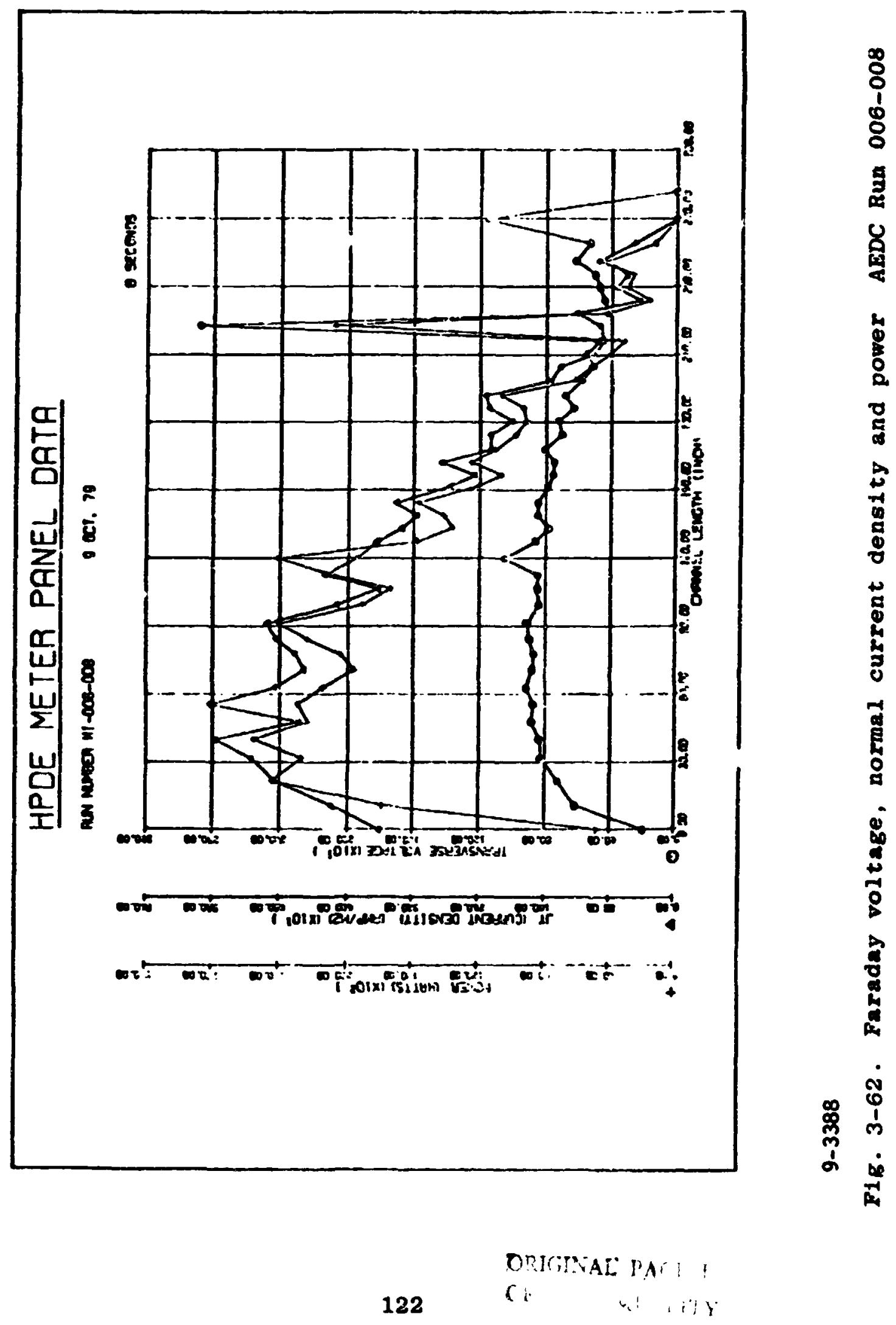


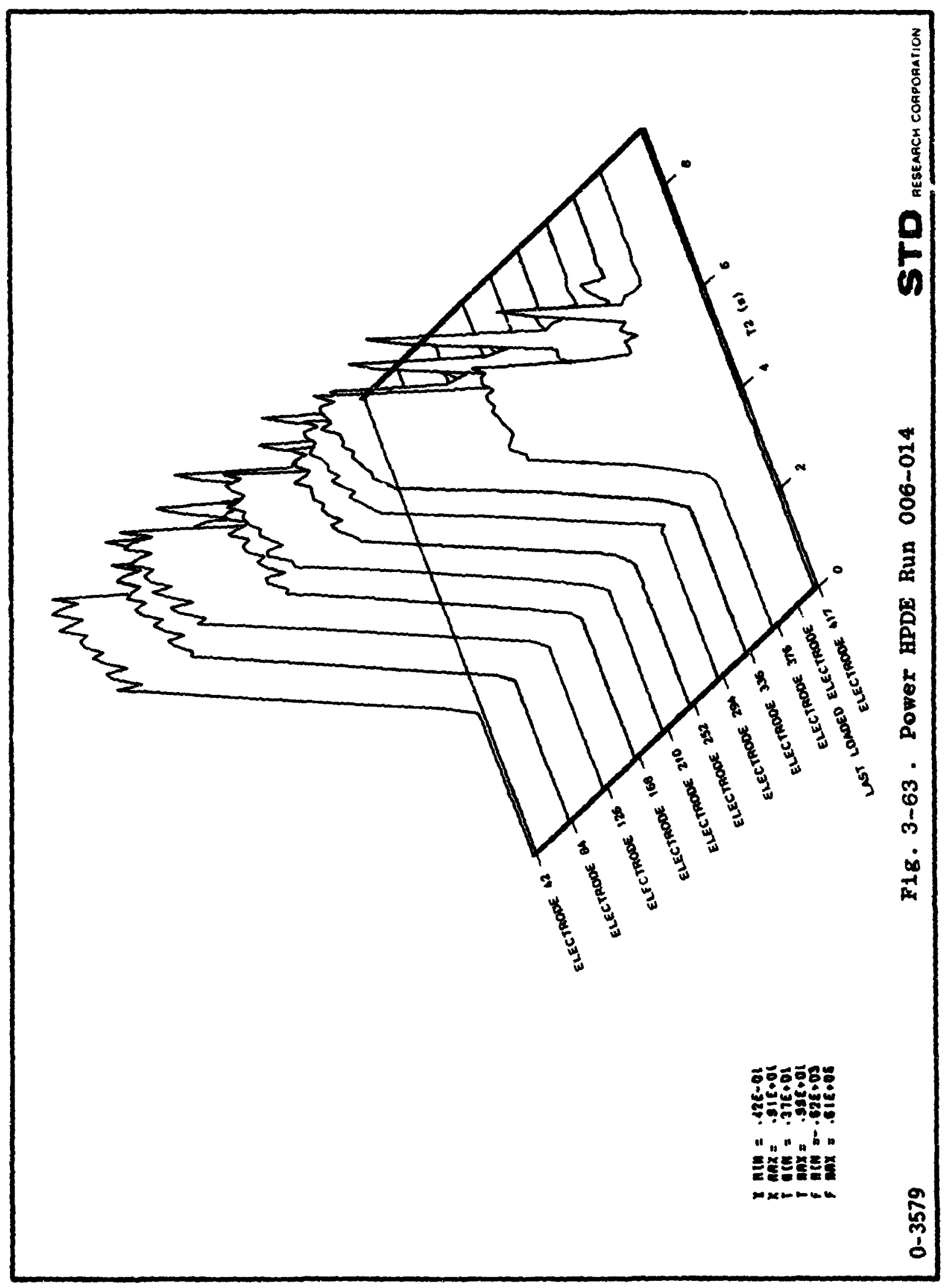




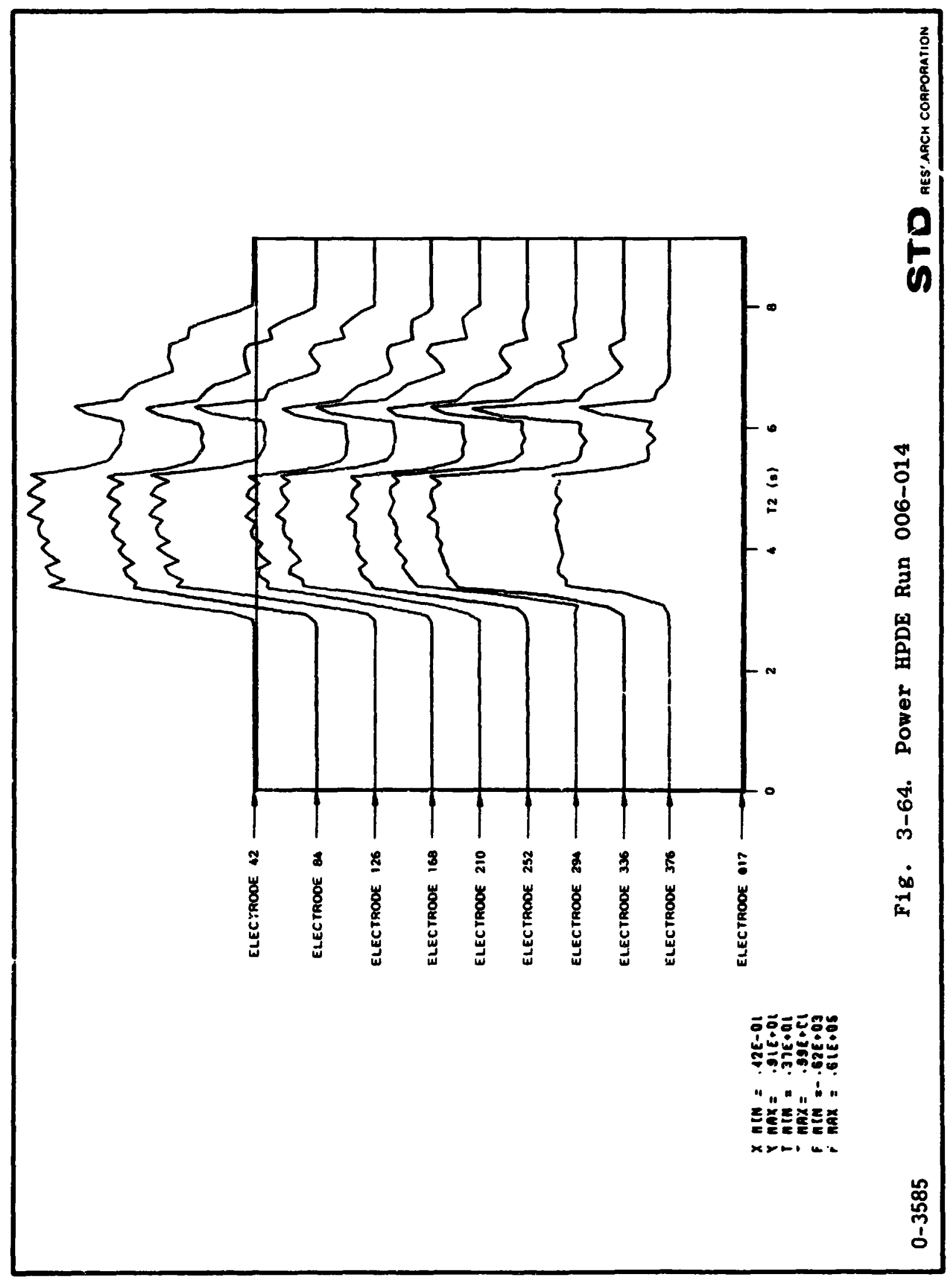




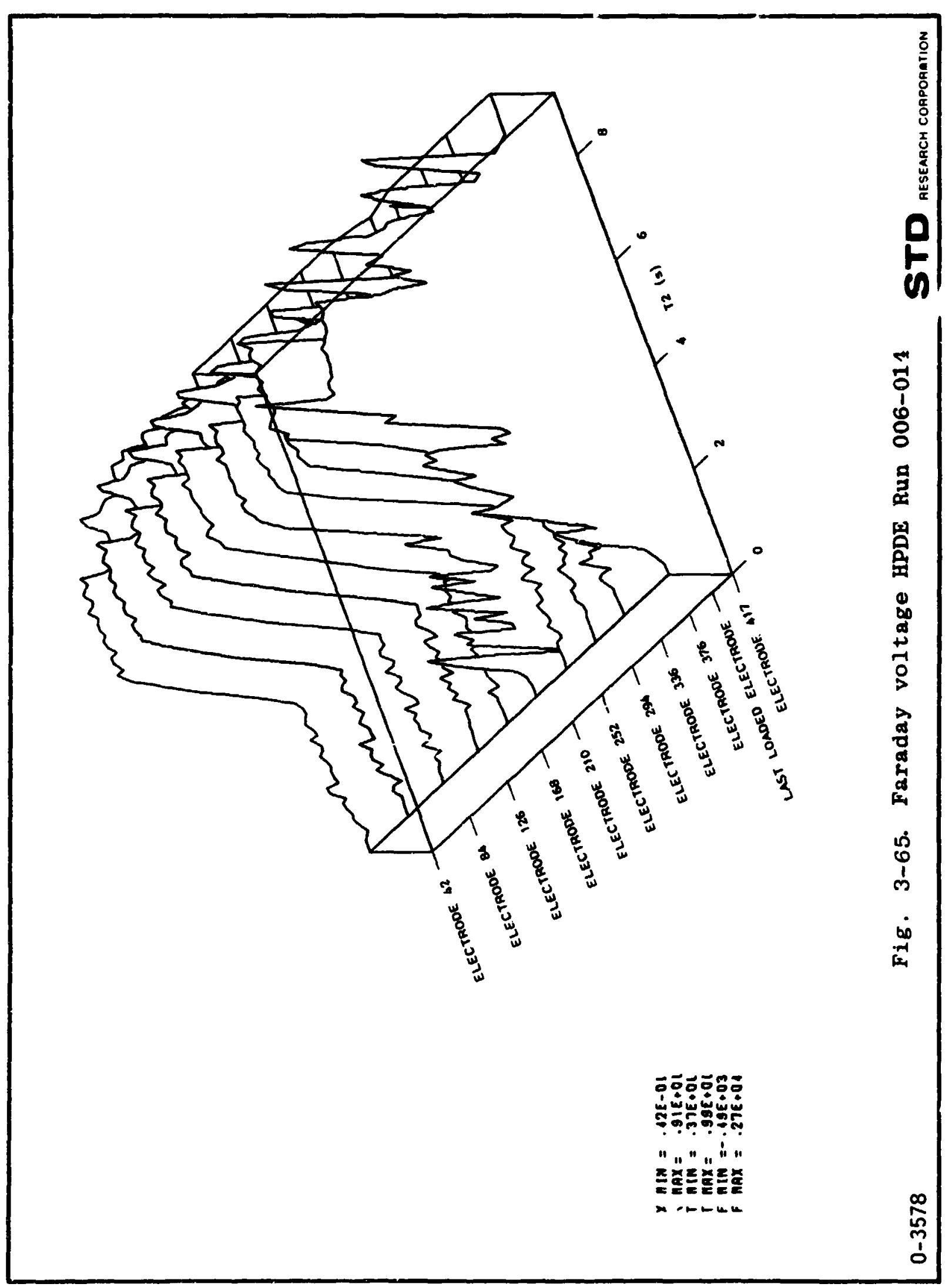




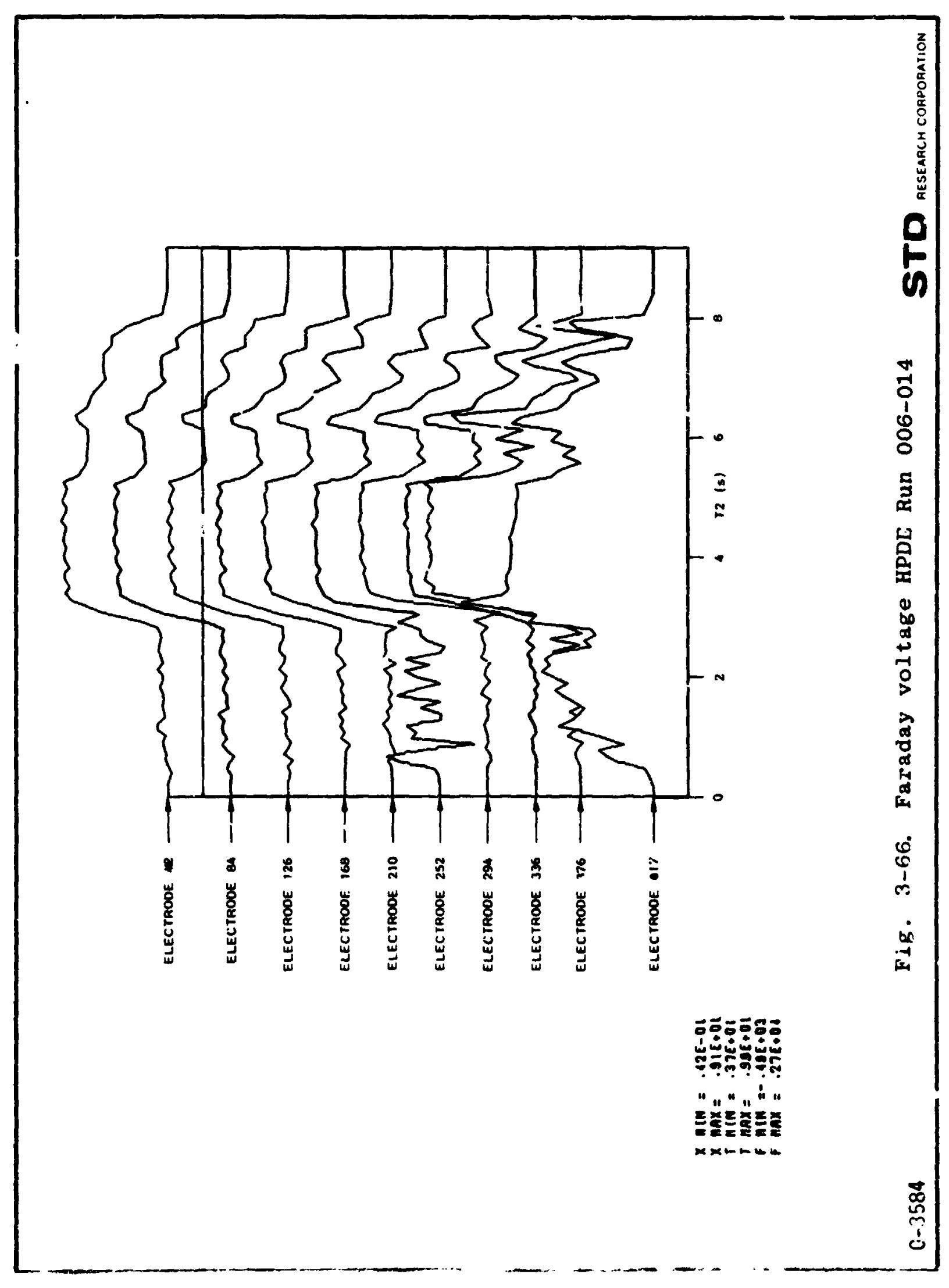




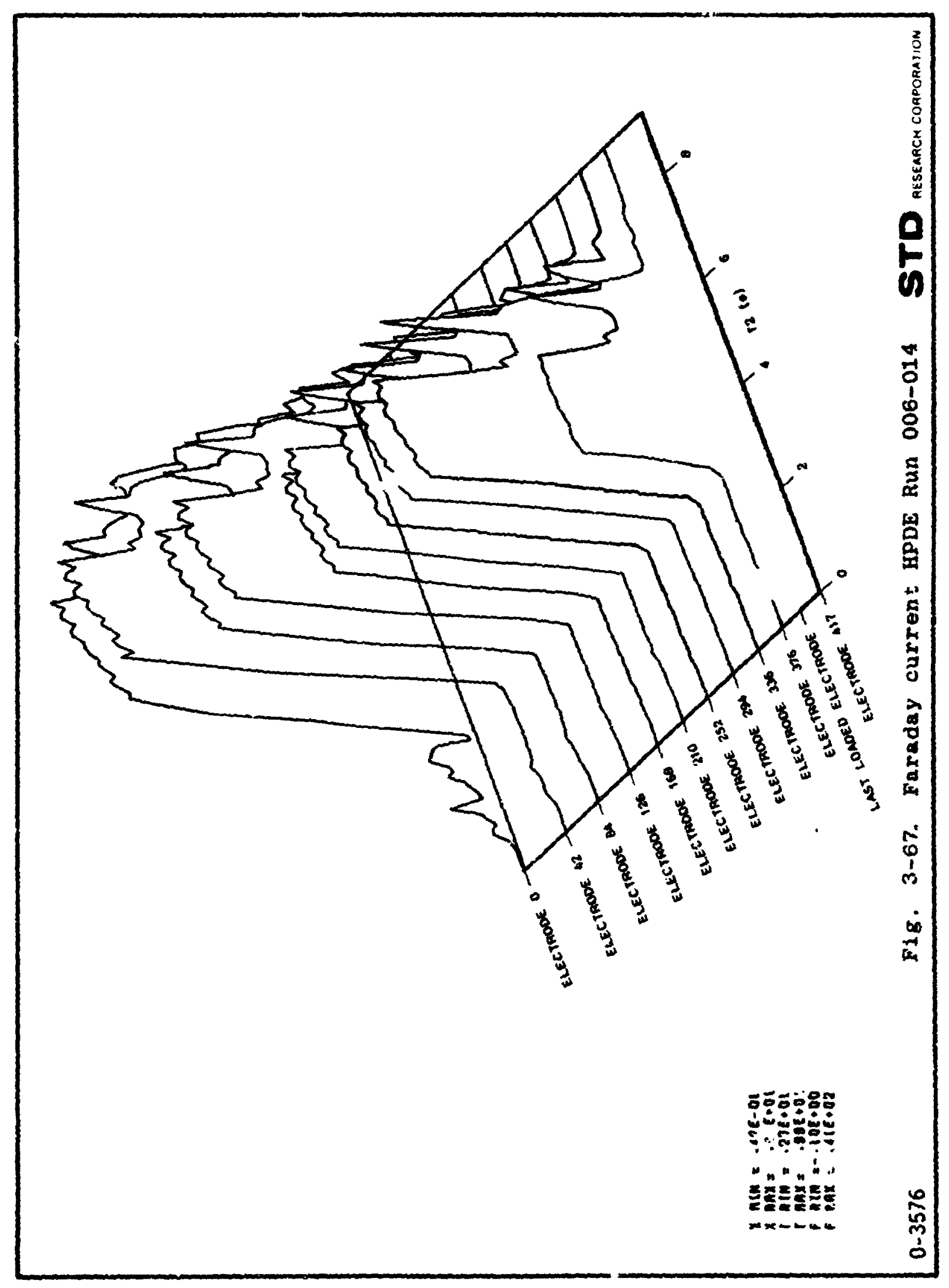




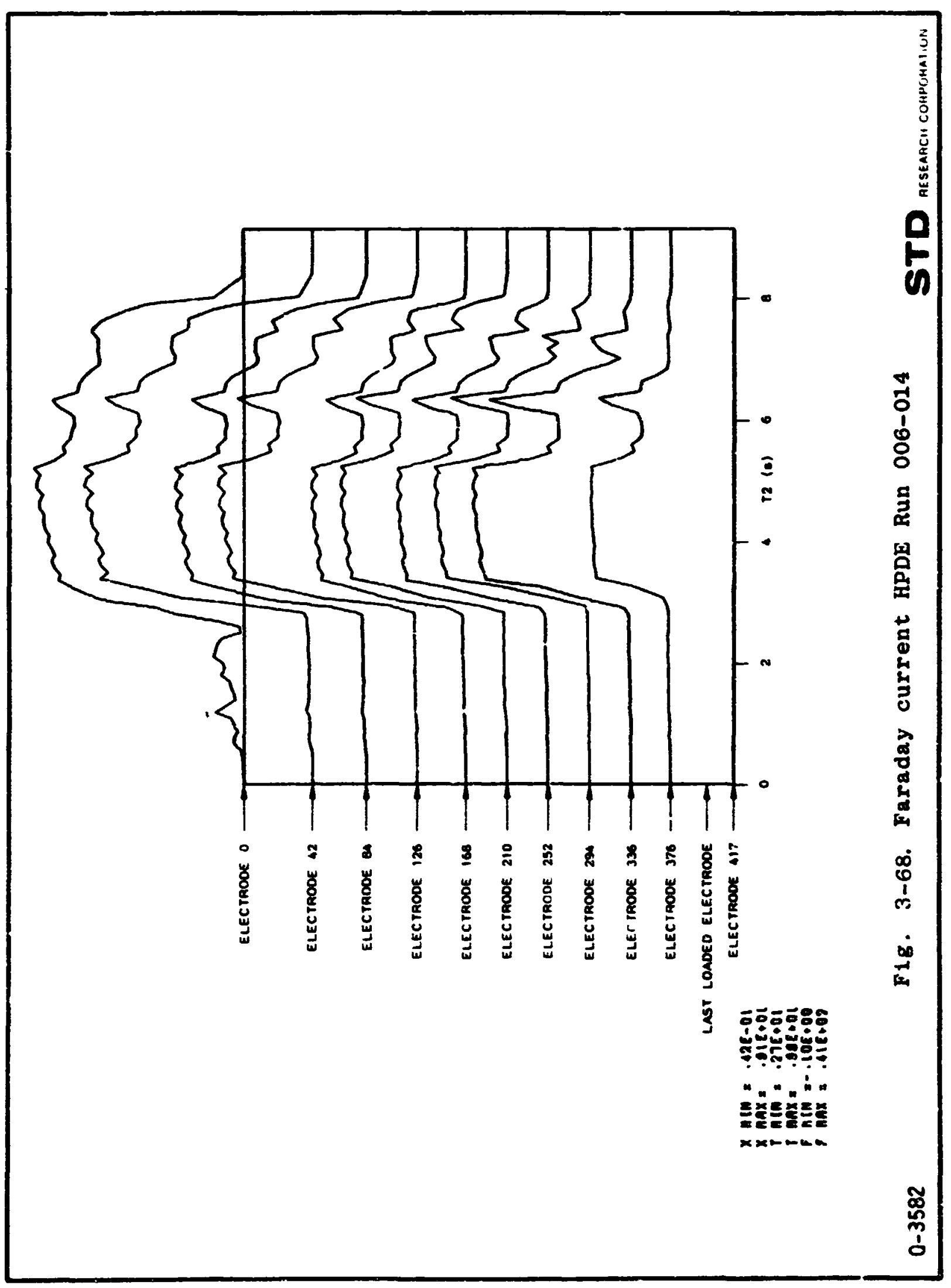




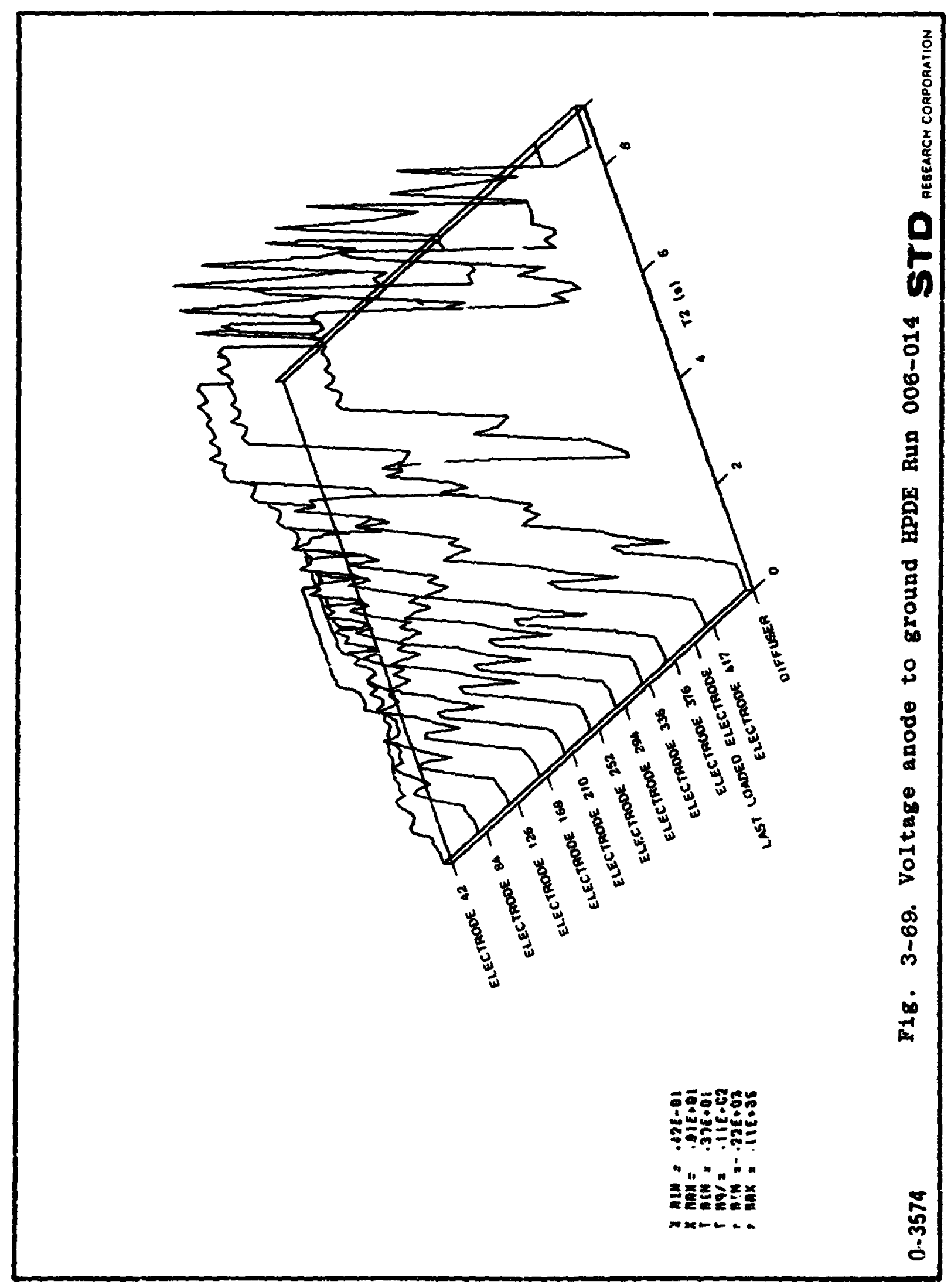




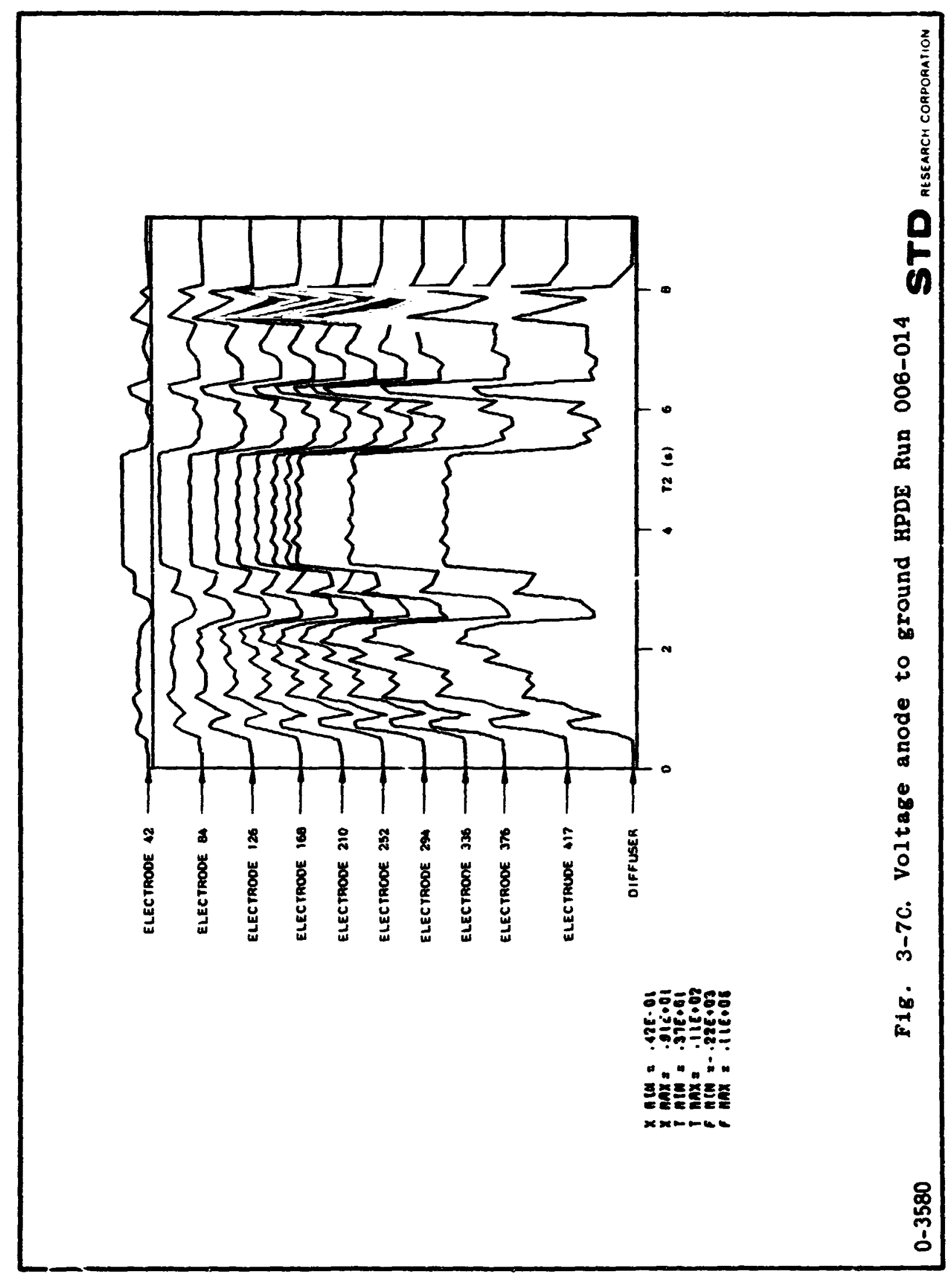




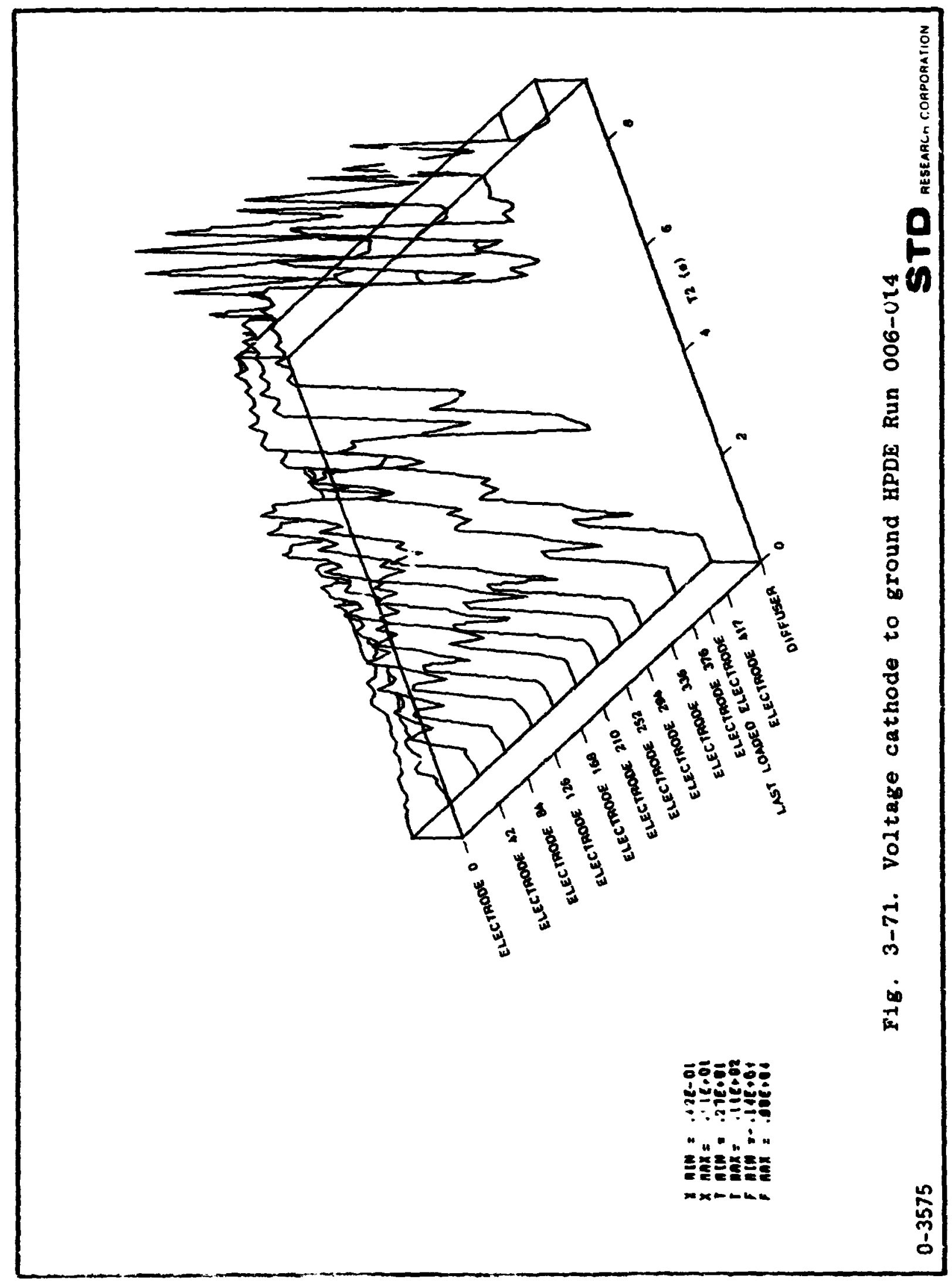




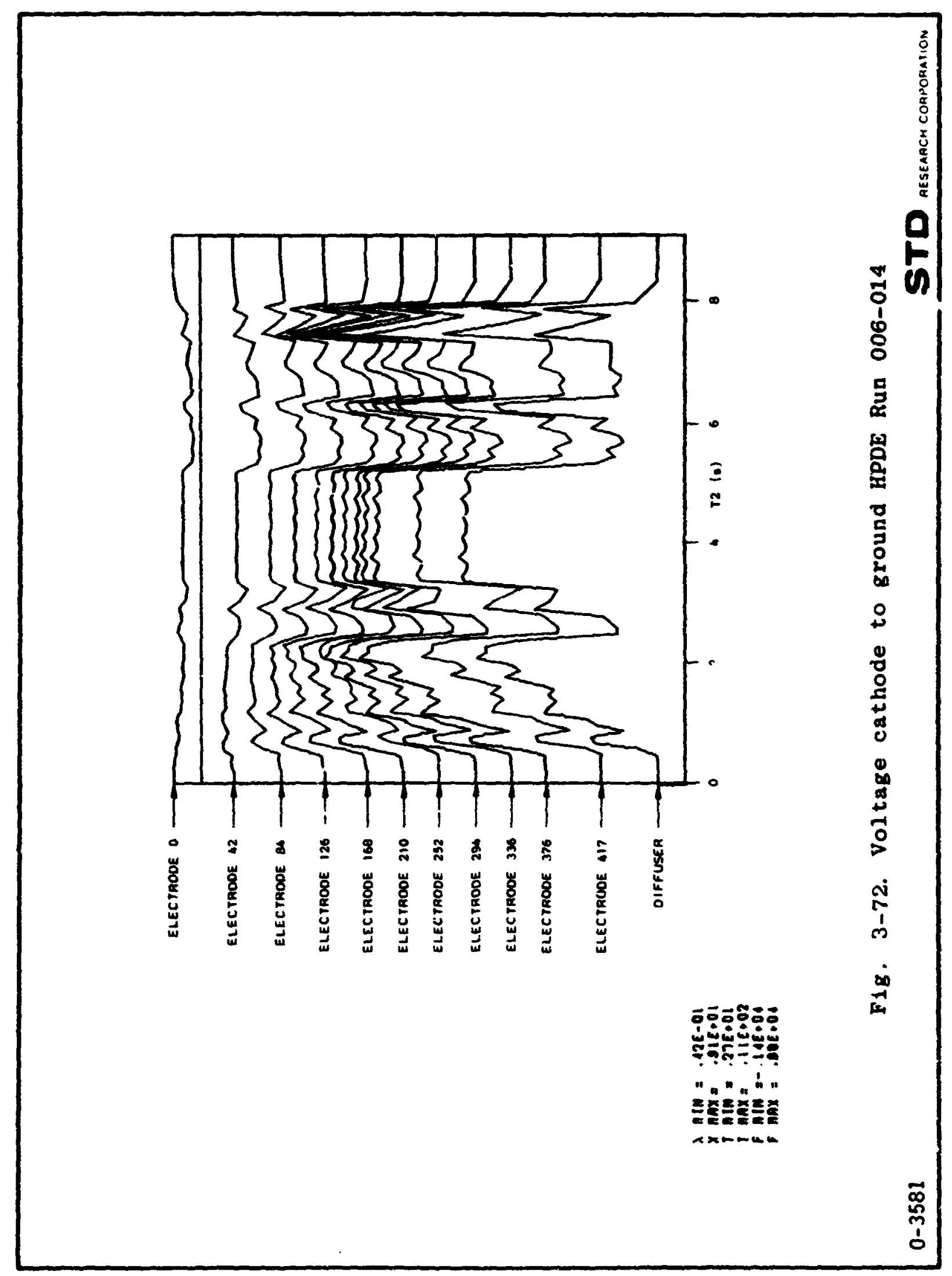


STD aeseahch corporation

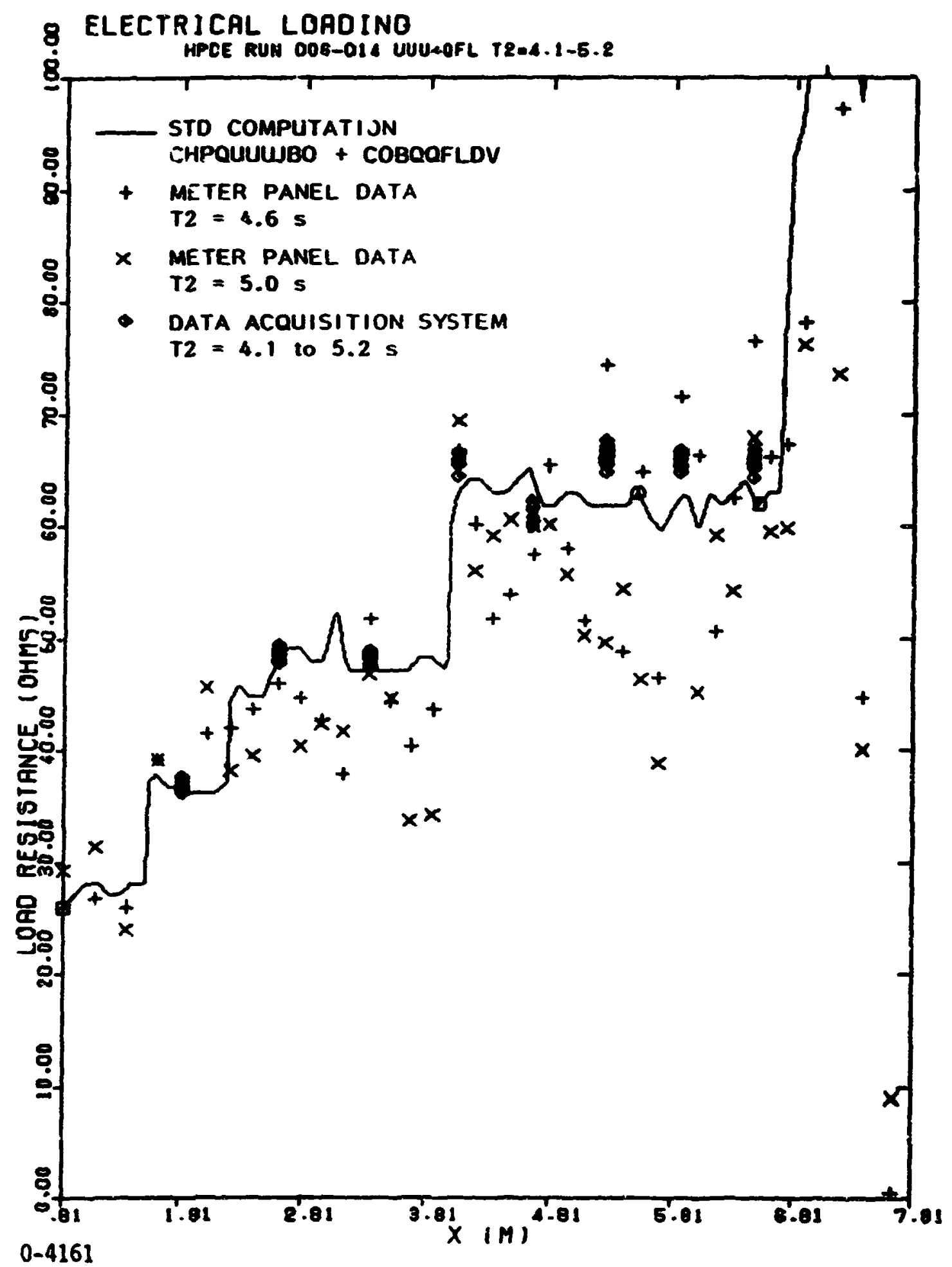

Fig. 3-73. Load resistance for HPDE Run 006-014 
STD hesearch conporation

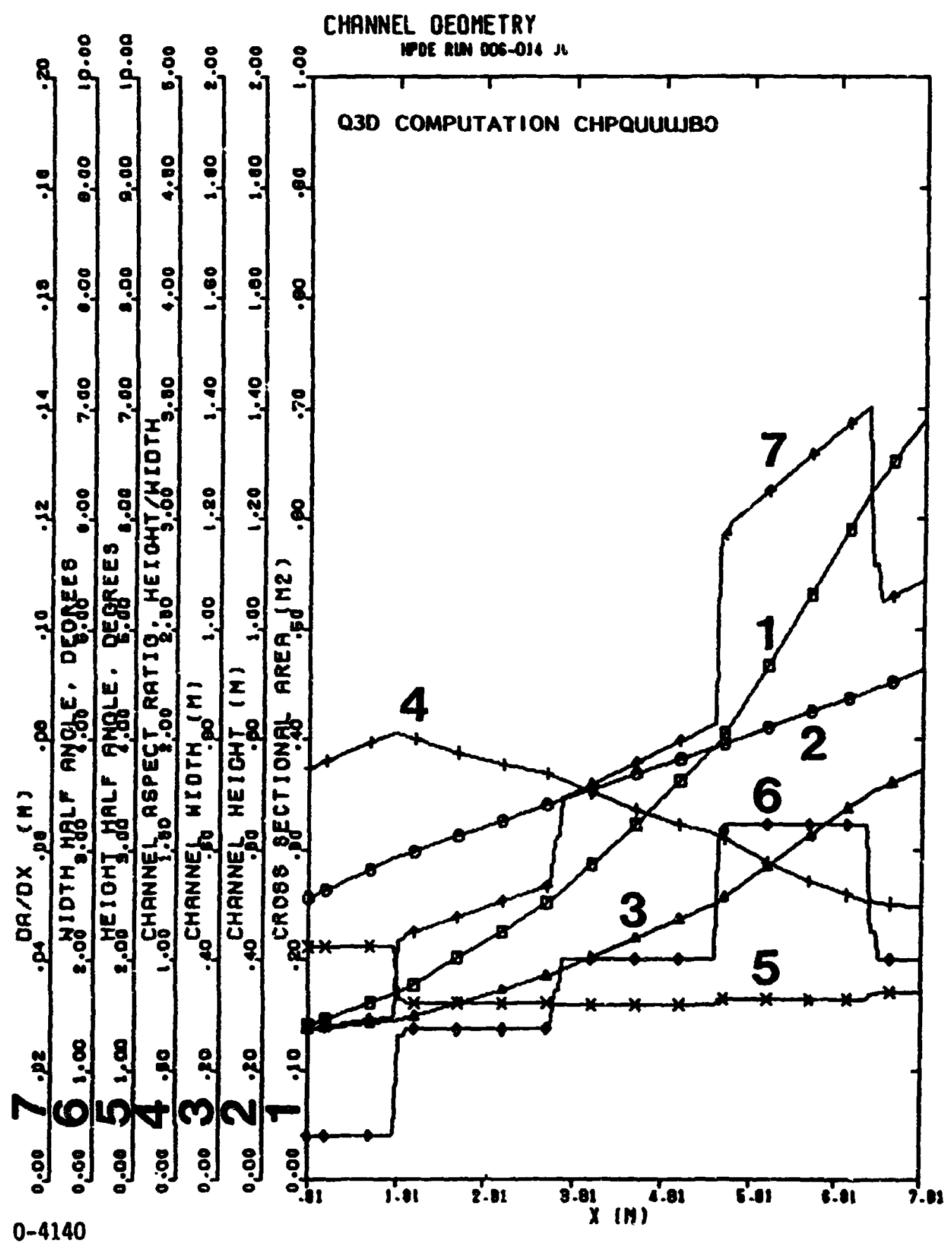

Fig. 3-74. Channel geometry HPDE Run 006-014 
STD aEstaAch Corpoaation

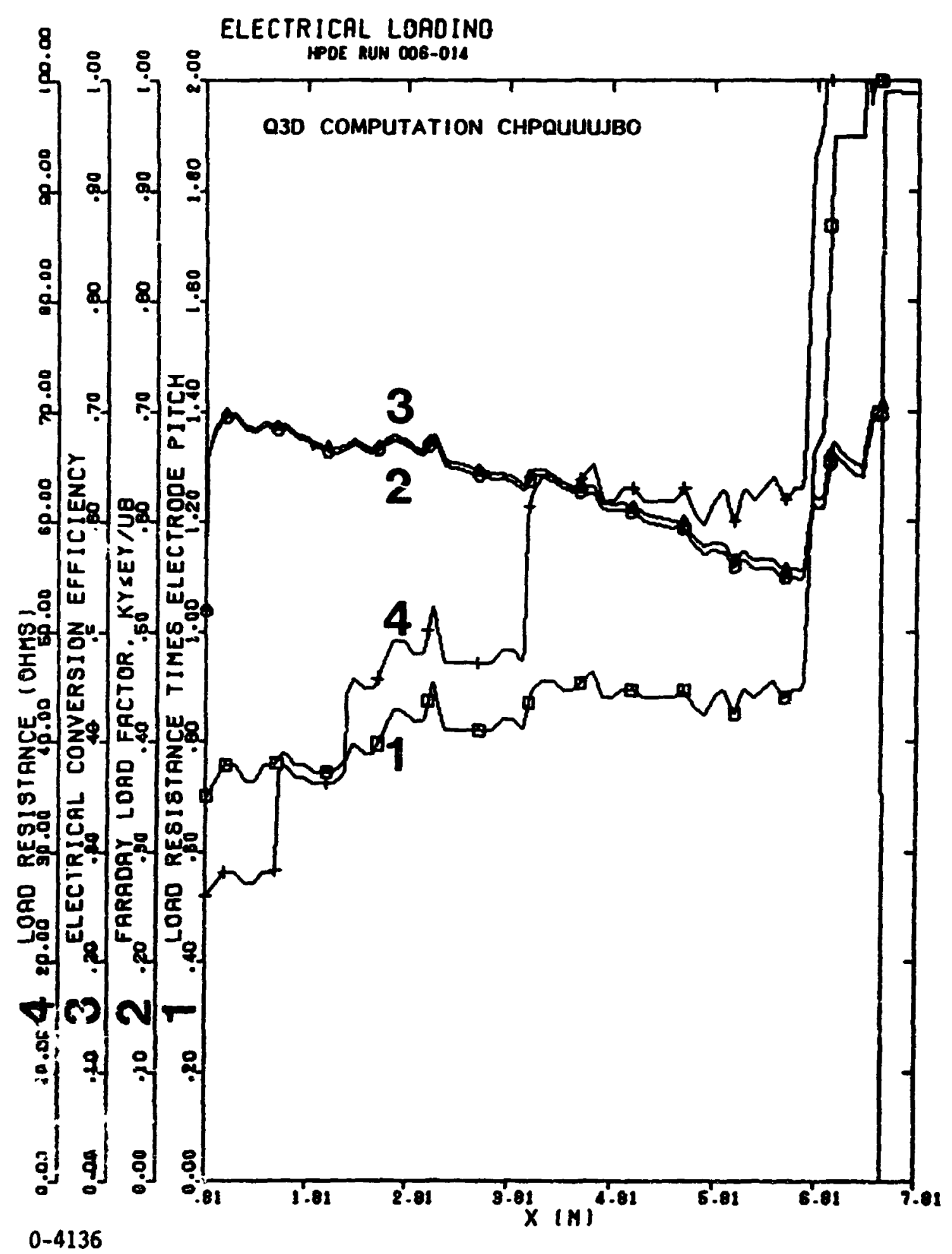

Fig. 3-75. Electrical loading HPDE Run 006-014 
STD RESEARCH CORPORATION

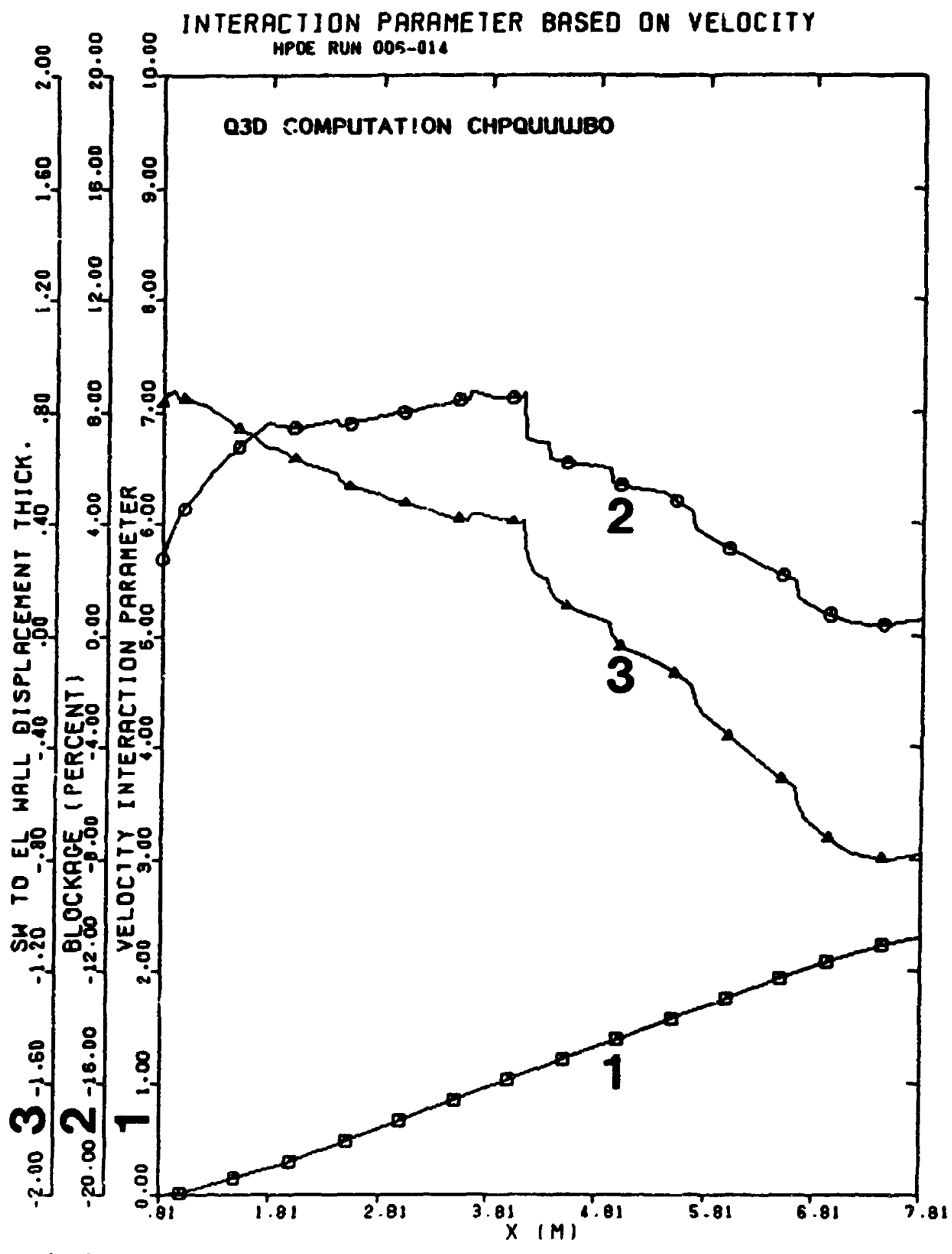

$0-4149$

Fig. 3-76. Interaction parameter based on velocity HPDE Run 
STD aeseabcr corporation

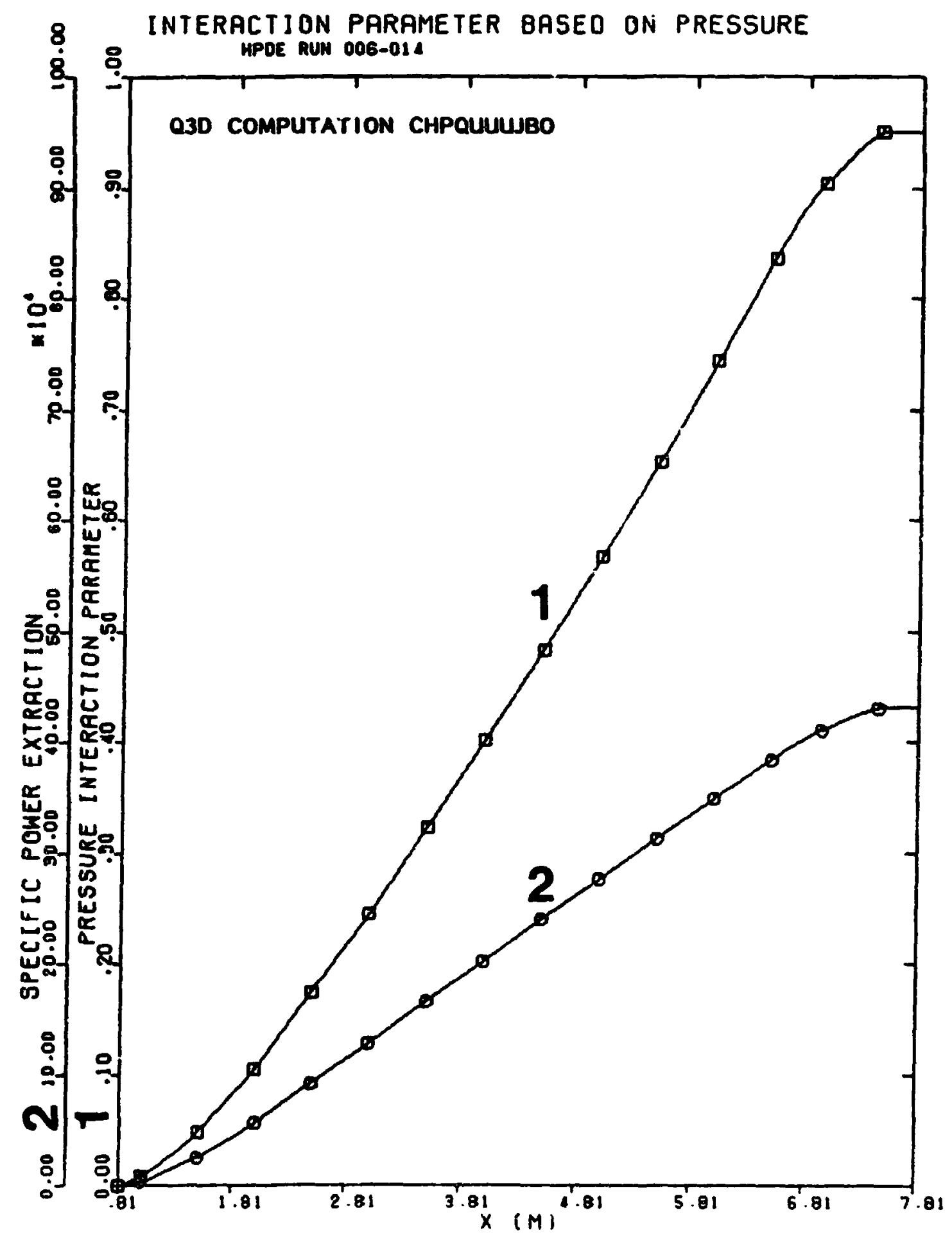

$0-4150$

Fig. 3-77. Interaction parameter based on pressure HPDE RuB 006-014 
STD aesearch coapoaAion

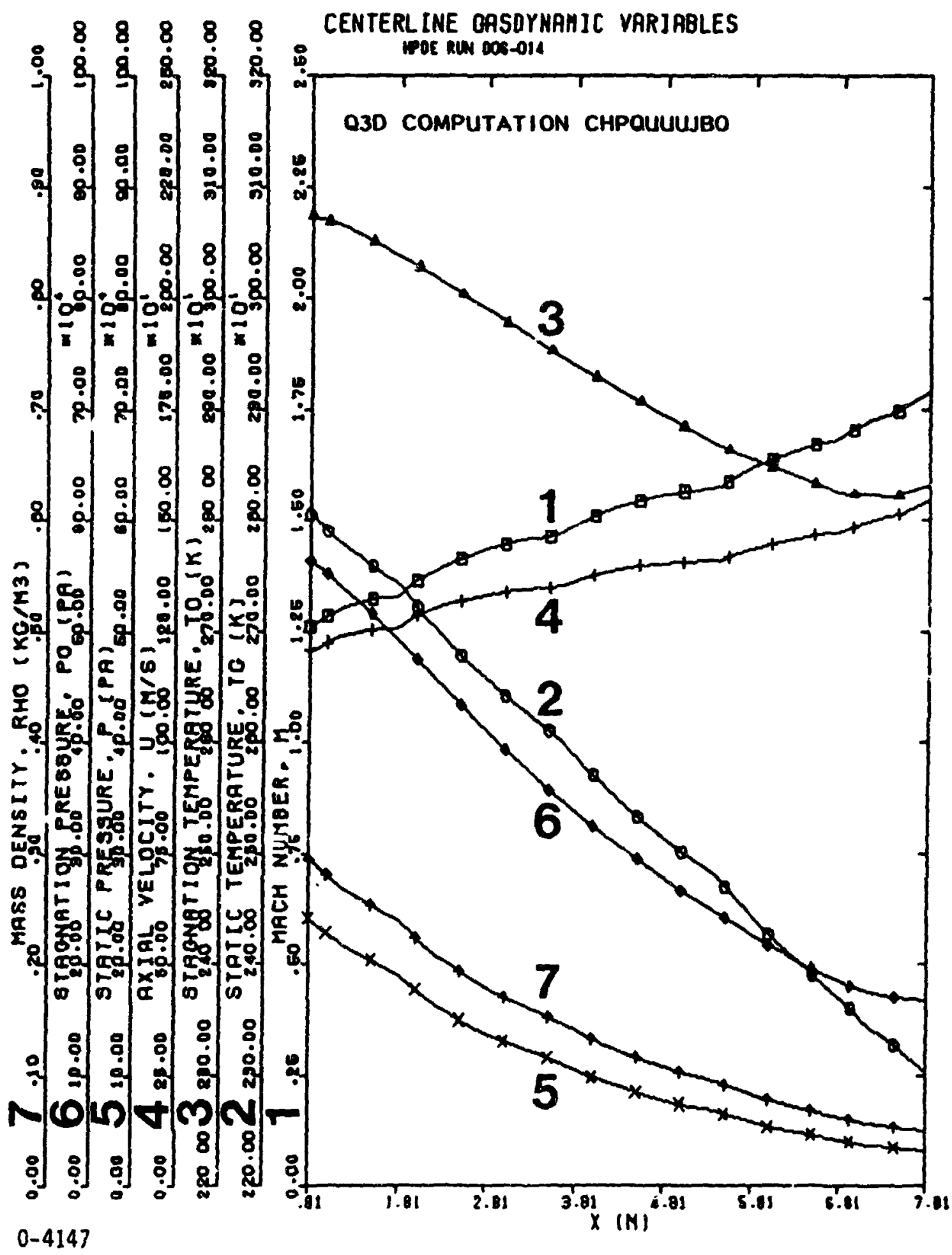

Fig. 3-78. Centerline gasdynamic variables HPDE Run 006-014 
STD mesearch copporation

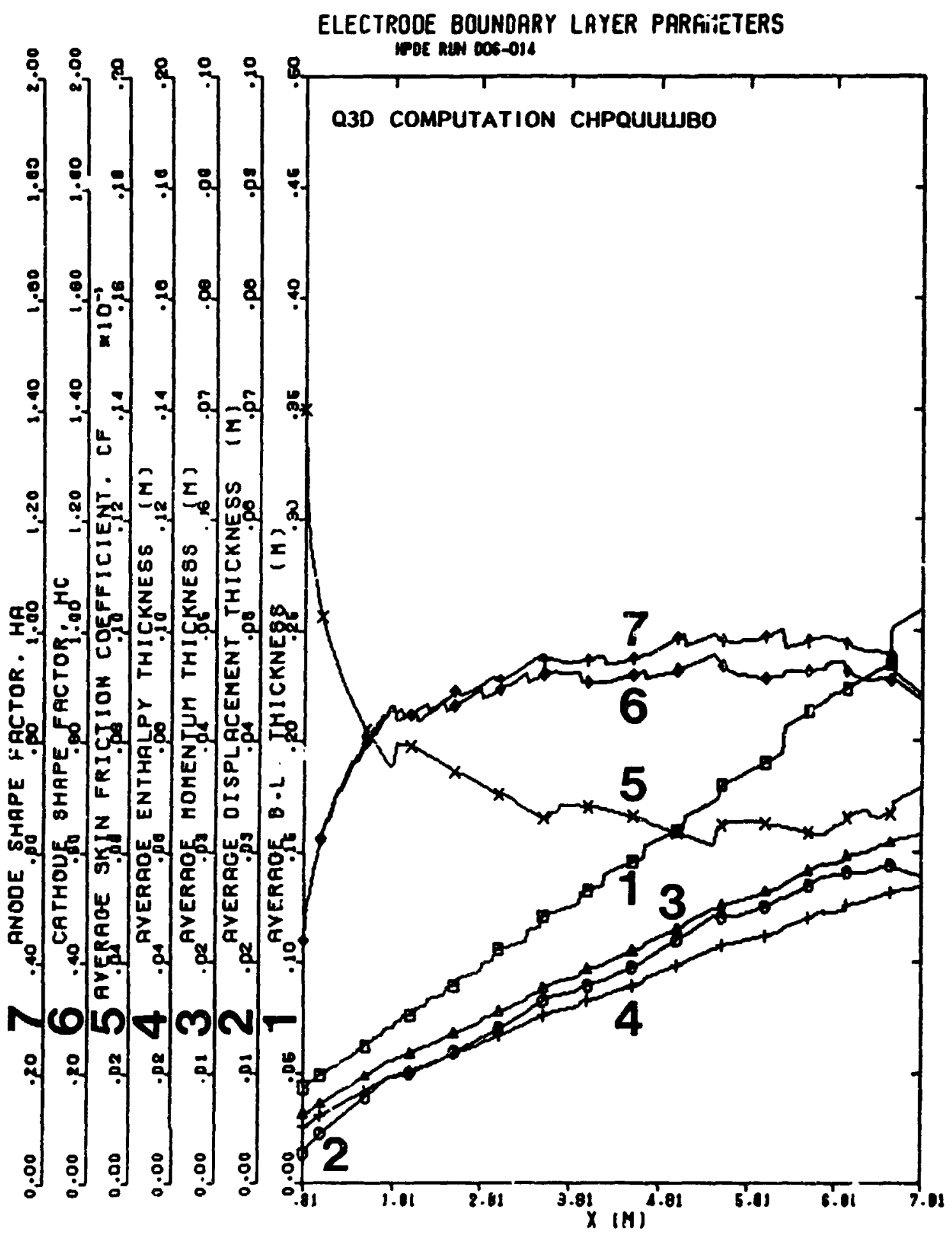

$0-4137$

Fig. 3-79. Electrode boundary layer parameters HPDE Run 006-014 
STD reseafim corpotation

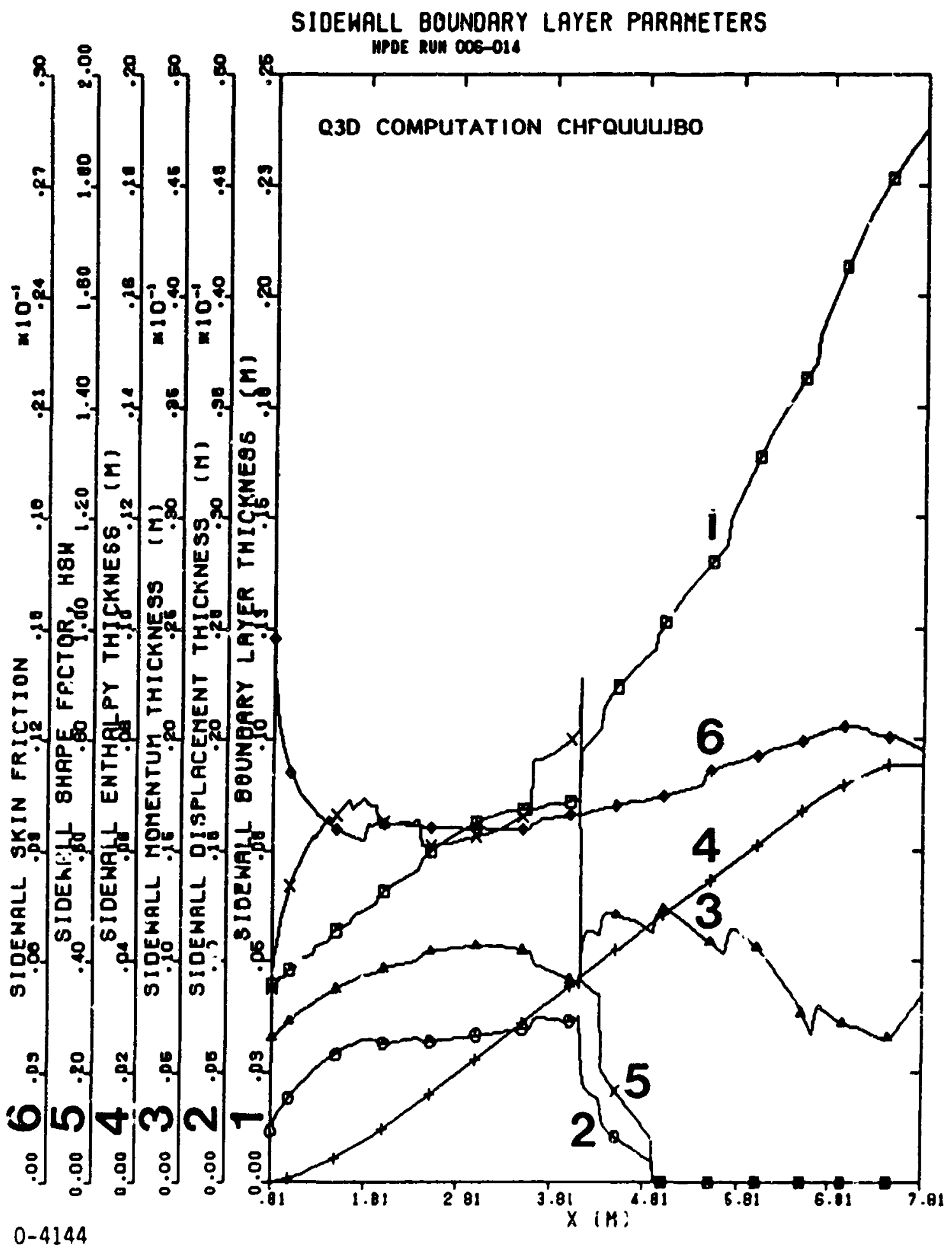

Fig. 3-8C. Sidewall boundary layer parameters HPDE Run 006-014 
STD aesencen conecouarion

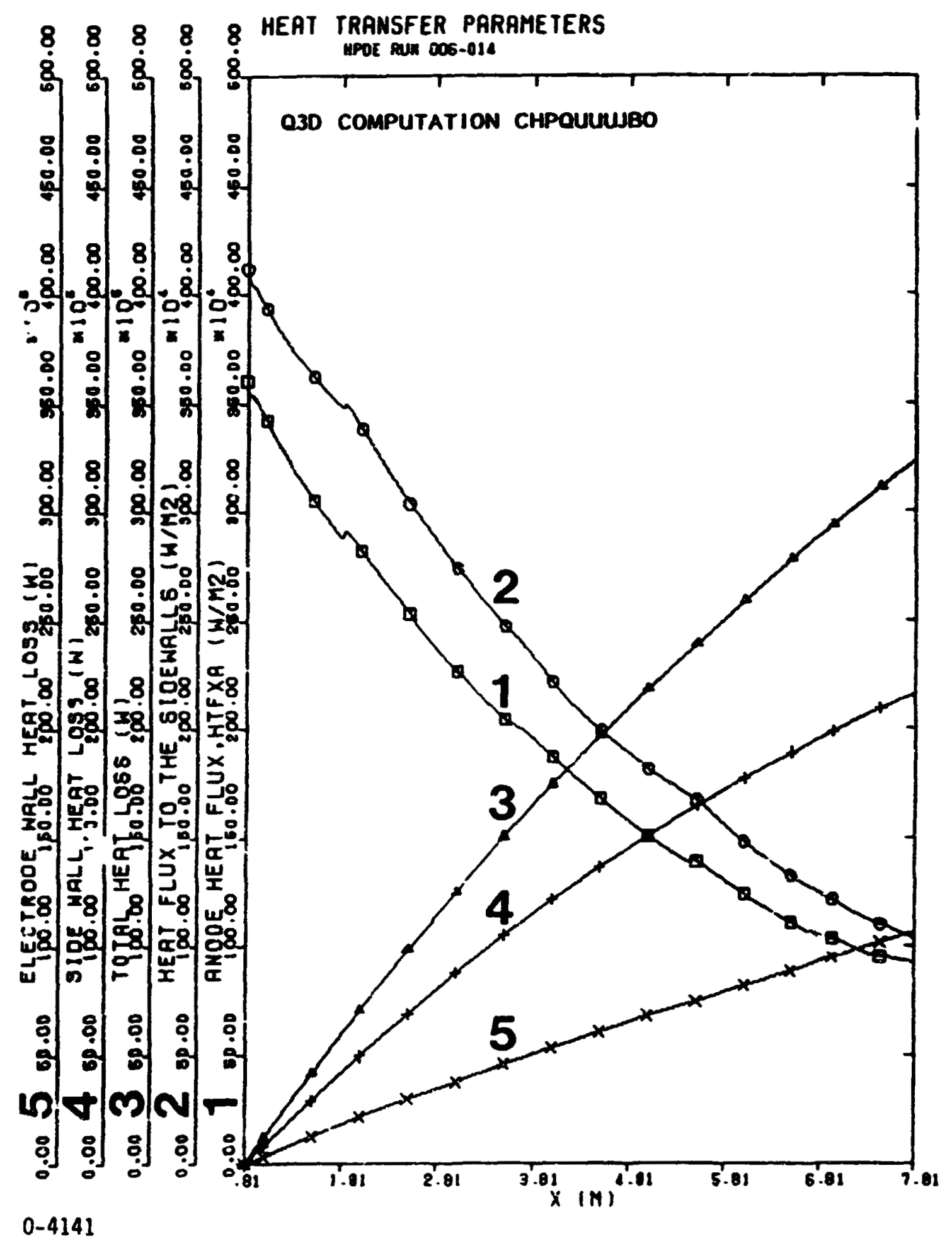

Fig. 3-81. Ho transfer paranters HPDE Kun 006-014 
STD heseascm corpopation

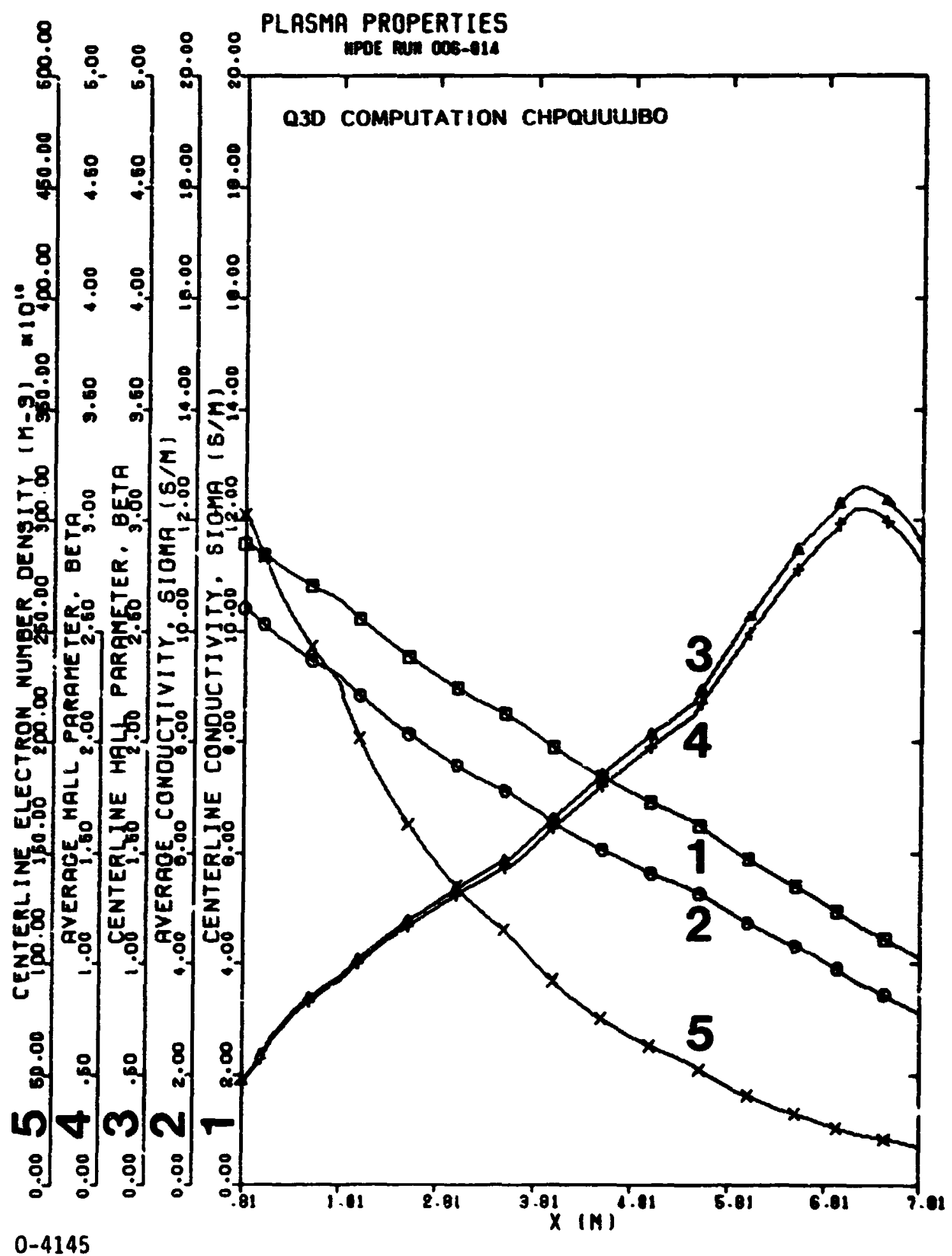

Fig. 3-82. Plasma proderties HPDE Run 006-014 
STD hesearcm comporation

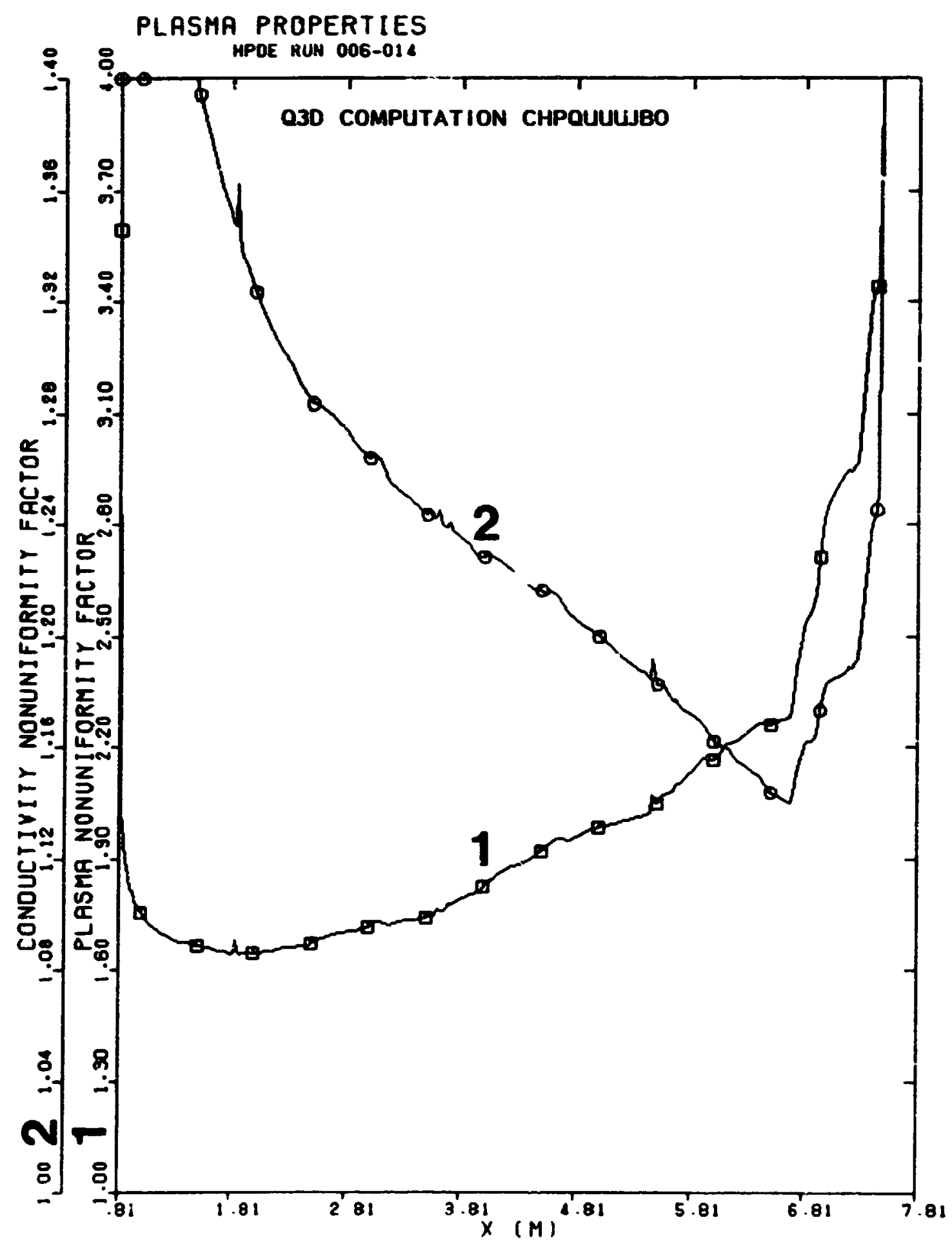

$0-, 151$

Fig. 3-83. Nonuniformity factors HPDE Run 006-014 
STD nesenacn CORporarion

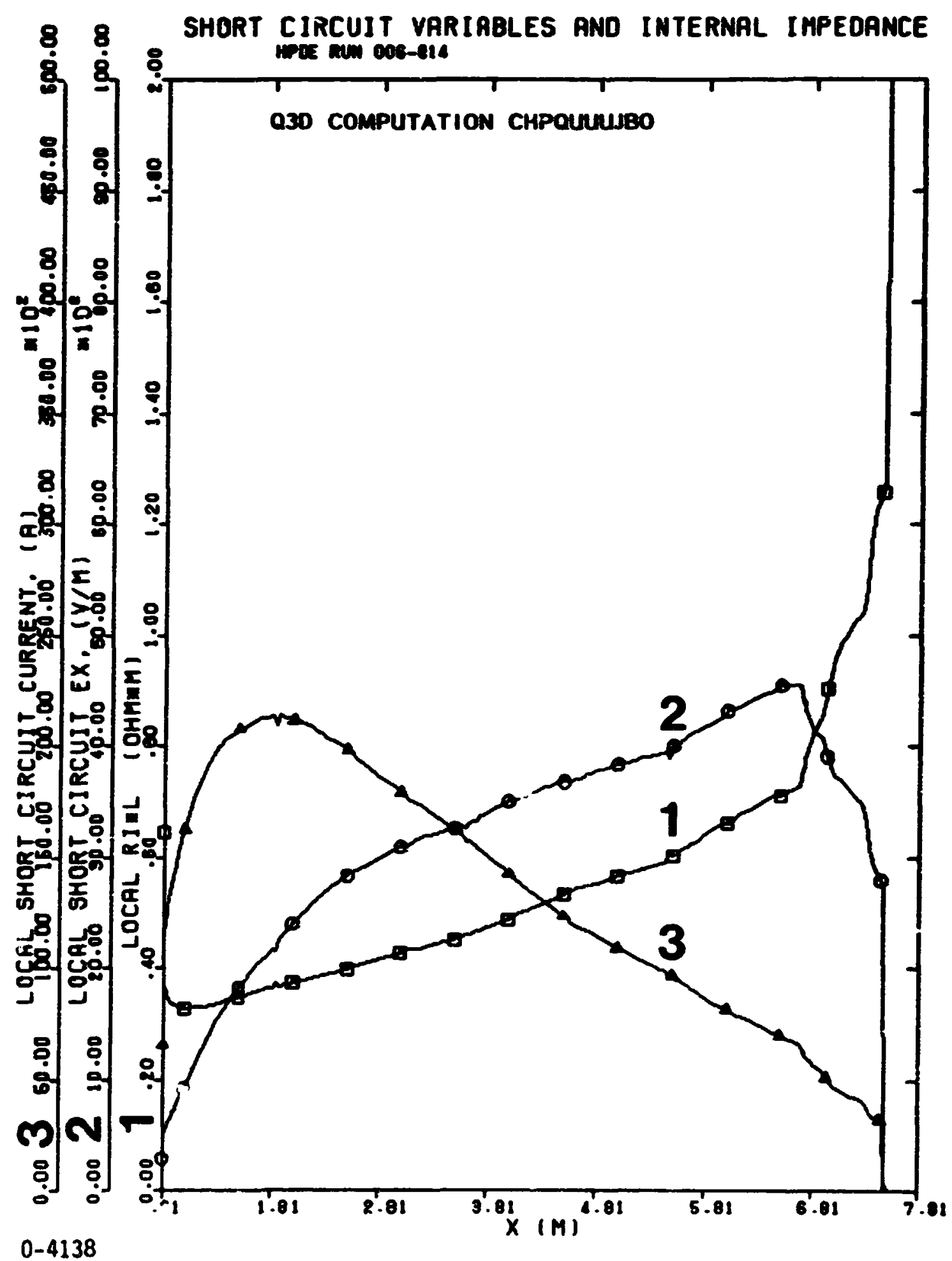

Tig. 3-84. Short circuit variables and internal impedance HPDE Run 006-014 
STD aEsearch COAmoRation

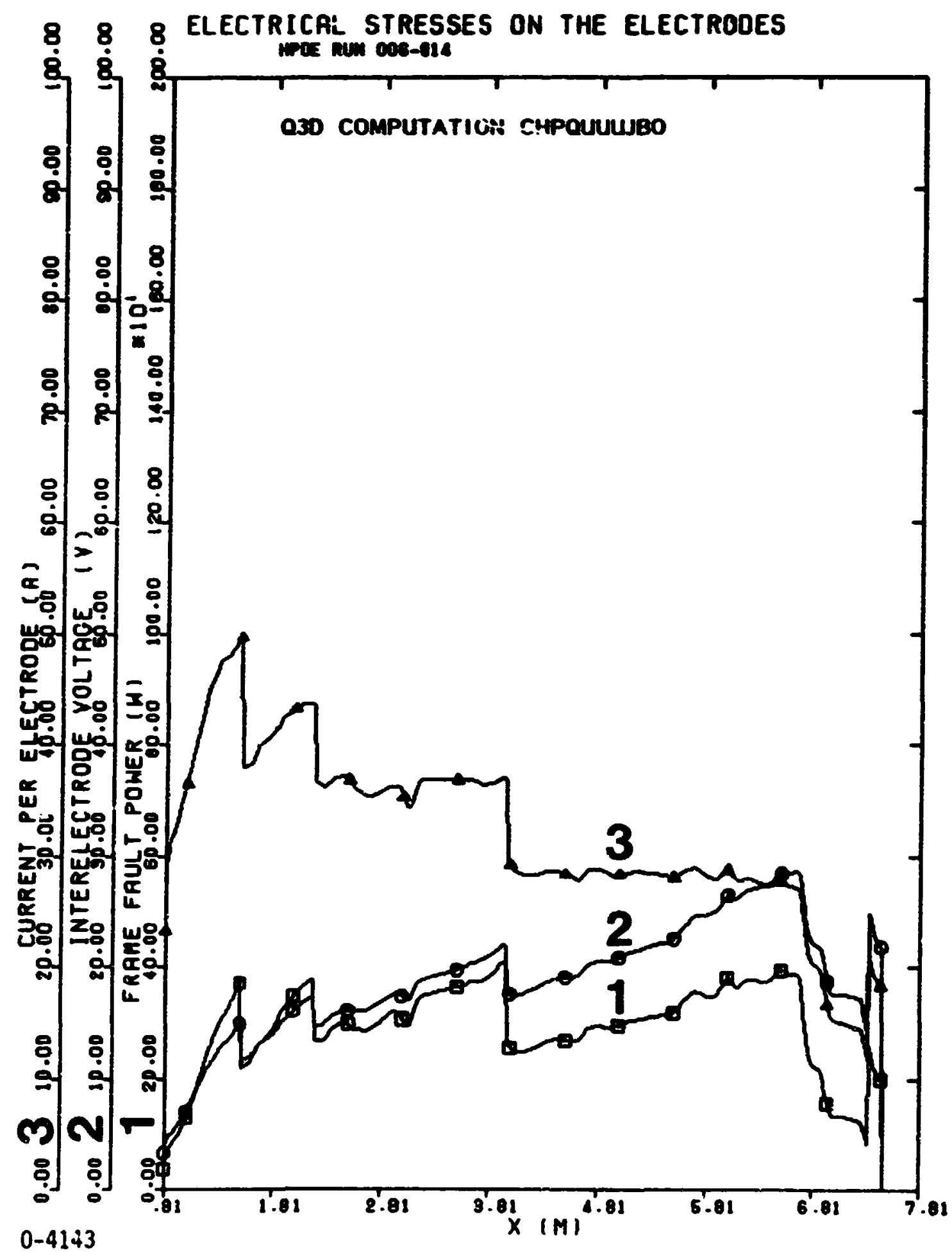

Fig. 3-85. Electrical stresses on the electrudes HPDE Rur 006-014 
STD aesearch Compohation

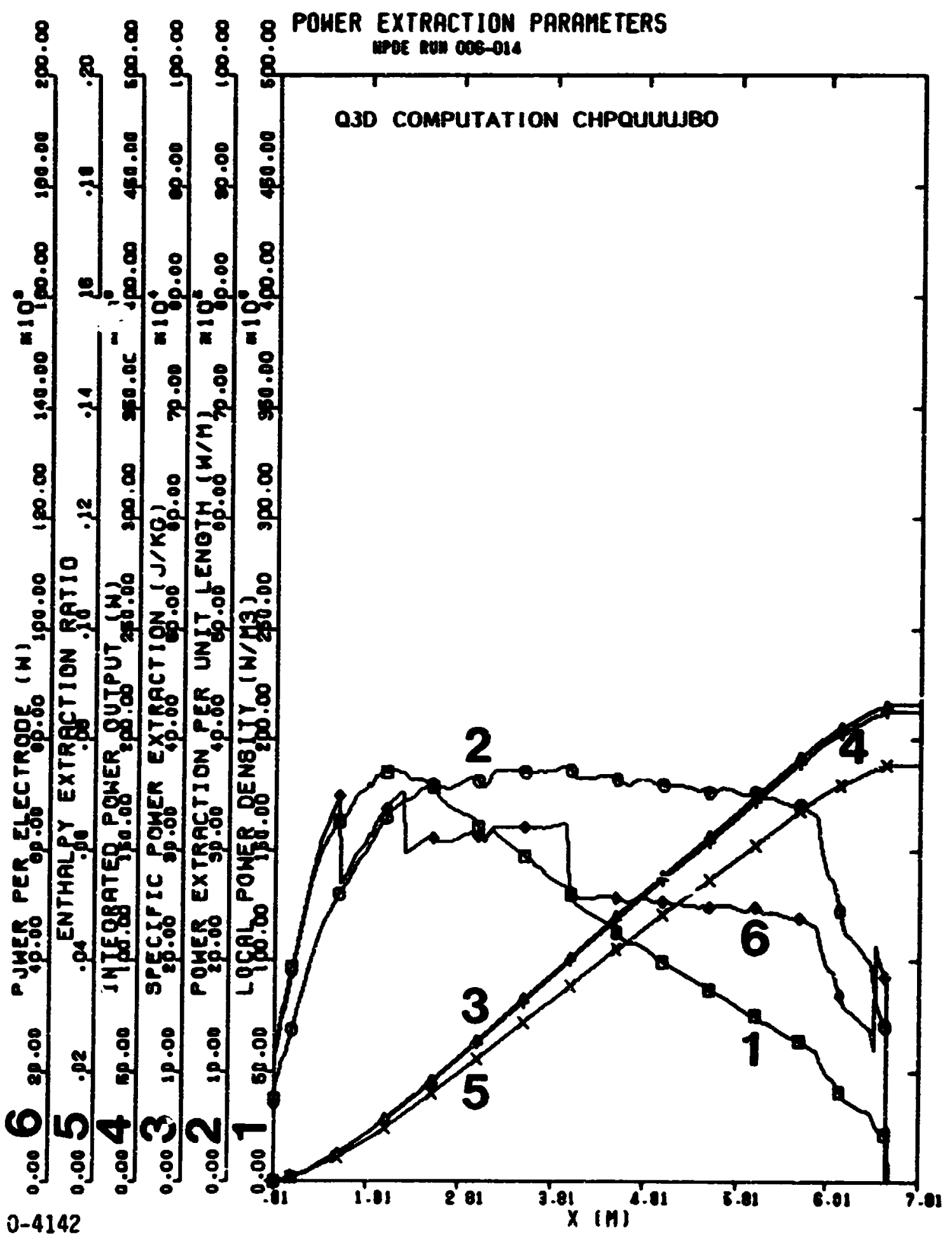

Fig. 3-86. Power extraction parameters HPDE Run 006-014 
STD heseafch componation

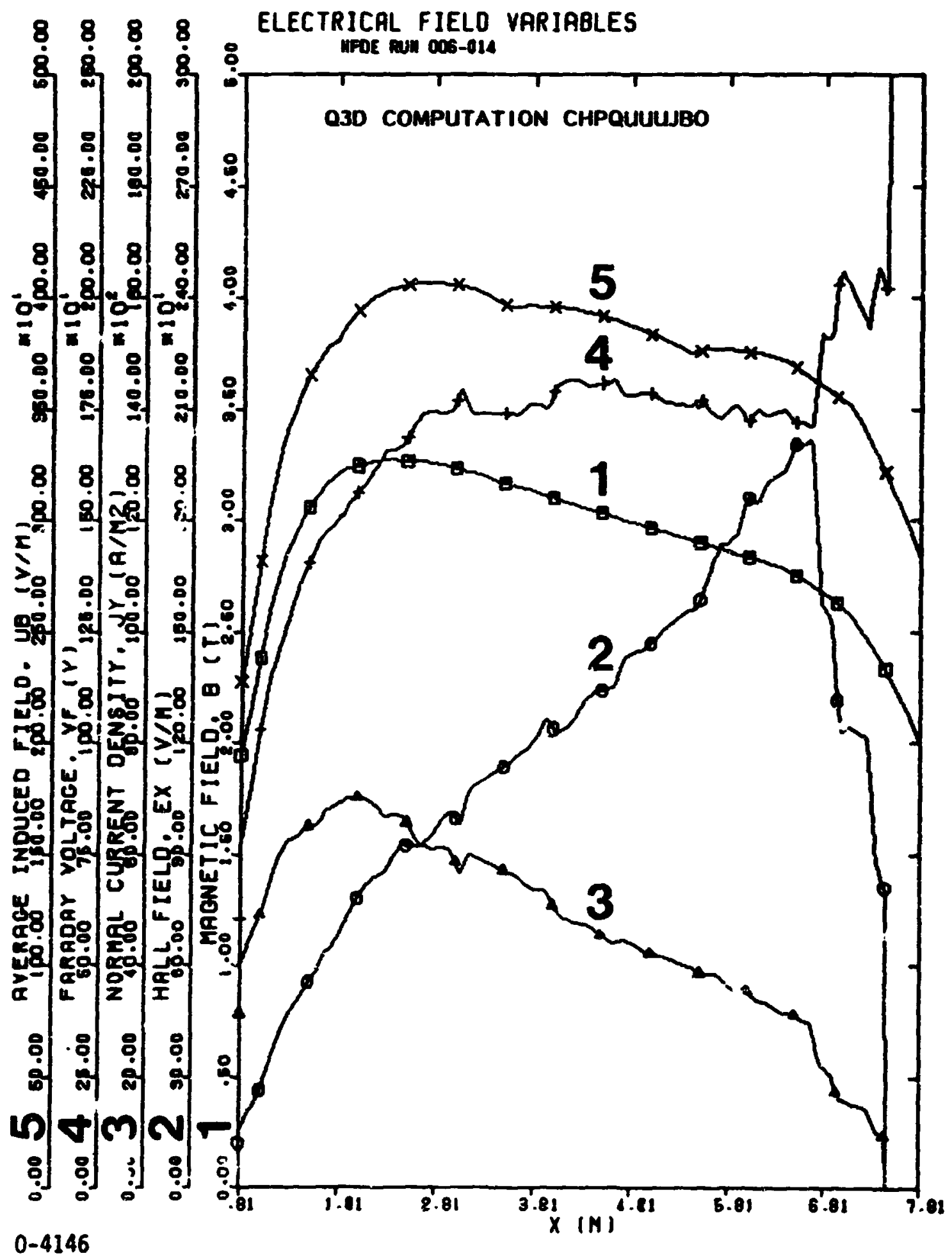

Fig. 3-87. Electrical field varlables HPDE Run 006-014 
STD aesfarch CORPOAATICN

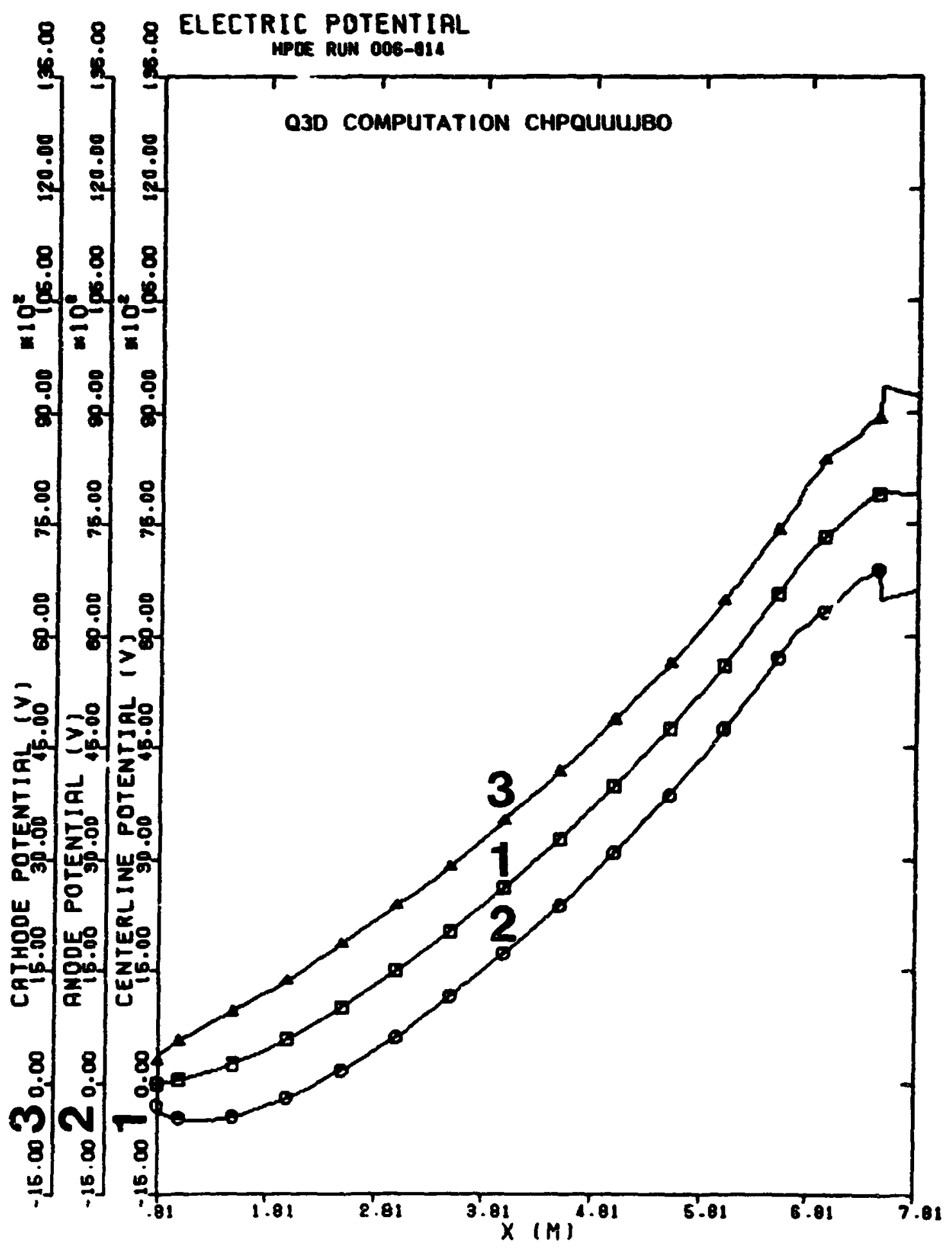

$0-4148$

Fig. 3-88. Electric potential HPDE Run 006-014 
STD

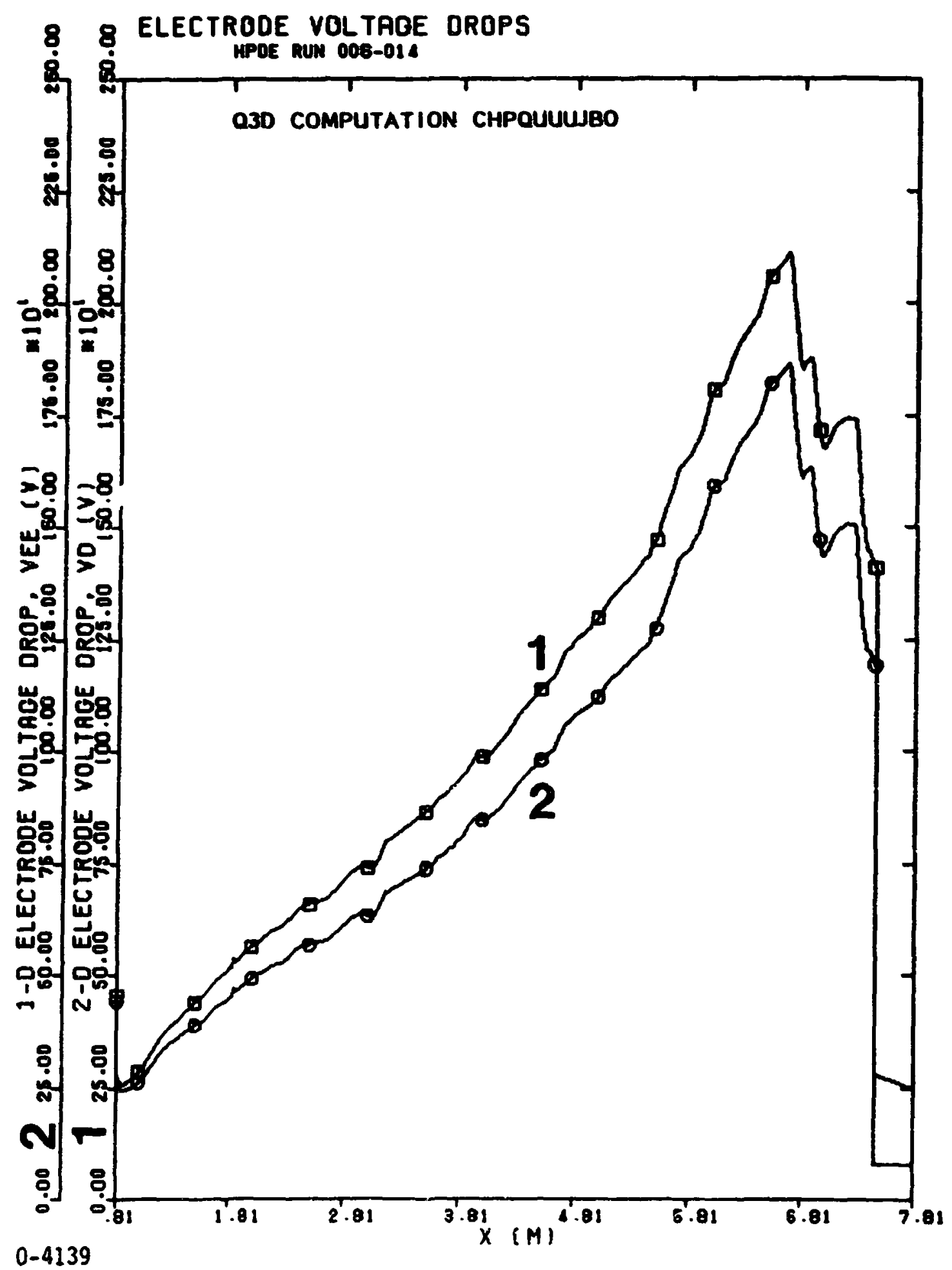

Fig. 3-89. Electrode voltage drops HPDE Run 006-014 


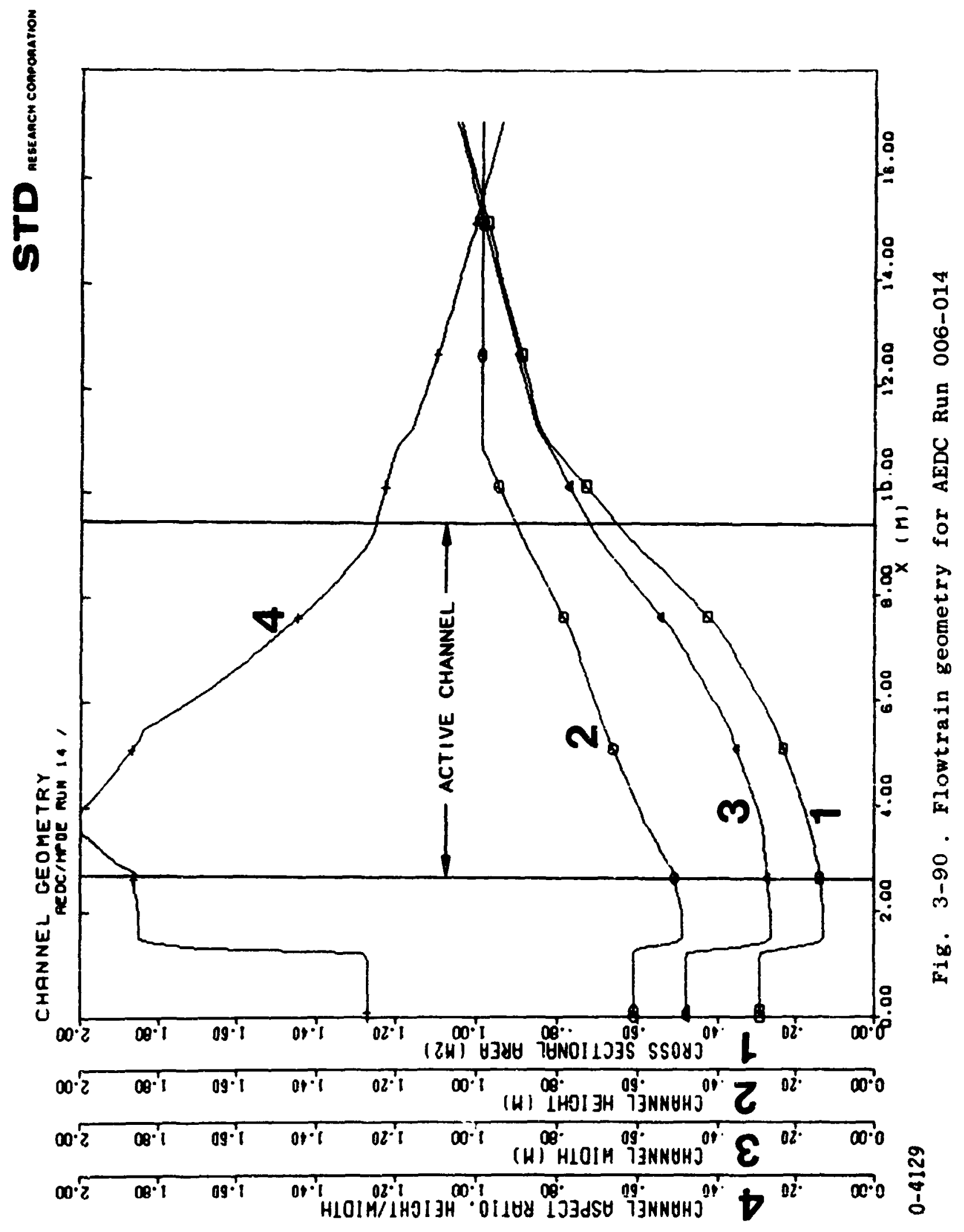




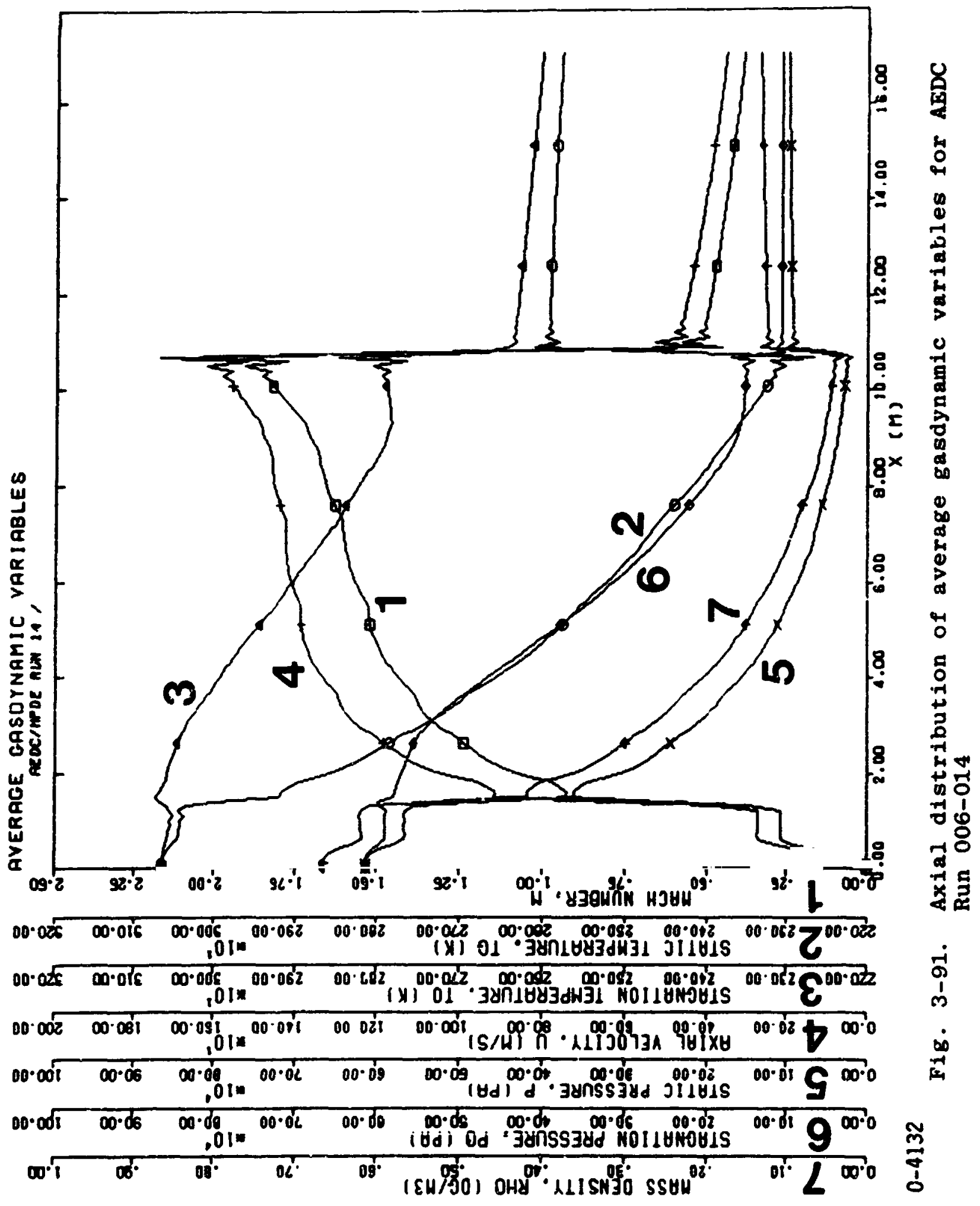




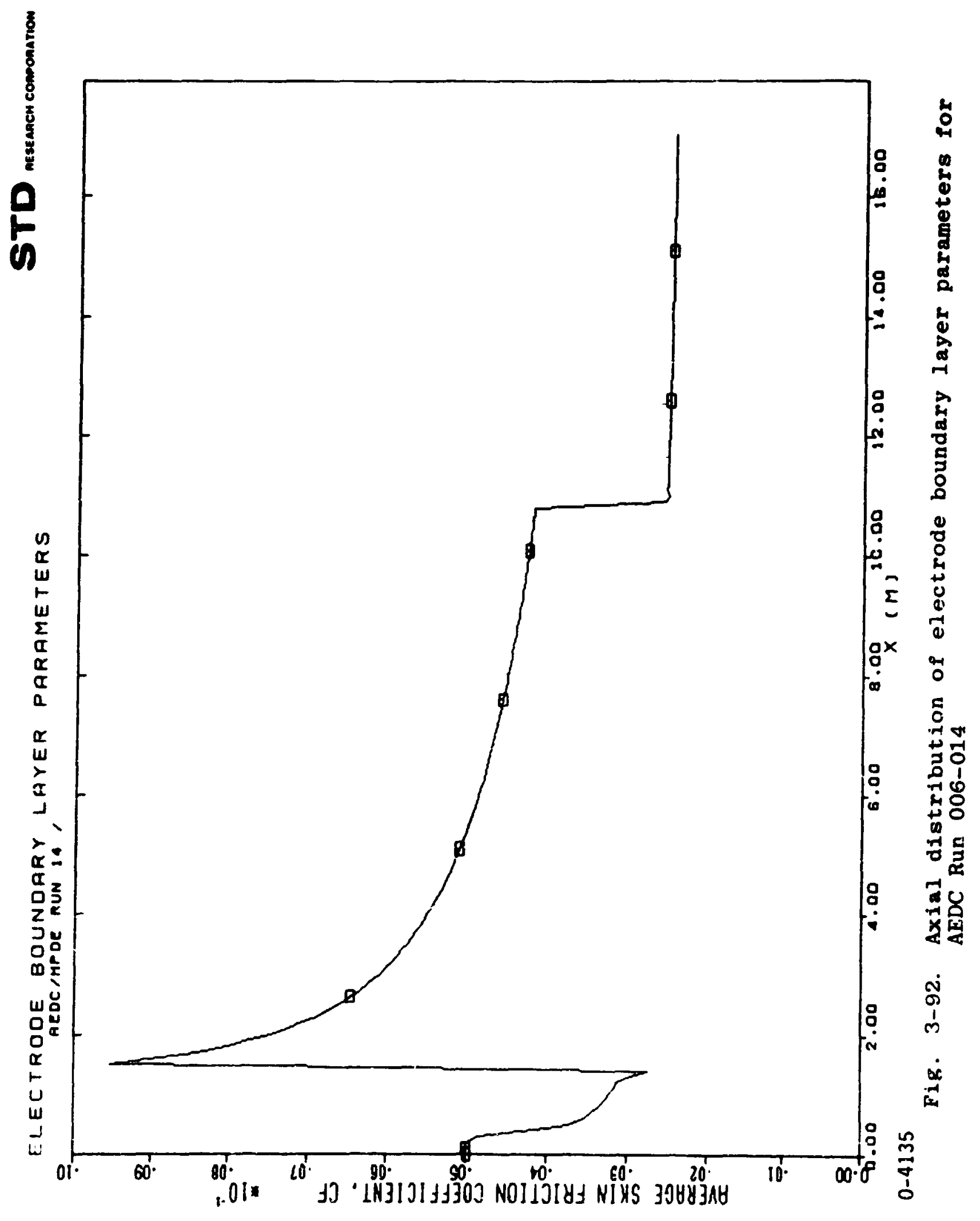




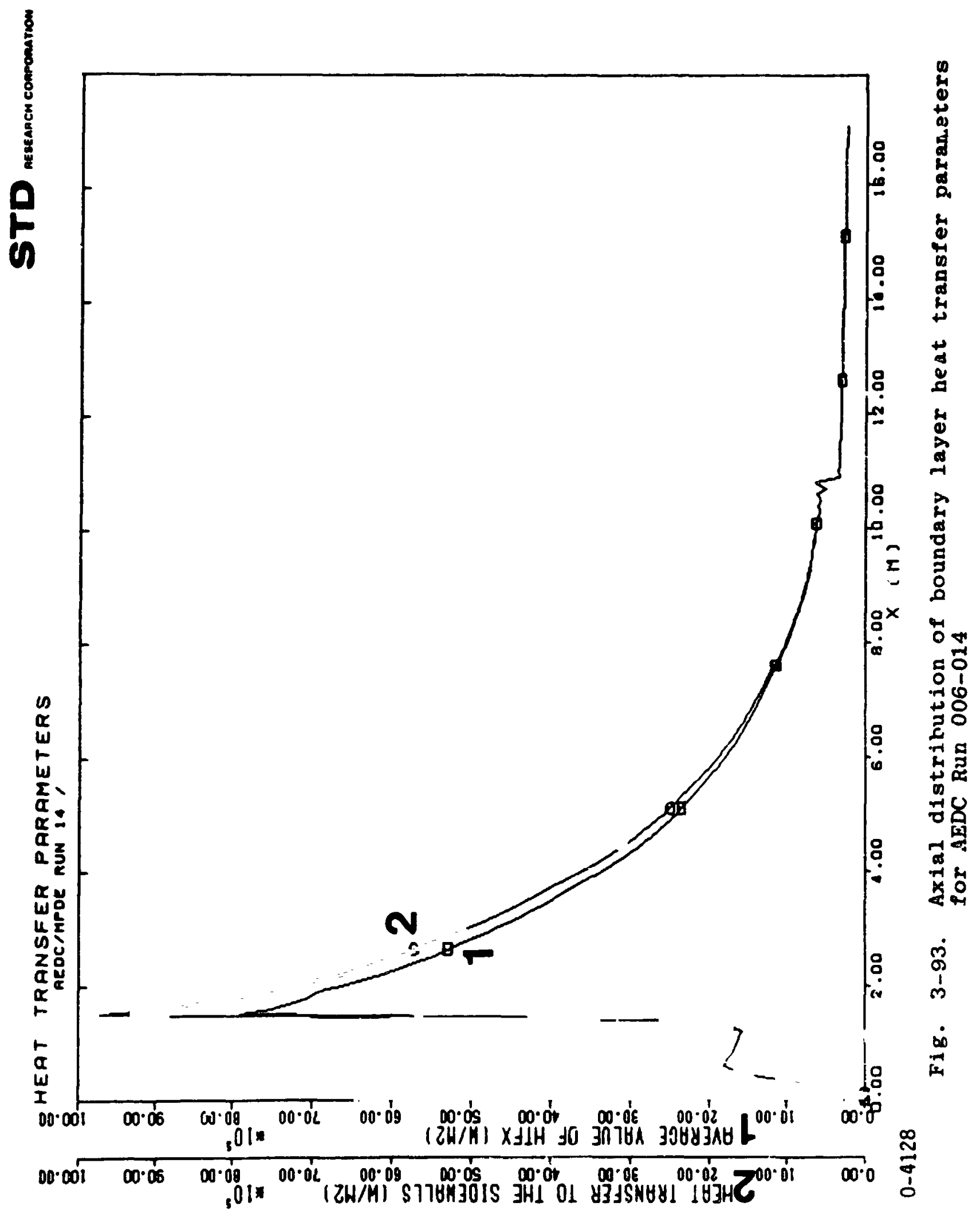




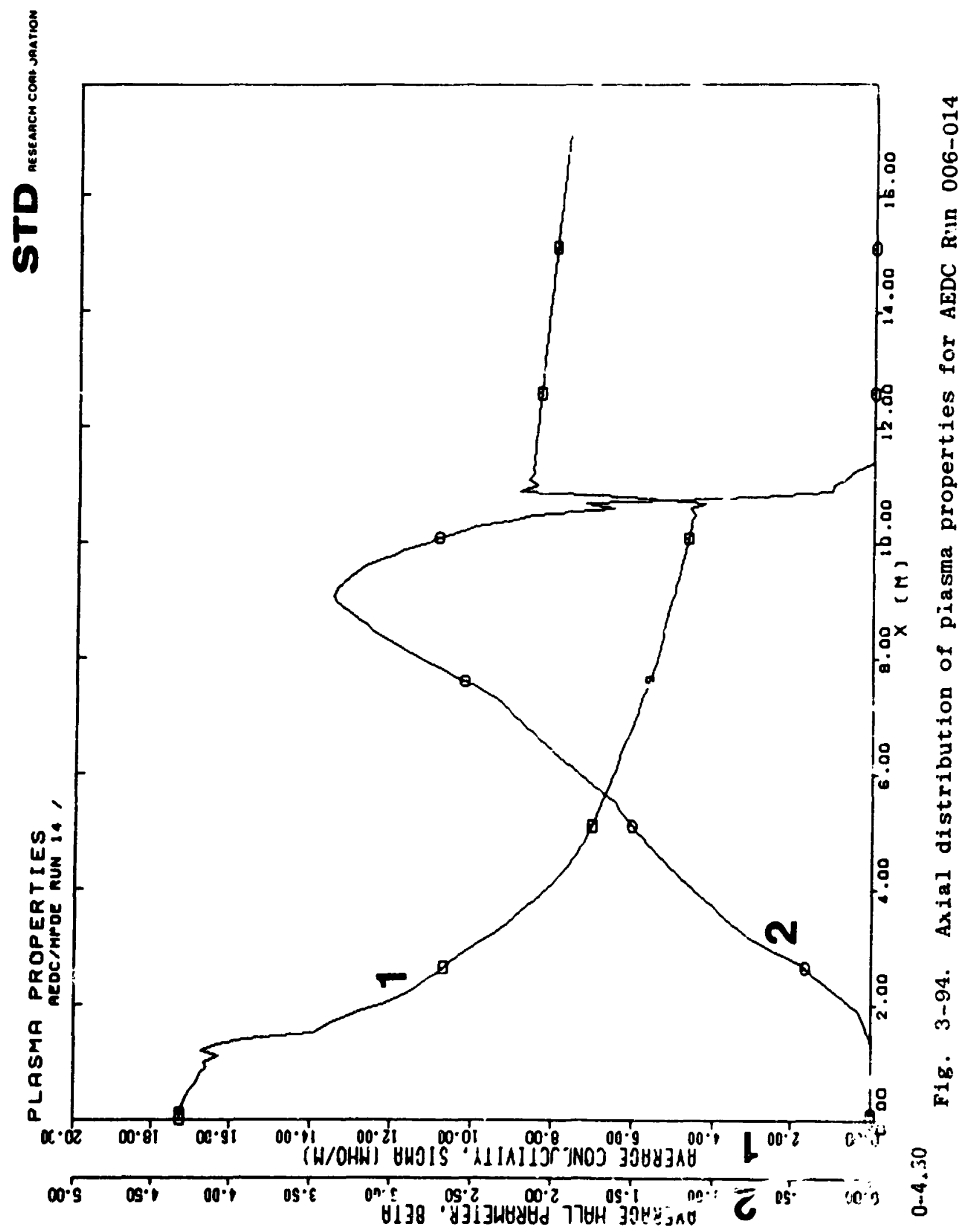




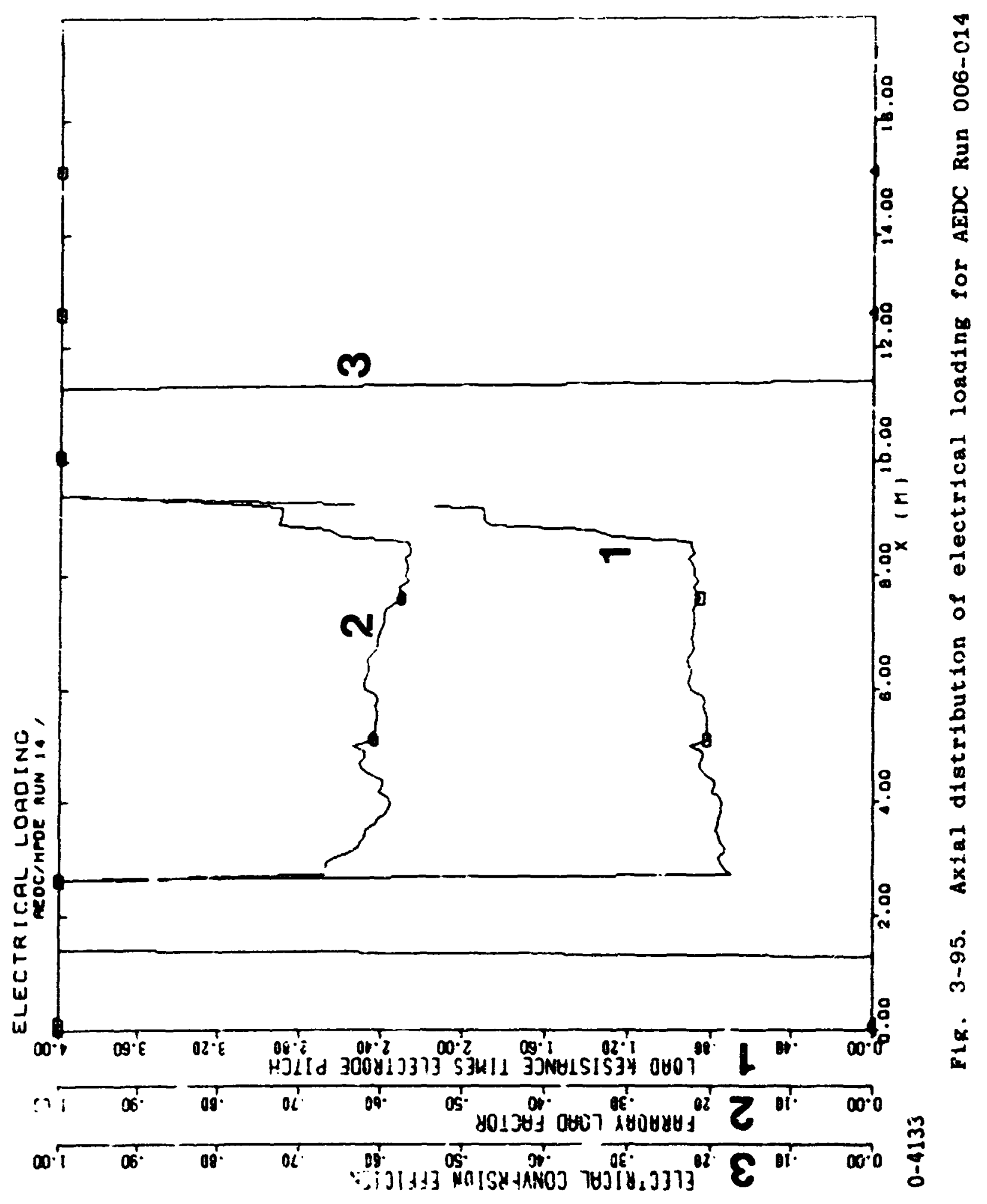




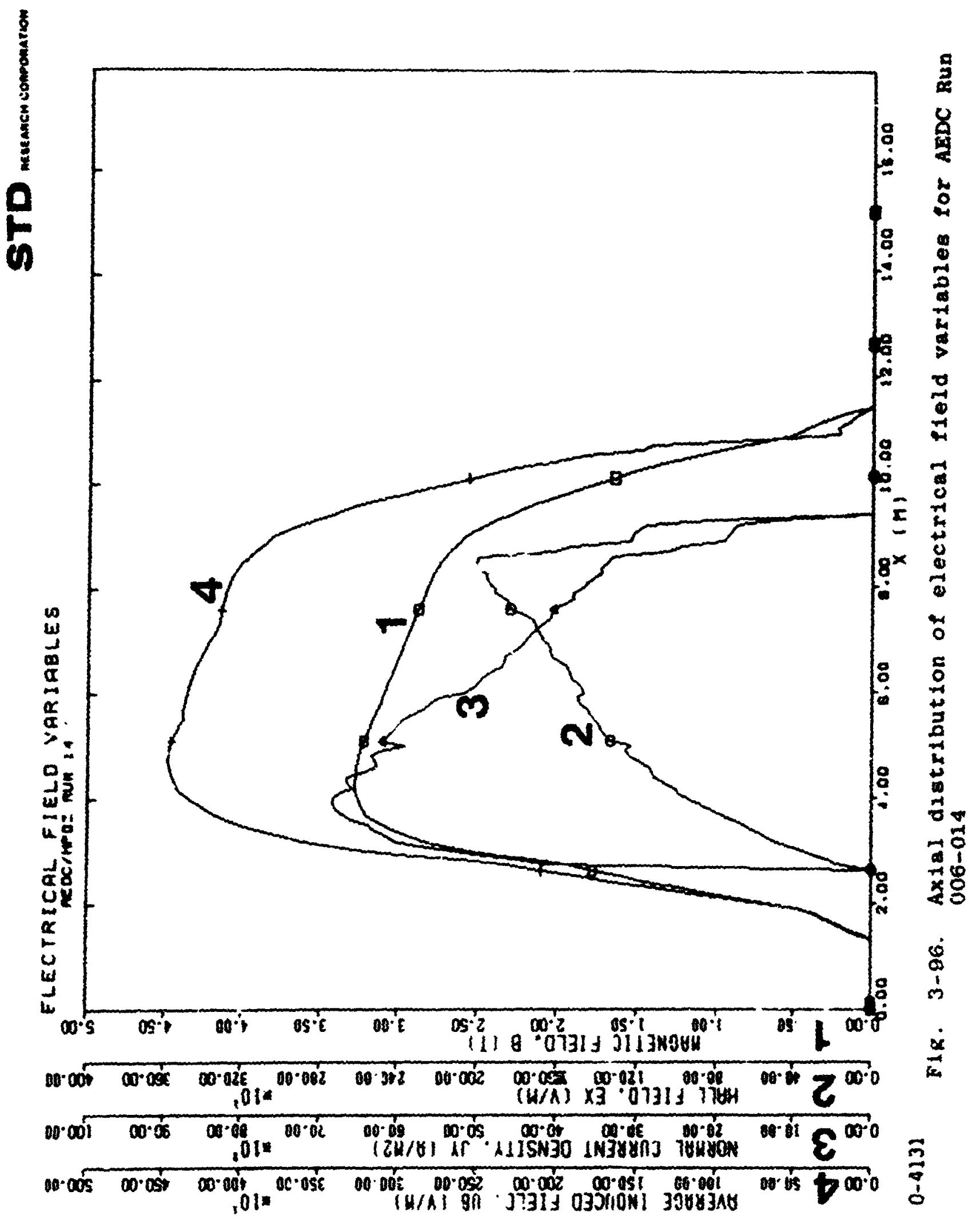




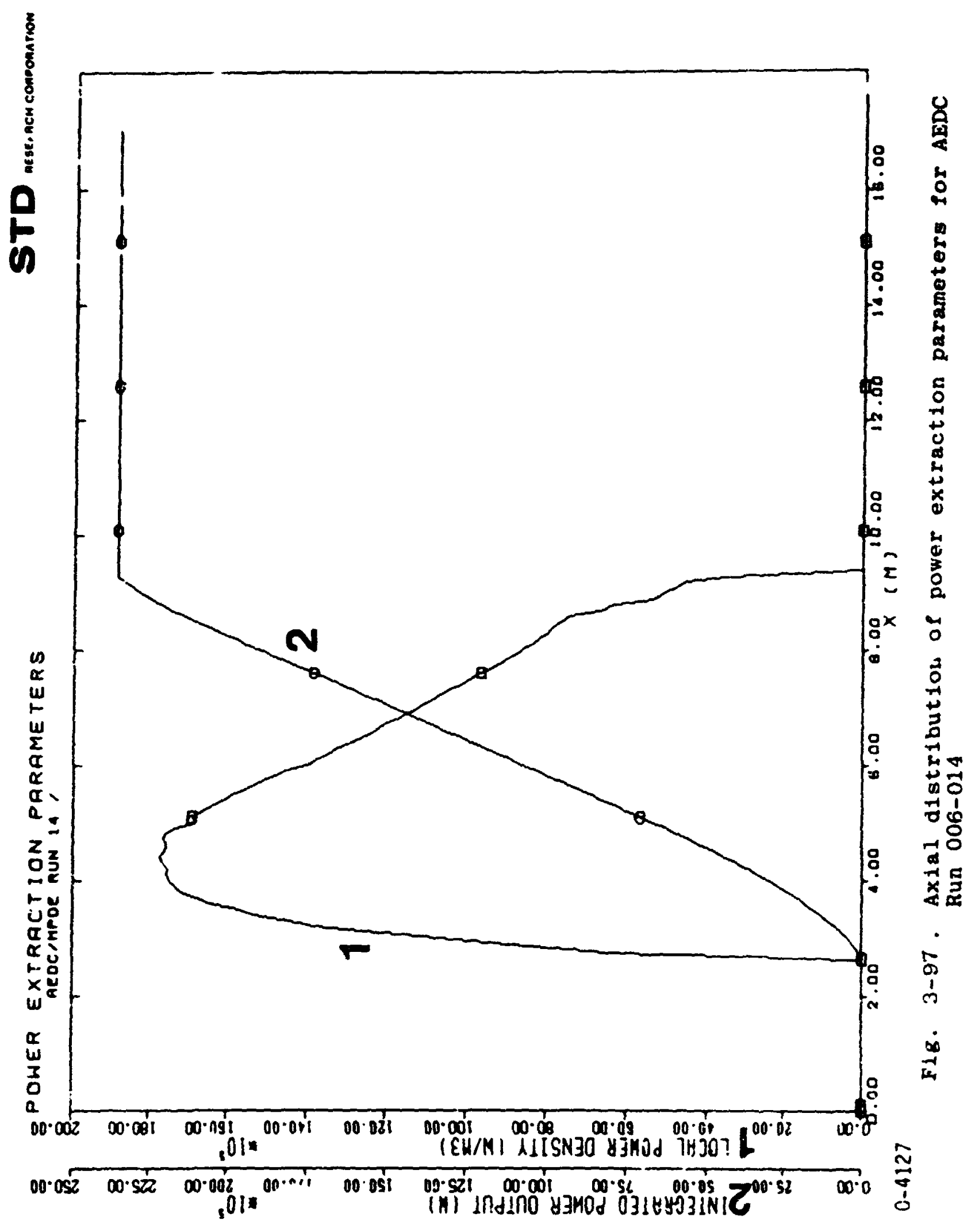




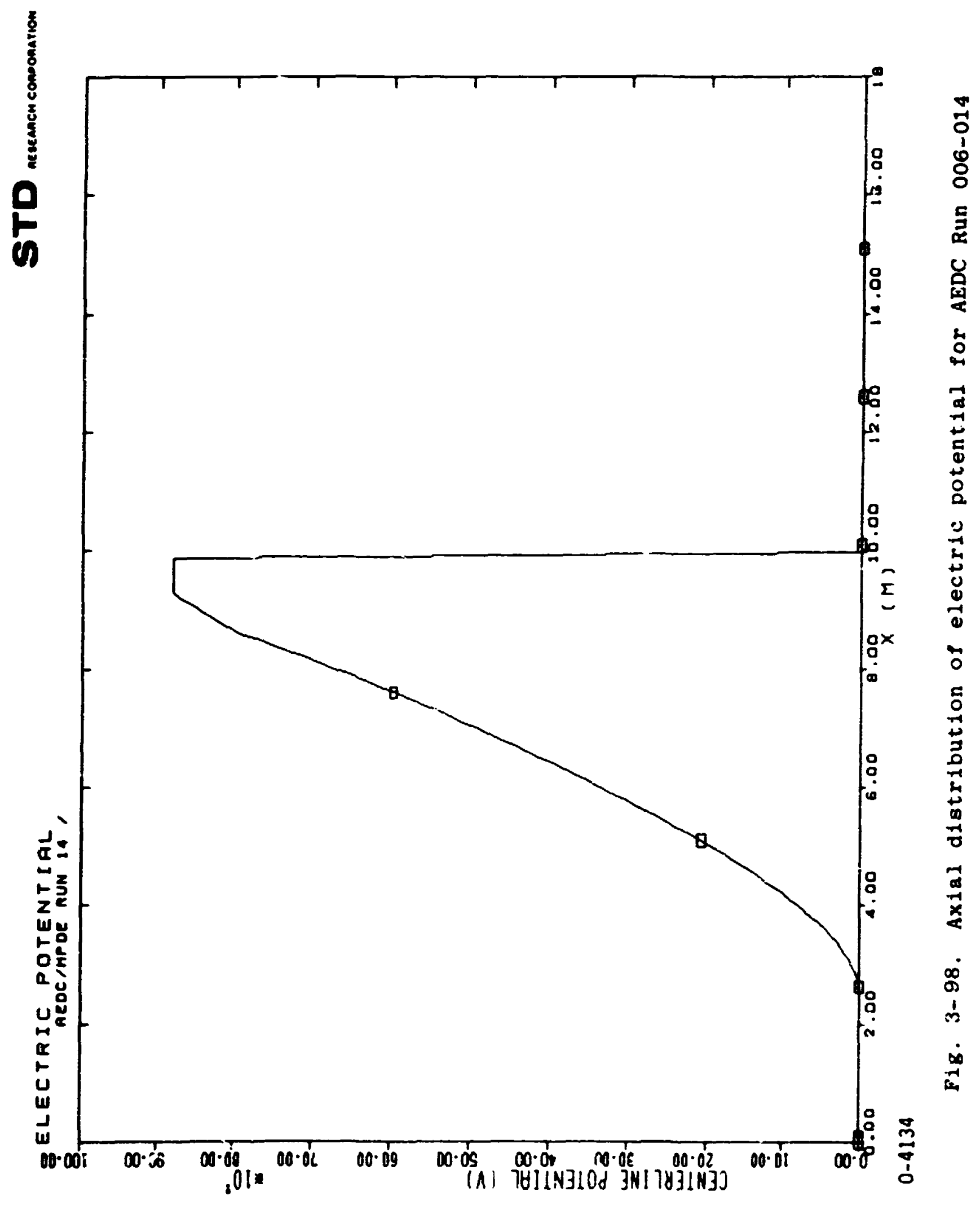


STD neseancu comonantion

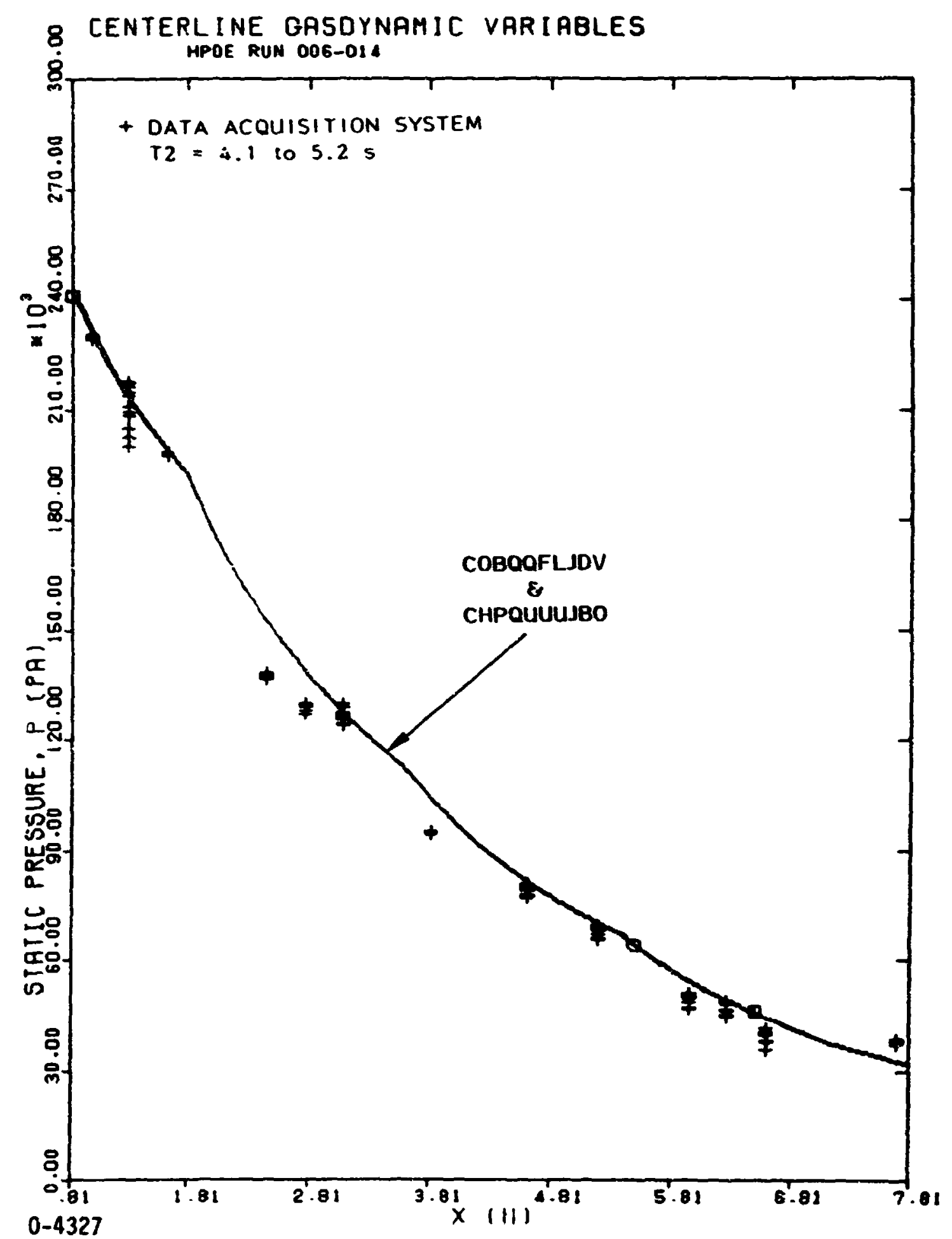

Fig. 3-99. Comparison of the axial distributions of static pressure from the data acquisition system and STD simulations of HPDE Run 006-014. 


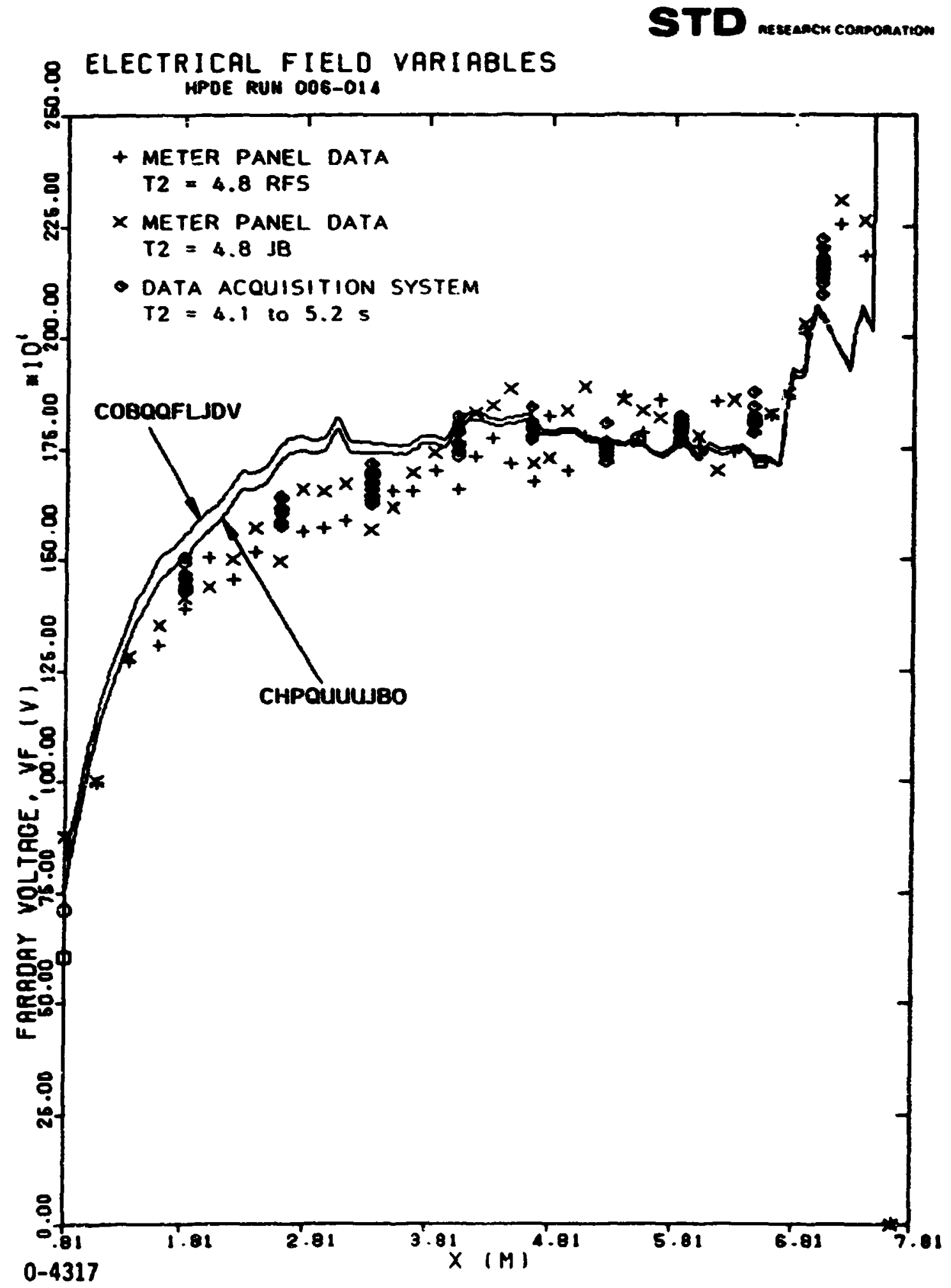

Fig. 3-100. Comparison of the axial distributions of Faraday voitage from the experimental data and from STD simulations of HPDE Run 006-014. 
STD neseacen conponarion

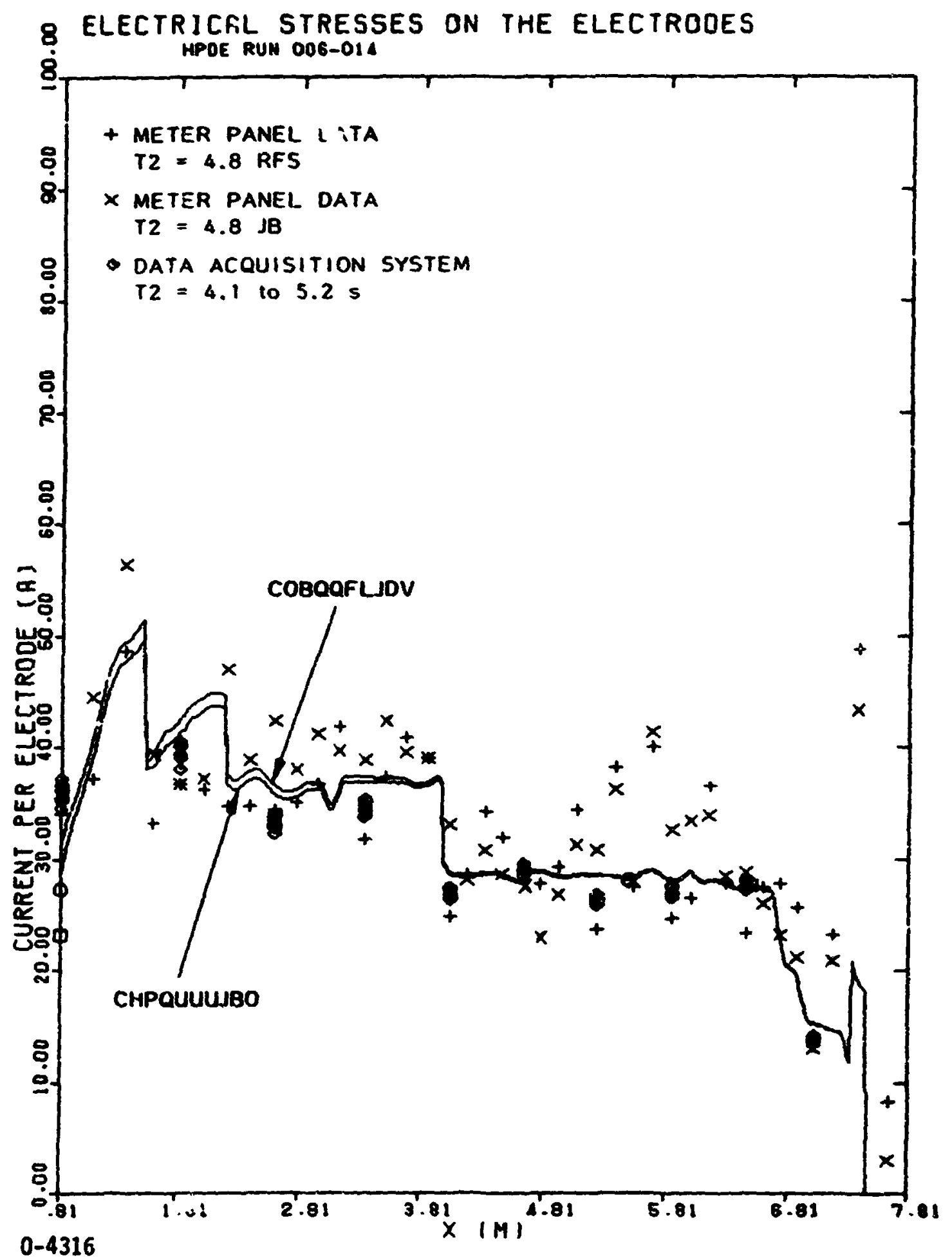

F1g. 3-101 . Comparison of the axial distributions of current per electrode from the experimental data and STD simulations of HPDE Run 006-j14. 
STD nesseancw conoonation

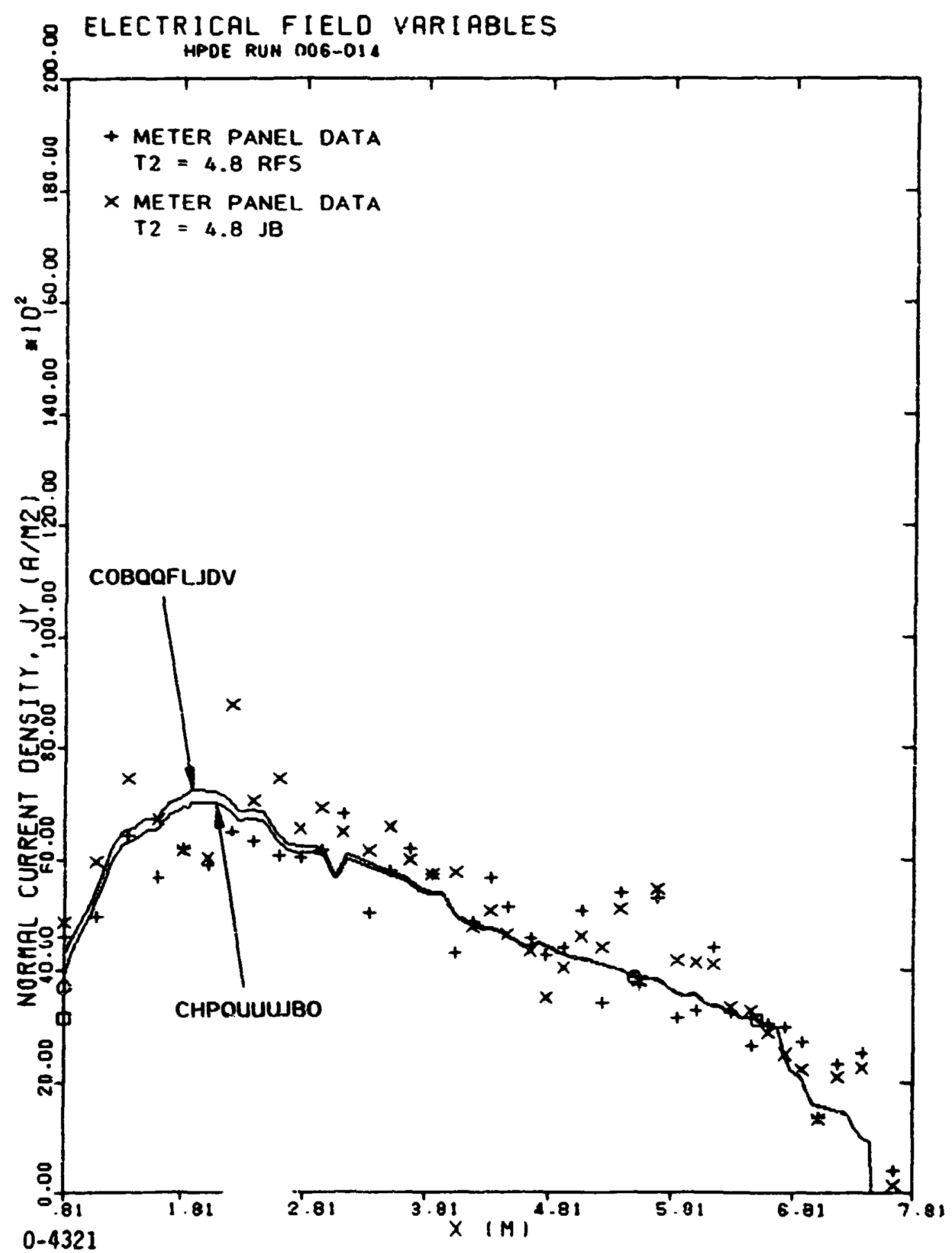

Fig. 3-102. Comparison of the axial distributions of normal current density from tl : experimental data and STD simulations of HPDE Run 0Ot - 014. 
STD ascencun conooanrow

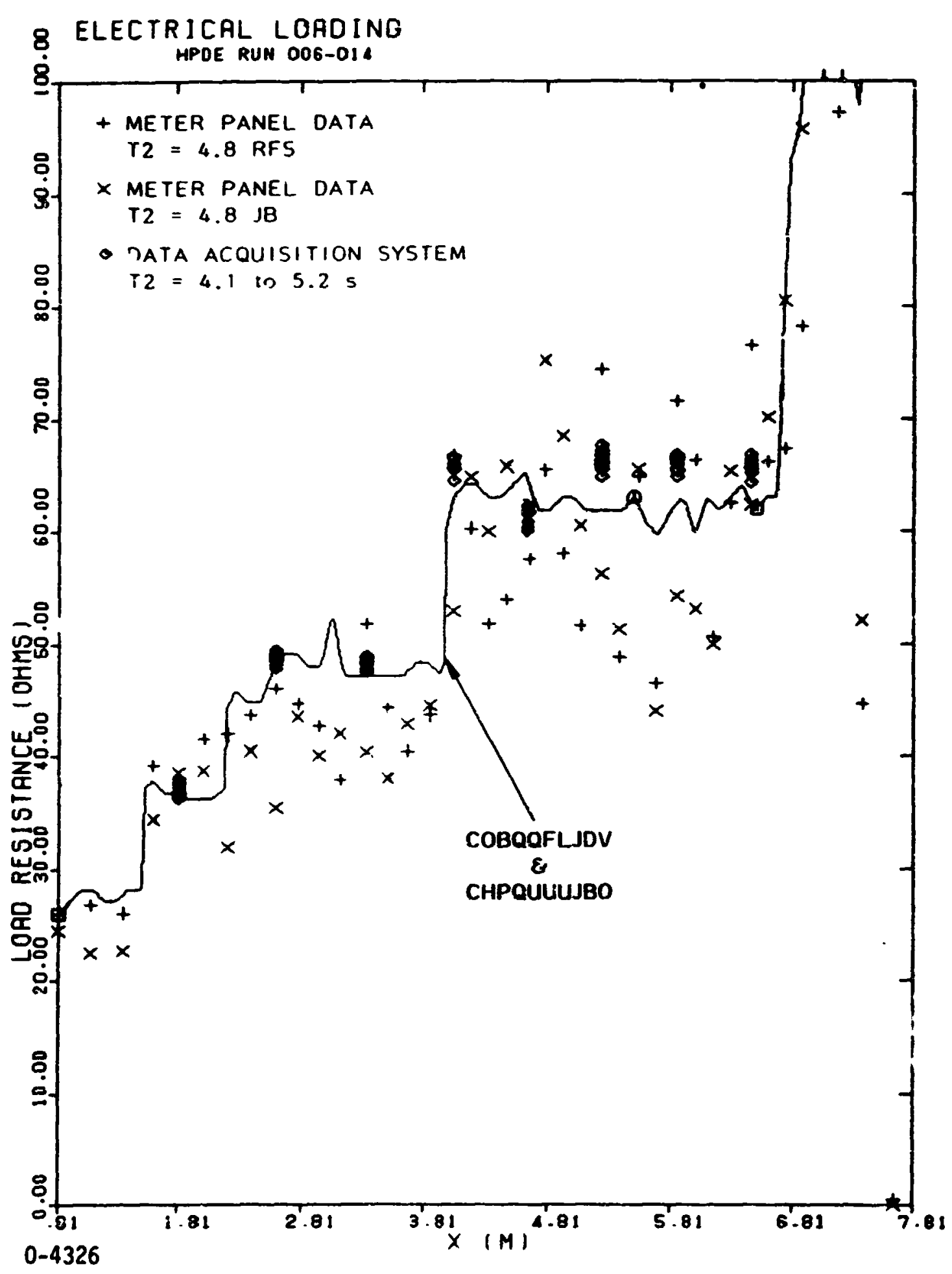

Fig. 3-103. Comparison of the axial distributions of load resistance from the experimental data and STD simulations of HPDE Run 006-014. 
STD nessancu conoonatrion

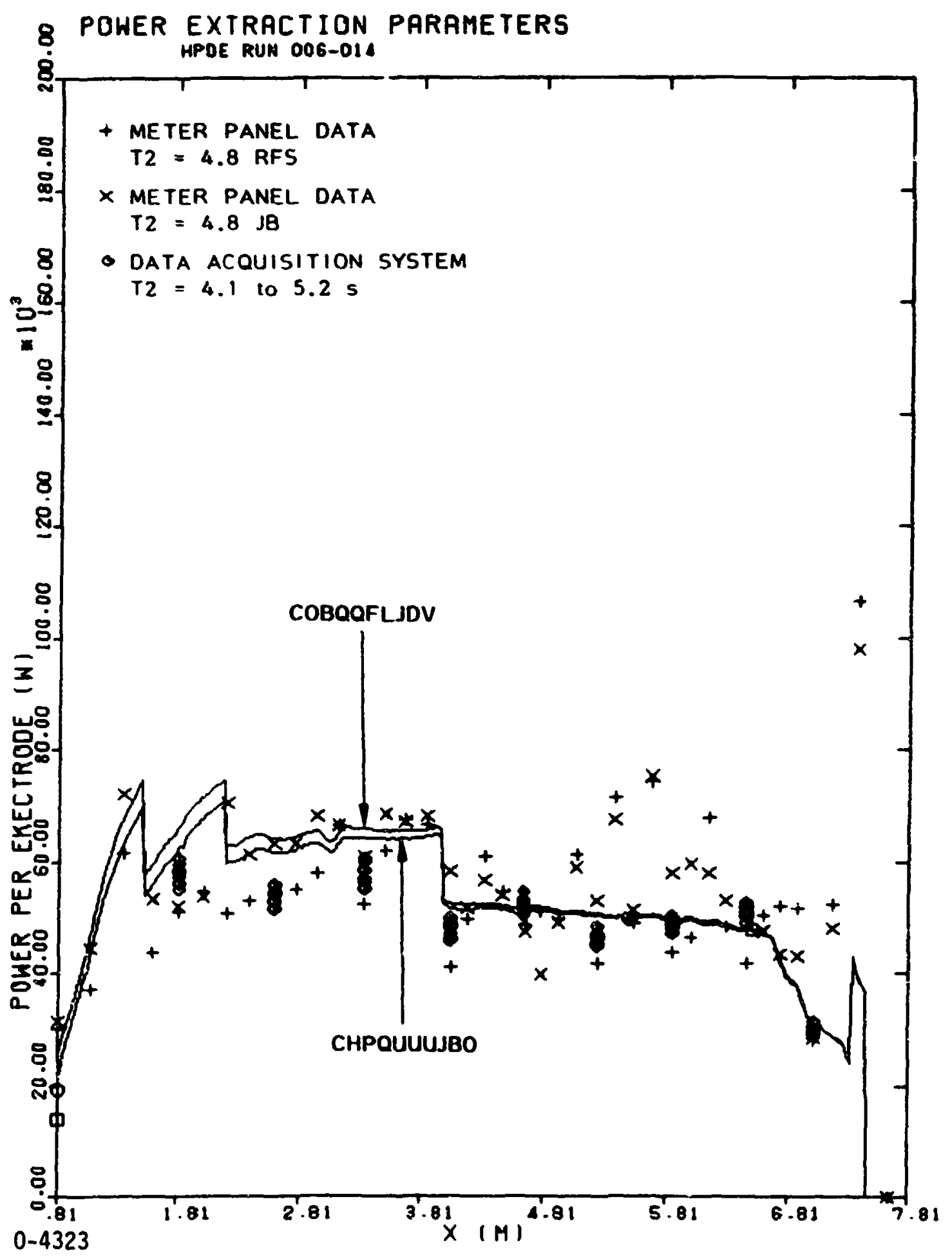

Fig. 3-104. Comparison of the axial distributions of power per electrode from the experimental data and STD simulations of HPDE Run 006-014. 
STD Aesenach conpuation

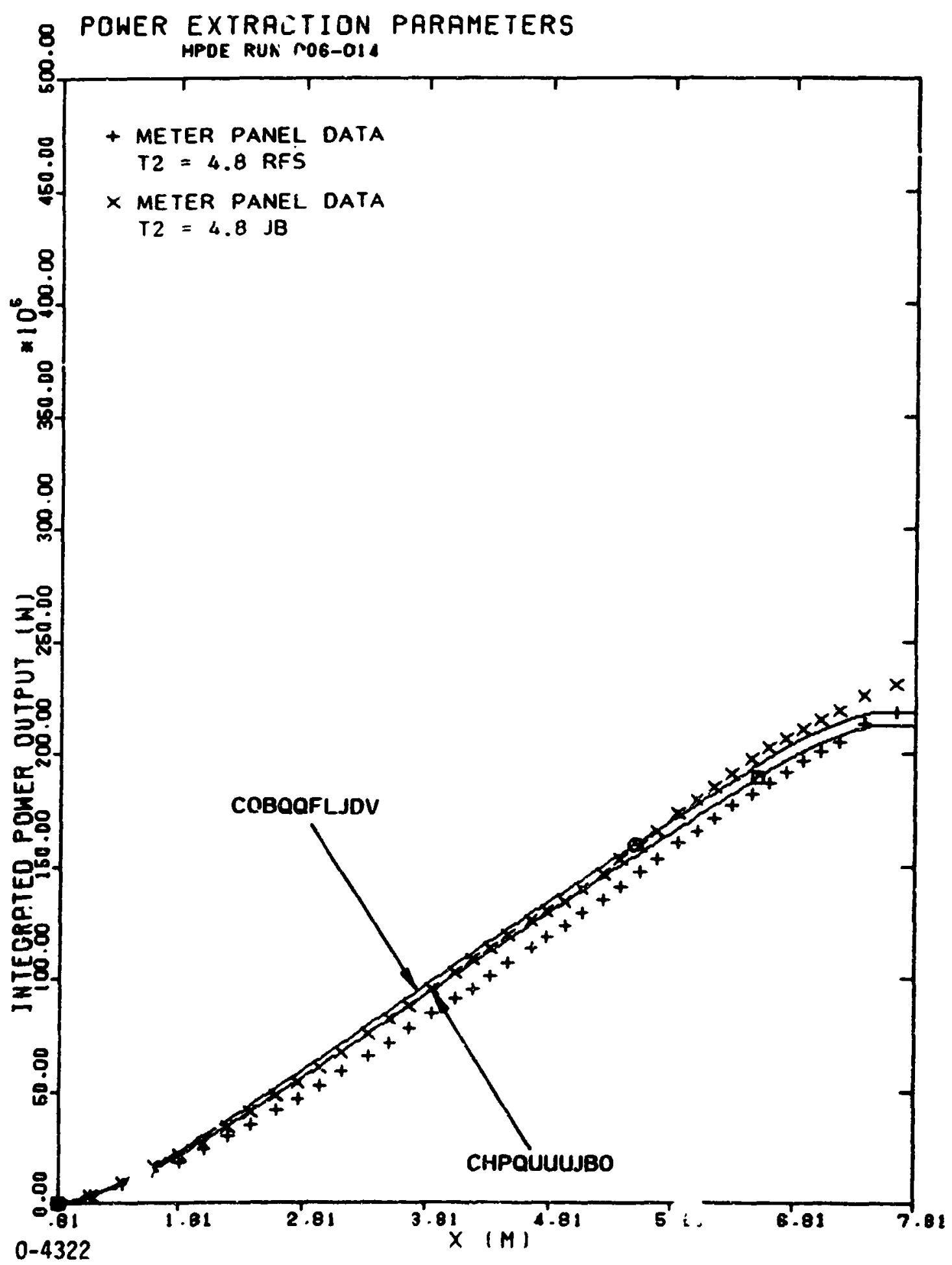

Fig. 3-10E. Comparison of the axial distributions of integrated power output from the experimental data and STD simulations of HPDE Run 006-014. 
STD arsearch cofimanion

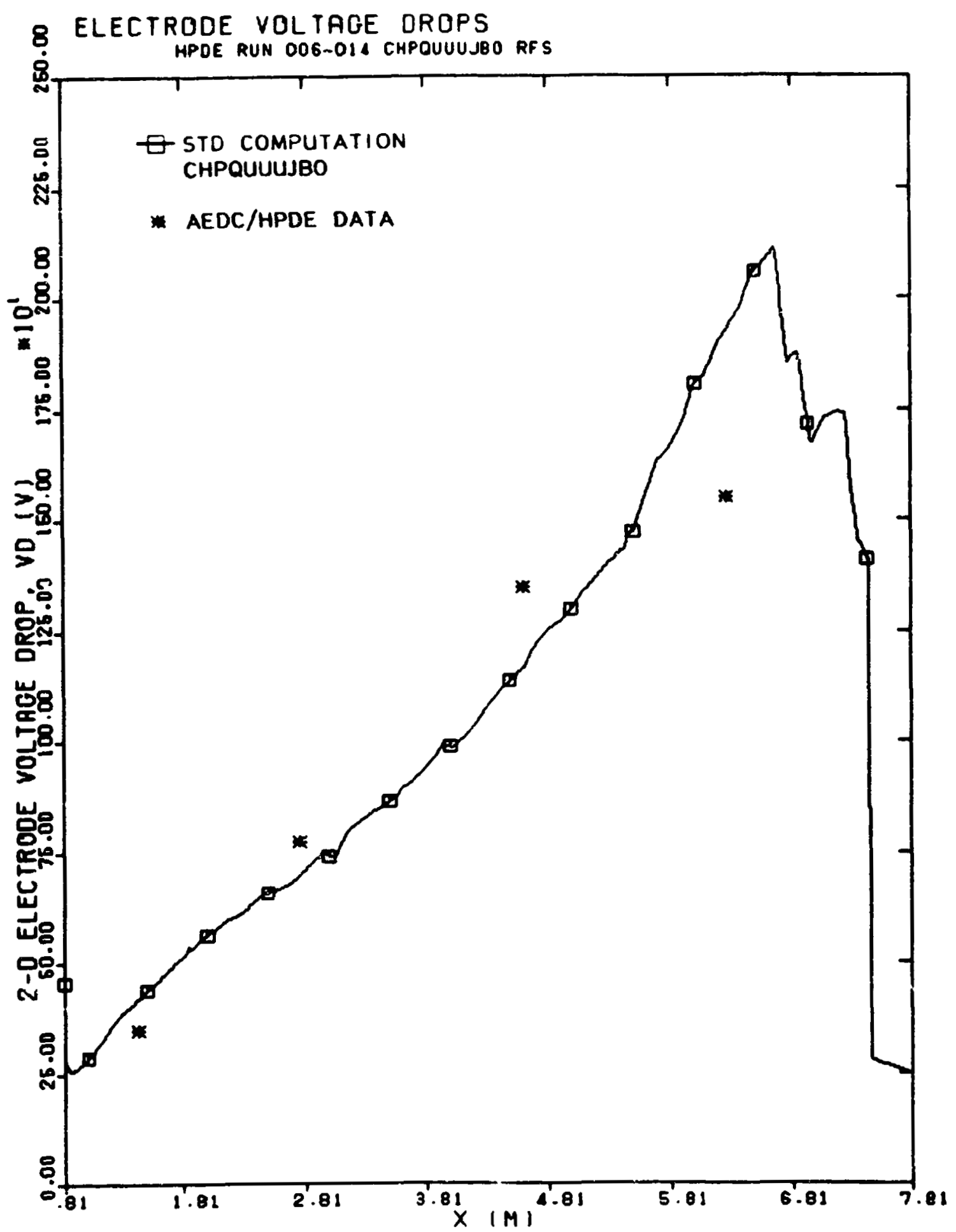

Fig. 3-106. Comparison of the axial distributions of 2-i electroie voltage drop VD(V) for HPDE run 006-014.

$0-3616$ 
STD afseancu coaroonation

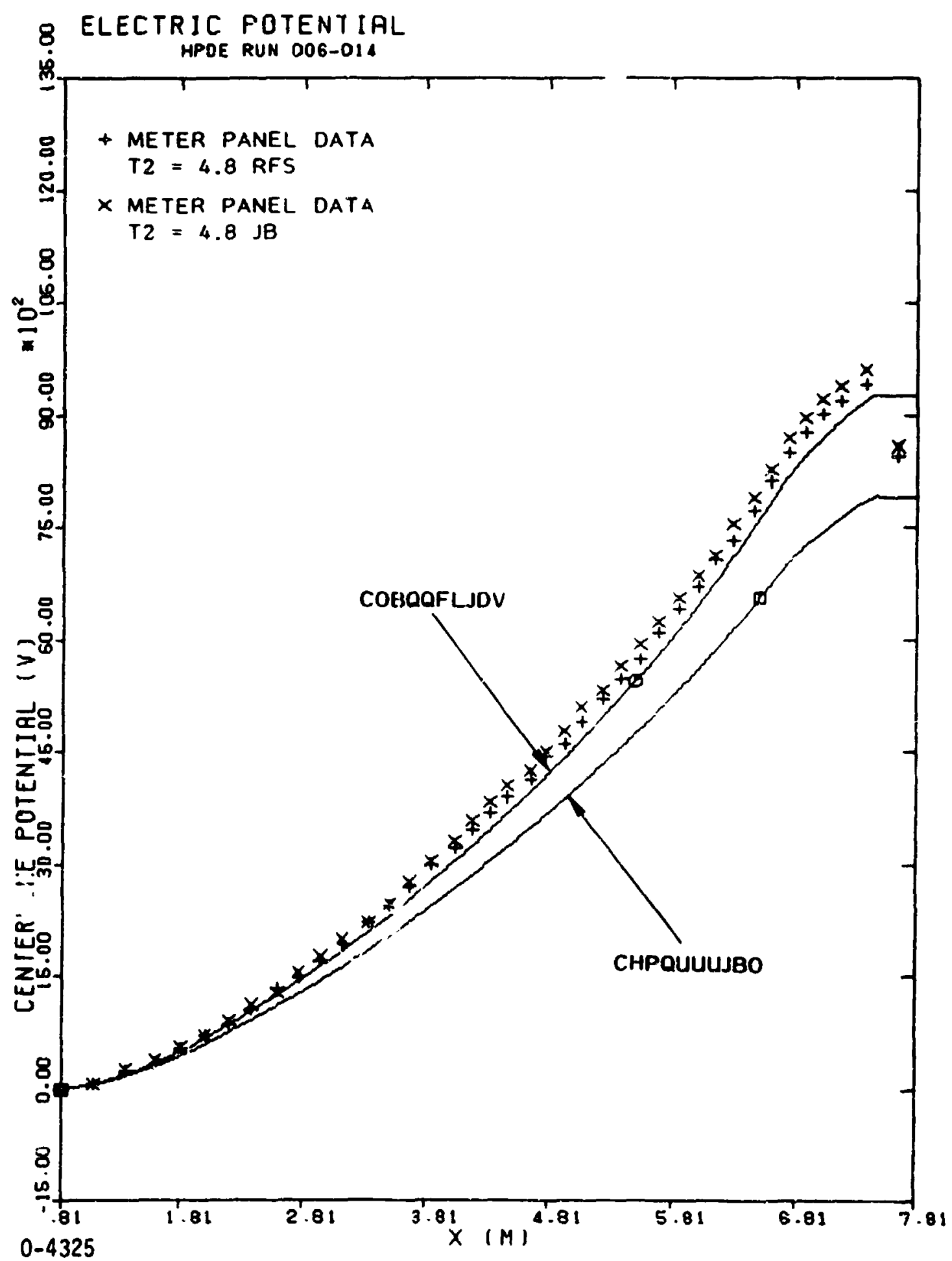

Fig. 3-107. Comparison of the axial distributions of centerine potential from the experimental data and STD simulations of HPDE Run 006-014. 
STD AzSEAACM COAPOAATION

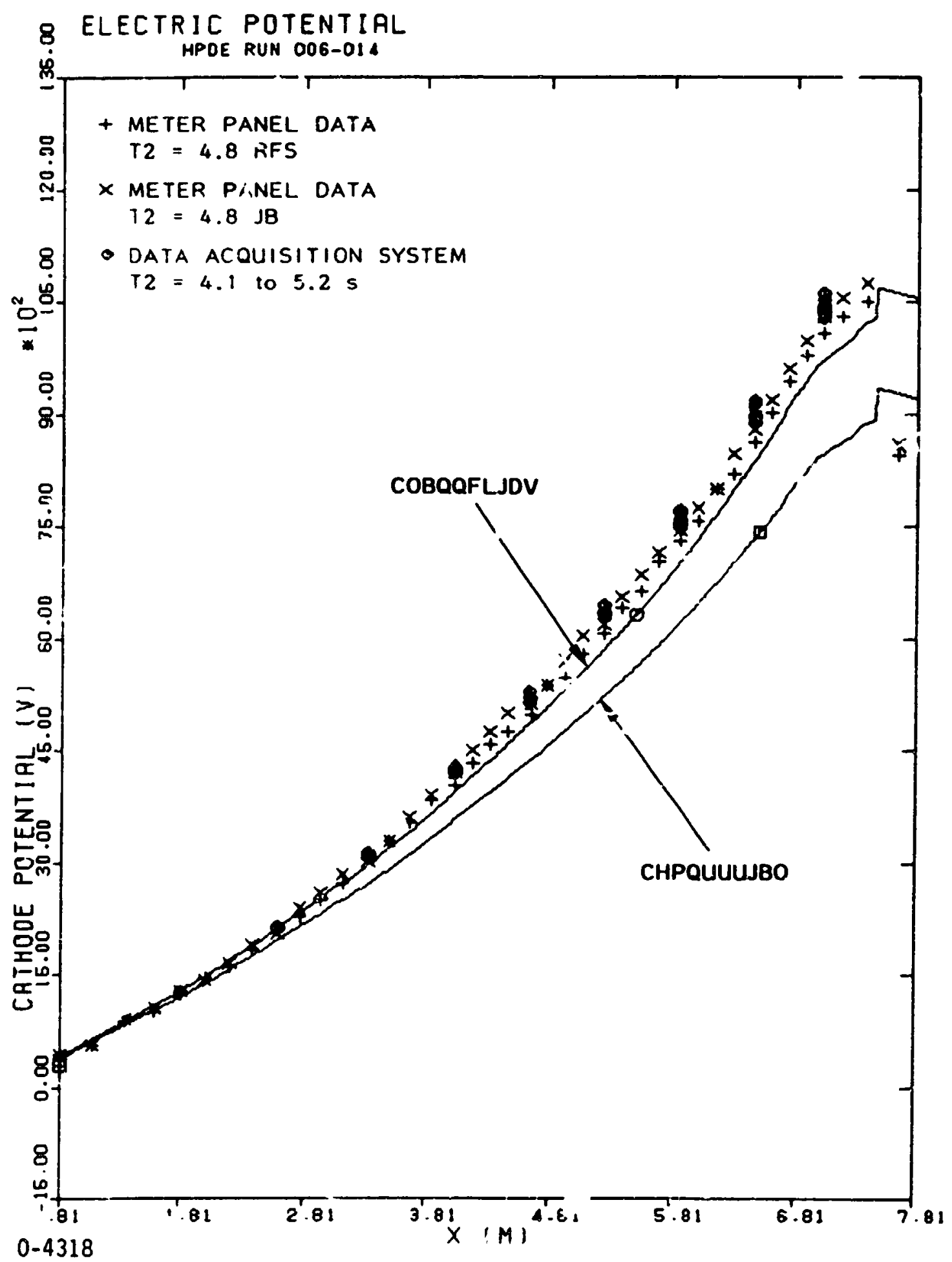

Fig. 3 108. mparison of the axial distributions of calhode (STD - ion potential from tie experimental lata and S idnutations of HPDE sun n06-014. 


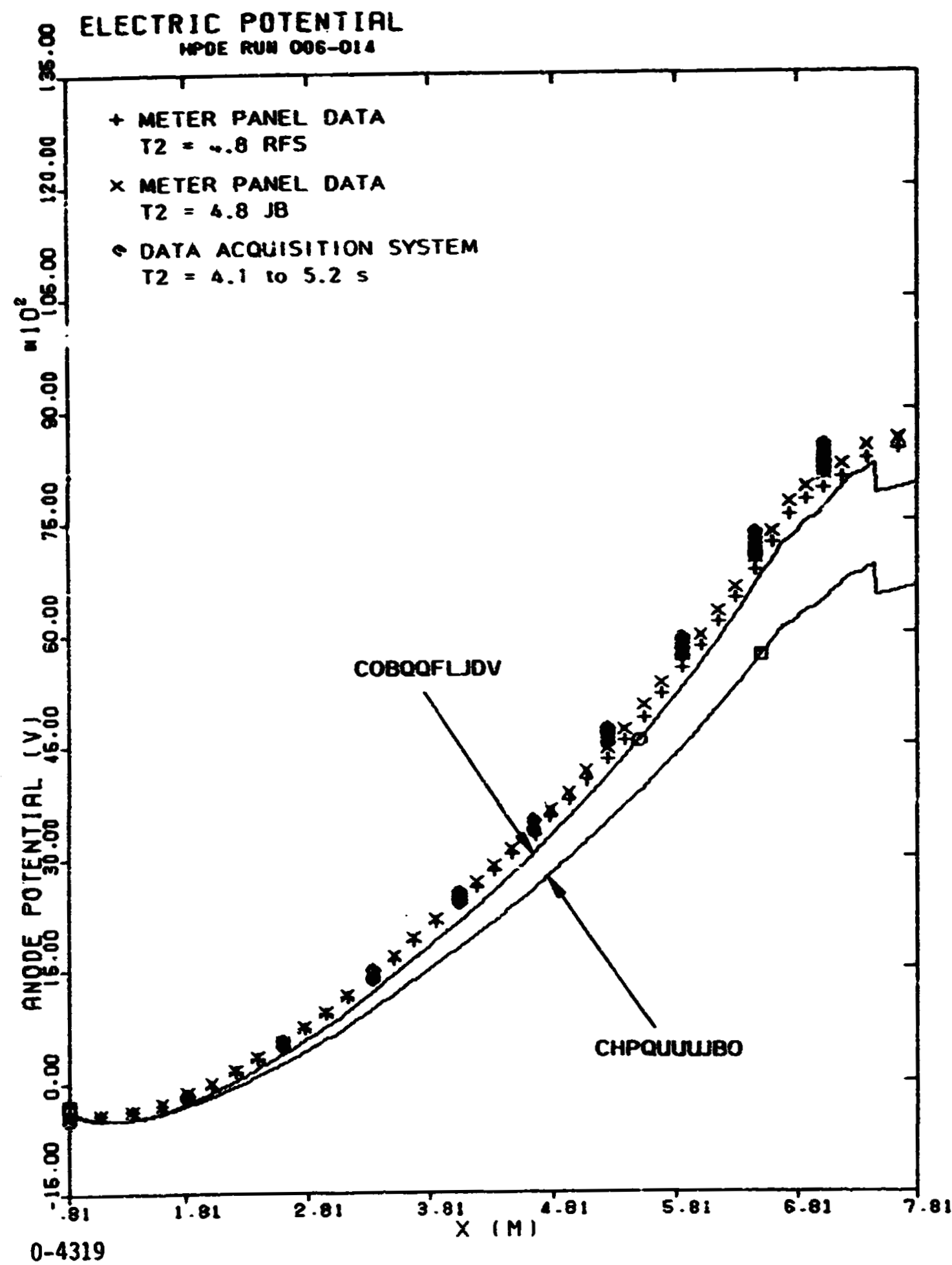

Fig. 3-109. Comparison of the axial distributions of anode (STD notation) potential from the experimental data and STD simulations of HPDE Run 006-014.

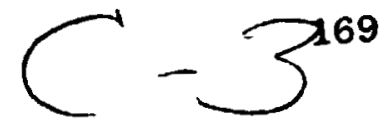


STD messencen comeonarious

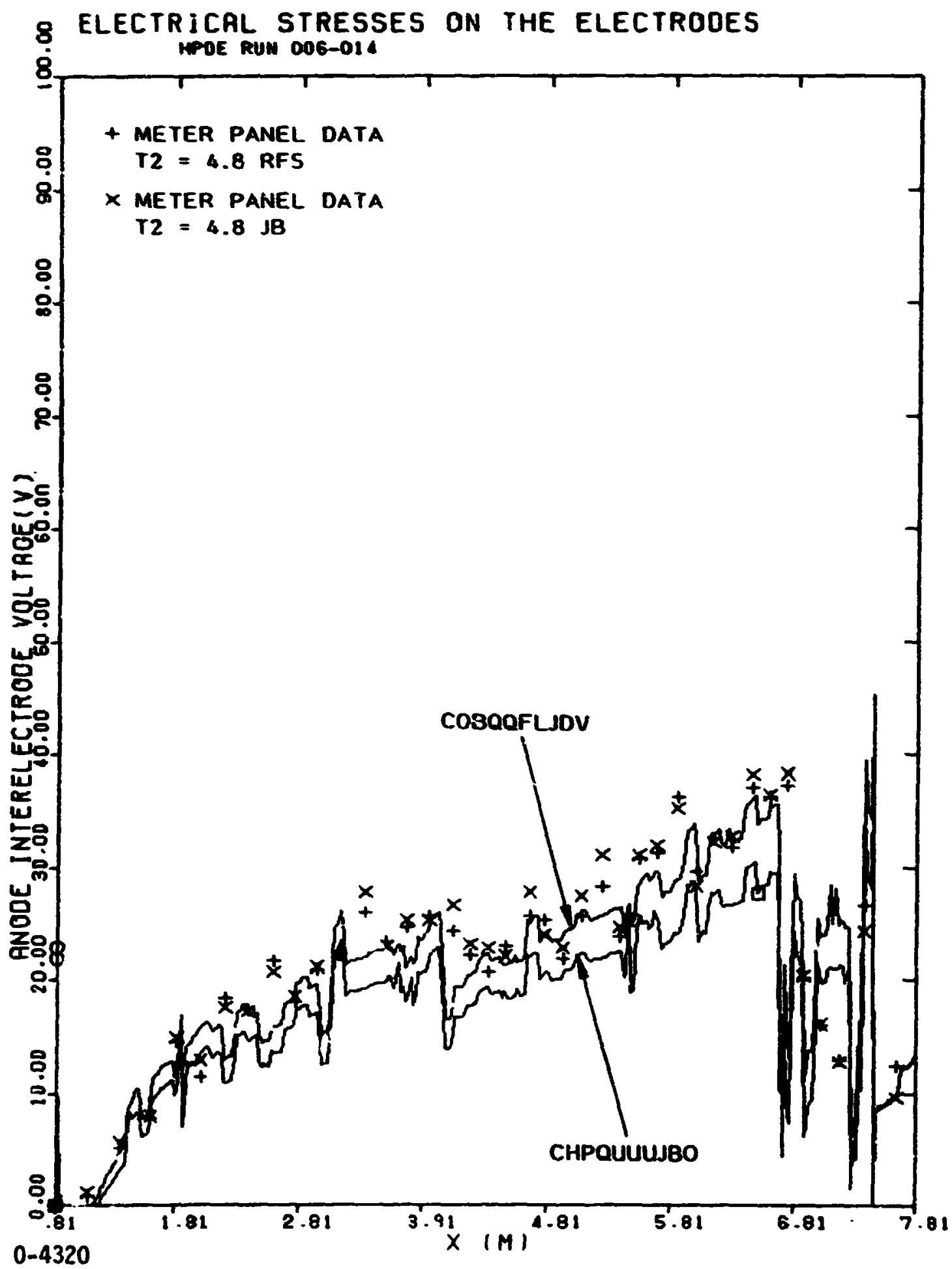

Fig. 3-110. Comparison of the axial distributions of anode interelectrode voltage from the experimental data and STD simulations of HPDE Run 005-014. Experimental data represent averages over 10 electroles. 
STD sescercen conocosartion

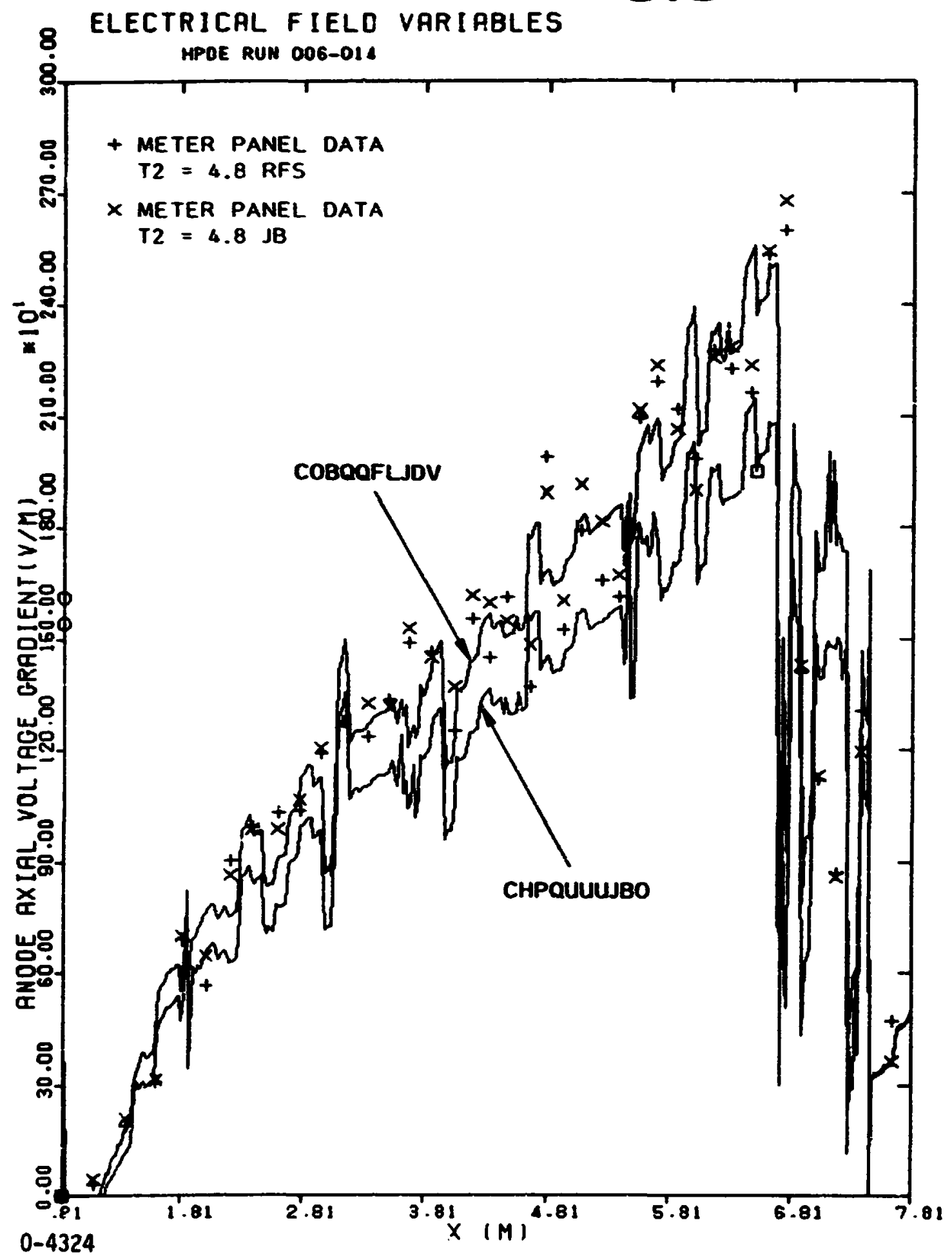

Fig. 3-111. Comparison of the axial distributions of the Anode Hall field from the experimental data and S'rD simulations of HPDE ilun 006-014. Expermiental data represents averages over 10 electrodes. 


\subsection{CRITICAL PHENOMENA IN THE AEDC/HPDE}

The experimental results of the AEDC/HPDE to date reveal the importance of a number of performance-controlling mechanisms in large-scale, high-interaction MHD power generators. Among these are boundary layer voltage drops and plasma nonuniformity, near-electrode phenomena including finite electrode effects, end effects, and the effects of various operating parameters on the optimization of the HPDE performance. This section is devoted primarily to the elucidation of these effects through comparisons with and parametric variations from the experimental operating conditions of HPDE Run 006-014.

\subsection{PARALETRIC VARIATIONS AROUND THE Q3D SIMULATION OF RUN 006-014}

The successful completion of the Run 006-014 simulations gives an opportunity to exploit the computer models to predict performance in other operating regines. Using the results of the detailed Q3D simulation of HPDE Run 006-014 described in Section 3.7 as a starting point, single-parameter variations in wall temperature, load factor, N/O ratio, mass flow rate, and magnetic field have been carried out. These computations demonstrate the sensitivity of HPDE performance to most of the operating conditions under the control of the experimentalists.

Fig. 4-1 presents the results of the parametric computations. All of the computations represented in Fig. 4-1 are variations from computation CHPQUUUJBO, which is the nominal, simulation of HPDE Run 006-014. The Faraday load 
factor distribution was altered to a constant value of 0.55 (computation COBQFRQJEA), 0.60 (computation COBQAVHJDY), and 0.70 (computation COBQBGDJDY . The electrode wall surface temperatures were increased to a constant value of $1250 \mathrm{~K}$ (computation COPQRPHJBJ), to a constant value of $1500 \mathrm{~K}$ (computation COBQJQKJDY), and to a constant value of $1750 \mathrm{~K}$ (computation COBQDTBJD1). Also shown is computation COBQAIYJE1, in which the electrode wall surface temperatures were assumed to rise to "20-sec" values, which were determined by the same method applied to obtain the " $5-\mathrm{sec}$ values of the nomical simulations. The peak magnetic field strength was increased to $4.02 \mathrm{~T}$ (computation COBQLRNJDZ) and to $4.28 \mathrm{~T}$ (computation COBQIJOJEA). The $N / O$ ratio was decreased to 0.80 (computation COBQMLZJDZ) and increased to 1.00 (computation COBQIFBJEA). In contrast to the other parametric computations, the N/O variations were centered about computation COBQAVWJDY with a constant load factor of 0.60 .

These new computations have shown that a constant Faraday load factor of 0.60 yields slightly better performance than the nominal simulation, which has a varying load schedule with an average load factor of approximately 0.61 . A constant Faraday load factor of 0.70 yields forse performance. A load factor of 0.55 increases enthalpy extraction at the expense of isentropic efficiency.

As shown in Fig. 4-1, an increase in wall temperature to $1500 \mathrm{~K}$ results in higher values for both enthalpy extraction and isentropic efficiency than for the nominal simulation or the simulation wilil 20 second wall temperatures, but lower values than for $1750 \mathrm{~K}$ walls. 
An increase in mass flow rate to $55 \mathrm{~kg} / \mathrm{s}$ results in lower isentropic efficiency and lower enthalpy extraction ratio. While a computation vith decreased mass flow rate was not carried out, it is clear that some improvement in the performance would probably be obtained by dropping the mass flow rate under the Run 006-104 conditions. The reason is that a reduction in the mass flow rate would drive the Mach number distribution toward the optimum, which in the case of the AEDC/HPDE appears to be transonic (see Sec. 4.4).

An increase in magnetic field strength (COBQLRNJDZ) results in higher values for both generator isentropic efficiency and enthalpy extraction ratio.

A variation of $N / O$ ratio to 0.80 leads to an increase in enthalpy extraction ratio, but a decrease in isentropic efficiency. The opposite is observed with an increase in $N / O$ ratio to 1.00 .

It is important to distinguish the genrator isentropic efficiency displayed in Fig. 4-1, which expresses how efficiently the generator made use of the total pressure drop across the generator, with the power train isentropic efficiency, which expresses how efficiently the generator made use of the pressure drop from the combustor to atmosphere. The latter quantity varies almost directly with the enthaipy extraction rate and is perhaps less informative than the generator efficiency. The results in Fig. 4-1 are easily interpreted if this distinction is kept in mind.

The conclusion from this survey is that, at least in the neighborhood of the Run 006-014 operating point, the pay-off from providing hot walls in this experiment is 
significant and justifies a change in operating procedures or hardware to achieve elevated electrode surface temperature. It is apparent that increases of the magnetic field and electrode surface temperatures are the primary means of attaining both the enthalpy extraction and isentropic efficiency goals of the HPDE.

There are methods for mitigating the voltage drops, even in cold-wall designs (e.g., load schedule optimization). The sensitivity of the electrode voltage drops to the parameters studied thus far are described in the following section. 
4.2 Parametric Dependence of Electrode Voltage Drops and Plusma Uniformity

The Q3D parametric survey of performance around the Run 006-014 conditions provides opportunity to investigete the sensitivity of the electrode voltage drops and the plasma nonuniformity to the important parameters of the experiment. In this section, the wall temperature, magnetic field, and load factor dependence of the total electrode voltage drop and two parameters, which characterize the effects of the plasma uniformity on generator performance, are examined. Before investigating the sensitivity of the total electrode voltage drop and the two nonuniformity factors to these three variables, it is useful to review the importance of the voltage drops and the nonuniformity factors in characterizing the performance of the MHD generator.

The primary importance of the electrode voltage drop is that it is a measurable quantity. The electrode voltage drop is measured by subtracting the Faraday voltage from the total transverse voltage one would obtain by extrapolating the core electric potential gradient all the way to the walls. clearly, high electrode voltage drops correspond to reduced power production. However, there is not a simple way of ralating the magnitude of the electrode voltage drops to the reduction in power output. Nevertheless, because the electrode voltage drops are measurable and give an indication of the power penaixy for given operating conditions, it is a useful parameter to compute and to compare with the experimental data. 
The contributing factors to the electrode voltage drop may be easily seen in the simplified MHD electrical equations. We can express the electrode yoltage drop in terms of the formula for the transverse electric field $E_{\mathbf{y}}$ :

$$
E_{y}=\frac{1+\beta^{2}}{\sigma} J_{y}+U B-\beta E_{x}
$$

It is seen from this formula that the voltage drops are equal to the difference between the centerline value of $E_{y}$ and the cross-sectional average value of $E_{y}$, since the Paraday voltage is simply the average transverse electric field, 〈 $\left.\mathrm{E}_{\mathrm{y}}\right\rangle$ multiplied by the transverse dimension of the channel, D. Thus the voltage drop is expressed as follows:

$$
\begin{aligned}
v_{d} & =D\left\{\left(E_{y}\right)_{\Phi}-\left\langle E_{y}\right\rangle\right\} \\
& \left.=D\left\{\left[\left(\frac{1+\beta^{2}}{\sigma}\right)_{\Phi}-\left\langle\frac{1+\beta^{2}}{\sigma}\right\rangle\right] J_{y}+\left[(U)_{\Phi}-\langle U\rangle\right] B-\left[(\beta)_{\Phi}, \beta\right\rangle\right] E_{x}\right\}
\end{aligned}
$$

It is seen from this formula that the electrode voltage drop can be considered to be composed of three components. These components are: the current density times the effective resistivity difference of the cross-section,

$$
\left[\left(\frac{1+\beta^{2}}{\sigma}\right)_{\epsilon}-\left\langle\frac{1+\beta^{2}}{\sigma}\right\rangle\right] J_{y}
$$

the induced field deficit in the boundary layers

$$
\left[(U)_{\Phi}-\langle U\rangle\right] B
$$

and the variation of the Hall parameter through the electrode boundary layers due to pressure and temperature gradients in the boundary layers, 


$$
\left[(\beta)_{\mathcal{E}}-\langle\beta\rangle\right] E_{x}
$$

It is perhaps interesting to tabulate these components of the electrode voltage drops under various conditions. However, the variation of these expressions does not yield a great deal of insight or quantitative information about the effects of experimental parameters upon the overall performance of the generator. Again, this is because the electrode voltage drop itself is not simply related to the performance of the MHD generazor. Also, because $J_{y}$ and $E_{x}$ are factors in these expressions which are themselves affected by the voitage drops, these expressions can hardly be called intrinsic characteristics of the channel or useful indications of the performance penalties associated with the voltage drops.

A much more useful quantity for expressing the effects of experimental parameters on the generator performance is the plasma nonuniformity factor. The plasma nonuniformity factor is defined as

$$
G \equiv\langle\sigma\rangle\left\langle\frac{1+\beta^{2}}{\sigma}\right\rangle-\langle\beta\rangle^{2}
$$

where the brackets indicate cross-sectional averages of the quantities enclosed within. The concept of the plasma nonuniformity factor is a generalization of the original expression by Rosa [4-1].

In the case where variations of the Hall parame:er in the cross-section are reak (usually a good assumption), the expression for the plasma nonuniformity factor can be simplified to the following expression:

$$
G \approx g+(g-1) \beta^{2}
$$


where

$$
g \equiv\langle\sigma\rangle\langle 1 / \sigma\rangle
$$

is the conductivity nonuniformity factor. Thus, the plasma nonuniformity factor, $G$, may be separated into contributions from the nonuniformity of the conductivity, $g$, and the strength of the Hall parameter, which is directly proportional to the magnetic field and approximately inversely proportional to the static pressure.

The plasma nonuniformity factor, $G$, is a useful parameter because $t$ directly divides the power density at any given station in a Paracay generator $\left\langle\left\langle J_{x}\right\rangle=0\right)$

$$
\langle\vec{J} \cdot \vec{E}\rangle=\frac{\langle\sigma\rangle\langle U\rangle^{2} B^{2}(1-K) K}{G}
$$

The conductivity unifority factor, $g$, is an intrinsic parameter which depends only on the distribution of conductivity in the channel cross-section, and not upon specific knowledge of the MHD interaction.

The scale-dependence of $g$ has been investigated by STD Research Corporation in [4-2]. There it was shown that a number of loss mechanisms, including cold bour lary layers, axial current leakage, surface voltage drops, and other effects could be summarized in this simple parameter, g. Also, as the scale of the generator increases (with fixed wall conditions), the value of $B$ was shown to generally decline as the inverse square root of the thermal input to the generator. In most applications, the dependence of the Hall parameter in the channel cross-sectiou is weak enough to make the parameter, $B$, 
a very useful characterization of the potential performance of the MHD generator duct.

The following sections examine the sensitivity of $v_{d}$, $G$, and $g$ to variations it the electrode wall temperature, the magnetic ijield strength, and the load factor. It is to be understood that the effacts of Hali parameter variation, induced field deficit, and effective resistivity variations are lumped into the total electrode voltage drop, $v_{d}$ in the fullowing sections.

\subsubsection{Dependence on Electrode Mall Temperature}

This section compares the total electrode voltage drops and the plasma and conductivity nonuniformity factors resulting from three parametric variations in electrode wall temperature around the nominal Run 006-014 simulation. The nominal calculation has been discussed in section 3.7. The three computations carried out with electrode wall temperature parametrically altered to constant values of $1250 \mathrm{~K}, 1500 \mathrm{~K}$, and $1750 \mathrm{~K}$ have been described in section 4.1. The conclusions regarding the sensitivity of electrode voltage drops and plasma uniformity presented in this section are based upon those four computations, and apply primarily to conditions in the neighborhood of the operating conditions of Run 006-014.

To display the sensitivity of the electode voltage drops and the plasma uniformity, we have chosen to tabulate their values at four stations in the HPDE. Three of these four stations were locations at which the HPDE instrumentation provided transverse profile measurements. The fourth station, which varies about 0.1 m from ccoputation to computation, was 
the location at which the maximum electrode voltage drop occurred in each STD Q3D computation.

The location of the three voltace profile measurement stations and the approximate location $w^{i}$ the marimum voltage drop computed by STD Research Corporation is shown in Fig. 4-2, which has been reproduced from $[4-3]$. Fig. 4-2 also presents a useful definition of the various coordinate systems used by STD Research Corporation and AEDC in iting positions in the channel. The lower axis in Fig. 4-2, which has been marked "STD Q3D coordinates" uses as a reference point the inlet flange of the MHD generator. The STD coordinate system also begins at the inlet flange of the MHD generator, but is expressed in metres rather than in inches. A second frame of reference has as its origin the first loaded electrode. This frame of reference in shown in the upper axis which contains the location and stations numbers, in inches, of the electrode voltage drop measurements made at the HPDE. The three stations at which the electrode voltage drops have been tabulated are, in STD coordicates, $x=1.43 \mathrm{~m}, x=2.78 \mathrm{~m}$, $:$ ind $x=4.62 \mathrm{~m}$. These locations correspond to STA 24.7 in., STA 77.4 in., and STA 215 in.

Fig. 4-3 illusir.tes the calculated variation of the total elejtrode voltage drop at the four stations in the MHD generator as a function of electrode surface temperature. It is noted that the maximum electrode voltage drop declines from approximately 2100 volts to approximately 1200 volts as the electrode surface temperature increases from the "5 sec" value of approximately $600 \mathrm{~K}$ to the assumed temperature $1750 \mathrm{~K}$. At the front of the generator, $x=1.43 \mathrm{~m}$, the electrode voltage 
drop declines from approximately 500 volts to approximately 300 volts as the surface temperature decreases from the 5 sec"value of approximately $1000 \mathrm{~K}$ to the assumed wall temperature of 1750 $K$. The decrease in electrode voltage drop is approximately licear with temperature at each station. For comparison the experimentally measured electrode voitage drops at stations 1.43, 2.78 , and 4.62 a are displayed at the same wall temperature that was assumed for the Q3D simulation of Run 006-014. The agreement between the measured and computed voltage drops appears to be within the experimental uncertainty of the measurements.

As noted above, the impact of the electrode wall surface temperature on the performance of the generator can be summarized by the value of the plasma nonuniformity factor $G$, which results from the nonuniformity of the conductivity fiald due to cold boundary layers. Fig. \$-4 presents the average value of the plasma nonuniformity factor throughout the generator as a function of the average electrode wall temperature. The error bars surrounding each average point correspond to the standard deviation of the value of wall temperature or plasma nonuniformity factor through the generator. For the "5 sec" walls conditions of the Run 006-014 simulation, the average conductivity nonuniformity factor is approximately 2. This means that any given station in the HPDE is operating with half the power density that would otherwise be obtained if the HPDE could provide perfect conductivity uniformity. Of course, doubling the local interaction at every station in the uHD generator would profoundly aiter the flowield in the generator, so that one cannot state categorically that the cold walls cause a $50 \%$ reduction in powel input. 
As the wall temperature increases, the average plasma nonuniformity factor declines to a value of 1.60 at $1250 \mathrm{~K}$, 1.38 at $1500 \mathrm{~K}$, and 1.22 at $1750 \mathrm{~K}$. These reductions in the plasma nonuniformity factor correspond to potential increases in the MHD interaction in the generator of $27 \%, 47 \%$, and $66 \%$, respectively, over the "5 sec" wall iemperature distribution.

The variation of the conductivity nonuniformity factor, which is an intrinsic characteristic of the channel flow, is shown in Fig. 4-5. As the wall temperature increases from the "5 sec" values to 1250,1500 , and $1750 \mathrm{~K}$, the average conductivity noruniformity factor, $g$, declines from 1.26 to 1.17, to 1.11 , and to 1.06 , respectively. As was shown in [4-2], values of this parameter implicit in the original design of the HPDE were typically or the order of 1.03 , corresponding to wall temperatures between $1900 \mathrm{~K}$ and $2100 \mathrm{~K}$. The "5 sec" wall temperature distribution is much more characteristic of small scale generators such as the Avco Mark VI and the UTSI channel.

\subsubsection{Dependence on Magnetic Field}

The dependence of the total electrode voltage drop upon the magnetic field is explained by two principal competing mechanisms. First, because of the increase in Hall parameter, the effects of conductivity nonuniformity are magnified by the contribution of the Hall parameter term in the plasma nonuniformity factor. Compensating this effect is tre fact that an increased magnetic field creates increased surrent density in the electrode boundary layers. This tends to heat the boundary layers through Joule disstpation and improve somewhat the overall uniformity of the conductivity in the cross-section. 
In the neighborhood of the HPDE Run 006-014 operating conditions, these two competing effects tend to slightly increase the electrode voltage drops as the magnetic field is increased to $4 \mathrm{~T}$. Fig. 4-6 illustrates the almost linear dependence of the electrode voltage drop at four stations in the MHD generator as the peak magnetic field is increased from the Run 006-014 value of $3.27 \mathrm{~T}$ to $4.0 \mathrm{~T}$ and to $4.25 \mathrm{~T}$.

Relative to the total induced voltage, however, the electrode voltage drop does not increase very dramatically as the magnetic field increases. Fig. 4-7 illustrates the near insensitivity of the relative voltage drop to the peak magnetic field value between $3.27 T$ and $4.25 T$. This result suggests that one-dimensional models which rely upon a reduction in the induced field in order to represent the electrode voltage drops may be adequate in the neighborhood of the HPDE Run 006-014 conditions as long as the axial variation of the reduction factor is accounted for.

The fact that the relative voltage drop is weakly dependent on peak magnetic field is related to the fact that the plasma nonuniformity factor is essentially constant with magnetic field, as is lllustrated in Fig. 4-8. Again, the competition between the declining conductivity nonuniformity factor due to Joule dissipation (shown in Fig. 4-9) and the increasing Hall parameter due to the increase in the peak magnetic field gives a nearly constant overall plasma nonuniformity factor variation.

The speculation that at high magnetic fields the voltage drops would be reduced by increased Joule dissipation in the boundary layers has not been borne out, at least in the neighborhood of the Run 006-014 operating conditions. The Hall parameter increase is an equal or even dominant effect over the g factor reduction due to Joule dissipation. 


\subsubsection{Dependence on Loat Factor}

The variation of the electrode voltage drops and the plasma uniformity with electrical load factor provides a vivid illustration of the influence of Joule dissipation in the boundary layers upon the performance of MHD generators. As the load factor increases and the generator goes more toward open circuit, the Joule dissipation in the boundary layer decreases. As the boundary layk.s become cooler, the plasma nonuniformity factor, G, and conductivity nonuniformity factor, $G$, increase substantially. In addition, with the reduction of current density associated with increasing load factor, less electron nonequilibrium can be sustained at the electrodes, so that the near electrode region becomes substantially more resistive.

The effect of increasing the Faraday load factor on the conductivity nonuniformity factor is shown in Fig. 4-10. At a load factor $K=0.55$, the average conductivity nonuniformity factor $g=1.22$. As the load factor increases to $0.60, \mathrm{~g}=1.24$. At the Run 006-014 simulation conditions, in which the average load factor is approximate'v 0.61 , the average value of $g$ increases to 1.26 . When the ad factor is held constant at 0.70 , the magnitude of $g$ increases to 1.34 . This is an important effect for cold wall generator designs which depend on Joule dissipation to alleviate some of the thermal gradients in the electrode wall boundary layers.

The effect of increasing load factor on the overall plasma nonuniformity factor, $G$, is illustrated in Fig. 4-11. The increase in $G$ is even more dramatic than the increase in $B$ because, as the channel goes toward open circuit, the flow velocity increases and the static pressure in the generator generally decreases. Hence, as the channel is unloaded, not 
only is there less Joule dissipation in the boundary layers, but the Hall parameter also tends to increase. This results in a steeply increasing plasma nonuniformity factor. The sensitivity of $G$ to load factor reemphasizes the need for calculational procedures which can account for the Joule dissipation in the boundary layers. A calculation which assumed that the value of $g$ and/or $G$ was independent of $B$ or $k$ would clearly be incorrect for this high interaction device.

The behavior of the total electrode voltage drops as a function of load factor reveals a very interesting phenomenon associated with the accumulation of Joule heat through the MHD generator. Fig. 4-12 illustrates the values of the electrode voltage drop at four stations in the AEDC/HPDE. Four calculations are presented. The Run 006-014 simulation, CHPQUUUJBO, and three parametric variations with constant load factor throughout the generator are shown. Also shown are the data from Run 006-014, assuming that the load factor at each measurement station is as it is computed by the simulation.

The computed dependence of the electrode voltage drop on loac factor is rather weak for the cases in which the load factor is held constant. (Of course, a constant voltage drop with increasing current density at lower load factors implies increased power losses.)

0. the other hand, the simulation of Run 006-014, which incorporated a variable load factor (approximately 0.69 at ie inlet to approximately 0.55 at the exit) deviates i.creasingly from the constant load factor trend lines toward the back end of the generator. The maximum voltage drop in the Run 006-014 simulation is approximately 2200 volts, whereas the maximum alectrode voltage drop with constant load factors 
occurs at a load factor of approximately 0.60 , and has a value of approximately 1800 volts. The implication of this result is that the declining load factor profile throughout the generator in the Run 006-014 conditions cause less Joule dissipation upstresm in the generator than would occur if the load factor were held constant at the average Run 006-014 values. This leads to generally colder electrode boundary layers in the downstream regions. This result suggests that the optimum load factor profile from the point of view of minimizing voltage drops would be one which begins initially lower than the average value and increases throughout the generator. While this is considered to be a second order effect on the overall performance of the device, it certainly bears further investigation for the future loading of the AEDC/HPDE.

\subsection{Parametric Variations Around the Transient Simulation of Run 006-014}

The response of the $\mathrm{HPDE}$ to parametric variations in the neighborhood of the Run 006-014 operating parameters was analyzed with the Q3D code family in the previous section. That study identified increases in the wall temperature and magnetic field as the principal means for achieving increased enthalpy extraction and isentropic efficiency of the generator. It is desirable to extend these results to the ultimate capabilities of the facility, i.e., $6 \mathrm{~T}$ operation. This section presents such results.

In the neighborhood of the Run 006-014 simulation studied with Q3D, the flow in the MHD generator is uniformly supersonic. Par from this neighborhood (1.e., at much higher magnetic fields or wall temperatures) the flow may become transonic or subsonic. Because of the additional expense and 
effort required for Q3D computations in the transonic or subsonic modes, which require iterative matching of downstream pressure conditions, it was decided to extend the parametric studies far from the neighborhood of the Run 006-014 conditions by using the quasi-steady option of the TRANSIENT family of codes.

To make such a parametric survey meaningful, it was necessary to incorporate as much of the information from the quasi-three-dimensional calculations using the Q3D family of codes into the TRANSIENT calculations. In order to include the effects of variable wall temperature on the electrical solution in the quasi-steady TRANSIENT calculation, values of the conductivity nonuniformity factor, $g$, obtained from Q3D simulations, were used as input. For the parametric survey of wall temperature, the $g$ values from Q3D Run CHPQUUUJBO were used for the "cold wall" or "5-sec" wall electrode wall conditions. Q3D calculation COBQFAVJDY was used to obtain a "hot wall" or constant $1500 \mathrm{~K}$ electrode wall temperature distribution of $g$ values.

The starting point for all of the TRANSIENT parametric studies was Run BRCYTRCJEB described in Section 3.8. Utilizing the $g$ factors corresponding to cold walls, the magnetic field distribution was increased progressively in steps from the nominal value of $3.27 \mathrm{~T}$ to $4 \mathrm{~T}, 5 \mathrm{~T}$ and then to $6 \mathrm{~T}$. At each step in the magnetic field, sufficient time was provided to obtain a steady-state solution before the next increase in magnetic field was applied. Similarly, the hot wall simulation was carried out by first instantaneously modifying the $g$ factor distribution to correspond to $1500 \mathrm{~K}$ walls of Q3D Run COBQFAVJDY, and then increasing the peak magnetic field 
strength in steps from $3.27 \mathrm{~T}$ to $4 \mathrm{~T}, 5 \mathrm{~T}$ and then to $6 \mathrm{~T}$. The rcsults of this parametric variation in wall temperature and magnetic field are shown in Fig. 4-13. Throughout all of these parametric variations, the load schedule was fixed according to the Run 006-014 distributions, and the $g$ distributions were held fixed at either the "hot wall" or "cold-wall" values. In view of the dependence of $g$ on magnetic flux density discussed in the above sections, the consequence of holding $g$ fixed is probably a slightly conservative estimate of performance.

As is clearly evident in Fig. 4-13, the reduction of the plasma nonuniformity factor due to the increase in wall temperature from the "5 sec" values to a constant $1500 \mathrm{~K}$ distribution makes the difference between achioving the enthalpy extraction goal of the HPDE and not achieving it. The $6 \mathrm{~T}$ operation of the HPDE with $1500 \mathrm{~K}$ walls is predicted to achieve nearly $15.5 \%$ enthalpy extraction with the Run 006-014 nominal load schedule. With "5 sec" walls the HPDE achieves less than $13 \%$ enthalpy extraction. A detailed list of the isentropic efficiency, enthalpy extraction ratio, and the exit Mach numbers are shown in Table 4-1.

It is seen from Table 4-1 that between 4 and $5 \mathrm{~T}$ with hot walls, there is a transition from supersonic to transonic flow with a shock in the generator. While the performance is predicted to increase despite the entry of the shock into the generator, the ramifications of operating a high-interaction MHD generator with a normal shock in the active portion of the generator remain to be investigated. 


\subsection{Optimal Load Schedules}

The goal of the HPDE is performance, not duration. Therefore, the optimization of the loading of the HPDE should be aimed at optimizing performance without the constraints of "rule of thumb" or conventional limitations on current density, Hall field, or other parameters.

For given conditions of velocity, conductivity, and magnetic field, the most important parameter in a Faraday generator is the electrical loading factor, $K=\left\langle E_{y}\right\rangle /\langle U B\rangle$, which defines the operating point on the load line of a local section of the Eenerator ( $K=1$ is open circuit, $K=0$ is short circuit). For given conditions of conductivity, velocity, and magnetic field, the maximum enthalpy extraction per unit length is achieved at a load factor of $K=0.5$. For maximum efficiency, the load factor is somewhat hig ser than 0.5.

In general, for generators of moderate and high interaction, the load-line is not linear. Changing the loading of the generator profoundy alters the distributions of conductivity and velocity in the generator, and multidimensional phenomena enhance the nonlinearity of the electrical performance. Therefore, in order to survey the performance of the device as a function of lnading, the model employed must be capable of accurately predicting the interaction between the MHD forces and the fluid. For the present studies of the HPDE loading, a code from the TRANSIENT code family is used in order to facilitate rapid surveys of the impact of the loading of the generator. The nonlinear coupling between the loading and the fluid behavior is described in this section. 
While sophisticated, constrained optimization procedures could be utilized for oftimizing the loading schedule of the HPDE. it is felt that the variation of the load factor in the axial directiun produce second order effects on the performance. The most important parameter is the average load factor. This conclusion is borne out by comparison with constant load factor calculations and variable load factor calculations, as was discussed in Section 4.2. Therefore, all of the load survey studies that have been conducted to date have been carried out with the specification of a constant load factor throughout the generator.

Many results have been presented in separate reports for the HPDE "nominal" operating conditions. Perhaps more pertinent are the results obta ned when parametric variations of the load factor around the simulation of an actual highpowered run of the HPDE are calculated. Section 4.1 illustrates the load factor influence around the operating conditions simulated by Q3D for the Run 006-014. It was shown in that sertion that the reduction of the load factor in the Run 006-014 experiment could have improved the enthalpy extraction somewhat, but at a penalty in isentropic efficiency. In Section 4.3 , the performance at various magnetic fields up to $6 \mathrm{~T}$ was compared with the TRANSIENT code with hot and cold wall assumptions. These studies utilized the load resistance schedule of Run 006-014.

Relative to the achievement of the performance goals of the HPDE, it was suggested that the variation in the load factor, particularly at the "hot wall", high magnetic field points in Section 4.3 , might allow both the enthalpy extraction and isentropic efficiency goals to be achieved simultaneously. Consequently, the electrical load factor was varied around each 
of the $4 \mathrm{~T}, 5 \mathrm{~T}$ and $6 \mathrm{~T}$ points with hot walls described in Section 4.3. The results of these load factor variations are shown in Figs. 4-14, 4-15, and 4-16 for $4 T, 5$, and 6 , respectively. Also shown on Figs. 4-14 through 4-16 are the points computed with the actual Run 006-014 load schedule. In each case the Run 006-014 load schedule yields slightly better performance than the maximum obtained with constant load factors. However, it is felt that there is not enough of an improvement to warrant a global optimization study with the quasi-one-dimensional TRANSIENT code. It would be more appropriate to carry out an optimization with a multidimensional code which could independently account for the boundary layer dissipation effects described in Section 4.3.3

The importance of operating the MHD generator near the optimum Mach number profile down the generator is emphasized by Figs. 4-14, 4-15 and 4-16. The flow regimes of the generator at the various load factors are indicated by the density of the trend curves. It is apparent that the enthalpy extraction of the generator optimizes in each case for 4,5 , and 6 T at transonic flow conditions. The optimum Mach number for maximum enthalpy extraction rate appears to be that load factor which provides transonic flow. At $4 \mathrm{~T}$, the optimum load factor is approximately 0.65 ; at $5 \mathrm{~T}$, it is approximately 0.72 ; and at 6 $T$, it is approximately 0.80 . The meaning of this is that, as the magnetic field increases, the load factor must also increase (i.e., ihe generator must be less and less heavily loaded) in order to keep the flow field distribution close to the optimum design values. This concept of maintaining the flow field at or near the optimum Mach number distribution is also central to the understanding of the part load and of $f$ design operation of generators of all scales, as is discussed in Section 7.2. 


\subsection{Near Electrode Phenomena with Finite Electrode}

Segmentation

In order to establish a basis for the Q3D current trassport models, near electrode phenomena were studied under the operating conditions of the AEDC/HPDE Run 006-014. Two principal code familes were utilized for the study of near electrode effects with finite electrode segmentation. The FIN family of codes computes two-rimensional distributions of electrical and plasma transport variables in the region between a single pair of electrodes. The assumption implicit in this code family is that the variation in behavior from electrode to electrode is sufficiently weak that periodic boundary conditions can be imposed on the upstream and downstream ends of the region being computed. On the other hand, the ARRAY family of codes considers a string of electrodes without the requirement of periodicity. The upstream and downstream boundary conditions are obtained from Q3D or from the imposition of Neumann boundary conditions specifying local 4 invariant electrical properties.

Two FIN calculations were performed at the $x=2.77 \mathrm{~m}$ station in the AEDC/HPDE. Input data for gasdynamic profiles and initial conditions were obtained from the Q3D Run CHPQUUUJBO, the nominal simulation run for the HPDE Run 006-014. Two computations were carried out. Computation BRCYICLJEA utilized the option with finite reaction rates. Computation BRCYLARJD4 utilized the option for instantaneous reaction rates for electron reactions. Table 4-2 shows a comparison between the Faraday voltage, total voltage drops, Hall field, and local power density, computed by the two FIN calculations and the Q3D simulation. We note that the inclusion of finte electrode effecis in the FIN calulations 
results in the reduction of the Faraday voltage by 68 volts. This Far tay voltage reduction is associated with an increase in the total electrode voltage drop of 128 volts. The Hall voli in the FIN calculations is approximately $50 \mathrm{~V} / \mathrm{m}$ higher in $m_{i}$ - de, and the overall power density at this station is slightly decreased $(4.5 \%)$ by the inclusion of the firite electrode effects. The relative small differences between the Q3D simulation CHPQUUUJBO and the FIN calculations indicates that the diffuse discharge model is suitable ior ulations under the Run 006-014 conditions.

On the other hand it is possible that the inclusion of finite electrode effects and near electrode effects may explain differences between the Run 006-014 measurements and the Q3D computation CHPQUUUJPO which were discussed in Section 3.9. It is anticipated that, as the boundary layers grow and the bulk nonuniformity of the boundary layers begins to dominate the near-electrode effects on the voltage drops, the imporance of near-electrode effects in the back end of the generator will be less. Insufficient resources were available to investigate the near-electrode behavior throughout the entire MHD generator under the present effort. It would be of interest to complete th: analysis thrcughout the entire length of the generator under the Run 006-014 simulation conditions.

Two ARRAy calculations were carried out to investigate near-electrode phenomena without the requirement of periodicity. Computation BRCYYsVJEC also considered the nominal conditions of operation in the neighborhood of $x=2.77$ $m$ in the AEDC/Hil? Run 006-014. A region of 2.5 channel diameters around $x=2.77 \mathrm{~m}$ was chosen for simulation. An expanded view of the current pattern in the near-electrode regions computed by the ARRAY family of codes at $x=2.77 \mathrm{~m}$ is 
shown in Figs. 4-17 and 4-18 for the cathode region and anode region. Instabilities in the near-anode region is apparent in Fig. 4-17. The operating characteristics of the electrodes at $x=2.77 \mathrm{~m}$ is quite comparable to the $F I^{\circ}$ results discussed above and with the measurements of Run $006 \cdot 014$.

Fig. 4-19 illistrates the current pattern in the entire computational region of computetion BRCYYMVJEC. In this figure, the details of the near-electrode behavior is suppressed by the coarseness of the plotting grid.

Fig. 4-20 shows the corresponding equipotentia's in tnis ARRAY computation. It is noted that the equipotentials are rather uniform. The steep inclination of the equipotential in the core in due to the relatively low Hall parameter (1.2) at this station. The curvature of the equipotentials near the walls reflects the presence of vol age drops due to the imposed gasdynamic boundary layer profiles.

The importance of relaring the periodicity assumption is emphasized in Figs. $1-21$ and 4-22. These Figures illustrate the result of calculation BRCYYQPJEC, in which the exact conditions of th previous ARRAY calculation were varied only by assuming that a single electrode pair went to short-circuit. The imposition of a short-circuit on this region causes a gross distortion of the equipotential pattern of Fig. 4-20, as shown in Fig. 4-21. Walls which no:mally operate at a potential difference of $1700 \mathrm{~V}$ or more are suddenly forced, in a very small region, to operate at the same potential. Very considerable electric fields developed due to this condition are likely to cause severe electrical breakdowns in the reighborhood of the Faraday short. 
In addition, all of the power being produced in a region approximately one or two diameters upstream and downstream of the aHD generator is avaliable to be coupled Into the Paraday short. As a result, the surge current through the Faraday short increases by two orders of magnitude over the normal operating current of the electrode pair. It is evident that the increase in the current to the external load circuit rould be enough to cause either mechanicai or thermal damage to the load circuit connections. This and similar faults must be protected against if tuture HPDE operations.

The result of this "Paraday catastrophe" vividiy illustrate, that fine electrode segmentation is both a blessing and a curse. Fine electrode segmentation increases the overall current uniformity and decreases Hall effect reductions in the power output of the generator. On the other hand, if Faraday faults do occur, they will occur with more damaging consequences with finer electrode

\subsection{Current Distribution in the End Fegions}

The HPDE inlet eddy currents were investigated, utisizing a two-dimensional, finite electrode, finite reaction rate code from the INLET famtly of codes. The operating conditions were specified from the outputs of the Run 006-014 simulation by Q3D (CHPQUUUJBO). The presence of open circuit electrodes in the magnetic field gradient of the end regions of the HPDE leads to internal circulating currents in the plasma. These are depicted in Fig. 4-23 for the computation BRCYZGGJBC. Because the passage of current in the downstream end of the current loops is through a higher magnetic field than the return current through the upstream portion of the loops, there is a net reduction in the total pressure of the power train. 
This loss of pressure does not produce useful work. Under the Run 006-014 operating conditions, it is estimated, based on this computation, that this pressure drop is of the order of 0.07 a tmospheres.

Fig. 4-24 shows the equipotentials associated with the current pattern in Fig. 4-23. It is interesting to note that the lack of current through the upstream boundary iayers make these boundary layers very good insulators. The cold upstream boundary layers effectively shield the core from the open-circuit electrodes, which serve as insulators, and promote the circulation of current inside the plasma. It is noted that the voltage in the core at the first loaded electrode persists for a considerable distance upstream of the generator. 


\section{REFERENCES}

[4-1] R.J. Rosa, Magnetohydrodynamic Power Generation, McGraw-Hil, New York, 1968.

[4-2] C.D. Maxwell, S.T. Demetriades, D.A. Oliver, A.A. Vetter, T.F. Swean, "Scale-up of Advanced MHD Generators", AIAA $18 \mathrm{th}$ Aerospace Sciences Meeting, Paper No. $A I A A=80=0179$, Pasadena, California, January 1980 .

[4-3] R.L. Lowery, "High Performance Demonstration Experiment Supersonic Faraday Performance Evaluation", Data Package: Propulsion Find Tunnel Facility, Arnold Engineering Development Center; Preliminary Data: Run MI-006-006, September 1979. 


\section{TABLE 4-1}

\section{Results of TRANSIENT Computations Performed for Task I Nominal Loading from HPDE Run 006-014 and Combustor Thermal Input of $303.2 \mathrm{kT}$}

\begin{tabular}{|c|c|c|c|c|c|c|}
\hline & & & Tall & & Enthalpy & \\
\hline Run & $\begin{array}{l}B_{\max } \\
(T) \\
\end{array}$ & $M_{\text {exit }}$ & $\begin{array}{l}\text { Temp } \\
(\mathrm{K}) \\
\end{array}$ & $\begin{array}{l}\text { Power } \\
\text { (MiT) } \\
\end{array}$ & $\begin{array}{c}\text { Extraction } \\
(\%) \\
\end{array}$ & $\begin{array}{l}\text { Efficie ucy } \\
\quad(\%)\end{array}$ \\
\hline \multirow[t]{4}{*}{ BRCYTRCJEB } & 3.274 & 1.794 & "5 s" & 23.60 & 8.24 & 37.46 \\
\hline & 4.0 & 1.648 & "5 s" & 28.95 & 10.11 & 40.72 \\
\hline & 5.0 & 0.651 & "5 s" & 34.17 & 11.94 & 12.25 \\
\hline & 6.0 & 0.654 & "5 s" & 36.39 & 12.71 & 45.11 \\
\hline \multirow[t]{3}{*}{ BRCYUXWJEB } & 4.0 & 1.124 & 1500 & 36.80 & 12.83 & 43.88 \\
\hline & 5.0 & 0.650 & 1500 & 41.75 & 14.56 & 51.56 \\
\hline & 6.0 & 0.699 & 1500 & 44.39 & 15.48 & 53.81 \\
\hline
\end{tabular}

$0-4361$ 


\section{TABLE 4-2}

Electrical Performance Parameters at $x=2.77 \mathrm{~m}$ in the AEDC/HPDE Run 006-014: Comparison Between FIN Calculation BRCYICLJEA and Q3D Calculation CHPQUUUJBC

\section{Quantity}

Run No.

Total Electruce

Voltage Drop, V

Faraday Voltage, $V$

Centerline Hall

Field, V/m

Local Power
Density, $\mathrm{m} / \mathrm{m}^{3}$ $\underline{\text { FIN }}$

Q3D

BRCY ICLJEA CHPQUUUJBO

830

702

1676

1743

984

959

16.05

16.78 
STD AESEACCH CORPOAAIION

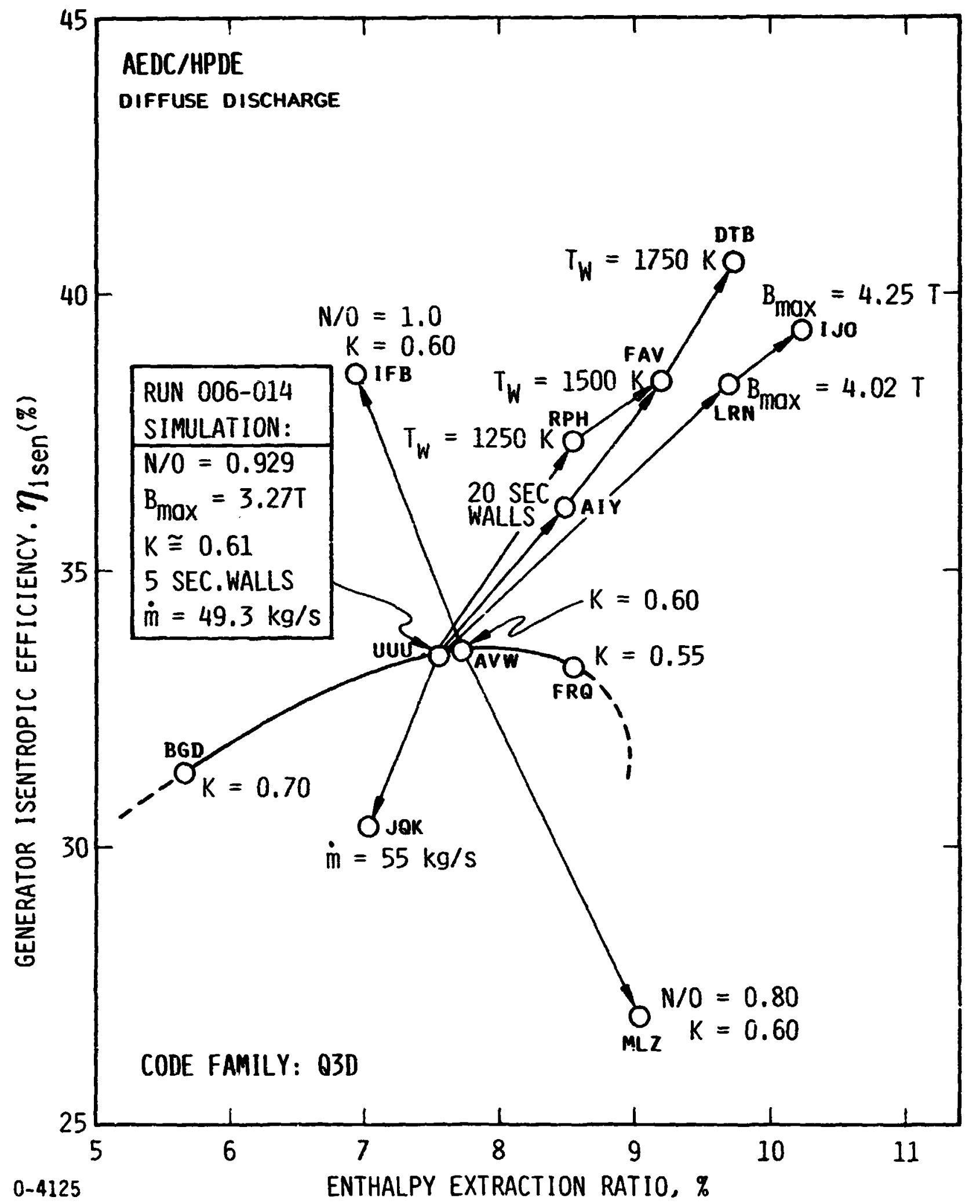

Fig. 4-1. Generator isentropic efficiency vs. enthalpy extraction ratio for simulation of HPDE Run 006-014 and simulations with variations in the conditions from those of Computation CHPQUUUJBO. Three-letter code by each point is the abbreviated STD Computation number. 


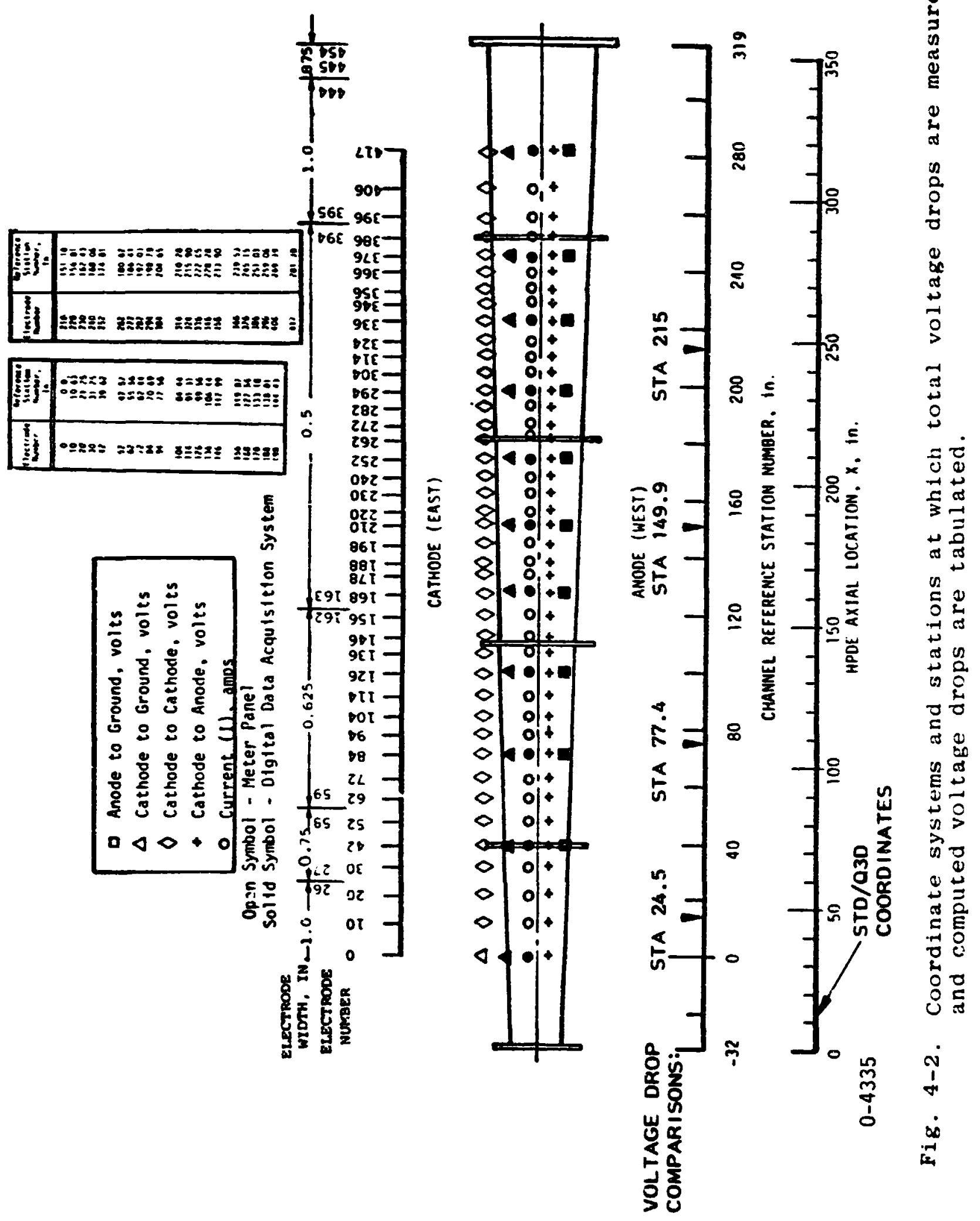


STD heseabch coppotation

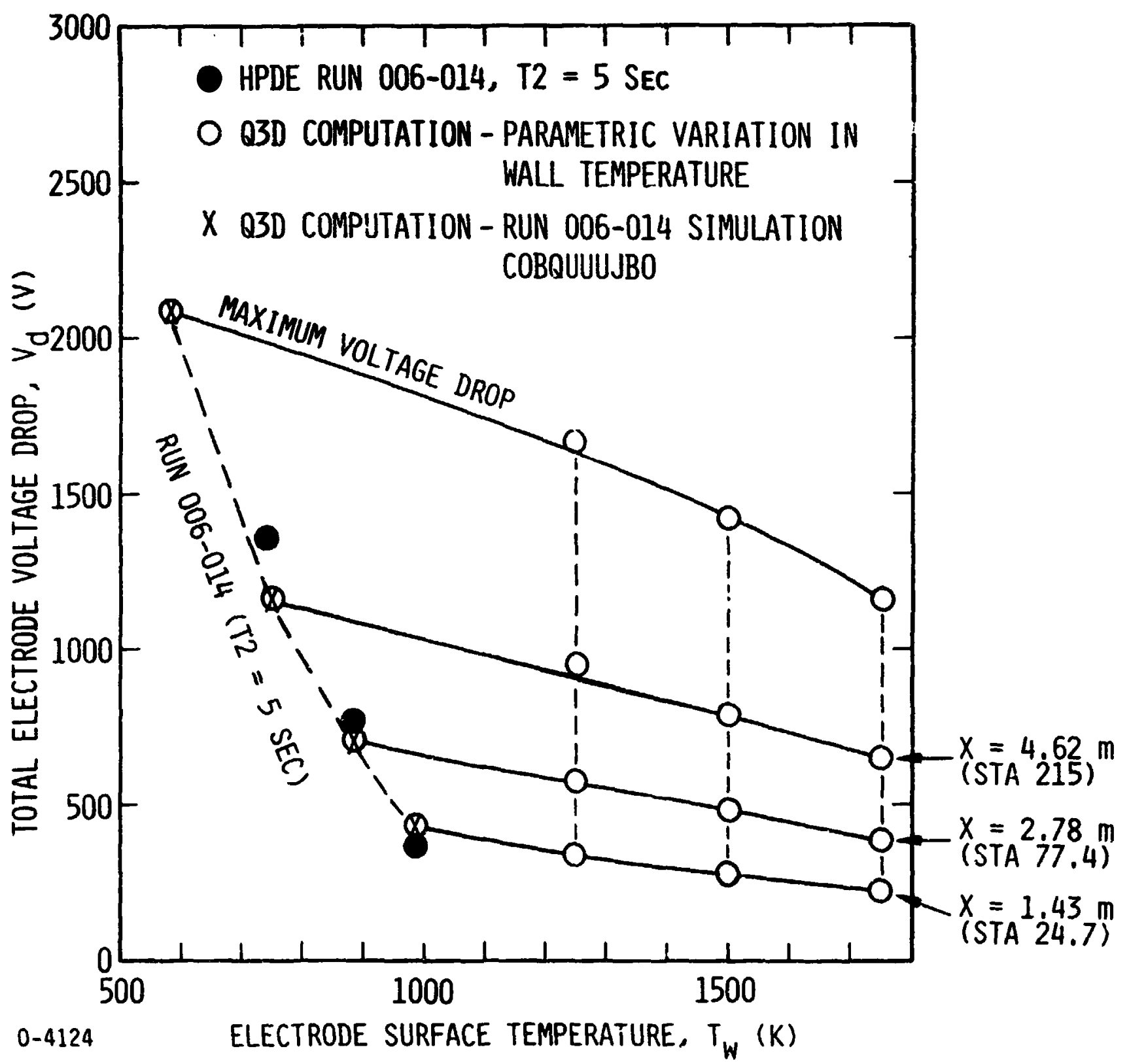

F1g. 4-3. Variation of total (anode + cathode) electrode voltage drop with electrode surface temperature under the conditions of the Q3D simulation COBQUUUJBO of the HPDE Run 006-014. 
STD heseaAch CORPohation

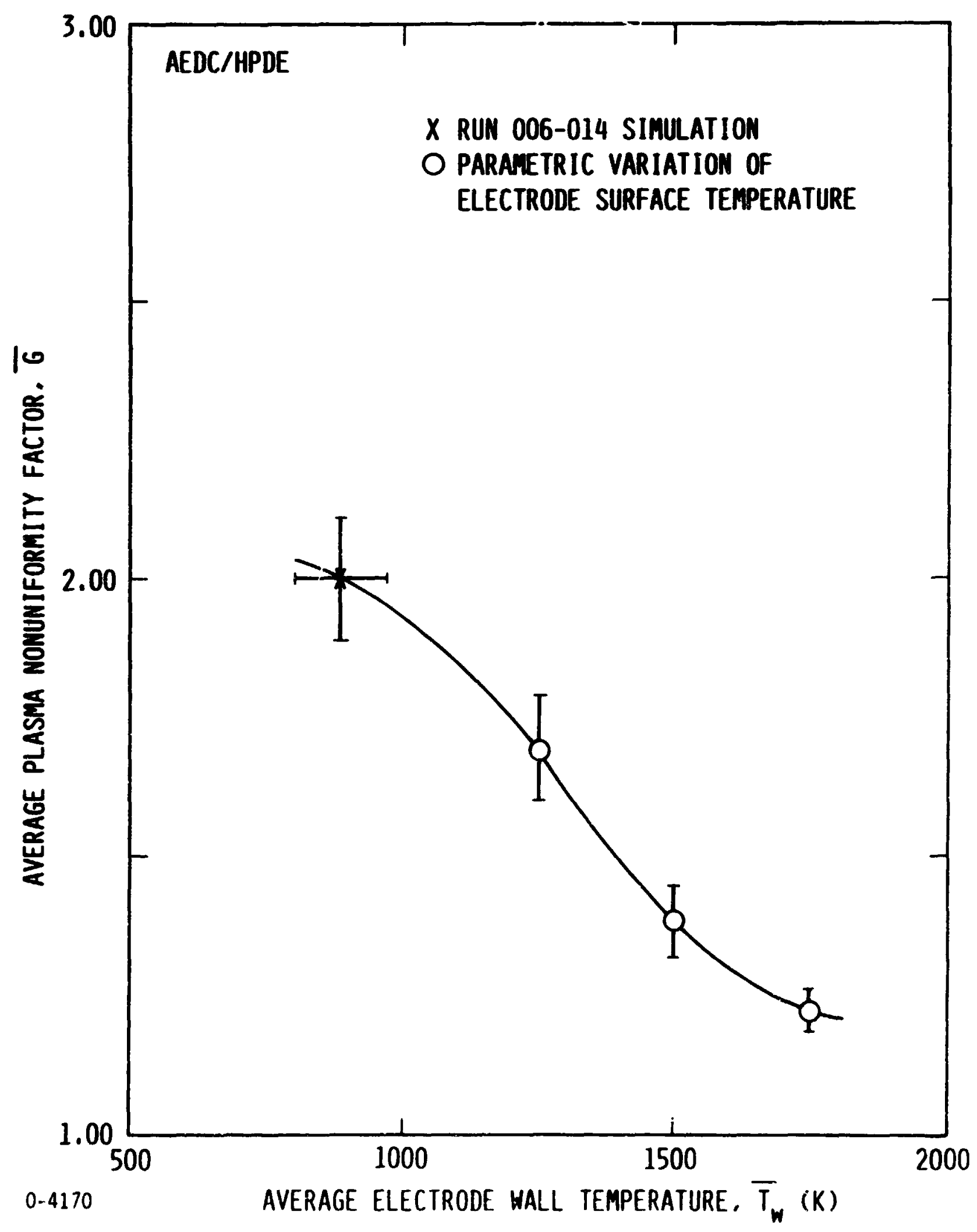

Fig. 4-4. Variation of the plasma nonuniformity factor $G$ with electrode wall temperature: Parametric variations around the AEDC/HPDE Run 006-014 simulation conditions. 


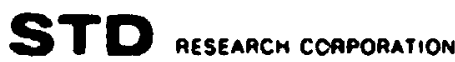

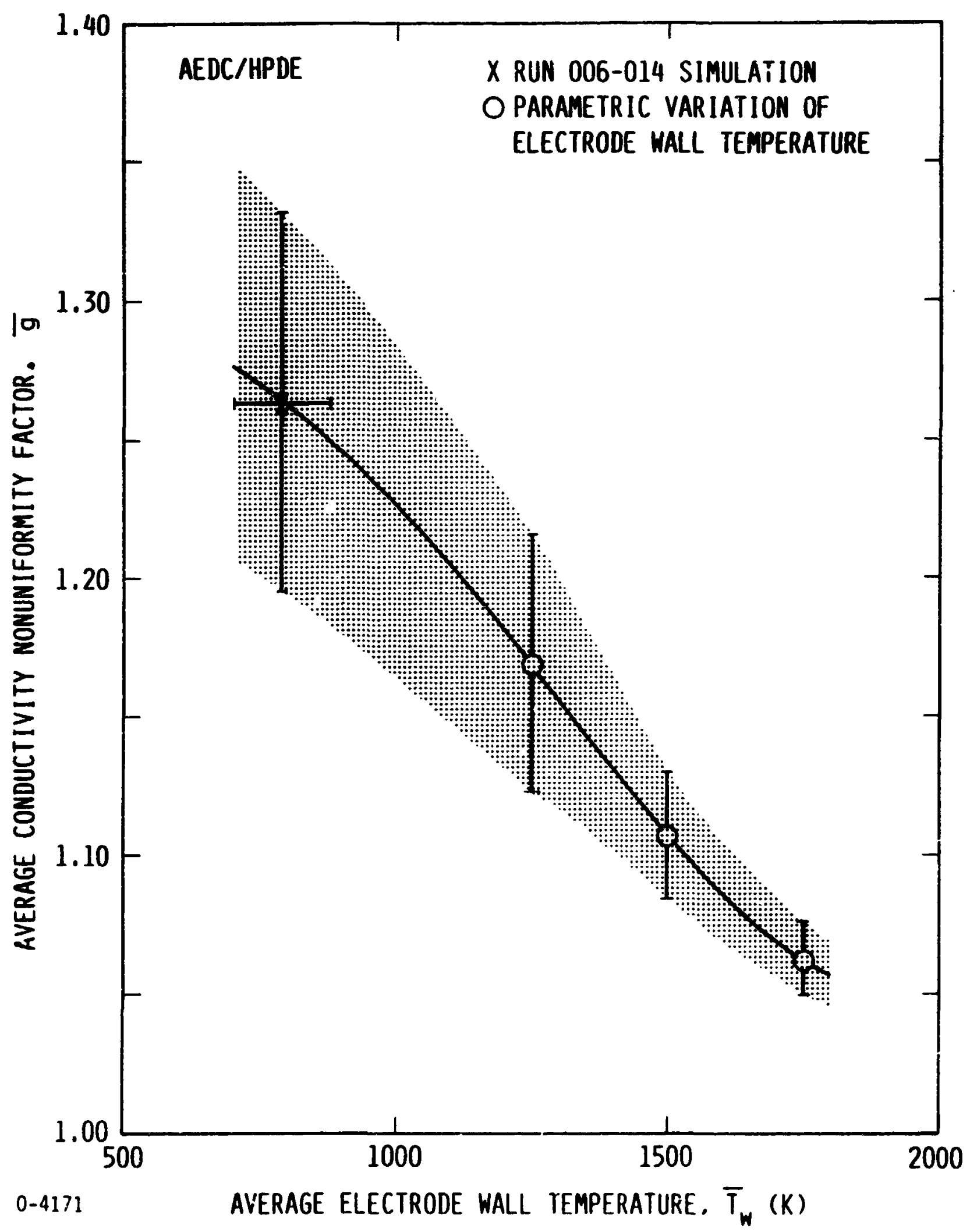

Fig. 4 5. Variation of the conductivity nonuniformity factor with electrode wall temperature: Parametric variations around the AEDC/HPDE Run 006-014 simulation conditions. 
STD hesearch Copropation

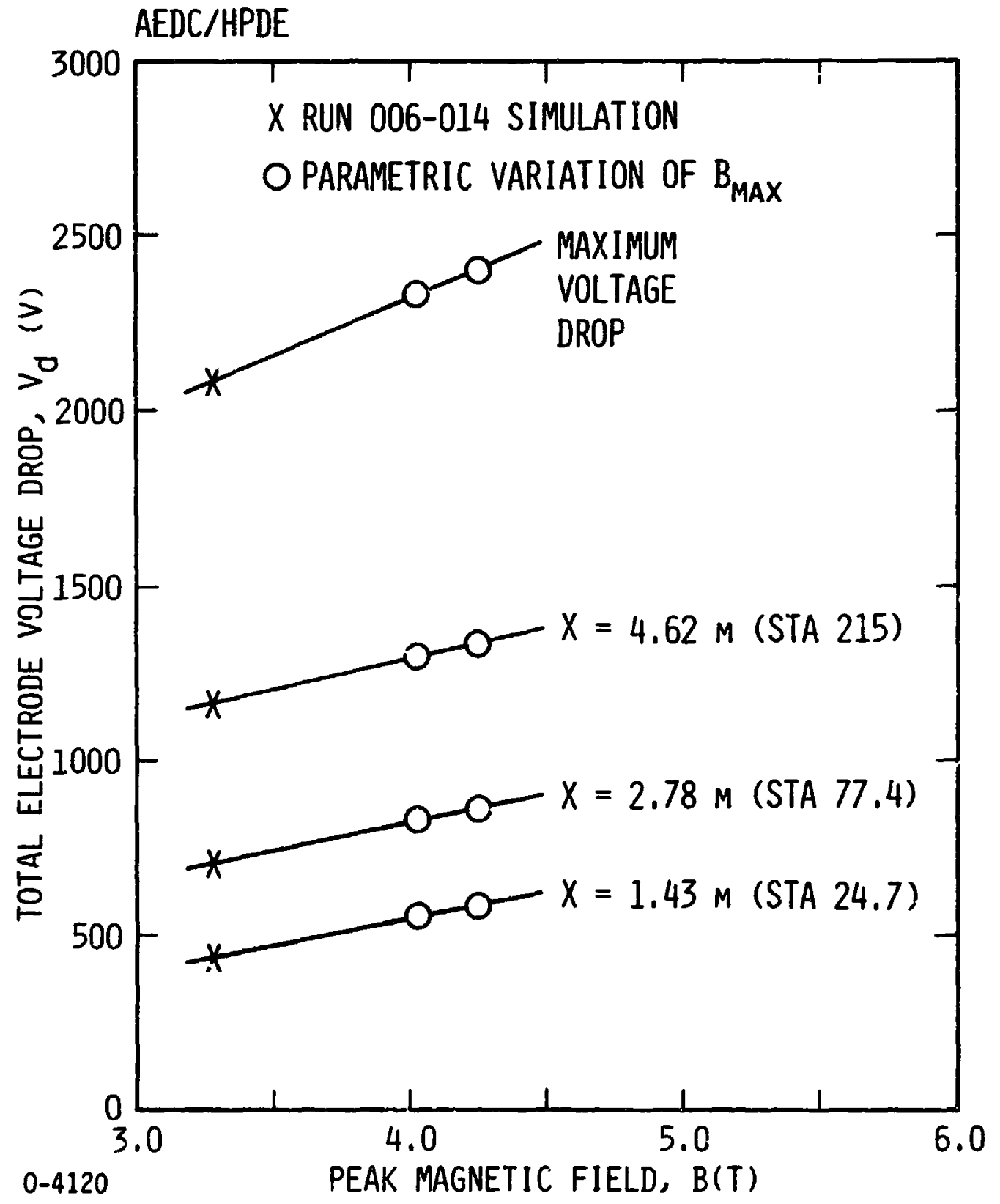

Fig. 4-6. Variation of the total electrode voltage drop with peak magnetic field at various stations in the AEDC/HPDE; parametric variations around the Q3D simulation of the HPDE Run 006-014 
OTD aeseareh conpuamion

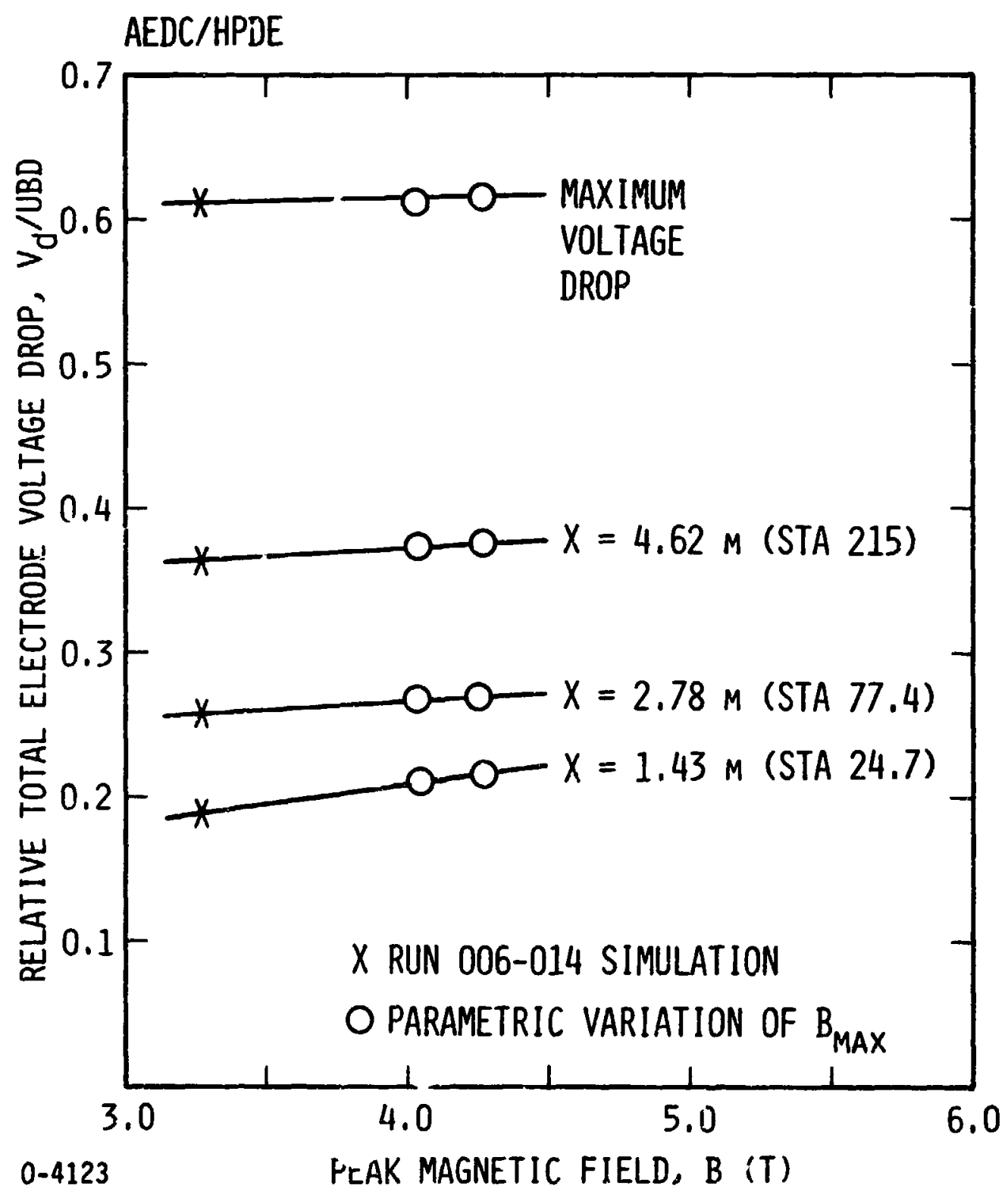

Fig. 4-7. Variation of the relative electrode voltage drops with peak magnetic field at various stations in the AEDC/HPDE: parametric variations around the Q3D simulation of the HPDE Run 006-014 
STD aeseahch Copporation

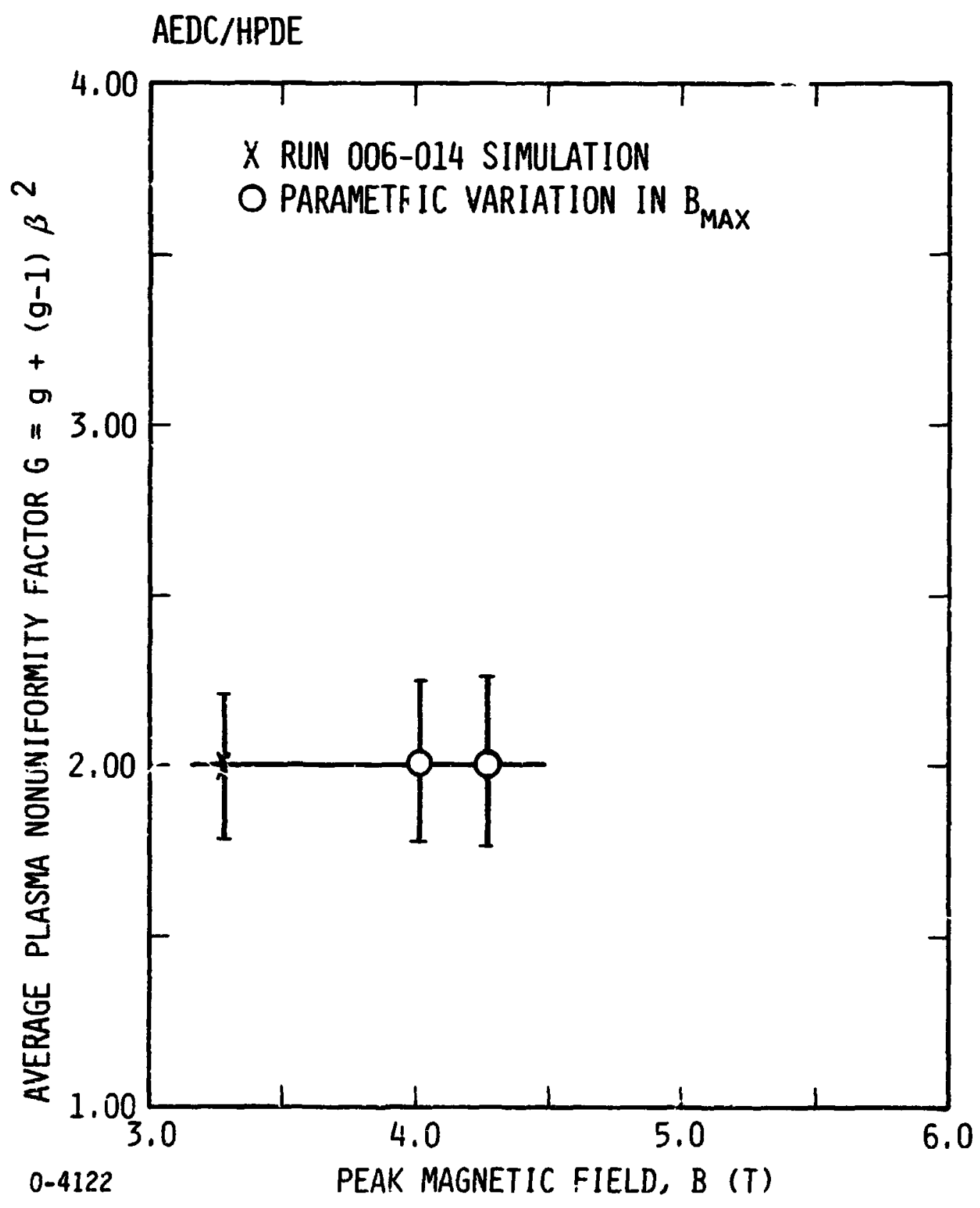

Fig. 4-8. Variation of the plasma nonuniformity factur with peak magnetic field in the AEDC/HPDE: parametric variations around the Q3D simulation of the HPDE Run 006-014 
$\$$ aesearch commation

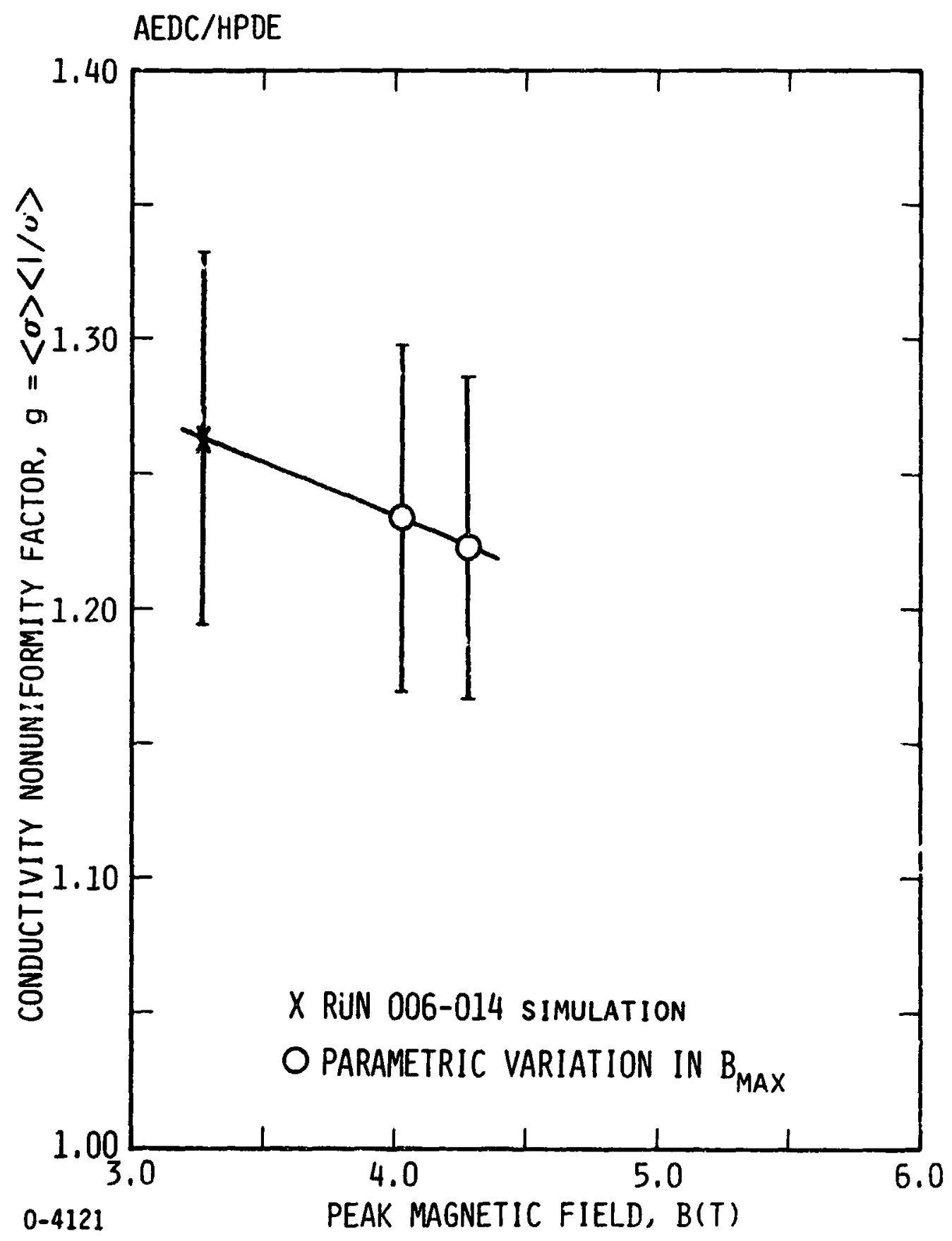

Fig. 4-9. Variation of the conductivity nonuniformity factor with peak magnetic field in the AFDC/HPDE: parametric variations arcund the Q3D simulation of the HPDE Run 006-014 
STD heseabch cohroasal un

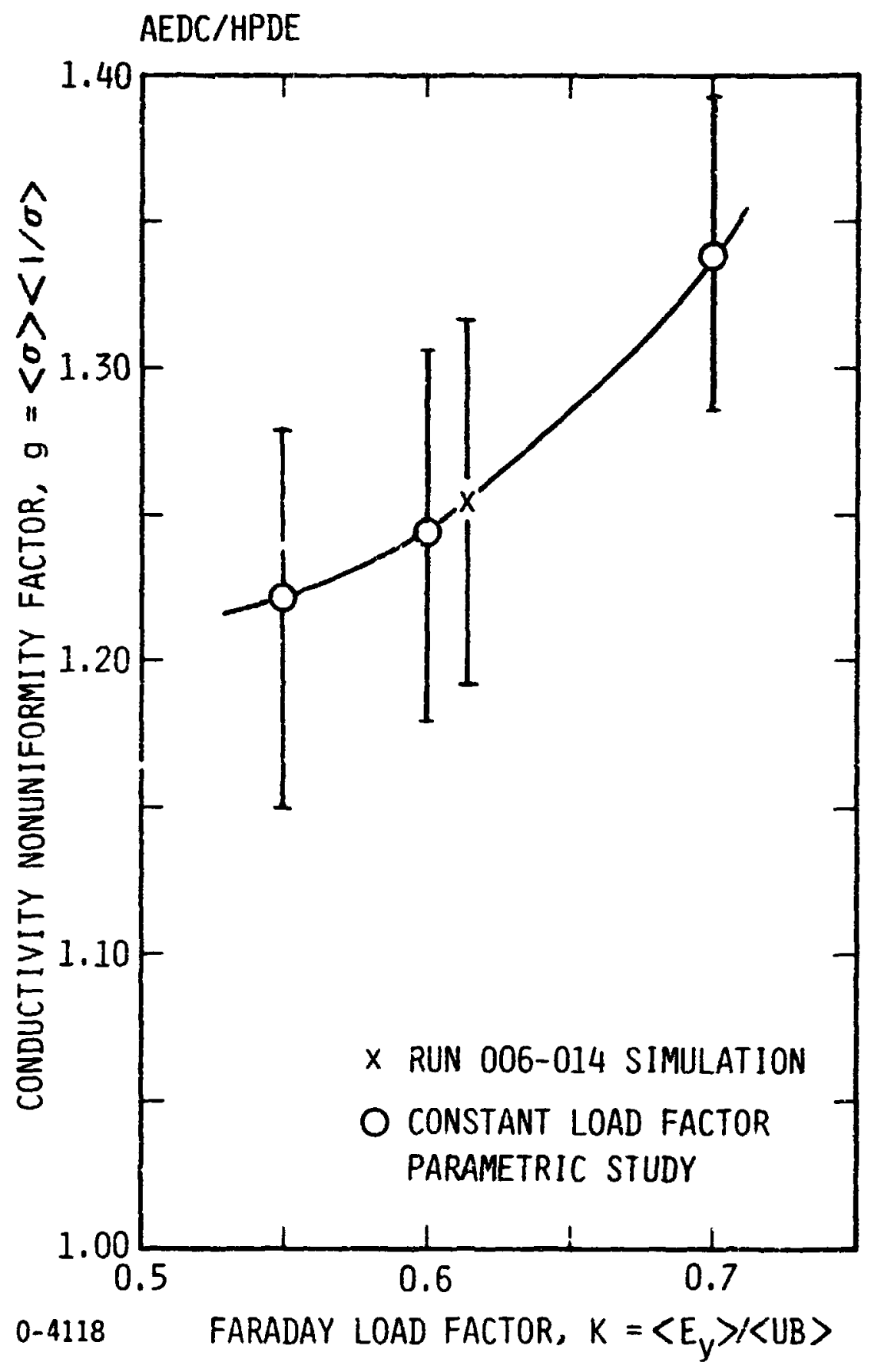

Fig. 4-10. Variation of the conductivity nonuniformity factor with Faraday load factor: parametric variations around the Q3D simulation of the AEDC/HPDE Run 006-014. 
STD aeseaAch coaporation

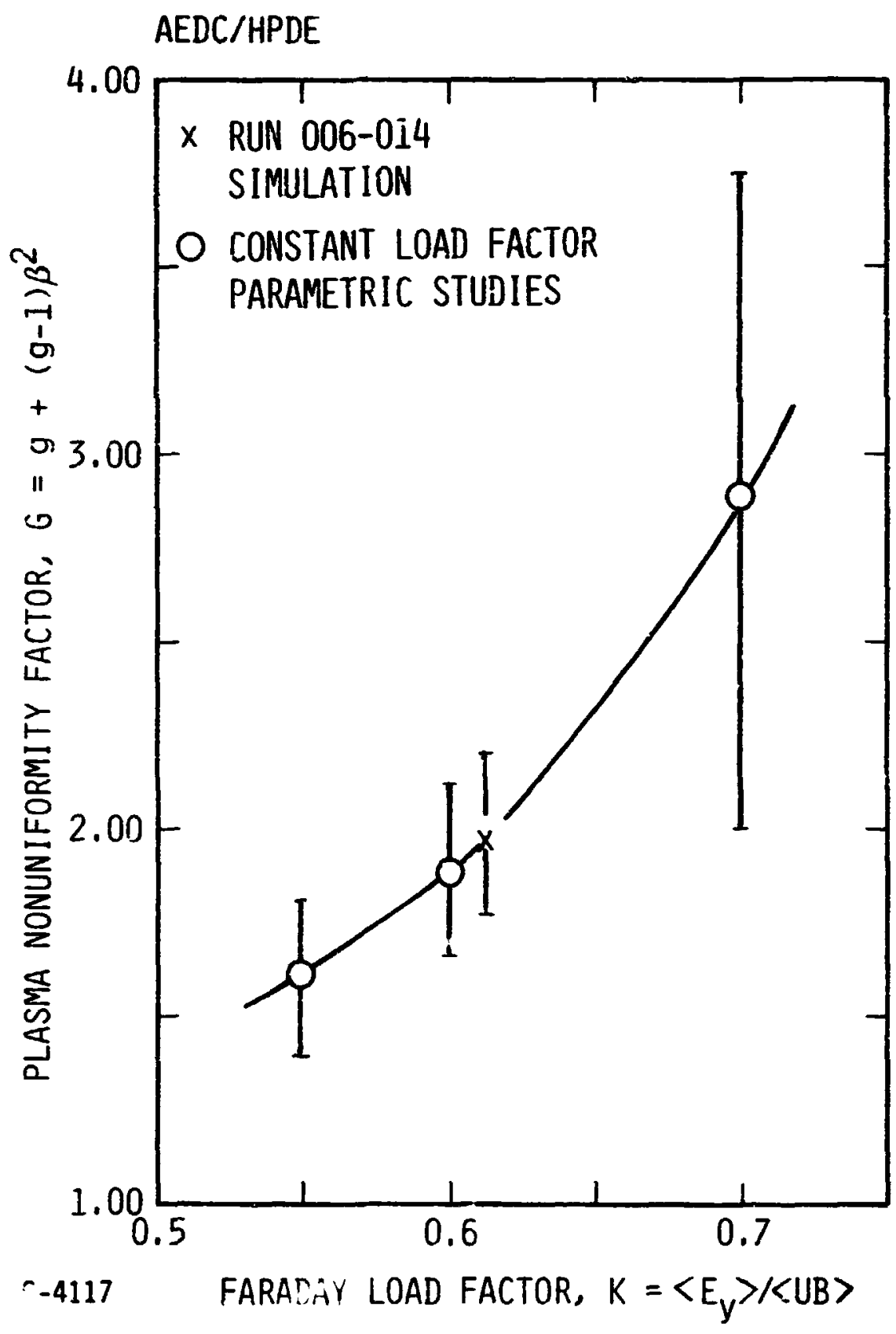

Fig. 4-11. Variation of the plasma nonuniformity factor with Faraday load factor: parametric variations around the Q3D simulation of the AEDC/HPDE Run 006-014. 


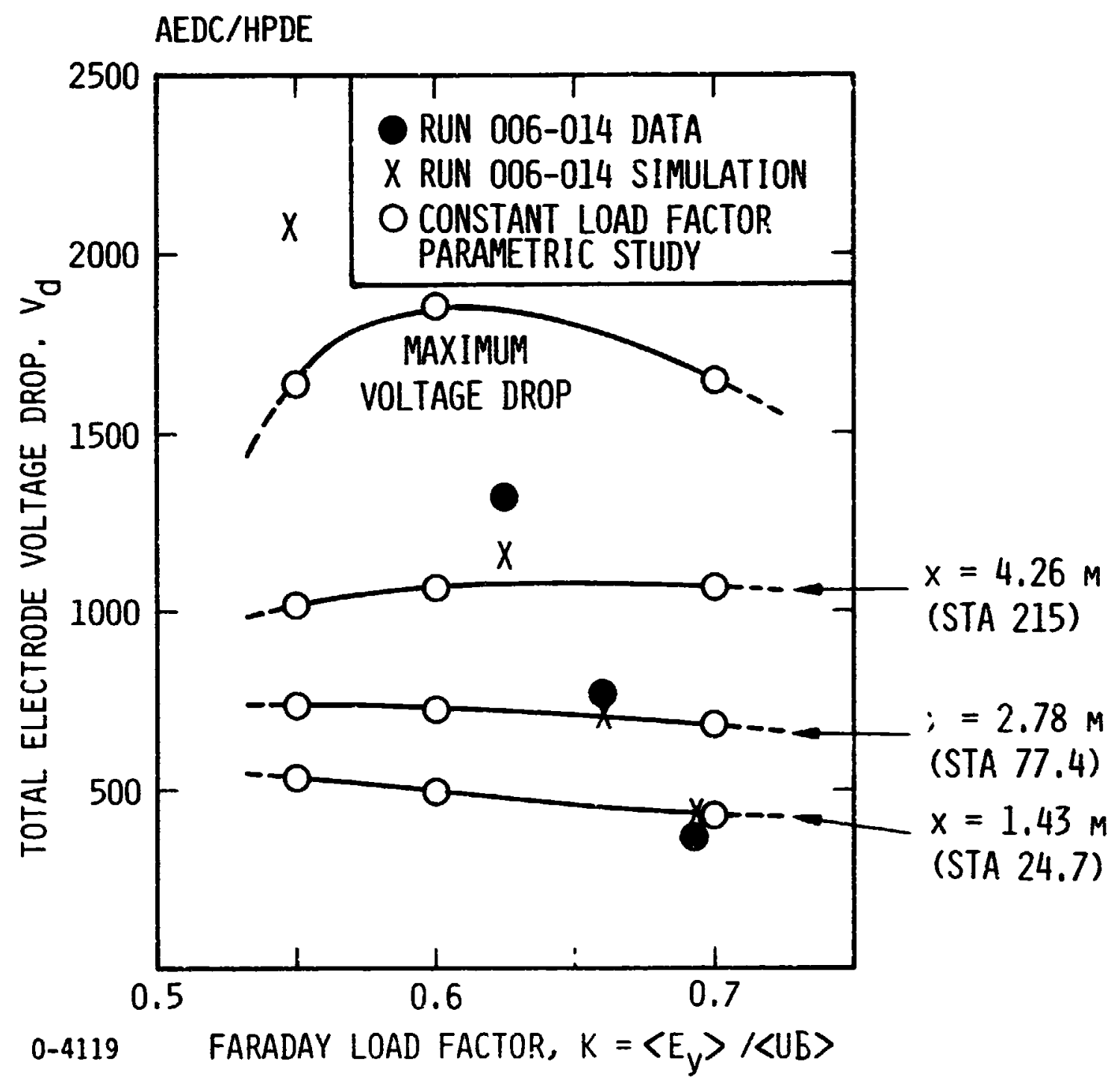

Fig. 4-12. Variation of total electrode voltage drop at various stations in the AEDC/HPDE with Faraday load factor: parametric variations around the Q3D simulation of Run 006-014. 
STD hesEATC, GOAPUHATION

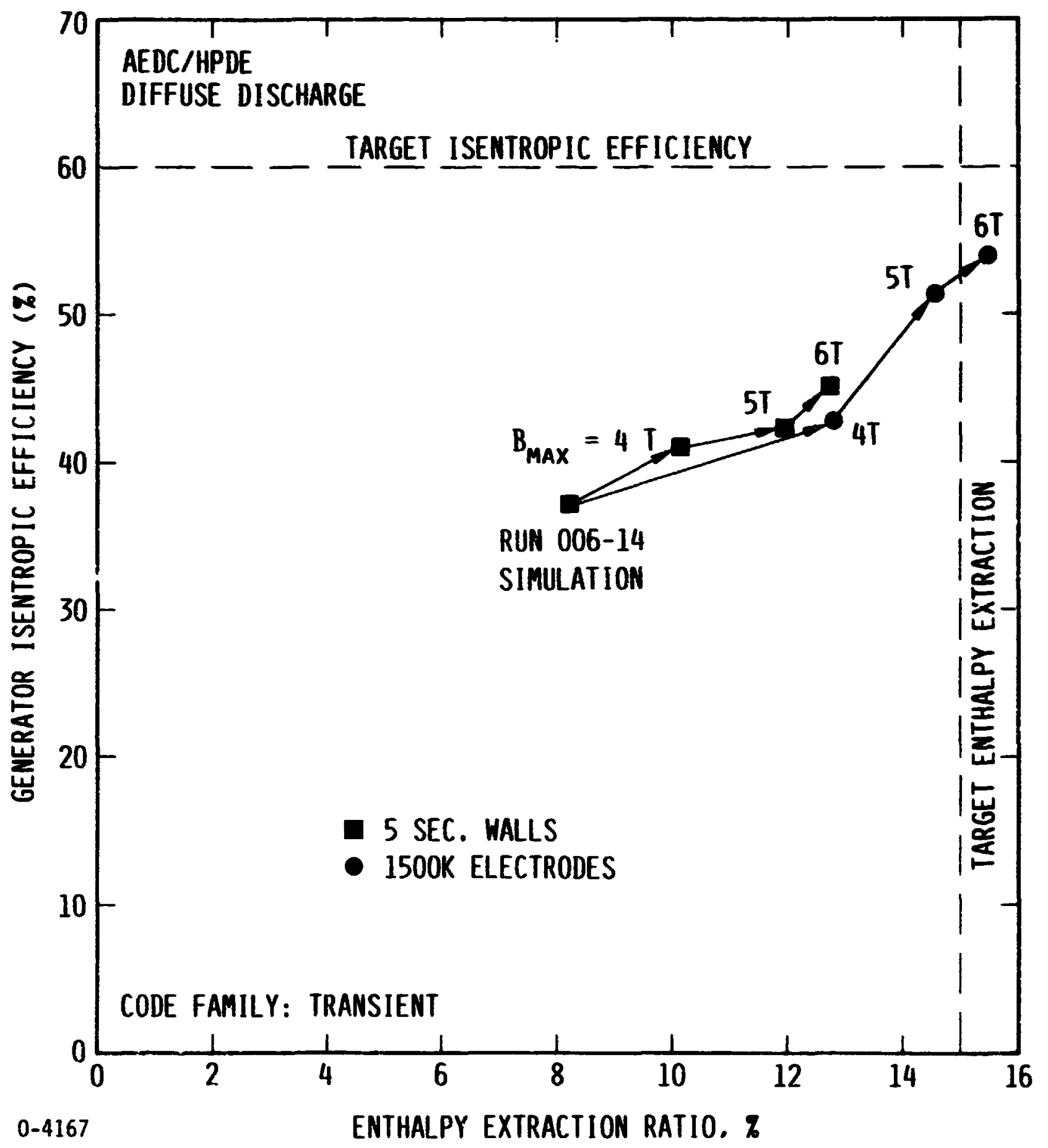

Fig. 4-13. Generator isentropic efficiency vs, enthalpy extraction ratio for simulation of HPDE Run 006-014 and simulations with variations in the magnetic field and wall temperature distribution 
STD geseaAch Conposerion

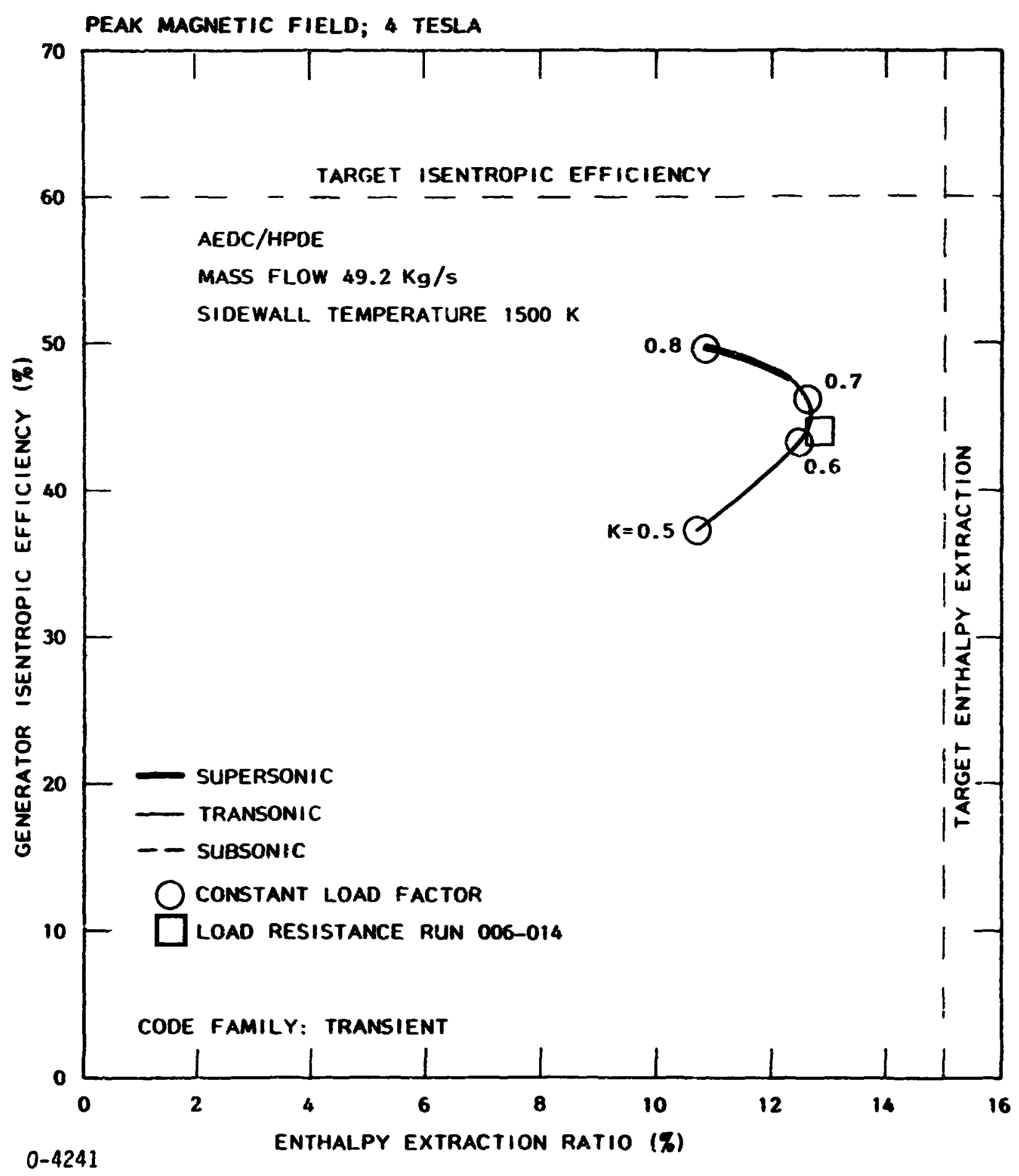

Fig. 4-14. Generator isentropic efficiency vs. enthalpy extraction ratio for simulation of AEDC/HPDE Run 006-014 with variations in load factor and a magnetic field of 4 tesia 


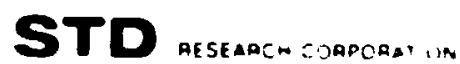

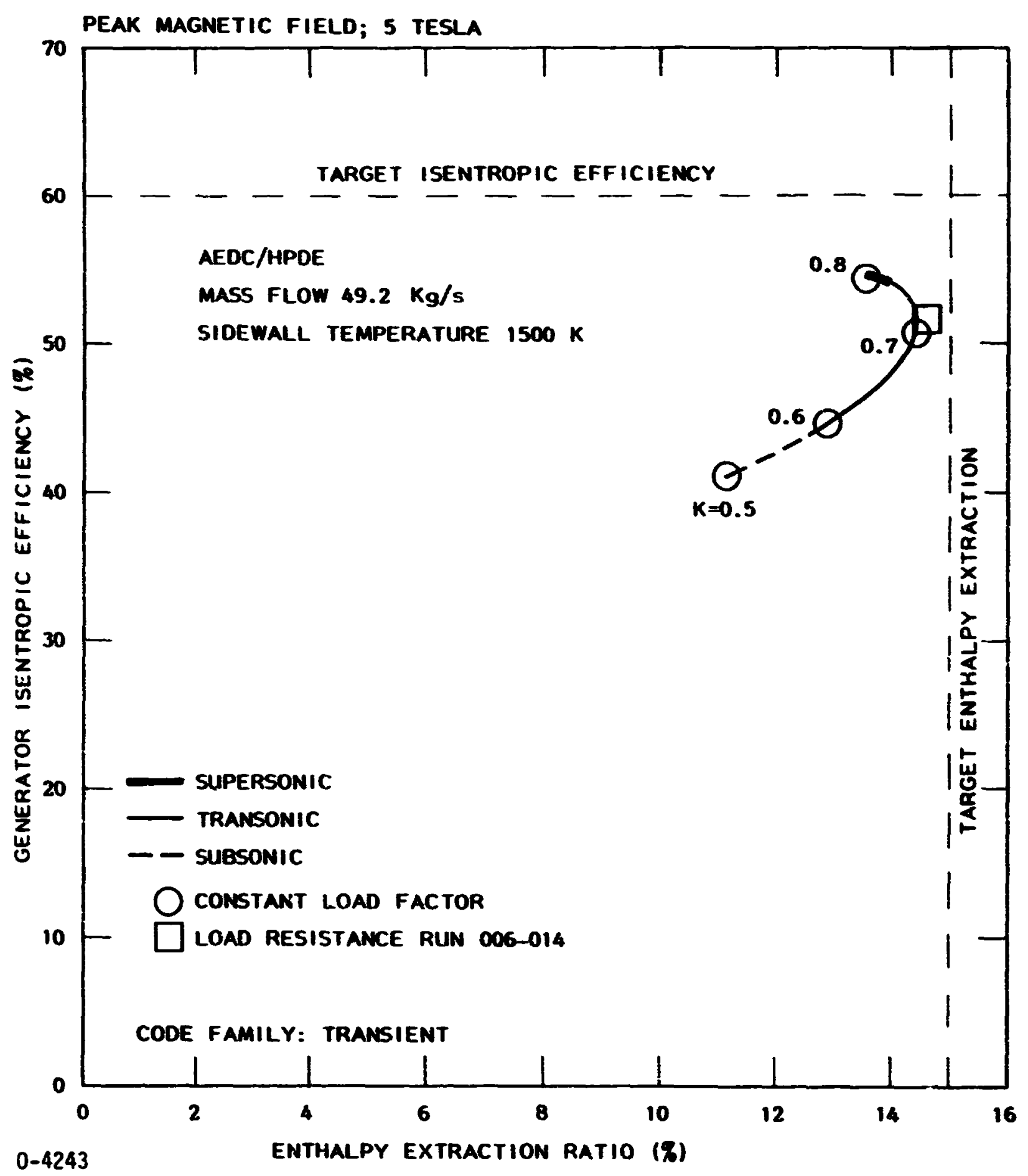

Fig. 4-15. Generator isentropic efficiency vs. enthalpy extraction ratio for simulation Of AEDC/HPDE Run 006-014 with variations in load factor and a magnetic field of 5 tesla 


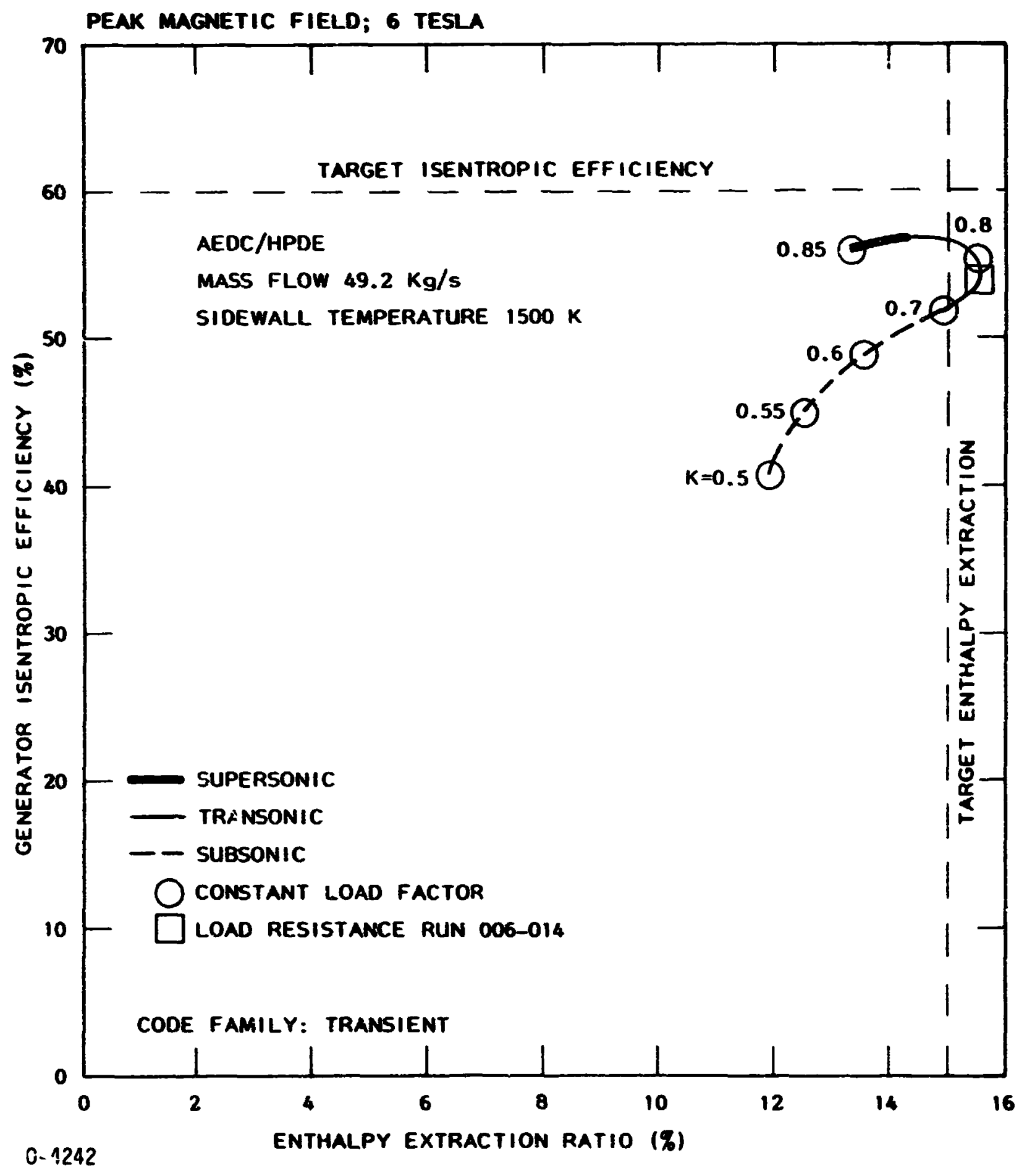

Fig. 4-16. Generator isentropic efficiency vs, enthalpy extraction ratio for simulation of AEDC/HPDE Run 006-014 with variations in load factor and a magnetic field of 6 tesla 


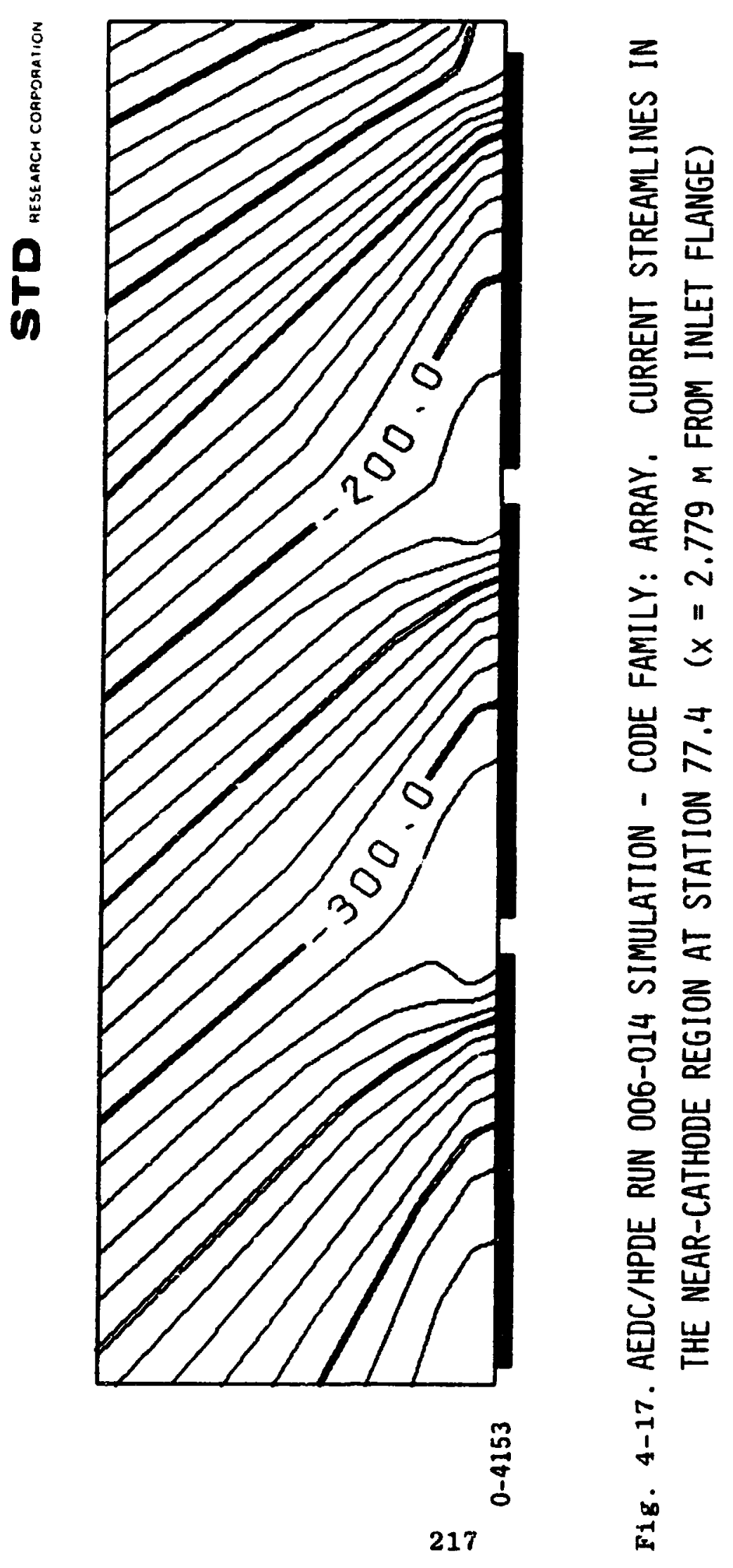



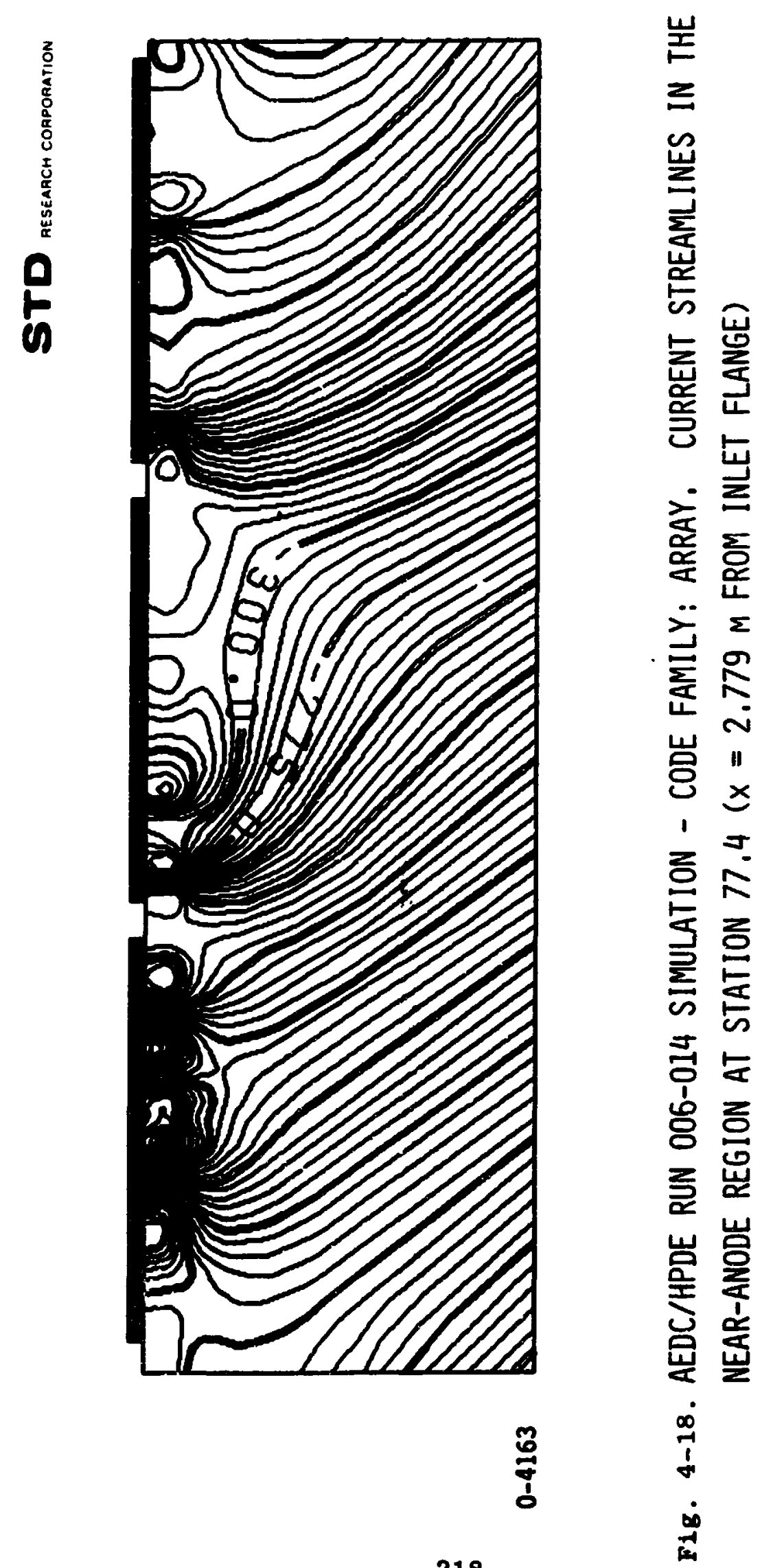


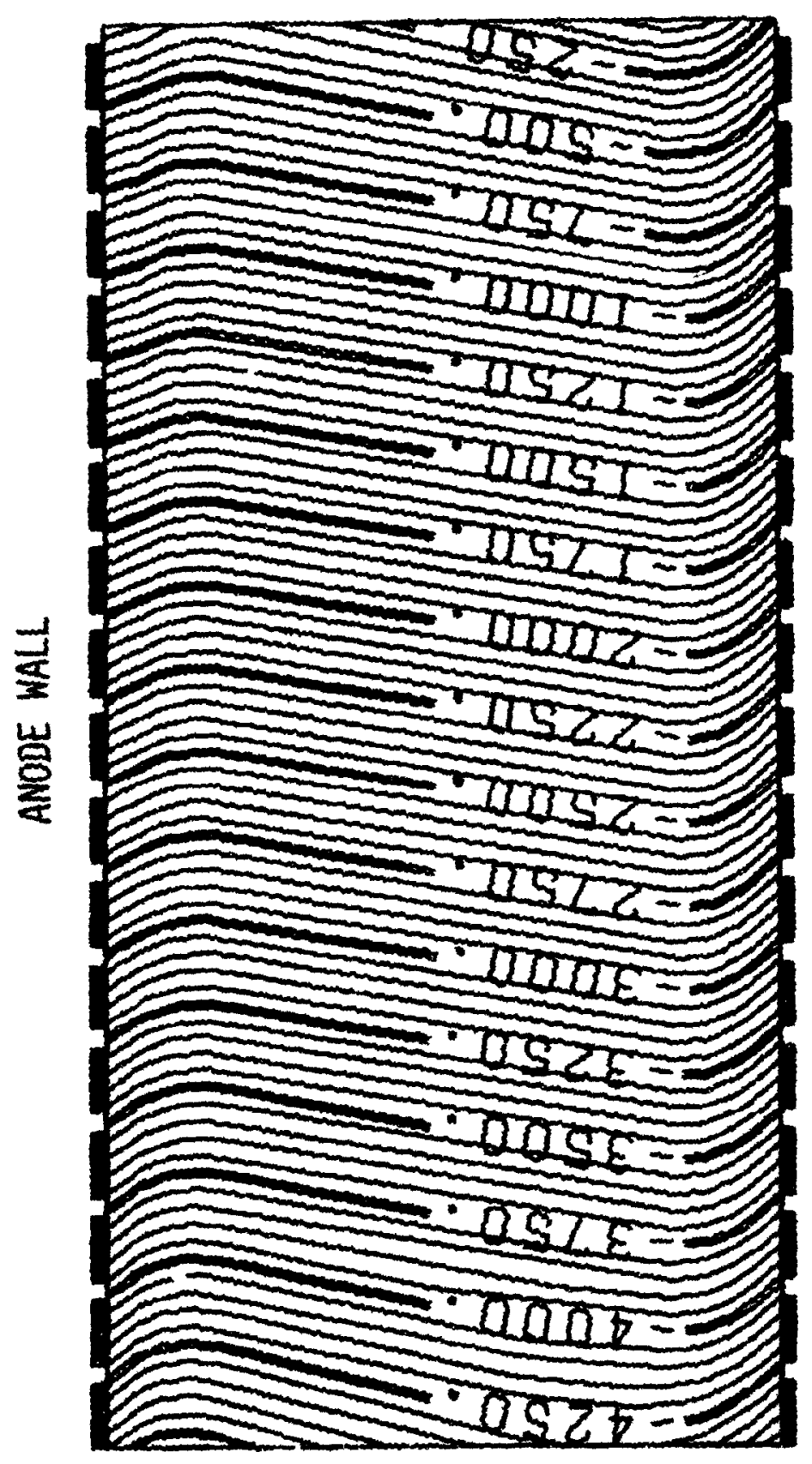

焉

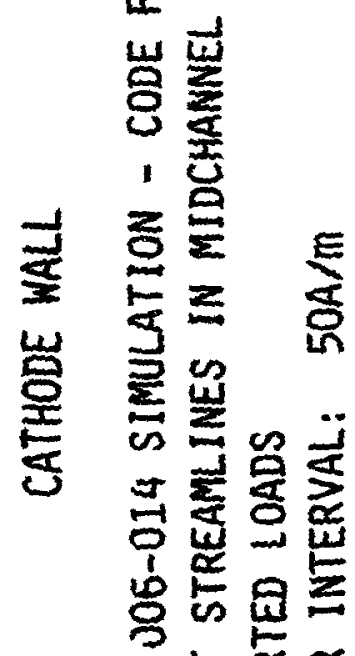
춘 웅 웅

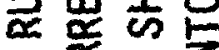
w实 도

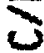

4

in 


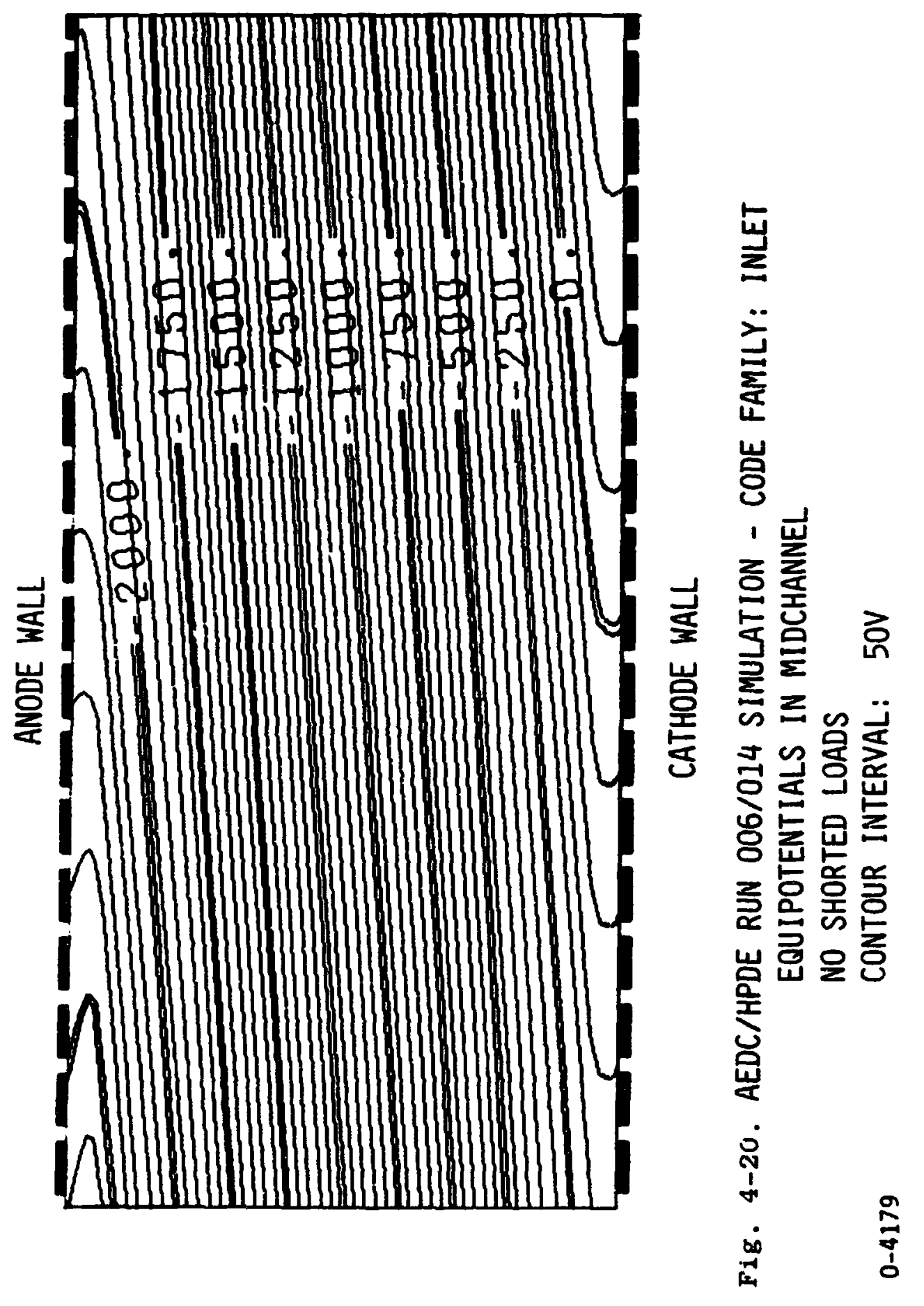




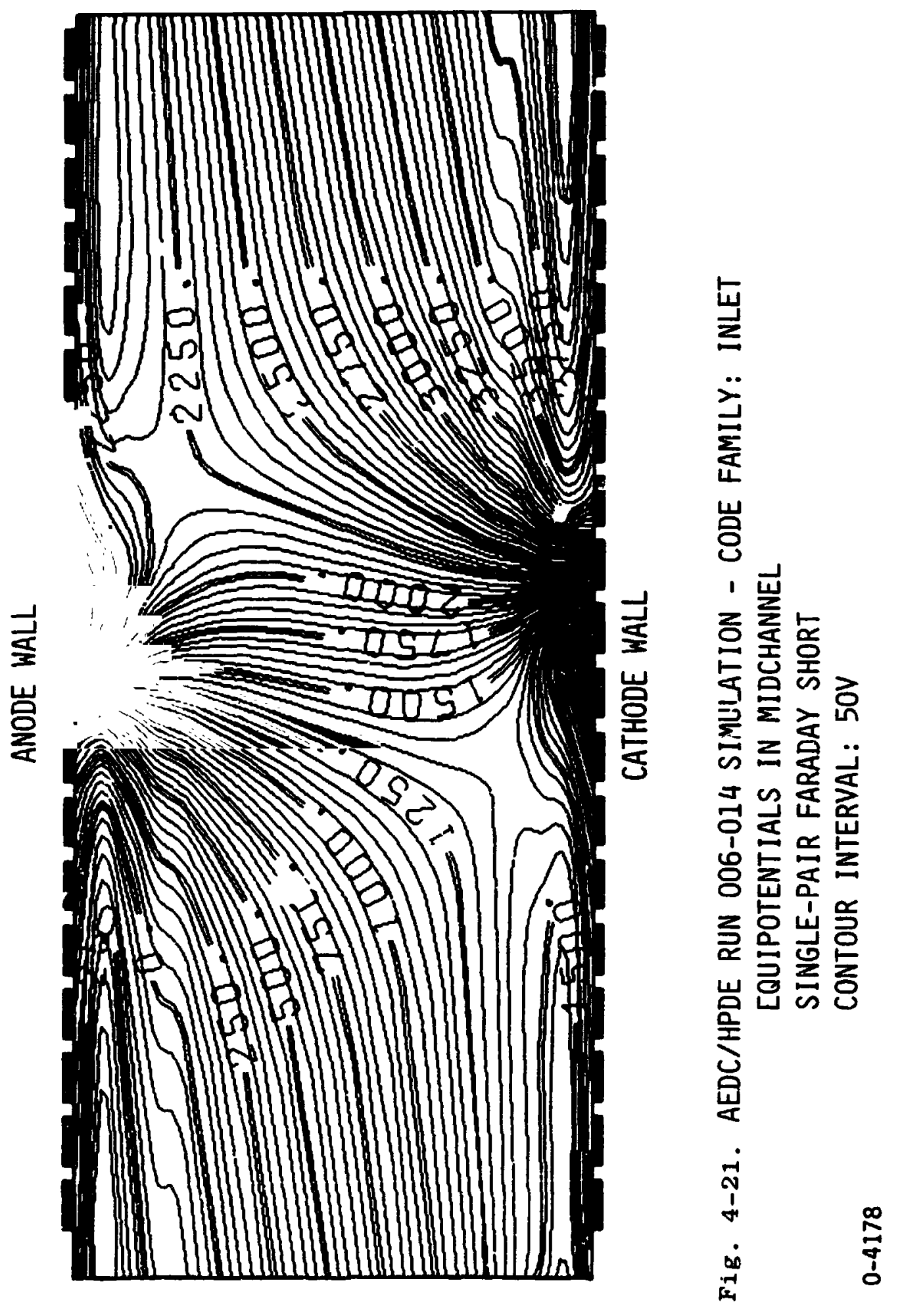

"Ricresia rare te 


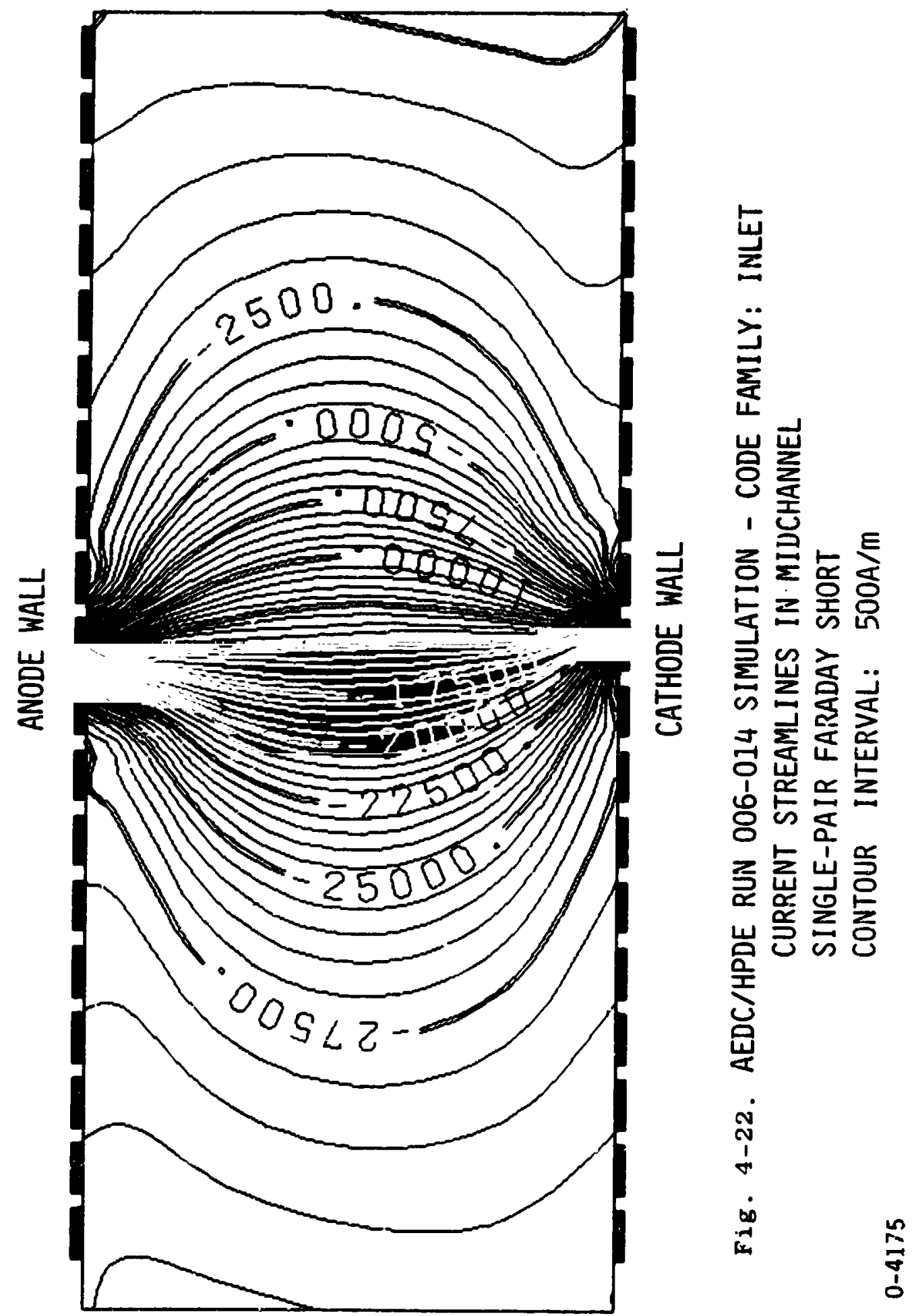




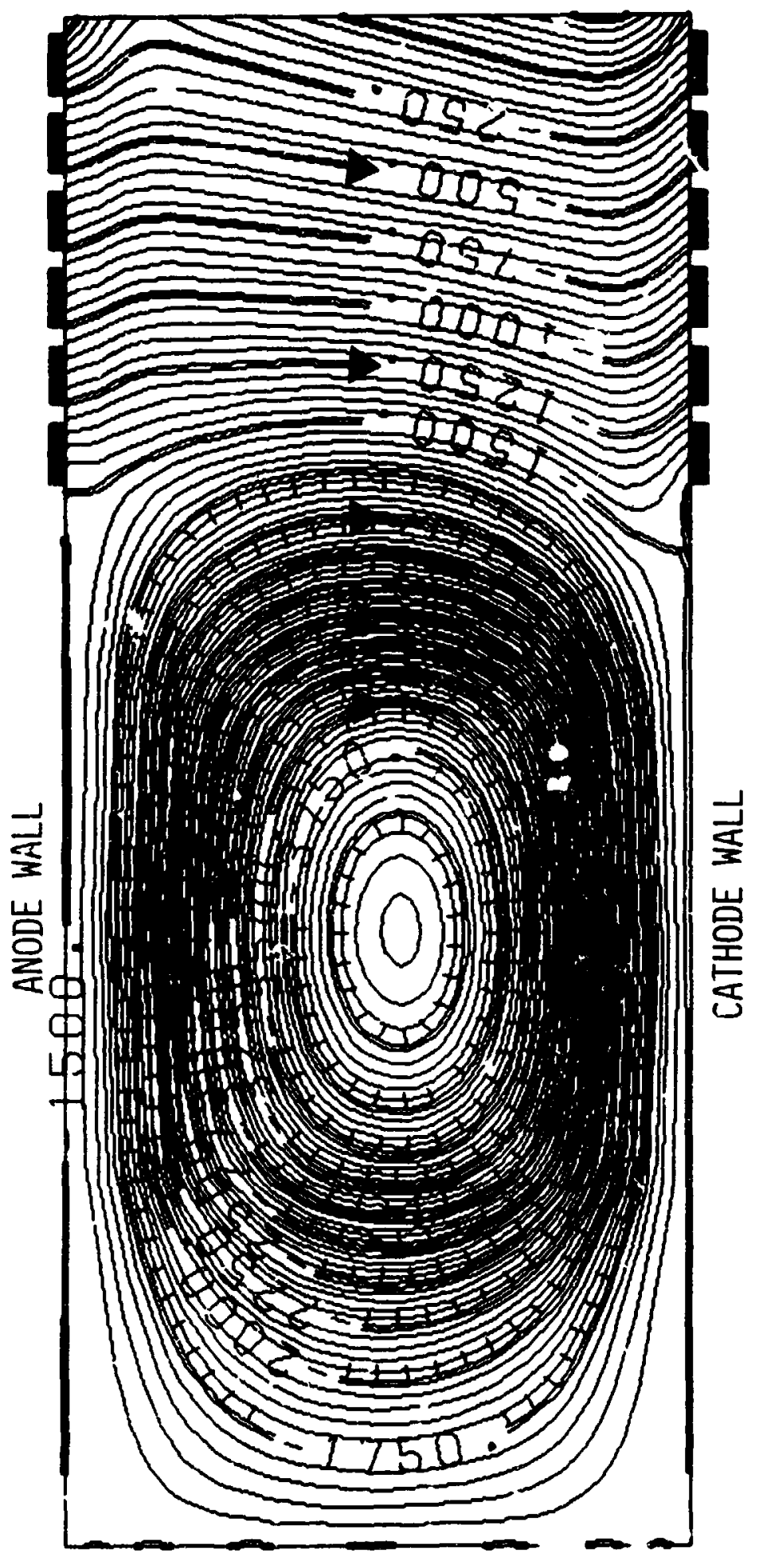

\author{
OrIgINAã page \\ OF POOR QUALITY
}

岂

崇岕

1 层

క.్ㅜ

的是

产领

的管

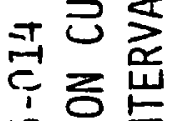

용은

8

뜽

늘

ำ

บ

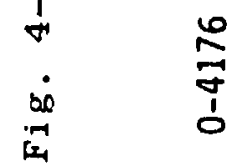




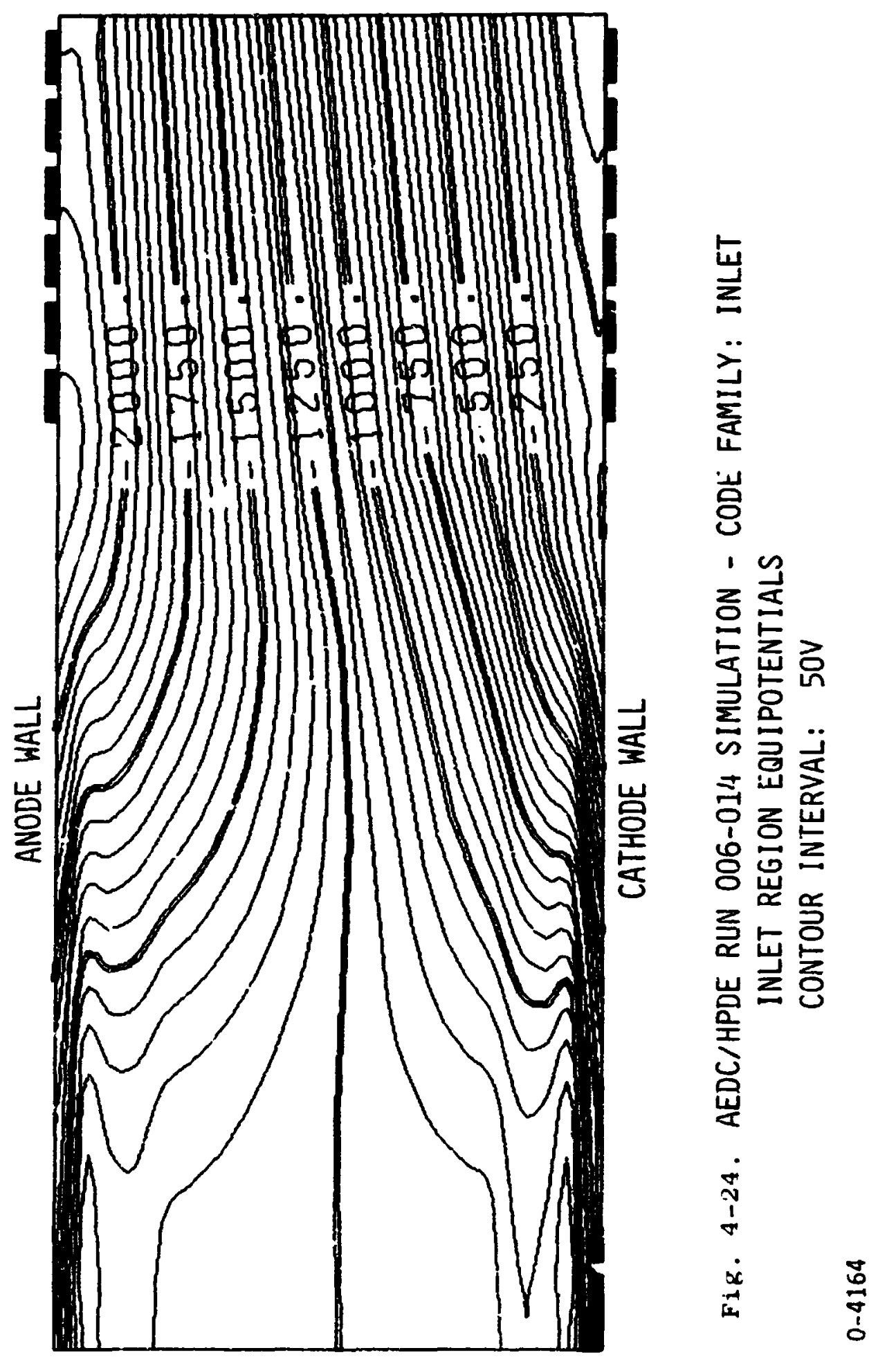


An MHD generator for installation in the Soviet U-25 facility was designed and fabricated in the United States as part of the joint U.S./U.S.S.R. cooperative MHD Program. STD has simulated the "as built" performance of the U.S. U-25 channel to determine the nominal operating performance and to analyze the critical phenomena of this experiment.

\subsection{Channel Cháracterization: U-25 Nominal Operating}

\section{Conditions}

The values of the physical and nominal operating parameters used for simulations of the U.S. U-25 channel are discussed in this section.

\subsubsection{Geometry}

The major components of the flow train sections of the U.S. U-25 experiment are the $\mathrm{kS}-3$ combustor (Soviet design and fabrication), the U.S. U-25 nozzle and channel assembly, and the U.S. U-25 diffuser assembly. The KS-3 combustor is a cylindrical unit with an inside diameter of $1.7 \mathrm{~m}[5-1]$. It is connected to the main oxidant feed line, which is of $1.5 \mathrm{~m}$ inside diameter, by a shcrt conical diffuser section. The combustion zone lies entirely within the conical divider, which is perforated to meter the oxidant flow to the upstream and downstream parts of the combustion zone. The combustion zone and the first exit transition piece is of circular section (1.2 $m$ in diameter) at the inlet and of rectangular section (640 $\mathrm{mm}$ by $760 \mathrm{~mm}$ ) at the exit. The nozzle of the U.S. U-25 flow train assembly is connected directly to the flange at the exit of the first transition piece. The nozzle inlet plan section is 624 $\mathrm{mm}$ by $744 \mathrm{~mm}[5-2]$. The nozzle contours are defined by the 
$753.7 \mathrm{~mm}$ tangent radius on the sidewall contour and a straight taper from $744 \mathrm{~mm}$ at inlet to $730 \mathrm{~mm}$ at nozzle exit on the electrode wall contour. The exit of the nozzle mates directlv with the inlet of the channel.

The channel frame number, width, height and diagonalization angle are given as functions of axial distance in Table 5-1 [5-3]. The origin of the axial distance coordinate is the 0-0 station, which corresponds to the front vertical edge of the magnet pole. This coordinate runs through the center of the channel, midway between both the electrode walls and the sidewalls. The U.S. U-25 generator is rectangular along its entire length.

\subsubsection{Magnetic Field}

The axial distribution of the magnetic flux density is giver in Table 5-2 [5-4].

\subsubsection{Loading}

The U.S. U-25 experiment is planned to be run with a single load at a nominal load current of 2840 A [5-5]. The loading is connected with distributed current collection through diodes (International Rectifier 501VFR200) on frames 1 through 67 on the front end and diodes (International Rectifier 501VFR200) on frames 366 through 432 in the back end |5-3|.

\subsubsection{Torking Fluid}

The working fluid for the U.S. U-25 experiment is defined in this section. The mass flow rate for the nominal conditions is $50 \mathrm{~kg} / \mathrm{s}[5-3]$. 
The composition of the fuel is given by volume as $[5-4],[5-6]$ :

$\begin{array}{ll}\mathrm{CH}_{4} & 92.4-94.5 \% \\ \mathrm{C}_{2} \mathrm{H}_{6} & 3.33-2.11 \% \\ \mathrm{C}_{3} \mathrm{H}_{8} & 1.38-0.87 \% \\ \mathrm{C}_{4} \mathrm{H}_{10} & 0.36-0.14 \% \\ \mathrm{CO}_{2} & 0.70-0.70 \% \\ \mathrm{~N}_{2} & 1.5-1.5 \% \\ \mathrm{C}_{4} & 0.3-0.2 \%\end{array}$

The fuel has a lower heating value of 45.98 to $46.51 \mathrm{~mJ} / \mathrm{kg}$ and is supplied to the combustor in the gaseous phase at ambient temperature $[5-4 j$.

The oxidizer is composed of air enriched with oxygen so that the total oxygen in the mixture is $40 \%$ by weight of the combined weight of the air and added oxygen [5-5]. The oxygen for enrichment is assumed to be pure $0_{2}$ [5-5]. The air is taken from the atmosphere and has a nominal moisture content of $0.89 \%$ by volume (equivalent to $50 \%$ relative humidity at $10^{\circ} \mathrm{C}$ ) [5-5]. The total oxidizer flow is preheated to $1200^{\circ} \mathrm{C}$ before it enters the combustor $[5-3],[5-5]$.

The seed is potassium carbonate in purified water solution [5-5]. The concentration of $\mathrm{K}_{2} \mathrm{CO}_{3}$ in the water is $1: 1$ by weight [5-5]. The flow rate of seed is such that $\mathrm{K}_{2} \mathrm{CO}_{3}$ is $2 \%$ of the total mass flow rate [5-5]. The seed solution is injected at ambient temperature [5-5].

For the nominal conditions the combustion is stoichiometric [5-3], [5-5]. 


\subsubsection{Dall Conditions}

The combustor wall temperature is $1773 \mathrm{~K}$ for all surfaces [5-5]. The nozzle, channel and difuser wall temperatures are $644[5-5]$, 044 to $672[5-5]$, and $514 \mathrm{~K}[5-3]$, respectively, for each wall and for the entire length of each section. The wall roughness is characterized by an equivalent sand roughness height of $3 \mathrm{~mm}$ in the combustor and channel and smooth walls in the nozzle and diffuser.

\subsubsection{Channel Inlet Conditions}

The conditions at the inlet of the U.S. U-25 are determined by the total mass flow rate, the thermodynamics of the working fluid, the inlet dimensions, and the enthalpy flux. The enthalpy flux was determined by assuming complete combustion and applying a 4.0\% heat loss [5-1] for the combustor.

\subsubsection{Other Conditions and Assumptions}

The design value of the diffuser recovery coefficlent is 0.45 . The pressure at the end of the diffuser is 2 to $3 \mathrm{kPa}$ below atmospheric [5-5]. 


\subsection{Steady-State Performance for Nominal Design}

The U-25 steady-state performance for the nominal desiga was simulated with the TRANSIENT code family in the quasi-steady mode of operation. The initial computations were performed under the optimistic condition of an assumed conductivity nonuniformity factor of 1.06. This value was taken from preliminary U-25 calculations with the Q3D family of codes performed previously to this contract and corresponds to arc-mode current transport mith a critical field of $12 \mathrm{kV} / \mathrm{m}$. Definitions of arc-mode current transport and critical electric field are given in Section 3.3. For the cold walls of the nominal operating conditions, the arc-mode current transport assumption is likely to yield the highest estimate of power output.

Under the nominal conditions, transonic flow is predicted for the U.S. U-25 experiment by computation BRCYKDXJGJ. The axial distributions of the gasdynamic and plasma property variables for the U-25 under the nominal conditions are given in Figs. 5-1 through 5-10. The variables displayed in these figures are grouped into the following classifications: (1) open and short circuit variables; (2) electrical loading, (3) plasma properties; (4) electrical field variables; (5) average gasdynamic variables; (6) electric potential; (7) channel geometry; (8) heat transfer parameters; (9) power extraction parameters; and (10) optimization variables. The origin of the axial coordinate in these figuris is the upstream end of the kS-3 combustor. For this axial coordinate system, station $0-0$ is located at an axial distance of $2.789 \mathrm{~m}$, the upstream end of the nozzle is located at an axial distance of $2.100 \mathrm{~m}$, the end of the channel is located at an axial distance of $9.851 \mathrm{~m}$, and the end of the diffuser is located ai an axial distance of $12.827 \mathrm{~m}$. 
Experience with simulations of the HPDE indicate a higher conductivity nonuniformity factor for cold wall channels than was utilized in computation BRCYKDXJGJ. Computation BRCYEHZJGK used the nominal conditions and, in addition, had an assumed conductivity nonuniformity factor of 1.24 , which was established from simulations of the HPDE under cold wall conditions. The results of computation BRCYEHzJGK are presented in Figs. 5-11 through 5-20. The variables presented in Figs. 5-11 through 5-20 correspond to the same variables presented in Figs. 5-1 through 5-10. The flow remains transonic and the power output decreases to $7.74 \mathrm{MW}$ (BRCYEHZJGK) from $10.97 \mathrm{MN}$ (BRCYKDXJGJ).

By decreasing the load current to obtain maximum power output, some of the power difference can be recovered. Computation BRCYJCGJGK assumed a conductivity nonuniformity factor of 1.24 and also assumed a load current of $2128 \mathrm{~A}$. The results of computation BRCYJCGJGK are presented in Figs. 5-21 through 5-30. The flow remains transonic, but the power increases to $9.44 \mathrm{MW}$, which is only slightly below the goal of $10 \mathrm{~km}$.

The isentropic efficiency is shown in Fig. 5-31 as a function of the gross channel power for these three simulations. This figure illustrates that of these three simulations of the vominal operating conditions only computation BRCYKDXJGJ predicts an output power above the goal of the experiment, but that simulation BRCYJCGJGK, with higher conductivity nonuniformity factor and lower load current, predicts that the output power will be very close to the goal. 


\subsection{Quasi-Steady Performance as a Function of Mass Flow Rate}

A series of five preliminary performance calculations with the nominal conditions, except for mass flow rate, were carried out with the TRANSIENT code family in the quasi-steady mode of operation. The range of mass flow rates was iaken to be from 35 to $55 \mathrm{~kg} / \mathrm{s}$. The performance of the U-25 over this mass flow rate range was evaluated with an assumed conductivity nonuniformity factor of 1.06. This value was taken from previous preliminary U-25 calculations with the Q3D family of codes and is typical of values obtained with the assumption of arc-mode current transport with a $12 \mathrm{kV} / \mathrm{m}$ breakdown criterion in the U-25. The arc-mode current transport assumption is likely to be the most favorable current transport mechanism for cold wall channels and is likely to yield the highest estimate of power output.

The five computations in this series are: A5MYISTIKM, 50 $\mathrm{kg} / \mathrm{s}$; A5MYNEPIKN, $45 \mathrm{~kg} / \mathrm{s}$; A5MYNGCIKN, $55 \mathrm{~kg} / \mathrm{s}$; A5MYONTIKN, 40 $\mathrm{kg} / \mathrm{s}$; and A5MYRAWI KN, $35 \mathrm{~kg} / \mathrm{s}$.

The transonic flow predicted by these calculations for all mass flow rates above $40 \mathrm{~kg} / \mathrm{sec}$ is illustrated by Fig. 5-32. Fig. 5-32 shows that the flow chokes from $3.2 \mathrm{~m}$ to $6.6 \mathrm{~m}$ downstream of the combustor backplate (the origin of axial coc dinate for these STD computations) and recovers to atmospheric pressure through a normal shock in the diffuser. Because the power extraction and MHD interaction in these computations are near maximum, it is expected that more conservative assumptions for the wall current transport mechanism will lead to even more supersonic flow conditions.

The power output computed in these preliminary calculations is presented in Fig. 5-33. It is significant that 
these prelim.nary calculations predict that power output in excess of $10 \mathrm{Mm}$ might be achieved even at a mass flow rate of $40 \mathrm{~kg} / \mathrm{s}$. It must be reemphasized, however, that these calculations are based on the assumption of the most favorable current transport mechanism for this cold wall channel.

The isentropic efficiency is given as a function of mass flow rate in Fig. 5-34. The maximum value of isentropic efficiency calculated was nearly $40 \%$ from A5MYNEPIKN. As is shown in Fig. 5-32, the shock enters the channel for the nominal conditions and for mass flow rates less than $44 \mathrm{~kg} / \mathrm{s}$. The decrease in isentropic efficiency caused by the shock entering the active channel is seen in Pig. 5-34. 


\section{REFERENCES}

[5-1] A. F. Sheindiin, The U-25 MHD pilot Plant: Major Components and the Long-Duration R-Channel, Institute of High Temperature, Moscow (1975)

[5-2] MEPPSCO Drawing U-25 SOD Channel Rev. N. attached to letter EET/ECP-369, dated 6 July 1979, from E. Doss (ANL) to D. Oliver (STD)

[5-3] Letter EET/ECP-369 and attachments thereto, dated $6 \mathrm{Ju}$ y 1979 from E. Doss (ANL) to D. Oliver (STD)

[5-4] Letter EET/ECP-386, dated $20 \mathrm{July} 1979$, from E. Doss (ANL) to D. Oliver (STD

[5-5] Coordination Meeting between ANL, NASA LeRC, and STD personnel held at ANL 3 October 1979

[5-6] Letter ENG/PE/4354, dated 1 November 1979 from $K$. D. Kuczen (ANL) to B. Phillips (NASA LeRC) 
TABLE 5-1. Design Data for the U.S. U-25 MHD Generator

\begin{tabular}{|c|c|c|c|c|}
\hline $\begin{array}{l}\text { FRAME } \\
\text { MUABEER }\end{array}$ & $\begin{array}{c}\text { AXIAL } \\
\text { OISTANCE } \\
(-)\end{array}$ & $\begin{array}{l}\text { MIOTH } \\
\text { (E) }\end{array}$ & $\begin{array}{c}\text { HE IGHI } \\
\text { (a) }\end{array}$ & $\begin{array}{c}\text { OIAGOMALIZATION } \\
\text { AMGLE } \\
\text { (radians) }\end{array}$ \\
\hline 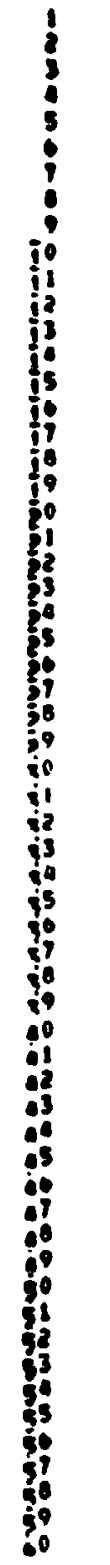 & 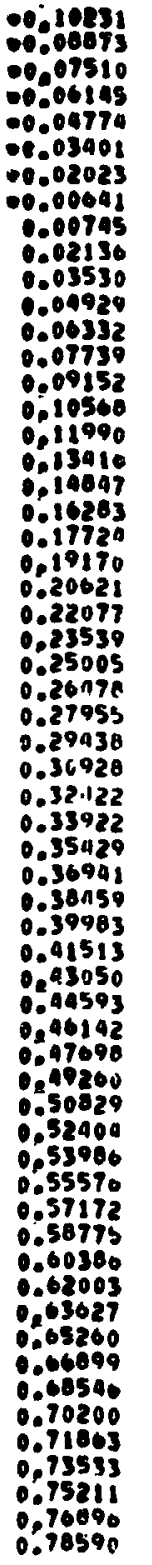 & 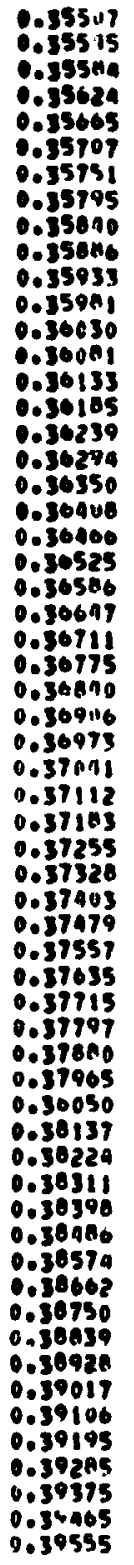 & $\begin{array}{l}0.73000 \\
0.73000 \\
0.73000 \\
0.73000 \\
0.73000 \\
0.73000 \\
0.73000 \\
0.73000 \\
0.73000 \\
0.73000 \\
0.73000 \\
0.73000 \\
0.73000 \\
0.73000 \\
0.73000 \\
0.73000 \\
0.73000 \\
0.73000 \\
0.73000 \\
0.73000 \\
0.73000 \\
0.73000 \\
0.73000 \\
0.73000 \\
0.73000 \\
0.73000 \\
0.73000 \\
0.73000 \\
0.731000 \\
0.73000 \\
0.73000 \\
0.73000 \\
0.73000 \\
0.73000 \\
0.73000 \\
0.73000 \\
0.73000 \\
0.73000 \\
0.73000 \\
0.73000 \\
0.73000 \\
0.73000 \\
0.73000 \\
0.73000 \\
0.73000 \\
0.73000 \\
0.73000 \\
0.73000 \\
0.73000 \\
0.73000 \\
0.73000 \\
0.73000 \\
0.73000 \\
0.73000 \\
0.73000 \\
0.73000 \\
0.73000 \\
0.73000 \\
0.73000 \\
0.73000 \\
0.73000\end{array}$ & 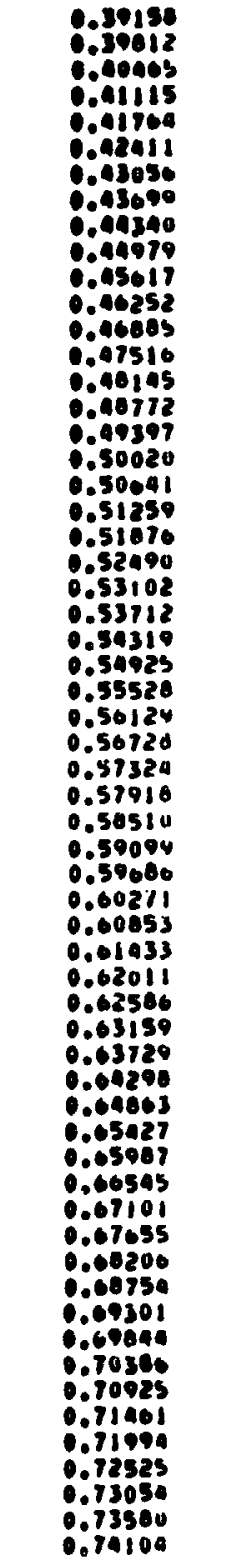 \\
\hline
\end{tabular}


TABiE 5-1. Design Data for the U.S. U-25 MHD Generator (cont)

\begin{tabular}{|c|c|c|c|c|}
\hline $\begin{array}{l}\text { FRAME } \\
\text { YUMBER }\end{array}$ & $\begin{array}{c}\text { AXIALL } \\
\text { DISTANCE } \\
\text { (a) }\end{array}$ & $\begin{array}{l}\text { M1OTH } \\
\text { (1) }\end{array}$ & $\begin{array}{c}\text { HEIGHT } \\
(n)\end{array}$ & $\begin{array}{c}\text { OIAGOMAL IZATIOH } \\
\text { AHGL! } \\
\text { (radians) }\end{array}$ \\
\hline 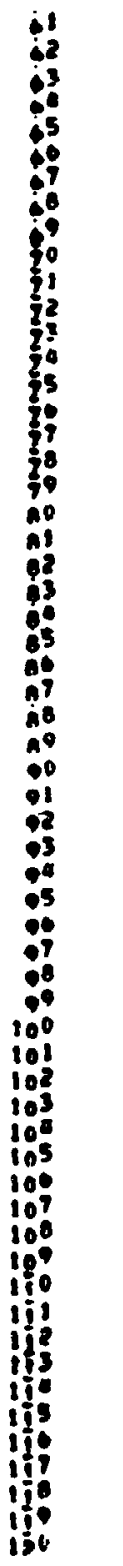 & 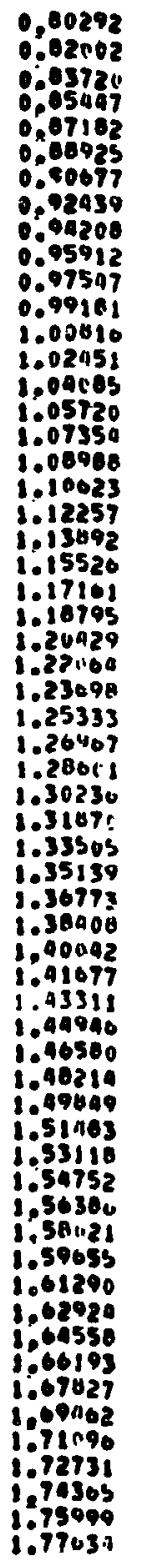 & 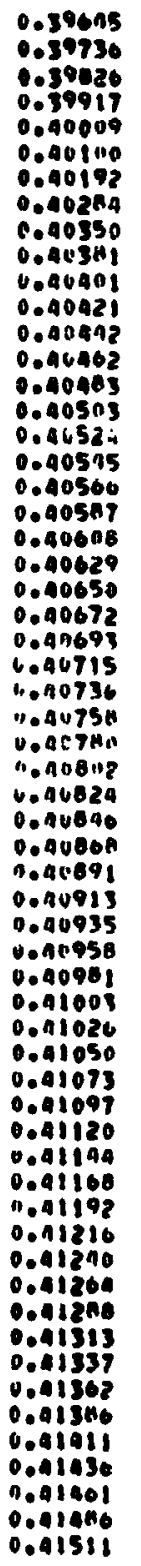 & 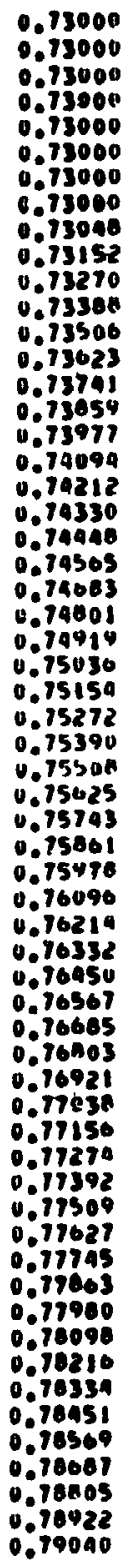 & 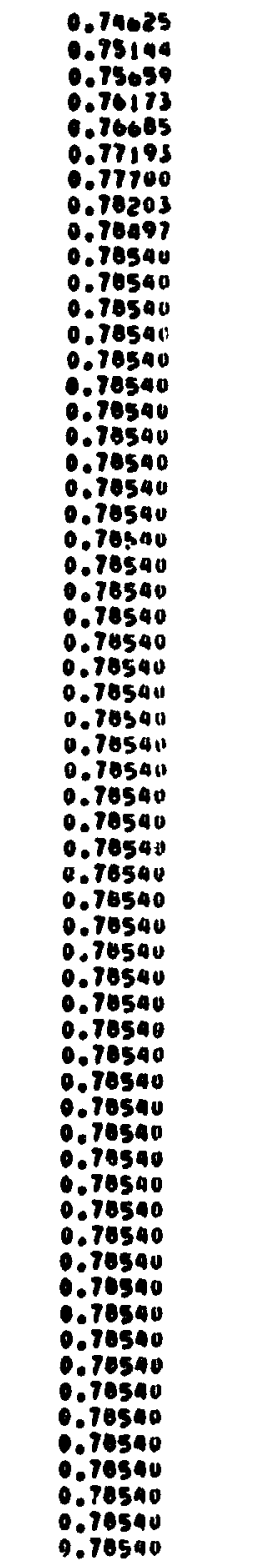 \\
\hline
\end{tabular}


TABLE 5-1. Design Data for the U.S. U-25 MHD Generator (cont)

\begin{tabular}{|c|c|c|c|c|}
\hline $\begin{array}{l}\text { F RAME } \\
\text { NUHBER }\end{array}$ & $\begin{array}{c}\text { AXIAL } \\
\text { DISTANCE } \\
(0)\end{array}$ & $\begin{array}{c}\text { WIOIH } \\
\text { (a) }\end{array}$ & $\begin{array}{c}\text { HE IGHT } \\
\text { (a) }\end{array}$ & $\begin{array}{c}\text { DIAGONAL IZATION } \\
\text { ANGLE } \\
\text { (radians) }\end{array}$ \\
\hline 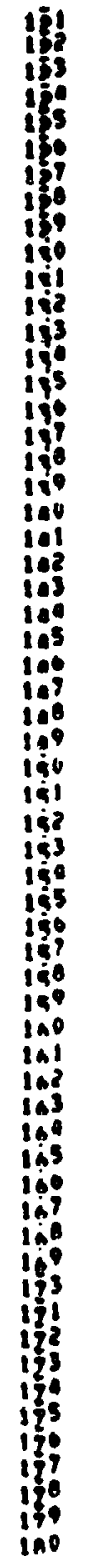 & 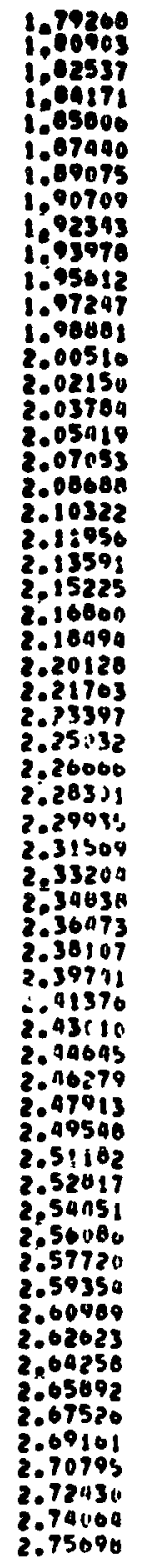 & 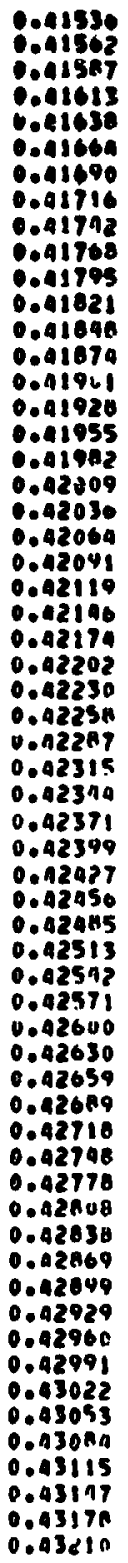 & 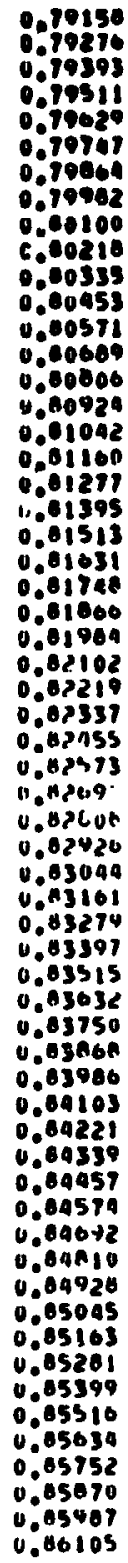 & 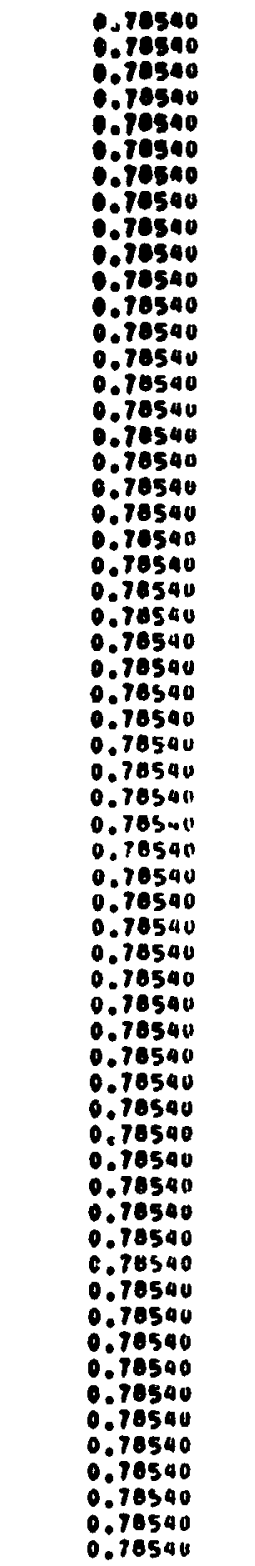 \\
\hline
\end{tabular}


TABLE 5-1. Design Data for the U.S. U-25 MHD Generator (cont)

FRAME

MUMBER (a)

2.77333
2.70867

8.60002

2,0230

2.03671

2.05505

8.07139

2000900

2.00408

2.03677

2.05311

8.06040

2.905en

3,00215

3.01648

$3.03 n 03$

3.05110

3.06752

3.08367

3.10021

3,11050

3.1329.9

3.10929

3.10559

3.19826

3.21402

3.23190

3.24731

3.26305

3. 5 100

$3.2963 a$

3.31200

3.32903

3.34537

3.36172

3.

3.41075

3.41075
3.42719

3.42719

3.45970

3.47013

3.49217

3.50661

3.52310

3.3413 .

3. 35743

3.57419

3.59053

3.60008

3.62322

3.63957

3.05591

.67225

3.68060

3.90190

3.72129 (a)

0.45272

cossera

0.43300

0.43371

0.43003

0.43430

0.43409

0.43501

$0.4353 a$

$0.4350 A$

0.43601

0.43635

0.43702

0.433

0.43736

0.0.

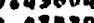

0.43439
0.03073

0.43673

0.43412

0.03975

0.44012

0.44017

0.447

0.04154

C.4714n

- 117220

0.41202

0044294

0.44335

0.14371

0.011408

0.44445

0.444 R?

0.455

0.44556

0.44594

0.44669

0.44707

0.44775

- 44793

0.Anci

0.49860

0.04899
0.09439

0.44979

0.45010

0.05050

0.43nga

0.45130

0.05210

0. 05200

$0.4526 n$

0.45312
$0.453 \mathrm{H}$
HE IGHI

(a)

ग.00223

0.6034,

0.0450

0.06694

0.06312

0.06929

0.07047

0,07105

0.072 as

0.67400

0.07518

0.87030

0.875

0.07409

0.06101

0.08225

0.08343

0.08460

0.08096

0.00411

0.08931

0.64044

n. 10203

1.

".niste

$0 . n 4320$

4.04038

0. Ay7so

0.09873

0.199491

0.00109

0.90227

$0.903 n n$

0.9040 ?

0.905811

0.90694

0.00815

0.90933

0.91051

0.91169

$0.912 a 6$

0.91409

0.91522

0.91040

0.91757

0.91875

0.91993

0.92111

0.92228

0.97346

0.92464

0.9258

0.92099

1.

0.4781 !

0.92935

0.93053
0.93170
DIAGONAL IZ2TIOK. AHGLE

iradians:

0.70540

0.70500

0.70590

0.10540

0.70540

0.18540

0.70540

0.18540

0.70540

0.18540

0.78540

0.18540

0.74540

0.7654 .1

0.705.

0.70540

0.16540

0.7 Eva

-.7csua

- ?.

0.18540

.74540

0.70540

0.78540

0.78540

0.70540

0.76540

0.70540

0.7854

0.70san

$0.785,010$

0.765411

0.70540

0.70540

0.78540

$0.7054^{\circ}$

0.76540

0.70540

(1059u

0.7050

(2)

0.70540

0.70sab

0.10540

0.73540

0.795411

0.16540

0.78540

0.70540

0.70590

0.70544

0.70540

0.118543

0.70540

0.70500

0.7500

0.70500

0.70500

0.78540 
TABLE 5-1. Design Data for the U.S. U-25 MHD Generator (cont)

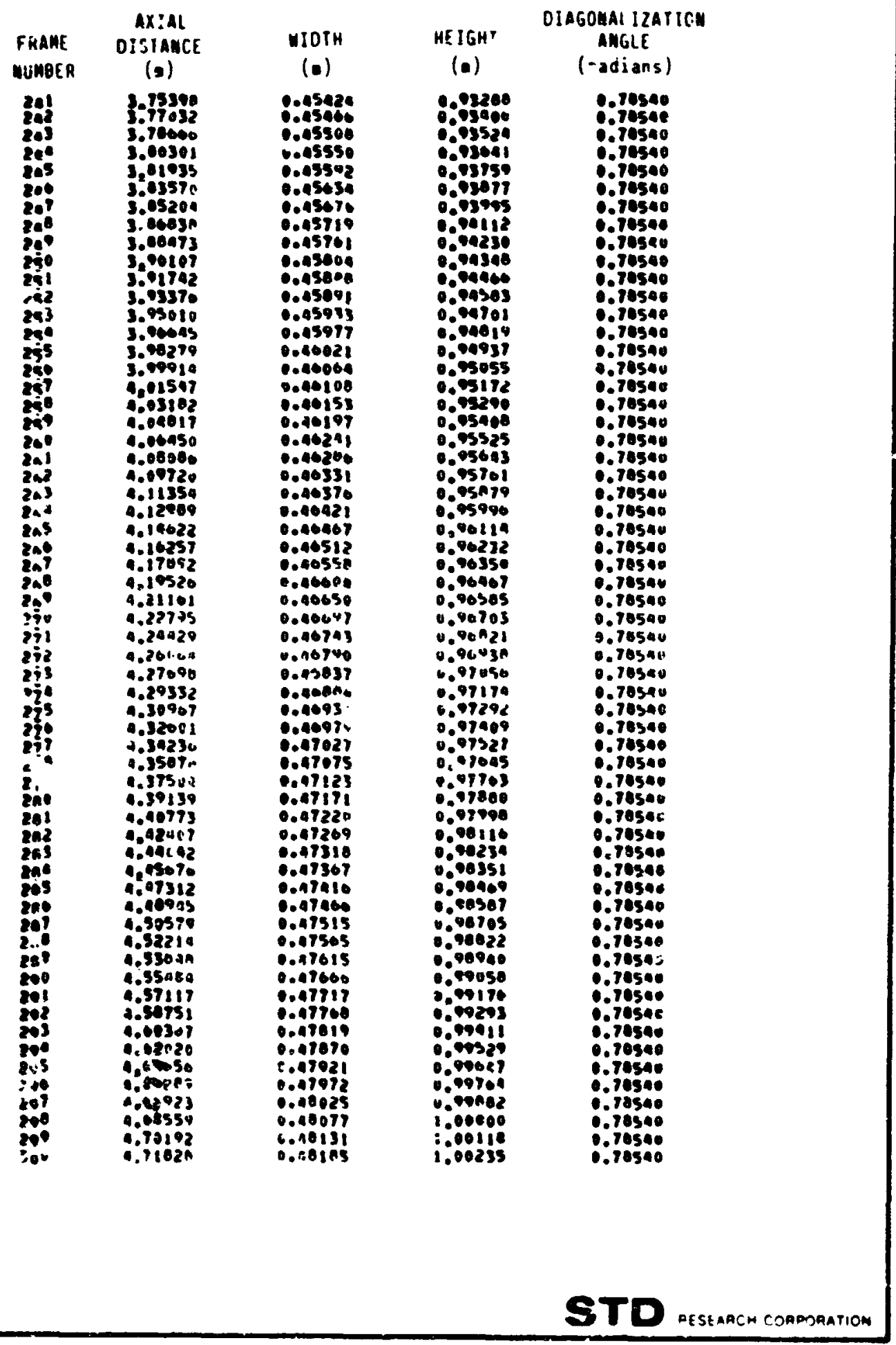


TABLE 5-1. Design Data for the U.S. U-25 MHD Generator (cont)

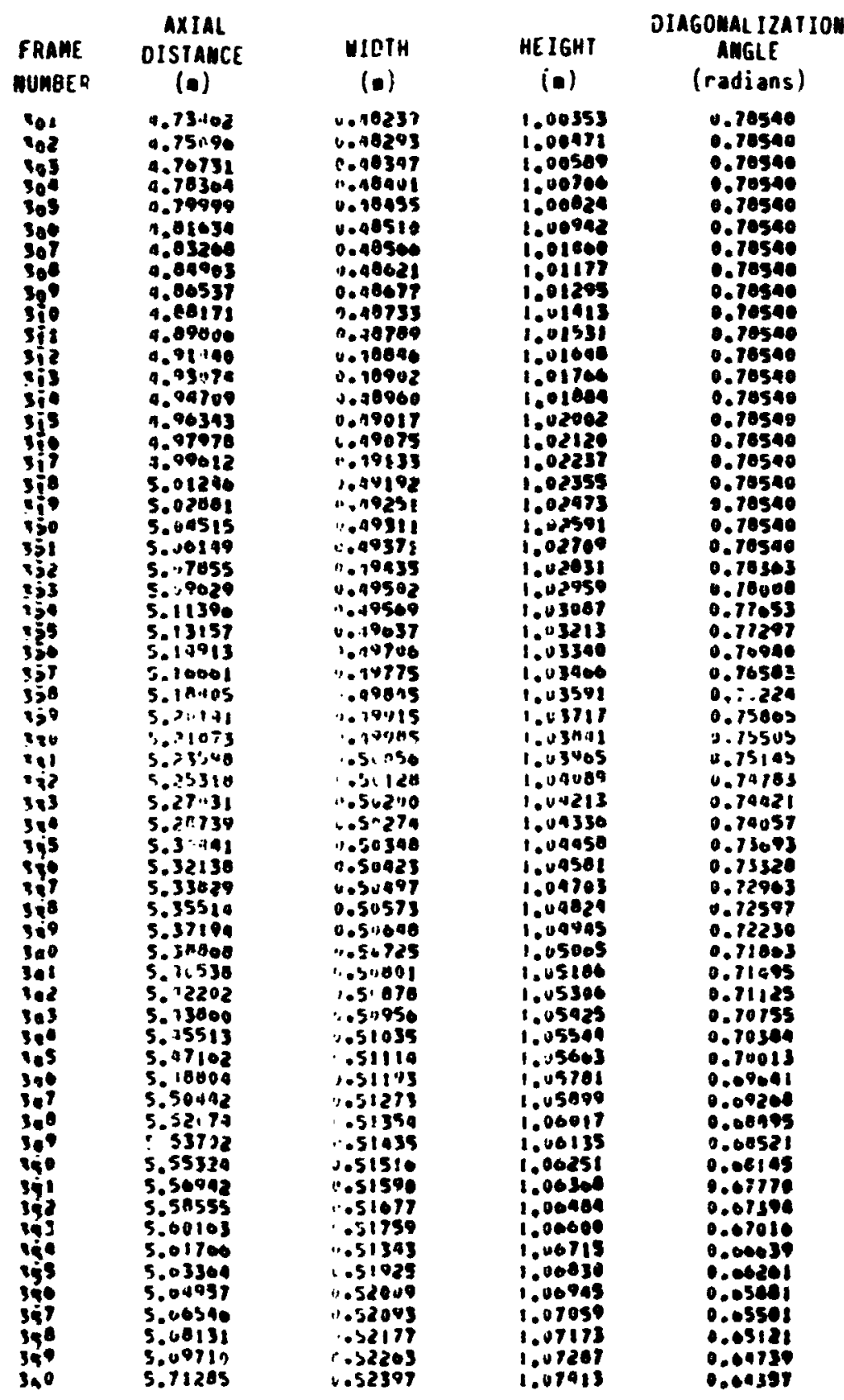


TABLE 5-1. Design Data for the U.S. U-25 MHD Generator (cont)

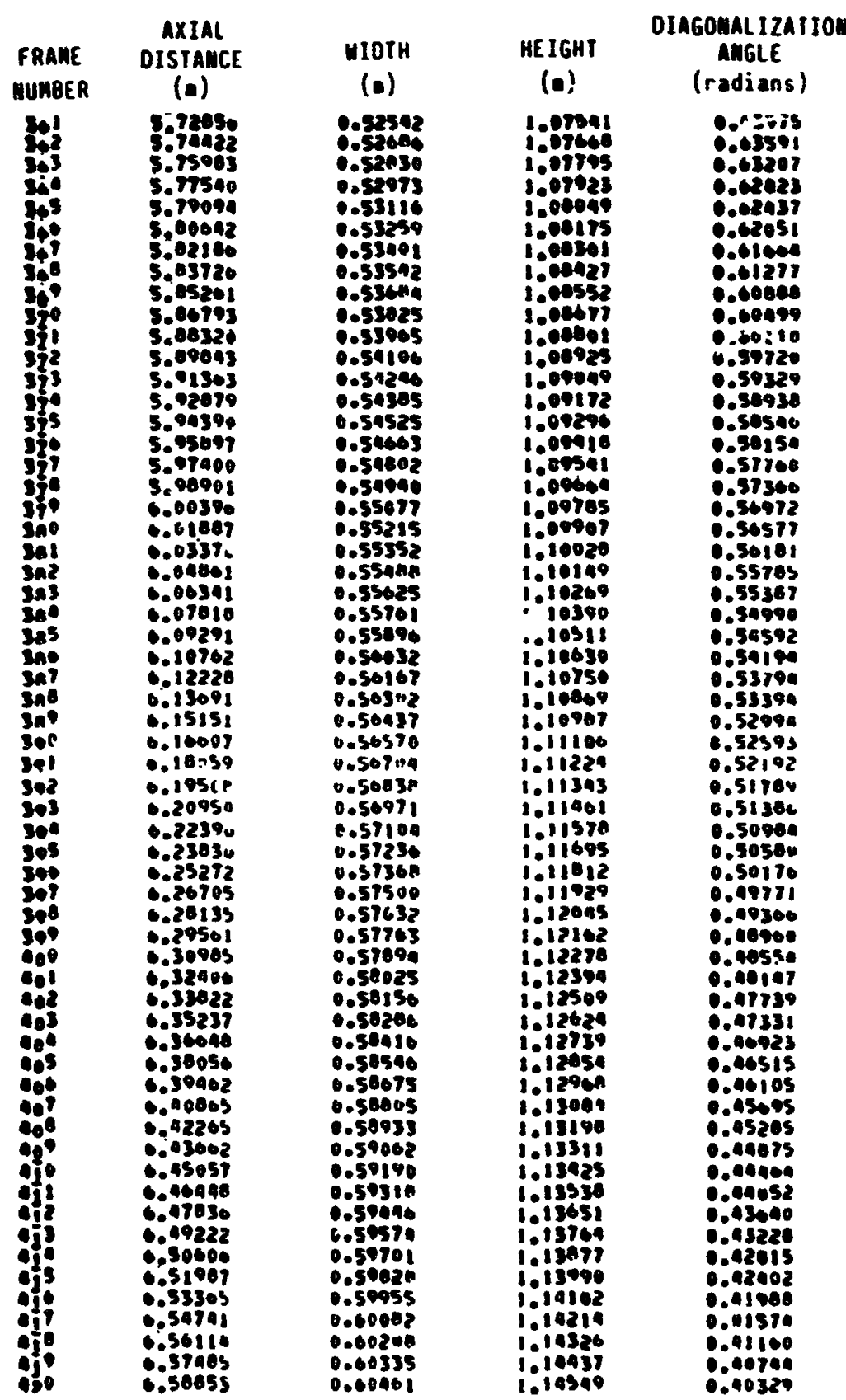


TABLE 5-1. Design Data for the U.S. U-25 MHD Generator (cont)

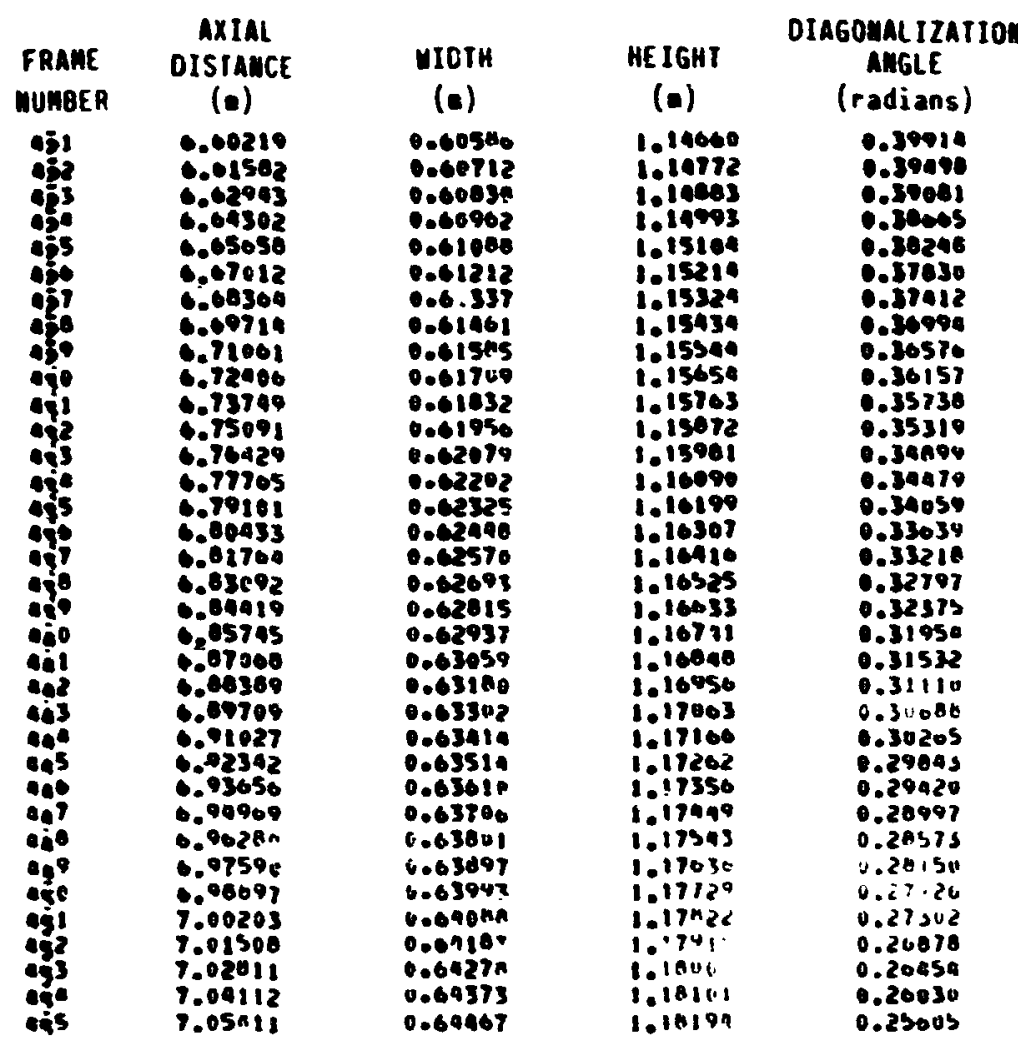




\section{TABLE 5-2}

The U.S. U-25 1-D Magnetic Field Distribution

\begin{tabular}{|c|c|c|c|}
\hline$X(m)$ & $B(T)$ & $X(\mathbf{n})$ & $B(T)$ \\
\hline-1.5 & 0.1 & 5.1 & 1.99 \\
\hline-1.4 & 0.11 & 5.2 & 1.98 \\
\hline-1.3 & 0.12 & 5.3 & 1.96 \\
\hline-1.2 & 0.14 & 5.4 & 1.92 \\
\hline-1.1 & 0.18 & 5.5 & 1.88 \\
\hline$-1 \cdot 0$ & 0.23 & 5.6 & 1.85 \\
\hline-0.9 & 0.3 & 5.7 & 1.75 \\
\hline-0.8 & 0.39 & 5.8 & 1.62 \\
\hline-0.7 & 0.52 & 5.9 & 1.48 \\
\hline-0.6 & 0.68 & 6.0 & 1.38 \\
\hline-0.5 & 0.83 & 6.1 & 1.22 \\
\hline-0.4 & 0.97 & 6.2 & 1.12 \\
\hline-0.3 & 1.13 & 6.3 & 1.0 \\
\hline-0.2 & 1.28 & 6.4 & 0.92 \\
\hline-0.1 & 1.4 & 6.5 & 0.86 \\
\hline 0.0 & 1.55 & 6.6 & 0.79 \\
\hline 0.1 & 1.72 & 6.7 & 0.73 \\
\hline 0.2 & 1.81 & 6.8 & 0.66 \\
\hline 0.3 & 1.88 & 6.9 & 0.6 \\
\hline 0.4 & 1.9 & 7.0 & 0.54 \\
\hline 0.5 & 1.94 & 7.1 & 0.5 \\
\hline 0.6 & 1.97 & 7.2 & 0.45 \\
\hline 0.7 & 1.98 & 7.3 & 0.41 \\
\hline 0.8 & 1.99 & 7.4 & 0.36 \\
\hline $0.9-5.0$ & 2.0 & 7.5 & 0.33 \\
\hline
\end{tabular}

$0-4.2 \times 3$ 


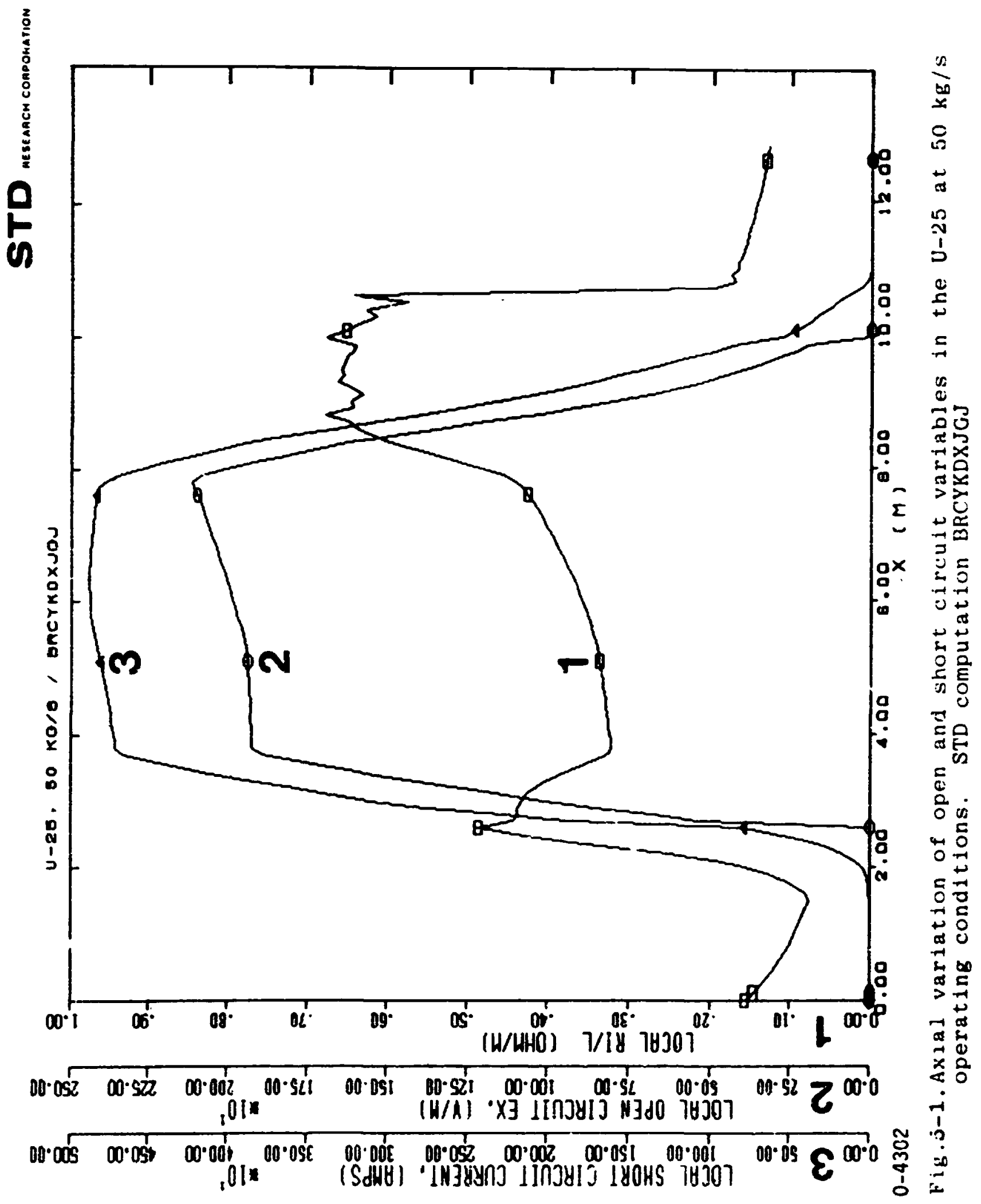




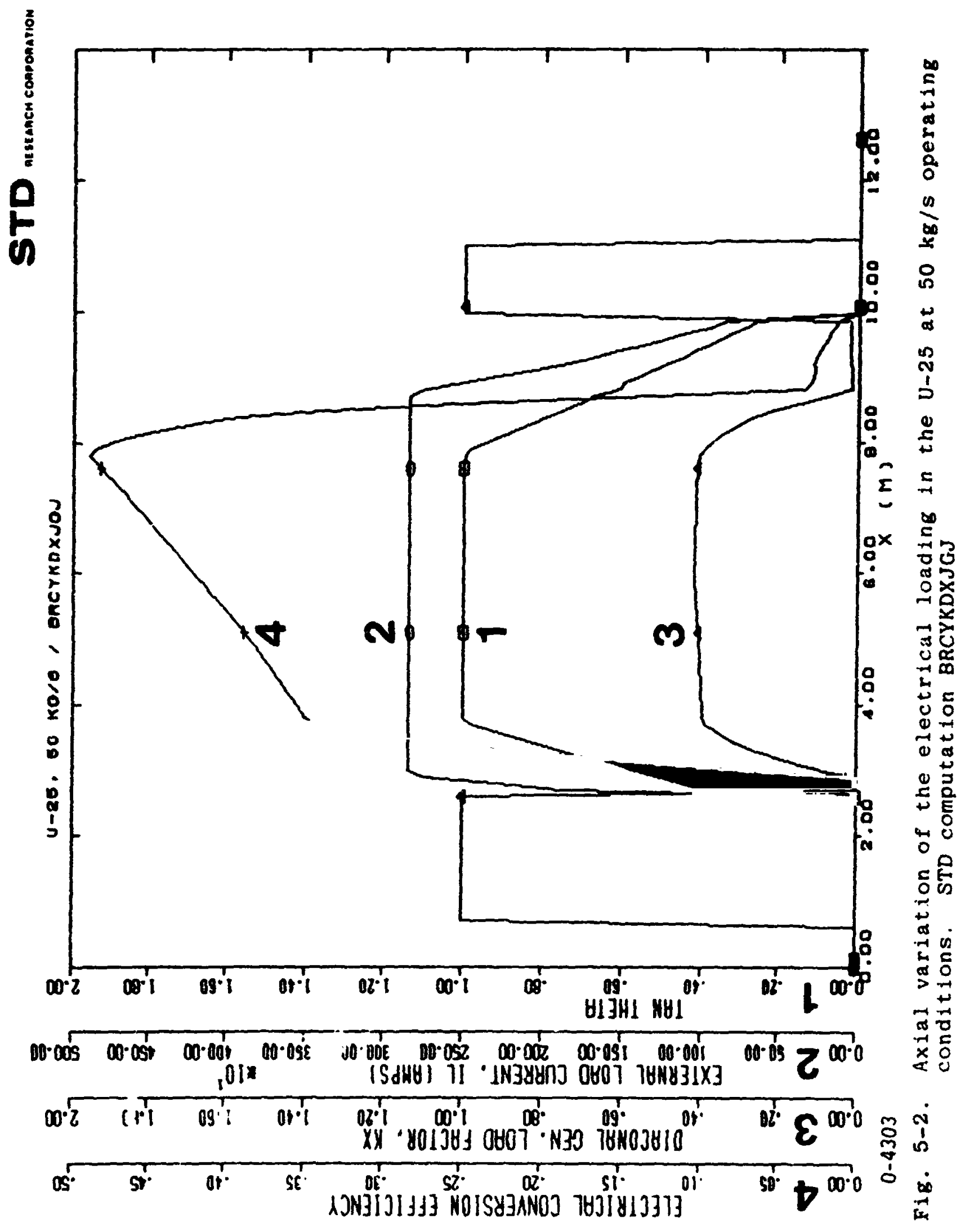




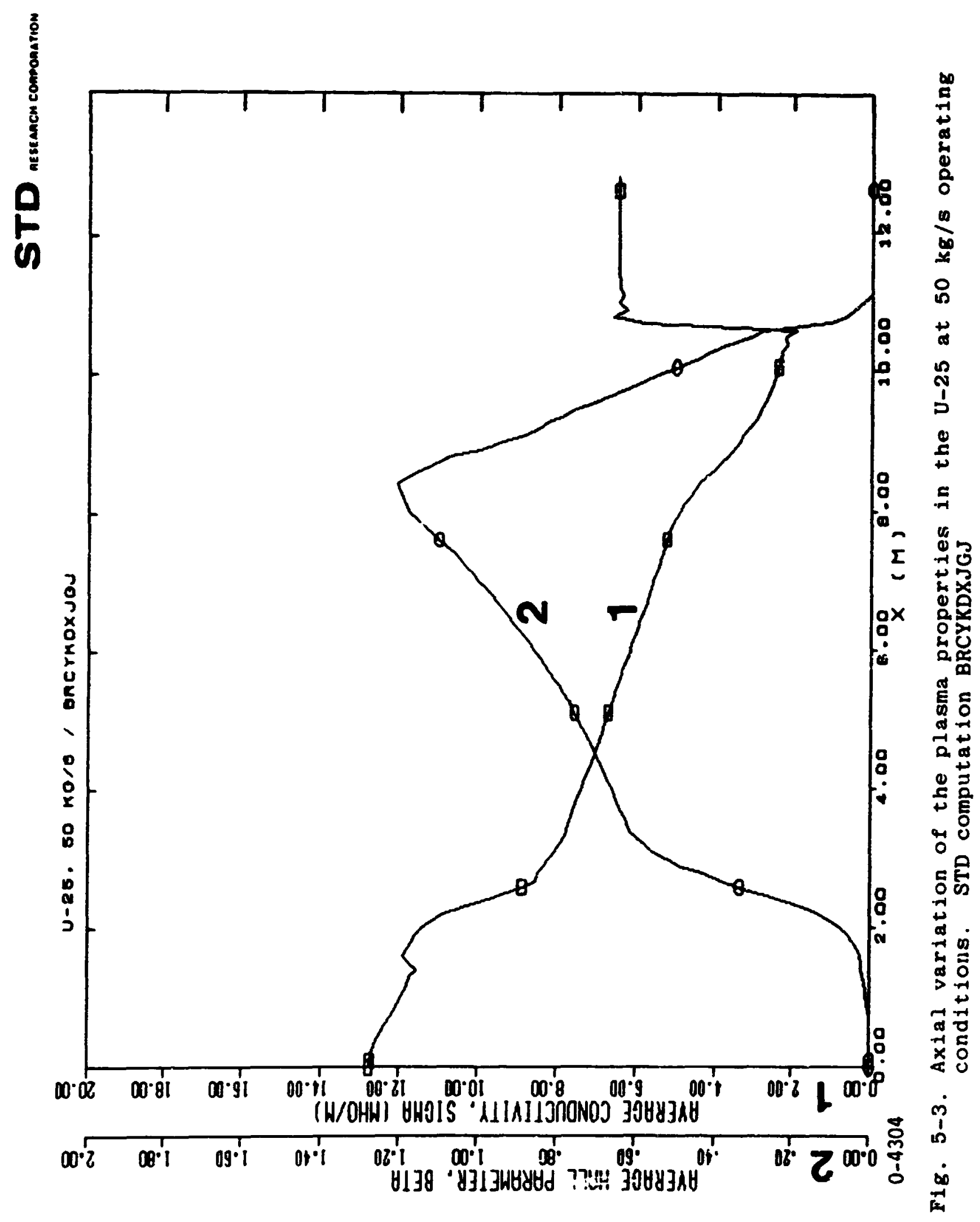




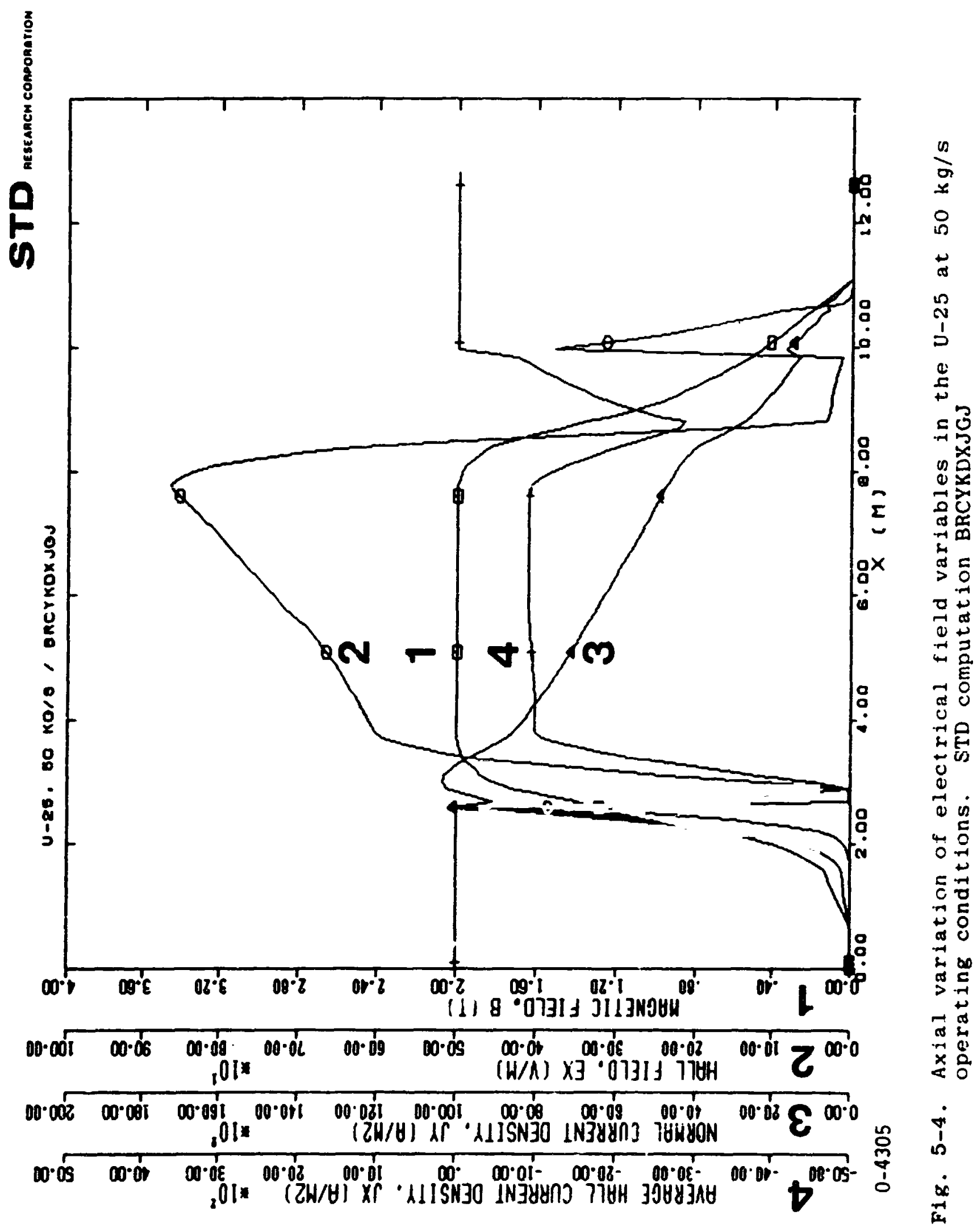




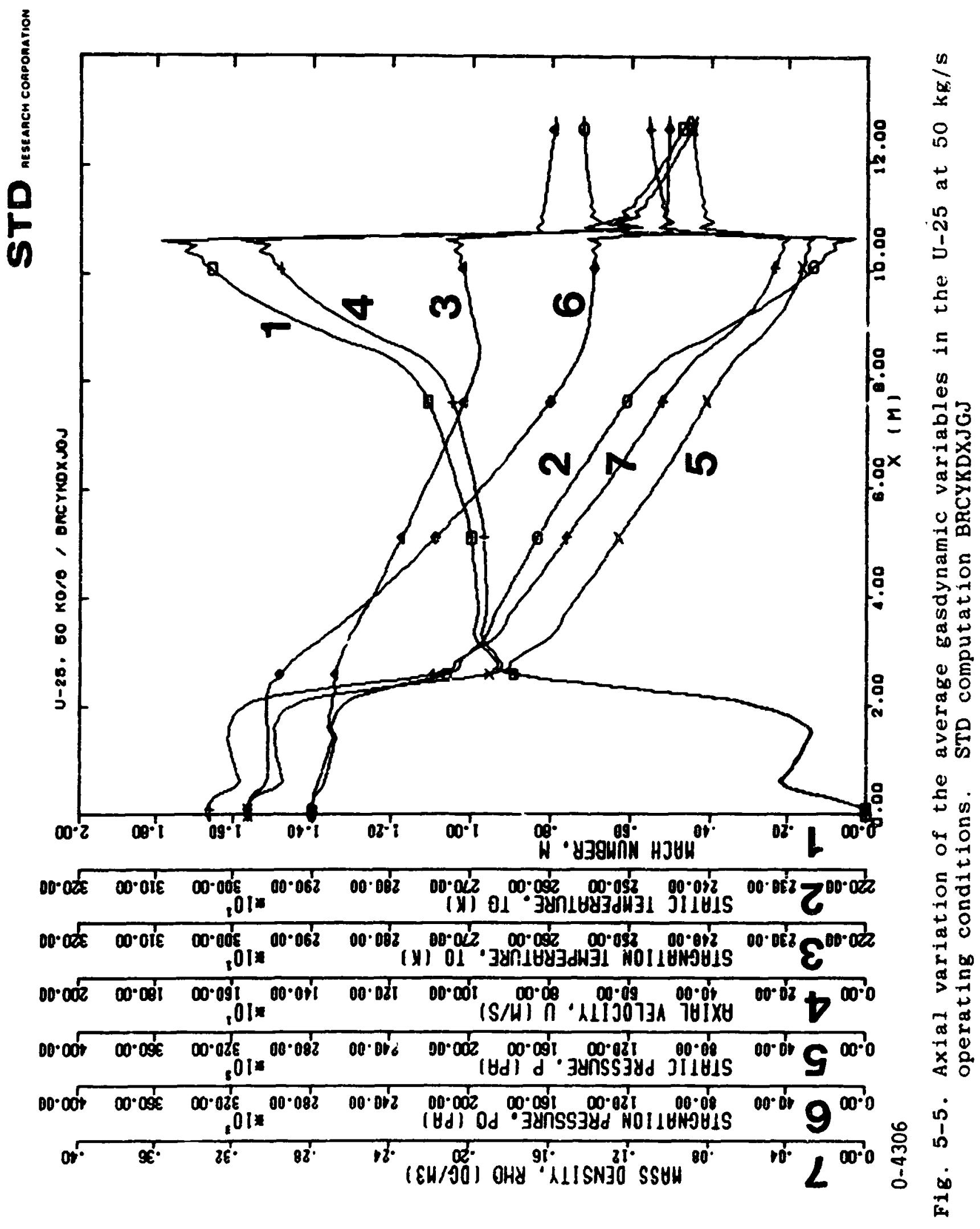




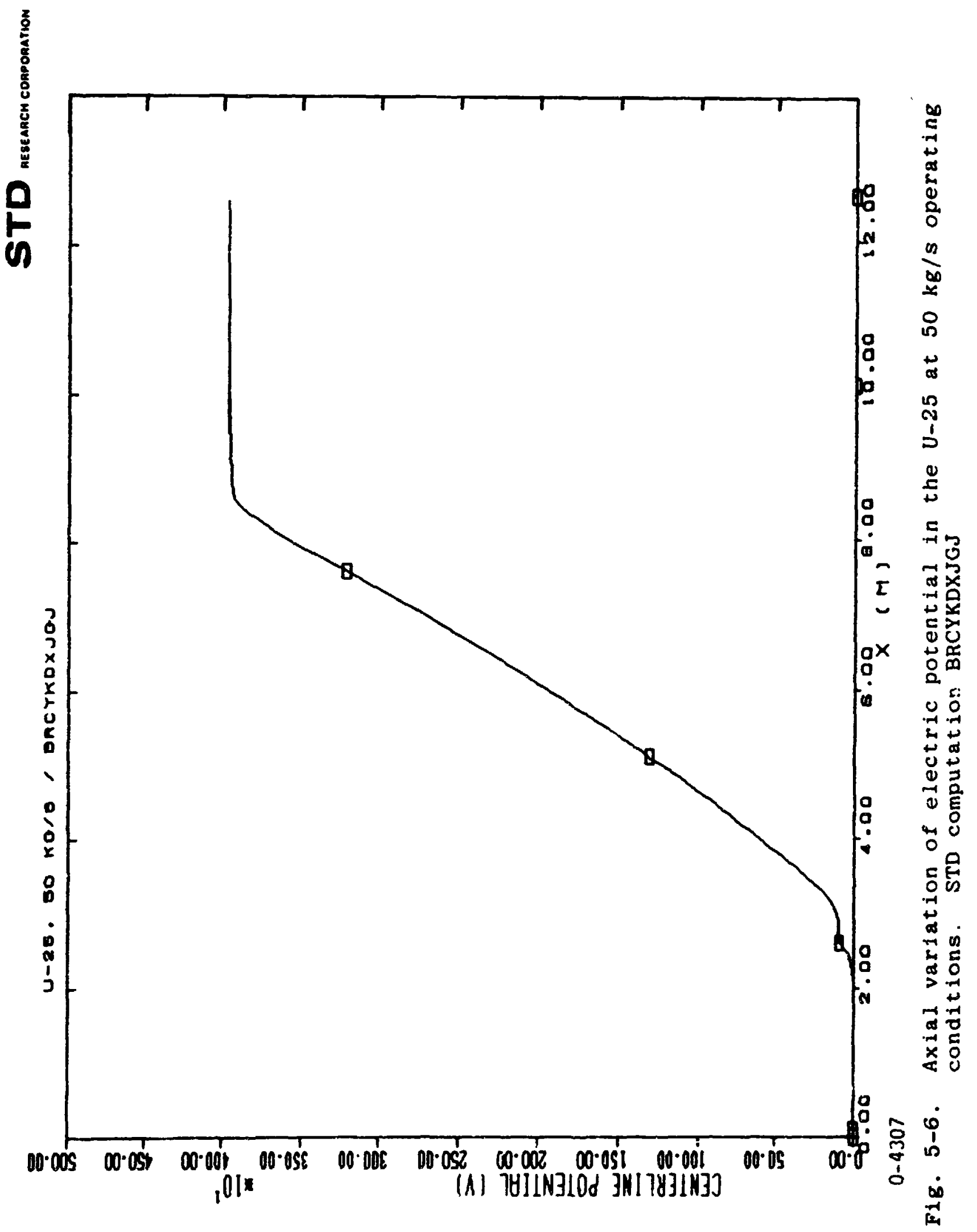




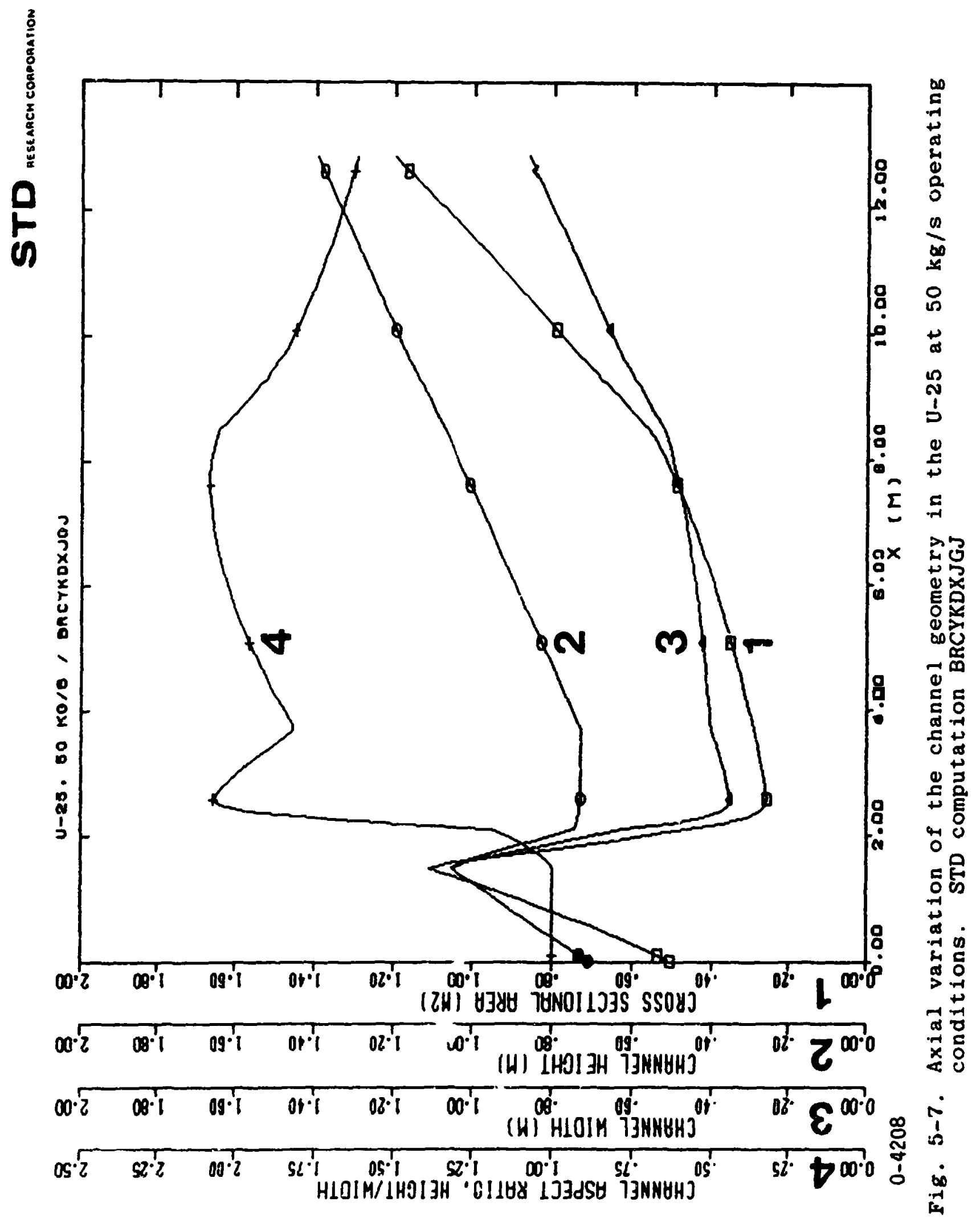




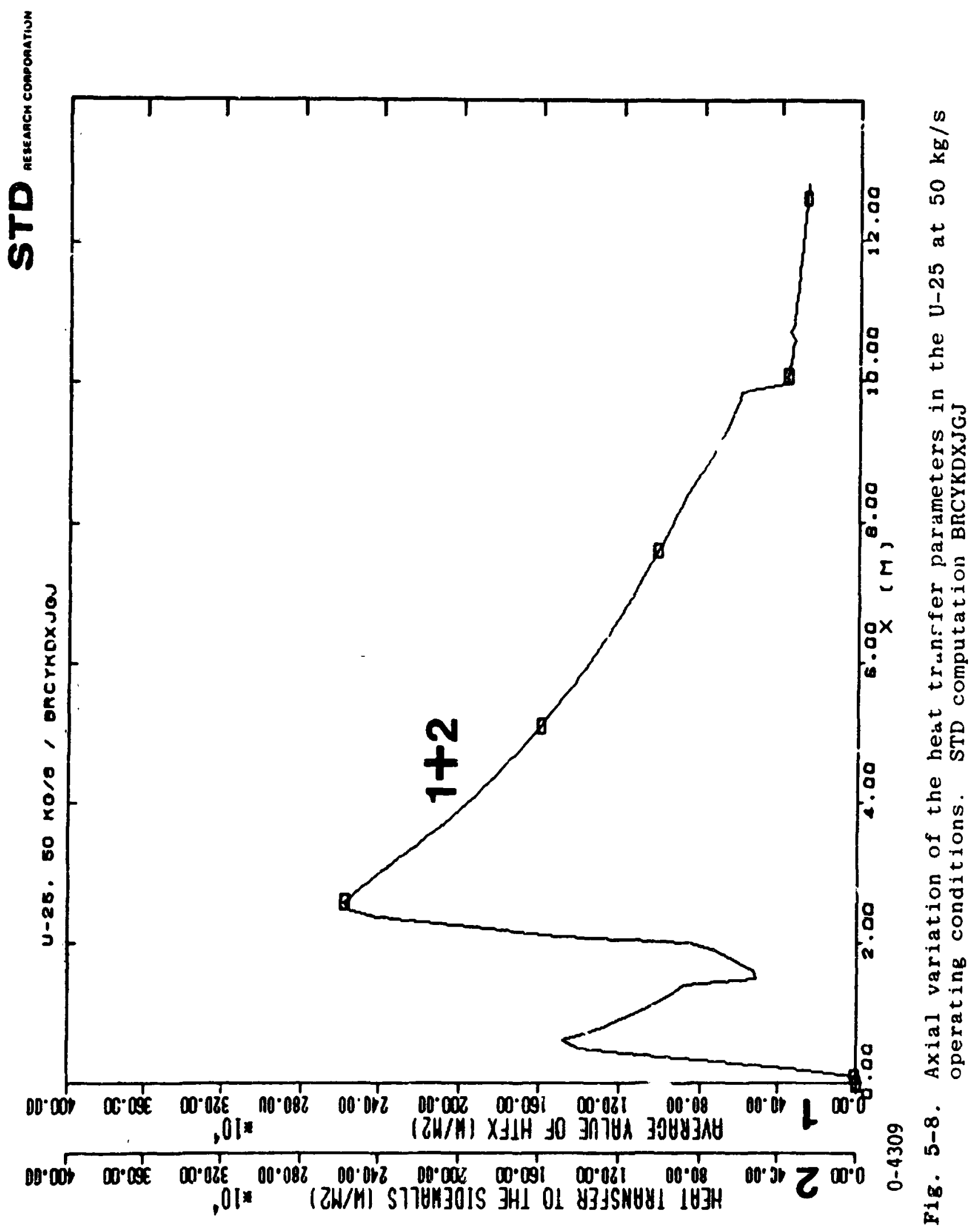




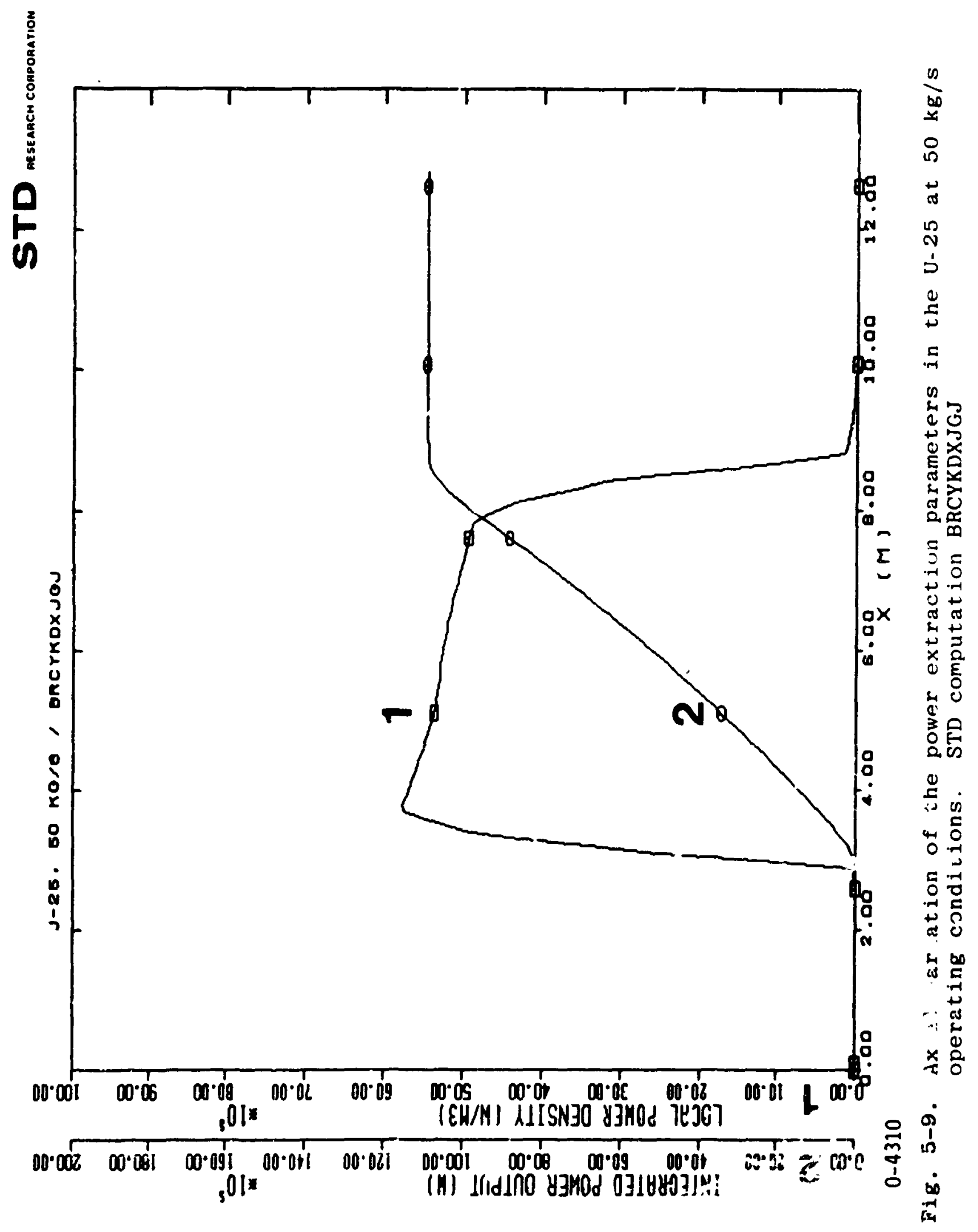




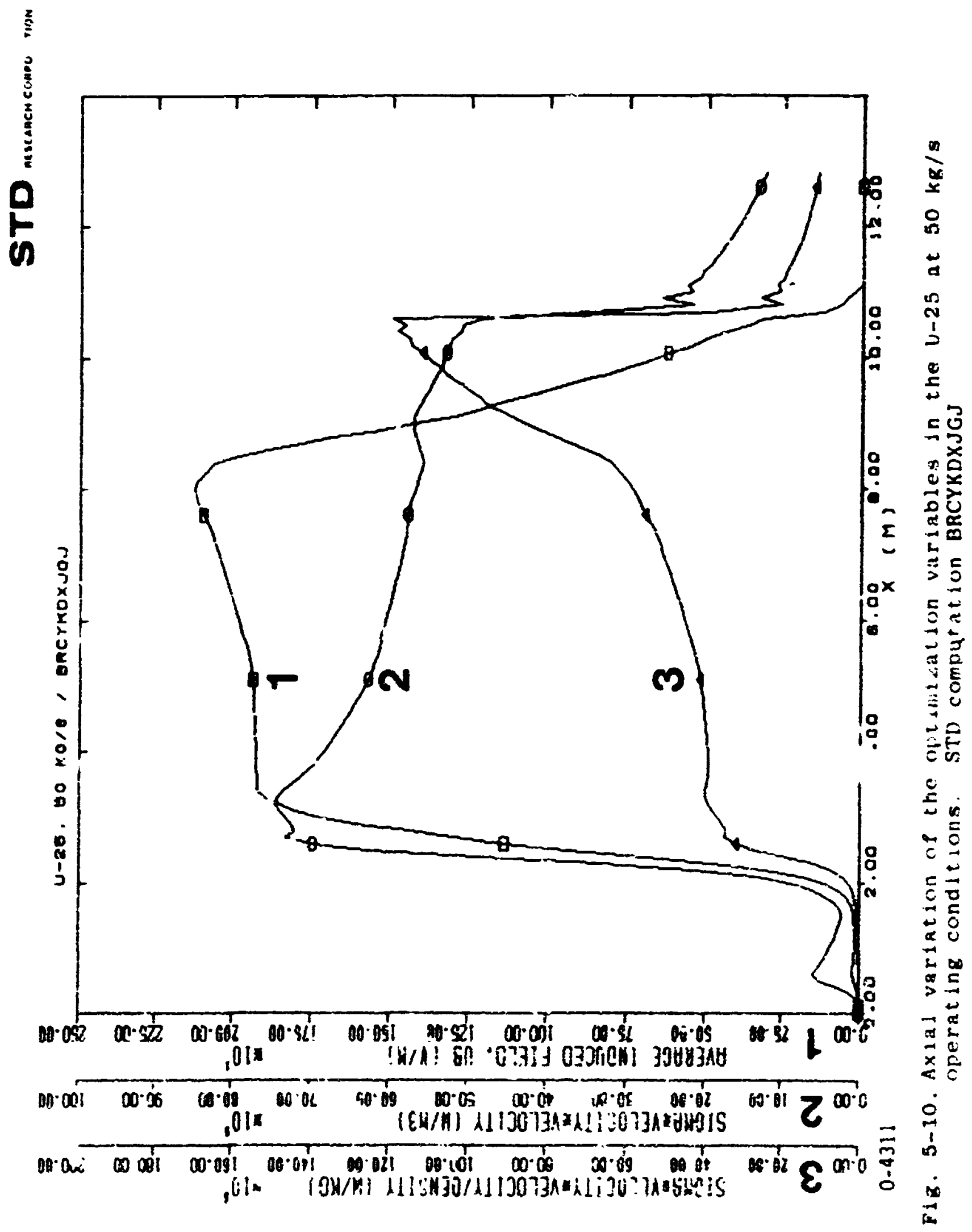




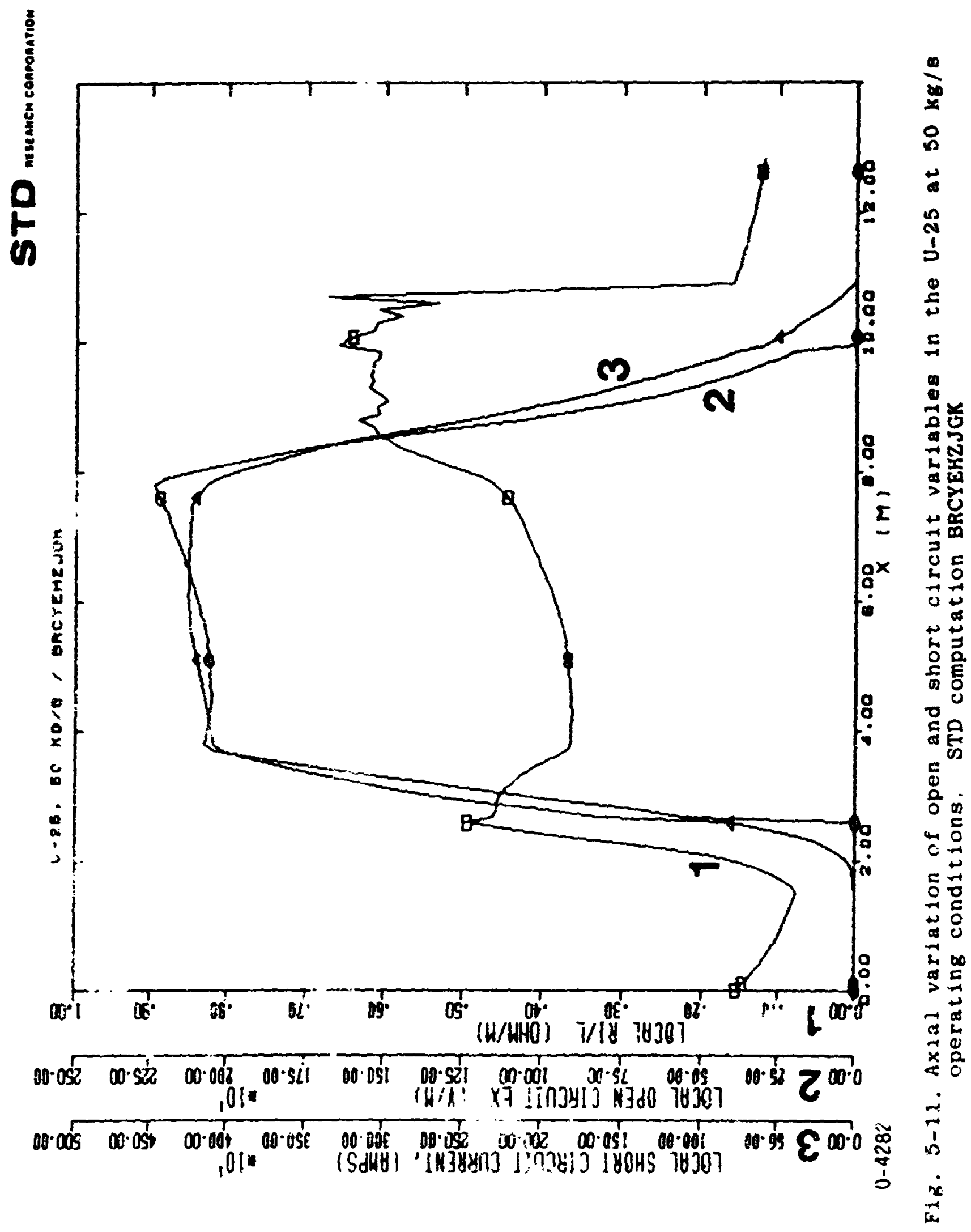




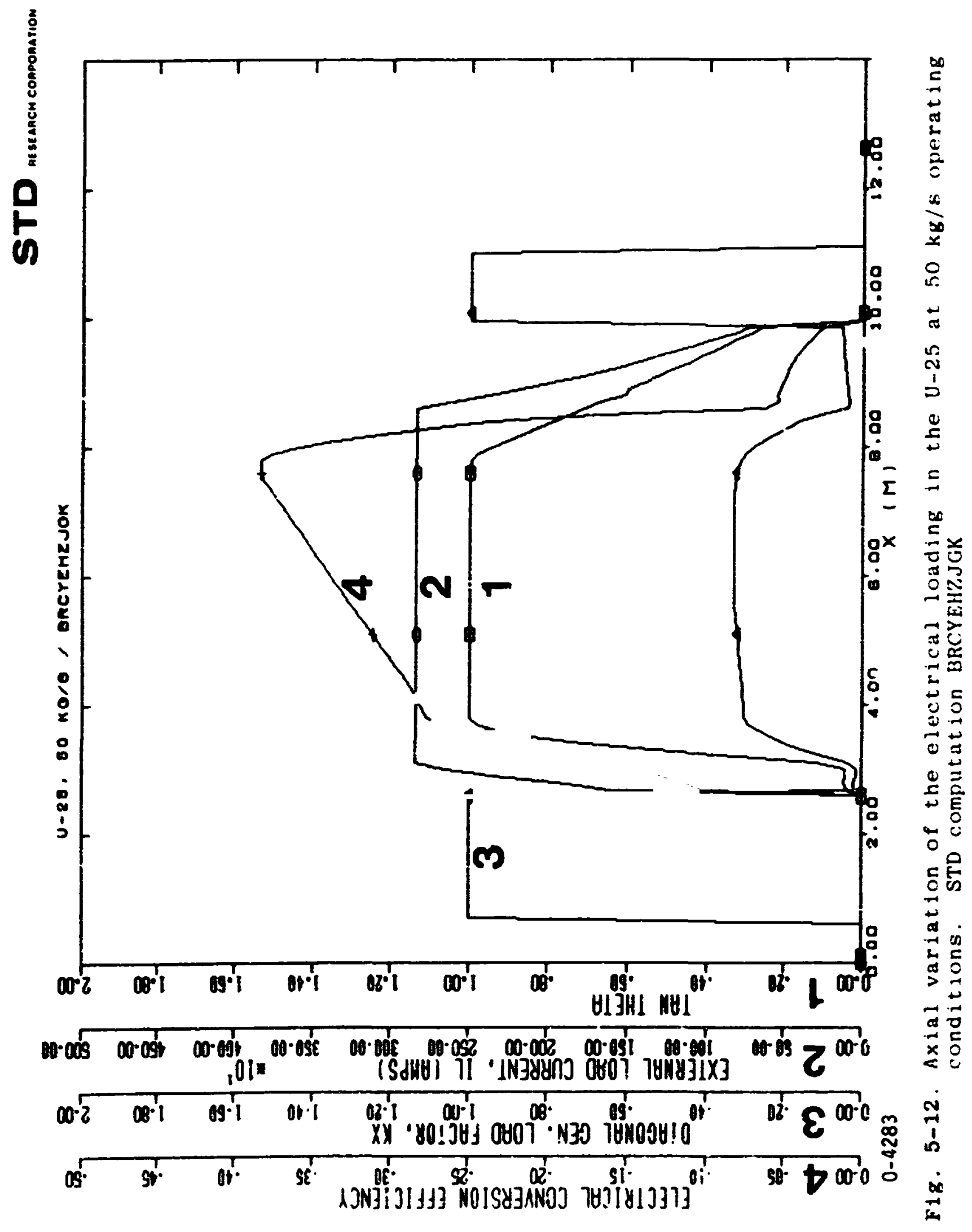




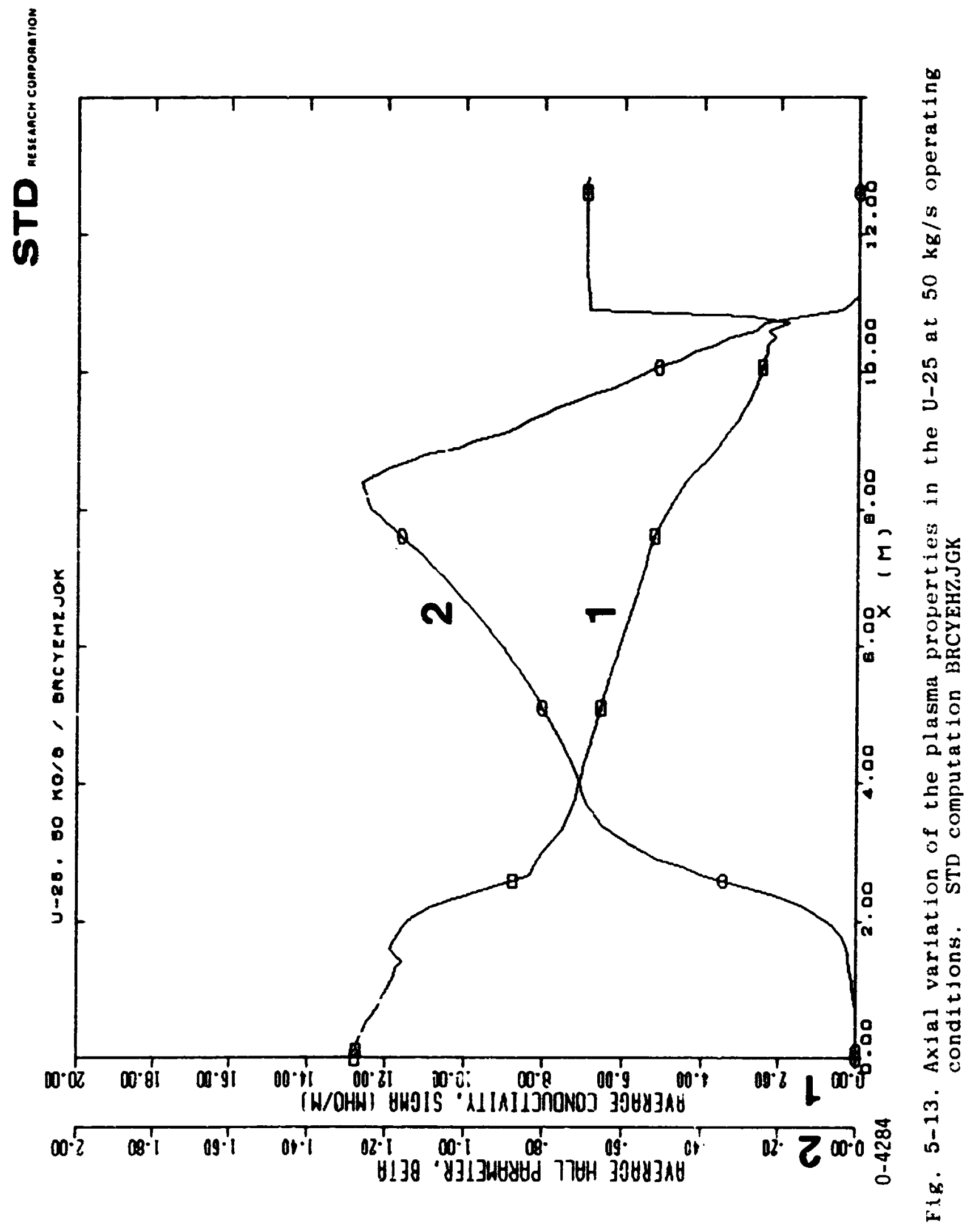




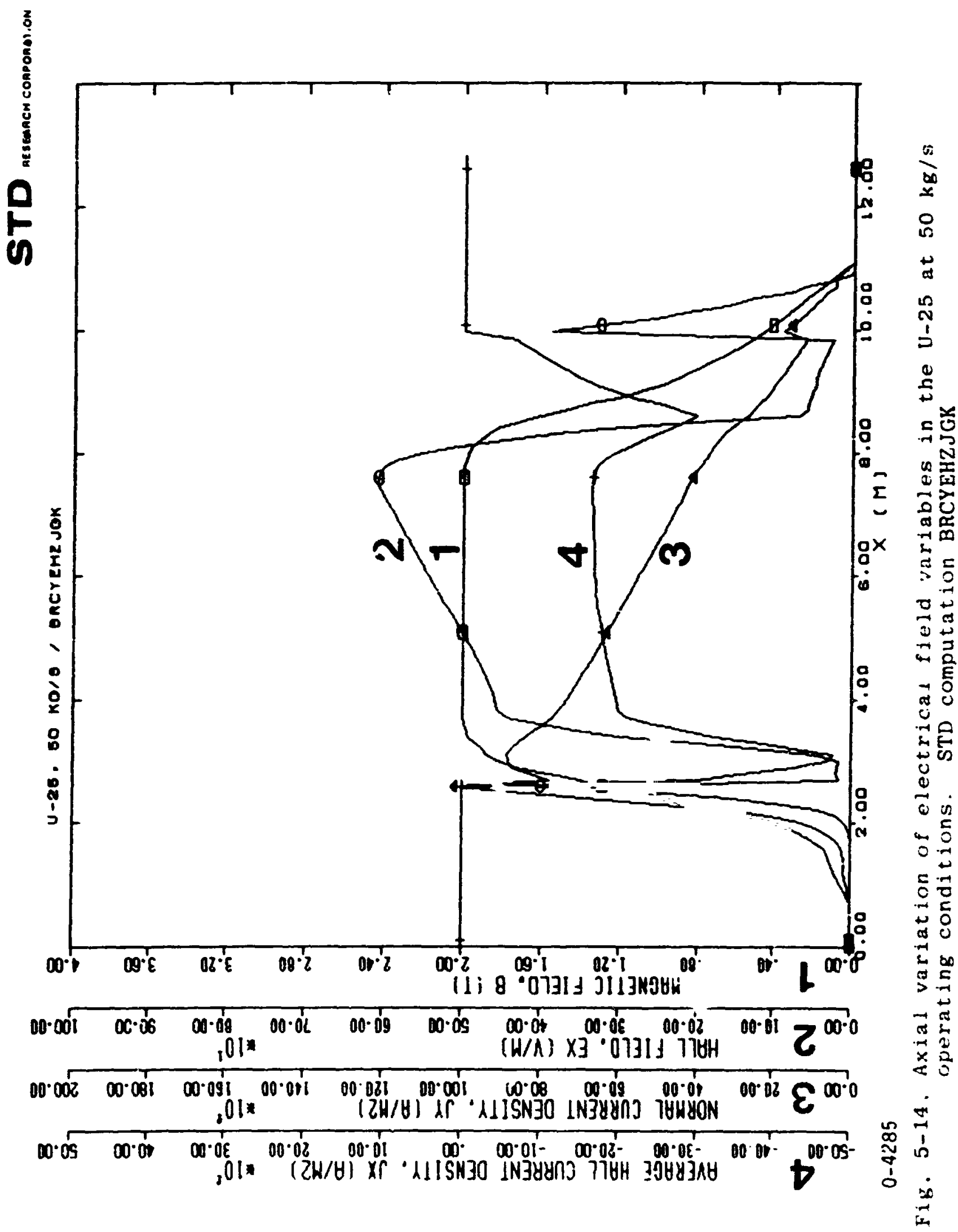




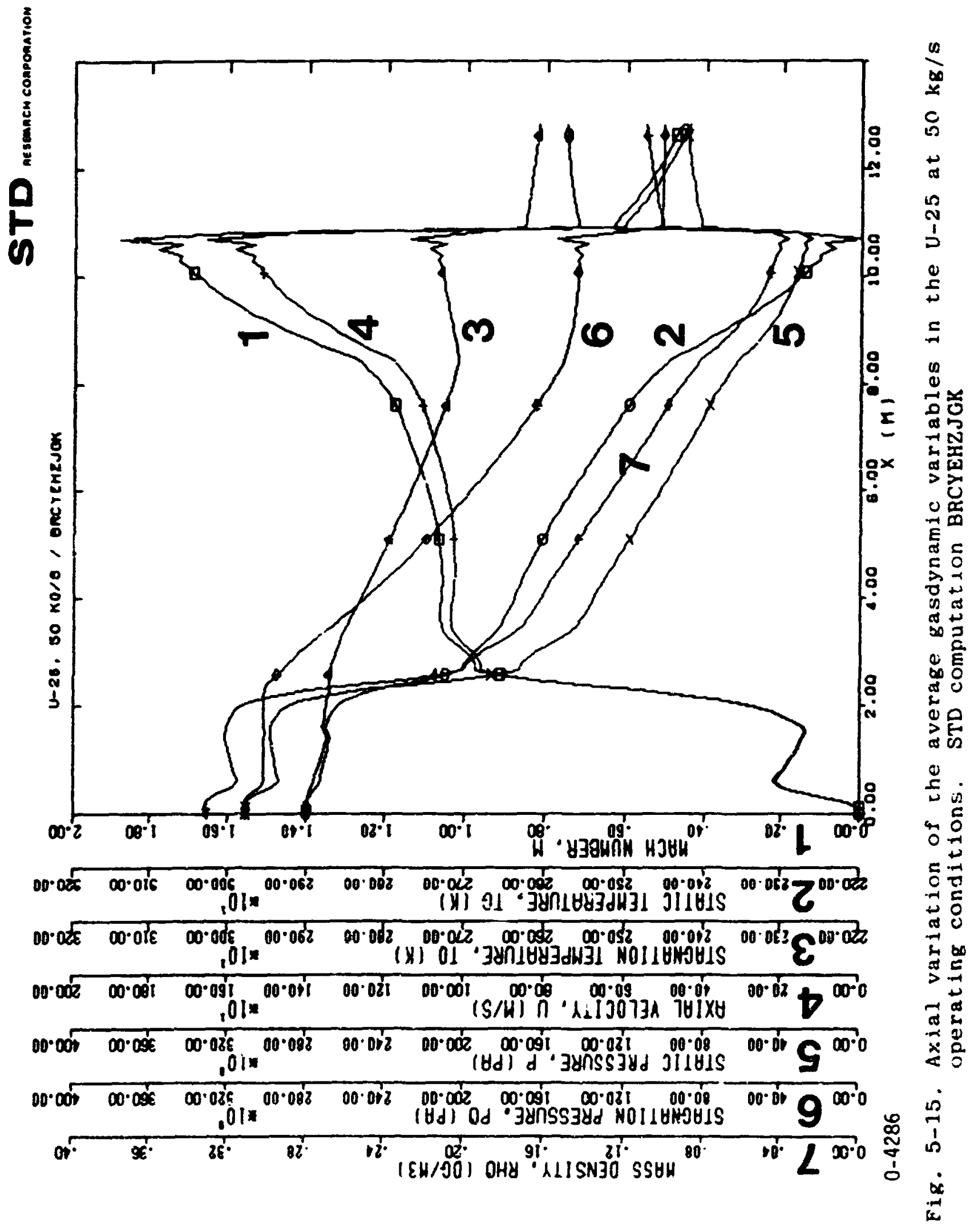




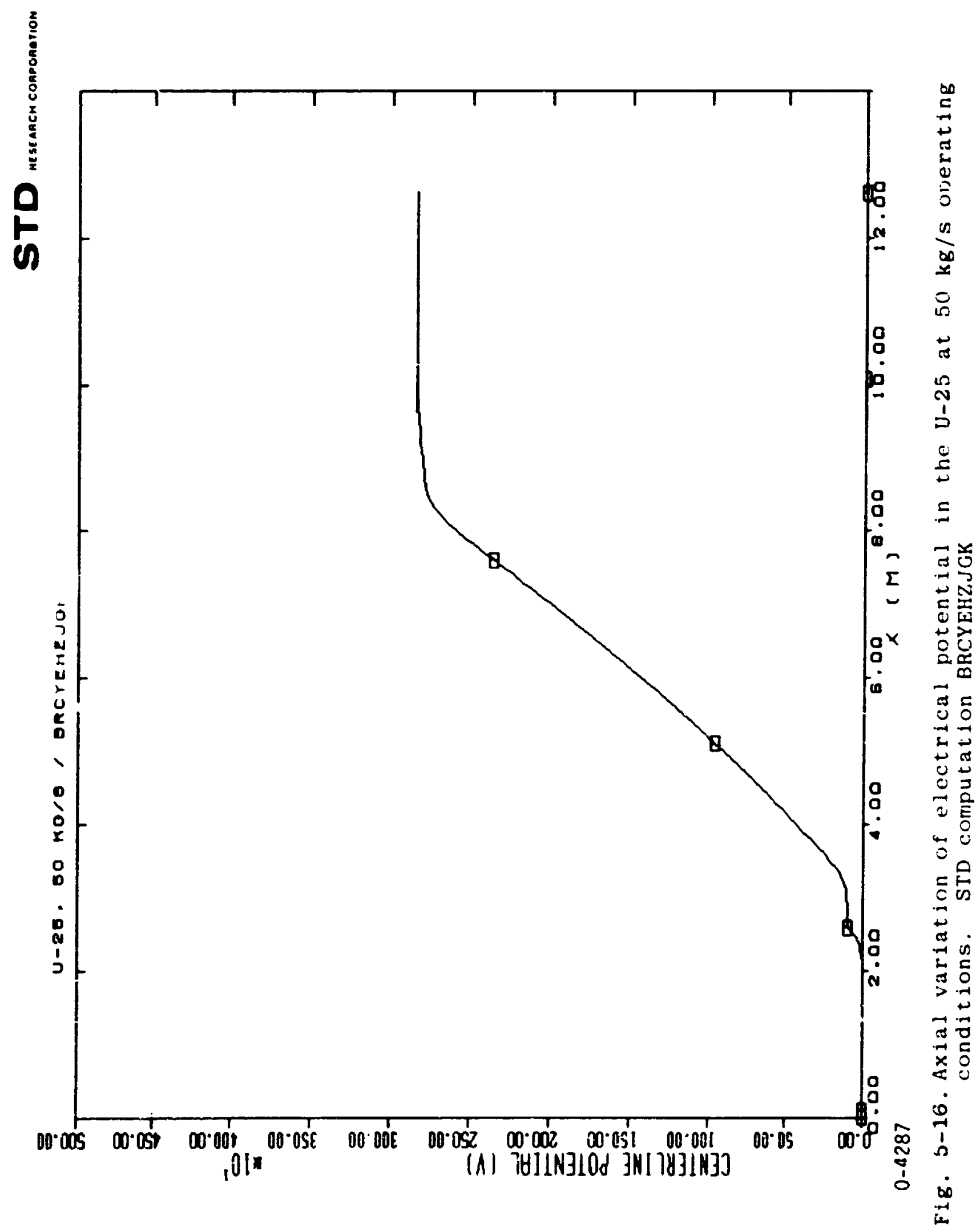




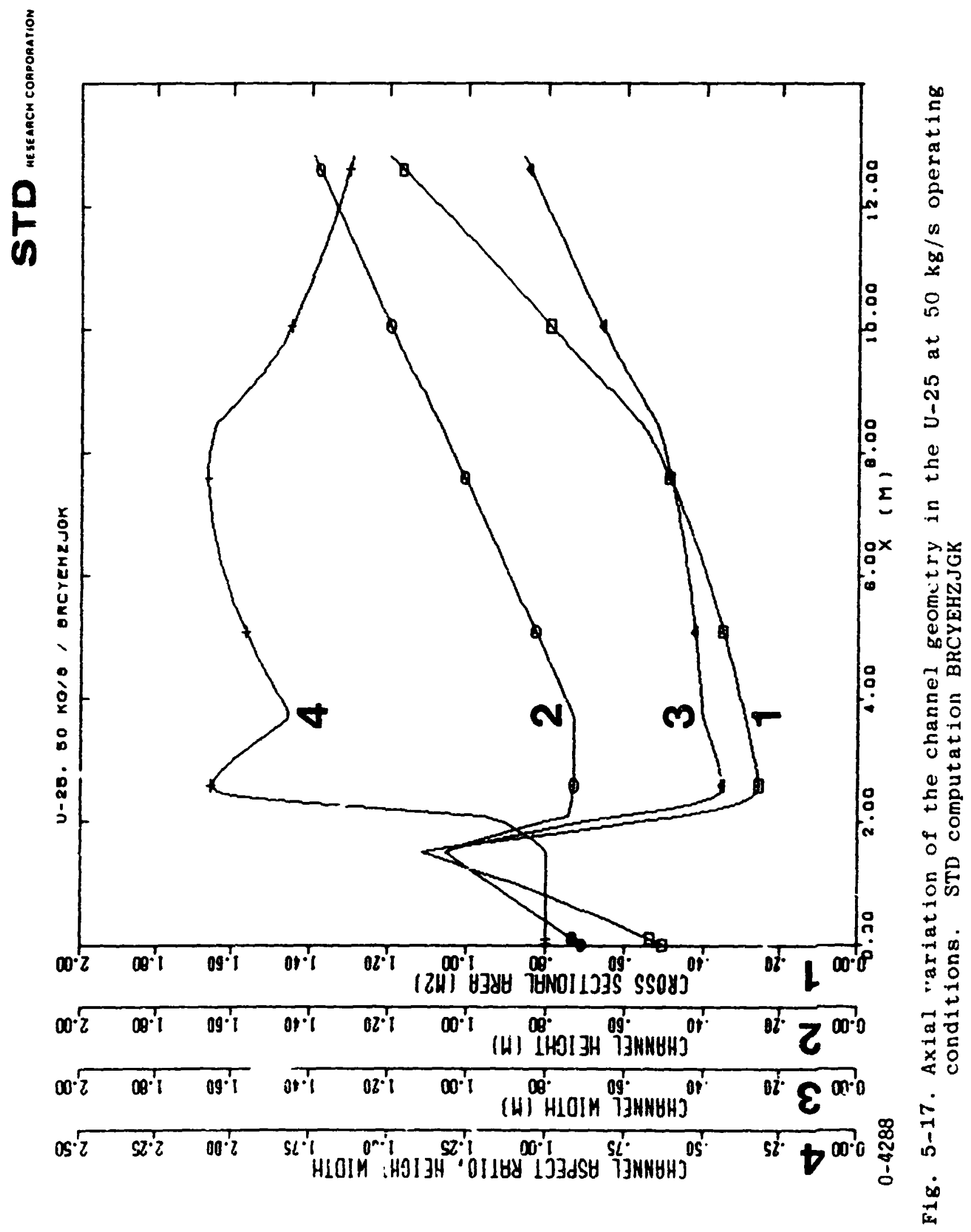




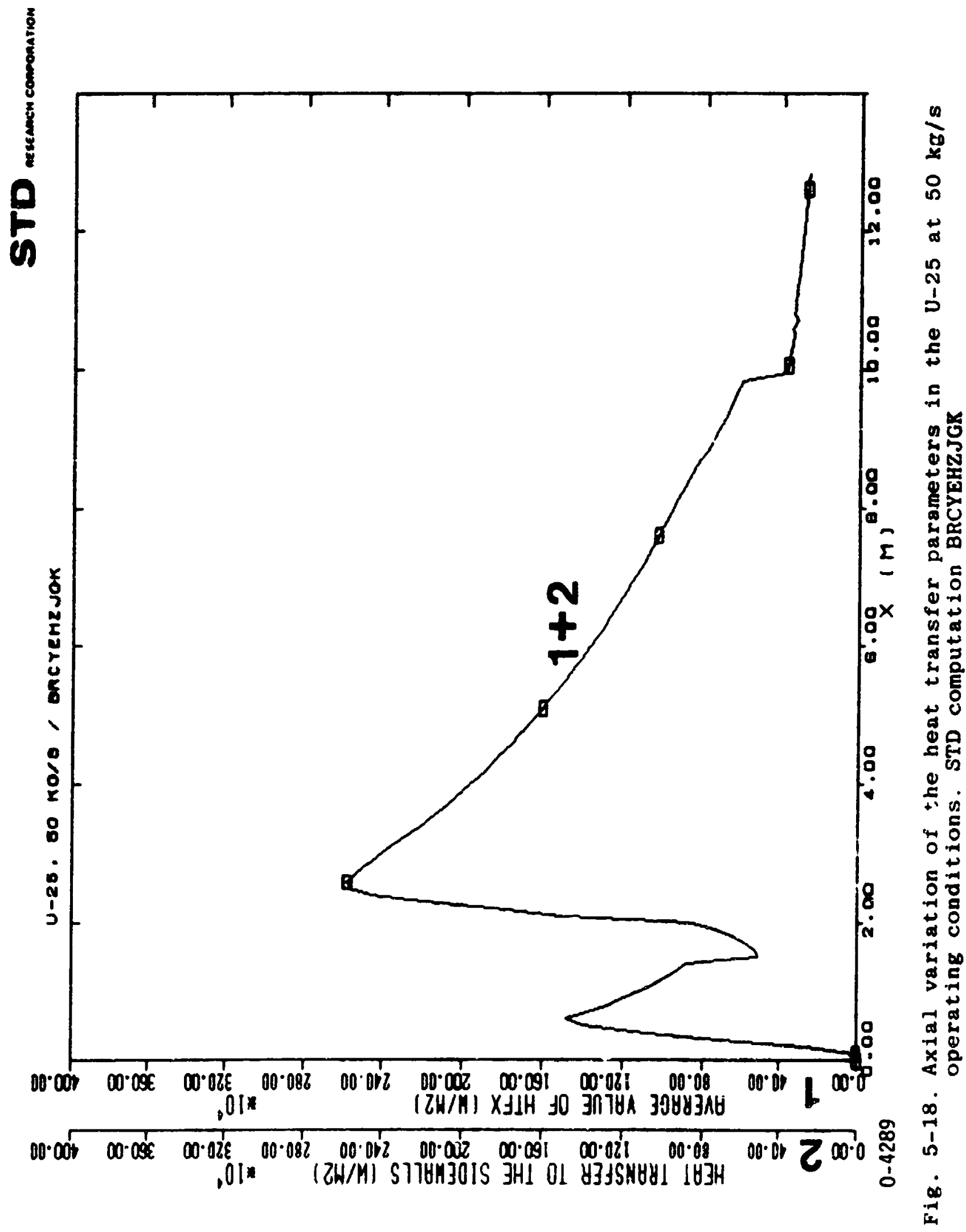




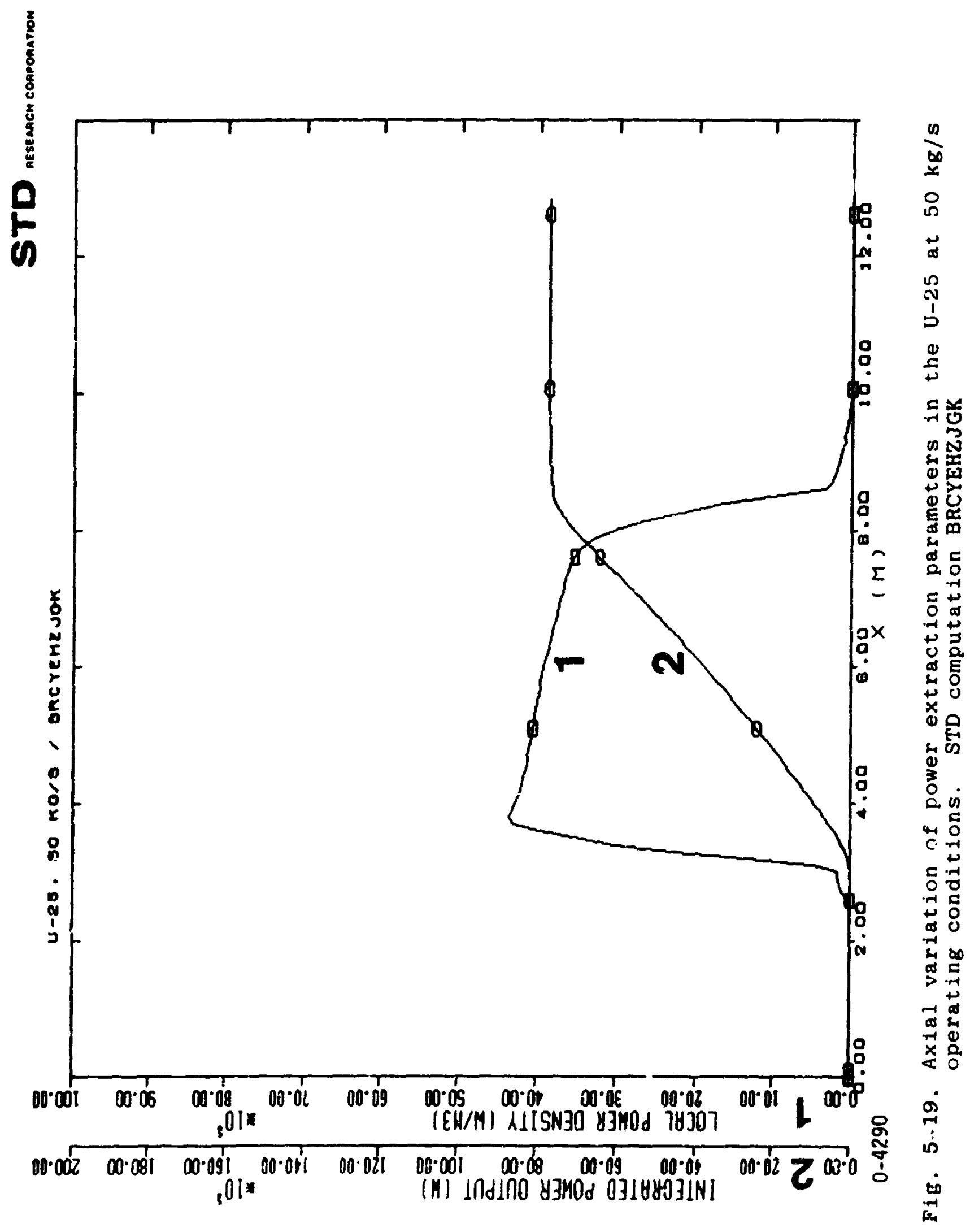




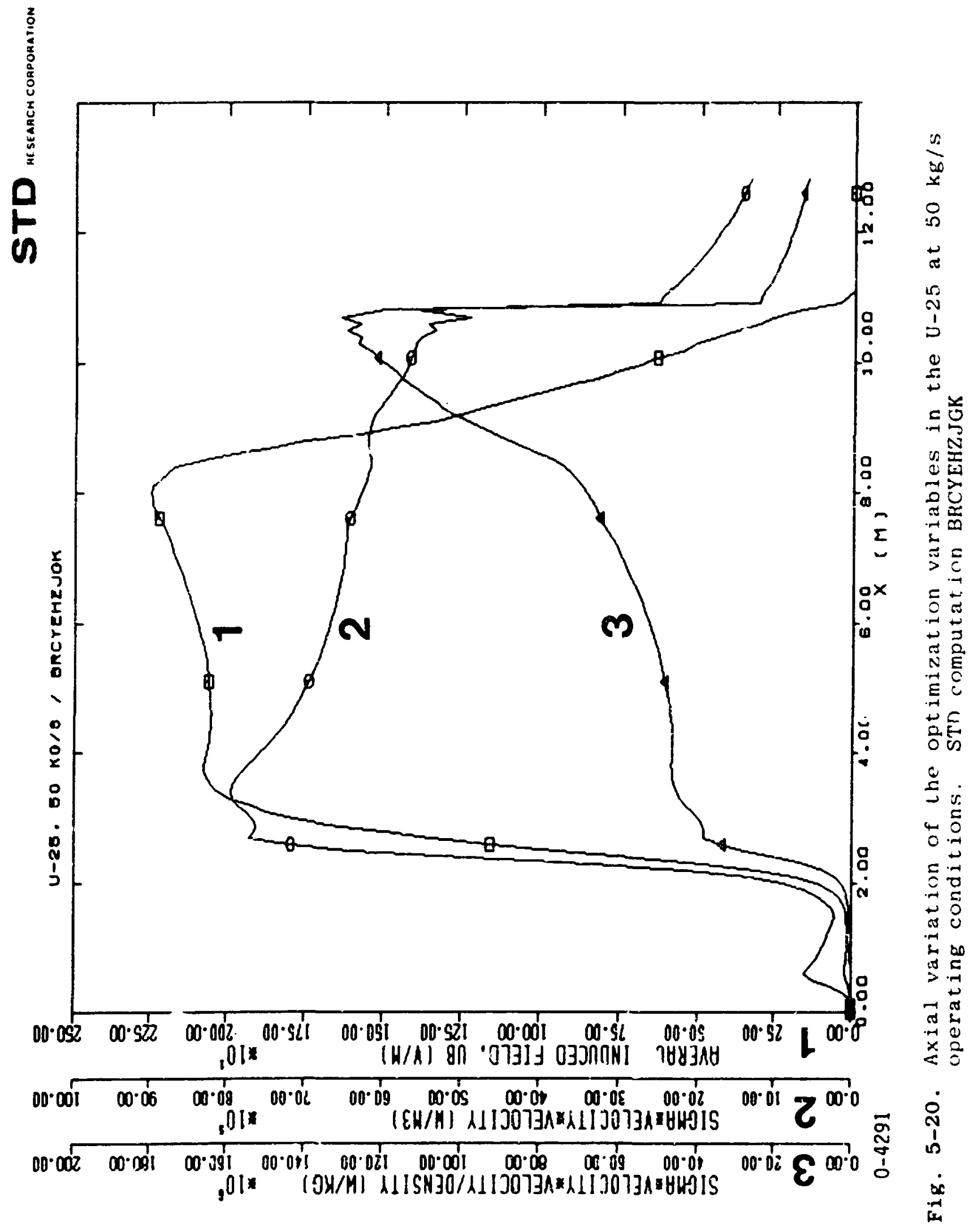




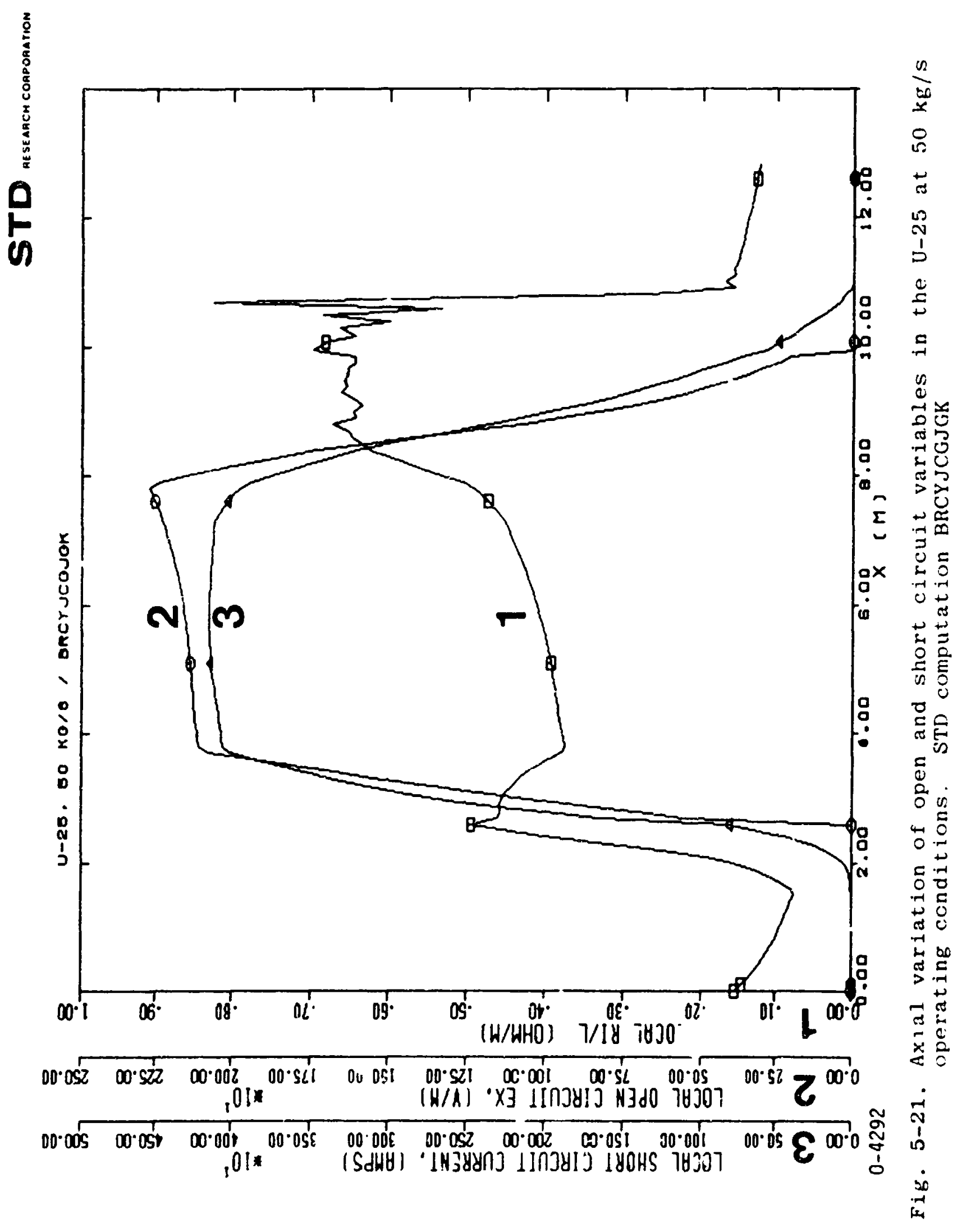




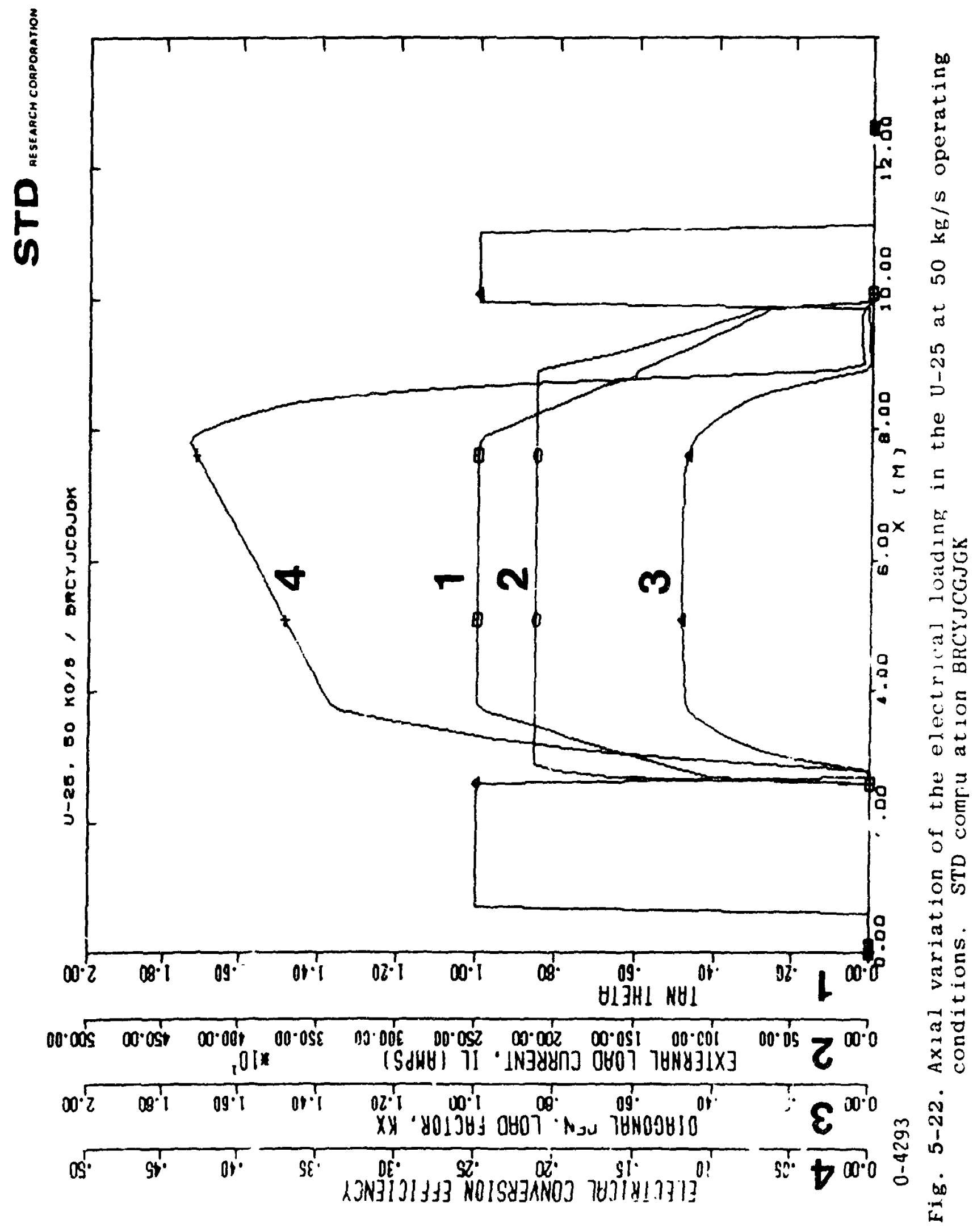




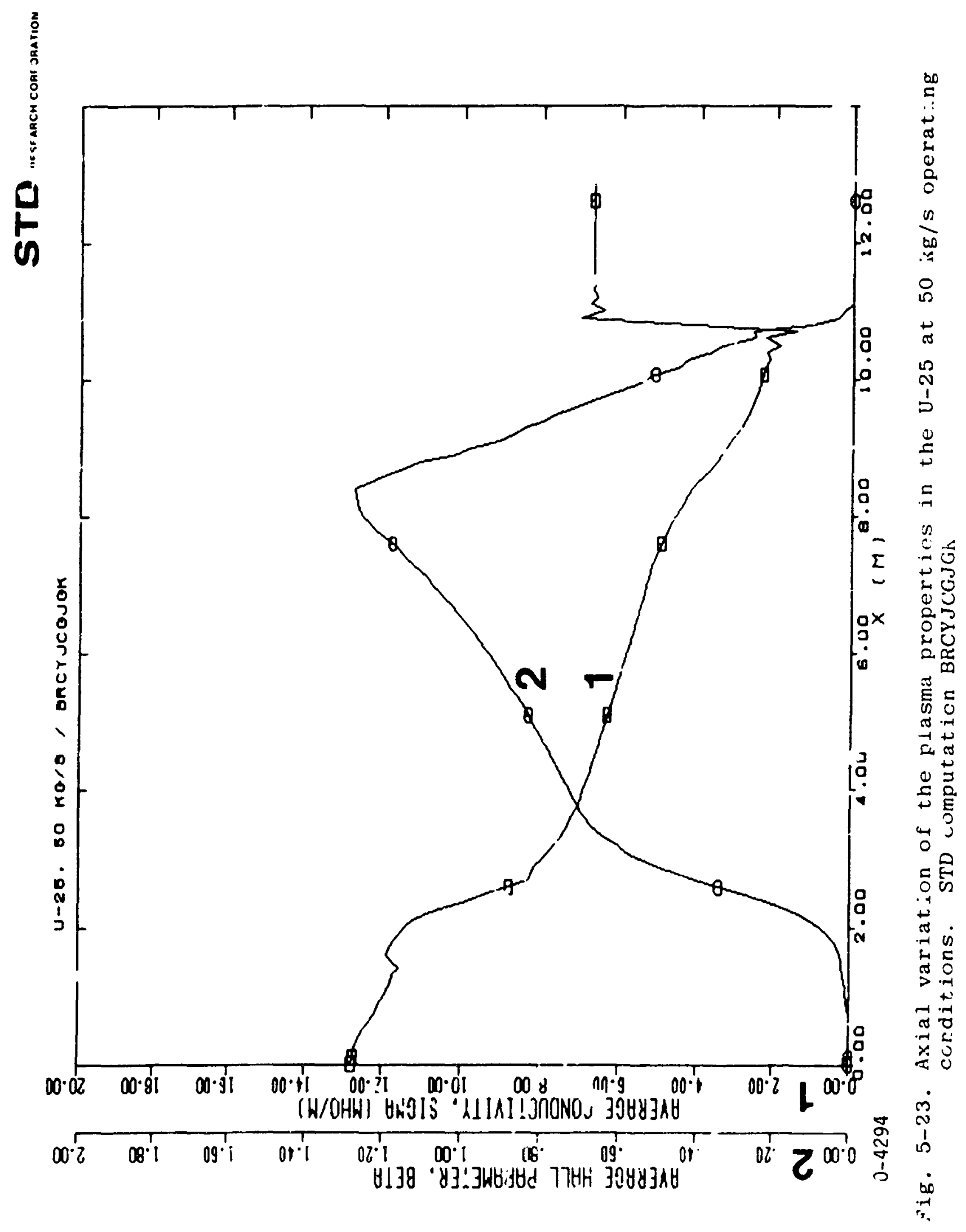




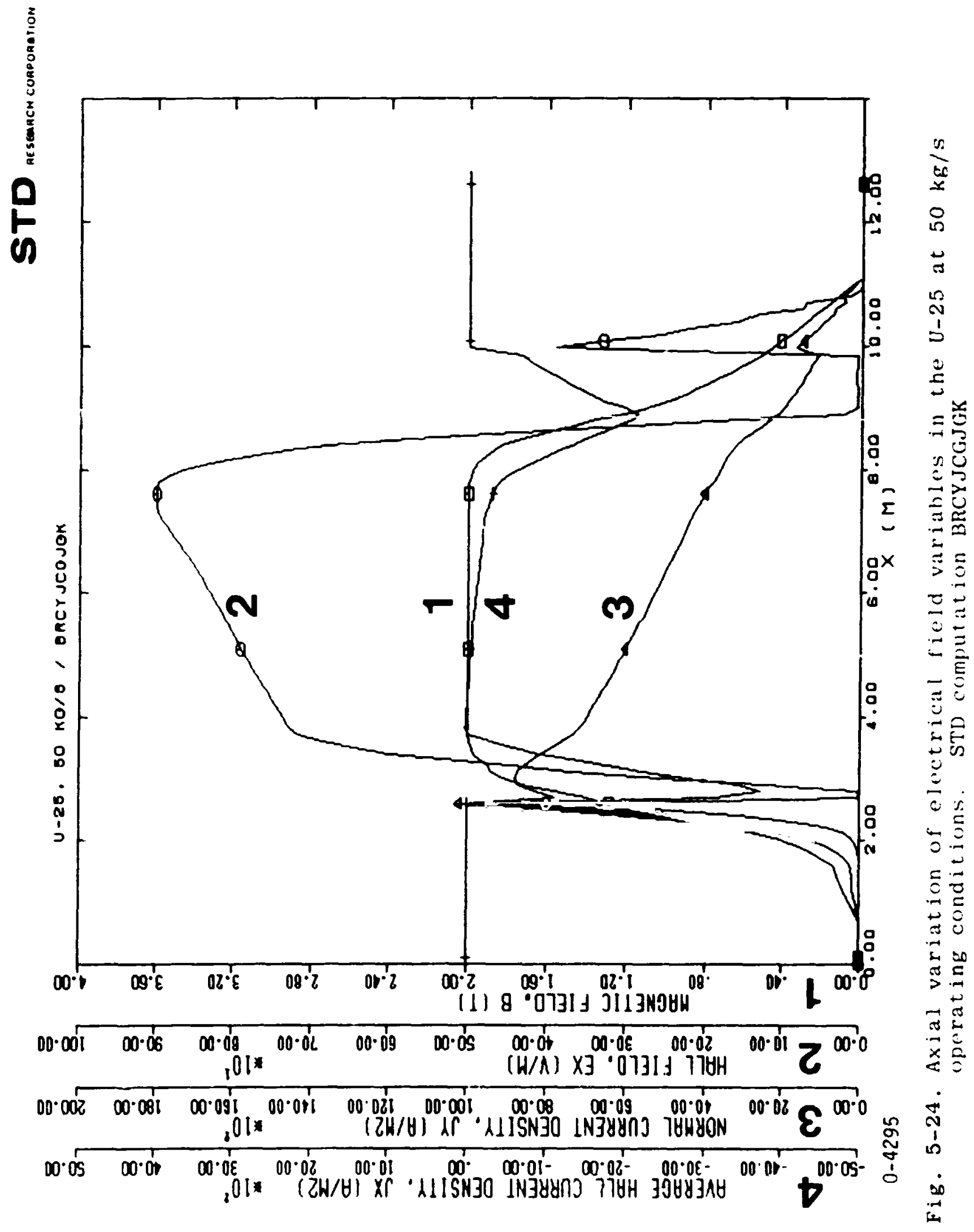




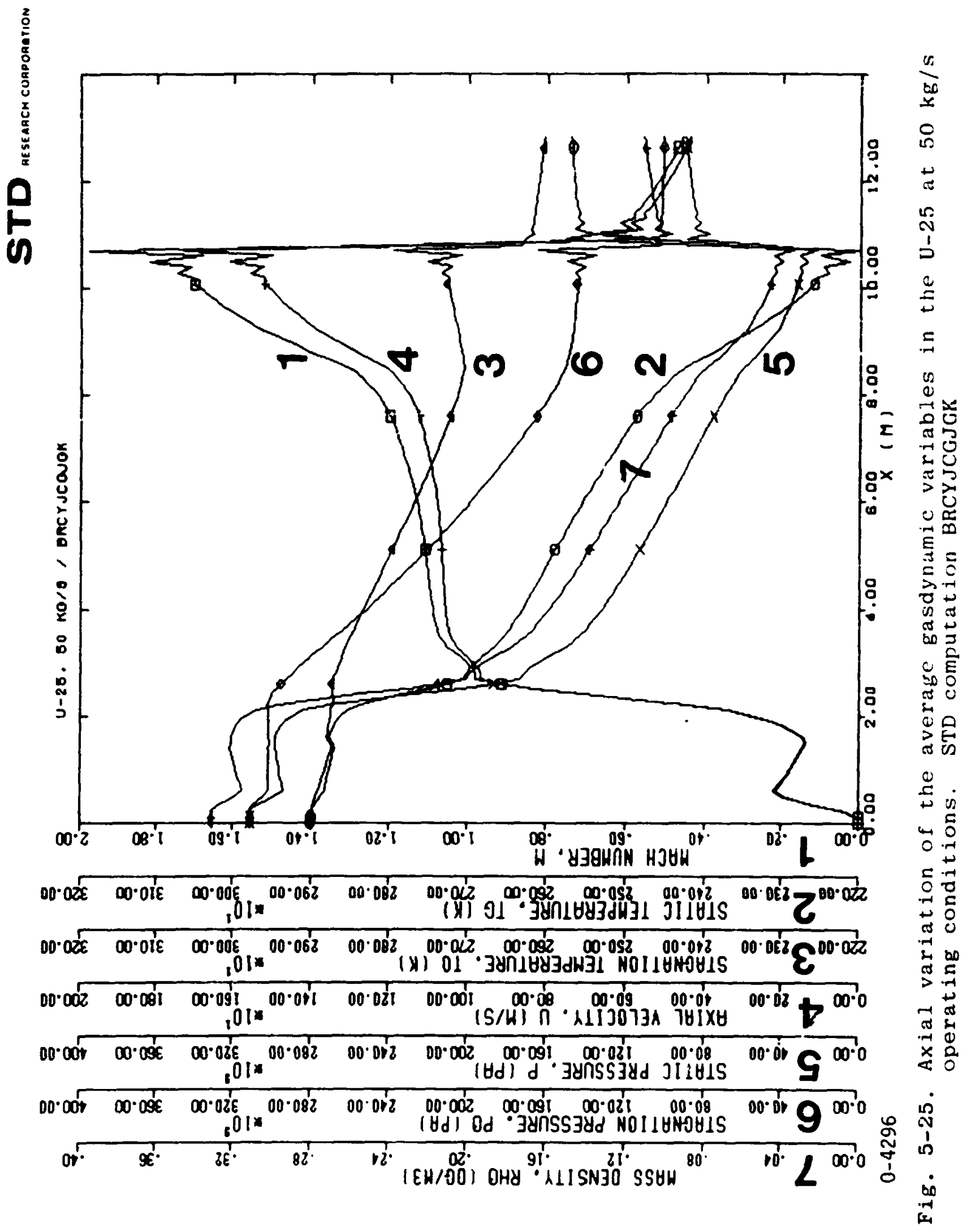




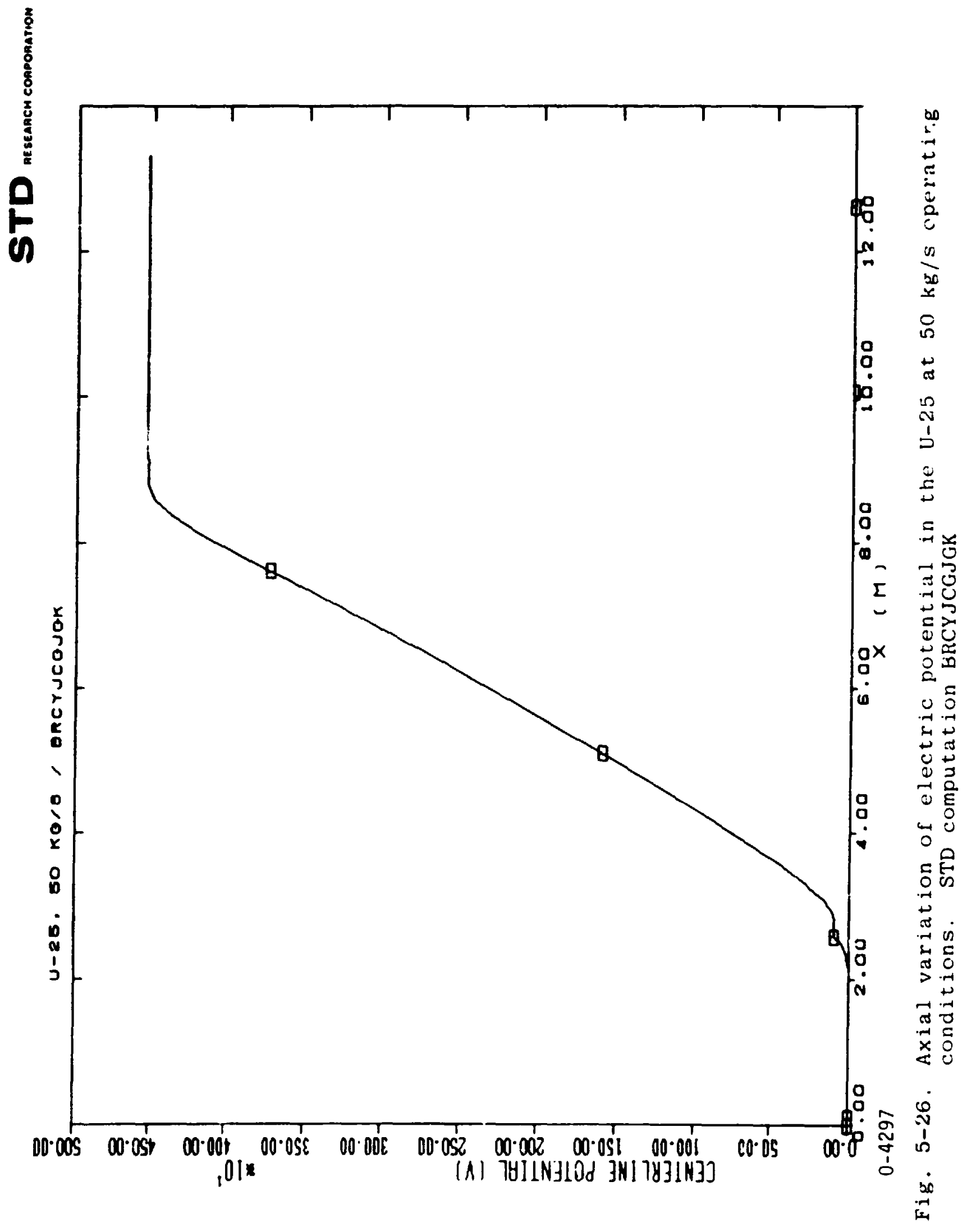




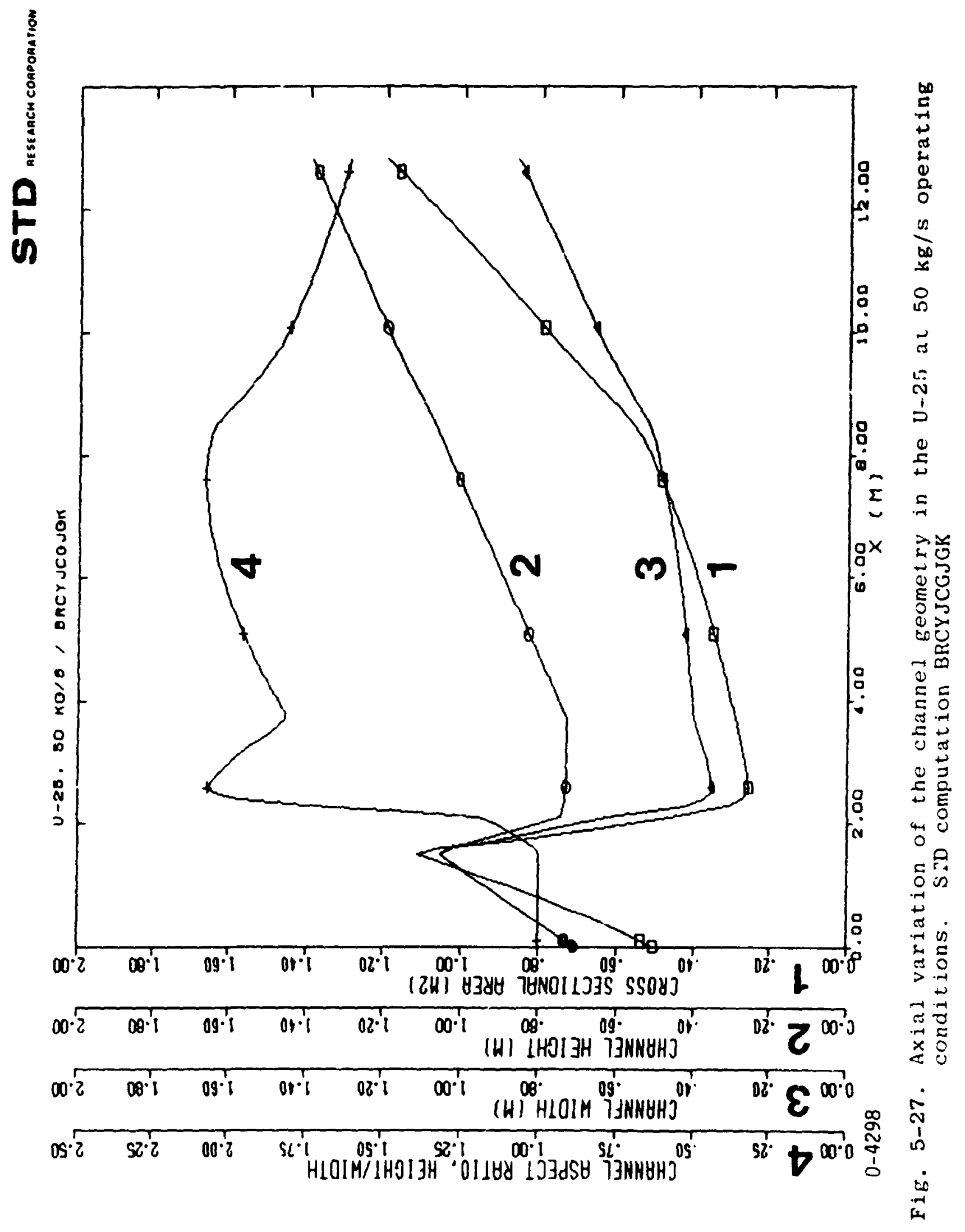




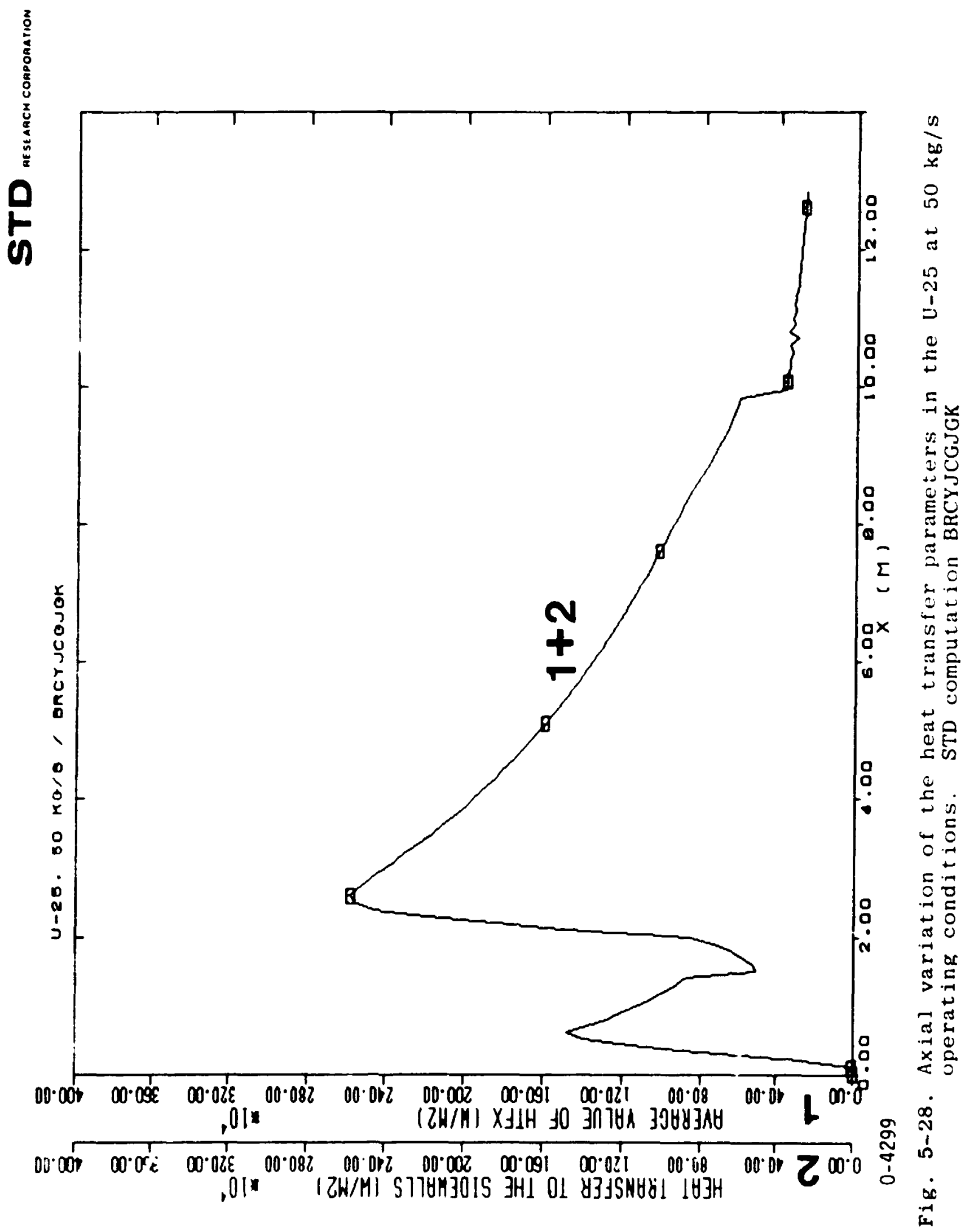




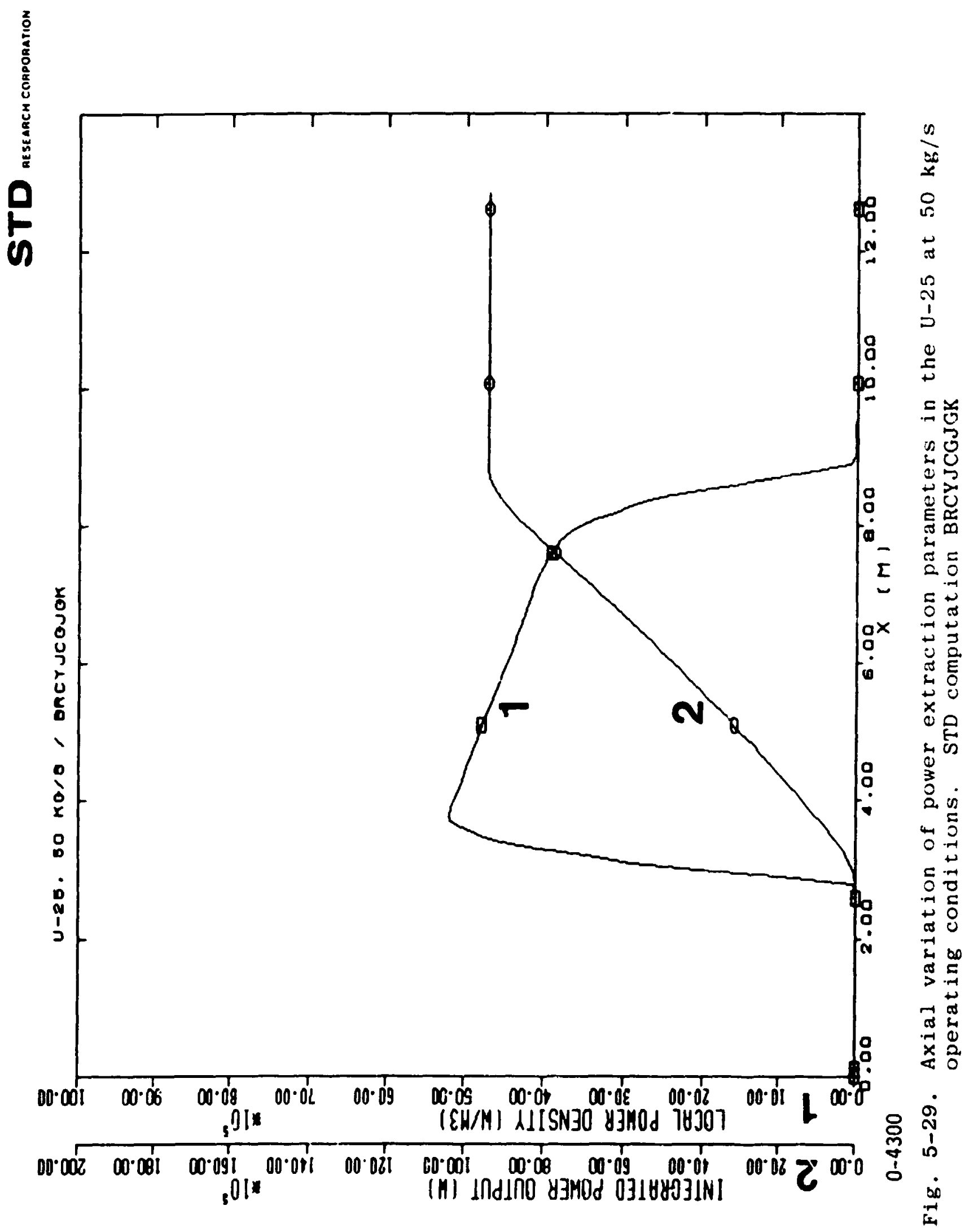




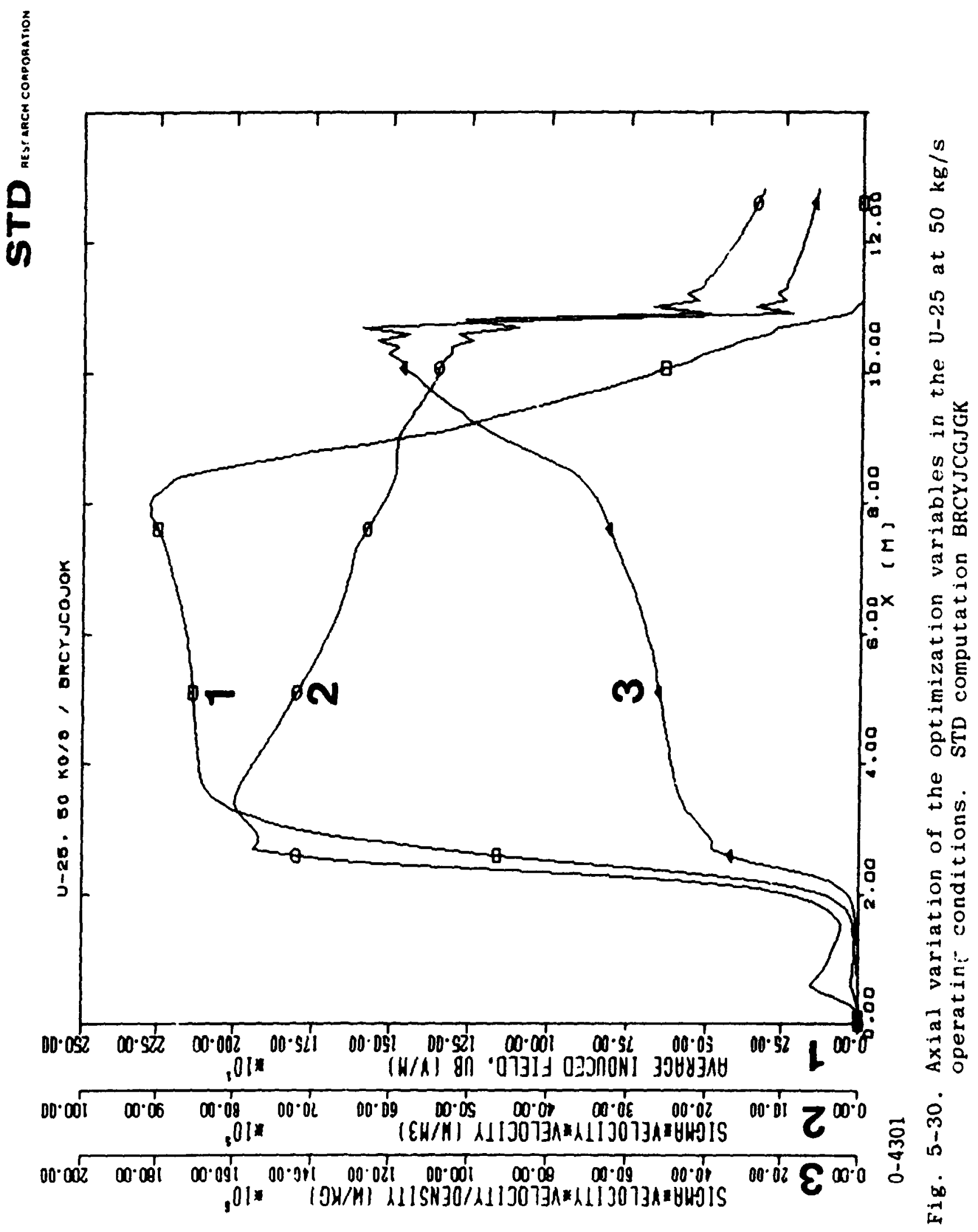




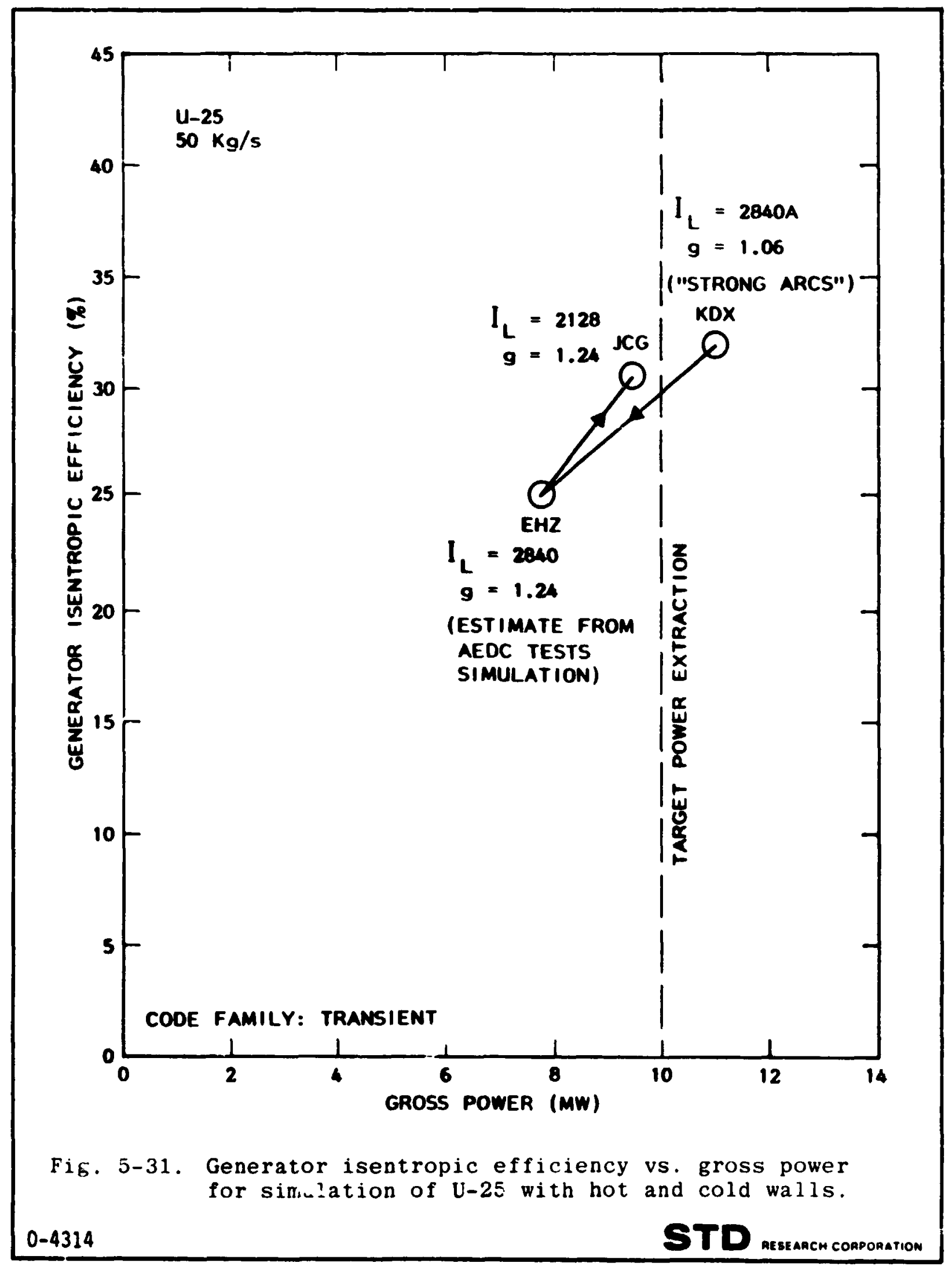




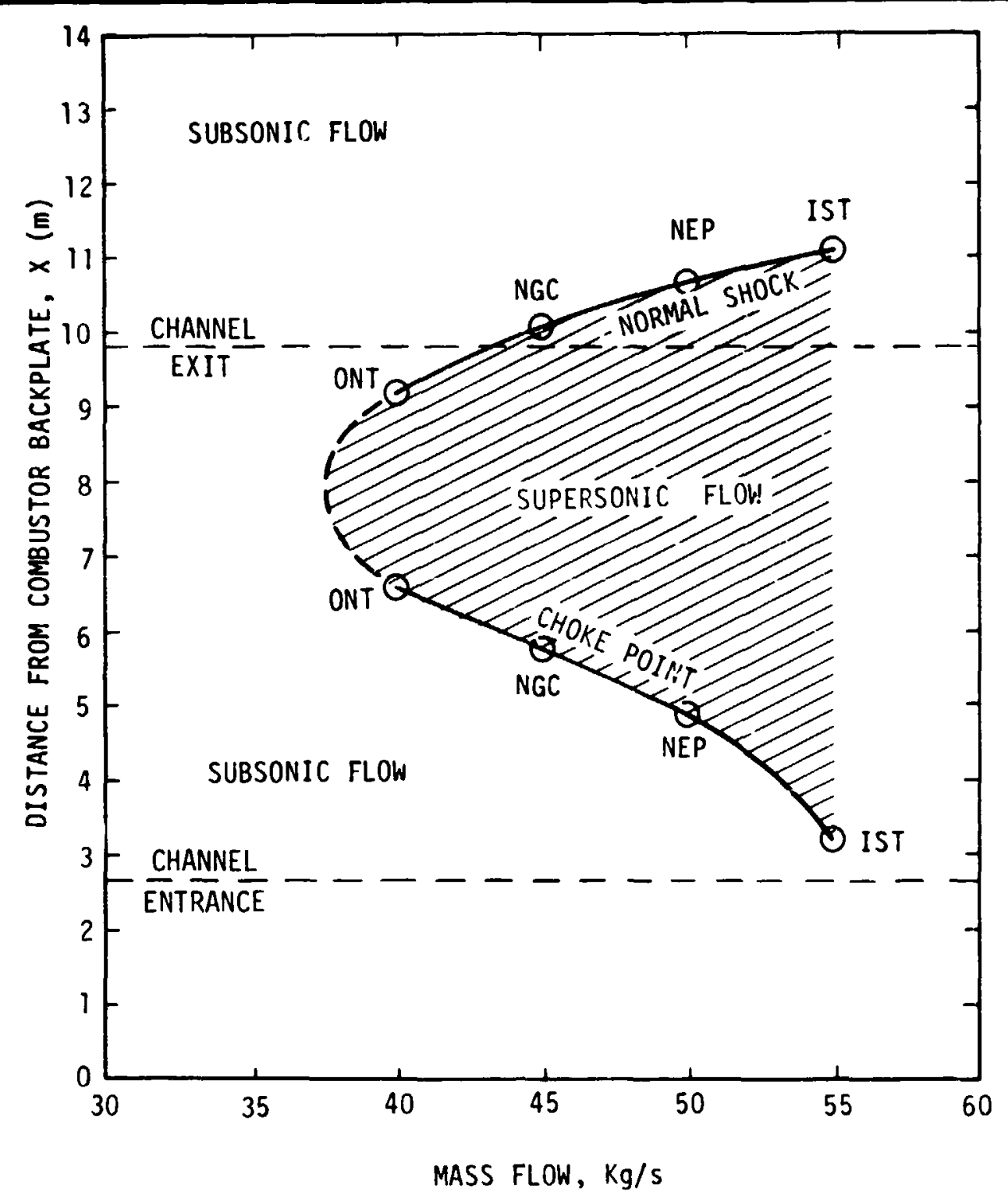

$9-3354$

Fig. 5-32. Shock location and choke points as a function of mass flowrate for the U-25 channe] 


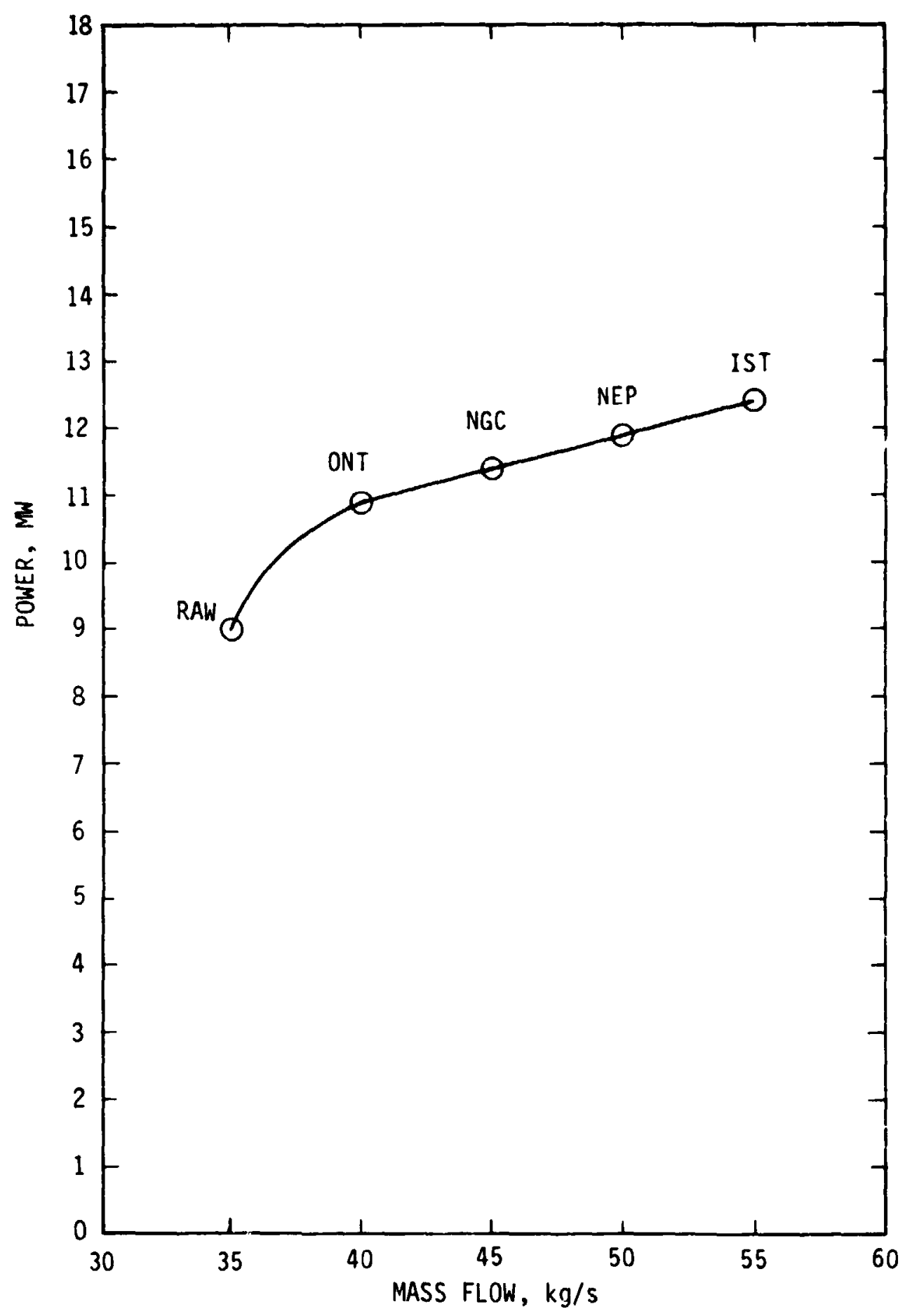
Fig. 5-33. Output power as a function of mass flowrate for the $9-3355$

S1D reseabih onhocanation 
STD aEseaAch Cofropation

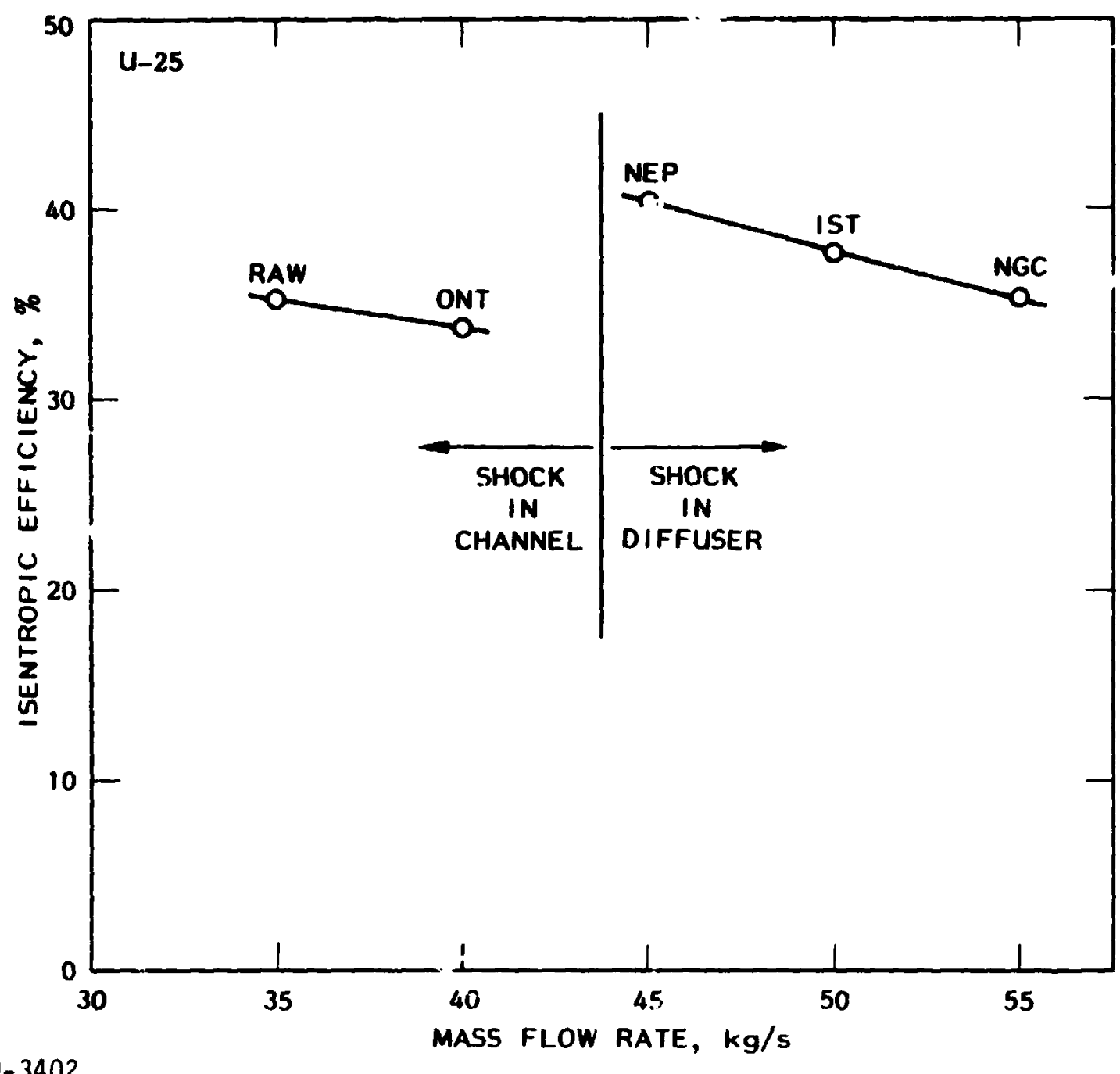

Fig. 5-34. Enthalpy extraction as a function of mass flow rate for the U-25 experiment. 


\subsection{PERFORMANCE OP NASA-SPECIFIED 500 MI(th) MHD GENERATOR \\ 6.1 Channel Characterization}

The full load operating characteristics of the NASAspeaified $500 \mathrm{MW}(\mathrm{th})$ MHD channel are given in Table 6-1. The

generator is defined to be square, i.e. rectangular with aspect ratio of 1.0 , from inlet to exit. The axial distribution of magnetic flux density increases from $4 \mathrm{~T}$ at the in:et to $6 \mathrm{~T}$ at $2.5 \mathrm{~m}$ from the inlet, then decreases linearly to $4.6 \mathrm{~T}$ at the exit of the generator. A list of the thermodynamic data to define the working fluid for this NASA-specified $500 \mathrm{Mm}$ (th) MHD channel are given in Table 6-2. The specifications contained in Tables 6-1 and 6-2 may not be consistent, i.e., it is possible that not all of these specifications can be attained simultaneously.

\subsection{Generator Inlet Plane Electrical Conductivity}

\section{Calculations}

The specifictions of the working fluid and inlet thermodynamic and fluid mechanical conditions, given in Tables 6-1 and 6-2, were reduced to species inlet mole ratio, listed in Table 6-3, and inlet thermodynamic conditions. The thermodynamic conditions are inlet static temperature of $2710 \mathrm{~K}$ and inlet static pressure of $4.35 \mathrm{~atm}$.

The inlet transport and thermodynamic properties for the NASA-specified $500 \mathrm{MW}(\mathrm{th})$ MHD generator have been computed with a zero-dimensional, equilibrium method using the STDNASP code from the STD THERMODYNAMICS family of codes. For thrse conditions, the inlet thermodynamic and transport data are electron concentration of $2.71 \times 10^{20}$ electrons/m 3 and electrical conductivity of $6.83 \mathrm{~s} / \mathrm{m}$. 
Even with equilibrium chemistry, the conductivity in the inlet plane is more realistically a function of the distribution of static temperatures and pressures and also a function of the distribution of current. Since the electrical conductivity is approximately an exponential function of temperature and the electrical current raises the electron temperature, both of these two important effects will raise the value of average ilectrical conductivity in the inlet plane above that computed with the zero-dimensional, equilibrium code.

For example, at the $0.813 \mathrm{~m}$ station (inlet plane) of the HPDE the average electrical conductivity from computation CHPQUUUJBO is $10.4 \mathrm{~s} / \mathrm{m}$, and the electrical conductivity calculated for the average static temperature and average static pressure is $9.46 \mathrm{~s} / \mathrm{m}$. Thus, for this example, the multidimensional, nonequilibrium code computes an average inlet conductivity which is $10.6 \%$ higher than would be calculated by a zero-dimensional method. In another example, taken from the inlet plane of the Mark VI-C simulation STD68830HI, the average conductivity is $12.4 \mathrm{~s} / \mathrm{m}$ and the zero-dimensional conductivity at the average static pressure and average static temperature is $12.2 \mathrm{~s} / \mathrm{m}$, a difference of only $2.0 \%$. 


\section{TABLE 6-1}

Full Load Operating Characteristics

For a NASA-Specified $500 \mathrm{MW}(\mathrm{th})$ MHD Channel

1. Channel mass flow rate: $97.5 \mathrm{~kg} / \mathrm{s}$

2. Channel inlet average stagnation pressure: $6.5 \mathrm{~atm}$

3. Channel inlet average stagnation temperature: $2837 \mathrm{~K}$

4. Channel inlet height: $0.51 \mathrm{~m}$

5. Channel inlet width: $0.51 \mathrm{~m}$

6. Channel length. $14.8 \mathrm{~m}$

7. Faraday load factor: $\mathbf{0 . 8 5}$

8. Channel inlet average mach number: 0.85

9. Burner and nozzle heat loss: $21 \mathrm{MW}$

10. Slag heat loss: $3.7 \mathrm{MW}$ 


\section{TABLE 6-2}

Thermodynamic Data to Define the Working Fluid For a NASA-Specified $500 \mathrm{Mm}(\mathrm{th})$ MHD Channel

1. Coal and Ash Analysis

A. Proximate analysis, weight percent

$\begin{array}{lr}\text { Moisture } & 22.7 \\ \text { Volatiles } & 29.4 \\ \text { Pixed Carbon } & 39.2 \\ \text { Ash } & 8.7\end{array}$

B. Ultimate Analysis, weight percent

$\begin{array}{lc}\text { Hydrogen } & 6.0 \\ \text { Carbon } & 52.1 \\ \text { Nitrogen } & 0.79 \\ \text { Oxygen } & 31.5 \\ \text { Sulfur } & 0.85\end{array}$

C. Ash analysis, weight percent

$\begin{array}{lr}\mathrm{SiO}_{2} & 37.6 \\ \mathrm{Al}_{2} \mathrm{O}_{3} & 17.3 \\ \mathrm{Fe}_{2} \mathrm{O}_{3} & 5.1 \\ \mathrm{TiO}_{2} & 0.7 \\ \mathrm{P}_{2} \mathrm{O}_{5} & 0.4 \\ \mathrm{CaO} & 11.0 \\ \mathrm{MgO} & 4.0 \\ \mathrm{Na}_{2} \mathrm{O} & 3.1 \\ \mathrm{~K}_{2} \mathrm{O} & 0.5 \\ \mathrm{SO}_{3} & 17.5 \text { (assumed redundant) }\end{array}$




\section{TABLE 6-2 (Continued)}

D. Heating values

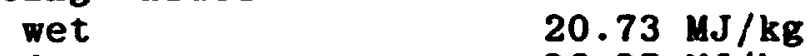

$20.73 \mathrm{MJ} / \mathrm{kg}$

$26.87 \mathrm{MJ} / \mathrm{kg}$

E. Coal dried to $5.0 \%$ moisture

F. Coal injected at $300 \mathrm{~K}$

G. Coal carrier gas: none

2. Oxidizer
A. Air composition of $300 \mathrm{~K}, 0.0132 \mathrm{~kg} \mathrm{H} \mathrm{H}_{2} \mathrm{O} / \mathrm{kg}$ dry air
B. Oxygen enriched to $35 \% \mathrm{O}_{2}$ by volume
C. Preheat to $922 \mathrm{~K}$

3. Seed
A. 1\% $\mathrm{K}$ by weight of channel mass flow rate
B. $0 \%$ by weight $\mathrm{K}_{2} \mathrm{SO}_{4}$
C. $100 \%$ by weight $\mathrm{K}_{2} \mathrm{CO}_{3}$
D. Anhydrous $\mathrm{K}_{2} \mathrm{CO}_{3}$
E. Seed temperature at $300 \mathrm{~K}$
F. Seed injected downstream of ash removal
G. Seed carrier fluid: none

4. Combusror
A. Ash removal of $65 \%$
B. Oxidizer/Fuel stoichiometric ratio of 0.90

5. Pliot
A. None

$0-4366$ 


\section{TABLE 6-3}

List of Species Used to Define the Working Fluid at the Inlet of the NASA-specified 500 MW(th) Generator

\section{$\underline{\text { Species }}$}

$\mathrm{C}_{5.3302}$

$\mathrm{SiO}_{2}$

$\mathrm{Al}_{2} \mathrm{O}_{3}$

MgO

$\mathrm{TiO}_{2}$

$\mathrm{CaO}$

$\mathrm{Fe}_{2} \mathrm{O}_{3}$

$\mathbf{P}$

$\mathrm{Na}_{2} \mathrm{O}$

$\mathrm{K}_{2} \mathrm{CO}_{3}$

$\mathrm{SO}_{3}$

$\mathrm{N}_{2}$

$\mathrm{O}_{2}$

$\mathrm{H}_{2} \mathrm{O}$
Mole Ratio

$\begin{array}{llll}H_{4.2192} & N_{0.0693} & S_{0.0326} & O_{0.8716}\end{array}$

1.0000

0.0269

0.0064

0.0037

0.00033

0.0074

0.0012

0.00022

0.0019

0.0708

0.0082

9.6694

5.3397

0.542 


\subsection{SCALE-UP CONSIDERATIONS FOR MHD POWER TRAINS}

In order to put the critical phenomena and the performance obtained to date in the power trains discussed above into the context of the overall development program for MHD power generation, it is necessary to consider the scale dependence of the performance and behavior of MHD power trains. In [7-1], STD Research Corporation outlined convenient interaction parameters for correlating the performance and behavior of high interaction MHD power trains. These interaction parameters and the appropriate measures of power train performance are defined in Table 7-1. Case F of [7-1] is the original "Revision 7-C" design conditions of the U-25 generator. Case \& of [7-1] pertained to the "Revision 4", "hot wall" design conditions of the AEDC/HPDE. The scale dependence of the performance of these two devices, relative to power trains ranging from the smallest (UTSI) to the largest commercial scale power train (the "STD Benchmark Power Train"), are depicted in Figs. 7-1 through 7-4 and 7-6 through 7-8, which are reproduced from [7-1].

In addition to the quasi-three-dimensional calculations discussed in [7-1], STD Research Corporation, under U.S. Department of Energy Contract AC-01-79ET15501 performed a series of calculations for cases B, C, F, and $G$ in order to elucidate the scale dependence of other critical phenomena besides those considered in $[7-1]$. These phenomena included quantitative calculations of the scale dependence of secondary flows transient behavior, part load operation, and the effects of alternate cross-sectional shapes on channel performance. While the results of these additional investigations into the scale dependence of these phenomena did not generally yield as 
simple and as elegant correlations as were obtained in [7-1], a number of interesting and important relationships were observed which will be described in the present section.

This section reviews the AEDC/HPDE performance to date in the context of the scaling parameters presented in Figs. 7-1 through 7-8 and reviews the additional investigations carried out under Contract AC-01-15501.

\subsection{AEDC/HPDE Performance to Date}

Figs. $7-1,7-2,7-3$, and $7-4$ summarize the electrical performance obtained by the AEDC/HPDE to date. It is observed that, at a magnetic field of $3.27 \mathrm{~T}$, the HPDE has achieved approximately half of its original design goals. ine enthalpy extraction rate is approximately $8 \%$ instead of $16 \%$. The isentropic efficiency is approximately $37 \%$ rather than approximately $60 \%$. The specific energy extraction is approximately $0.45 \mathrm{MJ} / \mathrm{kg}$ versus approximately $0.86 \mathrm{MJ} / \mathrm{kg}$. The interaction parameter based on pressure obtained to date is approximately 0.99, which is the highest interaction parameter based on pressure achieved in modern experiments aimed at commercial power genoration. This interaction parameter is compared to the original design interaction parameter of approx 1 mately 1.8 .

Fig. 7-5 shows the development of the axial velucity profiles between the electrode walls and between the sidewalls in the Run 006-014 Q3D simulation CHPQUUUJBO. (The first three profiles are offset from the main channel outline due to a plotter error.) The development of the $15 \%$ sidewall overshoots at the exit of this generator are consistent with the intcraction parameter based on velocity, $S_{u}$, of approximately 
2.2, which is obtained in this experiment. (As pointed out in [7-1], supersonic generators have lower interaction parameters based on velocity for a given interaction parameter based on pressure. The design value of interaction parameter based on velocity is in excess of 10 , because the original design was based on subsonic flow.) The ratio of the sidewall displacement thickness to the average electrode wall displacement thickness is exhibited in Fig. 7-6. It is clear that the AEDC/HPDE experiment has already achieved negative displacement thickness at the exit of the generator, according to the Q3D simulation. If properly instrumented with pressure probes, the experiment shoula be able to observe velocity overshoots under the Run 006-014 conditions. Fig. 7-7 shows the dependence of total channel blockage at the exit of the generator. It is seen that the AEDC generator performance to date has nearly passed the milestone of zero blockage at the exit of the channel. Finally, the average skin fric on coefficient throughout the generator is exhibited in Fig. 7-8 as a function of the interaction parameter based on velocity. It is seen that the sidewall friction, while greater than the electrode wall skin friction, has not reached the steep part of the curve associated with very strong velocity overshoots on the sidewalls.

It is evident that the AEDC/HPDE offers an early opportunity to study multidimensional phenomena in high interaction MHD devices. Many of the effects which are predicted by the STD/MHD codes will become important as the interaction level of the AEDC/HPDE is increased from its present levels. 
7.2 Optimum Mach Number Distribution and Its Effect on

Part-Load and Transient Behavior

The Mach number distribution determines performance because the power density falls dramatically on either side of an optimum Mach number value. The optimum Mach number is determined by the thermodynamic and transport properties of the working fluid and the stagnation conditions at each generator station. A series of part-load and off-design calculations performed by STD illustrate the impact of the Mich number distribution on generator performance.

Critical phenrmena related to the optimum Mach number distribution and its effect on part-load and transient behavior were deduced by analyses and evaluation of computations with the TRANSIENT family of codes which were completed under Contract AC-01-79ET15501. A diagram which schematically presents the logic of the variations of the part-load and unsteady cases which were completed with the ThansiENT code family under is given as Fig. 7-9. For each of four channels with thermal inputs of $20 \mathrm{MW}, 50 \mathrm{MW}, 300 \mathrm{MW}$, and $2000 \mathrm{MW}$, a nominal steady-state solution was computed. These steady-state solutions were the starting point of the following variations: (1) $5 \%$ step reduction in mass flow, (2) $\pm 5 \%$ sine wave input in mass flow; (3) $30 \%$ quasi-steady mass flow reduction; and (4) $30 \%$ instantaneous load reduction. The electrical connection of eich channel was then redesigned from Faraday to diagonal or diagonal to Faraday and the $30 \%$ quasi-steady mass flow reduction cases were calculated. A list of the final computations performed for these cases is presented in Table 7-2. 
The Mach number distributions for these channels during mass flow rate reduction are displayed in Figs. 7-10 through 7-17 for both the nominal electrical connections and the alternate electrical connections. For the 300 MW Faraday connected channel, the mass flow linearly decreases from $100 \%$ to $70 \%$ over the $300 \mathrm{~ms}$ time ringe of the computation, as shown in Fig. 7-14. For this case, the flow siarts out almost entirely subsonic. As the mass flow rate decreases, the small supersonic region becotues subsonic and the Mach number at each station in the entire flow field decreases.

The Mach number distribution for the 50 MW Faraday connected channel is shown in Fig. 7-13. This channel is initially completely supersonic with a shock near the end of the diffuser. As the mass flow rate decreases to $70 \%$ of its initial value, tile Mach number throughout the channel decreases. The Mach number at the channel entrance decreases only $0.3 \%$ but the Mach number at the channel exit decreases by $12.5 \%$.

A correlation of the normalized gross power to normalized mass flow rate for the four channels is shown in Figs. 7-18 and 7-19. The reduction in power follows very closely the reduction in mass flow, except in the case of the $50 \mathrm{MW}(\mathrm{th})$ channel. The power output of the $50 \mathrm{MW}(\mathrm{th})$ channel remains high as the mass flow decreases because its profile does not go far from optimum.

As the mass flow rate varies, so does the combustor pressure and the required compressor power. The combustor pressure is given as a function of normalized mass flow rate for the 20. 50, 300, and $2000 \mathrm{MW}(\mathrm{th})$ flow trains in Fig. 7-20 for the nominal electrical hookup and in Fig. 7-21 for the 
alternate electrical hookup. The net power is given as a function of normalized mass flow rate for the 20 and $50 \mathrm{MW}(\mathrm{th})$ flow trains in Figs. 7-22 and 7-23. The normalized net power is given as a function of the normalized mass flow rate for the 300 and $2000 \mathrm{MW}(\mathrm{th})$ ilow trains in Figs. 7-24 and 7-25.

The relationships between gross power per unit mass flow rate (specific energy extraction) and the product of interaction parameter and electrical efficiency are shown in Figs. 7-26 and 7-27. For each channel, a trace is shown which describes the result of linearly decreasing the nass flow from its nominal value to $70 \%$ of the nominal. For the two subsonic channels, nominally $300 \mathrm{MW}(\mathrm{th})$ and $2000 \mathrm{MW}(\mathrm{th})$, both the specific energy extraction and the interaction decrease as the mass flow rate is reduced. This is attributed to the departure of the channel Mach number distrioution further from the optimum distribution. Conversely, for the two supersonic channels, nominally $20 \mathrm{MW}(\mathrm{th})$ and $50 \mathrm{MW}(\mathrm{th})$, tre specific energy extraction and the interaction increase with decreasing mass flow. This effect is attributed to the fact that tre Mach number distribution for the supersonic designs of the small channels approaches the optimum Mach number distribution as the mass flow rate is reduced. These results point to the maintenance of the optimum Mach number distribution as an important consideration for MHD generator load following.

The relationship between power output and the specified mass flow which varies sinusoidally with a $\pm 5 \%$ amplitude, 15 given for the nominal $20 \mathrm{MW}(\mathrm{th})$, Faraday-connected case in Fig. 7-28 and for the nominal $300 \mathrm{MW}(\mathrm{th})$, Faraday-connected case in Fig. 7-29. These figures show both the relative amplitudes and the phase difference between the mass fluctuation and the resulting power fluctuations. 


\subsection{Effect of Alternate Cross-Sectional Shapes on Channel}

Performance

The effects of alternate cross-sectional shapes on c. 1 performance were deduced by analysis and evaluation of ccur udions completed under Contract AC-01-79ET15501 with codes from the Q3DYZ of the Q3D Family of codes. A diagram which represents schematically the variations of the cases which were completed is presented as Fig. $7-30$. Fcr eaci of four channels with nominal thermal inputs o1 $20 \quad 50 \mathrm{MW}, 300$ $M W$, and $2000 \mathrm{MW}$, three axial stations were chosen anulysis. These three axial stations were chosen to be representative of the front end, middle, and the back end of the channels. At each of these stations and for each channel, three different cross-sectional shapes, rectangular, hexagonal, and elliptical, were considered. Therefore, there were 36 cases completed. A list of these computations is given in Table 7-3.

The Q3DYZ code has been applied to analyze Faraday, diagonal-concucting-wall, and insulating-wall-diagonal channels. For the computations listed in Table 7-3, the $20 \mathrm{MW}$ and $2000 \mathrm{MW}$ generators are Faraday-connected, the $50 \mathrm{MW}(\mathrm{th})$ generator is a diagonal-conducting-wall generator, and the 300 $\because W$ generator is an insulating-wall-diagonal.

At each station in each channel, the load factor, enthalpy flux, magnetic field strength, average static pressure, wall temperature, anode-center boundary layer thickness, cathode-center boundary layer thickness, sidewal 1center boundary layer thickness, electrode center wall temperature, ana sidewall-center wall temperature were the same for each different geometry. At each station in the 20,50 and $300 \mathrm{MW}(\mathrm{th})$ channels, the cross-section.l area and aspect ratio were also the same for each geometry. 
The geometrical definitions used for the hexagonal, rectangular, and elilptical shapes of these computations are given in Fig. 7-31. For all hexagonal shaped channels the included electrode angle was 120 degrees. For the 20, 50, and $300 \mathrm{MW}(\mathrm{th})$ channels, th? angles subtendec iy the electrodes for the elliptical shapes were the same angles, as measured from the center point in the channel, as for the corresponding rectangular shapes. For the $2000 \mathrm{MW}(\mathrm{th})$ channel, the hexagonal cases were regular hexagons and the $\epsilon l l i p t i c a l$ cases had aspect rátios of unity (i.e., circular).

The data which have been compiled for the 36 cases are listed in Tables 7-4,7-5, 7-6, and 7-7 for the 2?, 50, 300, and $2000 \mathrm{MW}(\mathrm{th})$ channels, respectively. The data which are listed in these tables are run identifier, shape, axial location, magnetic field, load factor, diagonalization angle (for the diagonally-connected channel), aspect ratio, cros:sectional area, cross-sectior perimeter, enthalpy flow, enthalpy flux, average static pressure, average static temperature, core static temperature, average axial velocity, core axial velocity, average electrical conductivity, core electrical conductivity, average Hall paramcter, electrode rall temperature, sidewall temperature, anode boundary layer thickness, cathude boundary layer thickness, sidewall boundary layer thickness, Hall field, Faraday voltage, load currer:, power output, internal resistance, plasma nonuniformity factor, and two power production parameters, $C_{25}$ and $C_{26}$. The power production parameter $\mathrm{C}_{25}$ is defined as the power divided by the product of load factor, one minus the lnad factor, corc electrical conductivity, magnetic field squared, core a.:ial velocity squared, and the cross-sectiona' urea, and this parameter represenis the performance at the station relative to an ideal channel with no thermal or velocity gradients. The power production parameter $C_{26}$ is defined as the power divided 
by the product of load factor, one minus the load factor, average elsctrical conductivity, magnetic field squared, average axial velocity squared, and the cross-sectional area: this parameter represents the performance at the station relative to a simplified one-dimensional model.

The analyses and interpretations of the computations with alternate cross-sections have resulted in the following inplications:

(1) Some of the inefficiency caused by conductivity nonuniformity can be removed by appropriate choice of a channel cross-section. The larger the nonuniformity factor in a particular rectangular channel, the larger the potential gain which can be obtained with a nonrectangular geometry. As the nonuniformity factor increases with axial distance down the channel, the improvement in power obtained from nonrectangular geometry should also increase with axial distance.

In order to obtain the potential increase in performance, it is necessary to choose a geometry so that the boundary layer voltage drop is minimized, but not a geometry which causes the power producing core to become shorted out. An incorrect choice of geomrtry for a particular case can result in none of the potential gain from being exploited. In most cises, the potential loss from an inappropriate choice of geometry is much greater than the potential gain from an appropriate choice.

An example of the reduction of the boundary layer voltage drop is given with assistance from Figs. 7-32 through 7-35. The isopotentials and current streamlines for the $2 \mathrm{~m}$ axial station of the nominal $50 \mathrm{MW}(\mathrm{th})$ channel are given for the rectangular cross-section in Figs. 7-32 and 7-33, 
respectively. The isopotentials and current streamlines for the corresponding elliptical cross-section are shown in Figs. 7-34 and 7-35, respectively. The region of voltage drop near the anode and cathode is much larger for the rectangular case, Fig. 7-32, than for the eliptical case, Fig. 7-34. The peak potential (at the zero field point) for the cathode region in the rectangular case is $32 \mathrm{~V}$, and the peak potential for the cathode region in the elliptical case is $17 \mathrm{~V}$. That is a difference of about a factor of two.

(2) The geometry (cross-sectional shape) that improves the performance of one particular channel can yield poorer performance than the rectangular geometry for a different channel.

The optimum geometric shape is a complex function of many parameters (boundary layer thicknesses, wall temperatures, Hall parameter, to name a few) and the substitution of this shape into a different channel with different values for these important parameters will yield different results. An illustration of this can be found with the hexagonal shapes for the $20 \mathrm{MW}(\mathrm{th})$ and $300 \mathrm{MW}(\mathrm{th})$ channels. For each of the stations for the $20 \mathrm{MW}(\mathrm{th})$ channel, the hexagonal case had better performance than the corresponding rectangular case, but for each of the stations for the $300 \mathrm{MW}(\mathrm{th})$ channel, the hexagonal case had worse performance than the corresponding rectangular case.

(3) As shown by the results for the $50 \mathrm{Mm}(\mathrm{th})$ channel cases, compared to the results from the three other channels, the electrical performance of a completely diagonal-conductingwall channel is not as strong a function of cross-sectional shape as for the insulating wall Faraday-connected channel. since the diagonal-conducting-wall channel has all walls as 
conductors, the current can enter the walls where it is most efficient for the particular geometry and distributions of plasma properties. However, this does not say that the DCW channel is better than a Faraday-connected channel.

(4) Geometries which have less surface-to-area ratios and larger hydraulic diameters than the rectangular crosssectional shape, but have the same area, have smaller losses due to heat transfer to the walls, wall shear stresses, and viscous effects. For particular cases, each of these three losses can be simultaneously decreased by over $10 \%$.

(5) Geometries which allow the current to enter the electrodes parallel to the magnetic field have a lower internal plasma impedance. The lower internal plasma impedance can be utilized to improve the performance of the channel.

An example of the effect of lower plasma impedance is given with assistance from Figs. 7-36 through 7-39. The isopotentials and current streamlines for the $1 \mathrm{~m}$ station of the nominal $20 \mathrm{MW}(\mathrm{th})$ channel are given for the rectangular cross-section case in Figs. 7-36 and 7-37, respectively. The isopotentials and current streamlines for the corresponding hexagonal cross-sections are shown in Figs. 7-38 and 7-39, . espectively. As shown on Fig. 7-37, the current enters the electrode nearly perpendicular to the magnetic field for the rectangular case and, as shown on Fig. 7-39, the current enters the electrode nearly parallel to the magnetic field for the hexagonal case. The internal resistance of the hexagonal case, illustrated by Figs. 7-38 and 7-39, is $32 \%$ less than that for the rectangular case. At the same Faraday load factor of 0.5 (for maximum power production), the Paladay voltage is 13\% lower for the hexagon, but the load current is $28 \%$ higher, so 
that the power output is $12 \%$ higher for the hexagon than for the rectangle.

Although the cases represented in Table 7-3 and Fig. 7-30 define a systematic approach to analysis of the effects of cross-sectional geometry, the detailed analyses performed to date have not resulted in complete and unambiguous interpretations which are applicable in all cases. These calculations of performance with variable cross-sectional shapes did not include sufficient parametric variation and were applied over too great a range of conditions to obtain regular trends with scale and position in the channel. It is probable that many of the elliptical or hexagonal cross-sections with lower performance than the rectangular design values could be optimized to outperform the rectangle when additional systematic parametric variations are considered. In addition. other cross-sectional shapes (e.g., octagonal) may provide better performance than any of the three shapes considered. 


\section{REFERENCES}

[7-1] C.D. Maxwel1, S.T. Demetriades, D.A. Oliver, A.A. Vetter, T.F. Swean, "Scale-up of Advanced MHD Generators", AIAA $18 \mathrm{th}$ Aerospace Sciences Meeting, Paper No. AIAA-80-0179, Pasadena, California, January 1980. 
TABLE ?-1

Interaction Parameters and Performance Indices for Evaluation of MHD Generator Performaice Scaling

$$
\begin{aligned}
& \text { Interaction parameter based on pressure } \\
& \qquad I_{p} \equiv \int_{0}^{L} \frac{J_{y}^{B}}{p} d x \\
& \text { Interaction parameter based on velocity } \\
& S_{u} \equiv L \frac{\sigma B^{2}}{\rho \mathrm{T}}
\end{aligned}
$$$$
\text { Generalized nonuniformity factor }
$$

$$
G \approx g+\left(g-1\left(\beta^{2}\right)\right)
$$

where

$$
g=\langle\sigma\rangle\langle 1 / \sigma\rangle
$$

$$
\begin{aligned}
& \text { Electrical conversion efficiency } \\
& \eta_{e}=\frac{\vec{J} \cdot \vec{E}}{\vec{J} \cdot(\vec{U} \times \vec{B})} \\
& \text { Isentropic efficiency } \\
& \frac{\mathbb{P}}{(\Delta H)_{\text {isentropic }}} \\
& \text { Enthalpy extraction ratio } \\
& \frac{\mathbb{P}}{\mathrm{mH}_{0}} \frac{\text { power out }}{\text { thermal power in }} \\
& \text { Specific energy extra.tion } \\
& \frac{\mathbb{P}}{\dot{m}}: \text { power per unit mass flow rate }
\end{aligned}
$$




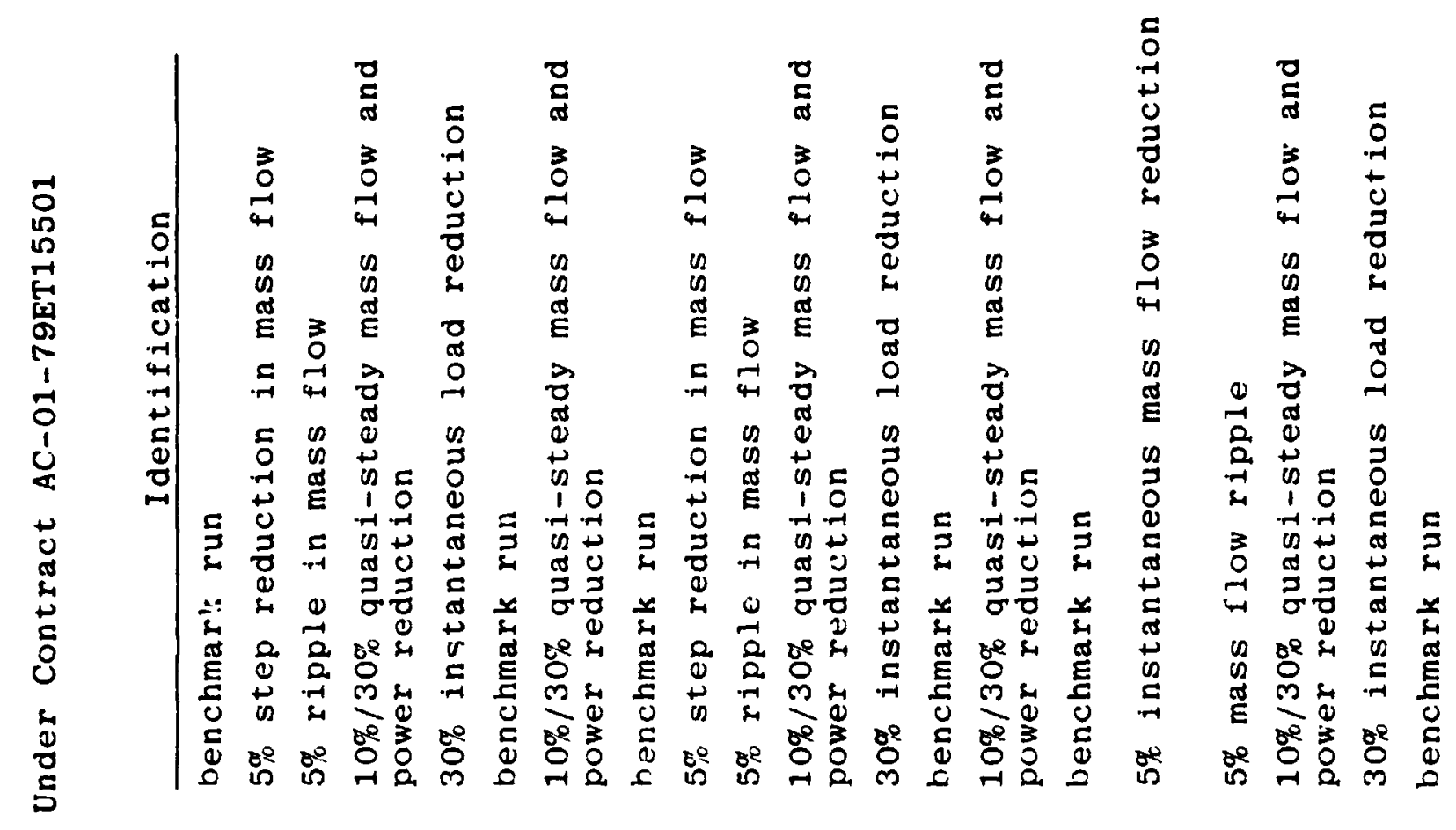

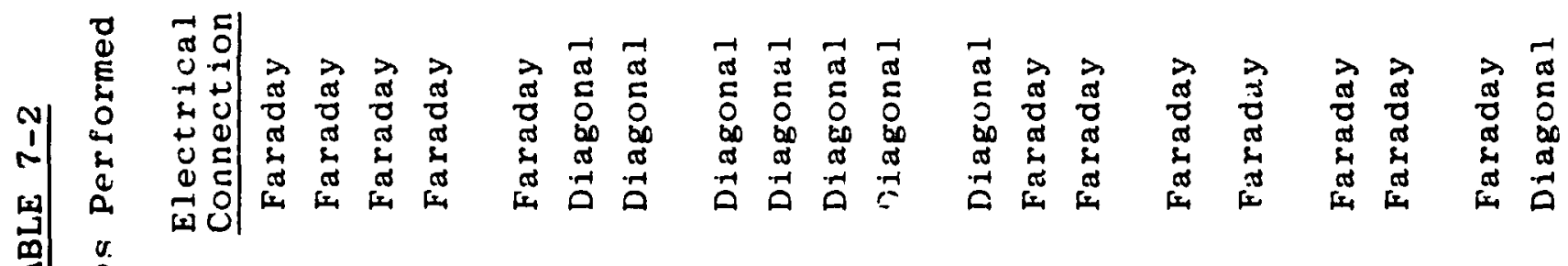

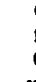

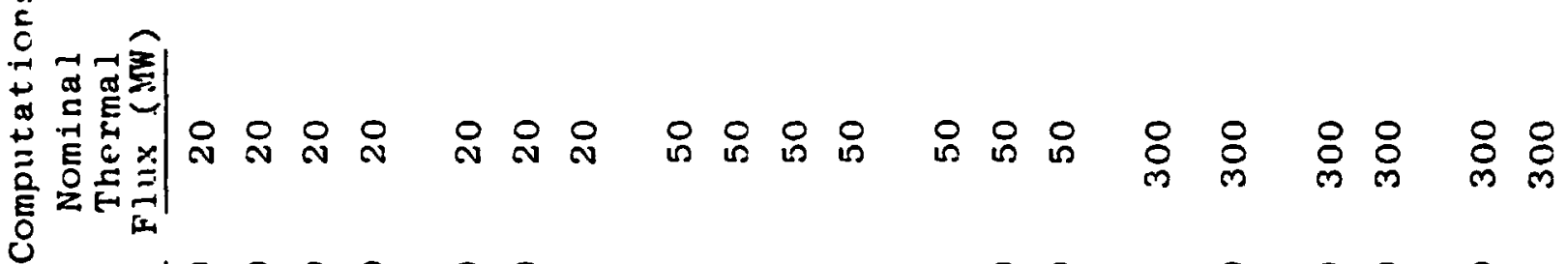

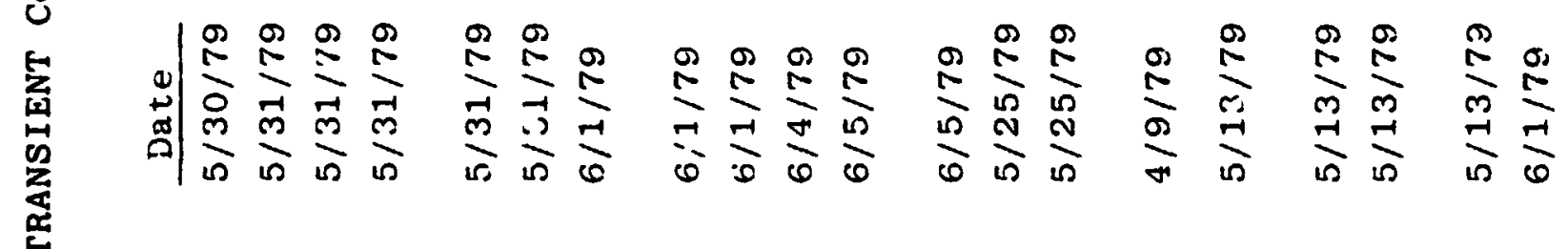

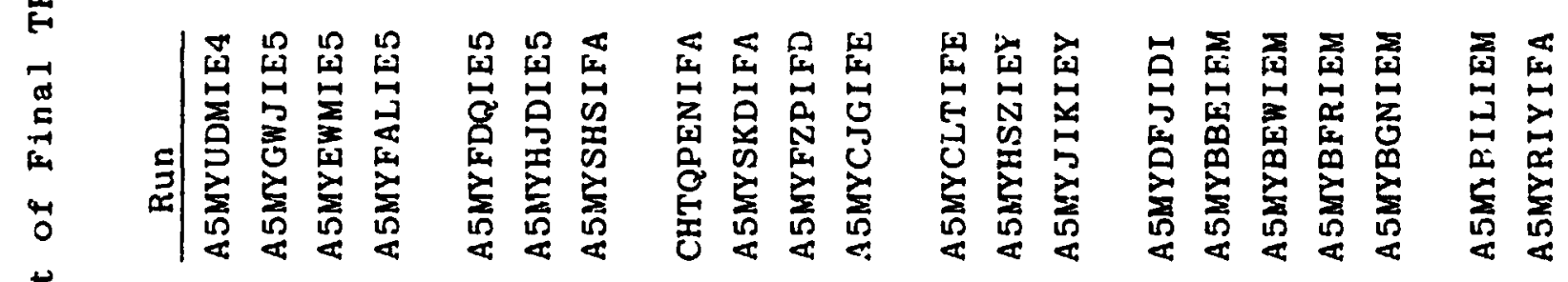
苟

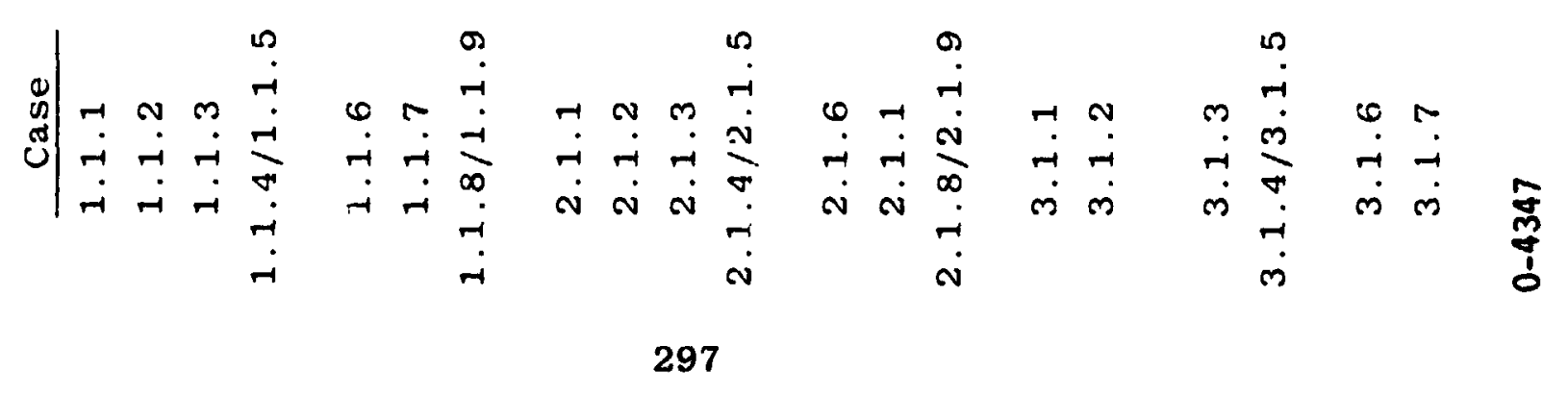




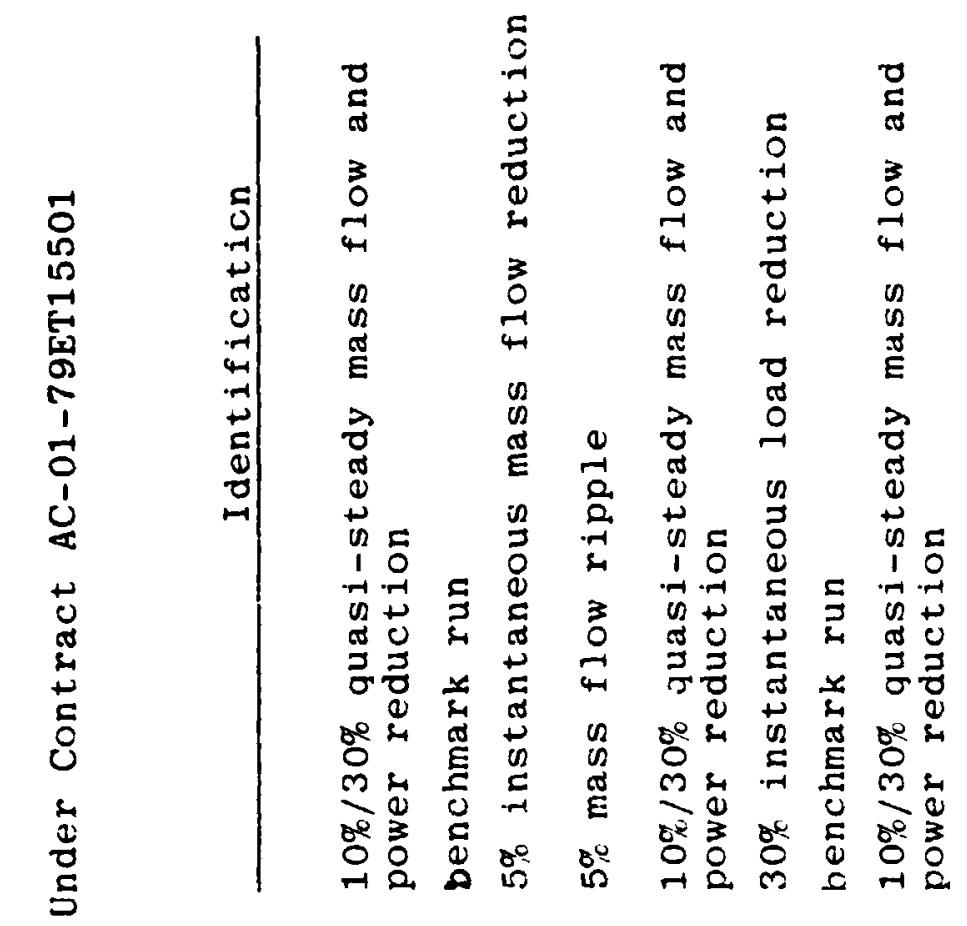

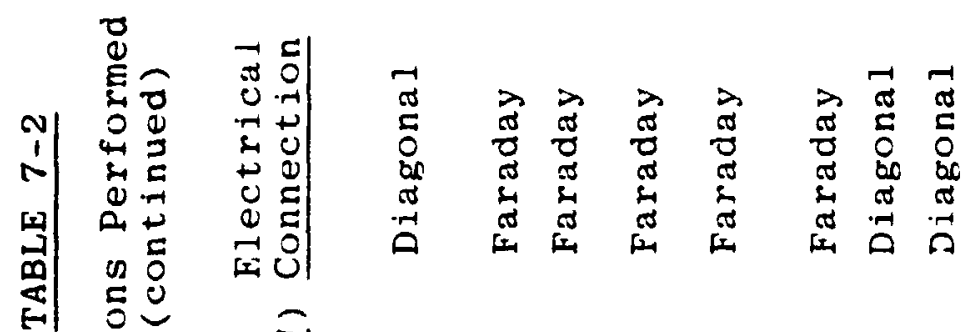

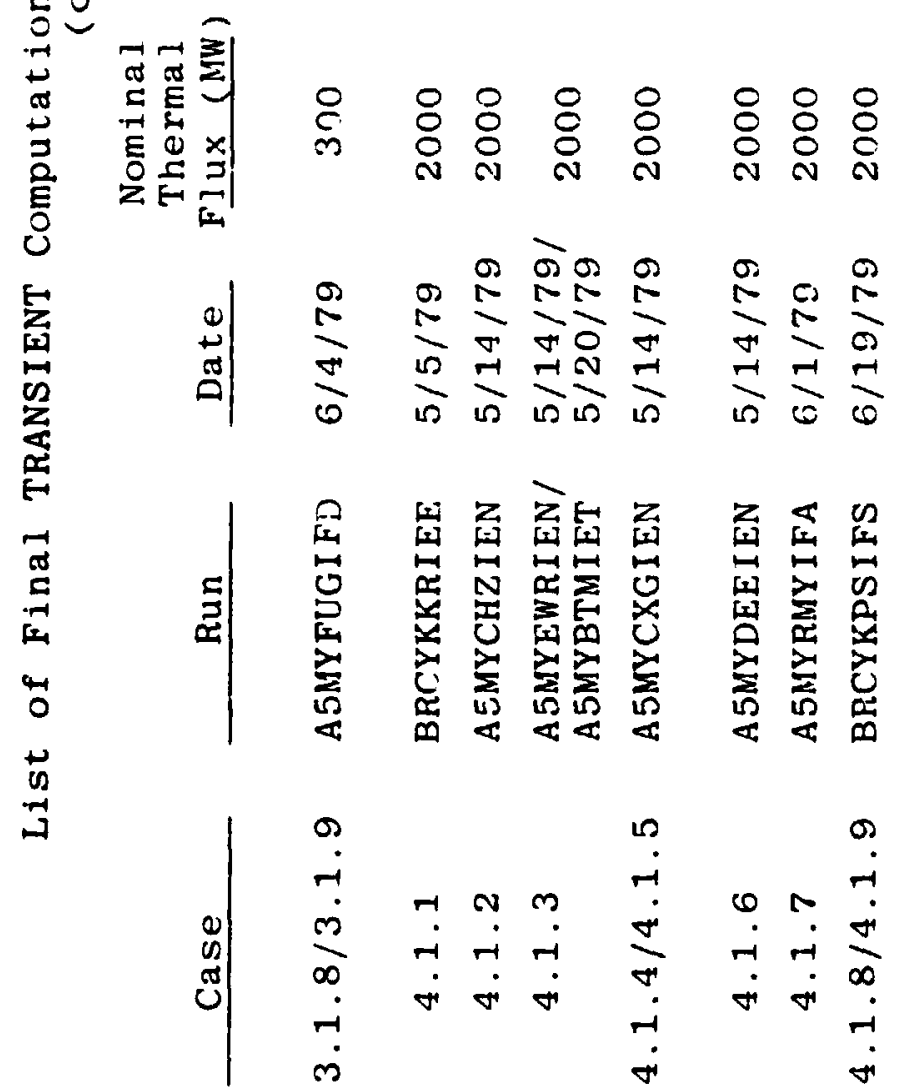


TABLE $7-3$

List of Final Q3DYZ Computations Performed Under Contract AC-01-79ET 15501

\begin{tabular}{|c|c|c|c|c|c|c|}
\hline Run & \multicolumn{3}{|c|}{ Date } & $\begin{array}{c}\text { Nominal } \\
\text { Thermal } \\
\text { Flux } \\
(\mathbf{M W}) \\
\end{array}$ & $\begin{array}{c}\text { Cross- } \\
\text { Sectional } \\
\text { Shape } \\
\end{array}$ & $\begin{array}{l}\text { Axial } \\
\text { Location } \\
\text { (m) } \\
\end{array}$ \\
\hline $\begin{array}{l}\text { BRCYCHP I FG } \\
\text { BRCYC } \mathcal{A} \text { I FQ }\end{array}$ & $\begin{array}{l}17 \\
17\end{array}$ & $\begin{array}{l}\text { June } \\
\text { June }\end{array}$ & $\begin{array}{l}1979 \\
1979\end{array}$ & $\begin{array}{l}20 \\
20\end{array}$ & $\begin{array}{l}\text { rectangle } \\
\text { rectangle }\end{array}$ & $\begin{array}{l}0.50 \\
1.00\end{array}$ \\
\hline BRCYCJCIFQ & 17 & June & 1979 & 20 & rectangle & 1.49 \\
\hline BRCYCISI FQ & 17 & June & 1979 & 20 & hexagon & 0.50 \\
\hline BRCYCIUIFQ & 17 & June & 1979 & 20 & hexagon & 1.00 \\
\hline BRCYCJKIFQ & 17 & June & 1979 & 20 & hexagon & 1.49 \\
\hline BRCYCIQIFQ & 17 & June & 1979 & 20 & ellipse & 0.50 \\
\hline BRCYCIWIFQ & 17 & June & 1979 & 20 & ellipse & 1.00 \\
\hline BRCYCJF I FQ & 17 & June & 1979 & 20 & ellipse & 1.49 \\
\hline BRCYB I E I FQ & 17 & June & 1979 & 50 & rectangle & 1.00 \\
\hline BRCYB I I I FQ & 17 & June & 1979 & 50 & rectangle & 2.00 \\
\hline BRCYBIQIFQ & 17 & June & 1979 & 50 & rectangle & 3.00 \\
\hline BRCYBHF IFQ & 17 & June & 1979 & 50 & hexagon & 1.00 \\
\hline BRCYBILIFQ & 17 & June & 1979 & 50 & hexagon & 2.00 \\
\hline BRCYBIM IFQ & 17 & June & 1979 & 50 & hexagon & 3.00 \\
\hline BRCYBHQIFQ & 17 & June & 1979 & 50 & ellipse & 1.00 \\
\hline BRCYBRN IFQ & 17 & June & 1979 & 50 & ellipse & 2.00 \\
\hline BRCYBIOIFQ & 17 & June & 1979 & 50 & ellipse & 3.00 \\
\hline BRCYBGL I FQ & 17 & June & 1979 & 300 & rectangle & 2.00 \\
\hline BRCYBGB I FQ & 17 & June & 1979 & 300 & rectangle & 3.50 \\
\hline BRCYBFZ IFQ & 17 & June & 1979 & 300 & rectangle & 5.00 \\
\hline BRCY BGH I FQ & 17 & June & 1979 & 300 & hexagon & 2.00 \\
\hline $\begin{array}{l}\text { BRCY BGF I FQ } \\
\text { BRCYBFL IFD }\end{array}$ & 17 & June & 1979 & 300 & hexagon & $\begin{array}{l}3.50 \\
3.00\end{array}$ \\
\hline BRCYBFLIFQ & 17 & June & 1979 & 300 & hexagon & 3.00 \\
\hline BRCYBG.I I FQ & 17 & June & 1979 & 300 & ellipse & 2.00 \\
\hline BRCYBGDIFQ & 17 & June & 1979 & 300 & ellipse & 3.50 \\
\hline BRCYBFXIFQ & 17 & June & 1979 & 300 & ellipse & 5.00 \\
\hline CHRQBUE I F3 & 29 & June & 1979 & 2000 & rectangle & 4.00 \\
\hline CHRQLN I I F3 & 29 & june & 1979 & 2000 & rectangle & 7.50 \\
\hline CHRQBVF IF' & 29 & June & 1979 & 2000 & rectangle & 11.00 \\
\hline CHRQEHR I F 3 & 29 & June & 1979 & 2000 & hexagon & 4.00 \\
\hline BRCYEHP I F3 & 27 & June & 1979 & 2000 & hexagon & 7.50 \\
\hline CHRQBXJ IF 3 & 29 & June & 1979 & 2000 & hexagon & \pm 1.00 \\
\hline CHRQDWB I F2 & 28 & June & 1979 & 2000 & ell1pse & 4.00 \\
\hline BRCYHQJ I F2 & 28 & June & 1979 & 2000 & ellipse & 7.50 \\
\hline BRCY I NQ I F2 & 28 & June & 1979 & 2000 & elljpse & 11.00 \\
\hline
\end{tabular}




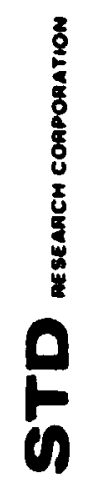

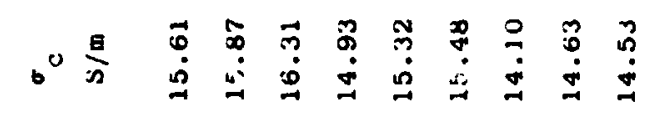

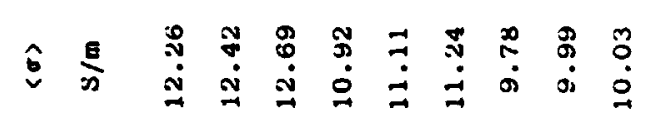

ว

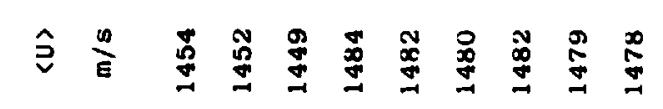

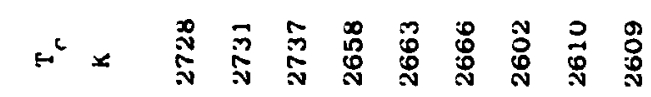

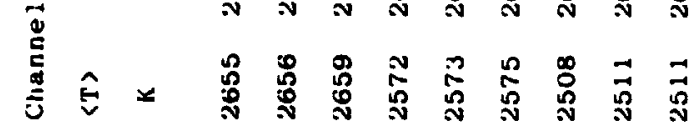

产焉

แ้อง

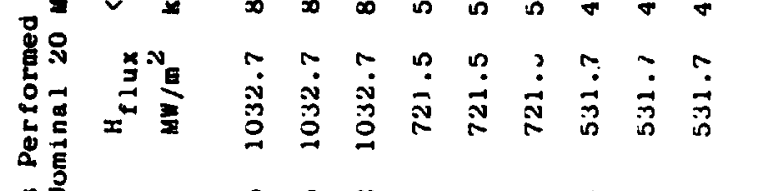

int

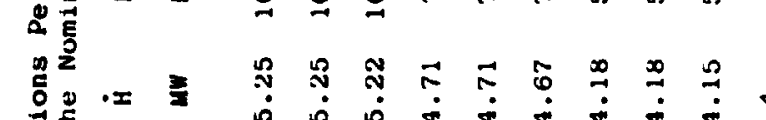

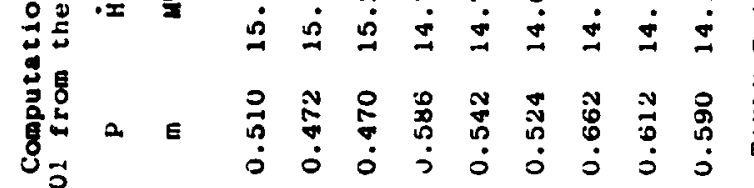

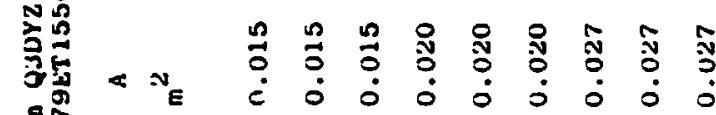

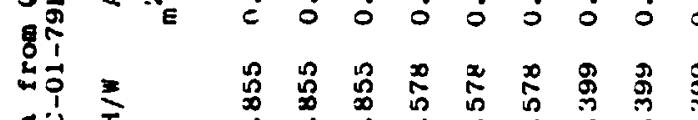

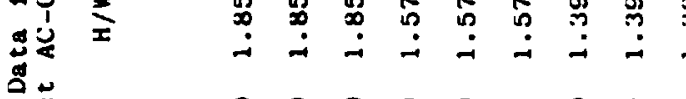

는

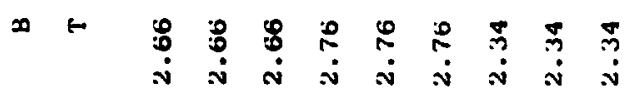

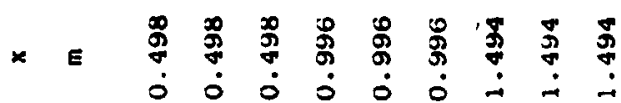

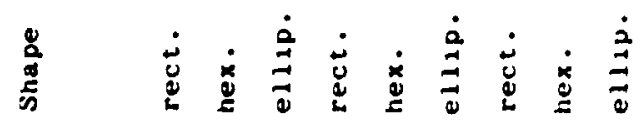

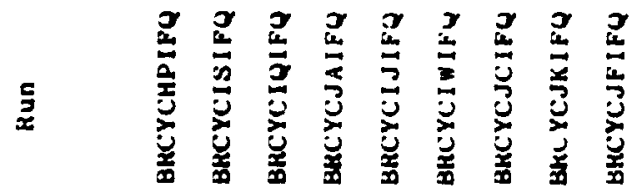

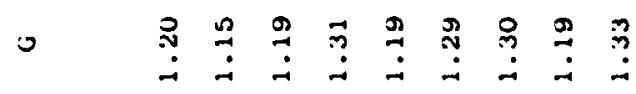

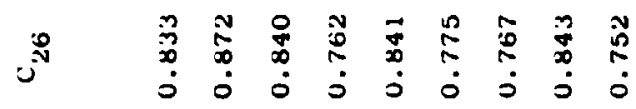

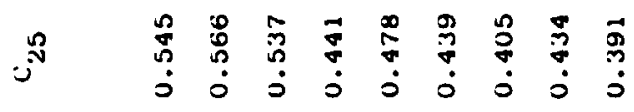

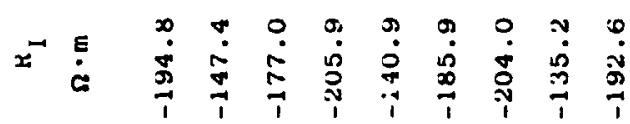

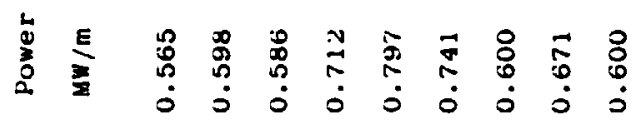

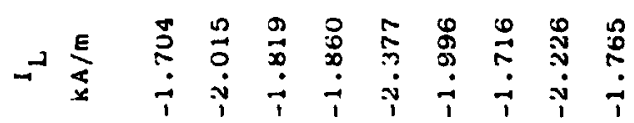

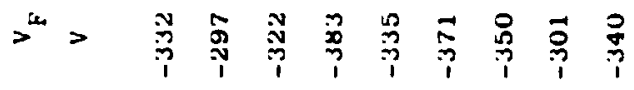

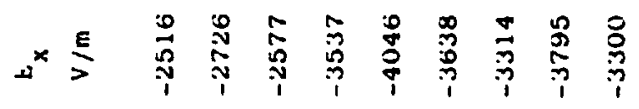

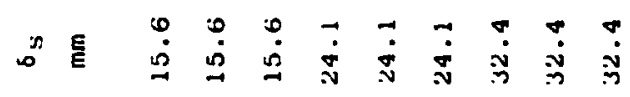

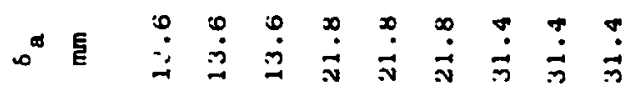

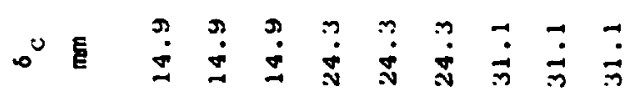

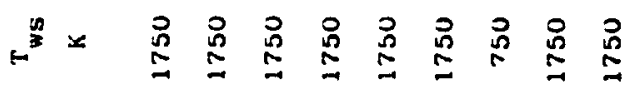

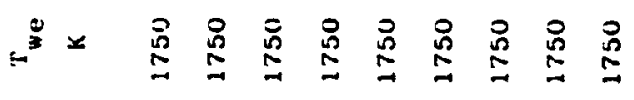

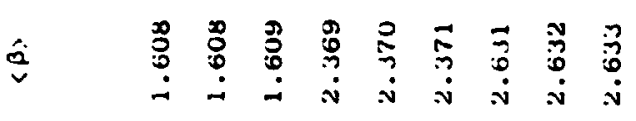

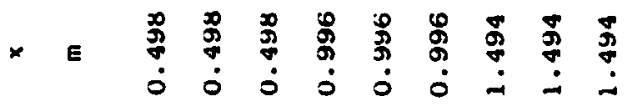

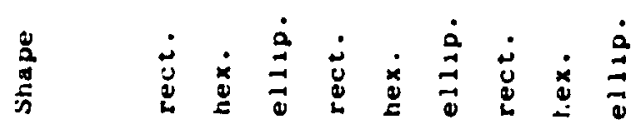

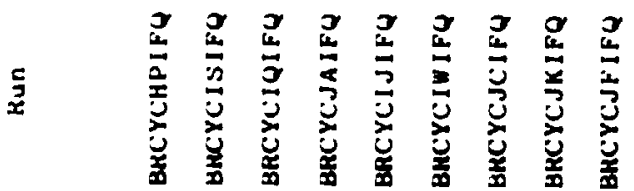

ORIGNAE PAGE $\mathrm{A}$ 


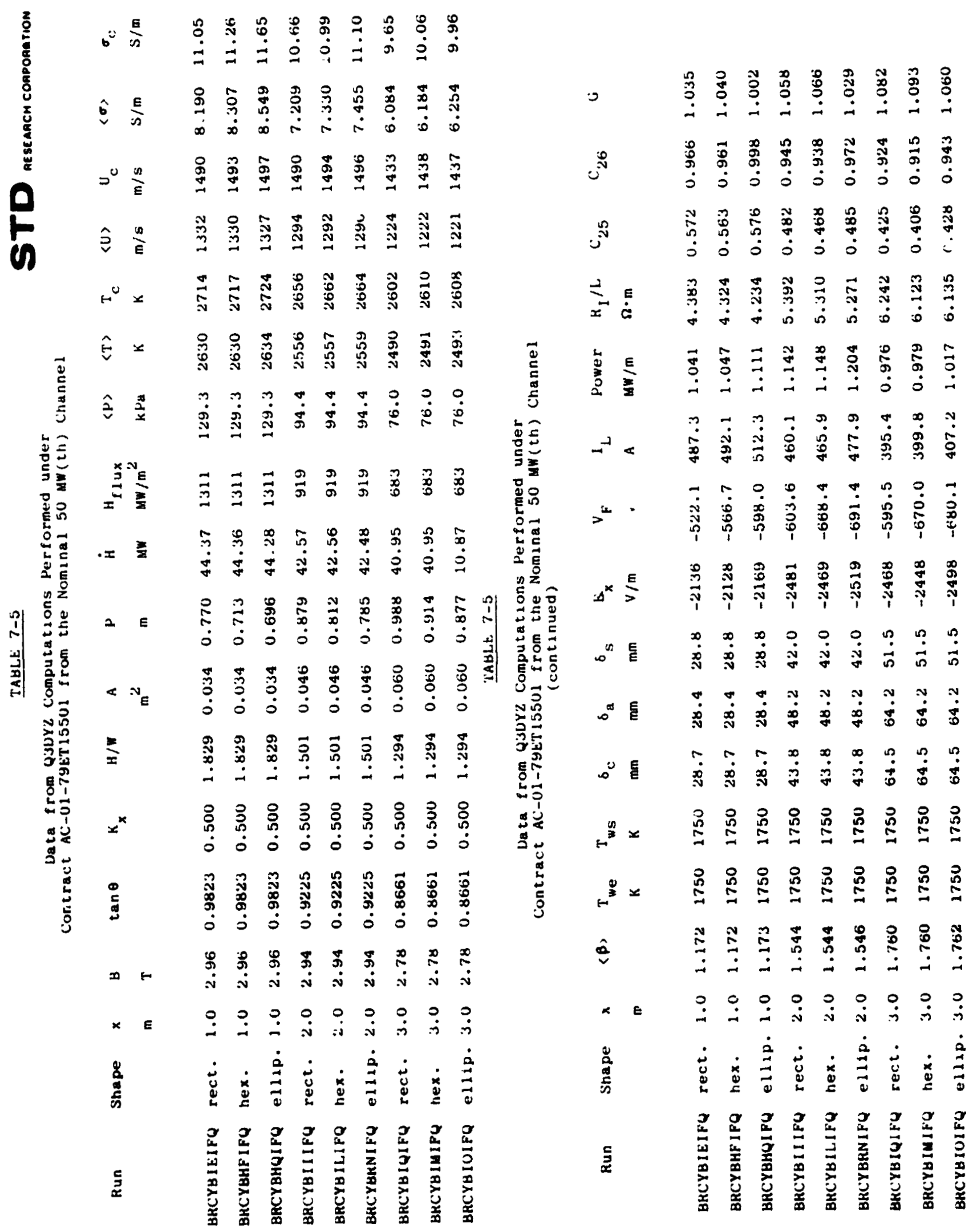




$$
\begin{array}{ccc}
4 \\
4
\end{array}
$$


م

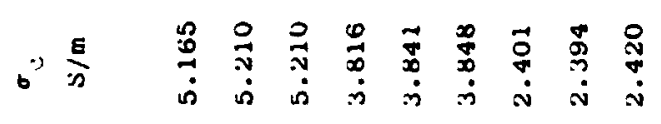

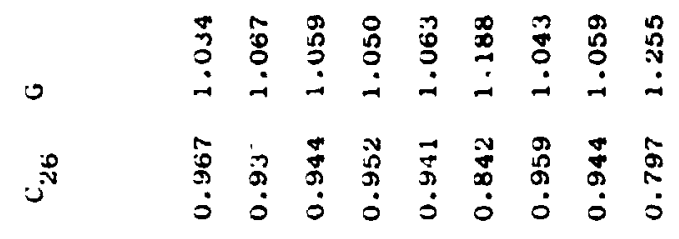

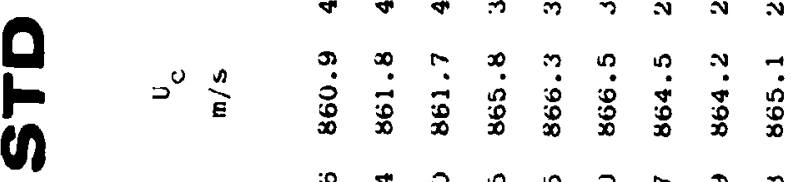

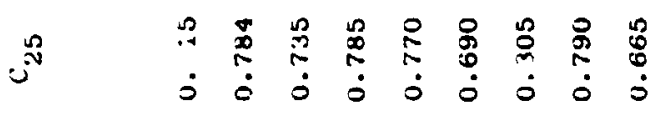

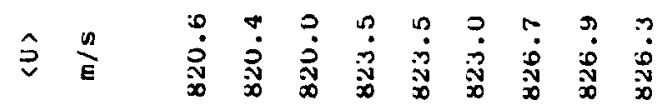

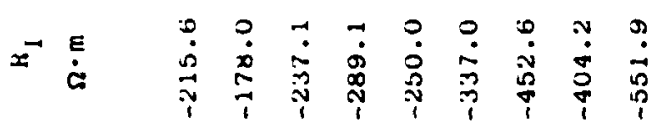

包

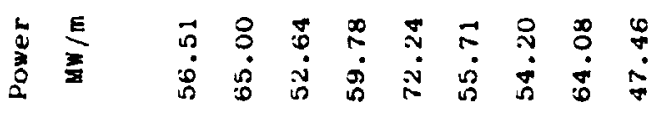

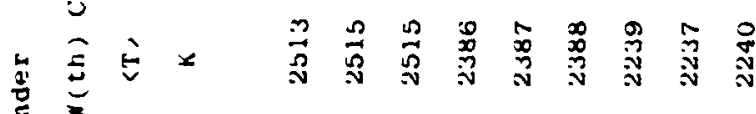

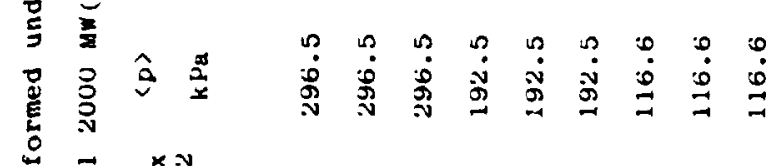

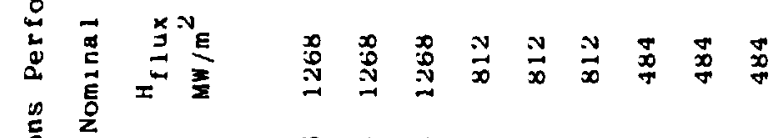

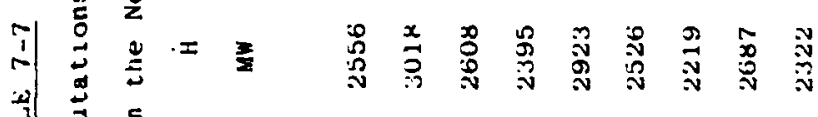

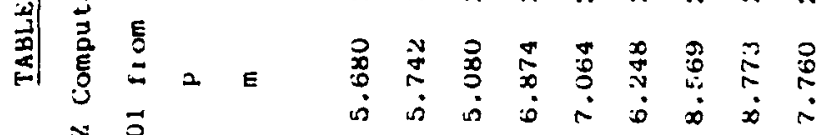

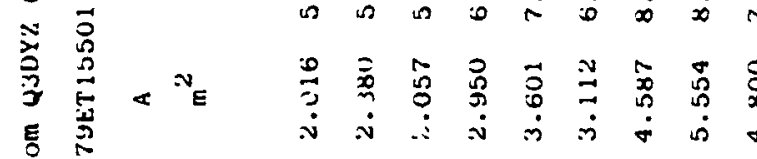

告

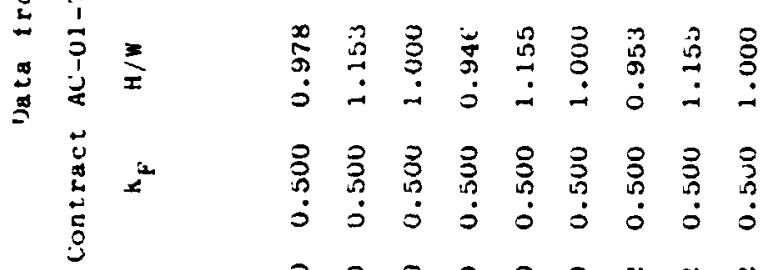

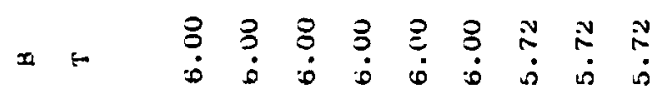

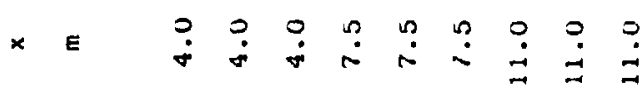

(1) ${ }^{n} \hat{D}^{\circ}$

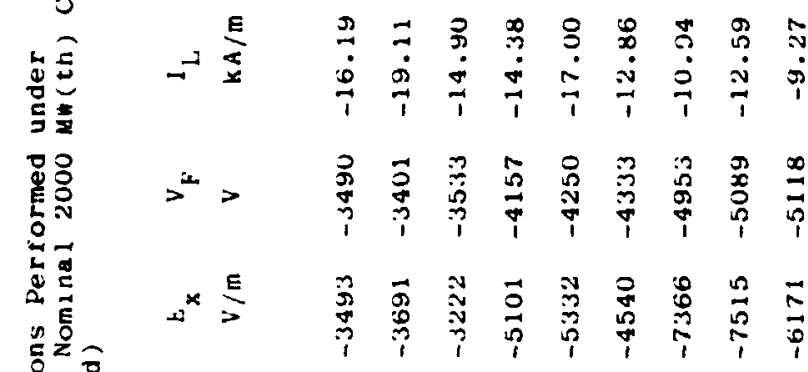

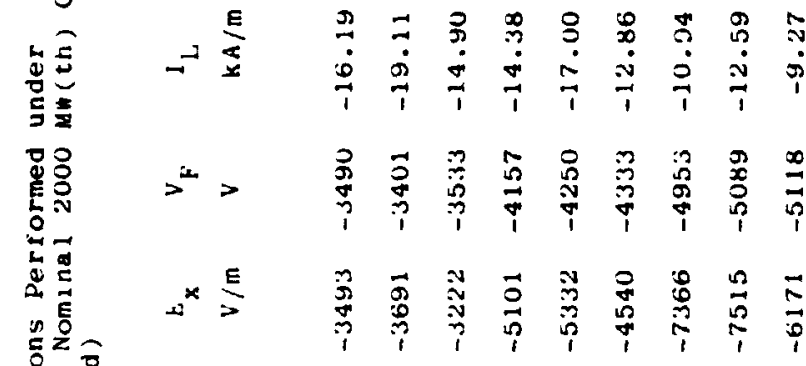

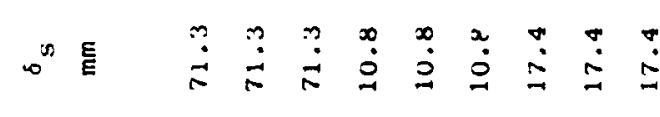

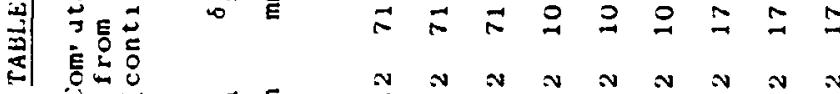

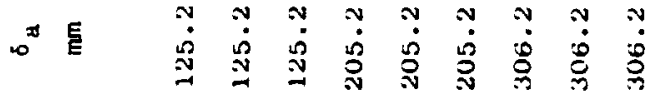

ㄱํㄱำ

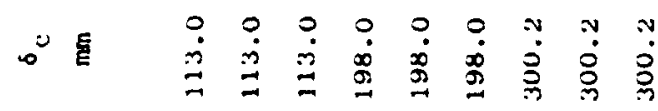

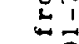

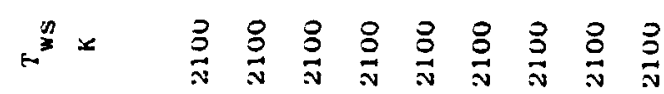

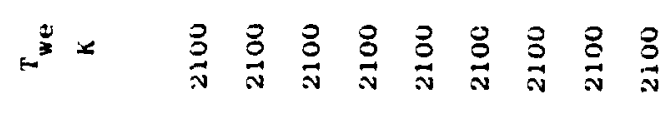

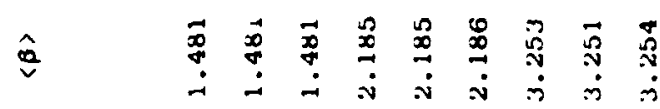

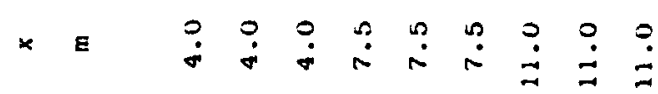

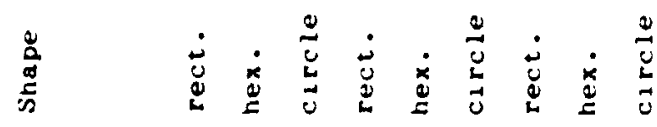

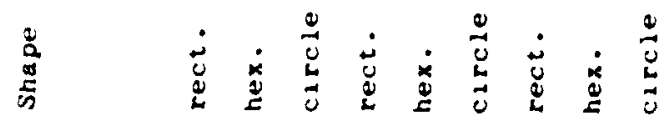

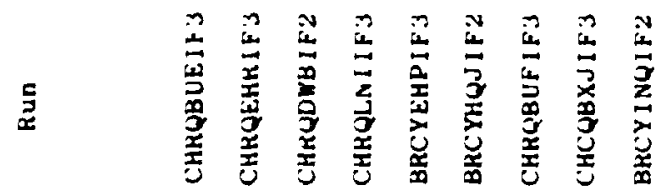

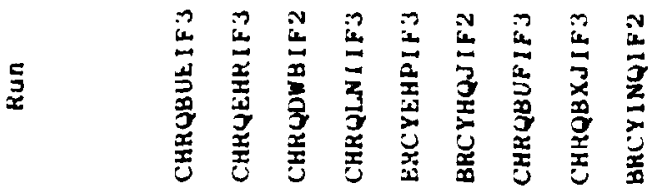




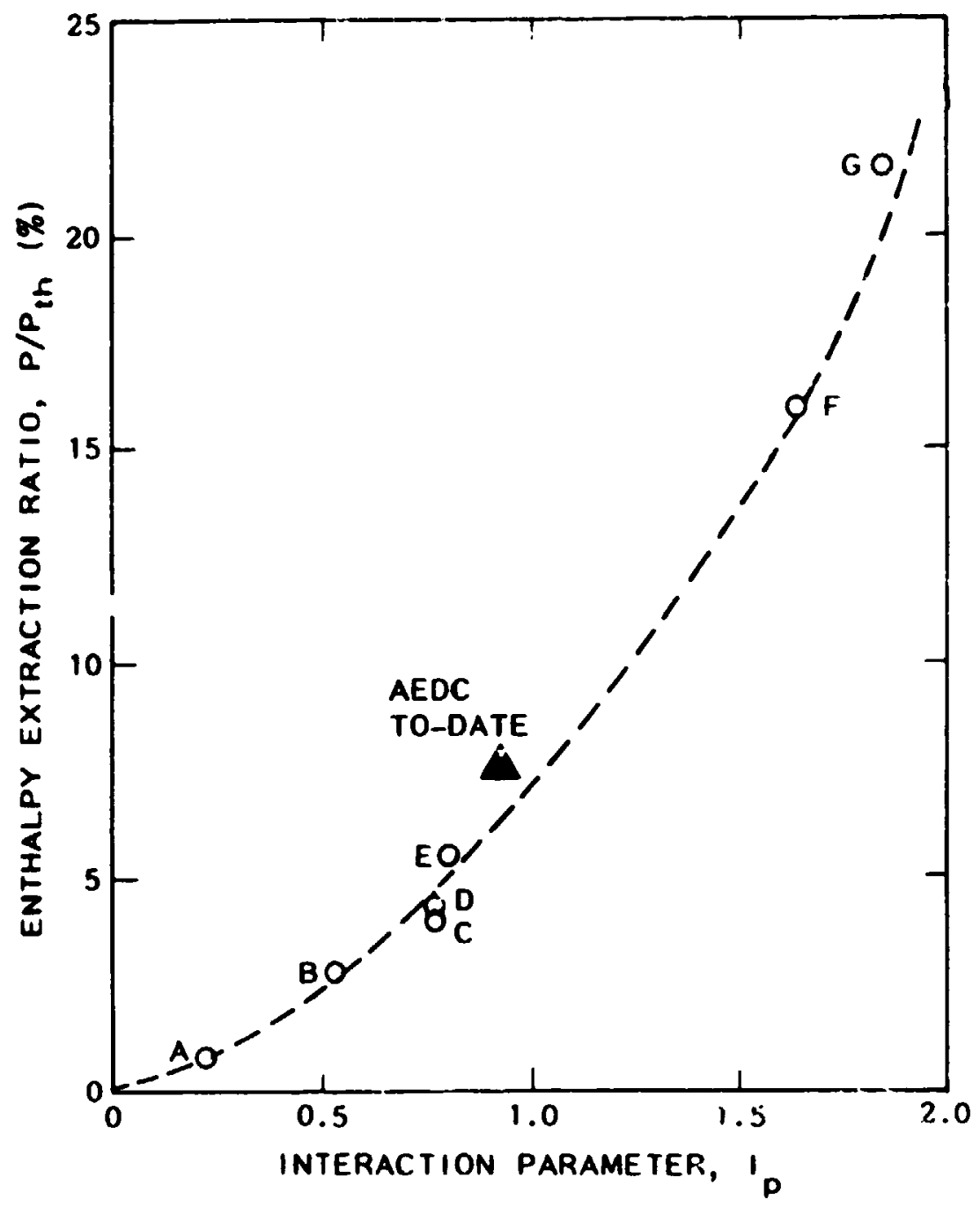

Fig. 7-1. Variation of erthalpy extraction rate with the interaction parameter based on pressure.

$0-3633 a$ 
STD

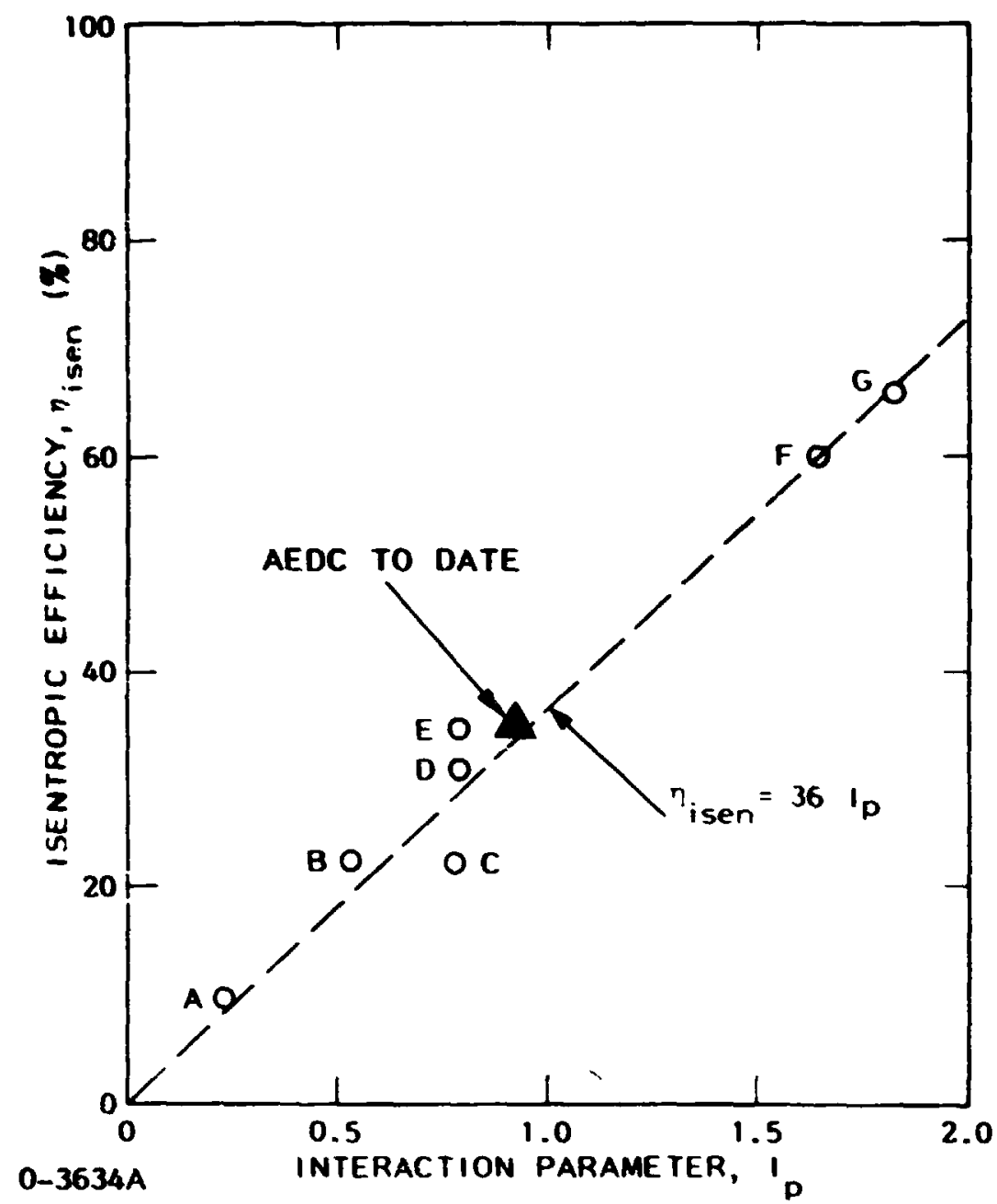

Fig. 7-2. Vartation of 1sentropic eff 1 clency with interact' on parameter based on pressure. 


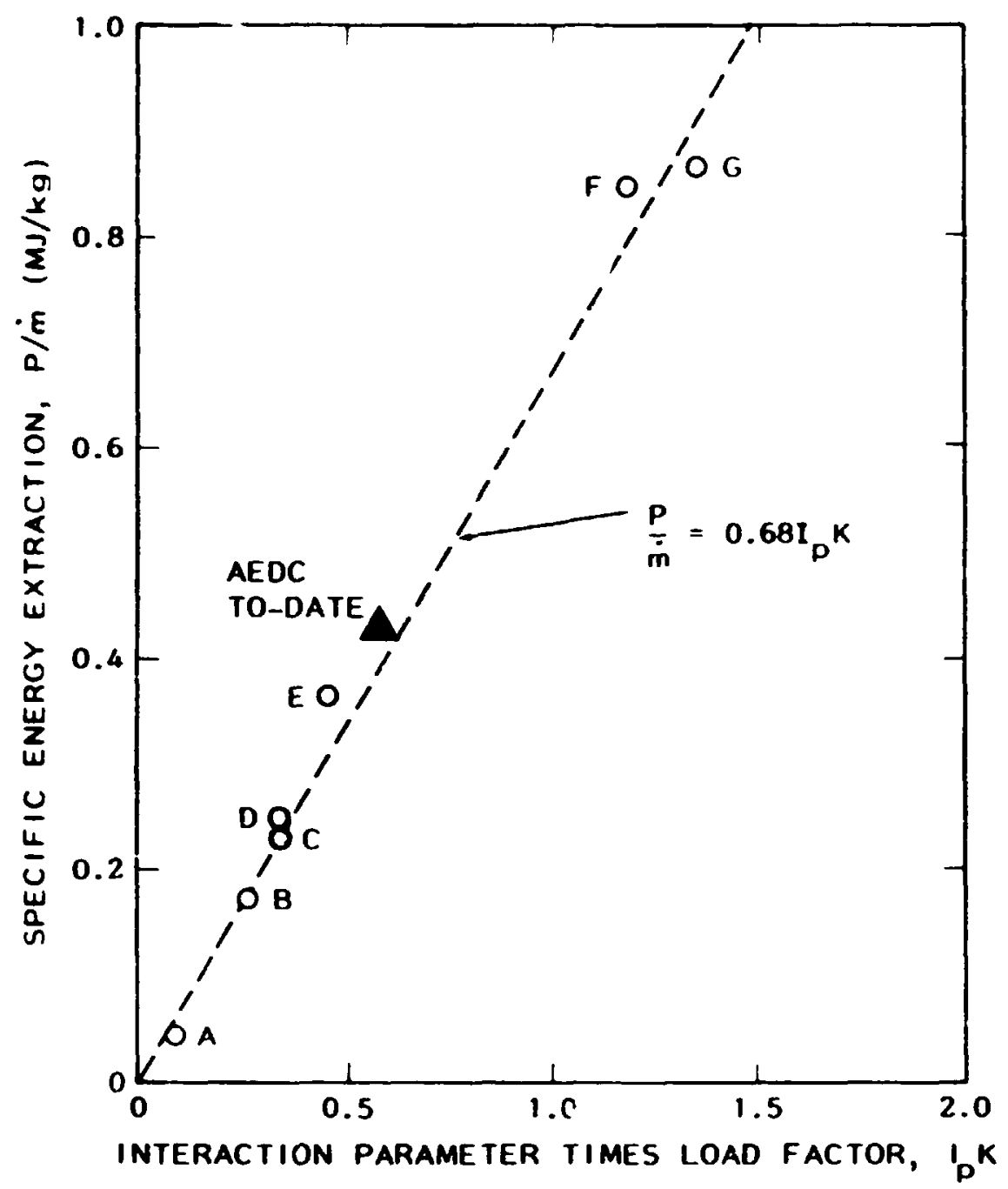

Fig. 7-3. Variation of specific energy extraction with the product of interaction parameter based on pressure and the Faraday load factor $K$ or diagonal generator electrical conversion efficiency $n_{e}$.

$u-3632 a$ 


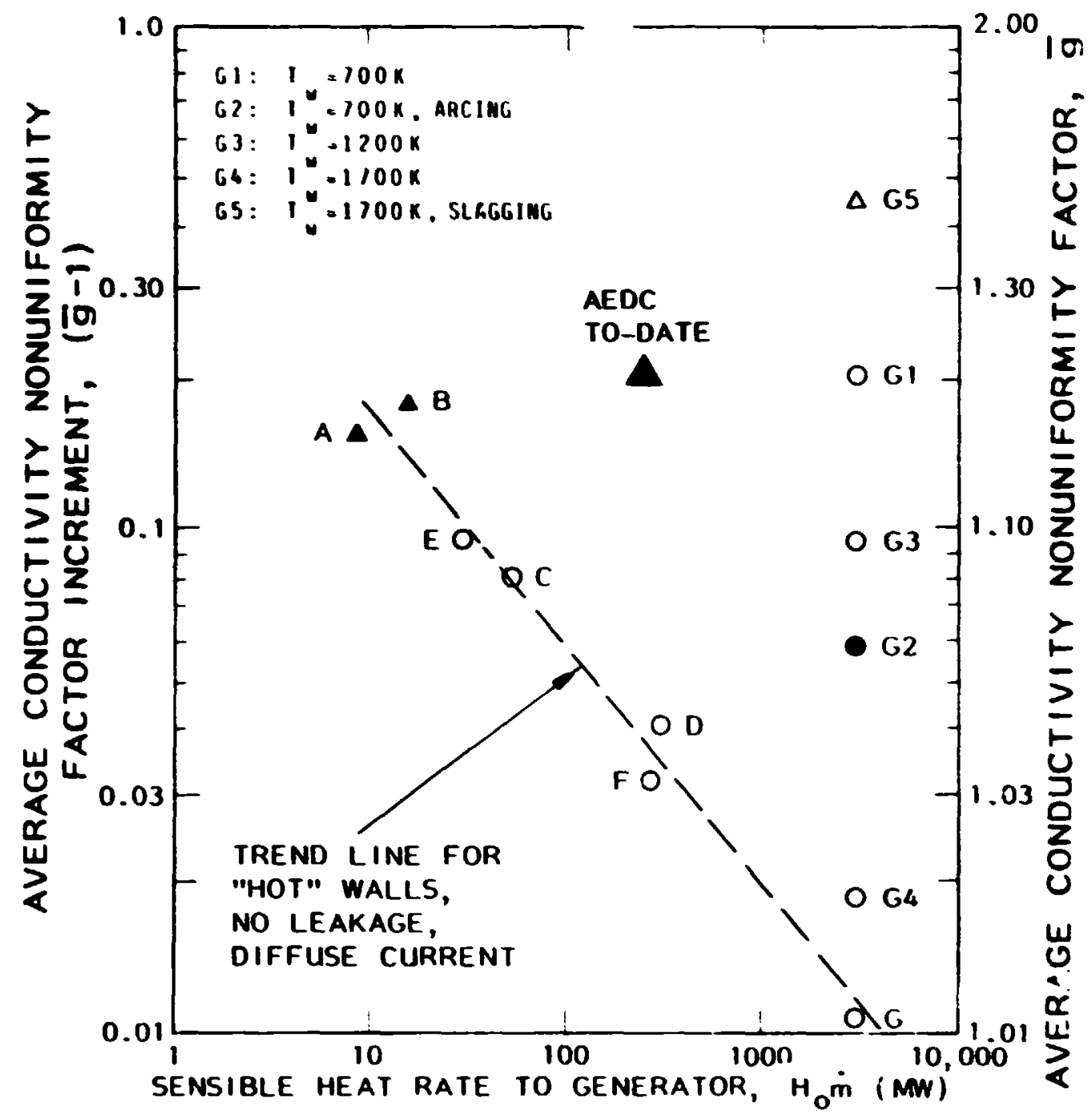

Fig. 7-4. Variation of conductivity nonuniformity factor $g$, averaged over the channel length, with sensible heat rate or thermal input tc the MHD generator. Solid symbols denote arc-mode current transport, and triangles denote slagging walls.

$0-3635 a$ 


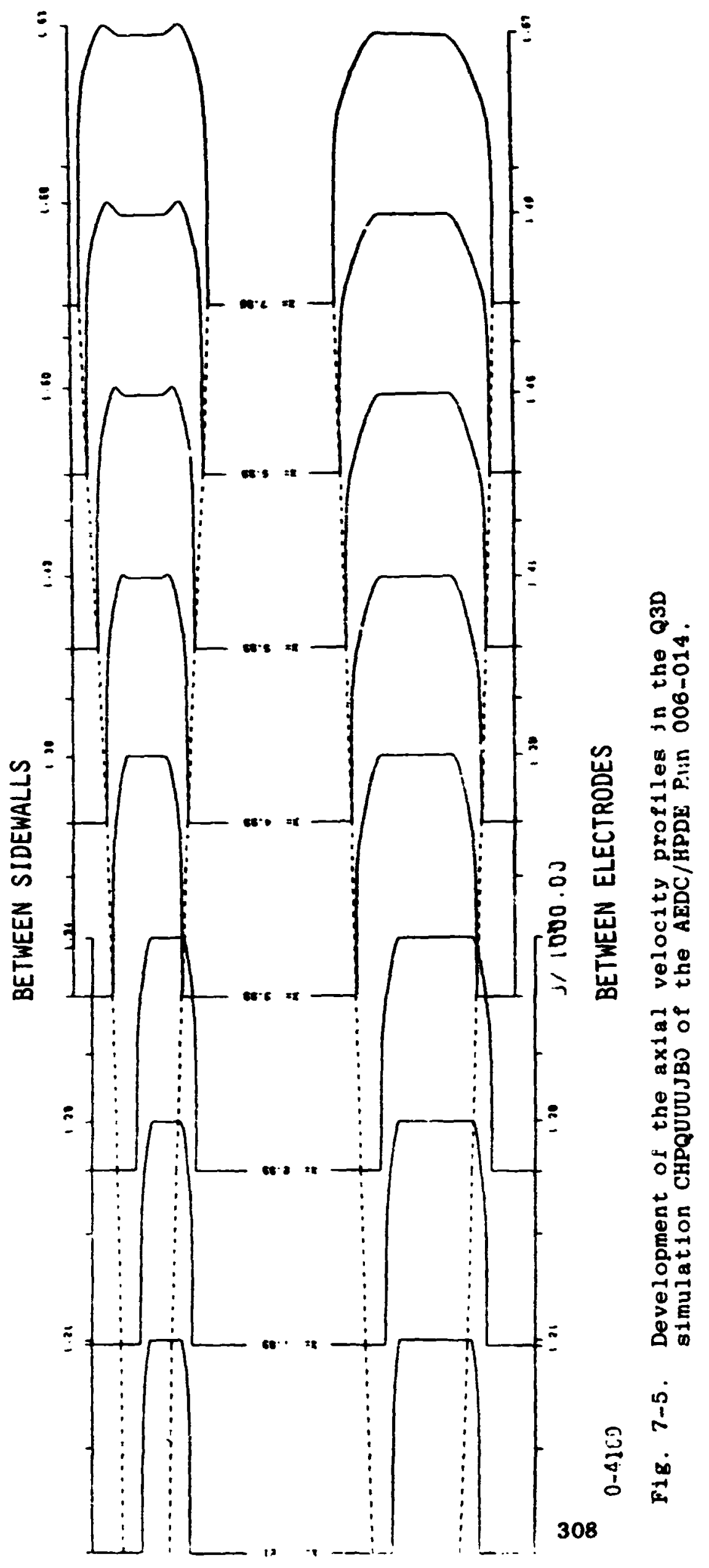




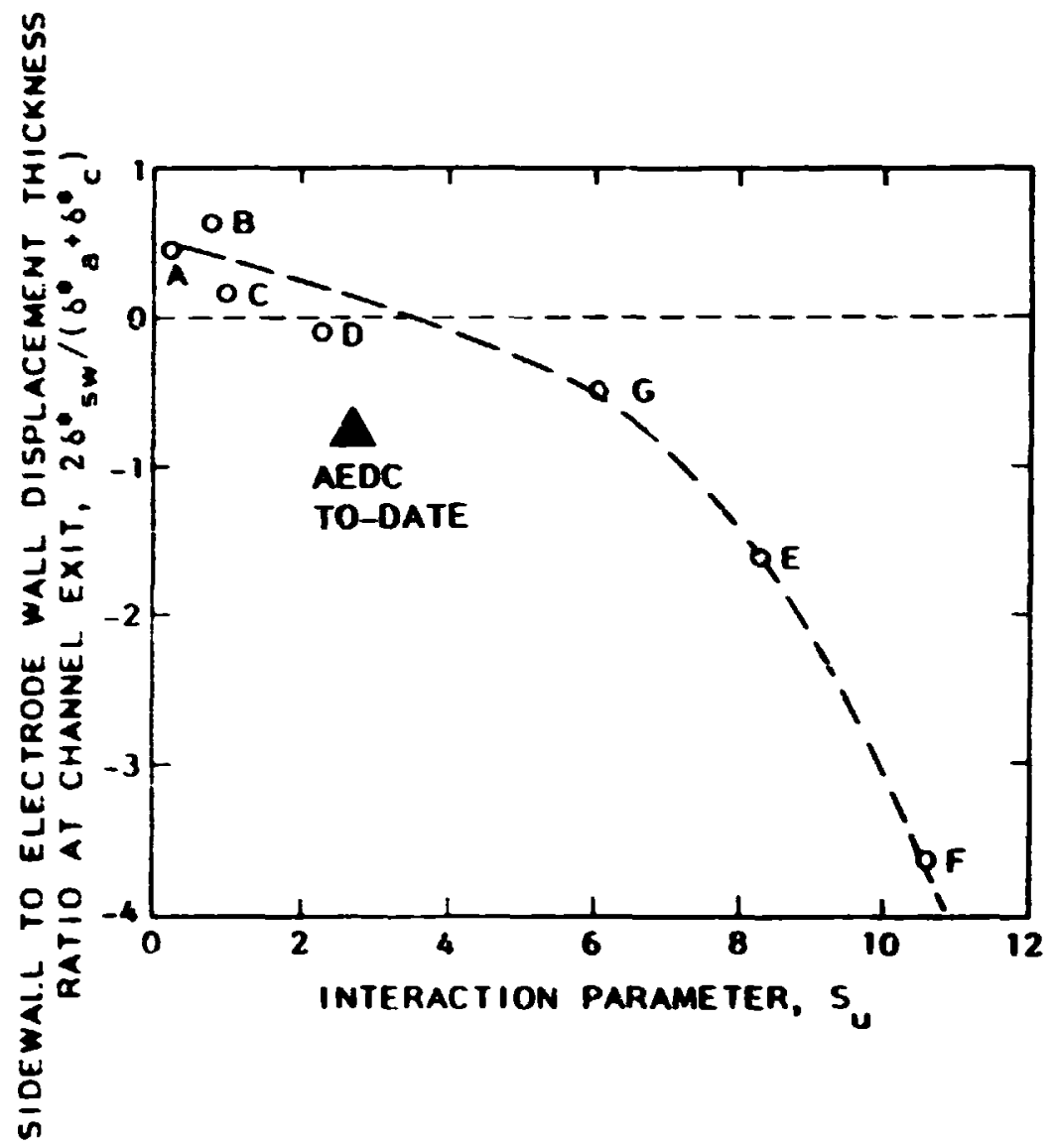

Fig. 7-6. Varlation of the ratio of sidewall to average electrode wall displacement thickness with the interaction parameter based on velocity.

$0-3630 a$ 


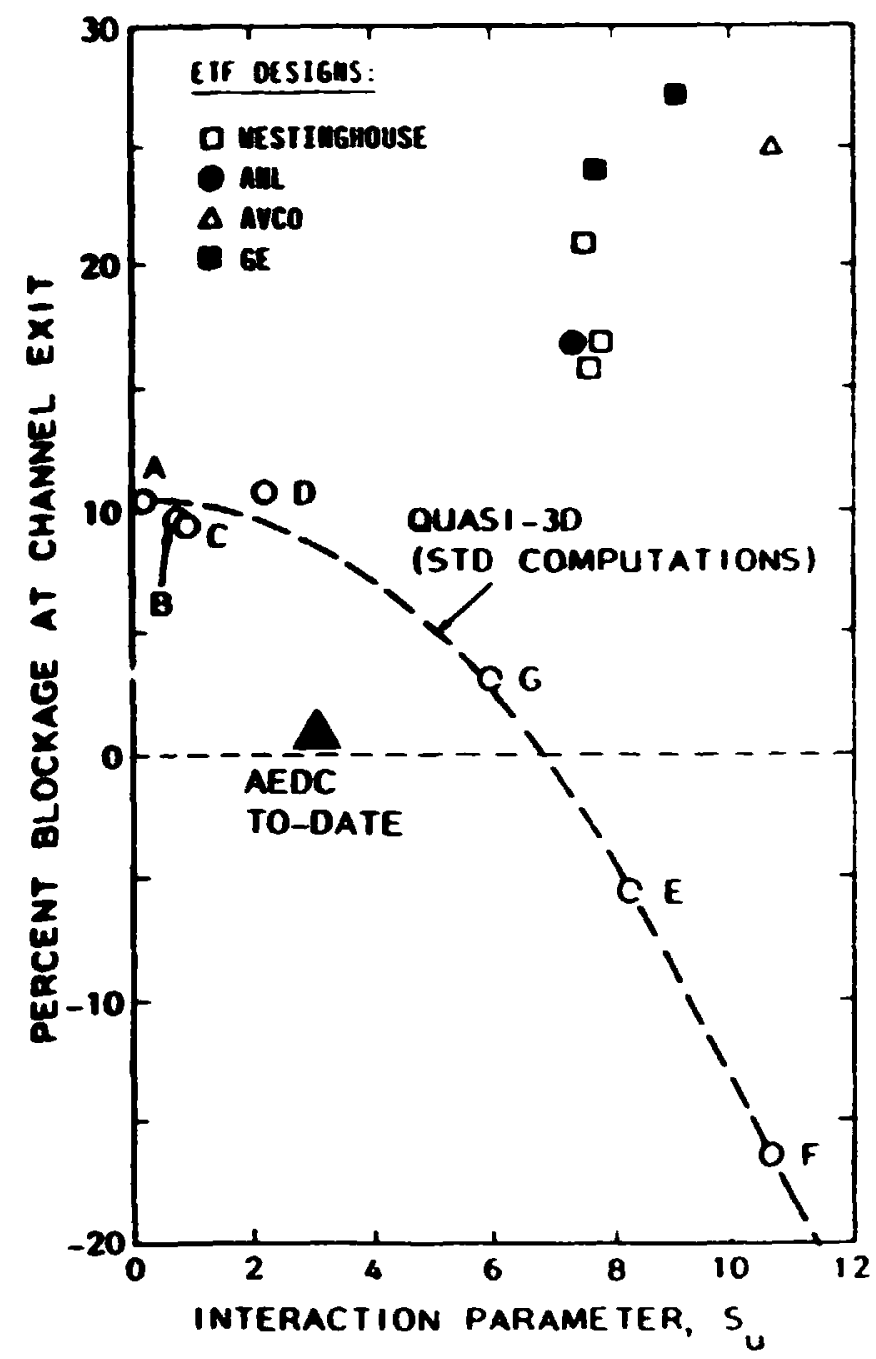

F1g. 7-7. Variation of the MHD generator exit blockage with interaction parameter based on velocity. The STD computational Cases A-G are compared with the estimates used by the ETF conceptual design contractors and the AEDC performance to date.

$0-3631$ a 


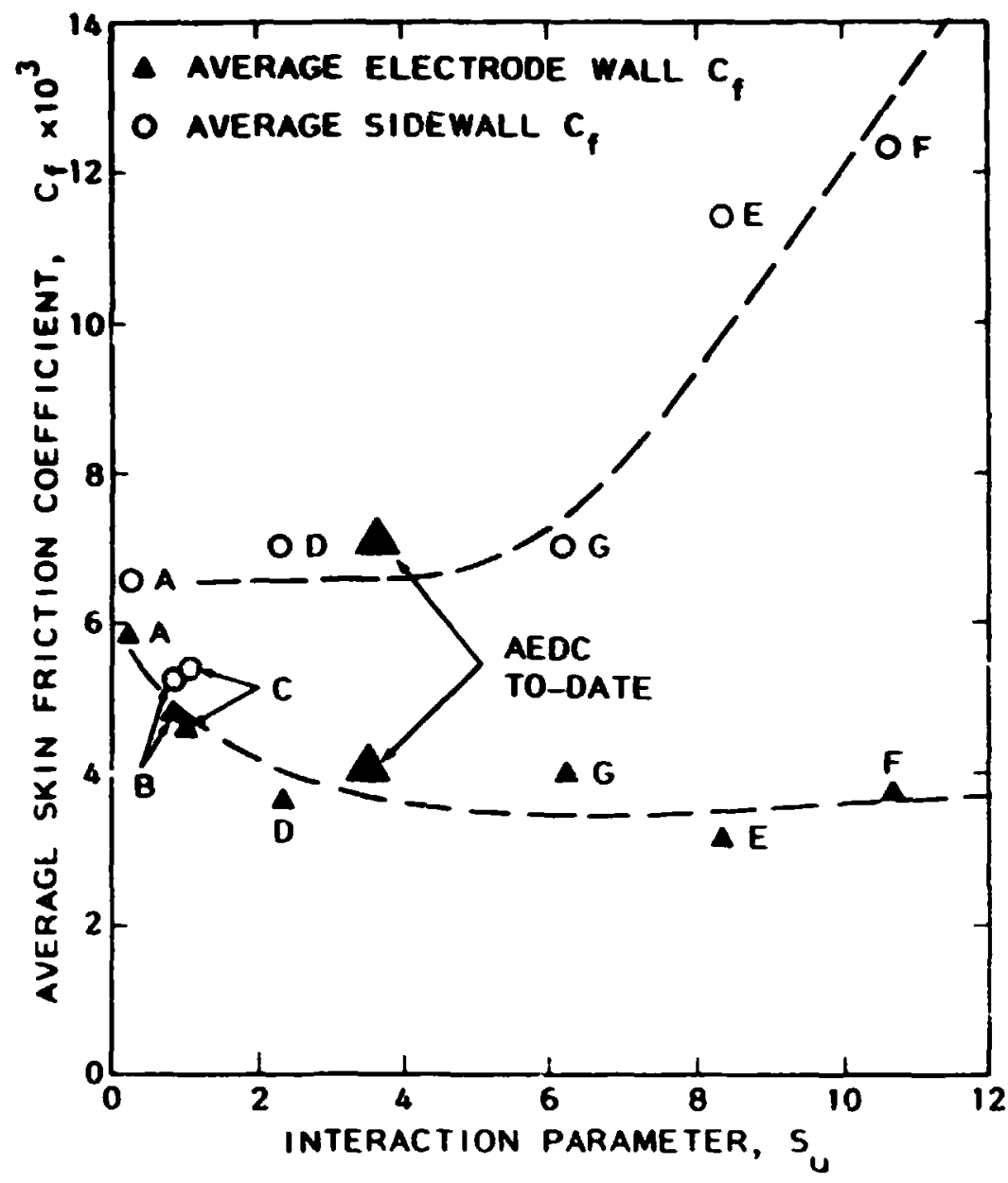

Fig. 7-8. Average skin friction coefficient on the electrode walls and sidewalls as a function of the interaction parameter based on velocity.

$0-3629$ a 


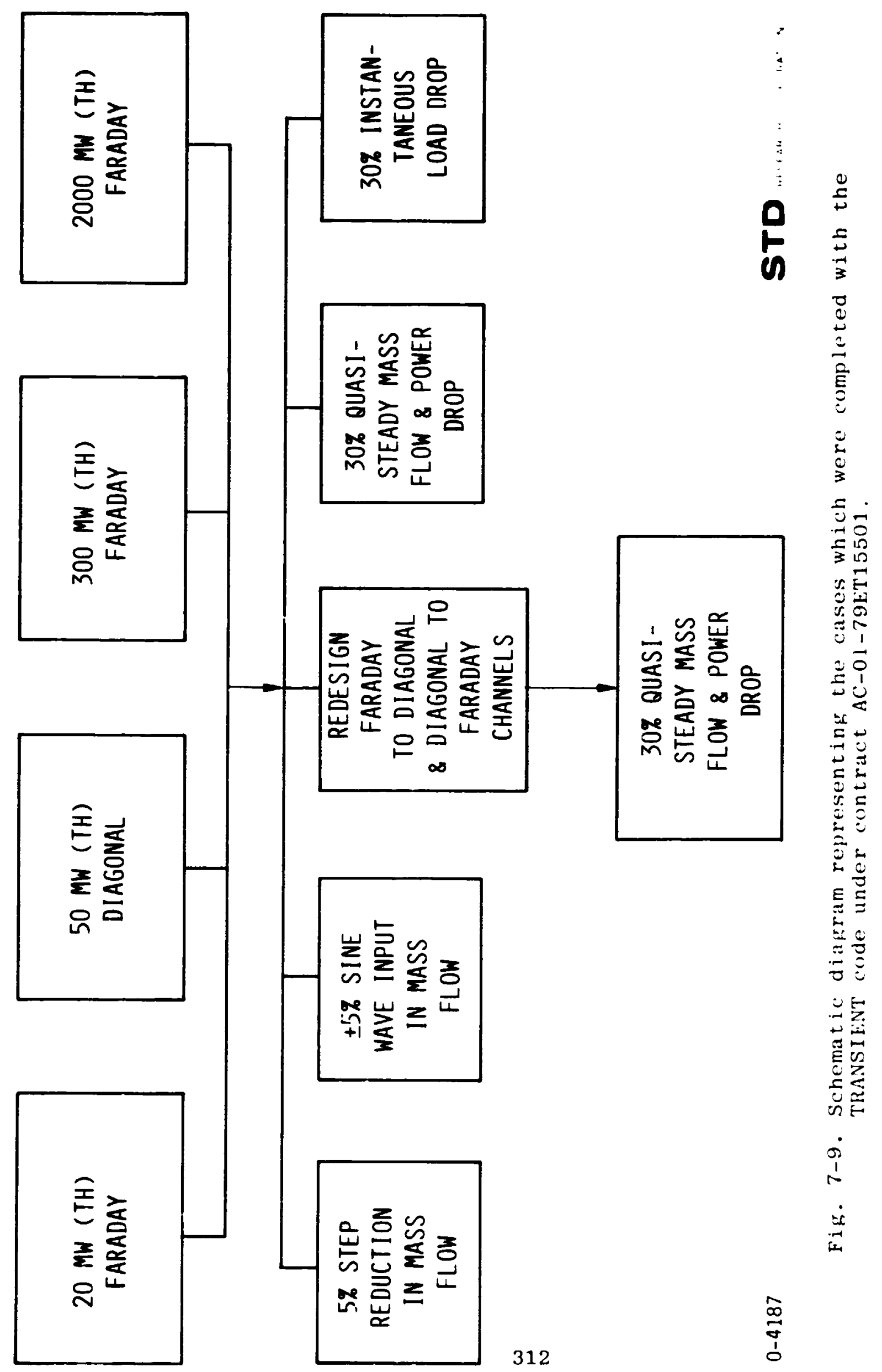




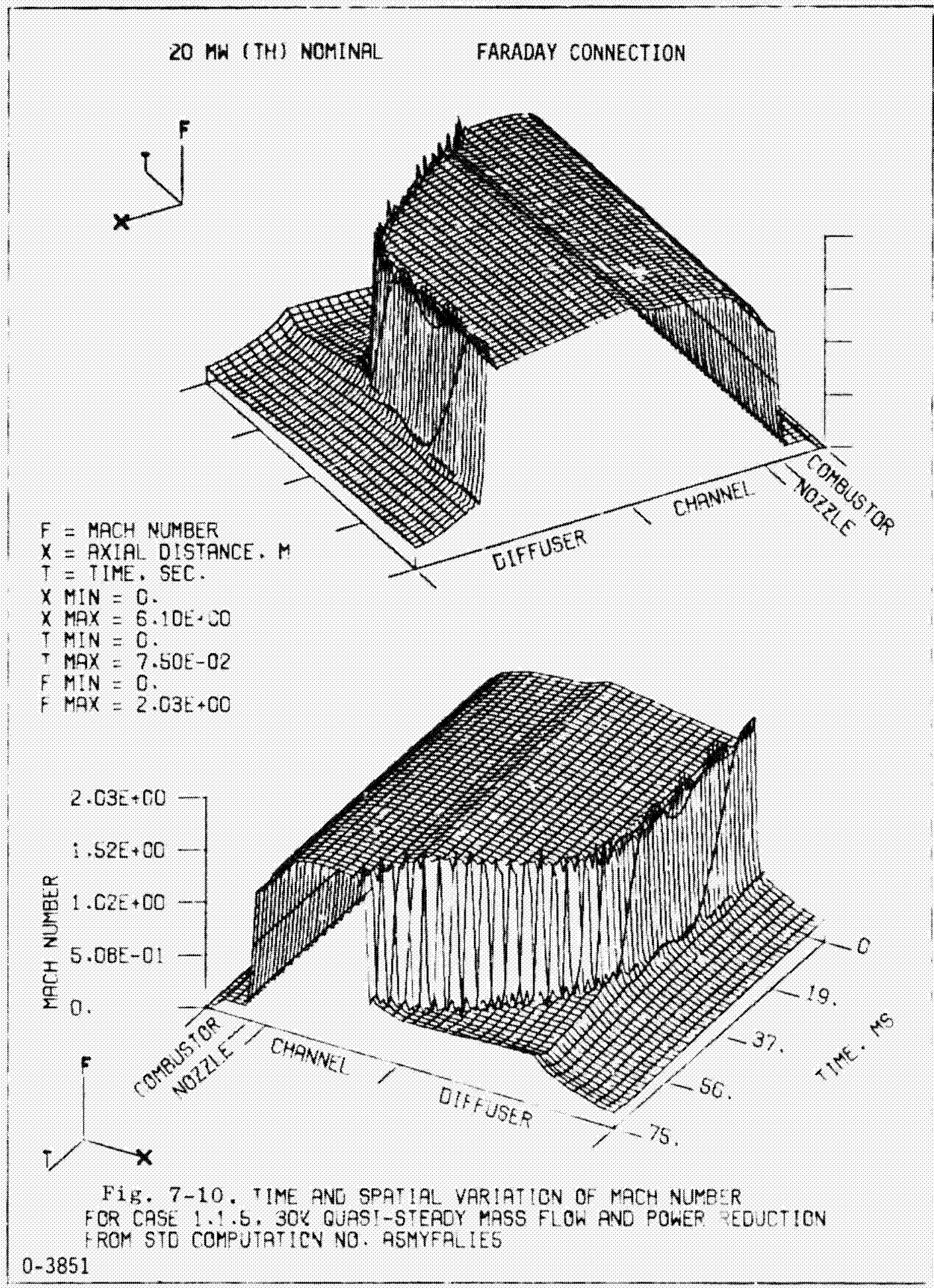




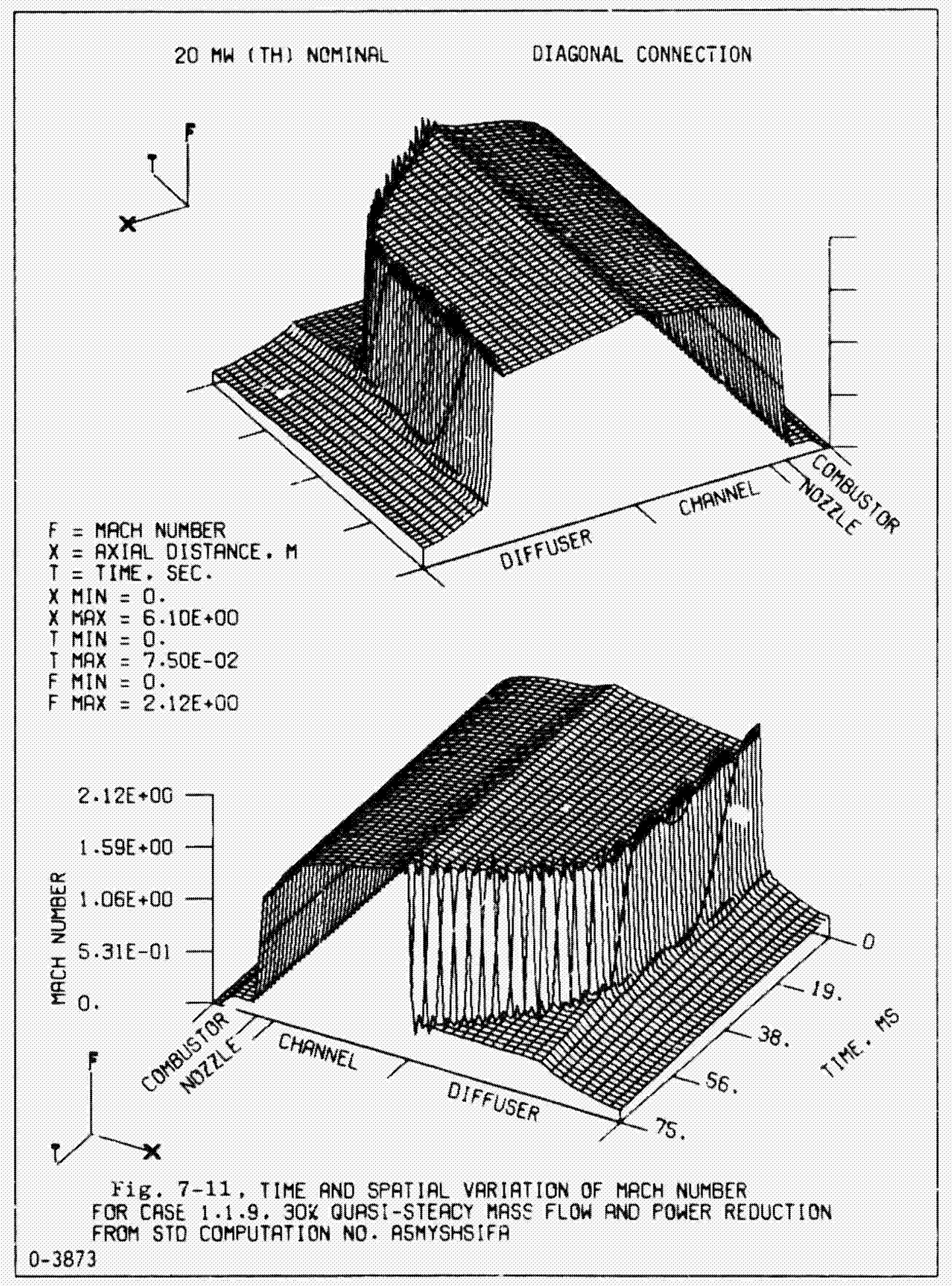




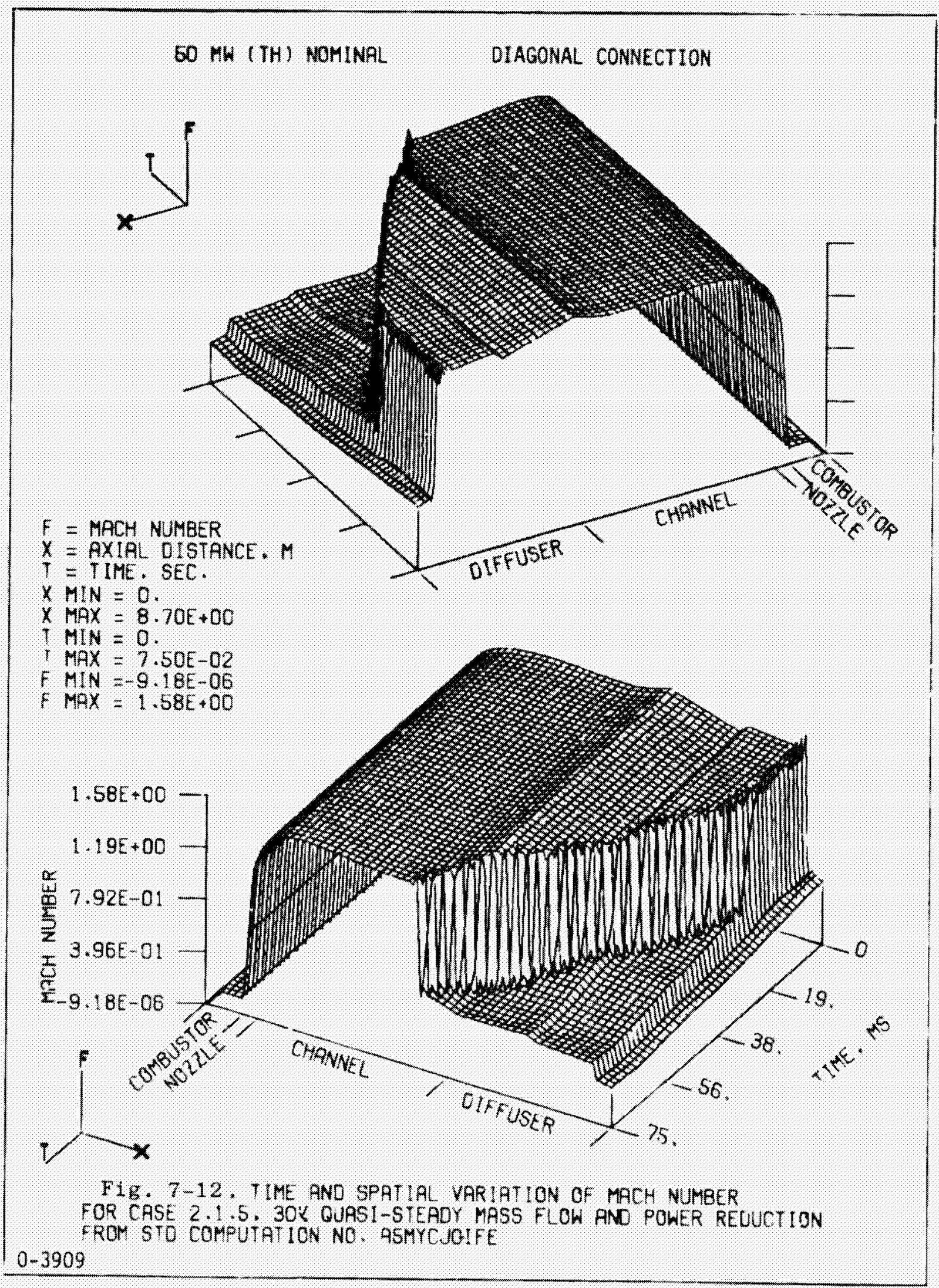




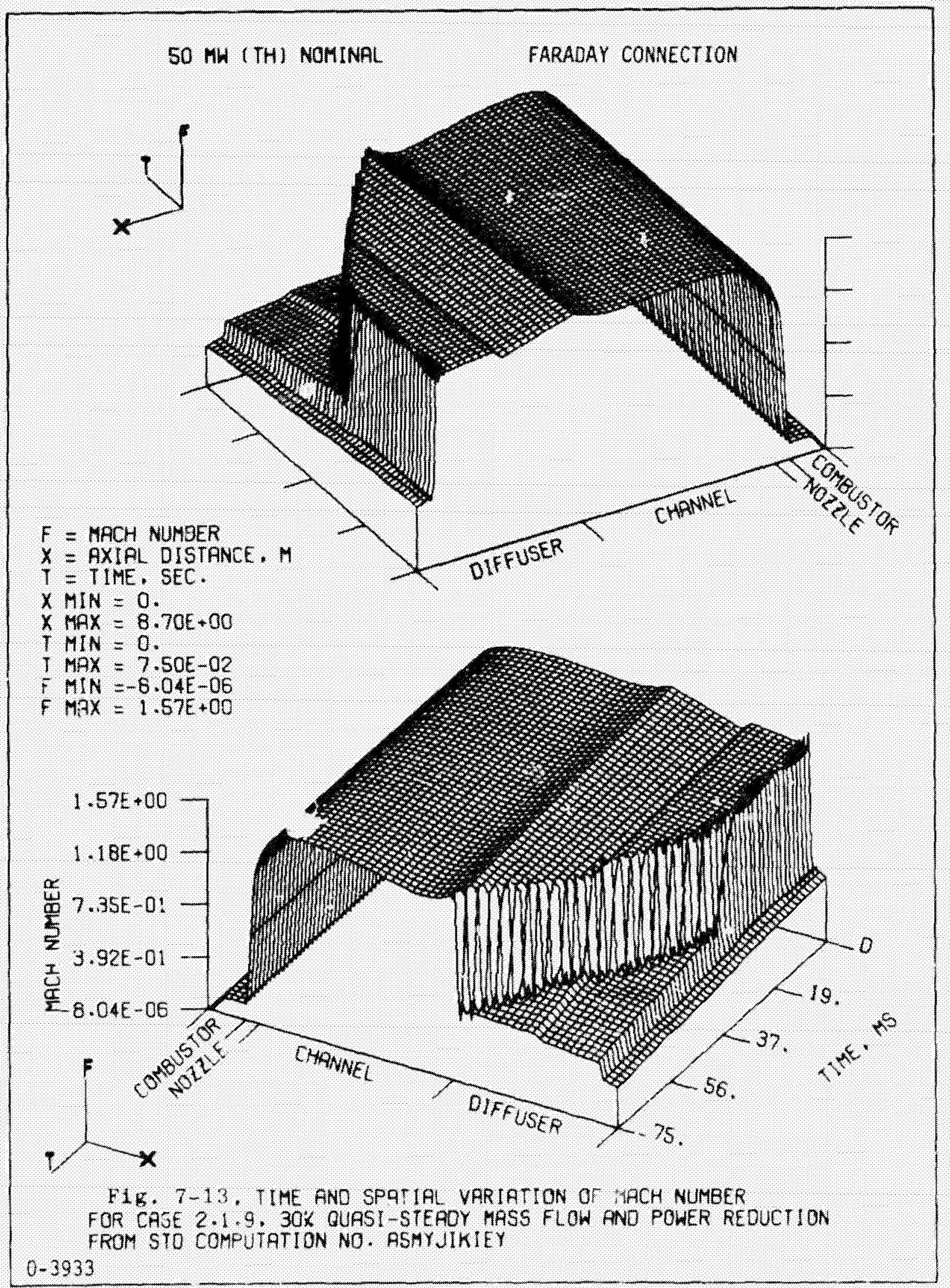




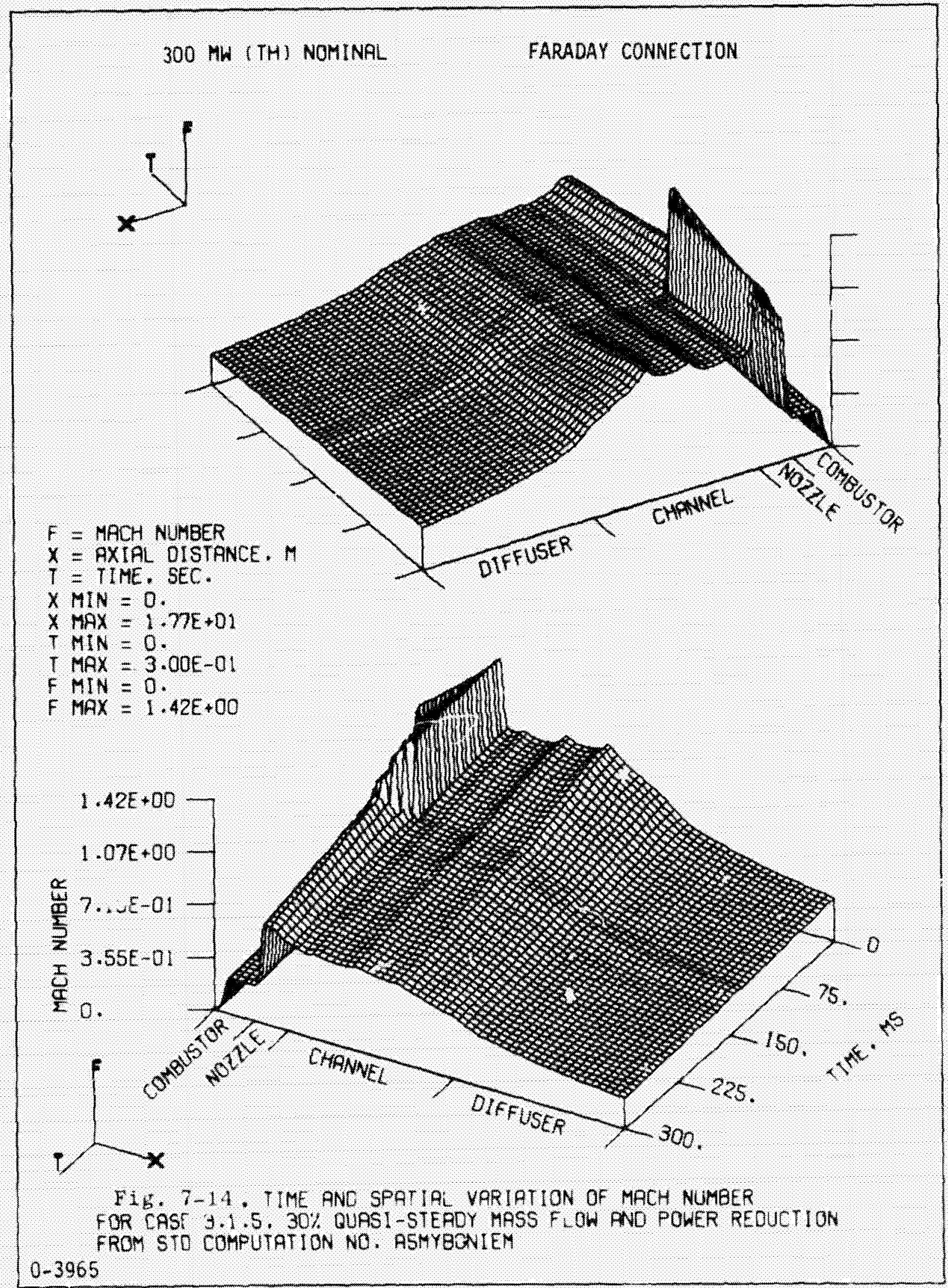




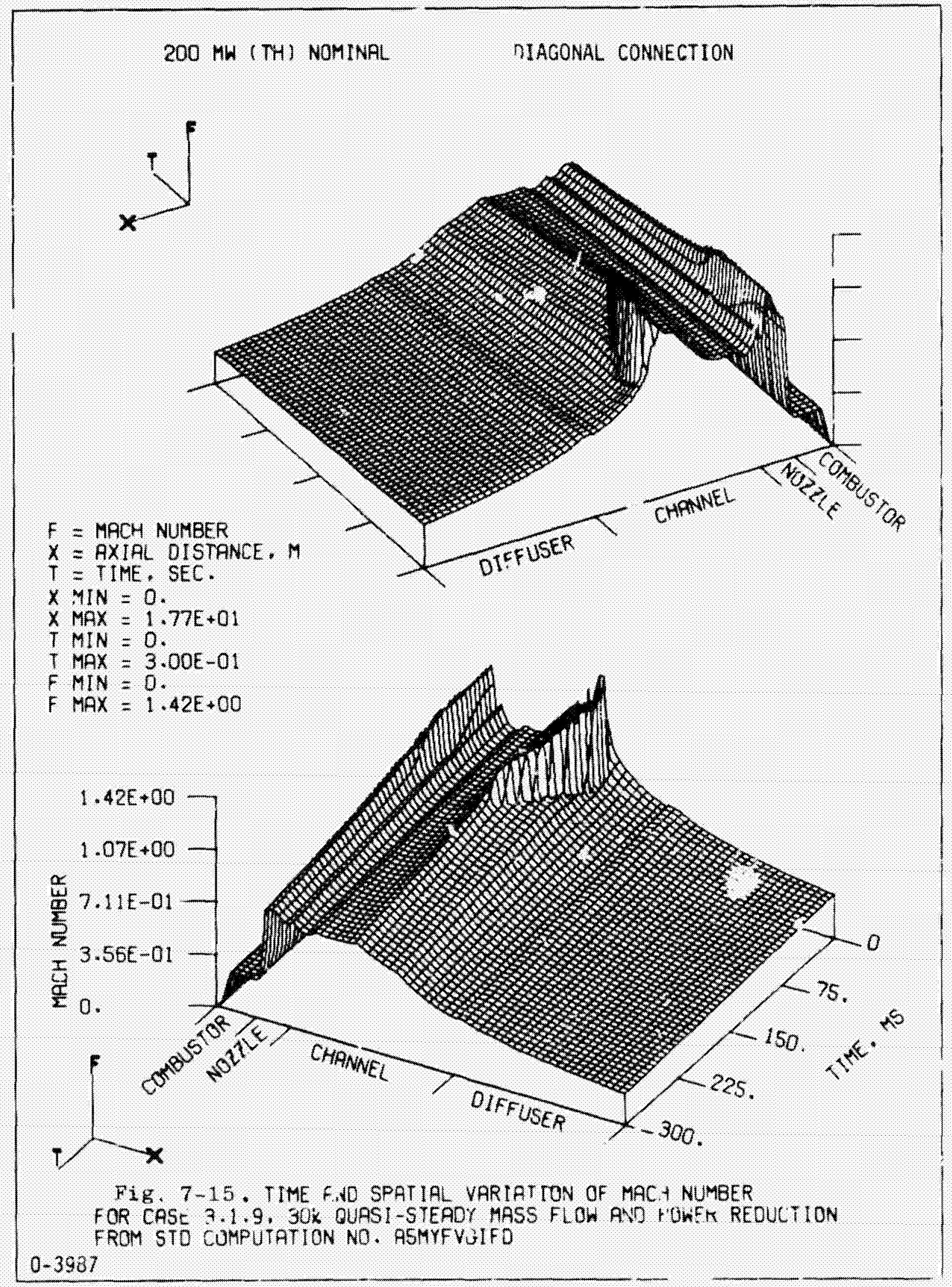




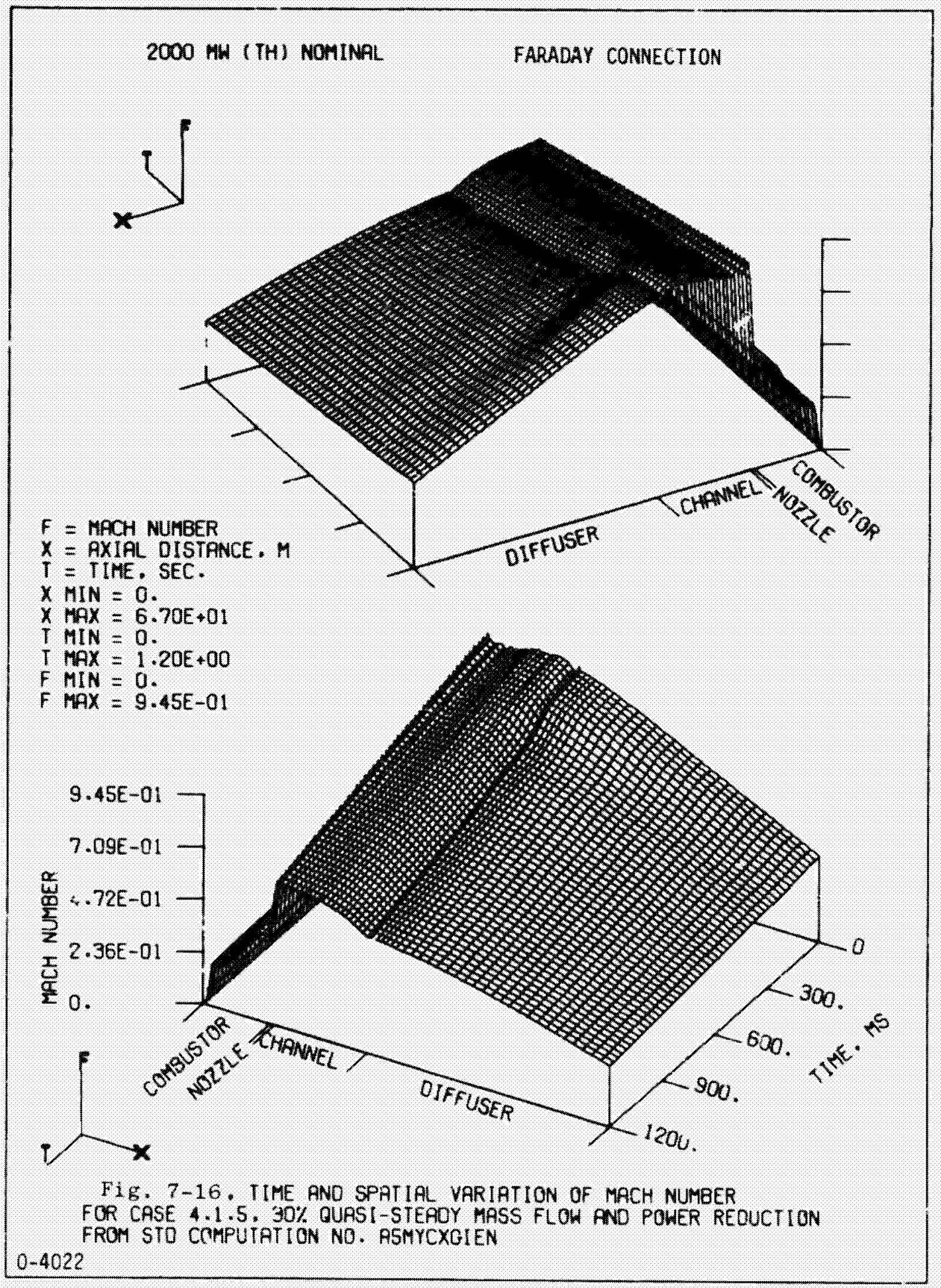




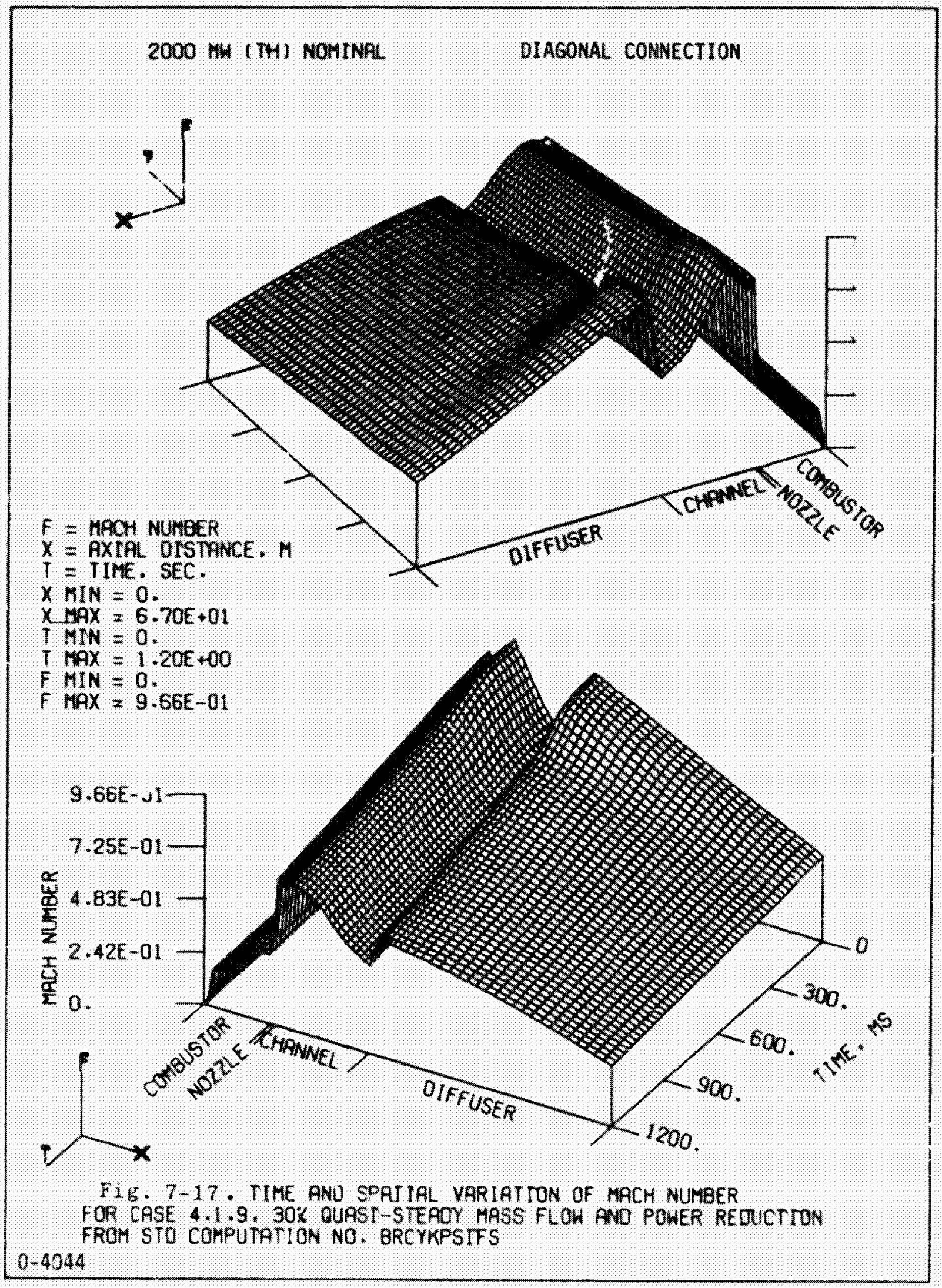


STD

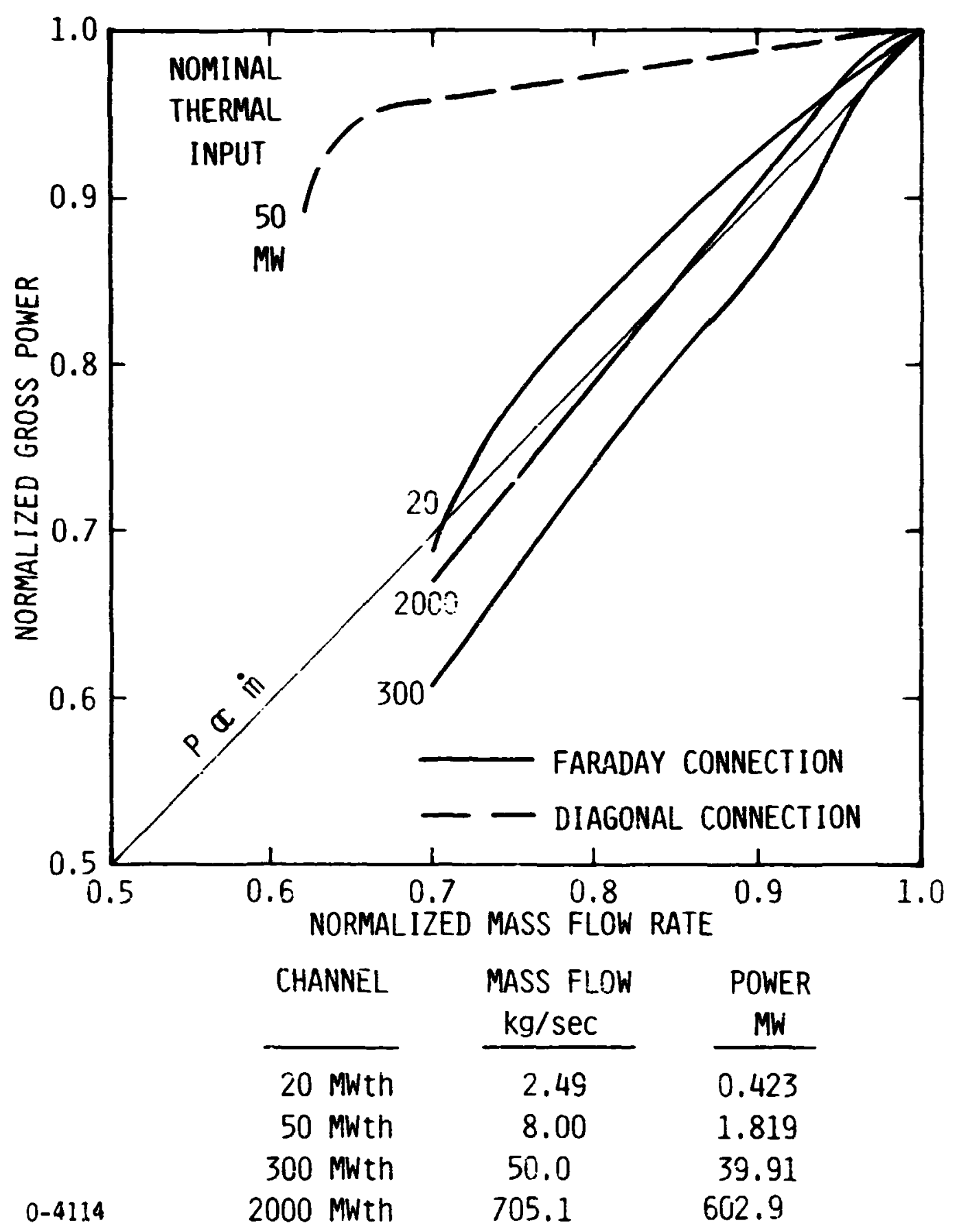

Fig. 7-18 Normalized gross power vs. normalized mass flow rate for channels with their nominal electrical hook-up. 
STD atreas - ar a...

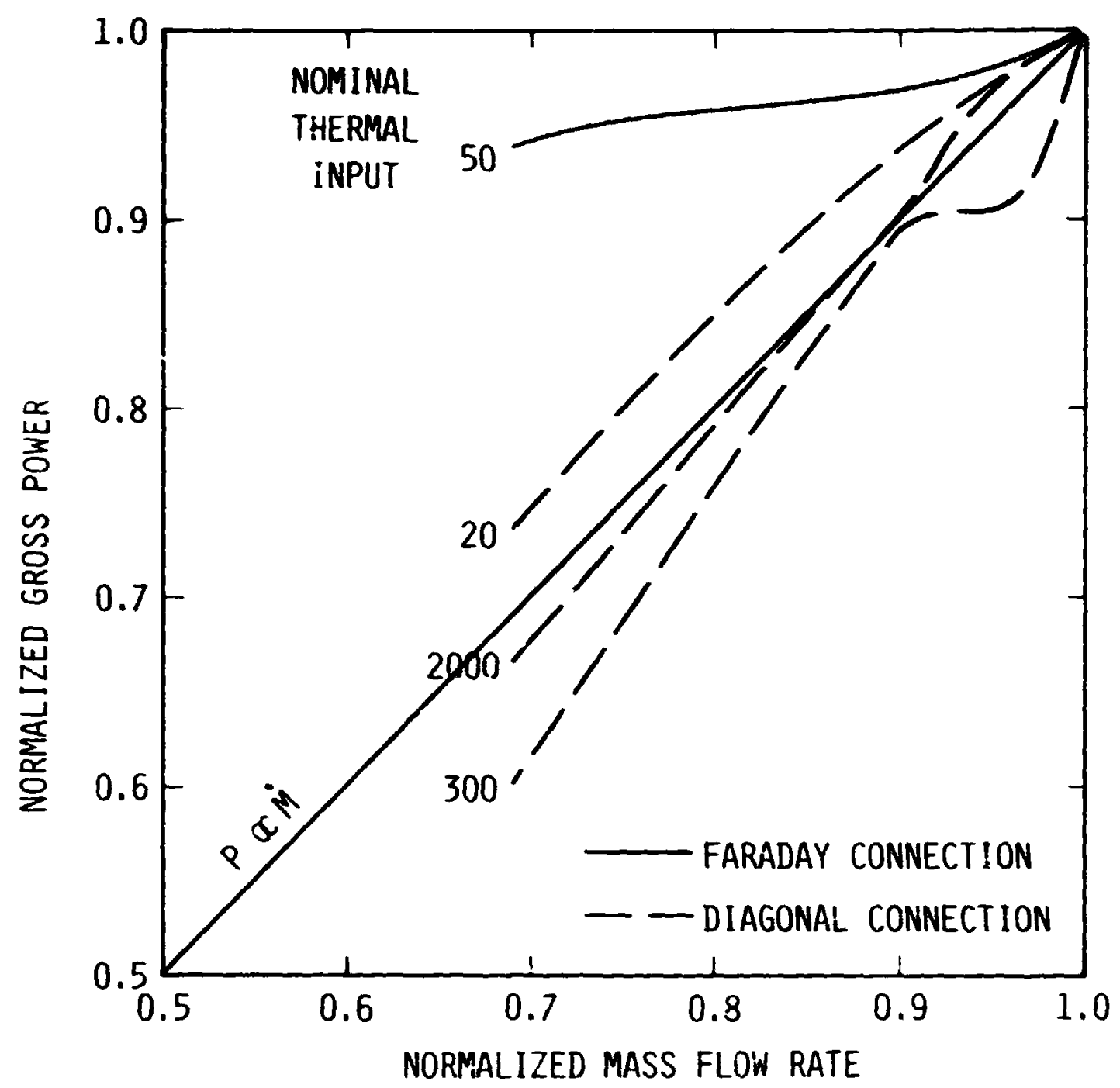

\begin{tabular}{|c|c|c|c|}
\hline & CHANNEL & $\begin{array}{l}\text { MASS FLOW } \\
\mathrm{kg} / \mathrm{sec} \\
\end{array}$ & POWER \\
\hline & 20 MWth & 2.49 & 0.502 \\
\hline & 50 MWth & 8.00 & 1.777 \\
\hline & 300 MWth & 50.0 & 36.67 \\
\hline $0-4111$ & $2000 \mathrm{MWth}$ & 705.9 & 522.9 \\
\hline
\end{tabular}

Fi. 7-19 Normalized gross power vs. normalized mass flow rate for 4 channels with their alternate electrical hook-up. 
STD atseabch rinatan....

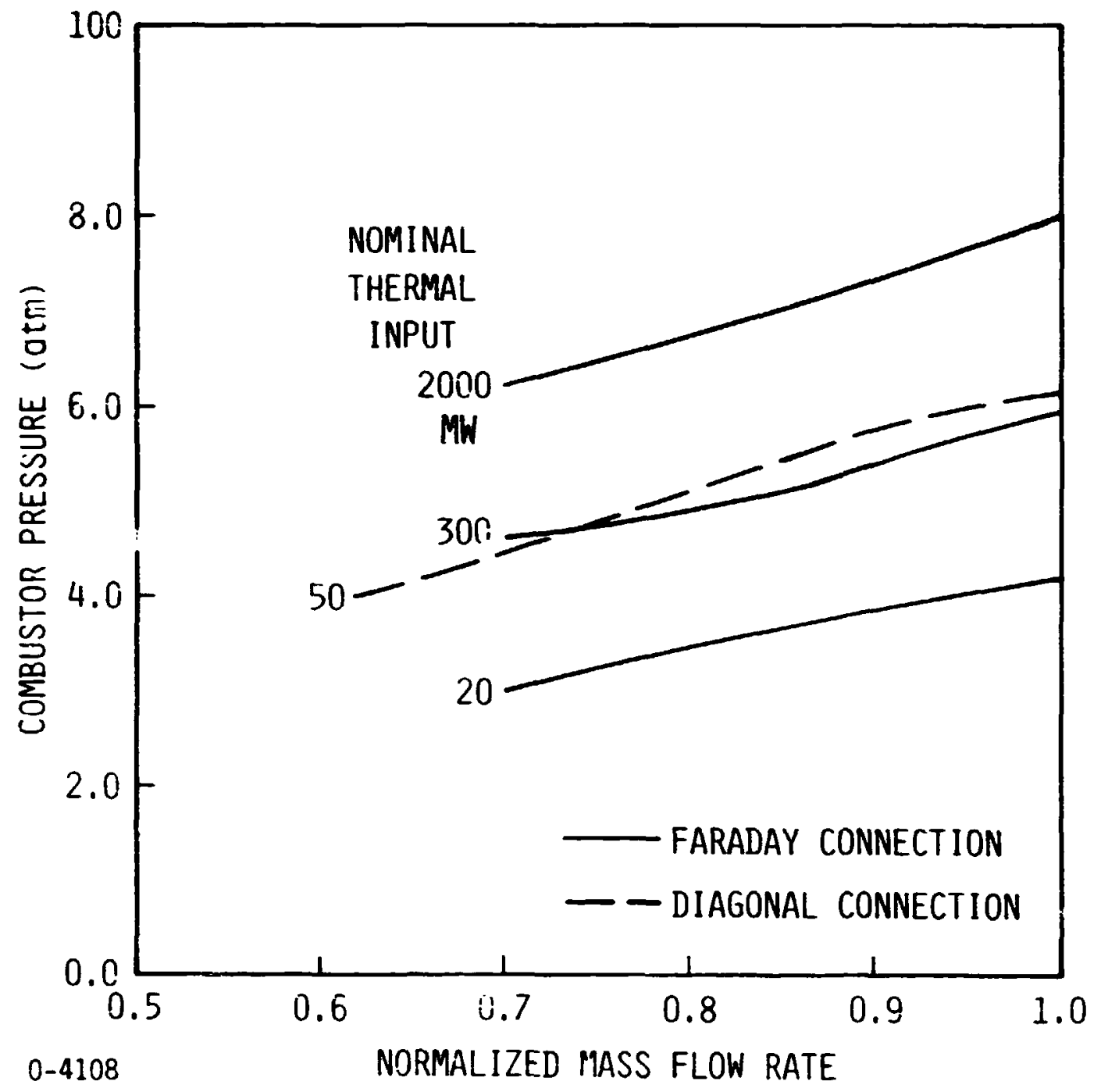

Fig. 7-20. Combustor stagnativi pressure vs, normalized mass flow rate for different size generators with mass flow varying between $70 \%$ and $100 \%$ at nominal design value. 
\&TO aeseapch coppoant on

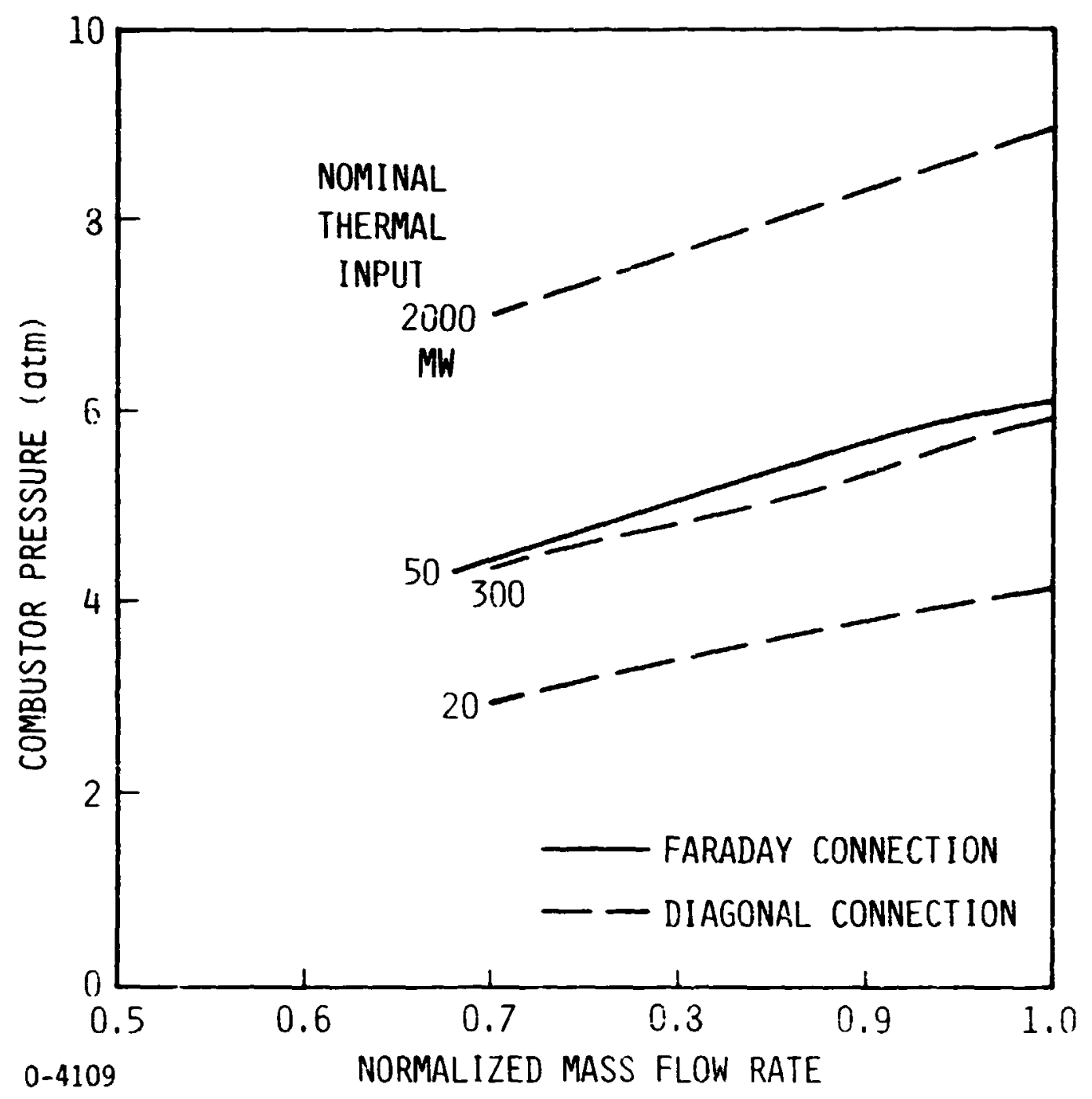

Fig. 7-21. Combustor stagnation pressure vs. normalized mass flow rate for different size generators with mass flow varying between $70^{r}$ and $100^{\circ}$ of nominal design value. 
STD aesfrach corporanion

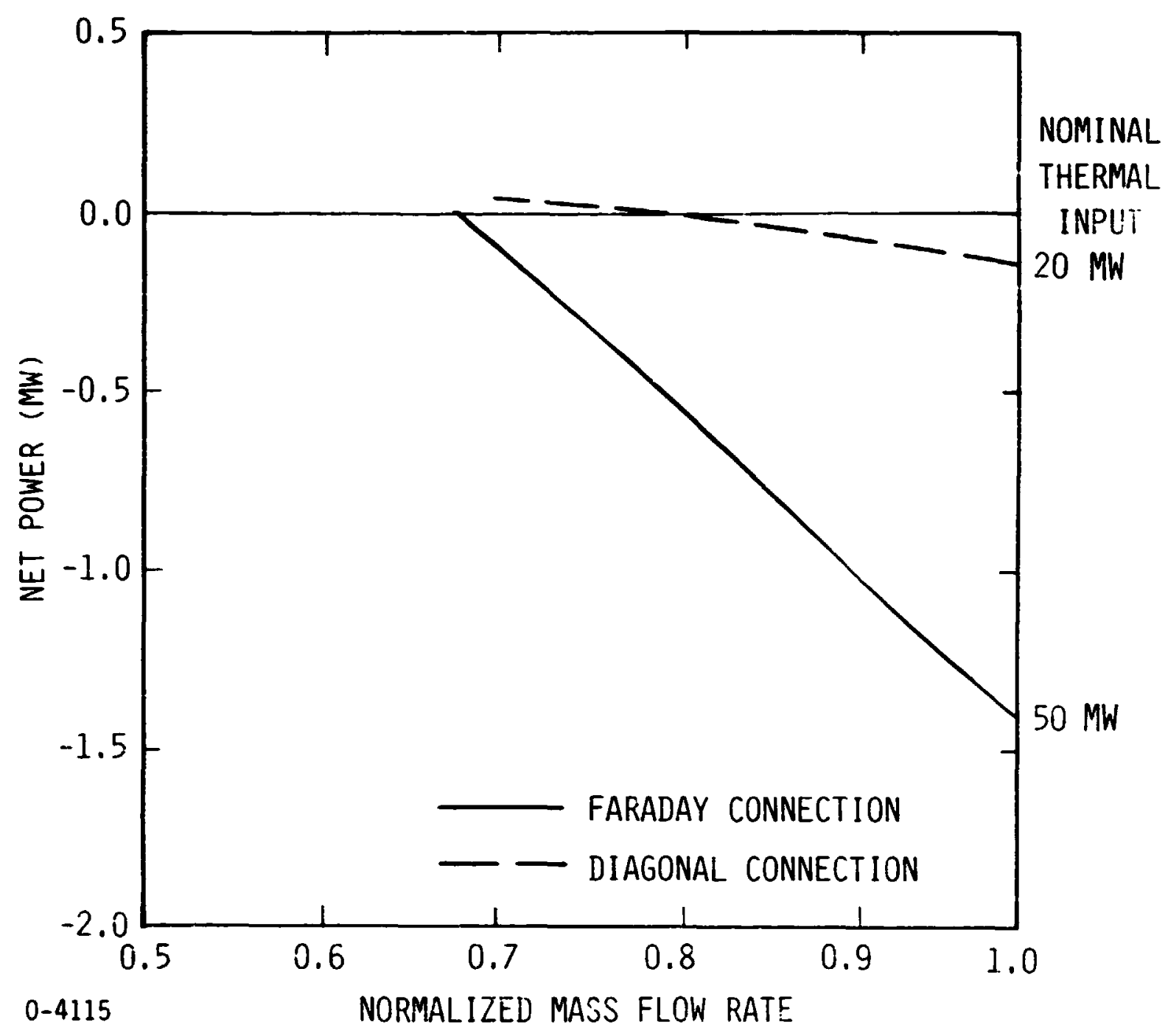

Fig. 7-22. Net power vs. normalized mass flow rate for two generators with mass flow varying between $70^{c}$ to $100 \%$ of nominal design value. 


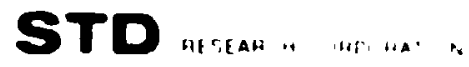

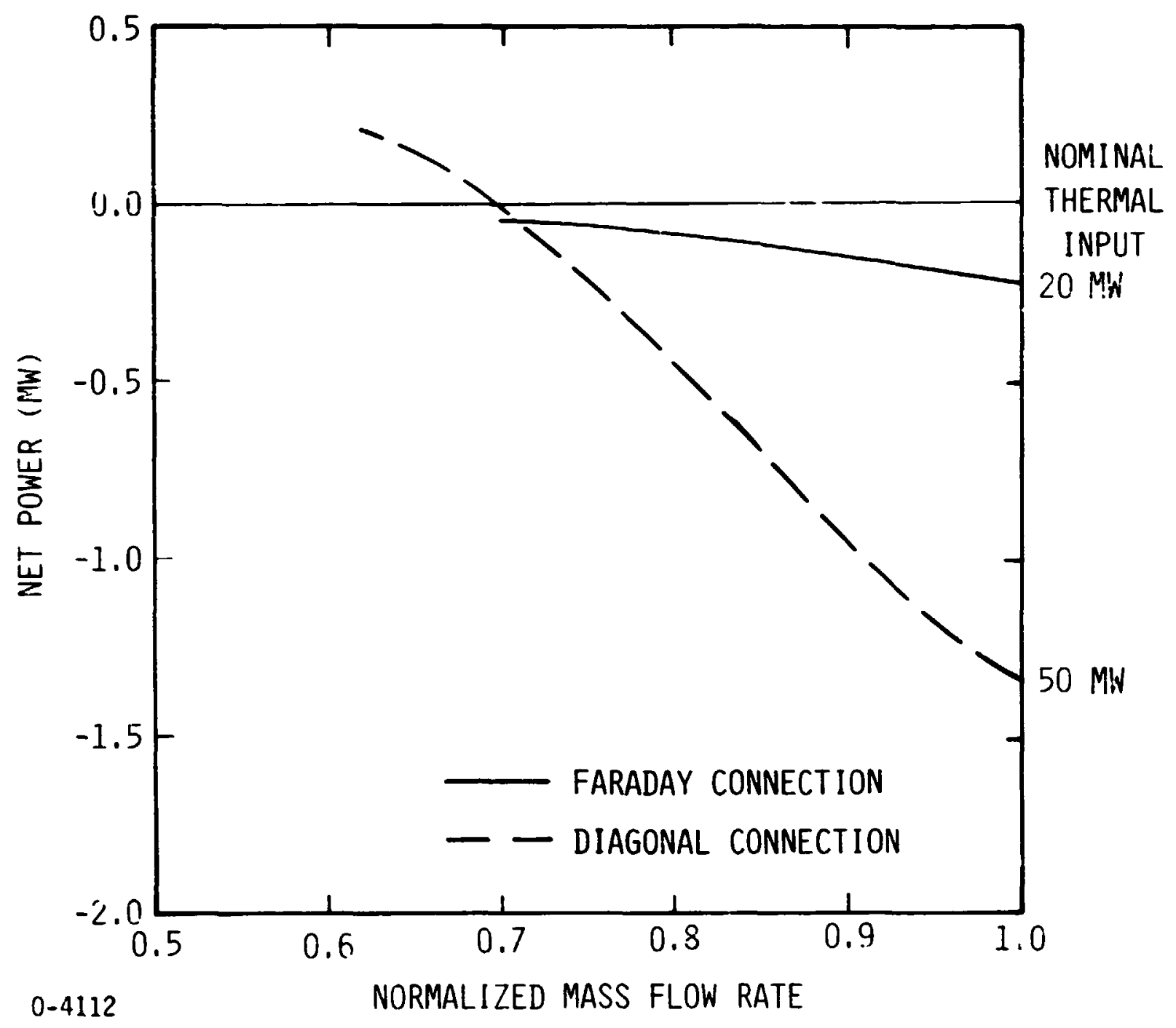

Fig. 7-23. Net power vs. normalized mass flow rate for two generators with mass flow varying between $70 \%$ to $100 \%$ of nominal design value. 


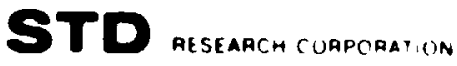

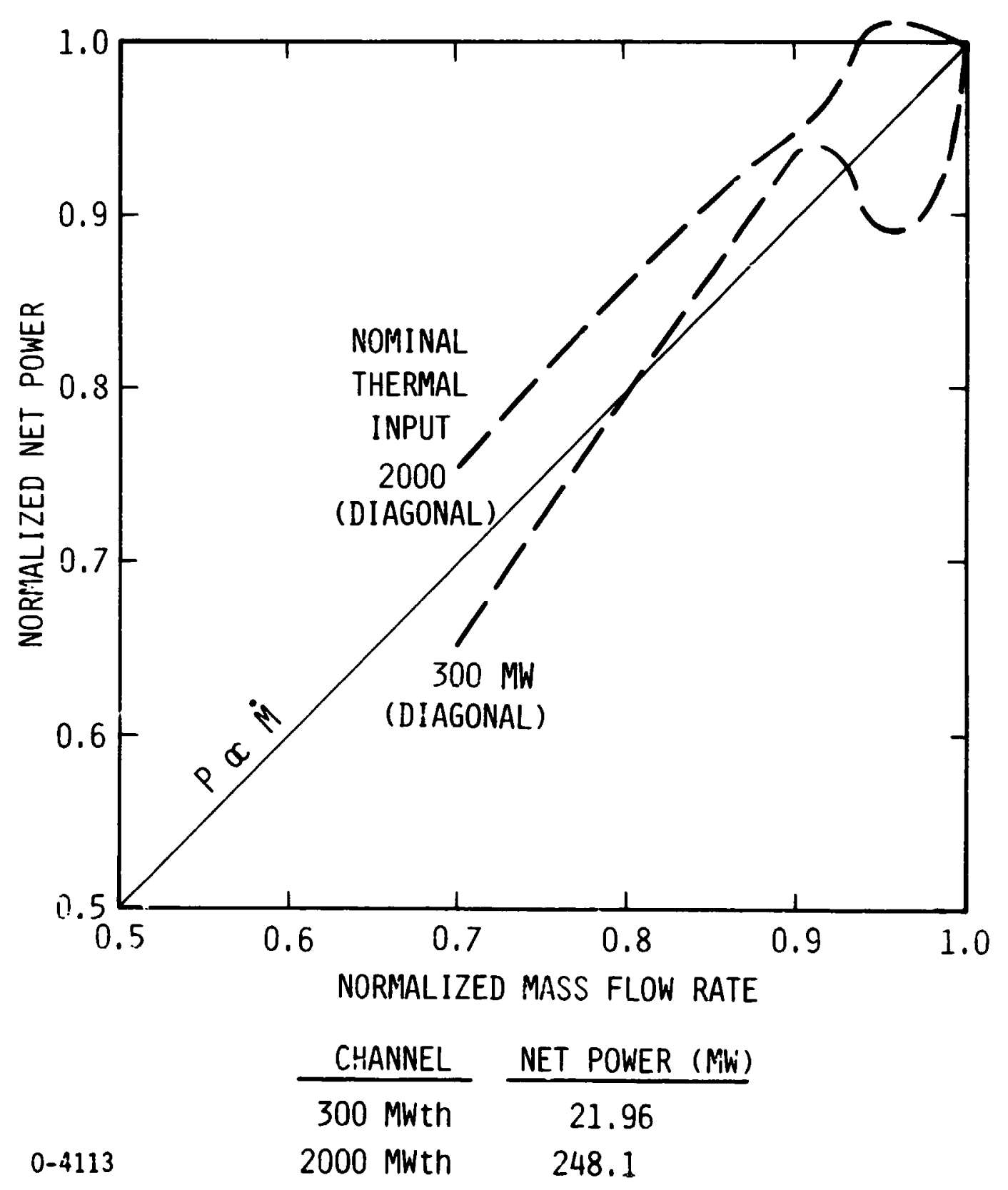

Fig. 7-24. Normalized net power vs. rormalized mass flow rate for two generators with mass flow varying between $70 \%$ and $100 \%$ of nominal design value. 
BDD afseahcm cumporation

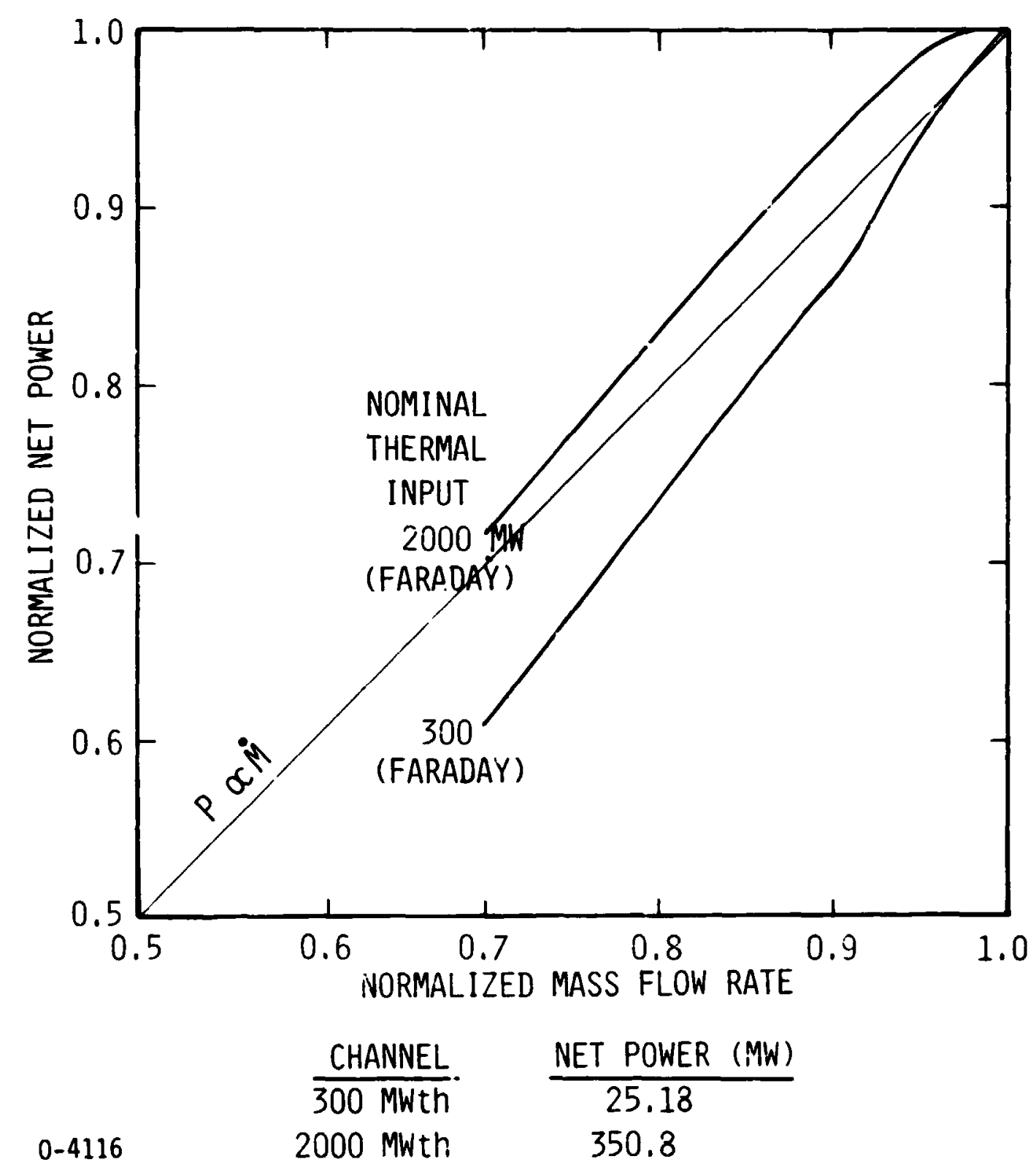

Fig. 7-25. Normalized net power vs. normalized mass flow rate for two generators with mass flow varying between $70 \%$ and $100 \%$ of the nominal design value. 
QTD aeseapch curroration

ALTERNATE ELECTRICAL HOOK-UP

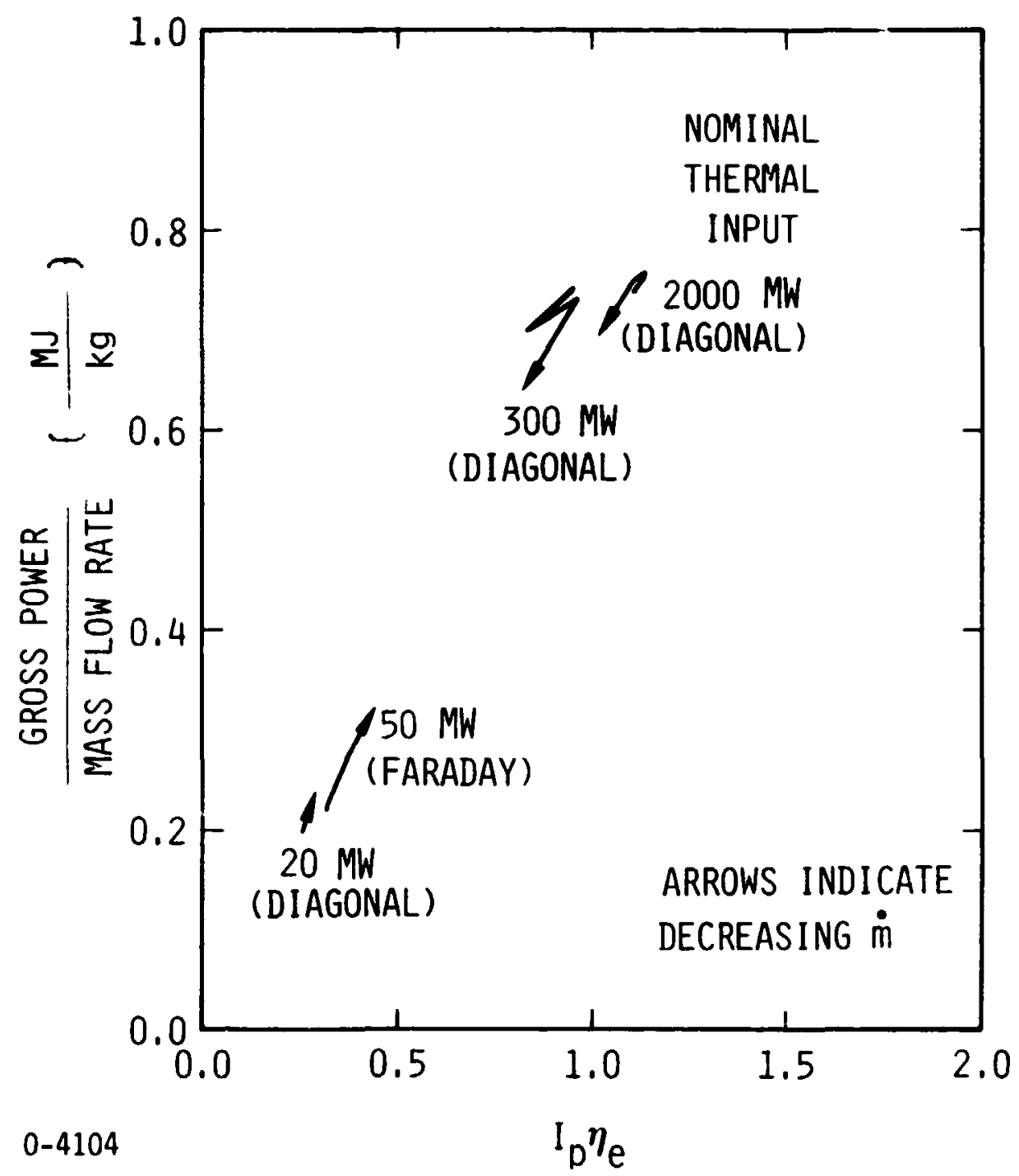

Fig. 7-26. Gross power/mass flow rate vs. interaction parameter times electrical efficiency for four different size channels with mass flow varying from design to $70 \%$ of design. 


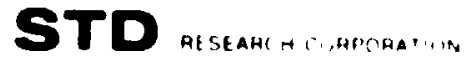

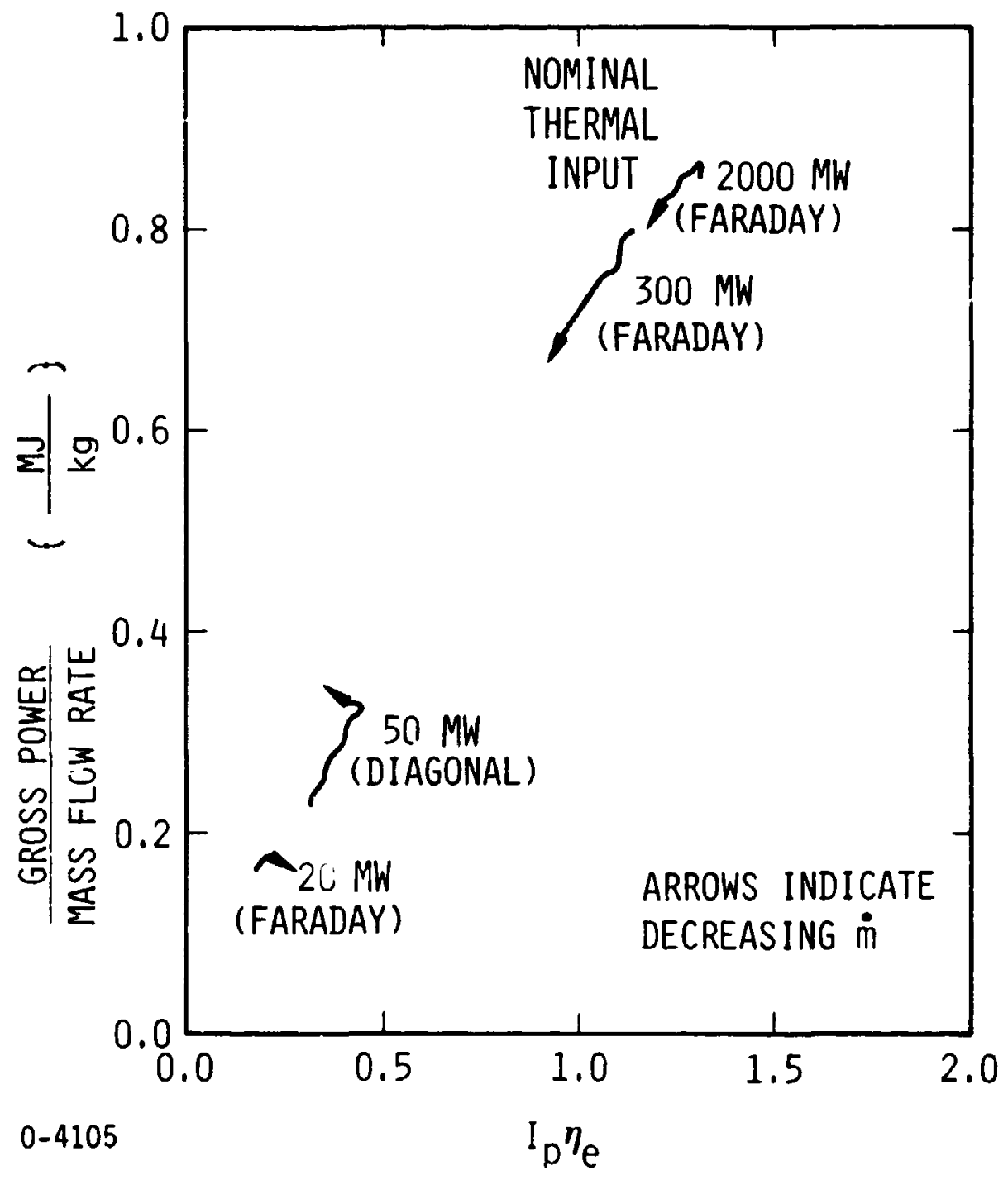

Fig. 7-27. Gross power/mass flow rate vs. Interaction para:neter times electrical efficiency for four different size channels with mass flow varying from design to $70 \%$ of design. 
STD ReseaACM :ORPOAar ION
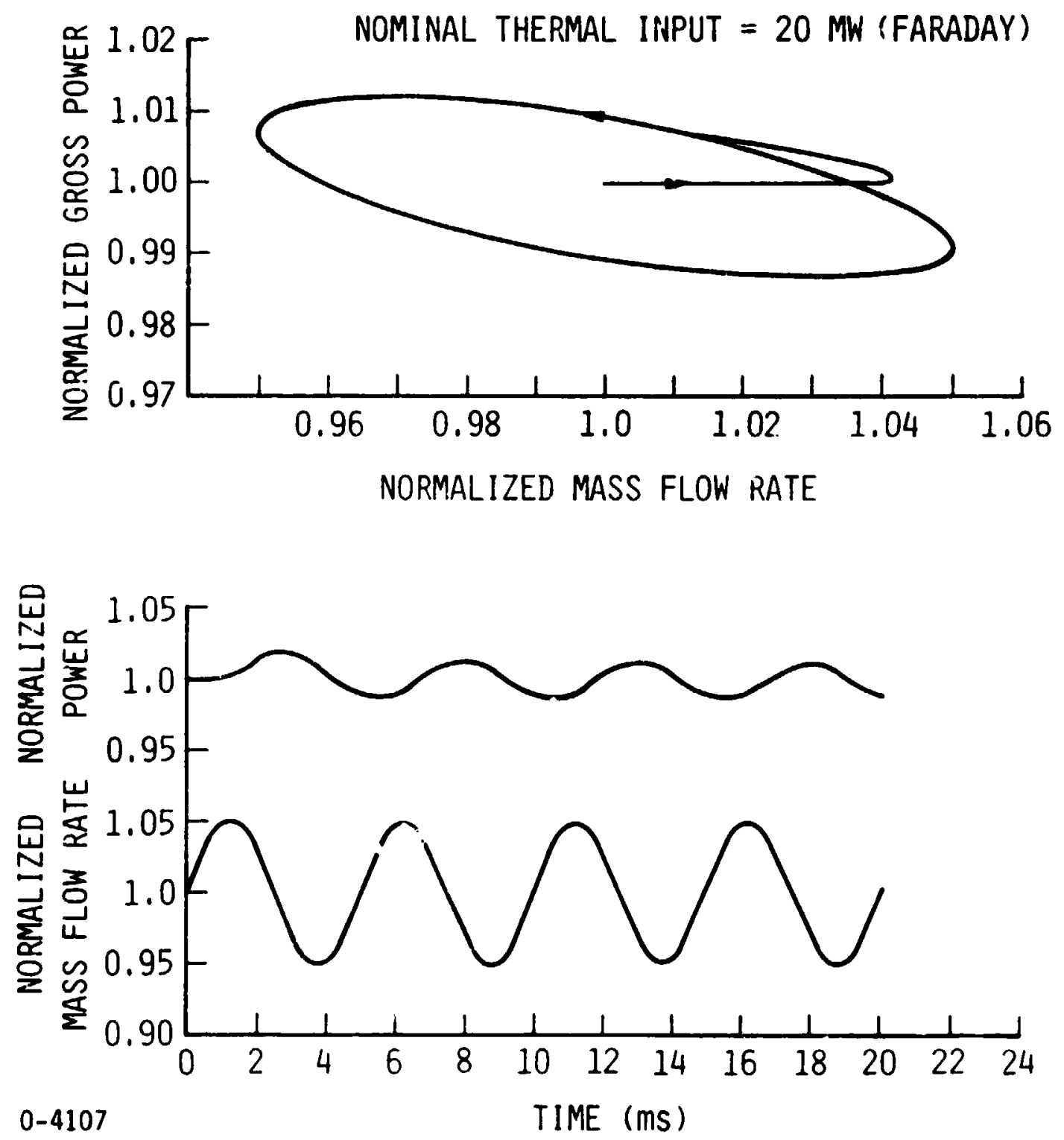

Fig. 7-28. Normalized gross power and normalized mass flow rate for an inputted $\pm 5 \%$ sine wave in mass flow for a $20 \mathrm{MWth}$ channel with Faraday connection. 
STD aESEAaCm COAponarion
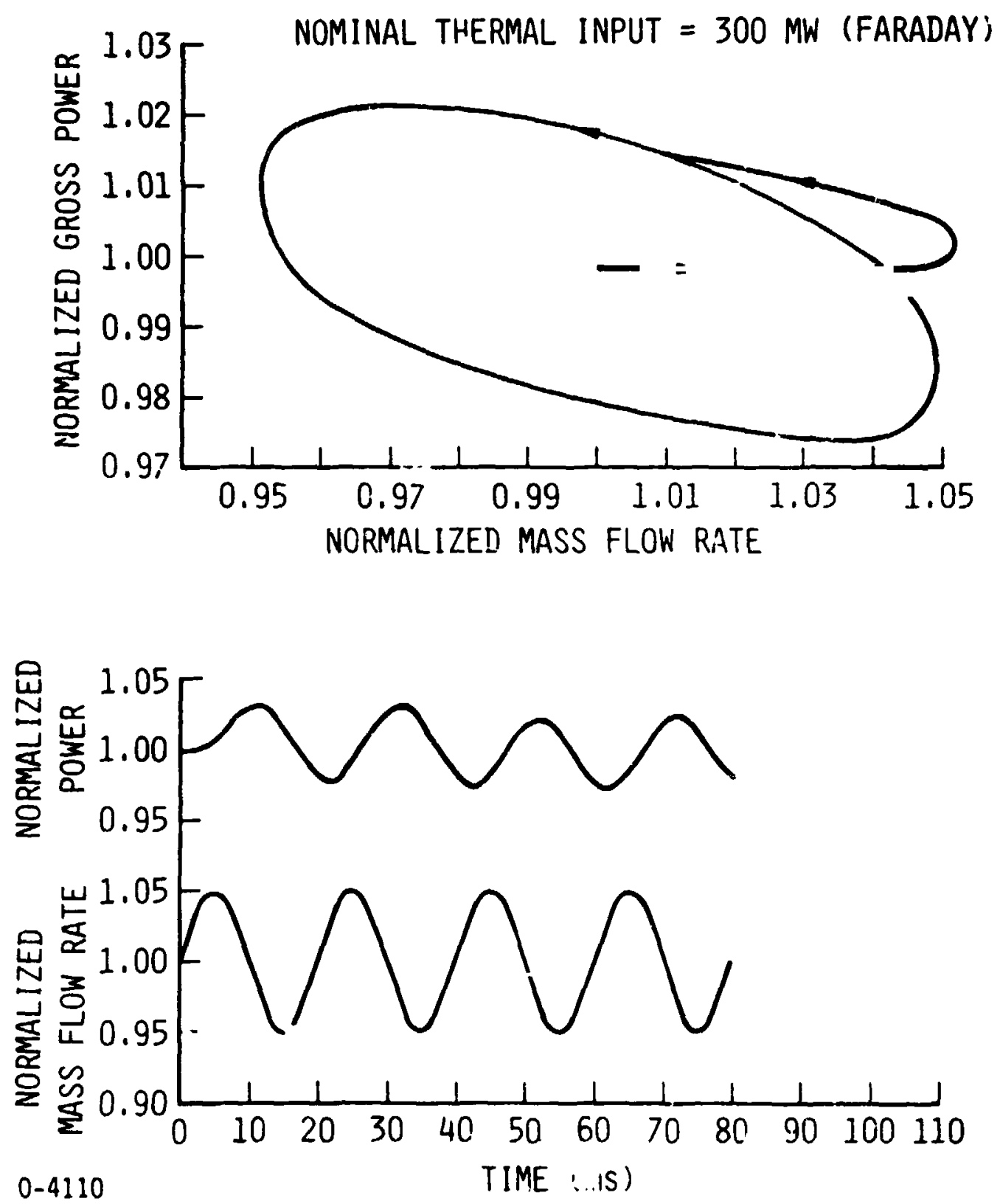

Fig. 7-29. Normalized gross power and norinalized mass flow rate for an inputted $\pm 5 \%$ sine wave in mass flow for a 300 wwth channel with Faraday connection. 


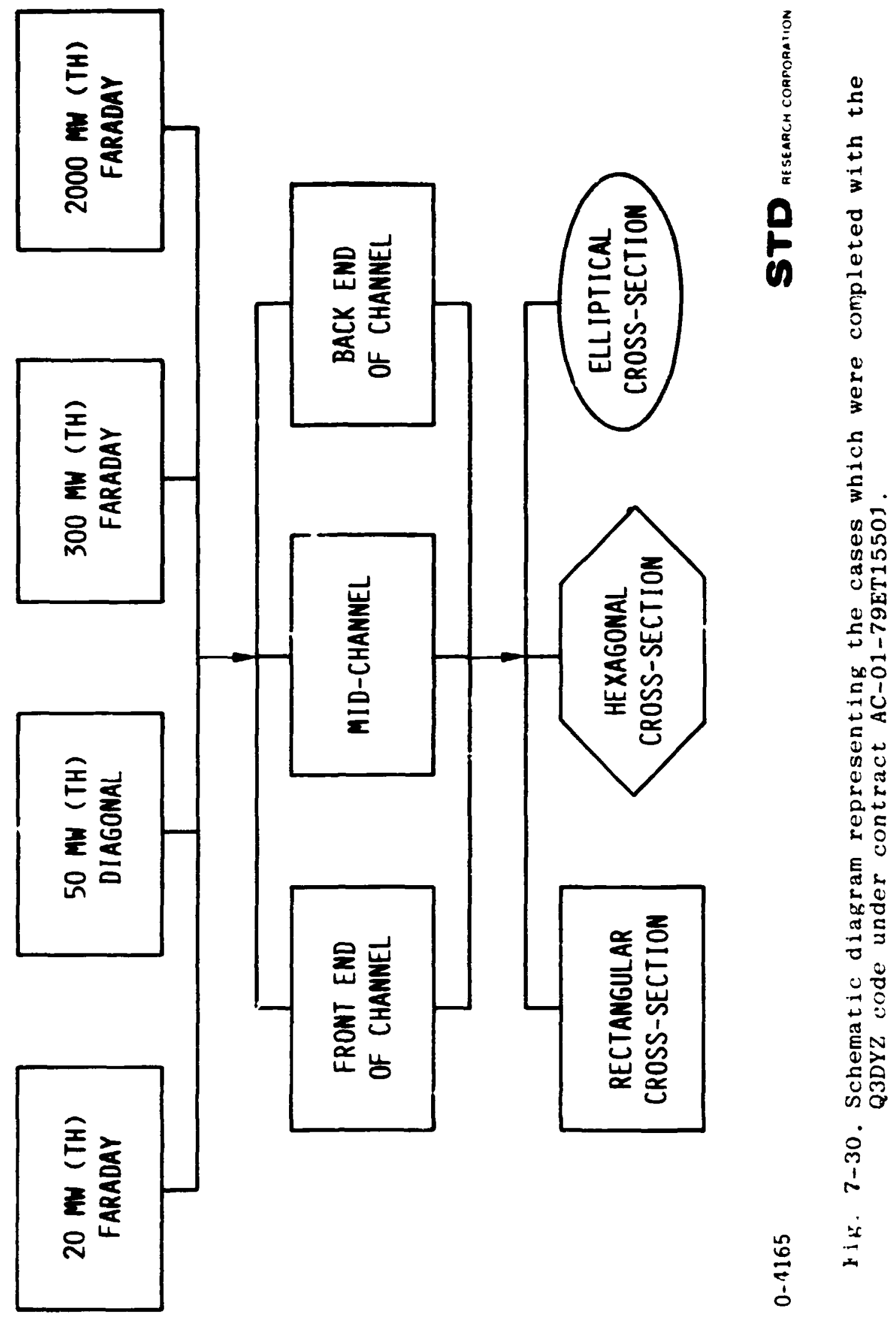




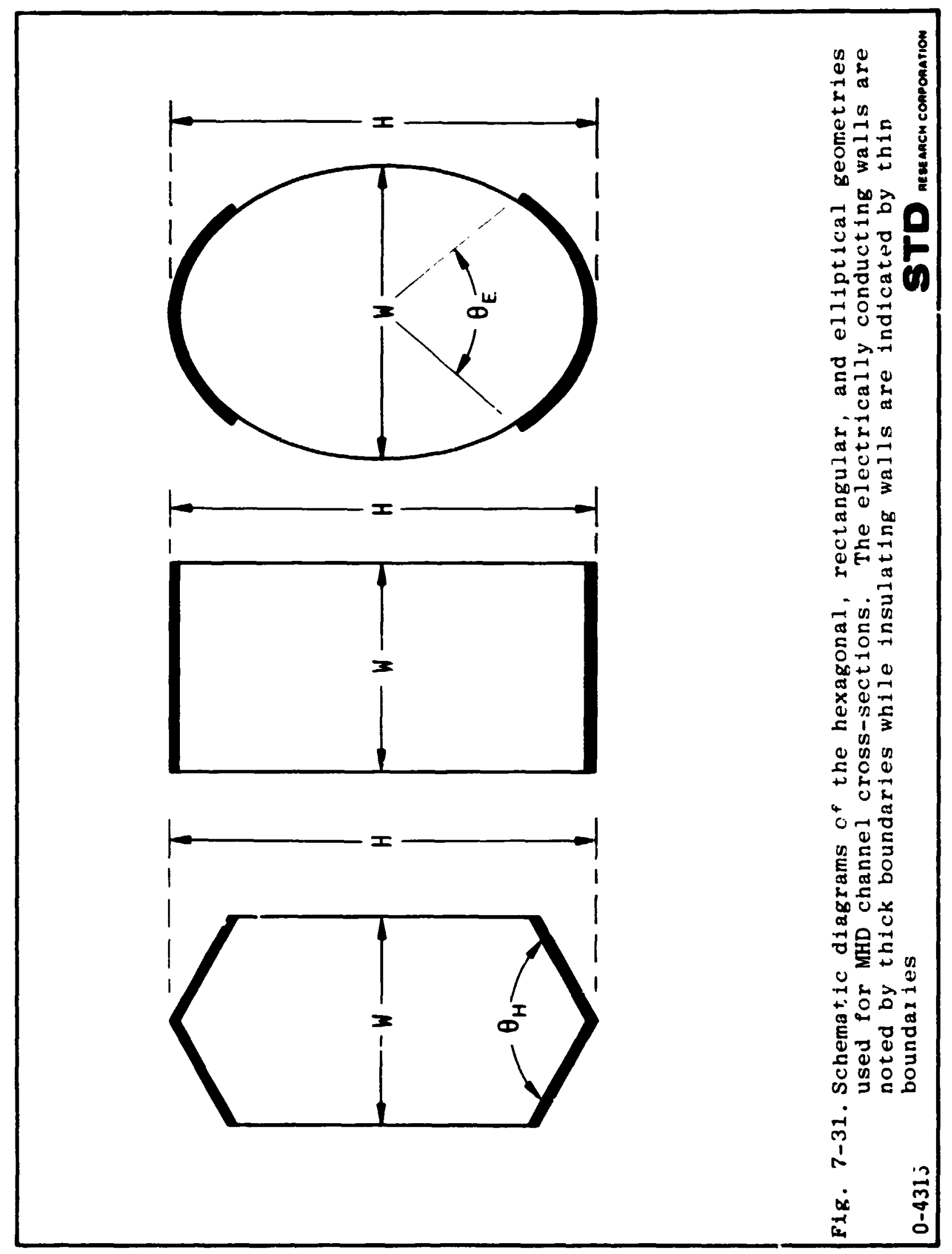




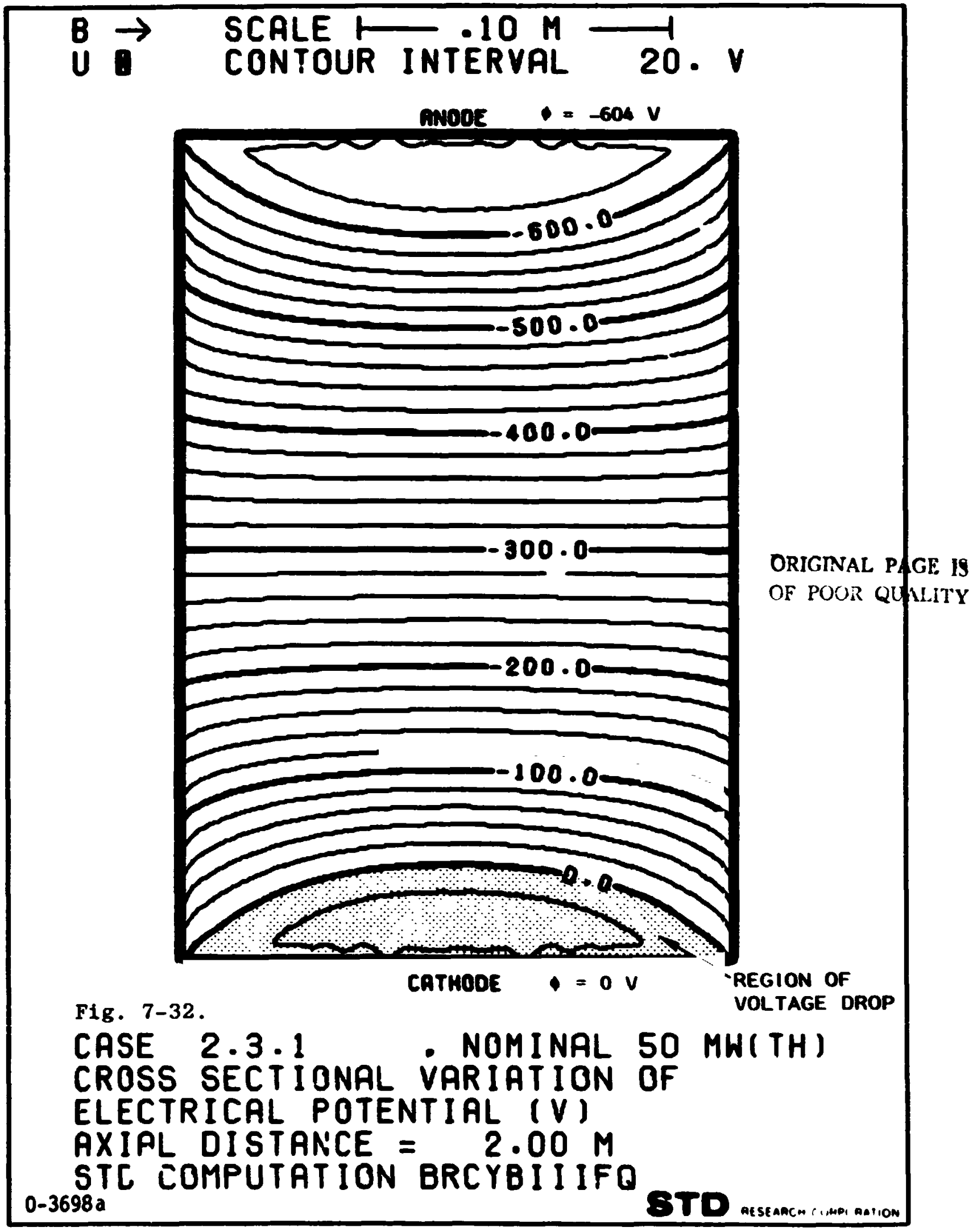




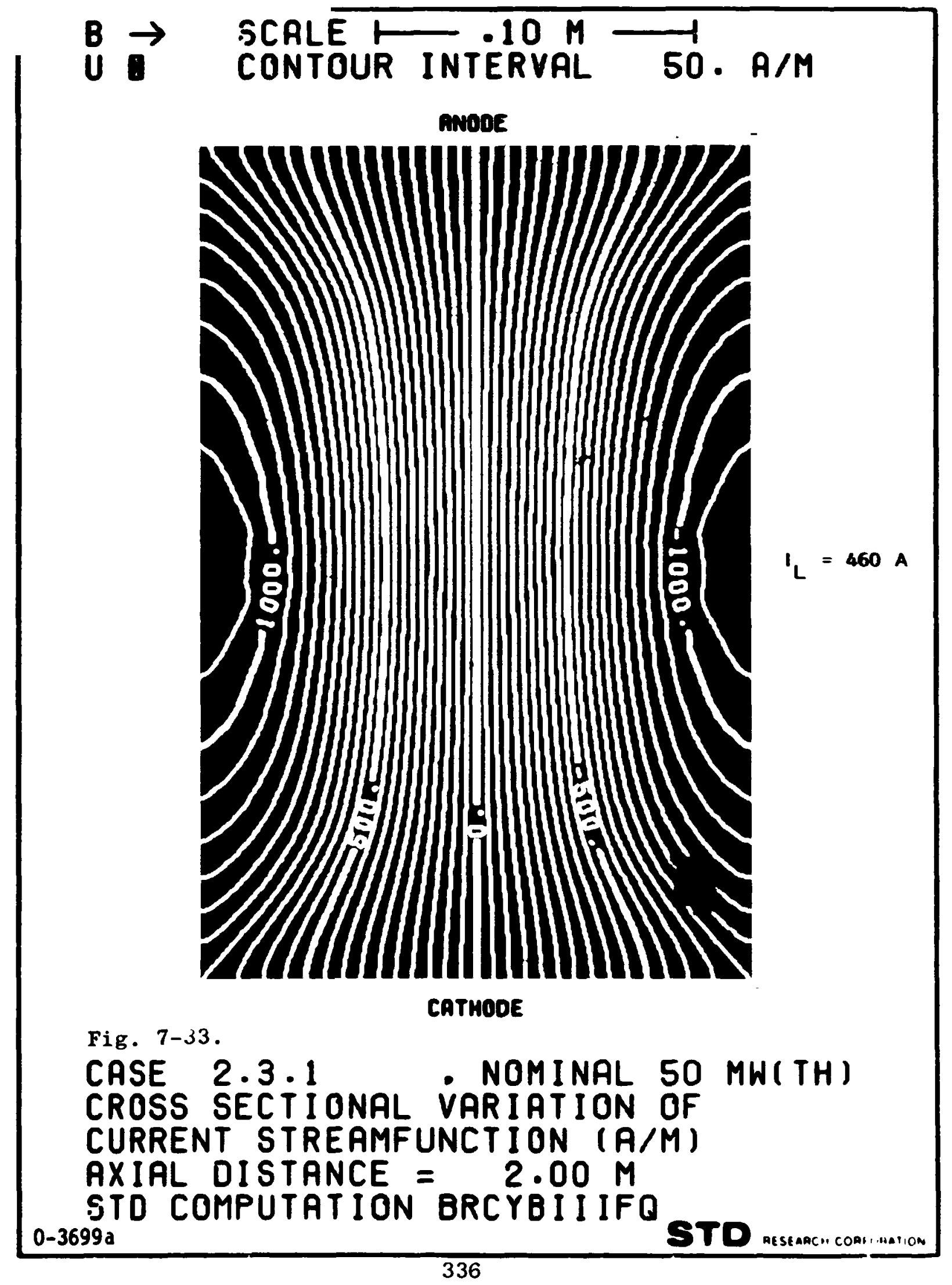




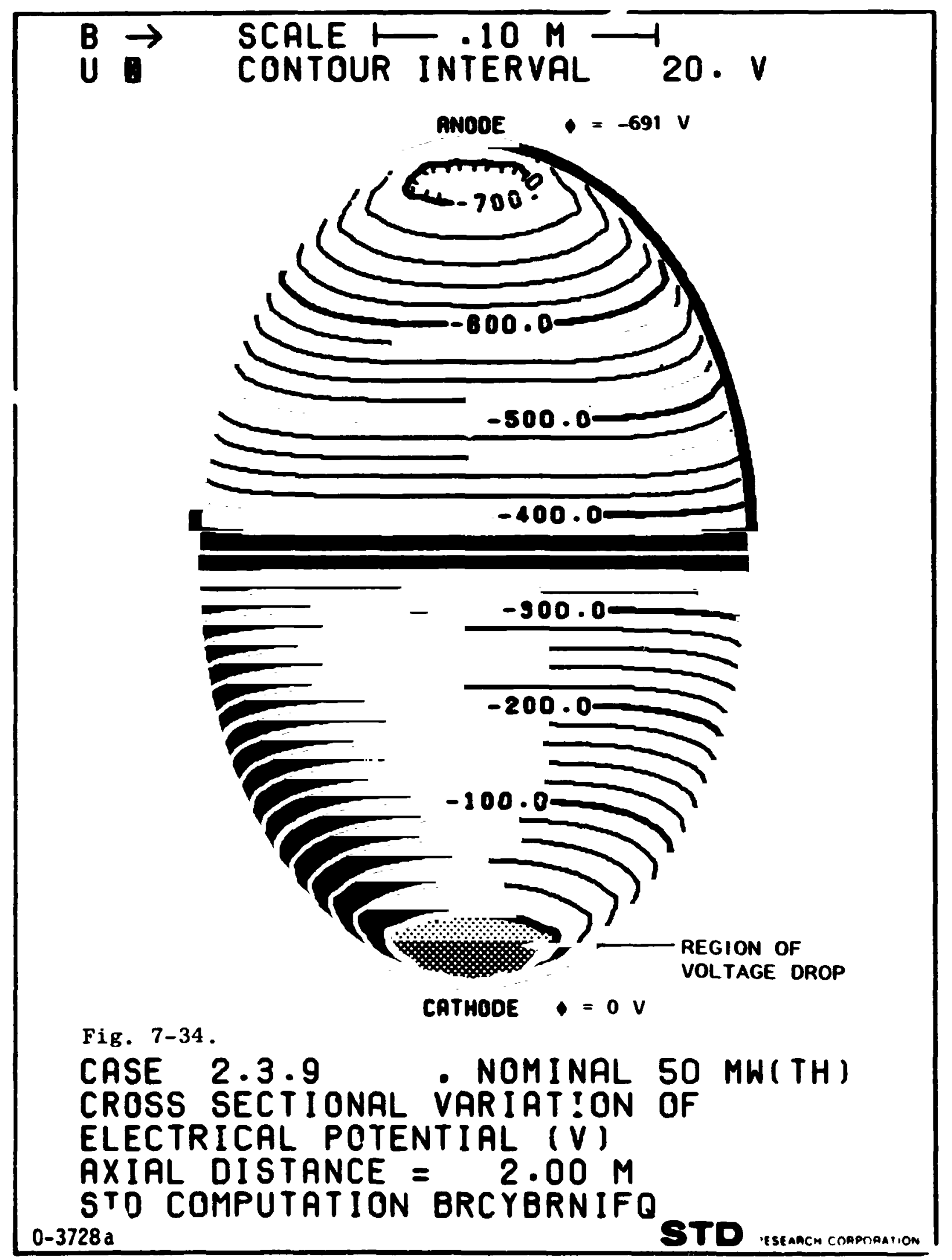




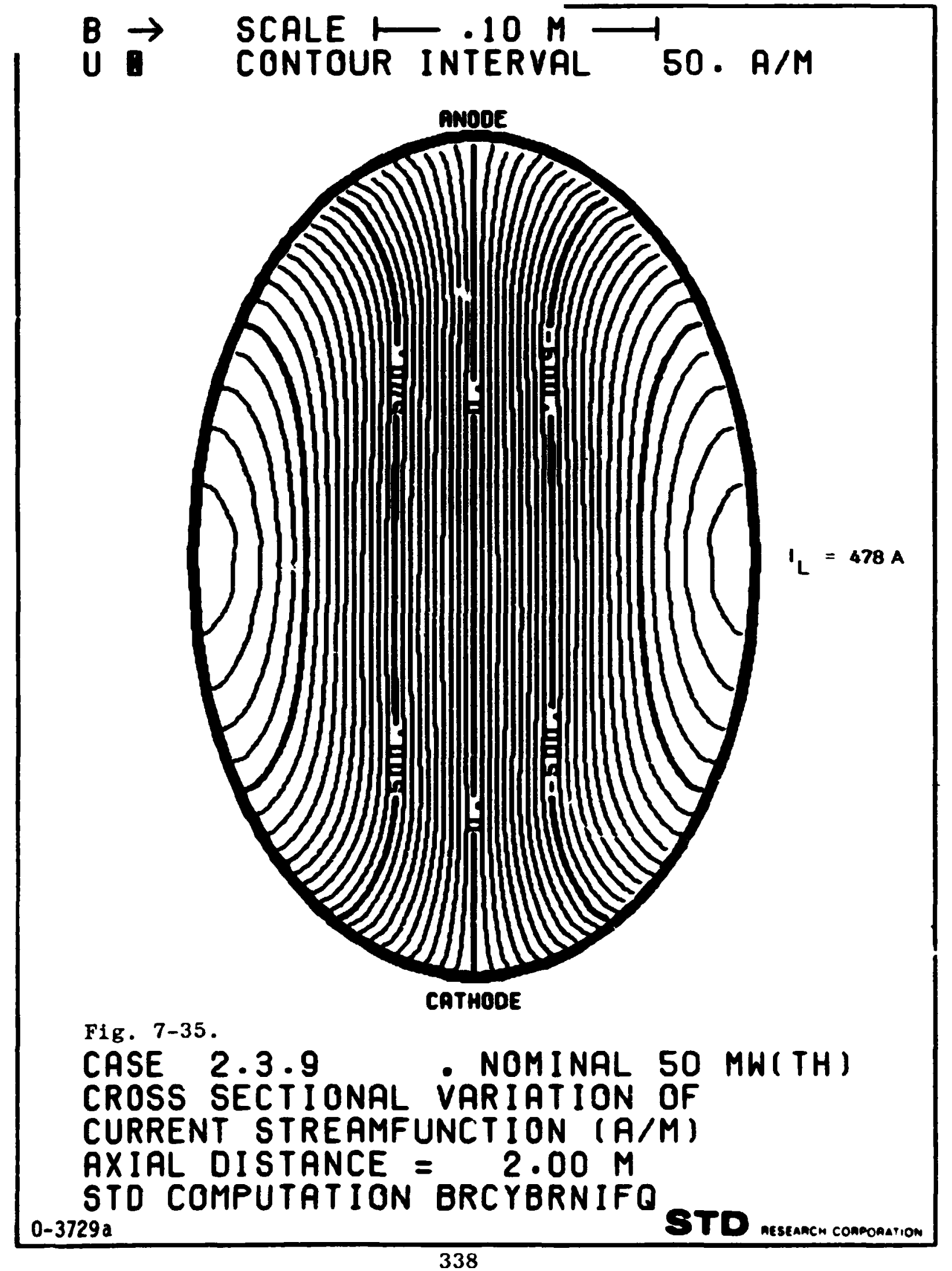




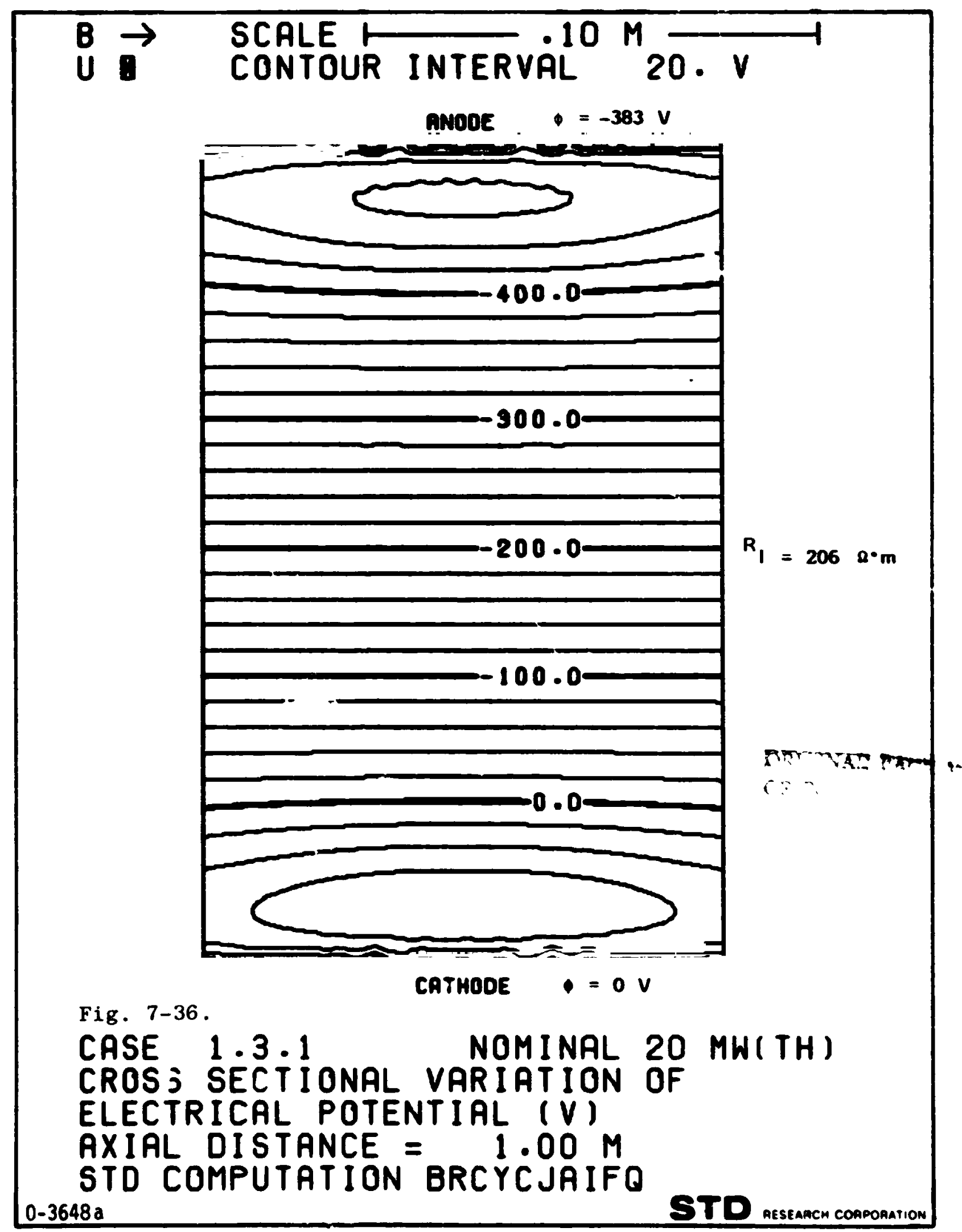




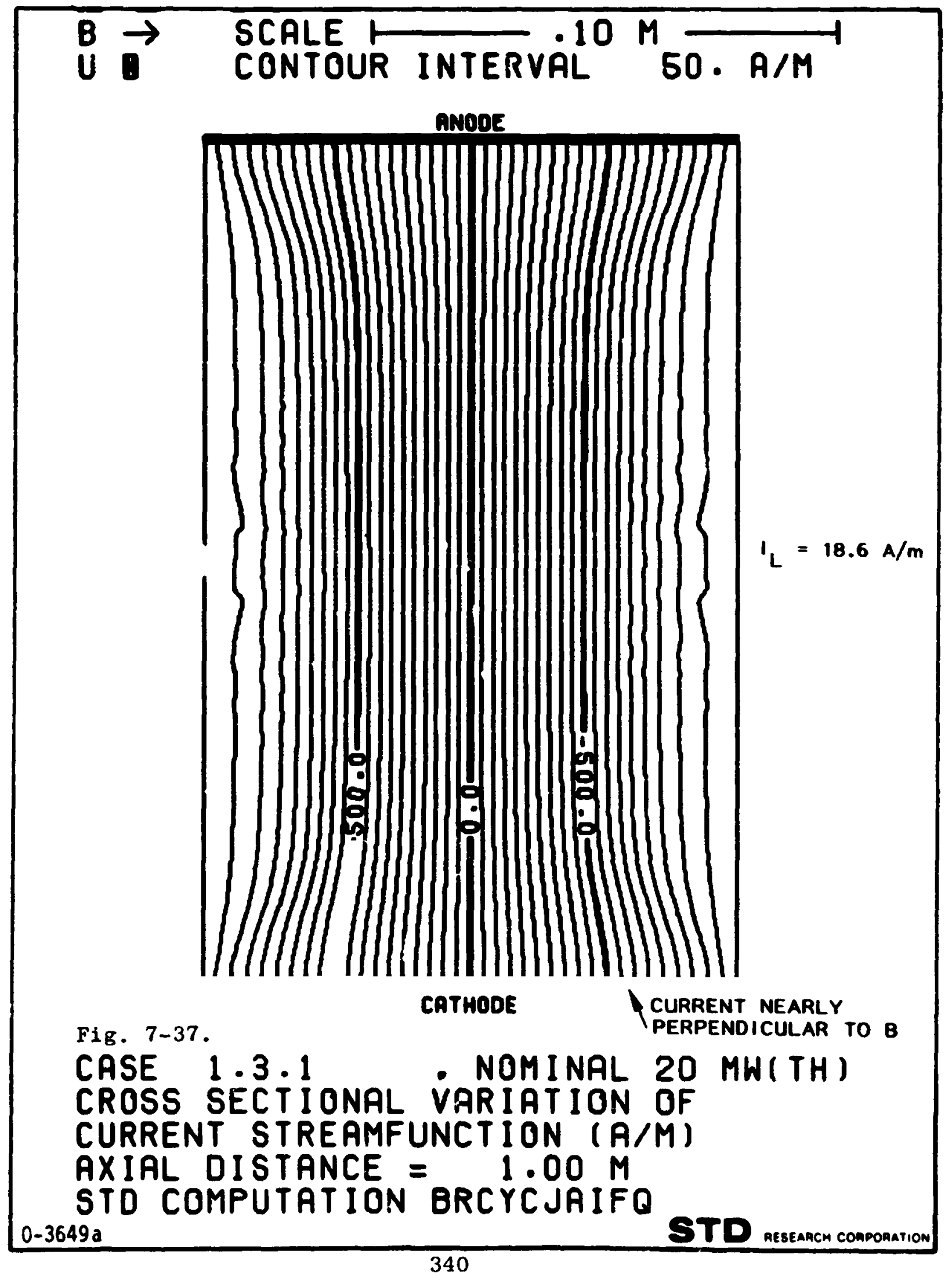




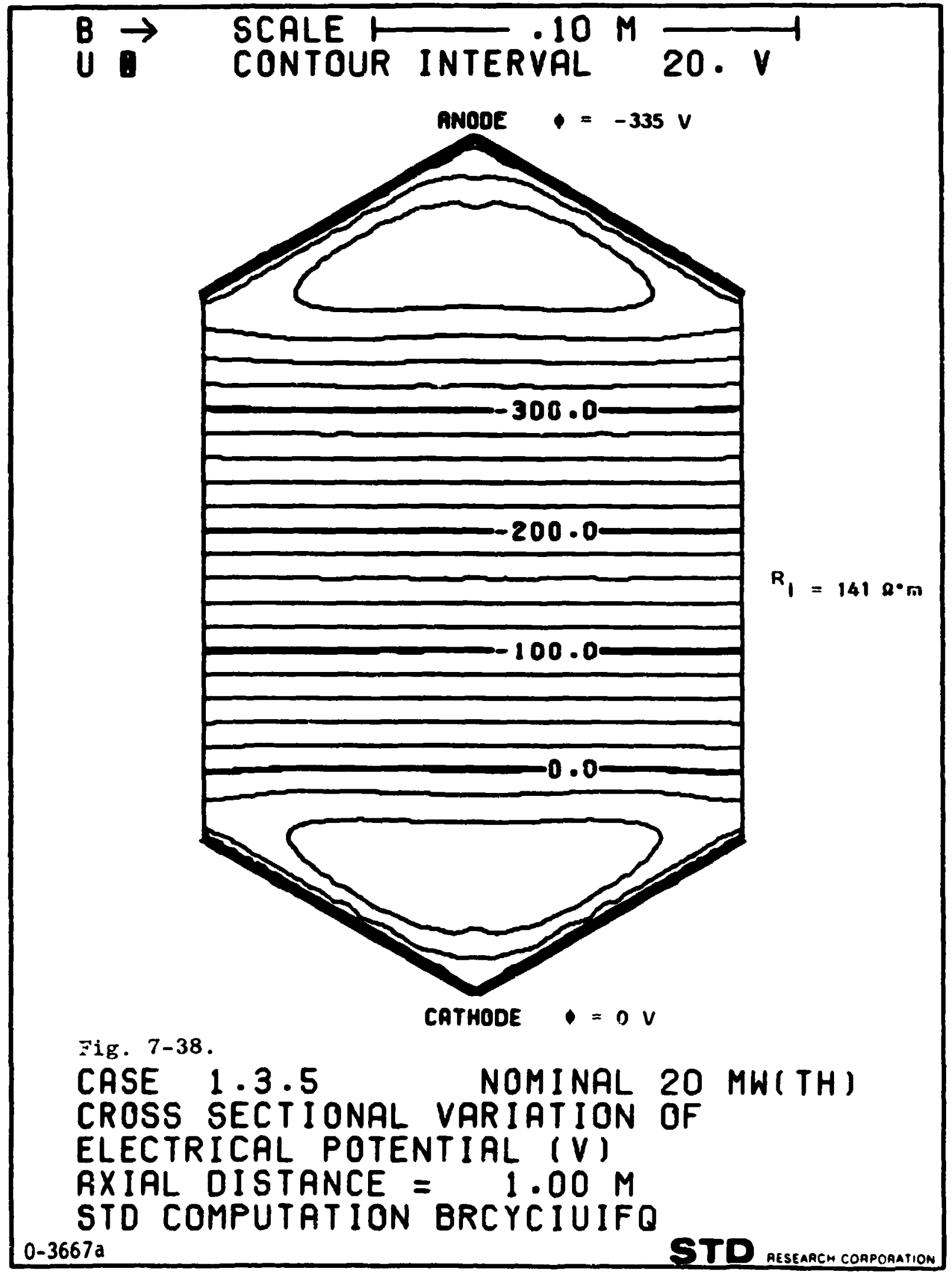




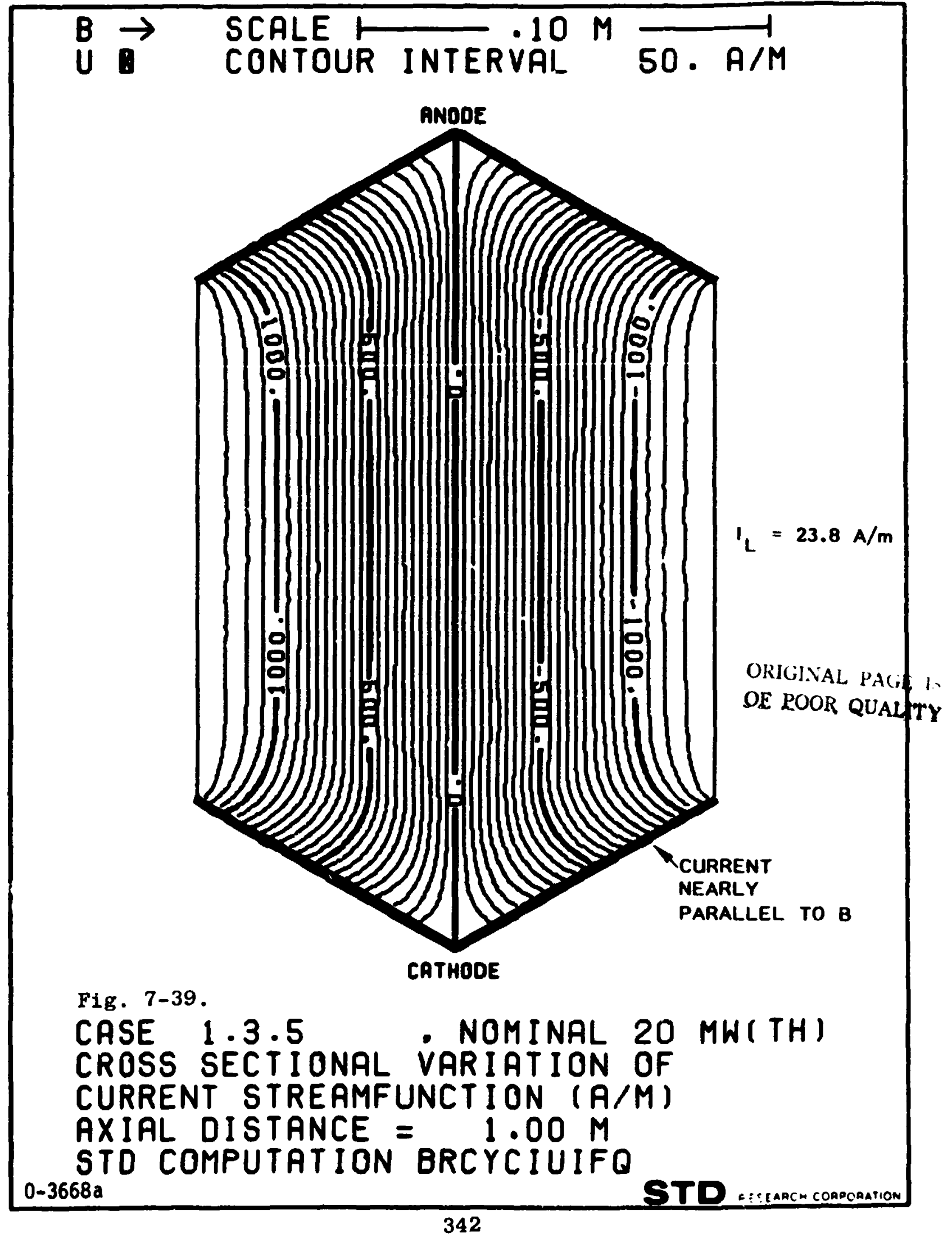


8.0 SUMMARY AND CONCLUSIONS

This section summarizes the major conclusions derived from the studies described in this report.

\subsection{AEDC/HPDE Data Analysis and Interpretation}

Attention has been concentrated on the data from two power producing runs of the AEDC/HPDE (Runs 006-008 and 006-014). Calculations have been performed with codes from the Q3D and TRANSIENT families in order to compare theory and experiment where possible.

The comparison of theory and experiment for Run 006-008 is incomplete because the experimental data were inadequate to accurately define the conditions that existed during the operation of that run. Nevertheless, tre qualitative agreement is sufficiently good to presume that if the Hall voltage across the channel were known, the discharge current cculd have been accurately cietermined and the comparison would have been excellent.

The comparison between theory and experiment was much better for Run 006-014. The data from the two recording systems were much more consistent for this run and are more reliable. On the whole, the predictions are well within the scatter for the data.

\subsection{Critical Phenomena in the AEDC/HPDE}

The power aissipation in the thermal boundary layers of the HPDE is much more important than was originally assumed in the design of the HPDE. However, the enthalpy extraction 
goal of the experiment can be met without providing the orlginal design values of electrode wall temperatures $(2100 \mathrm{~K}$ $1950 \mathrm{~K})$. On the basis of the calculations performed to-date, provision of a uniform $1500 \mathrm{~K}$ wall temperature would be sufficient to meet the enthalpy extraction goal of the experiment.

Under the operating conditions of Run 006-014, which represents the best-diagnosed, high-power run of the HPDE to date, the principal operational parameters available to increase the HPDE performance toward the goals of the experiment are the electrode surface timperature and magnetic field.

The central role of the Mach number distribution in achieving the performance goals of the experiment is demonstrated by the calculations performed to date. The optimum Mach number distribution in the AEDC/HPDE appears tc be transonic.

Finite electrode and near electrode effects appear to have a four to five percent impact on the performance of the MHD generator under the operating conditions of Run 006-014. The experimental data therefore are perhaps not sufficiently refined to discriminate between the differences calculated with some models for near electrode effects (small arcs vs. diffuse mode transport), and electrode segmentation. The data to date do appear to rule out the existence of very strong arcs and suggest that the severe losses associated with seed condensation on the elect. Jdes and seed depletion in the boundary layers are not controlling mechanisms. 
The AEDC/HPDE computations performed to date ildicate that the fluid mechanical behavior of the generator is approaching the interesting regime of zero blockage and measurabie sidewall velocity oversnoots. The data thus far arr not sufficiently complete to detect these effects. Howeve: the general agreement with the pressure predictions and the other electrical and gasdynamic predictions with the multidimensional $Q 3 D$ calculations suggests that the inclusion of these effects in the computations may be necessary. As the performance of the MHD generator improves, and the interaction level increases, the differences between simplified ana sophisticated models of the flow field will become more and more apparent. The AEDC/HPDE should be arforded additional instrumentation with which to diagnose the local fluid phenomena which are predicted by the analyses.

The unconventional fluid behavior will be magnified when the generator is operated in the subsonic mode. It would be possible, under conditions similar to HPDE Run 006-014, to achieve an interaction parameter based on velocity sufficient to exhibit many of the secondary flow and velocity overshoot effects predicted by the STD/MHD codes, if the mass flow rate were reduced sufficiently to provide subsonic flow throughout the generator. While this may not be the optimum enthalpy extraction and isentropic efficiency point, it would be possible, neverthess, to simulate conditions in a large, subsonic, baseload MHD generator, if the appropriate interaction parameter based on velocity is provided.

The inlet pressure loss was calculated fo one operating condition to be approximately $1.5 \%$ of the total pressure entering the generator. Such a pressure loss in the inlet and exit regions of the generator would not present a 
substantial penalty to the overall performance of an MHD plant operating with an MHD generator of the type represented by the AEDC/HPDE. The exit region pressure loss remains to be evaluated.

The electrode voltage drops are sensitive not only to the wall temperature but also to the amount of Joule dissipation heat deposited in the boundary layers. Second order improvement in performance may be achieved by tailoring the load factor distribut on to achieve high dissipation in the inlet regions of the generator. Analytical models which fail to account for Joul": dissipation effects in the electrode boundary layers will fail to predict the electrode voltage drops with accuracy.

\subsection{Critical Phenomena in the U.S. U-25 Experiment}

The nominal conditions of the U.S. U-25 are predicted io yield transonic behavior with a shock located in the channel. Even under these nonideal conditions, the U.S. U-25 experiment is predicted to be able to reach or come very close $t_{\text {, }}$ the goal of $10 \mathrm{MW}$ power output. The operation of this experiment can be altered by changing the mass flow rate, but simultaneous attainment of both fully subsoric $f l o w$ and at least $10 \mathrm{MW}$ power output was not predicted under the nominal operat,ng conditions.

\subsection{Electrical Conductivity Calculations i.. Coal Flames}

The calculation of electrical conductivity of the products of coal combustion is a subject of considerable uncertainty due to the lack of sufficiently accurate compositional and thermochemical data. Even for equilirien 
calcucations, the elemental composition of the products of combustion is dependent upon history of the combustion, particularly f.r the treaiment of ash and slag. In addition, for MHD generators the nonequilibrium effects of boundary layers, current transport, radiation transport, condensation, ind chemical kanetics must be taken into account.

Even when the coal crmposition is definet (defined for computational purposes), the values of the transport and thermodynamic soperties at the inlei to the generators is dependent upon the condicions in the combustor, separator, throat, nozzle, and mating sections of the flow train. It is particularly lmportant to know the distributions of heat flux and slay removal in these sections of the flow train in order to define the inlet con'llions.

For examp's. .ere are many ways of modeling slag removal: (a) all of the liquici and solid slag species can be removed at the flame temperature, ( $b$; the slag that is removed can be assumed + .. have the same composition as for the ash analysis; (c) the condensed phase slag can be renoved at the equiliorium composition of the gas-phase, condensed-phase interface temperature; or (d) any combination of these, i.e., some slag removed $a t$ one condition and some removed at another. since condensed phase matter is removed to the walls contiruously along each section of the flow train, the model of slag emoval must be zpplied as a function of axial distance ald for conditions which are fuactions of location in the flow $\operatorname{train}$. 


\begin{tabular}{|c|c|c|}
\hline $\begin{array}{l}\text { 1. REPOCI NO. } \\
\text { NASA CR-165143 }\end{array}$ & 2. GOVERMMENT ACCESSION MO. & 3. RELIPIENT'S IATLLZN \\
\hline \multirow{2}{*}{\multicolumn{2}{|c|}{$\begin{array}{l}\text { TITLE AMO JUBTILE } \\
\text { ANALYTICAL INVESTIGATION OF CRITICAL } \\
\text { PHENOMENA IN MHD POWER GENERATORS }\end{array}$}} & 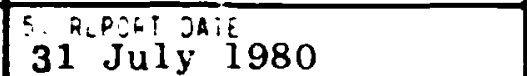 \\
\hline & & 6. PERFORMING ORJAMIZAT:JN \\
\hline \multicolumn{2}{|l|}{ 7. RUTHOR(S) } & $\begin{array}{l}\text { 8. PEAFORMIMG ORG. } \text { REFSF: } M_{i} \text {. } \\
\text { STDR-80-2द }\end{array}$ \\
\hline \multirow{2}{*}{\multicolumn{2}{|c|}{$\begin{array}{l}\text { 9. PERFORMIMG ORGAMIZATIOM MAME AMD ADDRESS } \\
\text { STD Research Corporation } \\
\text { P.O. BOX "C" } \\
\text { Arcadia, CA } 91006\end{array}$}} & 10. MJAK UNIT NJ. \\
\hline & & $\begin{array}{l}\text { 11. CONIRACT OF jFA4 } \\
\text { DEN3-179 }\end{array}$ \\
\hline \multirow{2}{*}{\multicolumn{2}{|c|}{$\begin{array}{l}\text { i2. SPQMSORMG "Fincr MAME AMp ApOCESS } \\
\text { U.S. Bep. Stment of Fnergy } \\
\text { Office of Magnetohydrodynamics } \\
\text { Washington, D.C. } 20545\end{array}$}} & \\
\hline & & $\begin{array}{l}\text { 16. SPOMSOR:ME AGEMCY FEPOR! MU. } \\
\text { DOE / NASA / 0179-1 }\end{array}$ \\
\hline \multicolumn{3}{|c|}{$\begin{array}{l}\text { 15. SUPPLEMEMTARY MOTES } \\
\text { Final Report. Prepared under Interagency Agreement EC-77-AA-012674. } \\
\text { Project Manager, Bert Phillips, Wind and Stationary Power Division } \\
\text { NASA Lewis Research Cenier, Cleveland, OH } 44133\end{array}$} \\
\hline \multicolumn{3}{|c|}{$\begin{array}{l}\text { 16. ABSTRACI } \\
\text { Critical phenomena in the Arnold Engineering Development Center } \\
\text { (AEDC) High Performance Demonstration Fxperiment (HPDE) and the } \\
\text { U.S. U-25 Experiment, are analyzed. Also analyzed are the } \\
\text { performance of a NASA-specified } 500 \text { NW(th) flow train and computa- } \\
\text { tions carried nut by STD Research under Contract AC-01-79ET15501 } \\
\text { concerning critical issues for the seale-up of MHD generators. } \\
\text { Critical phenomena analyzed inc ude Hall roltage overshoots, optima } \\
\text { load schedules, parametric dependence of the electrode voltage } \\
\text { drop:, boundary layer behavior, near electrode phenomena with } \\
\text { finite electrode segmentation, current distribution in the end } \\
\text { regions, scaie-up rules, optimum Mach number distribution, and the } \\
\text { eifects of alternative cross-sectional shapes. }\end{array}$} \\
\hline \multicolumn{3}{|c|}{\begin{tabular}{l|l} 
17. KEY MOROS (SUGGESTEO OY AUTHORIS:" & 18. OISTRIBUTIOM STATEMET \\
Magnetohydiodynamics & Lnclassified - Enlimited \\
& Star Categrry 44 \\
& DOE Category LC-90g
\end{tabular}} \\
\hline 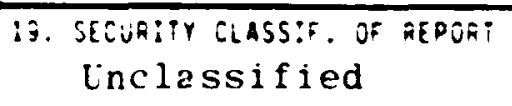 & $\begin{array}{l}\text { 20. Secinity classif. of iHis paus } \\
\text { linclassif i ed }\end{array}$ & \begin{tabular}{|c|c|c|} 
A. MO. OF PF. & $\therefore$ PQ:iS \\
\end{tabular} \\
\hline
\end{tabular}

